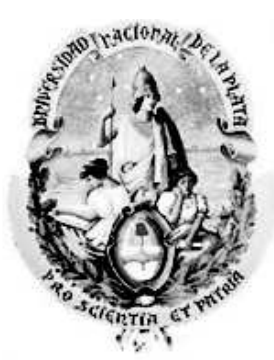

Universidad Nacional de La Plata

Facultad de Ciencias Exactas

Departamento de Matemática

\title{
Estimaciones a priori y a posteriori del error para problemas de autovalores
}

Tesis presentada para optar al título de

Doctor de la Facultad de Ciencias Exactas - Área Matemática

Anahí Dello Russo

Autor

Ricardo G. Durán

Director 

a Tomás, Bibiana, Néstor, Hilda y Fabián, mi familia 


\section{Agradecimientos}

Al Dr. Ricardo Durán por su colaboración generosa y su apoyo constante; sin ellos este trabajo no hubiera sido posible.

A la Dra. María Amelia Muschietti por su amable disponibilidad ante cada una de mis consultas.

A la Dra. Ana Alonso por la dedicación con la que leyó todas las páginas que forman parte de este trabajo y por sus valiosos comentarios.

A los Dres. José Luis Castiglioni, Marcela Zuccalli y Marisa Gutierrez por las gestiones que realizaron desde la Comisión de Grados Académicos.

Al Departamento de Matemática por brindarme un lugar de trabajo desde hace ya tantos años. 


\section{Índice general}

1. Introducción $\quad 1$

1.1. Convergencia y estimaciones del error . . . . . . . . . . . . . . . . . . . . 1

1.1.1. Problemas planteados en dominios poligonales . . . . . . . . . . . . . . . . . . 1

1.1.2. Problemas planteados en dominios con bordes curvos . . . . . . . . . . . . . . . . . 4 4

1.1.3. Problemas aproximados por métodos mixtos . . . . . . . . . . . . . . 5 5

1.1.4. Resultados obtenidos . . . . . . . . . . . . . . . . . . . . . 5

1.2. Análisis a posteriori del error . . . . . . . . . . . . . . . . . 8

1.2.1. Problemas de autovalores - formulación variacional clásica . . . . . . . . . . . . . . . 9 9

1.2.2. Problemas de autovalores - formulación variacional mixta . . . . . . . . . . . . . 10

1.3. Otros comentarios . . . . . . . . . . . . . . . . . . . . . . 12

2. Preliminares 13

2.1. Operadores acotados en espacios de Hilbert . . . . . . . . . . . . . . . . . . . . 13

2.2. Propiedades espectrales de un operador lineal y acotado . . . . . . . . . . . . . . . . 15

2.3. El gap entre subespacios invariantes . . . . . . . . . . . . . . . . . . . . . 18

2.4. Principios variacionales ........................... . . 19

3. Aproximación espectral no conforme para operadores acotados 21

3.1. Problemas de autovalores formulados variacionalmente . . . . . . . . . . . . . . . . 21

3.2. Definición de los operadores solución . . . . . . . . . . . . . . . . . . . 23

3.3. Resultados abstractos sobre aproximación espectral . . . . . . . . . . . . . . . . . 25

3.3.1. Convergencia de las autofunciones y de los subespacios invariantes . . . . . . . 27

3.3.2. Convergencia de las autovalores . . . . . . . . . . . . . . . . . 30

3.3.3. Estimaciones del error para las autofunciones en la norma $\|\cdot\|_{h} \ldots \ldots$. . . . . . 31

3.3.4. Estimaciones del error para los autovalores . . . . . . . . . . . . . . . 37

3.3.5. Convergencia y estimaciones del error para las autofunciones en la norma $|\cdot|_{X(\widehat{\Omega})}$. 42

3.4. Problemas de autovalores en forma mixta . . . . . . . . . . . . . . . . . . . . . . . . . . . . . . . .

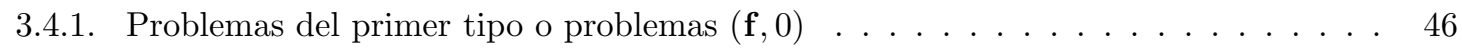

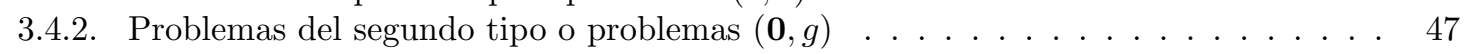

3.5. Problemas de autovalores no simétricos . . . . . . . . . . . . . . . . . . . 55

3.5.1. El problema de autovalores dual . . . . . . . . . . . . . . . . . . . . . . . . . . . . . . . . . 55

3.5.2. Discretización de los problemas . . . . . . . . . . . . . . . . . . 56

3.5.3. Estimaciones del error . . . . . . . . . . . . . . . . . . 57

4. Aplicaciones $\quad \mathbf{6 1}$

4.1. Dominios poligonales y espacios discretos no-conformes . . . . . . . . . . . . . . . 61

4.1.1. El problema de autovalores de Steklov . . . . . . . . . . . . . . . . . . . 62

4.1.2. Vibraciones en un medio elástico . . . . . . . . . . . . . . . . . . . . 72

4.2. Dominios curvos y espacios discretos conformes . . . . . . . . . . . . . . . . . . 87

4.2.1. Vibraciones en un sistema acoplado fluido-estructura . . . . . . . . . . . . . 89

4.3. Dominios curvos y espacios discretos no conformes . . . . . . . . . . . . . . . . . . . . . 110

4.3.1. El problema de autovalores de Laplace con condiciones de borde de Neumann . . . 110 
5.1. Preliminares . . . . . . . . . . . . . . . . . . . . . 126

5.2. El problema de autovalores de Steklov . . . . . . . . . . . . . . . . . . . . . 128

5.2.1. Indicadores locales del error. Confiabilidad de las estimaciones del error . . . . . . 129

5.2.2. Eficiencia de los indicadores de error . . . . . . . . . . . . . . . . . . . 131

5.2.3. Otros dos estimadores locales del error . . . . . . . . . . . . . . . . . . . 135

5.3. El problema de las vibraciones en un medio elástico . . . . . . . . . . . . . . . . . . . 139

5.3.1. Normas discretas . . . . . . . . . . . . . . . . . . . . . . . . 139

5.3.2. Método de post-procesamiento . . . . . . . . . . . . . . . . . . . 140

5.3.3. Indicadores locales del error para los tensores . . . . . . . . . . . . . . . . . . . . 141

5.3.4. Indicadores locales del error para los desplazamientos . . . . . . . . . . . . . . . 146

5.3.5. Estimador del error para los pares tensor/desplazamiento. Confiabilidad y eficiencia de los indicadores del error. . . . . . . . . . . . . . . . . 146

$\begin{array}{lr}\text { Apéndice } & 147\end{array}$

$\begin{array}{lr}\text { A. Resultados sobre la aproximación por elementos finitos. } & 147\end{array}$

A.1. Interpolaciones clásicas . . . . . . . . . . . . . . . . . . . . . . . . . . 147

A.2. Interpolación de Arnold-Winther . . . . . . . . . . . . . . . . . . . . . . . . . 149

A.3. Proyecciones $L^{2}$-ortogonales . . . . . . . . . . . . . . . . . . . . . . . . . . . . . . 150

A.4. Desigualdades de traza e inversa . . . . . . . . . . . . . . . . . . . . . 151

B. Cambios en la normalización de los autovectores y orden de convergencia de la aproximación.

C. Otras estimaciones a priori para el método de Arnold-Winther 153

C.1. Convergencia del método en la norma de la energía . . . . . . . . . . . . . . . . . . . 153

C.2. Estimaciones del error para la solución post-procesada . . . . . . . . . . . . . . . 156

$\begin{array}{lr}\text { D. Estimaciones básicas en dominios curvos } & 163\end{array}$

E. Propiedades de aproximación y compacidad discreta para problemas con interacción $\begin{array}{ll}\text { fluido-estructura } & 167\end{array}$

E.1. Descomposición de Helmhotz . . . . . . . . . . . . . . . . . . . . . . . . . . 167

E.2. Teorema de trazas para espacios de Sobolev definidos en $\Omega_{h}$. . . . . . . . . . . . . . . . . 168

E.3. Potencial escalar en $\widehat{\Omega}_{\mathrm{F}} \ldots \ldots \ldots \ldots \ldots \ldots \ldots \ldots$. . . . . . . . . . . . . . . . 174

E.4. Operadores de interpolación . . . . . . . . . . . . . . . . . . . . . . 177

E.5. Propiedad de compacidad discreta . . . . . . . . . . . . . . . . . . . . 179

E.6. Otras estimaciones . . . . . . . . . . . . . . . . . . . . . . . . . 184

$\begin{array}{lr}\text { Bibliografía } & 189\end{array}$ 
Although this may seem a paradox, all exact science is dominated by the idea of approximation.

Bertrand Russell in The Scientific Outlook, 1931. 


\section{Introducción}

Los problemas de autovalores juegan un rol central en numerosas áreas de la ciencia y de la ingeniería; aparecen frecuentemente en la dinámica de estructuras elásticas, acústica, electromagnetismo, mecánica cuántica, mecánica de fluidos, teoría de procesos aleatorios y biofísica. En la mayoría de las aplicaciones, el conocimiento de los autovalores permite, por ejemplo, estudiar la establidad de un sistema (físico/químico cuántico/biológico) en las inmediaciones de sus estados de equilibrio. La cuestión se vuelve crucial en el contexto de la mecánica cuántica (relativista o no-relativista) ya que el entendimiento de la mayoría de los fenómenos cuánticos depende fuertemente del conocimiento del espectro de ciertos operadores lineales.

Desde el punto de vista puramente matemático, la teoría espectral es también una fuente de problemas fascinantes. Varios de ellos están todavía sin resolver; en particular, algunas cuestiones relacionadas con el comportamiento de los autovalores cuando se producen variaciones en la geometría del dominio.

Para operadores con rango de dimensión infinita, el cálculo exacto de los autovalores y las autofunciones resulta, salvo raras excepciones, extremadamente complejo o directamente imposible.

Por tal motivo, existe una gran variedad de métodos numéricos para abordar este tipo de problemas; por ejemplo, los autovalores asociados a problemas de valores de contorno pueden calcularse aproximadamente utilizando métodos variacionales o diferencias finitas o métodos de interpolación.

Adoptado un determinado método de aproximación, una pregunta que surge naturalmente es la siguiente: cómo se relacionan los objetos espectrales del problema original con los objetos espectrales del problema aproximado?

\subsection{Convergencia y estimaciones del error}

\subsubsection{Problemas planteados en dominios poligonales}

En términos generales, para calcular numéricamente el espectro de un operador lineal $\mathbf{T}$, con dominio y rango en un espacio de Hilbert (separable) $X$, se sustituyen los autovalores y las autofunciones de $\mathbf{T}$ por los autovalores y las autofunciones de otro operador lineal $\mathbf{T}_{h}$, con dominio en $X$ y rango en un espacio de dimensión finita $V_{h}{ }^{1}$. Diremos que $\mathbf{T}_{h}$ es una aproximación de $\mathbf{T}$ si

$$
\mathbf{T}_{h} u \rightarrow \mathbf{T} u \text { para cualquier } \quad u \in X
$$

El espectro de cada operador $\mathbf{T}_{h}$ se obtiene resolviendo un problema matricial de autovalores generalizado. Uno de los objetivos principales de la teoría de aproximación espectral es analizar la convergencia de los valores propios y de los subespacios invariantes de la sucesión $\left\{\mathbf{T}_{h}\right\}_{h>0}$ a los correspondientes elementos de $\mathbf{T}$ a medida que $h \rightarrow 0$.

\footnotetext{
${ }^{1}$ Adoptaremos aquí la notación correspondiente a la versión $h$ del método de los elementos finitos; $h$ será siempre un parámetro positivo que tiende a cero.
} 
Qué características debería tener el espectro de $\mathbf{T}_{h}$ para ser considerado una aproximación correcta del espectro de $\mathbf{T}$ ?

Sea $\lambda$ un autovalor aislado de $\mathbf{T}$ con multiplicidad algebraica finita igual a $m$. Sea $\gamma$ una curva de Jordan alrededor de $\lambda$; elegida de manera tal que su interior no contenga otros puntos pertenecientes al espectro de $\mathbf{T}$. Para $z$ en el conjunto resolvente de $\mathbf{T}$, el operador $R_{z}(\mathbf{T}):=(\mathbf{T}-z \mathbf{I})^{-1}$ define la resolvente de $\mathbf{T}$ en $z$ y el operador

$$
\mathbf{E}_{\lambda}:=-\frac{1}{2 \pi i} \int_{\gamma} R_{z}(\mathbf{T}) d z
$$

es una proyección sobre el subespacio invariante $\mathbf{E}_{\lambda}(X)$, cuya dimensión es igual a $m$.

De la misma manera, para $z$ en el conjunto resolvente de $\mathbf{T}_{h}, R_{z}\left(\mathbf{T}_{h}\right):=\left(\mathbf{T}_{h}-z \mathbf{I}\right)^{-1}$ define al operador resolvente de $\mathbf{T}_{h}$ en $z$. Si $\gamma$ está incluido en el conjunto resolvente de $\mathbf{T}_{h}$,

$$
\mathbf{E}_{\lambda_{h}}:=-\frac{1}{2 \pi i} \int_{\gamma} R_{z}\left(\mathbf{T}_{h}\right) d z
$$

es la proyección espectral asociada con todos los autovalores de $\mathbf{T}_{h}$ (si es que existe alguno) que yacen en el interior de $\gamma$.

El método de aproximación será espectralmente correcto si, para h suficientemente chico,

- cualquier conjunto compacto incluido en el conjunto resolvente de $\mathbf{T}$ también estará incluido en el conjunto resolvente de $\mathbf{T}_{h}$,

- existen exactamente $m$ autovalores $\lambda_{1 h}, \cdots, \lambda_{m h}$ de $\mathbf{T}_{h}$ en el interior de $\gamma$ (repetidos según sus respectivas multiplicidades) y todos convergen a $\lambda$.

La condición (1.1), en general, no es suficiente para garantizar que el método sea espectralmente correcto. Ejemplos de esto pueden encontrarse en [82].

Qué condiciones se deberían agregar?

Siguiendo a Chatelin [85], asumiremos que la aproximación $\mathbf{T}_{h}$ es fuertemente estable; es decir, para $h$ suficientemente chico,

$$
\begin{aligned}
& \text { existe } M>0 \text { tal que }\left\|R_{z}\left(\mathbf{T}_{h}\right)\right\|_{\mathcal{L}(X)} \leq M \quad \text { para } z \in \gamma \\
& \operatorname{dim} \mathbf{E}_{\lambda}(X)=\operatorname{dim} \mathbf{E}_{\lambda_{h}}(X)
\end{aligned}
$$

Entonces $\lambda$ será estable en el sentido de Kato ([124], pag. 437); en consecuencia, $\mathbf{T}_{h}$ será una aproximación espectralmente correcta.

Qué propiedades de la aproximación podrían garantizar estas condiciones?

Cuando $V_{h} \subset X, \mathbf{T}$ es compacto y los operadores $\mathbf{T}_{h}$ convergen a $\mathbf{T}$ uniformemente; es decir,

$$
\left\|\mathbf{T}-\mathbf{T}_{h}\right\|_{\mathcal{L}(X)} \rightarrow 0
$$

$\mathbf{T}_{h}$ resulta estable en una vecindad de cualquier autovalor de $\mathbf{T}$ con preservación de las multiplicidades algebraicas. Más aún, se pueden obtener varios resultados (óptimos) sobre el orden con el que convergen los autovalores y autofunciones aproximados $[29,42]^{2}$. La hipótesis de compacidad es válida para muchos problemas de autovalores de interés; por ejemplo, la mayoría de los sistemas de ecuaciones diferenciales en la teoría lineal de la elasticidad están asociados con operadores que tienen inversas compactas.

\footnotetext{
${ }^{2}$ Las aproximaciones conformes de problemas de autovalores compactos han sido estudiadas en numerosos trabajos: Anselone-Palmer [11], Bramble-Osborn [49], Fix [105], Chatelin [82], Osborn [144], Chatelin-Lemordant [85], Kolata [130], Babǔska-Osborn [28], Beattie [31], Knyazev-Osborn [129], por mencionar solo algunas de las publicaciones más influyentes.
} 
Cuando el operador $\mathbf{T}$ no es compacto, ya no es posible (claramente) asumir propiedades como la (1.2). La no compacidad de $\mathbf{T}$ puede causar serias complicaciones. En primer lugar, el espectro esencial de $\mathbf{T}^{3}$ ya no está obligado a ser un subconjunto de $\{0\}$, como en el caso compacto ${ }^{4}$. Esto significa que el espectro $\sigma(\mathbf{T})$ de $\mathbf{T}$ podría ahora contener autovalores de multiplicidad infinita, puntos de acumulación de autovalores y un espectro continuo. Además, no hay garantías para la convergencia de los autovalores/autofunciones aproximados y, todavía peor, podrían aparecer autovalores espurios en la aproximación; es decir

$$
\lambda_{h} \in \sigma\left(\mathbf{T}_{h}\right) \quad \text { tal que } \quad \lim _{h \rightarrow 0} \lambda_{h}=\lambda \notin \sigma(\mathbf{T}) .
$$

En particular, el método de los elementos finitos, con funciones bases convencionales, origina la aparición de soluciones espurias cuando se lo utiliza para aproximar el espectro de operadores lineales no compactos. Este fenómeno (muy conocido) se presenta, por ejemplo, en problemas de magnetohidrodinámica [146], guías de ondas [120], acústica [115] y electromagnetismo [44].

En muchos casos, si el operador $\mathbf{T}$ es acotado, los autovalores espurios pueden removerse modificando la aproximación $\mathbf{T}_{h}$ de manera tal que se satisfagan las siguientes propiedades:

$$
\begin{aligned}
& \text { P1 para todo } u \in V \subset X \text { existe } u_{h} \in V_{h} \subset V \text { tal que } \lim _{h \rightarrow 0}\left\|u-u_{h}\right\|_{V}=0, \\
& \text { P2 } \quad \lim _{h \rightarrow 0} \sup _{\substack{u_{h} \in V_{h} \\
\left\|u_{h}\right\|_{V}=1}}\left\|\left(\mathbf{T}-\mathbf{T}_{h}\right) u_{h}\right\|_{V}=0 .
\end{aligned}
$$

Esencialmente, el método numérico se diseña para que $\mathbf{T}_{h}$ converja a $\mathbf{T}$, ya no uniformemente, sino en un sentido más débil; suficiente para garantizar que la aproximación sea espectralmente correcta (libre de modos espurios) para todo autovalor aislado de T. Estas condiciones fueron introducidas por DesclouxNassif-Rappaz [94]. Los mismos autores establecieron también estimaciones del orden con el que convergen los autovalores y autofunciones aproximados a los correspondientes autovalores y autofunciones de $\mathbf{T}$ pero solo para el caso en que $\mathbf{T}_{h}$ es una aproximación conforme obtenida por el método de Galerkin [95]. En este contexto, es interesante señalar lo siguiente:

si $\mathrm{P} 1$ se satisface, $\mathrm{P} 2$ implica que $\mathbf{T}_{h}$ aproxima compactamente a $\mathbf{T}$, en el sentido de Vainikko [164] ${ }^{5}$.

Esto sugiere que la teoría de Descloux-Nassif-Rappaz se apoya sobre cierta compacidad subyacente del operador T. Una estrategia similar para métodos mixtos puede encontrarse en [43].

Una característica interesante de la teoría de Descloux-Nassif-Rappaz es que puede ser adaptada para analizar aproximaciones no-conformes $\left(V_{h} \not \subset V\right)$ de problemas espectrales. Supongamos que podemos definir una norma $\|\cdot\|_{h}$ en $V_{h} \subset X$ con la siguiente propiedad (compatibilidad de normas):

$$
\|u\|_{h}=\|u\|_{V} \quad \text { para todo } u \in V .
$$

Entonces, asumiendo que las propiedades P1-P2 se satisfacen con la norma $\|\cdot\|_{h}$ reemplazando a la norma $\|\cdot\|_{V}$, es posible usar los argumentos en [94, 95] para probar que el método converge correctamente y estimar la rapidez de convergencia de la aproximación.

Esta idea fue utilizada por

Rodríguez-Solomin [150], para determinar el orden de convergencia de las frecuencias de vibración aproximadas, obtenidas usando un método de elementos finitos no-conforme, de un sistema con interacción fluido-estructura,

Antonietti-Buffa-Perugia [12], para analizar la aproximación discontinua de Galerkin del espectro del operador de Laplace con condiciones de borde de Dirichlet,

\footnotetext{
${ }^{3}$ Espectro esencial de $\mathbf{T}, \sigma_{e s}(\mathbf{T}):=\{\mu \in \mathbb{C}$ tal que $(\mathbf{T}-\mu \mathbf{I}): X \rightarrow X$ no es un operador de Fredholm. $\}$.

${ }^{4} \mathrm{Si} X$ es de dimensión infinita y $\mathbf{T}: X \rightarrow X$ es compacto, entonces $0 \in \sigma(\mathbf{T}) ; 0$ puede o no ser un autovalor de $\mathbf{T}$ y su espacio propio puede o no tener dimensión finita.

${ }^{5}$ Supongamos que $V_{h} \subset V$. Decimos que $\mathbf{T}_{h}$ es una aproximación compacta de $\mathbf{T}$ si la convergencia puntual es tal que para cualquier sucesión acotada $\left\{x_{h}\right\}$ de elementos de $V_{h}$, la sucesión $\left\{\left(\mathbf{T}-\mathbf{T}_{h}\right) x_{h}\right\}$ es relativamente compacta en $V$. La noción de convergencia compacta fue introducida por Vainikko en un contexto más general $\left(V_{h} \not \subset V\right)$. La prueba de la estabilidad fuerte de tal aproximación puede encontrarse en [164, 83].
} 
Buffa-Perugia [60], para analizar la aproximación discontinua de Galerkin del problema espectral de Maxwell.

En el primer caso, se asume que las formas bilineales continuas (definidas a partir de la formulación variacional del problema) y sus versiones aproximadas (determinadas por el método numérico) son iguales. En particular, $\|\cdot\|_{h}=\|\cdot\|_{V}$.

En los dos casos restantes, solo se analizan variantes del método discontinuo de Galerkin que resultan aproximaciones consistentes, en el sentido definido por Arnold-Brezzi-Cockburn-Marini ([19], pag. 10). Esto simplifica considerablemente el trabajo de estimar el orden con el que convergen las aproximaciones de los autovalores.

Aproximaciones no-conformes para problemas espectrales formulados variacionalmente también han sido analizadas en

$$
\text { Mercier-Osborn-Rappaz-Raviart [138]. }
$$

En este trabajo se asume que el operador $\mathbf{T}$ es compacto, que las formas bilineales aproximadas coinciden con las formas bilineales continuas y que

$$
\lim _{h \rightarrow 0}\left\|\mathbf{T}-\mathbf{T}_{h}\right\|_{\mathcal{L}(X)}=0 .
$$

\subsubsection{Problemas planteados en dominios con bordes curvos}

Supongamos que el problema que nos interesa resolver está definido sobre un dominio con bordes curvos. Una manera standard de abordar esta situación consiste en:

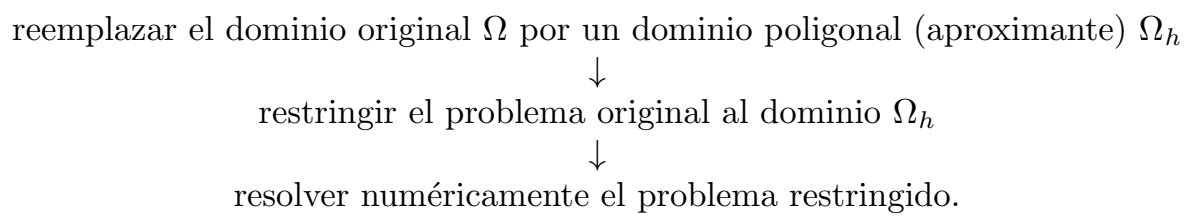

Este procedimiento es muy conveniente desde el punto de vista computacional ${ }^{6}$ pero introduce la siguiente (obvia) dificultad: el dominio sobre el que está definido el problema original es distinto al dominio sobre el que está definida la aproximación del problema; además, esta diferencia cambia con $h$. Diremos que la modificación que sufre el dominio es una perturbación si

$$
\left(\Omega-\Omega_{h}\right) \cup\left(\Omega_{h}-\Omega\right) \rightarrow 0 .
$$

Cómo estimar la influencia que tiene la perturbación del dominio en la aproximación del espectro?

Siendo $\Omega_{h} \neq \Omega, V_{h}\left(\Omega_{h}\right) \not \subset V(\Omega)$ y, por lo tanto, la aproximación será siempre no-conforme. Podríamos entonces intentar adaptar la teoría de Descloux-Nassif-Rappaz para analizar esta situación. Observemos que la aplicación de esta teoría implica la definición de operadores solución $\mathbf{T}$ (asociado con el problema original) y $\mathbf{T}_{h}$ (asociado con el problema aproximado) sobre un mismo espacio funcional.

Cómo se puede lograr esto?

El requisito anterior no es tan difícil de satisfacer cuando $\Omega$ es convexo ya que, en este caso, $\Omega_{h} \subset \Omega$ para todo $h$.

\footnotetext{
${ }^{6}$ En el contexto del método de los elementos finitos, la teoría de aproximación sobre dominios curvos ha sido extensamente desarrollada $[76,179,151,40]$. En la mayoría de estos trabajos, se utilizan elementos paramétricos: las funciones bases son obtenidas mapeando las bases polinomiales standard desde un elemento de referencia a un elemento curvo por medio de una transformación no-afín. Como consecuencia, los espacios de elementos finitos no serán estrictamente polinomiales. Este procedimiento es muy utilizado en la práctica y suficiente para muchos fines. Sin embargo, en algunas aplicaciones, es más ventajoso construir una familia de espacios de elementos finitos encajados definida sobre una jerarquía de grillas que se obtiene a partir de una grilla inicial más un proceso de refinamiento sistemático. Esta situación es típica de los métodos adaptativos. Los elementos paramétricos no permiten, en general, producir esta jerarquía de espacios discretos.
} 
Un ejemplo clásico: usar un método de los elementos finitos para aproximar el problema espectral de Laplace con condiciones de borde de Dirichlet.

Una técnica posible: imponerle a cada función en $V_{h}\left(\Omega_{h}\right)$ que se anule (fuerte o débilmente) en todos los grados de libertad que yacen sobre $\partial \Omega_{h}$ y, luego, extenderla por cero fuera de $\Omega_{h}$.

El resultado obtenido: las funciones así extendidas pertenecerán a $L^{2}(\Omega)$; por lo tanto, los operadores $\mathbf{T}$ y $\mathbf{T}_{h}$ podrán definirse con dominio en $L^{2}(\Omega)$ (en particular, si $V_{h}\left(\Omega_{h}\right) \subset H_{0}^{1}\left(\Omega_{h}\right)$, el método resultará conforme y podría aplicarse la teoría clásica para operadores compactos).

Cuando $\Omega_{h} \not \subset \Omega$, la aplicación de esta metodología no conduce a resultados de convergencia óptimos. Existen alternativas para tratar con la discrepancia entre $\Omega$ y $\Omega_{h}$ pero, en general, son específicas para cada clase de problemas. Por ejemplo, en el contexto del método de los elementos finitos,

- las aproximaciones del problema de autovalores de Laplace (usando los espacios de Lagrange que son $H^{1}\left(\Omega_{h}\right)$-conformes) fueron analizadas en:

Vanmaele-Z̆eníšek [165] y Lebaud [133] (condiciones de borde de Dirichlet),

Hernández-Rodríguez [116] (condiciones de borde de Neumann),

Acosta-Armentano [1] (condiciones de borde mixtas en un dominio no-Lipschitz),

- la aproximación del problema espectral del operador grad-div (usando el espacio de Raviart-Thomas de orden cero que es $H\left(\right.$ div,$\left.\Omega_{h}\right)$-conforme) fue analizada en:

Hernández-Rodríguez [117] (condiciones de borde nulas).

En todos estos trabajos, el problema estudiado es bi-dimensional. Además, salvo por Vanmaele-Z̆eníšek que utilizan la caracterización min-max, en los restantes trabajos se aplica la teoría de Babǔska-Osborn [29] para probar que las aproximaciones convergen con orden óptimo.

\subsubsection{Problemas aproximados por métodos mixtos}

Los métodos mixtos han sido extendidos exitosamente para aproximar los autovalores y las autofunciones de operadores diferenciales.

Algunas de las ideas fundamentales para analizar matemáticamente tales métodos fueron introducidas en los trabajos pioneros de Canuto [63], Osborn [145] y Mercier-Osborn-Rappaz-Raviart [138]; quienes, en particular, probaron resultados muy útiles para estimar la rapidez con la que convergen las soluciones aproximadas.

Un análisis alternativo se presenta en Boffi-Brezzi-Gastaldi [43] donde se establecen condiciones necesarias y suficientes para asegurar una convergencia espectralmente correcta de las aproximaciones vía métodos mixtos de problemas elípticos.

La característica común a todas estas teorías es que han sido desarrolladas para

- operadores compactos,

- problemas planteados sobre dominios poligonales,

- métodos conformes.

\subsubsection{Resultados obtenidos}

Uno de los objetivos de este trabajo es analizar las aproximaciones por elementos finitos no-standard de varios problemas de autovalores. Los problemas considerados son simétricos y están asociados a ecuaciones o a sistemas de ecuaciones diferenciales parciales. Consta de cuatro partes; desarrolladas a lo largo de los capítulos 3 y 4 .

En la primera parte (capítulo 3, secciones 3.1-3.3), se presenta una teoría de aproximación espectral para operadores lineales y acotados en espacios de Hilbert. La teoría es abstracta y fue desarrollada para 
estudiar las aproximaciones no-conformes de problemas de autovalores formulados variacionalmente. La no-conformidad de las aproximaciones puede deberse a

- las discontinuidades de los espacios discretos utilizados por el método numérico,

- que el problema de autovalores esté planteado en un dominio con interfaces y/o bordes curvos,

- una combinación de ambas situaciones.

La teoría se obtuvo como una generalización de la teoría de Descloux-Nassif-Rappaz [94, 95].

Una hipótesis crucial de la teoría propuesta es la existencia de operadores de extensión (uniformemente acotados) que actúan sobre las funciones pertenecientes a los espacios $V(\Omega)$ y $V_{h}\left(\Omega_{h}\right)$ para convertirlas en funciones

- con dominio en el conjunto unión $\widehat{\Omega}:=\Omega \cup \Omega_{h}$,

- pertenecientes al espacio suma $\left(V^{e}+V_{h}^{e}\right)(\widehat{\Omega}) \subset X(\widehat{\Omega})$,

- satisfaciendo las siguientes propiedades:

$$
\begin{aligned}
& \text {. si } u \in V(\Omega),\left.\quad u^{e} \in V(\widehat{\Omega}) \mathrm{y} u^{e}\right|_{\Omega}=u \text {, } \\
& \text {. si } u \in V_{h}\left(\Omega_{h}\right),\left.\quad u^{e} \in X(\widehat{\Omega}) \mathrm{y} u^{e}\right|_{\Omega_{h}}=u \text {. }
\end{aligned}
$$

Como veremos, estas extensiones nos permitirán definir operadores lineales y acotados sobre un mismo espacio funcional y cuyos espectros estarán directamente relacionados con los espectros de los operadores $\mathbf{T}$ y $\mathbf{T}_{h}$.

Asumiendo que el método numérico satisface las propiedades P1-P2 (adecuadamente modificadas) en una norma $\|\cdot\|_{h}$ compatible, probaremos que quedan garantizadas las siguientes propiedades:

- la aproximación es libre de autovalores espurios,

- los autoespacios aproximados tienen la dimensión correcta.

En otras palabras, las propiedades P1-P2 garantizan una convergencia espectralmente correcta.

Concentrándonos en problemas de autovalores simétricos formulados variacionalmente, estableceremos estimaciones para el error en la aproximación de los autovalores y las autofunciones. Aquí, no asumiremos que las formas bilineales continuas y discretas son iguales. Nuestras hipótesis (basadas en el análisis de los métodos de elementos finitos de mayor uso) imponen:

- una condición de compatibilidad entre las formas bilineales cuando se las testea con funciones del continuo,

- la existencia de una norma $\|\cdot\|_{*}$, definida sobre el espacio suma y equivalente a la norma $\|\cdot\|_{h}$ sobre los espacios discretos, que permite acotar a las formas bilineales aproximadas,

- comportamiento asintótico a cero de las autofunciones del operador $\mathbf{T}$ (extendidas a $\widehat{\Omega}$ ) sobre los dominios $\Omega-\Omega_{h}$ y $\Omega_{h}-\Omega$.

Cabe señalar que casi todos los resultados a los cuales hemos hecho referencia anteriormente ([12, 60, $116,117,150,165])$ se pueden obtener utilizando esta teoría; en algunos casos, incluso, con cálculos más simples $([116])$.

En la segunda parte (capítulo 3, sección 3.4), se indica como adaptar la teoría propuesta para analizar las aproximaciones por métodos mixtos no-conformes de un problema de autovalores planteado sobre un dominio con bordes curvos.

Siguiendo los argumentos de Mercier-Osborn-Rappaz-Raviart [138], analizaremos dos tipos de problemas: 
- de primera clase (también conocidos como problemas tipo (f, 0$)$ );

Encontrar $\mu \in \mathbb{C},(\mathbf{u}, p) \in V(\Omega) \times Q(\Omega),(\mathbf{u}, p) \neq(\mathbf{0}, 0)$, tal que

$$
\left\{\begin{array}{l}
a(\mathbf{u}, \mathbf{v})+\overline{b(\mathbf{v}, p)}=\mu c(\mathbf{u}, \mathbf{v}) \quad \forall \mathbf{v} \in V(\Omega) \\
b(\mathbf{u}, q)=0 \quad \forall q \in Q(\Omega)
\end{array}\right.
$$

- de segunda clase (o problemas tipo $(\mathbf{0}, g)$ );

Encontrar $\mu \in \mathbb{C},(\mathbf{u}, p) \in V(\Omega) \times Q(\Omega),(\mathbf{u}, p) \neq(\mathbf{0}, 0)$, tal que

$$
\left\{\begin{array}{l}
a(\mathbf{u}, \mathbf{v})+\overline{b(\mathbf{v}, p)}=0 \quad \forall \mathbf{v} \in V(\Omega) \\
-b(\mathbf{u}, q)=\mu d(p, q) \quad \forall q \in Q(\Omega)
\end{array}\right.
$$

En ambas situaciones, asumiremos que se satisfacen las condiciones de Brezzi [56] (aseguran la existencia, unicidad y estabilidad de la solución del problema fuente asociado) y probaremos que las propiedades P1-P2 (en versiones adecuadas) son suficientes para garantizar una convergencia libre de autovalores espurios.

Probaremos también, para cada tipo de problemas, estimaciones del error cometido en la aproximación de los autovalores y las autofunciones.

Observemos que, a efectos de aplicar la teoría propuesta, no es necesario determinar la compacidad (o la falta de compacidad) del operador solución subyacente. Esta característica de la teoría es muy atractiva ya que, como fuera señalado por Boffi-Brezzi-Gastaldi [43], chequear propiedades de compacidad es una tarea delicada; en especial, cuando el problema está planteado en forma mixta.

En la tercera parte (capítulo 3, sección 3.5), se indica como extender la teoría propuesta con el fin de analizar las aproximaciones no-conformes de problemas espectrales no-simétricos planteados sobre dominios no necesariamente poligonales.

Los operadores no-autoadjuntos aparecen naturalmente en numerosas aplicaciones (un ejemplo: problemas del tipo convección-difusión y absorción que describen muchos fenómenos multi-físicos, como aquellos que se presentan en la dinámica de fluidos/gases, láseres y scattering nuclear). El comportamiento espectral de estos operadores es considerablemente más complejo que el de los operadores autoadjuntos. En particular, los autovalores ya no estarán confinados al eje real y podrían tener multiplicidades algebraica y geométrica diferentes.

Los resultados obtenidos en esta sección aplican a problemas no-simétricos formulados variacionalmente. Entre estos resultados, las estimaciones del error en la aproximación de los autovalores generalizan las ya obtenidas por Descloux-Nassif-Rappaz [95] y Rodríguez-Solomin [150].

En la cuarta parte (capítulo 4), finalmente, se aplica la teoría propuesta para estudiar la aproximación de varios problemas de autovalores por métodos de elementos finitos no-conformes.

Los resultados enunciados en este resumen forman parte de los siguientes trabajos:

Spectral approximation of variationally posed eigenvalue problems by nonconforming methods, A. Alonso and A. Dello Russo, Journal of Computational and Applied Mathematics, 223 (2009) 177-197

Spectral approximation of variationally formulated eigenvalue problems on curved domains, A. Alonso and A. Dello Russo, Electronic Transactions on Numerical Analysis 35 (2009) 69-87.

Finite element approximation of Maxwell eigenproblems on curved Lipschitz polyhedral domains, A. Dello Russo and A. Alonso, Applied Numerical Mathematics 59 (2009) 1796-1822.

Hybrid finite element analysis of fluid-structure systems with coupling on curved interfaces, A. Dello Russo and A. Alonso, IMA Journal of Numerical Analysis, 31 (2011), 1636-1682.

Mixed finite element analysis of eigenvalue problems on curved domains, A. Dello Russo and A. Alonso, Computational Methods in Applied Mathematics, 14 (2014) 1-34.

Eigenvalue approximation by mixed nonconforming finite element methods. The determination of the vibrational modes of a linear elastic solid, A. Dello Russo, Calcolo, 51 (2014), 563-597. 


\subsection{Análisis a posteriori del error}

Cómo obtener una solución aproximada de un problema diferencial (fuente o de autovalores) con una exactitud determinada (dentro de una tolerancia prescripta) y con el menor costo computacional posible?.

Básicamente, hay dos tipos de procedimientos para estimar el error cometido en una aproximación:

- el análisis a priori; solo brinda información sobre el comportamiento asintótico del error y sus resultados dependen fuertemente de la regularidad de la solución exacta,

- el análisis a posteriori; la información sobre el error se extrae de la solución aproximada y de los datos del problema.

Claramente, las estimaciones a posteriori son las que nos permitirán un control cuantitativo del error. Más aún, son la base de los (importantísimos) métodos de refinamiento adaptativo [170]. De acuerdo con Garau-Morin-Zuppa [106], el objetivo fundamental de los métodos adaptativos es equidistribuir el error y el esfuerzo computacional para obtener una sucesión de grillas de cálculo de complejidad óptima.
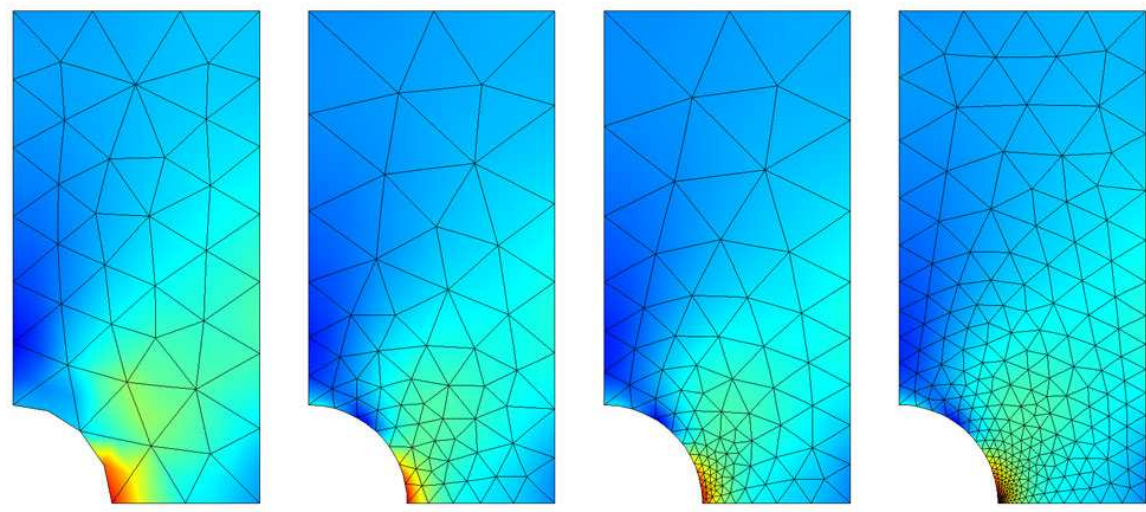

Figura 1.1: Sucesivos refinamientos sobre una grilla inicial obtenidos por un método adaptativo (ejemplo tomado de Multiphysics Cyclopedia [78]).

En el contexto del método de los elementos finitos, el análisis a posteriori del error en la aproximación de ecuaciones en derivadas parciales (muchas de las cuales aparecen con frecuencia en las aplicaciones de la física o la ingeniería) constituye un área de investigación en constante avance; de hecho, el progreso alcanzado durante las últimas décadas ha sido enorme. Sin embargo, la mayor parte del trabajo realizado hasta la fecha está relacionado con problemas fuente.

Debido a la naturaleza no lineal de los problemas espectrales, las técnicas desarrolladas para problemas fuente no pueden extenderse de manera directa a los problemas de autovalores (principalmente, por la pérdida de la ortogonalidad de Galerkin ${ }^{7}$ ).

El primer análisis a posteriori para las aproximaciones por elementos finitos de un problema de autovalores elíptico de segundo orden se debe a:

Verfürth [169]; asimiló el problema a una ecuación no lineal dependiente de un parámetro.

Posteriormente, el mismo problema espectral fue considerado en varios trabajos y desde varios puntos de vista; entre ellos:

\footnotetext{
${ }^{7}$ Una propiedad importante de la aproximación vía el método de los elementos finitos es la ortogonalidad de Galerkin. Esta propiedad implica que el error en la aproximación es, en algún sentido, ortogonal al espacio discreto. Gracias a esta propiedad, es posible introducir la interpolada de alguna función adecuada en la ecuación del error y, así, probar (usando resultados standard sobre interpolaciones) que las estimaciones a posteriori proveen una cota superior global del error.
} 
Larson [132]; combina los análisis a priori y a posteriori para obtener estimaciones que resultan óptimas pero solo válidas para autofunciones muy suaves,

Durán-Padra-Rodríguez [101]; definen estimadores de tipo residual y asintóticamente equivalentes a la norma $H^{1}$ del error,

Grubišic-Ovall [110]; usan bases jerárquicas para obtener estimaciones del error asintóticamente exactas.

Una vasta experiencia computacional indica que los métodos adaptativos basados en estos estimadores dan resultados excelentes; incluso, con estrategias de refinamiento muy simples. Sobre la convergencia de algunos métodos adaptativos para problemas de autovalores elípticos, pueden consultarse los trabajos de Giani-Graham [108], Garau-Morin-Zuppa [106] y Carstensen-Gedicke [69].

En cuanto a las aproximaciones por métodos mixtos, los resultados publicados no son tantos:

Durán-Gastaldi-Padra [98]; definen un estimador a posteriori para la solución del problema espectral de Laplace obtenida usando el espacio de Raviart-Thomas de orden cero [147],

Gardini [107]; considera el problema de determinar los modos de oscilación de un fluido ideal contenido por un recipiente rígido (problema de las vibraciones acústicas) y define un estimador $a$ posteriori para la solución obtenida usando los espacios de Brezzi-Dougas-Marini [56],

Lovadina-Lyly-Stenberg [136]; introducen un estimador de tipo residual para las aproximaciones del problema de Stokes obtenidas usando pares de espacios discretos estables.

En todos los casos, los estimadores propuestos son equivalentes a una norma (adecuada) del error salvo términos de orden superior.

Estas técnicas, desarrolladas para métodos conformes, podrían ser extendidas a métodos no-conformes?

La dificultad principal son los términos de consistencia que aparecerán en la ecuación del error. Estos términos de consistencia dependerán de la solución exacta y no pueden ser despreciados.

\subsubsection{Problemas de autovalores - formulación variacional clásica}

Asumamos que

- el espacio no-conforme $V_{h}$ contiene como subespacio a un espacio conforme $V_{h}^{c} \subset V_{h} \cap V$ (muchos de los espacios de elementos finitos no-conformes que se utilizan en las aplicaciones satisfacen esta propiedad),

- existe un operador lineal de transferencia (o de post-procesamiento) $\mathbf{R}_{h}: V_{h} \rightarrow V_{h}^{c}$.

Entonces, el error en la aproximación puede acotarse como sigue:

$$
\left\|u-u_{h}\right\|_{h} \leq \underbrace{\left\|u-\mathbf{R}_{h} u_{h}\right\|_{h}}_{\begin{array}{c}
\text { parte conforme } \\
\text { del error }
\end{array}}+\underbrace{\left\|\left(\mathbf{I}-\mathbf{R}_{h}\right) u_{h}\right\|_{h}}_{\begin{array}{c}
\text { parte no-conforme } \\
\text { del error }
\end{array}} .
$$

El primer término puede estimarse usando las técnicas desarrolladas para el análisis a posteriori del error en las aproximaciones conformes. El tratamiento del segundo término, en cambio, depende de la definición concreta del operador $\mathbf{R}_{h}$.

Usando este procedimiento, definimos varios estimadores del error a posteriori para las aproximaciones, obtenidas usando el método no-conforme de Crouzeix-Raviart [81], del problema de autovalores de Steklov. Estos estimadores son simples de calcular y, además, pueden calcularse localmente; por lo tanto, son aptos para definir métodos de refinamiento adaptativo. Más aún, son equivalentes a la norma $H^{1}$-quebrada del error, salvo términos de orden superior. Estos resultados se presentan en el capítulo 5, sección 5.2 de este trabajo.

La metodología aquí descripta puede compararse con la que proponen Dari-Durán-Padra [87], desarrollada para operadores elípticos a partir de técnicas conocidas para problemas fuente $[86,100]$. 


\subsubsection{Problemas de autovalores - formulación variacional mixta}

En el capítulo 5, sección $\mathbf{5 . 3}$ de este trabajo se introduce un estimador de error a posteriori para las aproximaciones de los modos de vibración de un cuerpo elástico obtenidas usando el método no-conforme de Arnold-Winther [24]. Salvo, términos de orden superior, el estimador propuesto resulta equivalente a una norma discreta del error y robusto; incluso, para materiales casi incompresibles.

La metodología utilizada para obtener este estimador es muy flexible, en el sentido que puede ser aplicada a distintos métodos de aproximación (conformes o no-conformes), no requiere regularidad adicional de la solución débil ni hipótesis de saturación y no utiliza una descomposición de Helmholtz para la variable tensorial.

Dado que el problema de autovalores asociado al operador de Laplace es (matemáticamente) muy similar al problema espectral de la elasticidad lineal, expondremos las ideas básicas del método considerando el problema espectral de Laplace con condiciones de borde de Dirichlet. Sabemos que, introduciendo

$$
\sigma=\nabla u,
$$

obtendremos la formulación variacional mixta correspondiente a este problema; es decir:

encontrar $\lambda \in \mathbb{R} y(\boldsymbol{\sigma}, u) \in H(\operatorname{div} ; \Omega) \times L^{2}(\Omega),(\boldsymbol{\sigma}, u) \neq(\mathbf{0}, 0)$, tal que

$$
\begin{array}{cl}
\int_{\Omega} \boldsymbol{\sigma} \cdot \boldsymbol{\tau}+\int_{\Omega} \operatorname{div} \boldsymbol{\tau} u=0 & \forall \boldsymbol{\tau} \in H(\operatorname{div} ; \Omega), \\
-\int_{\Omega} \operatorname{div} \boldsymbol{\sigma} v=\lambda \int_{\Omega} u v & \forall v \in L^{2}(\Omega) .
\end{array}
$$

Dados los espacios de dimensión finita $\boldsymbol{\Sigma}_{h}(\Omega) \times V_{h}(\Omega) \subset H($ div $; \Omega) \times L^{2}(\Omega)$ (asumiremos que satisfacen las condiciones de estabilidad usuales para los esquemas mixtos), aproximaremos el problema anterior por medio de las soluciones del siguiente problema discreto:

encontrar $\lambda_{h} \in \mathbb{R} y\left(\boldsymbol{\sigma}_{h}, u_{h}\right) \in \boldsymbol{\Sigma}_{h}(\Omega) \times V_{h}(\Omega),\left(\boldsymbol{\sigma}_{h}, u_{h}\right) \neq(\mathbf{0}, 0)$, tal que

$$
\begin{array}{cl}
\int_{\Omega} \boldsymbol{\sigma}_{h} \cdot \boldsymbol{\tau}_{h}+\int_{\Omega} \operatorname{div} \boldsymbol{\tau}_{h} u_{h}=0 & \forall \boldsymbol{\tau}_{h} \in \boldsymbol{\Sigma}_{h}(\Omega), \\
-\int_{\Omega} \operatorname{div} \boldsymbol{\sigma}_{h} v_{h}=\lambda_{h} \int_{\Omega} u_{h} v_{h} & \forall v_{h} \in V_{h}(\Omega) .
\end{array}
$$

El análisis a posteriori de la aproximación está basado en la observación de una característica distintiva de los métodos mixtos: la variable $\boldsymbol{\sigma}$ se aproxima utilizando bases polinomiales de un grado mayor que las utilizadas para aproximar a la variable $u$; esto parece indicar que no existe un análogo discreto de la relación $\sigma=\nabla u$.

Siguiendo a Lovadina-Stenberg [137], calcularemos, mediante un método de post-procesamiento local, una nueva aproximación $u_{h}^{*}$ de $u$ (que converge a $u$ más rápido que $u_{h}$ ) en un espacio de elementos finitos $V_{h}^{*}(\Omega)$ que satisface la inclusión $V_{h}(\Omega) \subset V_{h}^{*}(\Omega)$; típicamente, $V_{h}^{*}(\Omega)$ contiene funciones polinomiales de un grado mayor que las utilizadas para aproximar a $\sigma$. La idea será reemplazar a $u_{h}$ por $u_{h}^{*}$ en las estimaciones; esto puede interpretarse como un intento de recuperar o de imitar la relación $\sigma_{h} \approx \nabla u_{h}$ en cada elemento de la partición.

Siguiendo a Kim [128], consideremos la siguiente descomposición del error:

$$
\boldsymbol{\sigma}-\boldsymbol{\sigma}_{h}=\nabla(u-\varphi)+\left(\nabla \varphi-\sigma_{h}\right),
$$

siendo $\varphi \in H_{0}^{1}(\Omega)$ la solución del siguiente problema:

$$
\int_{\Omega} \nabla \varphi \cdot \nabla v=\int_{\Omega} \boldsymbol{\sigma}_{h} \cdot \nabla v \quad \forall v \in H_{0}^{1}(\Omega) .
$$


Gracias a la ortogonalidad de Galerkin $\int_{\Omega}\left(\nabla \varphi-\sigma_{h}\right) \cdot \nabla(u-\varphi)=0$, obtendremos

$$
\left\|\boldsymbol{\sigma}-\boldsymbol{\sigma}_{h}\right\|_{0}^{2}=\underbrace{\|\nabla(u-\varphi)\|_{0}^{2}}_{\begin{array}{c}
\text { parte continua } \\
\text { del error }
\end{array}}+\underbrace{\left\|\nabla \varphi-\boldsymbol{\sigma}_{h}\right\|_{0}^{2}}_{\begin{array}{c}
\text { parte discreta } \\
\text { del error }
\end{array}} .
$$

La parte continua del error puede estimarse acotando el residuo

$$
r(v):=\lambda \int_{\Omega} u v-\lambda_{h} \int_{\Omega} u_{h} v \quad \forall v \in H_{0}^{1}(\Omega),
$$

mientras que la parte discreta del error puede estimarse por medio de la expresión

$$
\left\|\nabla \varphi-\sigma_{h}\right\|_{0} \leq \inf _{v \in H_{0}^{1}(\Omega)}\left\|\nabla v-\sigma_{h}\right\|_{0},
$$

que mide cuan próximo está $\boldsymbol{\sigma}_{h}$ del gradiente de una función en $H_{0}^{1}(\Omega)$. De aquí que, para completar la acotación de la parte discreta del error, bastará con especificar una función $\widetilde{u} \in H_{0}^{1}(\Omega)$.

Cómo elegir $\widetilde{u}$ de manera que el estimador, que de esa elección se obtiene, sea localmente eficiente?

La elección más apropiada será (claramente) aquella para la cual $\nabla \widetilde{u}$ esté lo más cerca posible de $\boldsymbol{\sigma}_{h}$. Con esta idea como guía y teniendo en cuenta que la solución post-procesada $u_{h}^{*} \in V_{h}^{*} \not \subset H_{0}^{1}(\Omega)$, elegimos:

$$
\widetilde{u}=\mathbf{R}_{h} u_{h}^{*} \in H_{0}^{1}(\Omega) .
$$

Es interesante mostrar como esta metodología nos conduce a estimaciones del error que son equivalentes a las obtenidas usando una descomposición de Helmholtz para el error en la variable vectorial.

Identifiquemos a $\boldsymbol{\Sigma}_{h}(\Omega)$ con el espacio de Raviart-Thomas de orden cero y a $V_{h}(\Omega)$ con el espacio de las funciones constantes a trozos. A partir de los resultados de Durán-Gastaldi-Padra [98], es posible establecer la siguiente estimación

$$
\left\|\lambda u-\lambda_{h} u_{h}\right\|_{0} \leq 2 \lambda_{h}\left\|\eta_{1}\right\|_{0}+\text { h.o.t. }
$$

donde

- $\left.\eta_{1}\right|_{T}:=-\frac{1}{|T|}\left(\int_{T} \boldsymbol{\sigma}_{h}\right) \cdot(\mathbf{x}-\widehat{\mathbf{x}})+\frac{1}{2|T|}\left(\frac{20}{9} b_{T}-1\right) \int_{T} \boldsymbol{\sigma}_{h} \cdot(\mathbf{x}-\widehat{\mathbf{x}})$,

- $T$ es un elemento de $\mathcal{T}_{h}$; la triangulación del dominio $\bar{\Omega}$ usada para el cálculo de $\lambda_{h}$ y $\left(\boldsymbol{\sigma}_{h}, u_{h}\right)$,

- $\widehat{\mathbf{x}}$ es el baricentro de $T$,

- $b_{T}$ es la función burbuja cúbica que toma el valor 1 en $\widehat{\mathbf{x}}$,

- h.o.t. representan términos de orden superior comparados con el error.

Sea $\eta_{2}:=\left\|\nabla \widetilde{u}-\sigma_{h}\right\|_{0}$. Entonces,

$$
\left\|\boldsymbol{\sigma}-\boldsymbol{\sigma}_{h}\right\|_{0}^{2} \leq 4 \lambda_{h}^{2}\left\|\eta_{1}\right\|_{0}^{2}+\left\|\eta_{2}\right\|_{0}^{2}+\text { h.o.t. }
$$

expresión que provee una cota superior para el error global que es completamente calculable; es decir, sin constantes genéricas difíciles de estimar. Además,

- de los resultados de Durán-Gastaldi-Padra [98], se obtiene

$$
\left\|\eta_{1}\right\|_{0}^{2} \leq C_{1} \sum_{T \in \mathcal{T}_{h}}|T|\left\|\boldsymbol{\sigma}_{h}\right\|_{0, T}^{2}
$$


- siguiendo argumentos similares a los propuestos por Kim [128], se llega a

$$
\left\|\eta_{2}\right\|_{0}^{2} \leq C_{2} \sum_{\ell \in \mathcal{E}_{h}}|\ell|\left\|\left[\left[\boldsymbol{\sigma}_{h} \cdot \mathbf{t}_{\ell}\right]\right]\right\|_{0, \ell}^{2},
$$

donde $\mathcal{E}_{h}$ es el conjunto de todos los lados $\ell$ en $\mathcal{T}_{h}, \mathbf{t}_{\ell}$ es un vector unitario tangente a $\ell$ y $[[\cdot]]$ denota el salto de la función escalar $\boldsymbol{\sigma}_{h} \cdot \mathbf{t}_{\ell}$ a través de $\ell$.

Es decir, el estimador hallado es equivalente al propuesto por Durán-Gastaldi-Padra [98]. Luego, también será eficiente.

La metodología aquí descripta puede considerarse una versión generalizada para problemas de autovalores del método propuesto por Vohralík [171] - Kim [128] para problemas fuente y aproximaciones conformes. En este trabajo, también extenderemos estas ideas para tratar con métodos no-conformes.

Los resultados expuestos en este resumen pueden encontrarse en los siguientes trabajos:

A posteriori error estimates for nonconforming approximations of Steklov eigenvalue problems, A. Dello Russo and A. Alonso, Computers and Mathematics with Applications 62 (2011) 4100- 4117.

A posteriori error analysis of the non-conforming Arnold-Winther method for linear elasticity eigenvalue problems, A. Dello Russo, en redacción.

\subsection{Otros comentarios}

Al comienzo, en el capítulo 2 , se introducen notación, terminología y resultados básicos que se utilizarán a lo largo de todo este trabajo. En particular, se resumen las propiedades espectrales de los operadores lineales acotados en espacios de Hilbert.

Finalmente, en el Apéndice, se recordarán algunos resultados standard de las aproximaciones obtenidas usando el método de los elementos finitos y se presentarán las demostraciones de varios lemas técnicos que fueron omitidas en el desarrollo de los capítulos para mayor claridad de la exposición. 


\section{Preliminares}

En esta sección recordaremos definiciones básicas y algunos de los resultados principales de la teoría espectral de operadores en espacios de Hilbert que usaremos posteriormente. Como todos estos resultados son clásicos, no incluiremos aquí sus demostraciones las cuales pueden encontarse en los libros de K. Yosida [178] y T. Kato [124].

\subsection{Operadores acotados en espacios de Hilbert}

Sean $X$ e $Y$ dos espacios de Hilbert sobre $\mathbb{C}$ con normas $\|\cdot\|_{X} \mathrm{y}\|\cdot\|_{Y}$, respectivamente.

Se dice que un operador $\mathbf{T}: \mathcal{D}(\mathbf{T}) \subseteq X \rightarrow Y$ es lineal si

- $\mathcal{D}(\mathbf{T})$ es un subespacio de $X$,

- $\mathbf{T}(\alpha x+\beta y)=\alpha \mathbf{T} x+\beta \mathbf{T} y \quad \forall \alpha, \beta \in \mathbb{C}, \quad \forall x, y \in \mathcal{D}(\mathbf{T})$.

Un operador lineal $\mathbf{T}: \mathcal{D}(\mathbf{T}) \subseteq X \rightarrow Y$ se dice acotado si existe una constante $C>0$ tal que

$$
\|\mathbf{T} x\|_{Y} \leq C\|x\|_{X} \forall x \in \mathcal{D}(\mathbf{T})
$$

En lo que sigue, a menos que se diga explícitamente lo contrario, asumiremos que $\mathcal{D}(\mathbf{T})=X^{1}$.

A la colección de todos los operadores lineales y acotados $\mathbf{T}: X \rightarrow Y$ se la denotará $\mathcal{L}(X, Y)$. Puede demostrarse que $\mathcal{L}(X, Y)$ dotado con la norma

$$
\|\mathbf{T}\|_{\mathcal{L}(X, Y)}:=\sup _{x \in X} \frac{\|\mathbf{T} x\|_{Y}}{\|x\|_{X}}
$$

es un espacio de Banach.

Dado un operador $\mathbf{T} \in \mathcal{L}(X, Y)$, quedan definidos los siguientes conjuntos:

$\mathcal{N}(\mathbf{T}):=\{x \in X: \mathbf{T} x=0\}$, denominado núcleo o espacio nulo de $\mathbf{T}$,

$\mathcal{R}(\mathbf{T}):=\{y \in Y: y=\mathbf{T} x$ para algún $x \in X\}$, denominado rango o espacio imagen de $\mathbf{T}$.

\footnotetext{
${ }^{1}$ Si el operador $\mathbf{T}$ es acotado, entonces puede ser extendido a la clausura de $\mathcal{D}(\mathbf{T})$. Si $\mathcal{D}(\mathbf{T})$ no fuera denso en $X$, se puede definir $\mathbf{T} x=0$ para todo $x \in \overline{\mathcal{D}(\mathbf{T})}^{\perp} \mathrm{y}$, por linealidad, extender $\mathbf{T}$ a todo $X$. Dicha extensión estará también acotada y su norma será igual a la norma de $\mathbf{T}$.
} 
Un operador $\mathbf{T} \in \mathcal{L}(X, Y)$ se dice acotado por abajo si existe una constante $C>0$ tal que

$$
\|x\|_{X} \leq C\|\mathbf{T} x\|_{Y} \forall x \in X .
$$

Un operador $\mathbf{T} \in \mathcal{L}(X, Y)$ está acotado por abajo si, y solo si, $\mathcal{N}(\mathbf{T})=\left\{0_{X}\right\}$ y $\mathcal{R}(\mathbf{T})$ es cerrado en $Y$. De este resultado, se infiere que, si $\mathbf{T} \in \mathcal{L}(X, Y)$ y está acotado por abajo, entonces existe un operador $\mathbf{T}^{-1} \in \mathcal{L}(\mathcal{R}(\mathbf{T}), X)$, llamado operador inverso de $\mathbf{T}$, tal que

$$
\begin{array}{ll}
\text { - } \mathbf{T}^{-1} \mathbf{T} x=x & \forall x \in X, \\
\text { - } \mathbf{T T}^{-1} y=y & \forall y \in \mathcal{R}(\mathbf{T}) .
\end{array}
$$

En este trabajo, trataremos casi exclusivamente con endormorfismos continuos definidos sobre $X$; es decir, con aplicaciones $\mathbf{T} \in \mathcal{L}(X, X)=: \mathcal{L}(X)$. De aquí en adelante, $(\cdot, \cdot)$ indicará el producto interno en $X$.

Sean $\mathbf{I}$ el operador identidad en $X$ y $\mathbf{T}$ un operador en $\mathcal{L}(X)$ tal que $\|\mathbf{T}\|_{\mathcal{L}(X)}<1$. Entonces, $(\mathbf{I}-\mathbf{T})^{-1}$ existe y puede representarse de la siguiente manera:

$$
(\mathbf{I}-\mathbf{T})^{-1}=\sum_{k \geq 0} \mathbf{T}^{k},
$$

donde la serie (de von Neumann) es absolutamente convergente en $\mathcal{L}(X)$. Además,

$$
\left\|(\mathbf{I}-\mathbf{T})^{-1}\right\|_{\mathcal{L}(X)} \leq \frac{1}{1-\|\mathbf{T}\|_{\mathcal{L}(X)}} .
$$

Sea $\mathbf{T} \in \mathcal{L}(X)$. Claramente, la expresión $(\mathbf{T} x, y)$ es lineal en $x$, conjugada-lineal en $y$ y acotada. Luego, de acuerdo con del teorema de representación de Riesz ${ }^{2}$, existe una única aplicación $\mathbf{T}^{*} \in \mathcal{L}(X)$ tal que

$$
(\mathbf{T} x, y)=\left(x, \mathbf{T}^{*} y\right) \quad \forall x, y \in X .
$$

El operador $\mathbf{T}^{*}$ recibe el nombre de operador adjunto de $\mathbf{T}$ y satisface:

- $\left\|\mathbf{T}^{*}\right\|_{\mathcal{L}(X)}=\|\mathbf{T}\|_{\mathcal{L}(X)}$,

$-\left(\mathbf{T}^{*}\right)^{*}=\mathbf{T}$,

- si $\mathbf{T}_{1}, \mathbf{T}_{2} \in \mathcal{L}(X)$ y $\alpha \in \mathbb{C}, \quad\left(\alpha \mathbf{T}_{1}+\mathbf{T}_{2}\right)^{*}=\bar{\alpha} \mathbf{T}_{1}^{*}+\mathbf{T}_{2}^{*}$,

- si $\mathbf{S} \in \mathcal{L}(X),(\mathbf{S T})^{*}=\mathbf{T}^{*} \mathbf{S}^{*}$,

- si $\mathbf{T}$ es invertible, entonces $\left(\mathbf{T}^{*}\right)^{-1}=\left(\mathbf{T}^{-1}\right)^{*}$.

Un operador $\mathbf{T} \in \mathcal{L}(X)$ se dirá autoadjunto o hermitiano si $\mathbf{T}=\mathbf{T}^{*}$.

Supongamos que exista un subespacio $Z$ de $X$, cerrado y no trivial, tal que $\mathbf{T}(Z) \subset Z$. Se dice, entonces, que $Z$ es invariante con respecto a $\mathbf{T}$ o, simplemente, $\mathbf{T}$-invariante. En este caso, puede definirse la restricción $\left.\mathbf{T}\right|_{Z}:=\mathbf{T}: Z \rightarrow Z$ como un operador lineal sobre $Z$.

Supongamos ahora que $X=Y \oplus Z$, siendo $Y$ y $Z$ subespacios T-invariantes. Entonces, para todo $x \in X$, tendremos

$$
\begin{aligned}
& \text { - } x=y+z \quad \text { con } y \in Y, z \in Z, \\
& \text { - } \mathbf{T} x=\left.\mathbf{T}\right|_{Y} y+\left.\mathbf{T}\right|_{Z} z .
\end{aligned}
$$

\footnotetext{
${ }^{2}$ Sean $V$ un espacio de Hilbert, $V^{\prime}$ el espacio dual de $V$ y $f: V \rightarrow \mathbb{C}$ una forma lineal y continua (i.e., $f(v) \leq\|f\|_{V^{\prime}}\|v\|_{V}$ ). Entonces, existe un único $u \in V$ tal que $(u, v)_{V}=f(v)$ para todo $v \in V$. Más aún, $\|u\|_{V}=\|f\|_{V^{\prime}}$.
} 
Además, si $Z$ es invariante respecto de $\mathbf{T}, Z^{\perp}$ (el complemento ortogonal de $Z$ en $X$ ) será $\mathbf{T}^{*}$-invariante. Luego, si $\mathbf{T} \in \mathcal{L}(X)$ es autoadjunto, la acción de $\mathbf{T}$ sobre $X$ puede determinarse por la acción de $\mathbf{T}$ sobre sus subespacios invariantes ortogonales.

Los operadores compactos constituyen una clase muy importante de operadores acotados (su origen se remonta a la teoría de las ecuaciones integrales de segunda especie); suponen la generalización natural de los operadores cuyos rangos tienen dimensión finita.

Un operador $\mathbf{T} \in \mathcal{L}(X)$ es compacto si, para todo conjunto acotado $U \subset X$, el conjunto $\overline{\mathbf{T}(U)}$ es compacto.

Las siguientes propiedades caracterizan a los operadores compactos en espacios de Hilbert (de dimensión infinita):

- $\mathbf{T} \in \mathcal{L}(X)$ es compacto si, y solo si, para cada sucesión $\left\{u_{n}\right\}$, acotada en $X$, que converge débilmente a $u$, se verifica que la sucesión $\left\{\mathbf{T} u_{n}\right\}$ converge en norma a $\mathbf{T} u$,

- si $\mathbf{T} \in \mathcal{L}(X)$ es un operador compacto, $\mathcal{R}(\mathbf{T})$ no contiene subespacios cerrados de dimensión infinita,

- $\mathbf{T} \in \mathcal{L}(X)$ es compacto si, y solo si, es el límite en norma de una sucesión de operadores lineales con rango de dimensión finita.

El siguiente resultado es importante en la resolución de ecuaciones que involucran operadores compactos (Teorema de Schauder):

- $\mathbf{T} \in \mathcal{L}(X)$ es compacto si, y solo si, $\mathbf{T}^{*}$ es también un operador compacto.

\subsection{Propiedades espectrales de un operador lineal y acotado}

El conjunto resolvente de un operador $\mathbf{T} \in \mathcal{L}(X)$, que denotaremos $\rho(\mathbf{T})$, se define como el conjunto de los números complejos $\lambda$ tal que $(\mathbf{T}-\lambda \mathbf{I})$ tiene inversa acotada en $X$; equivalentemente,

$$
\rho(\mathbf{T})=\{\lambda \in \mathbb{C}: \mathcal{N}(\mathbf{T}-\lambda \mathbf{I})=\{0\} \text { y } \mathcal{R}(\mathbf{T}-\lambda \mathbf{I})=X\}
$$

Los números $\lambda \in \rho(\mathbf{T})$ se denominan puntos regulares de $\mathbf{T}$. Puede probarse que $\rho(\mathbf{T})$ es un subconjunto abierto de $\mathbb{C}$ que contiene al exterior del disco $|\lambda| \leq\|\mathbf{T}\|_{\mathcal{L}(X)}$.

Si $\lambda \in \rho(\mathbf{T})$, se define el operador $R_{\lambda}(\mathbf{T}):=(\mathbf{T}-\lambda \mathbf{I})^{-1}$, denominado resolvente de $\mathbf{T}$ en $\lambda$ o, simplemente, resolvente de $\mathbf{T}$. Las siguientes son propiedades importantes de la resolvente de $\mathbf{T}$ :

- $R_{\lambda}(\mathbf{T}) \in \mathcal{L}(X)$,

- $\mathbf{T} \circ R_{\lambda}(\mathbf{T})=R_{\lambda}(\mathbf{T}) \circ \mathbf{T}$,

- $R_{\lambda}(\mathbf{T}) \circ R_{\mu}(\mathbf{T})=R_{\mu}(\mathbf{T}) \circ R_{\lambda}(\mathbf{T})$, para cualquier $\mu \in \rho(\mathbf{T})$,

- $R_{\lambda}(\mathbf{T})$, considerada como función de $\lambda$, es localmente holomorfa en $\rho(\mathbf{T})^{3}$.

De acuerdo con los desarrollos de von Neumann, $R_{\lambda}(\mathbf{T})$ admite la siguiente representación:

$$
R_{\lambda}(\mathbf{T})=-\frac{1}{\lambda}\left(\mathbf{I}-\frac{\mathbf{T}}{\lambda}\right)^{-1}=-\frac{1}{\lambda} \sum_{k \geq 0}\left(\frac{\mathbf{T}}{\lambda}\right)^{k}
$$

que converge si, y solo si, $|\lambda|>\|\mathbf{T}\|_{\mathcal{L}(X)}$ y prueba que $R_{\lambda}(\mathbf{T})$ es holomorfa en el infinito. En particular,

$$
\left\|R_{\lambda}(\mathbf{T})\right\|_{\mathcal{L}(X)} \leq \frac{1}{|\lambda|\left(1-\left\|\frac{\mathbf{T}}{\lambda}\right\|_{\mathcal{L}(X)}\right)} \rightarrow 0 \quad \text { si } \quad|\lambda| \rightarrow \infty
$$

\footnotetext{
${ }^{3}$ Dados un conjunto $\mathcal{D}$ abierto en $\mathbb{C}$ y $\mathbf{S}_{\lambda}: \mathcal{D} \rightarrow \mathcal{L}(X)$, una función cuyos valores son operadores en $X$, se dice que $\mathbf{S}_{\lambda}$ es localmente holomorfa en $\mathcal{D}$ si para todo $x \in X, f \in X^{*}$, la función $h(\lambda):=f\left(\mathbf{S}_{\lambda} x\right)$ es holomorfa en $\mathcal{D}$.
} 
El espectro de $\mathbf{T}$ es el conjunto de puntos definido por

$$
\sigma(\mathbf{T}):=\mathbb{C} \backslash \rho(\mathbf{T})=\{\lambda \in \mathbb{C}: \mathcal{N}(\mathbf{T}-\lambda \mathbf{I}) \neq\{0\} \text { o } \mathcal{R}(\mathbf{T}-\lambda \mathbf{I}) \neq X\} ;
$$

es decir, son los $\lambda \in \mathbb{C}$ tales que $(\mathbf{T}-\lambda \mathbf{I})$ no tiene inversa en $X$. Como consecuencia de los resultados anteriores, el espectro de un operador lineal y acotado en un espacio de Hilbert $X$ es un conjunto cerrado, no vacío, y satisface

$$
\sigma(\mathbf{T}) \subset\left\{\lambda \in \mathbb{C}:|\lambda| \leq\|\mathbf{T}\|_{\mathcal{L}(X)}\right\}
$$

A los números $\lambda \in \sigma(\mathbf{T})$ se los denomina puntos espectrales de $\mathbf{T}$. Los puntos espectrales de $\mathbf{T}$ se pueden clasificar de la siguiente manera:

- un punto $\lambda$ pertenece al espectro puntual $\sigma_{p}(\mathbf{T})$ de $\mathbf{T}$ si $(\mathbf{T}-\lambda \mathbf{I})$ no es inyectivo; $\lambda$ es un autovalor de $T$,

- un punto $\lambda$ pertenece al espectro continuo $\sigma_{c}(\mathbf{T})$ de $\mathbf{T}$ si $(\mathbf{T}-\lambda \mathbf{I})$ es inyectivo pero no suryectivo y $\mathcal{R}(\mathbf{T}-\lambda \mathbf{I})$ es denso en $X$,

- un punto $\lambda$ pertenece al espectro residual $\sigma_{r}(\mathbf{T})$ de $\mathbf{T}$ si $(\mathbf{T}-\lambda \mathbf{I})$ es inyectivo pero no suryectivo y $\mathcal{R}(\mathbf{T}-\lambda \mathbf{I})$ no es denso en $X$.

De la clasificación anterior se deduce que

$$
\mathbb{C}=\rho(\mathbf{T}) \cup \sigma(\mathbf{T})=\rho(\mathbf{T}) \cup \sigma_{p}(\mathbf{T}) \cup \sigma_{c}(\mathbf{T}) \cup \sigma_{r}(\mathbf{T})
$$

y que la unión es disjunta, obteniéndose así una partición de $\mathbb{C}$.

Existe una relación (relativamente simple) entre los conjuntos espectrales correspondientes a $\mathbf{T}$ y a su operador adjunto. En efecto, si $\mathbf{T}^{*} \in \mathcal{L}(X)$ es el operador adjunto de $\mathbf{T} \in \mathcal{L}(X)$, entonces [131]

- $\lambda \in \rho(\mathbf{T})$ si, y solo si, $\bar{\lambda} \in \rho\left(\mathbf{T}^{*}\right)$,

- $\lambda \in \sigma(\mathbf{T})$ si, y solo si, $\bar{\lambda} \in \sigma\left(\mathbf{T}^{*}\right)$,

- $\lambda \in \sigma_{c}(\mathbf{T})$ si, y solo si, $\bar{\lambda} \in \sigma_{c}\left(\mathbf{T}^{*}\right)$,

- si $\lambda \in \sigma_{r}(\mathbf{T}), \bar{\lambda} \in \sigma_{p}\left(\mathbf{T}^{*}\right)$,

- si $\lambda \in \sigma_{p}(\mathbf{T}), \bar{\lambda} \in \sigma_{p}\left(\mathbf{T}^{*}\right) \cup \sigma_{r}\left(\mathbf{T}^{*}\right)$.

Si bien $\sigma(\mathbf{T}) \neq \emptyset$, cualquiera de las partes disjuntas que componen el espectro de $\mathbf{T}$ podría ser vacía. Por ejemplo,

- si $\mathbf{T} \in \mathcal{L}(X)$ es autoadjunto, $\sigma(\mathbf{T})$ es real y, por lo tanto, $\sigma_{r}(\mathbf{T})=\emptyset$.

En general, si $\sigma_{p}(\mathbf{T}) \neq \emptyset$, cualquier conjunto de vectores propios asociados con valores propios distintos será linealmente independiente.

Asumamos ahora que $\lambda \neq 0$ es un punto aislado del espectro de T. Sea $\gamma$ una curva de Jordan cerrada en $\rho(\mathbf{T})$ alrededor de $\lambda$. Supongamos que el origen y todos los puntos pertenecientes a $\sigma(\mathbf{T})$ distintos de $\lambda$ están en el exterior de $\gamma$. La proyección espectral asociada con $\mathbf{T}$ relativa a $\lambda$ se define mediante la integral de Dunford ${ }^{4}$

$$
\mathbf{E}_{\lambda}:=-\frac{1}{2 \pi i} \int_{\gamma} R_{z}(\mathbf{T}) d z
$$

Claramente, la definición no depende de $\gamma$. Además, $\mathbf{E}_{\lambda} \in \mathcal{L}(X)$ y se verifican

\footnotetext{
${ }^{4}$ Sea $H$ un espacio de Hilbert complejo. Sea $\mathbf{A}$ un operador acotado sobre $H$. Sea $f$ es una función analítica en $\Delta$, un subconjunto abierto de $\mathbb{C}$ tal que $\sigma(\mathbf{A}) \subset \Delta$. Entonces $f(\mathbf{A})$ denotará al operador en $H$ definido por la integral de Dunford-Taylor [124]

$$
f(\mathbf{A}):=\frac{1}{2 \pi i} \int_{C} f(z)(z \mathbf{I}-\mathbf{A})^{-1} d z
$$

donde $C$ es una colección de curvas de Jordan en $\Delta$, orientadas positivamente, tales que $\sigma(\mathbf{A})$ queda en el interior de $C$.
} 
$-\mathbf{T} \circ \mathbf{E}_{\lambda}=\mathbf{E}_{\lambda} \circ \mathbf{T}$,

$-\mathbf{E}_{\lambda} \circ \mathbf{E}_{\lambda}=\mathbf{E}_{\lambda}$

- $\mathbf{E}_{\lambda} \circ \mathbf{E}_{\mu}=0 \quad$ si $\lambda \neq \mu$,

- $\mathcal{R}\left(\mathbf{E}_{\lambda}\right)$ es $\mathbf{T}$-invariante,

- $\mathcal{N}\left(\mathbf{E}_{\lambda}\right)$ es $\mathbf{T}$-invariante.

Hagamos $X_{1}=\mathcal{R}\left(\mathbf{E}_{\lambda}\right)$ y $X_{2}=\mathcal{N}\left(\mathbf{E}_{\lambda}\right)$, entonces

- $X=X_{1} \oplus X_{2}$

- $\left.\mathbf{T}\right|_{X_{j}} \in \mathcal{L}\left(X_{j}\right) \quad j=1,2$,

- $\sigma\left(\left.\mathbf{T}\right|_{X_{1}}\right)=\{\lambda\}$,

- $\sigma(\mathbf{T})=\{\lambda\} \cup \sigma\left(\left.\mathbf{T}\right|_{X_{2}}\right)$,

- si $\mu \in \rho(\mathbf{T})=\rho\left(\left.\mathbf{T}\right|_{X_{1}}\right) \cap \rho\left(\left.\mathbf{T}\right|_{X_{2}}\right), R_{\mu}\left(\left.\mathbf{T}\right|_{X_{j}}\right)=\left.R_{\mu}(\mathbf{T})\right|_{X_{j}} \quad j=1,2$.

El proyector espectral $\mathbf{E}_{\lambda}$ es el operador idempotente de Riesz correspondiente a $\lambda$ [80].

A continuación, introduciremos un subconjunto de $\sigma(\mathbf{T})$ que jugará un rol importante en lo que sigue. Definamos

$$
\sigma_{0}(\mathbf{T}):=\left\{\lambda \in \sigma(\mathbf{T}):(\mathbf{T}-\lambda \mathbf{I}) \text { es un operador de Fredholm con índice cero }{ }^{5}\right\},
$$

que también puede escribirse como

$$
\sigma_{0}(\mathbf{T})=\left\{\lambda \in \sigma_{p}(\mathbf{T}): \overline{\mathcal{R}(\mathbf{T}-\lambda \mathbf{I})}=\mathcal{R}(\mathbf{T}-\lambda \mathbf{I}) \neq X \quad \mathrm{y} \operatorname{dim} \mathcal{N}(\mathbf{T}-\lambda \mathbf{I})=\operatorname{dim} \mathcal{N}\left(\mathbf{T}^{*}-\bar{\lambda} \mathbf{I}\right)\right\} .
$$

Tendremos $[80,131]$,

- $\lambda \in \sigma_{0}(\mathbf{T})$ si, y solo si, $\bar{\lambda} \in \sigma_{0}\left(\mathbf{T}^{*}\right)$,

- si $\lambda$ es un punto aislado de $\sigma(\mathbf{T}), \lambda \in \sigma_{0}(\mathbf{T})$ si, y solo si, $\mathbf{E}_{\lambda}$ tiene rango finito.

Designemos por $\pi_{0}(\mathbf{T})$ al siguiente conjunto:

$$
\pi_{0}(\mathbf{T}):=\left\{\lambda \in \sigma_{p}(\mathbf{T}): \lambda \text { es un punto aislado de } \sigma(\mathbf{T}) \mathrm{y} \operatorname{dim} \mathcal{R}\left(\mathbf{E}_{\lambda}\right)<\infty\right\} .
$$

Entonces, es posible escribir

$$
\sigma_{0}(\mathbf{T})=\tau_{0}(\mathbf{T}) \cup \pi_{0}(\mathbf{T}),
$$

donde $\tau_{0}(\mathbf{T})$ es un conjunto abierto del plano complejo que está incluido en $\sigma_{p}(\mathbf{T})$. El conjunto $\pi_{0}(\mathbf{T})$ se denomina conjunto de puntos de Riesz; también es conocido como el conjunto de autovalores aislados de T con multiplicidad algebraica finita (ver más adelante).

El núcleo del operador $\mathbf{T}-\lambda \mathbf{I}$ se denomina subespacio propio de $\mathbf{T}$ asociado con $\lambda$ o, brevemente, $\lambda$-espacio de $\mathbf{T}$. Es inmediato que

- $\mathcal{N}(\mathbf{T}-\lambda \mathbf{I})$ es un subespacio vectorial cerrado de $X$

$-\mathcal{N}(\mathbf{T}-\lambda \mathbf{I}) \neq 0$ si, y solo si, $\lambda \in \sigma_{p}(\mathbf{T})$,

- $\mathcal{N}(\mathbf{T}-\lambda \mathbf{I})$ es $\mathbf{T}$-invariante.

\footnotetext{
${ }^{5}$ Un mapeo $\mathbf{A} \in \mathcal{L}(X)$ es un operador de Fredholm si: i) $\mathcal{R}(\mathbf{A})$ es cerrado, ii $) \operatorname{dim} \mathcal{N}(\mathbf{A})<\infty$ y $\operatorname{codim} \mathcal{R}(\mathbf{A})<\infty$. El índice de un operador de Fredholm es el número entero definido por
}

$$
\operatorname{Ind}(\mathbf{A}):=\operatorname{dim} \mathcal{N}(\mathbf{A})-\operatorname{codim} \mathcal{R}(\mathbf{A})
$$


Supongamos que $\lambda \in \pi_{0}(\mathbf{T})$. Se define la multiplicidad ascendente de $\lambda$ como el menor número entero $l$ tal que $\mathcal{N}\left((\mathbf{T}-\lambda \mathbf{I})^{l+1}\right)=\mathcal{N}\left((\mathbf{T}-\lambda \mathbf{I})^{l}\right)$. A la dimensión del espacio $\mathcal{N}\left((\mathbf{T}-\mathbf{I} \lambda)^{l}\right)$ se la denomina multiplicidad algebraica de $\lambda$ y los elementos de $\mathcal{N}\left((\mathbf{T}-\lambda \mathbf{I})^{l}\right)$ son los vectores propios generalizados de $\mathbf{T}$ asociados con $\lambda$. Un vector propio generalizado se dice de orden $k$, si pertenece a $\mathcal{N}\left((\mathbf{T}-\lambda \mathbf{I})^{k}\right)$ pero no pertenence a $\mathcal{N}\left((\mathbf{T}-\lambda \mathbf{I})^{k-1}\right)$. Los vectores propios generalizados de orden 1 se denominan autovectores de $\mathbf{T}$ asociados con $\lambda$. La dimensión del espacio $\mathcal{N}(\mathbf{T}-\lambda \mathbf{I})$ define lo que se conoce como la multiplicidad geométrica de $\lambda$. Si $m$ y $g$ representan a las multiplicidades algebraica y geométrica, respectivamente, tendremos

$$
1 \leq l \leq m \quad \mathrm{y} \quad g \leq m
$$

Para $\lambda \neq 0$, autovalor aislado de $\mathbf{T}$ con multiplicidad algebraica finita $m$, tendremos

- $\mathcal{R}\left(\mathbf{E}_{\lambda}\right)=\mathcal{N}\left((\mathbf{T}-\lambda \mathbf{I})^{m}\right)$,

- para cada $k=1, \cdots, m,(\mathbf{T}-\lambda \mathbf{I})^{k}$ es un operador de Fredholm con índice cero; esto implica que $\operatorname{dim} \mathcal{N}\left((\mathbf{T}-\lambda \mathbf{I})^{k}\right)=\operatorname{dim} \mathcal{N}\left(\left(\mathbf{T}^{*}-\bar{\lambda} \mathbf{I}\right)^{k}\right)$ para cada $k \mathrm{y}$, por lo tanto, las multiplicidades ascendente, algebraica y geométrica de $\bar{\lambda} \in \sigma_{0}\left(\mathbf{T}^{*}\right)$ serán iguales a las respectivas multiplicidades ascendente, algebraica y geométrica de $\lambda \in \sigma_{0}(\mathbf{T})$.

En particular, si $\mathbf{T}$ es autoadjunto, la multiplicidad ascendente de cada autovalor será igual a uno. Esto implica que todos los vectores propios generalizados serán autovectores y que las multiplicidades algebraica y geométrica coinciden. Más aún, autovectores correspondientes a distintos autovalores serán ortogonales.

El espectro de un operador compacto en $X$ tiene una estructura simple, análoga a la de un operador que actúa en un espacio de dimensión finita. En efecto, sea $\mathbf{T} \in \mathcal{L}(X)$ un operador compacto, entonces

- 0 es el único punto de acumulación posible de $\sigma(\mathbf{T})$,

- si $\lambda \in \sigma(\mathbf{T}) \backslash\{0\}, \lambda$ es un punto aislado de $\sigma(\mathbf{T})$,

- $\sigma(\mathbf{T}) \backslash\{0\}$ es un conjunto discreto de $\mathbb{C}$,

- $\sigma(\mathbf{T})$ es un conjunto numerable.

Del análisis anterior, surge que (en ausencia de hipótesis de compacidad) un operador $\mathbf{T} \in \mathcal{L}(X)$ podría no tener autovalores o podría tener autovalores pero no aislados. Sin embargo, desde el punto de vista de las aplicaciones, las situaciones más interesantes están relacionadas con los autovalores aislados de ciertos operadores lineales.

\subsection{El gap entre subespacios invariantes}

A fin de evaluar la distancia entre un $\lambda$-espacio de $\mathbf{T}$ y su aproximación, se necesita primero dar una definición precisa de la noción de distancia entre autoespacios. Dados dos subespacios cerrados $U$ y $V$ de $X$, la proximidad entre ambos puede medirse en términos del gap entre $U$ y $V$ que definiremos por

$$
\widehat{\delta}(U, V):=\operatorname{máx}\{\delta(U, V), \delta(V, U)\},
$$

siendo

$$
\delta(U, V):=\sup _{\substack{u \in U \\\|u\|_{X}=1}} \operatorname{dist}(u, V)=\sup _{\substack{u \in U \\\|u\|_{X}=1}} \inf _{v \in V}\|u-v\|_{X} .
$$

Las siguientes resultados son propiedades importantes que usaremos más adelante (ver también [123]):

- $\delta(U, V)=0$ si, y solo si, $U \subset V$,

- $\delta(U, V)<1$ implica $\operatorname{dim} U \leq \operatorname{dim} V$,

- $\widehat{\delta}(U, V)<1$ implica $\operatorname{dim} U=\operatorname{dim} V$,

- $\operatorname{dim} U=\operatorname{dim} V<\infty$, entonces $\delta(U, V) \leq \delta(V, U)(1-\delta(V, U))^{-1}$. 


\subsection{Principios variacionales}

Supongamos que tenemos un espacio vectorial normado $V$, equipado con la norma $\|\cdot\|_{V}$, una forma bilineal continua $a: V \times V \rightarrow \mathbb{R}$, una forma lineal continua $f: V \rightarrow \mathbb{R}$ y un subconjunto $U$, no vacío, del espacio $V$. Con estos datos, podemos definir los siguientes problemas variacionales.

- Encontrar $u \in U$ tal que

$$
a(u, v-u) \geq f(v-u) \quad \forall v \in U
$$

- Encontrar $u \in U$, siendo $U$ un cono con vértice en 0, tal que

$$
\left\{\begin{array}{l}
a(u, v) \geq f(v) \quad \forall v \in U \\
a(u, u)=f(u),
\end{array}\right.
$$

- Encontrar $u \in U$, siendo $U$ un subespacio de $V$, tal que

$$
a(u, v)=f(v) \quad \forall v \in V .
$$

Cada uno de estos problemas tiene una, y solo una, solución si el espacio $V$ es completo, el subconjunto $U$ de $V$ es cerrado y convexo y la forma bilineal $a$ es continua, $V$-elíptica y simétrica. Si $a$ no fuera simétrica, el problema variacional planteado tendría solución única si el espacio $V$ es un espacio de Hilbert. El siguiente resultado es importante por su amplio rango de aplicabilidad. La prueba puede encontrarse en el libro de P. Ciarlet [75] o de K. Yosida [178].

Teorema 2.1 (Lax-Milgram) Sea $V$ un espacio de Hilbert, a una forma bilineal y $f$ una forma lineal. Supongamos que

- la forma bilineal a es continua; es decir, existe una constante positiva $M$ tal que, para todo $u, v \in V$, $|a(u, v)| \leq M\|u\|_{V}\|v\|_{V}$

- la forma bilineal a es $V$-elíptica; es decir, existe una constante positiva $\alpha$ tal que, para todo $u \in V$, $a(u, u) \geq \alpha\|u\|_{V}^{2}$,

- la forma lineal $f$ es continua; es decir, existe una constante positiva $C$ tal que, para todo $v \in V$, $|f(v)| \leq C\|v\|_{V}$.

Entonces, existe una única $u \in V$ tal que

$$
a(u, v)=f(v) \quad \forall v \in V .
$$

En el caso de espacios de Hilbert complejos y problemas variacionales a valores complejos, el Teorema de Lax-Milgram sigue siendo válido para una forma $a$ bilineal o sesquilineal. La suposición de $V$-elípticidad puede ser relajada a

$$
\operatorname{Re}(a(u, u)) \geq \alpha\|u\|_{V}^{2} \quad \forall u \in V .
$$

El Teorema de Lax-Milgran también puede generalizarse para formas bilineales débilmente coercivas. La siguiente versión fue introducida por J. Nečas [141] y, luego, popularizada por I. Babuška en el contexto de la teoría de los elementos finitos [26].

Teorema 2.2 (Nečas-Babuška) Sean $U$ y $V$ dos espacios de Hilbert, a $U \times V \rightarrow \mathbb{R}$ una forma bilineal continua y $f: V \rightarrow \mathbb{R}$ una forma lineal y continua. Supongamos que existe una constante positiva $M$ tal que

$$
\begin{aligned}
& \text { - } \sup _{\substack{u \in U \\
u \neq 0}} \frac{|a(u, v)|}{\|u\|_{U}} \geq M\|v\|_{V} \quad \forall v \in V, \\
& \text { - } \sup _{v \in V}|a(u, v)|>0 \quad \forall u \in U, \text { con } u \neq 0 .
\end{aligned}
$$


Entonces, existe una única $u \in U$ que satisface

$$
a(u, v)=f(v) \quad \forall v \in V .
$$

Sea $V^{\prime}$ el espacio dual de $V$. El mapeo $V^{\prime} \rightarrow V, f \mapsto v$, introducido en el teorema anterior, es lineal y continuo. A partir de la continuidad, tendremos

$$
\|u\|_{U} \leq \frac{1}{M}\|f\|_{V^{\prime}}
$$

lo que prueba que el problema variacional (2.2) está bien planteado; en el sentido siguiente: la solución existe, es única y varía continuamente con el dato $f$. Las mismas conclusiones son válidas para el problema (2.1).

Si la forma bilineal a fuera simétrica, entonces la solución del problema variacional (2.1) sería también la única solución del siguiente problema de minimización

$$
J(u)=\inf _{v \in V} J(v) \quad \text { donde } \quad J(v):=\frac{1}{2} a(v, v)-f(v) .
$$

En Mecánica, esta funcional representa frecuentemente a la energía del sistema y su minimización sigue del principio de Dirichlet. Más aún, en este caso, las correspondientes ecuaciones de Euler-Lagrange o primera variación (2.1), representan el principio de los trabajos virtuales. 


\section{CAPÍTULO 3}

\section{Aproximación espectral no conforme para operadores acotados}

\subsection{Problemas de autovalores formulados variacionalmente}

Sea $\Omega$ un dominio abierto y acotado en $\mathbb{R}^{n}, n=2$ o 3 , en general, no convexo, con un borde $\Gamma:=\partial \Omega$ Lipschitz-continuo. Asumiremos que $\Gamma$ es suave a trozos.

Sean $X(\Omega)$ y $V(\Omega)$ dos espacios de Hilbert complejos con normas $|\cdot|_{X(\Omega)}$ y $\|\cdot\|_{V(\Omega)}$, respectivamente. Supondremos que $V(\Omega)$ es un subespacio de $X(\Omega)$ y que la inclusión $V(\Omega) \hookrightarrow X(\Omega)$ es continua. Sean $a: V(\Omega) \times V(\Omega) \rightarrow \mathbb{C}$ y $b: X(\Omega) \times X(\Omega) \rightarrow \mathbb{C}$ dos formas sesquilineales continuas. Supondremos que $a$ es coerciva sobre $V(\Omega)$; es decir, existe $\alpha>0$ tal que

$$
\operatorname{Re} a(v, v) \geq \alpha\|v\|_{V(\Omega)}^{2} \quad \forall v \in V(\Omega) .
$$

Consideremos el siguiente problema de autovalores:

Encontrar $\mu \in \mathbb{C}, u \in V(\Omega), u \neq 0$, tal que

$$
a(u, v)=\mu b(u, v) \quad \forall v \in V(\Omega) .
$$

Sea $\mathbf{T}$ el operador lineal definido por

$$
\begin{aligned}
\mathbf{T}: X(\Omega) & \rightarrow V(\Omega) \hookrightarrow X(\Omega) \\
x & \mapsto u,
\end{aligned}
$$

siendo $u \in V(\Omega)$ la solución del siguiente problema variacional

$$
a(u, y)=b(x, y) \quad \forall y \in V(\Omega) .
$$

Como $a$ es coerciva, $b$ es continua y $V(\Omega) \hookrightarrow X(\Omega)$, la aplicación del Teorema de Lax-Milgram nos permite concluir que $\mathbf{T}$ es un operador acotado. A partir de (3.2), es fácil probar que

- $\mu$ es un autovalor de (3.1) si, y solo si, $\lambda=1 / \mu$ es un autovalor del operador $\mathbf{T}$,

- las autofunciones $u$ correspondientes a los autovalores $\lambda$ y $\mu$ coinciden.

Sea $\Omega_{h}$ una aproximación poligonal del dominio $\bar{\Omega}$, construida de manera tal que

- cada vértice del borde $\Gamma_{h}:=\partial \Omega_{h}$ es también un elemento de $\Gamma$,

- cada punto donde $\Gamma$ deja de ser suave es un vértice de $\Gamma_{h}$. 


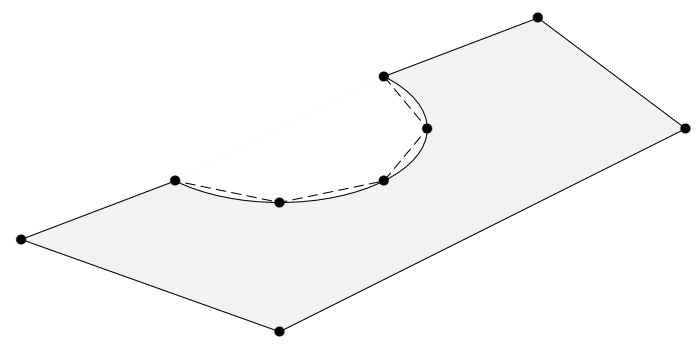

Figura 3.1: Dominio de cálculo $\Omega_{h}$ y dominio original $\Omega$.

A continuación, nos veremos obligados a trabajar con funciones definidas sobre los dominios discretos $\Omega_{h}$. Observemos que, en general, $\Omega_{h} \not \subset \Omega$.

Consideraremos primero los espacios de funciones $X\left(\Omega_{h}\right)$ y $V\left(\Omega_{h}\right)$. Definiremos a estos espacios como extensiones naturales de los espacios $X(\Omega)$ y $V(\Omega)$, en el siguiente sentido: los elementos de $X\left(\Omega_{h}\right)$ y $V\left(\Omega_{h}\right)$ son funciones complejas que tienen la misma regularidad que las funciones pertenecientes a $X(\Omega)$ y $V(\Omega)$, respectivamente, pero que están definidas sobre los dominios $\Omega_{h}$. Estos espacios quedan naturalmente equipados con las normas $|\cdot|_{X\left(\Omega_{h}\right)}$ y $\|\cdot\|_{V\left(\Omega_{h}\right)}$, respectivamente, y verifican la inclusión $V\left(\Omega_{h}\right) \hookrightarrow X\left(\Omega_{h}\right)$.

Consideraremos ahora una familia de espacios de dimensión finita $\left\{V_{h}\left(\Omega_{h}\right)\right\}_{0<h<1}$ que satisface la inclusión $V_{h}\left(\Omega_{h}\right) \subset X\left(\Omega_{h}\right)$ para todo $h$. Típicamente, la función $N(h):=\operatorname{dim} V_{h}\left(\Omega_{h}\right)$ crece de manera monótona a medida que el parámetro $h$ decrece. Supondremos que cada espacio $V_{h}\left(\Omega_{h}\right)$ está equipado con la norma $\|\cdot\|_{V_{h}\left(\Omega_{h}\right)}$ y que se verifican

$$
\begin{aligned}
\|v\|_{V_{h}\left(\Omega_{h}\right)} & =\|v\|_{V\left(\Omega_{h}\right)} \quad \forall v \in V\left(\Omega_{h}\right), \\
\left(V_{h}\left(\Omega_{h}\right),\|\cdot\|_{V_{h}\left(\Omega_{h}\right)}\right) & \hookrightarrow\left(X\left(\Omega_{h}\right),|\cdot|_{X\left(\Omega_{h}\right)}\right) \quad \text { uniformemente en } h .
\end{aligned}
$$

Sean $a_{h}: V_{h}\left(\Omega_{h}\right) \times V_{h}\left(\Omega_{h}\right) \rightarrow \mathbb{C}$ y $b_{h}: X\left(\Omega_{h}\right) \times X\left(\Omega_{h}\right) \rightarrow \mathbb{C}$ las formas sesquilineales obtenidas mediante la discretización de las formas continuas $a$ y $b$, respectivamente. A continuación asumiremos que existen constantes positivas $\widehat{\alpha}, \widehat{\beta}$ y $\widehat{\gamma}$, independientes de $h$, tales que

$$
\begin{gathered}
\operatorname{Re} a_{h}\left(u_{h}, u_{h}\right) \geq \widehat{\alpha}\left\|u_{h}\right\|_{V_{h}\left(\Omega_{h}\right)}^{2} \quad \forall u_{h} \in V_{h}\left(\Omega_{h}\right), \\
\left|a_{h}\left(u_{h}, v_{h}\right)\right| \leq \widehat{\beta}\left\|u_{h}\right\|_{V_{h}\left(\Omega_{h}\right)}\left\|v_{h}\right\|_{V_{h}\left(\Omega_{h}\right)} \quad \forall u_{h}, v_{h} \in V_{h}\left(\Omega_{h}\right), \\
\left|b_{h}\left(u_{h}, v_{h}\right)\right| \leq \widehat{\gamma}\left|u_{h}\right|_{X\left(\Omega_{h}\right)}\left|v_{h}\right|_{X\left(\Omega_{h}\right)} \quad \forall u_{h}, v_{h} \in X_{h}\left(\Omega_{h}\right),
\end{gathered}
$$

Consideremos el problema de autovalores discreto:

Encontrar $\mu_{h} \in \mathbb{C}, u_{h} \in V_{h}\left(\Omega_{h}\right), u_{h} \neq 0$, tal que

$$
a_{h}\left(u_{h}, v_{h}\right)=\mu_{h} b_{h}\left(u_{h}, v_{h}\right) \quad \forall v_{h} \in V_{h}\left(\Omega_{h}\right) .
$$

Definimos el operador discreto $\mathbf{T}_{h}$, análogo al operador solución $\mathbf{T}$, como sigue:

$$
\begin{aligned}
\mathbf{T}_{h}: X\left(\Omega_{h}\right) & \rightarrow V_{h}\left(\Omega_{h}\right) \\
x & \mapsto u_{h},
\end{aligned}
$$

siendo $u_{h} \in V_{h}\left(\Omega_{h}\right)$ la solución del siguiente problema variacional

$$
a_{h}\left(u_{h}, y_{h}\right)=b_{h}\left(x, y_{h}\right) \quad \forall y_{h} \in V_{h}\left(\Omega_{h}\right) .
$$

Aplicando nuevamente el Teorema de Lax-Milgram, podemos concluir que el operador $\mathbf{T}_{h}$ es un operador lineal y acotado. Debido a las propiedades (3.4)-(3.7), $\mathbf{T}_{h}$ es acotado uniformemente en $h$. Como en el caso continuo, es simple probar que 
- $\mu_{h}$ es un autovalor de (3.8) si, y solo si, $\lambda_{h}=1 / \mu_{h}$ es un autovalor del operador $\mathbf{T}_{h}$,

- las autofunciones $u_{h}$ correspondientes a $\lambda_{h} \mathrm{y} \mu_{h}$ coinciden.

Observación 3.1 Para poder aproximar numéricamente el problema (3.1), fue necesario primero aproximar el dominio $\Omega$ mediante un dominio poligonal $\Omega_{h}$. En general, $\Omega_{h} \not \subset \Omega$ pero $\Omega_{h} \rightarrow \Omega$ a medida que $h$ va a cero ya que hemos asumido que $\Gamma$ es suave a trozos, que los vértices de $\Gamma$ son también vértices de $\Gamma_{h}$ y que todos los vértices de $\Gamma_{h}$ están sobre $\Gamma$. En esta situación, decimos que $\Omega_{h}$ es una aproximación externa de $\Omega$ (la terminología aqui empleada se debe a las definiciones introducidas en [165] y las referencias allí citadas). Como consecuencia de esta perturbación en el dominio, $V_{h}\left(\Omega_{h}\right) \not \subset V(\Omega)$. Por lo tanto, (3.8) representará siempre una aproximación no conforme de (3.1).

En lo que sigue, mientras no se especifique lo contrario, $C$ denotará una constante positiva genérica, no necesariamente la misma cada vez que aparezca, pero siempre independiente de $h$.

\subsection{Definición de los operadores solución}

Al tratar de analizar la convergencia del problema (3.8) surgen las siguientes dificultades:

- el operador $\mathbf{T}$ es acotado pero no necesariamente compacto,

- los operadores $\mathbf{T}$ y $\mathbf{T}_{h}$ están definidos sobre dominios distintos,

- el dominio de definición del operador $\mathbf{T}_{h}$ cambia con $h$.

Para poder avanzar en nuestro estudio, necesitaremos considerar operadores $\mathbf{A}$ y $\mathbf{A}_{h}$ que estén definidos sobre un mismo dominio y cuyos espectros se relacionen directamente con los espectros de los operadores T y $\mathbf{T}_{h}$, respectivamente. Esto puede lograrse introduciendo operadores de extensión adecuados.

Comenzaremos con la siguiente definición. Para cada aproximación $\Omega_{h}$ del dominio $\Omega$, definimos

$$
\widehat{\Omega}:=\Omega \cup \Omega_{h} .
$$

Sobre los dominios $\widehat{\Omega}$, consideraremos los espacios de funciones $X(\widehat{\Omega})$ y $V(\widehat{\Omega})$ definidos como extensiones naturales (en el sentido dado en la sección anterior) de los espacios $X(\Omega)$ y $V(\Omega)$, respectivamente.

Introduciremos ahora la norma discreta $\|\cdot\|_{h}$ que asumiremos definida sobre funciones con dominio $\widehat{\Omega}$. Denotaremos por $\|\cdot\|_{h, \mathcal{D}}$ a la restricción canónica de la norma $\|\cdot\|_{h}$ sobre cualquier conjunto abierto $\mathcal{D}$ contenido en $\widehat{\Omega}$. A esta nueva norma la pensaremos inducida por la norma $\|\cdot\|_{V_{h}\left(\Omega_{h}\right)}$, definiéndola con las siguientes propiedades:

$$
\begin{aligned}
& \text { - }\|v\|_{h, \Omega_{h}} \simeq\|v\|_{V_{h}\left(\Omega_{h}\right)} \quad \text { si }\left.\quad v\right|_{\Omega_{h}} \in V_{h}\left(\Omega_{h}\right), \\
& \text { - }\|v\|_{h, \Omega} \simeq\|v\|_{V(\Omega)} \quad \text { si }\left.\quad v\right|_{\Omega} \in V(\Omega), \\
& \text { - }\|v\|_{h} \simeq\|v\|_{V(\widehat{\Omega})} \quad \text { si } \quad v \in V(\widehat{\Omega}) .
\end{aligned}
$$

A continuación, asumiremos que existe un operador de extensión, que actúa sobre las funciones del continuo, con las siguientes propiedades:

$$
\begin{aligned}
& \text { - } \mathbf{P}: V(\Omega) \rightarrow V(\widehat{\Omega}), u \mapsto u^{e}, \\
& \text { - }\left.u^{e}\right|_{\Omega}=u, \\
& \text { - }\left|u^{e}\right|_{X(\widehat{\Omega})} \leq C\left\|u^{e}\right\|_{h} \leq C\|u\|_{V(\Omega)}
\end{aligned}
$$

De manera análoga, asumiremos que existe un operador de extensión, que actúa sobre las funciones de los espacios discretos, con las siguientes propiedades: 


$$
\begin{aligned}
& \text { - } \mathbf{P}_{h}: V_{h}\left(\Omega_{h}\right) \rightarrow X(\widehat{\Omega}), u_{h} \mapsto u_{h}^{e}, \\
& \text { - }\left.u_{h}^{e}\right|_{\Omega_{h}}=u_{h}, \\
& \text { - }\left|u_{h}^{e}\right|_{X(\widehat{\Omega})} \leq C\left\|u_{h}^{e}\right\|_{h} \leq C\left\|u_{h}\right\|_{V_{h}\left(\Omega_{h}\right)} .
\end{aligned}
$$

Ahora, para cada aproximación $\Omega_{h}$, definimos

$$
\begin{aligned}
V^{e}(\widehat{\Omega}) & :=\mathbf{P}(V(\Omega)) \subset V(\widehat{\Omega}), \\
V_{h}^{e}(\widehat{\Omega}) & :=\mathbf{P}_{h}\left(V_{h}\left(\Omega_{h}\right)\right) \subset X(\widehat{\Omega}), \\
W(\widehat{\Omega}) & :=\left(V^{e}+V_{h}^{e}\right)(\widehat{\Omega}),
\end{aligned}
$$

y equipamos al espacio suma $W(\widehat{\Omega})$ con la norma $\|\cdot\|_{h}$ que (por construcción) satisface

$$
\begin{aligned}
\|v\|_{h} & \simeq\|v\|_{V(\widehat{\Omega})} \quad \forall v \in V(\widehat{\Omega}), \\
\left(W(\widehat{\Omega}),\|\cdot\|_{h}\right) & \hookrightarrow\left(X(\widehat{\Omega}),|\cdot|_{X(\widehat{\Omega})}\right) \quad \text { uniformemente en } h .
\end{aligned}
$$

Consideremos el operador lineal definido por:

$$
\begin{aligned}
\mathbf{A}: X(\widehat{\Omega}) & \rightarrow V^{e}(\widehat{\Omega}) \subset W(\widehat{\Omega}) \hookrightarrow X(\widehat{\Omega}) \\
x & \mapsto u^{e}=\mathbf{P} \circ \mathbf{T}\left(\left.x\right|_{\Omega}\right) .
\end{aligned}
$$

Observemos que como $\mathbf{T} \in \mathcal{L}(X(\Omega), V(\Omega))$, entonces $\mathbf{A} \in \mathcal{L}(X(\widehat{\Omega}))$. En efecto, para cualquier $x \in X(\widehat{\Omega})$, $\mathbf{A} x \in W(\widehat{\Omega})$. Luego, usando (3.11), la definición de $\mathbf{A}$ y las propiedades de $\mathbf{P}$, obtenemos

$$
|\mathbf{A} x|_{X(\widehat{\Omega})} \leq C\|\mathbf{A} x\|_{h}=C\left\|\mathbf{P} \circ \mathbf{T}\left(\left.x\right|_{\Omega}\right)\right\|_{V(\widehat{\Omega})} \leq C\left\|\mathbf{T}\left(\left.x\right|_{\Omega}\right)\right\|_{V(\Omega)} \leq C|x|_{X(\widehat{\Omega})} .
$$

También es posible probar que $\mathbf{A} \in \mathcal{L}(W(\widehat{\Omega}))$. A partir de la desigualdad anterior y usando una vez más la propiedad (3.11), tenemos

$$
\|\mathbf{A} x\|_{h} \leq C|x|_{X(\widehat{\Omega})} \leq C\|x\|_{h} .
$$

La contrapartida discreta del operador $\mathbf{A}$ se define de manera análoga:

$$
\begin{aligned}
\mathbf{A}_{h}: X(\widehat{\Omega}) & \rightarrow V_{h}^{e}(\widehat{\Omega}) \subset W(\widehat{\Omega}) \hookrightarrow X(\widehat{\Omega}) \\
x & \mapsto u_{h}^{e}=\mathbf{P}_{h} \circ \mathbf{T}_{h}\left(\left.x\right|_{\Omega_{h}}\right) .
\end{aligned}
$$

Como en el caso continuo, dado que el operador $\mathbf{T}_{h} \in \mathcal{L}\left(X\left(\Omega_{h}\right), V_{h}\left(\Omega_{h}\right)\right)$, $\mathbf{A}_{h}$ será un operador acotado. En efecto, para cualquier $x \in X(\widehat{\Omega}), \mathbf{A}_{h} x \in W(\widehat{\Omega})$. Luego, usando (3.11), la definición de $\mathbf{A}_{h}$ y las propiedades de $\mathbf{P}_{h}$,

$$
\left|\mathbf{A}_{h} x\right|_{X(\widehat{\Omega})} \leq C\left\|\mathbf{A}_{h} x\right\|_{h}=C\left\|\mathbf{P}_{h} \circ \mathbf{T}_{h}\left(\left.x\right|_{\Omega_{h}}\right)\right\|_{h} \leq C\left\|\mathbf{T}_{h}\left(\left.x\right|_{\Omega_{h}}\right)\right\|_{V_{h}\left(\Omega_{h}\right)} \leq C|x|_{X(\widehat{\Omega})} .
$$

Finalmente, si $x \in W(\widehat{\Omega})$, tendremos

$$
\left\|\mathbf{A}_{h} x\right\|_{h} \leq C|x|_{X(\widehat{\Omega})} \leq C\|x\|_{h} .
$$




\subsection{Resultados abstractos sobre aproximación espectral}

Consideremos las restricciones $\boldsymbol{\Upsilon}:=\left.\mathbf{T}\right|_{V(\Omega)}$ y $\boldsymbol{\Lambda}:=\left.\mathbf{A}\right|_{W(\widehat{\Omega})}$. A continuación probaremos que toda la información que necesitemos sobre el espectro de $\boldsymbol{\Upsilon}$ podremos obtenerla analizando el espectro de $\boldsymbol{\Lambda}$. La prueba que aquí se presenta sigue los argumentos usados en la prueba del Lema 4.1 en [33].

Lema 3.2 Los espectros de $\boldsymbol{\Upsilon}$ y $\boldsymbol{\Lambda}$ satisfacen la siguiente relación

$$
\sigma(\mathbf{\Upsilon}) \cup\{0\}=\sigma(\boldsymbol{\Lambda}) .
$$

Prueba. Elijamos $z \notin \sigma(\mathbf{\Upsilon}), z \neq 0$. Vamos a probar que $(z-\boldsymbol{\Lambda}) \in \mathcal{L}(W(\widehat{\Omega}))$ es biyectiva. Luego, por el Teorema de la aplicación abierta ${ }^{1},(z-\boldsymbol{\Lambda})^{-1} \in \mathcal{L}(W(\widehat{\Omega}))$ (ya que la inversa de una aplicación abierta biyectiva es continua) y, por lo tanto, $z \notin \sigma(\boldsymbol{\Lambda})$.

Escojamos $x \in W(\widehat{\Omega})$ de manera tal que $(z-\boldsymbol{\Lambda}) x=0$. Como $\boldsymbol{\Lambda} x \in V^{e}(\widehat{\Omega})$, para todo $x \in W(\widehat{\Omega})$, tendremos directamente que

$$
x=\frac{1}{z} \boldsymbol{\Lambda} x \in V^{e}(\widehat{\Omega}) .
$$

Entonces, $(z-\boldsymbol{\Lambda}) x=0$ equivale a $\left(z-\left.\boldsymbol{\Lambda}\right|_{V^{e}(\widehat{\Omega})}\right) x=0$. De la definición del espacio $V^{e}(\widehat{\Omega})$ y del operador A, obtenemos

$$
0=z x-\mathbf{P} \circ \mathbf{\Upsilon}\left(\left.x\right|_{\Omega}\right)=(z-\mathbf{\Upsilon})\left(\left.x\right|_{\Omega}\right) \chi(\Omega)+\left(\mathbf{P} \circ(z-\mathbf{\Upsilon})\left(\left.x\right|_{\Omega}\right)\right) \chi(\widehat{\Omega}-\Omega),
$$

donde $\chi(\Omega)$ y $\chi(\widehat{\Omega}-\Omega)$ son las funciones características de los subconjuntos $\Omega$ y $\widehat{\Omega}-\Omega$, respectivamente. Luego, $(z-\mathbf{\Upsilon})\left(\left.x\right|_{\Omega}\right)=0$. Esto implica que $\left.x\right|_{\Omega}=0$ pues, como $z \notin \sigma(\mathbf{\Upsilon}), z-\boldsymbol{\Upsilon}$ es una aplicación uno a uno. Finalmente, de las propiedades del operador $\mathbf{P}$, concluimos que $x=0$ en $\widehat{\Omega}$. Entonces, $z-\boldsymbol{\Lambda}$ es una aplicación inyectiva.

Ahora, dado $y \in W(\widehat{\Omega})$, tendremos que $\Lambda y \in V^{e}(\widehat{\Omega})$. Esto nos permite definir las siguientes funciones:

$$
\begin{gathered}
x=\left.(z-\mathbf{\Upsilon})^{-1}(\boldsymbol{\Lambda} y)\right|_{\Omega} \in V(\Omega), \\
\widehat{x}=\frac{1}{z}(y+\mathbf{P} x) \in W(\widehat{\Omega}) .
\end{gathered}
$$

Teniendo en cuenta que $\left.\mathbf{P} x\right|_{\Omega}=x$, es fácil comprobar que $(z-\boldsymbol{\Lambda}) \widehat{x}=y$. Como $y$ es un elemento arbitrario de $W(\widehat{\Omega})$, entonces, $z-\boldsymbol{\Lambda}$ es una aplicación exhaustiva.

Recíprocamente, supongamos $z \notin \sigma(\boldsymbol{\Lambda})$. Observemos primero que, necesariamente, $z \neq 0$ ya que $z-\boldsymbol{\Lambda}$ no define una aplicación exhaustiva si $z=0$ (recordemos que $\boldsymbol{\Lambda}(W(\widehat{\Omega})) \subset V(\widehat{\Omega})$ ). Ahora, dado $y \in V(\Omega)$, existe un único elemento $x \in W(\widehat{\Omega})$ tal que $(z-\boldsymbol{\Lambda}) x=\mathbf{P} y$ ya que, por hipótesis, $z \notin \sigma(\boldsymbol{\Lambda})$. Es claro que

$$
x=\frac{1}{z}(\mathbf{P} y+\mathbf{\Lambda} x) \in V^{e}(\widehat{\Omega}) .
$$

Luego, existe un único $\left.x\right|_{\Omega} \in V(\Omega)$ tal que

$$
(z-\mathbf{\Lambda}) x=\left(z x-\mathbf{P} \circ \mathbf{\Upsilon}\left(\left.x\right|_{\Omega}\right)\right)=\mathbf{P} \circ(z-\mathbf{\Upsilon})\left(\left.x\right|_{\Omega}\right)=\mathbf{P} y .
$$

Esto implica que $(z-\mathbf{\Upsilon}): V(\Omega) \rightarrow V(\Omega)$ es invertible y, por lo tanto, $z \notin \sigma(\mathbf{\Upsilon})$.

Debido a que el dominio $\widehat{\Omega}$ depende de $h$, el espacio $W(\widehat{\Omega})$ podría depender de $h$ y, en ese caso, el operador $\boldsymbol{\Lambda}$ dependerá de $h$ también. Observemos primero que, para $z$ fijo en $\rho(\boldsymbol{\Lambda})$ y cualquier $\widehat{x}$ en $W(\widehat{\Omega})$, tendremos

$$
\|(z-\boldsymbol{\Lambda}) \widehat{x}\|_{h} \leq|z|\|\widehat{x}\|_{h}+\|\boldsymbol{\Lambda} \widehat{x}\|_{h} \leq(|z|+C)\|\widehat{x}\|_{h}
$$

ya que $\boldsymbol{\Lambda}$ es un operador acotado en $W(\widehat{\Omega})$ con constante independiente de $h$ (ver estimación (3.12)). Luego, $z-\boldsymbol{\Lambda}$ define una aplicación en $W(\widehat{\Omega})$ acotada uniformemente en $h$. En el próximo lema probaremos que la inversa de $z-\boldsymbol{\Lambda}$ también está acotada uniformemente en $h$.

\footnotetext{
${ }^{1}$ Sean $X$ e $Y$ dos espacios de Banach y sea $\mathcal{T}: X \rightarrow Y$ una aplicación lineal, acotada y suryectiva. Entonces, $\mathcal{T}$ mapea conjuntos abiertos de $X$ en conjuntos abiertos de $Y$.
} 
Lema 3.3 Sea $z \in \rho(\boldsymbol{\Lambda}), z \neq 0$, fijo. Existe una constante positiva $C$, que depende de $\Omega y|z|$, tal que

$$
\left\|(z-\boldsymbol{\Lambda})^{-1}\right\|_{h} \leq C .
$$

Prueba. Fijemos $z \in \rho(\boldsymbol{\Lambda}), z \neq 0$ y elijamos $\widehat{y} \in W(\widehat{\Omega})$ arbitrariamente. Procediendo como en la prueba del lema anterior, es simple probar que

$$
\widehat{x}=\frac{1}{z}\left(\widehat{y}+\mathbf{P} \circ(z-\mathbf{\Upsilon})^{-1}\left(\left.\boldsymbol{\Lambda} \widehat{y}\right|_{\Omega}\right)\right),
$$

es el único elemento de $W(\widehat{\Omega})$ que verifica $(z-\boldsymbol{\Lambda}) \widehat{x}=\widehat{y}$. Como $\boldsymbol{\Lambda} \widehat{y}$ pertenece a $V^{e}(\widehat{\Omega})$, tendremos que $(z-\boldsymbol{\Upsilon})^{-1}\left(\left.\boldsymbol{\Lambda} \widehat{y}\right|_{\Omega}\right) \in V(\Omega)$ y, por lo tanto, podremos escribir

$$
\left\|\mathbf{P} \circ(z-\mathbf{\Upsilon})^{-1}\left(\left.\boldsymbol{\Lambda} \widehat{y}\right|_{\Omega}\right)\right\|_{h} \leq C\left\|(z-\mathbf{\Upsilon})^{-1}\left(\left.\boldsymbol{\Lambda} \widehat{y}\right|_{\Omega}\right)\right\|_{V(\Omega)} .
$$

A partir de este resultado, obtendremos

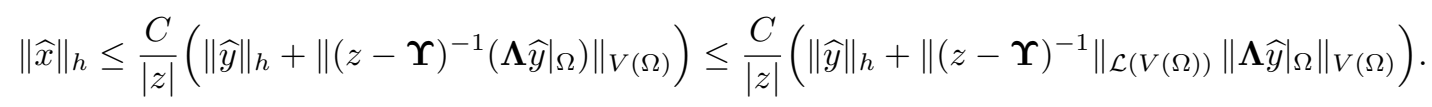

Finalmente, podemos usar (3.12) para obtener

$$
\left\|\left.\boldsymbol{\Lambda} \widehat{y}\right|_{\Omega}\right\|_{V(\Omega)} \leq\|\boldsymbol{\Lambda} \widehat{y}\|_{V(\widehat{\Omega})} \simeq\|\boldsymbol{\Lambda} \widehat{y}\|_{h} \leq C\|\widehat{y}\|_{h} .
$$

La combinación de este resultado con la desigualdad anterior, nos permite completar la prueba.

Corolario 3.4 Sea $\mathcal{G}$ un subconjunto cerrado de $\rho(\boldsymbol{\Lambda})$. Entonces, existe una constante positiva $C$, independiente de $h$, tal que

$$
\left\|(z-\boldsymbol{\Lambda})^{-1}\right\|_{h} \leq C \quad \forall z \in \mathcal{G} .
$$

Prueba. A partir del resultado anterior, la aplicación $z \rightarrow\left\|(z-\boldsymbol{\Lambda})^{-1}\right\|_{h}$ es uniformemente continua para cada $z \in \rho(\boldsymbol{\Lambda})$. Además, como $\boldsymbol{\Lambda} \in \mathcal{L}(W(\widehat{\Omega})),\left\|(z-\boldsymbol{\Lambda})^{-1}\right\|_{h}$ tiende a cero cuando $|z| \rightarrow \infty$. Luego, es claro que $\left\|(z-\boldsymbol{\Lambda})^{-1}\right\|_{h}$ puede acotarse uniformemente, con respecto a $h$ y a $z$, en cualquier conjunto cerrado incluido en $\rho(\boldsymbol{\Lambda})$.

Sea ahora $\lambda$ un autovalor aislado de $\boldsymbol{\Lambda}$, distinto de cero, con multiplicidad algebraica $m$. Sea $\gamma$ un círculo en el plano complejo, centrado en $\lambda$ y contenido en $\rho(\boldsymbol{\Lambda})$, de manera tal que no encierre ningún otro punto pertenenciente a $\sigma(\boldsymbol{\Lambda})$.

Podemos definir los siguientes proyectores espectrales relativos a $\lambda$ :

- asociado con $\boldsymbol{\Lambda}, \mathbf{E}_{\lambda}: W(\widehat{\Omega}) \rightarrow W(\widehat{\Omega})$,

$$
\mathbf{E}_{\lambda}:=\frac{1}{2 \pi i} \int_{\gamma} R_{z}(\boldsymbol{\Lambda}) d z
$$

- asociado con $\boldsymbol{\Upsilon}, \mathcal{E}_{\lambda}: V(\Omega) \rightarrow V(\Omega)$,

$$
\mathcal{E}_{\lambda}:=\frac{1}{2 \pi i} \int_{\gamma} R_{z}(\mathbf{\Upsilon}) d z .
$$

Lema 3.5 Los proyectores espectrales asociados a $\boldsymbol{\Lambda}$ y $\mathbf{T}$ satisfacen la siguiente relación:

$$
\mathbf{E}_{\lambda}(W(\widehat{\Omega}))=\mathbf{P}\left(\mathcal{E}_{\lambda}(V(\Omega))\right) .
$$


Prueba. Recordemos que un vector $\widehat{y} \in \mathbf{E}_{\lambda}(W(\widehat{\Omega}))$ si existe $k$ tal que $(\lambda-\boldsymbol{\Lambda})^{k} \widehat{y}=0$ pero $(\lambda-\boldsymbol{\Lambda})^{k-1} \widehat{y} \neq 0$. Utilizando la definición del operador $\Lambda$, tendremos

$$
\widehat{y}_{1}:=(\lambda-\mathbf{A}) \widehat{y}=\lambda \widehat{y}-\mathbf{P}\left(\mathbf{T}\left(\left.\widehat{y}\right|_{\Omega}\right)\right)=\lambda \mathbf{P}\left(\left.\widehat{y}\right|_{\Omega}\right)-\mathbf{P}\left(\mathbf{T}\left(\left.\widehat{y}\right|_{\Omega}\right)\right)=\mathbf{P}\left((\lambda-\mathbf{T})\left(\left.\widehat{y}\right|_{\Omega}\right)\right) \neq 0,
$$

Procediendo de manera análoga,

$$
\begin{aligned}
\widehat{y}_{2} & :=(\lambda-\mathbf{A})^{2} \widehat{y}=(\lambda-\mathbf{A}) \widehat{y}_{1}=\mathbf{P}\left((\lambda-\mathbf{T})\left(\left.\widehat{y}_{1}\right|_{\Omega}\right)\right)=\mathbf{P}\left((\lambda-\mathbf{T})^{2}\left(\left.\widehat{y}\right|_{\Omega}\right)\right) \neq 0, \\
\cdot & \cdot \\
\cdot & :=(\lambda-\mathbf{A})^{k-2} \widehat{y}=(\lambda-\mathbf{A}) \widehat{y}_{k-2}=\mathbf{P}\left((\lambda-\mathbf{T})\left(\left.\widehat{y}_{k-2}\right|_{\Omega}\right)\right)=\mathbf{P}\left((\lambda-\mathbf{T})^{k-1}\left(\left.\widehat{y}\right|_{\Omega}\right)\right) \neq 0, \\
\widehat{y}_{k-1} & :=(\lambda)=\mathbf{P}\left((\lambda-\mathbf{T})^{k}\left(\left.\widehat{y}\right|_{\Omega}\right)\right)=0 .
\end{aligned}
$$

Dado que $\left.\widehat{y}\right|_{\Omega}=\left.(\mathbf{P} y)\right|_{\Omega}=y \in V(\Omega)$, concluimos que existe $k$ tal que $(\lambda-\mathbf{T})^{k-1} y \neq 0$ y $(\lambda-\mathbf{T})^{k} y=0$. Luego, $y \in \mathcal{E}_{\lambda}(V(\Omega))$.

Recíprocamente, sea $y \in \mathcal{E}_{\lambda}(V(\Omega))$. Sabemos que existe $k$ tal que $(\lambda-\mathbf{T})^{k} y=0$ pero $(\lambda-\mathbf{T})^{k-1} y \neq 0$. Entonces tendremos

$$
\begin{aligned}
\mathbf{P} y_{1} & :=\mathbf{P}((\lambda-\mathbf{T}) y)=\lambda \mathbf{P} y-\mathbf{P}(\mathbf{T} y)=(\lambda-\mathbf{A})(\mathbf{P} y)=(\lambda-\mathbf{A}) \widehat{y} \neq 0, \\
\mathbf{P} y_{2} & :=\mathbf{P}\left((\lambda-\mathbf{T})^{2} y\right)=\mathbf{P}\left((\lambda-\mathbf{T}) y_{1}\right)=(\lambda-\mathbf{A})\left(\mathbf{P} y_{1}\right)=(\lambda-\mathbf{A})^{2} \widehat{y} \neq 0, \\
\cdot & \\
\cdot & . \\
\mathbf{P} y_{k-1} & :=\mathbf{P}\left((\lambda-\mathbf{T})^{k-1} y\right)=\mathbf{P}\left((\lambda-\mathbf{T}) y_{k-2}\right)=(\lambda-\mathbf{A})\left(\mathbf{P} y_{k-2}\right)=(\lambda-\mathbf{A})^{k-1} \widehat{y} \neq 0, \\
\mathbf{P} y_{k} & :=\mathbf{P}\left((\lambda-\mathbf{T})^{k} y\right)=\mathbf{P}\left((\lambda-\mathbf{T}) y_{k-1}\right)=(\lambda-\mathbf{A})\left(\mathbf{P} y_{k-1}\right)=(\lambda-\mathbf{A})^{k} \widehat{y}=0 .
\end{aligned}
$$

Luego, claramente, $\widehat{y}=\mathbf{P} y \in \mathbf{E}_{\lambda}(W(\widehat{\Omega}))$.

\subsubsection{Convergencia de las autofunciones y de los subespacios invariantes}

En esta sección vamos a generalizar la teoría desarrollada por Descloux-Nassif-Rappaz en [94]. Esto nos permitirá analizar, en particular, la convergencia de las aproximaciones obtenidas mediante métodos noconformes de un operador lineal y acotado definido sobre un dominio curvo $\Omega$. Para probar los resultados aquí presentados, seguiremos argumentos similares a los utilizados en [94].

Ya hemos señalado que el espacio $W(\widehat{\Omega})$ podría depender de $h$. Como consecuencia de esto, los vectores propios generalizados $x \in \mathbf{E}_{\lambda}(W(\widehat{\Omega}))$ podrían variar con $h$ también. Sin embargo, como $\left.x\right|_{\Omega} \in \mathcal{E}_{\lambda}(V(\Omega))$ es independiente de $h$ y $\Omega_{h} \rightarrow \Omega$ a medida que $h$ va a cero, $x$ será asintóticamente igual a $\left.x\right|_{\Omega}$.

Asumiremos que las siguientes propiedades se satisfacen:

P1: para $x \in \mathbf{E}_{\lambda}(W(\widehat{\Omega}))$, existe $x_{h} \in V_{h}^{e}(\widehat{\Omega})$ tal que $\left\|x-x_{h}\right\|_{h} \leq \eta_{1 h}\|x\|_{h}$, con $\eta_{1 h} \rightarrow 0$ para $h \rightarrow 0$,

P2: $\lim _{h \rightarrow 0}\left\|\left.\left(\mathbf{A}-\mathbf{A}_{h}\right)\right|_{V_{h}^{e}(\widehat{\Omega})}\right\|_{h}=0$.

El siguiente lema muestra que la aplicación $z \rightarrow\left\|\left(z-\left.\mathbf{A}_{h}\right|_{V_{h}^{e}(\widehat{\Omega})}\right)^{-1}\right\|_{h}$ es uniformemente continua para cada $z \in \rho(\boldsymbol{\Lambda})$, siempre y cuando $h$ sea lo suficientemente chico. Entonces, la aproximación $\mathbf{A}_{h}$ resultará estable en $z$.

Lema 3.6 Sea $z \in \rho(\boldsymbol{\Lambda}), z \neq 0$, fijo, y supongamos que la propiedad $\mathbf{P 2}$ se satisface. Existen constantes positivas $h_{0}$ y $C$, que dependen de $\Omega$ y $|z|$, tales que

$$
\left\|\left(z-\left.\mathbf{A}_{h}\right|_{V_{h}^{e}(\widehat{\Omega})}\right)^{-1}\right\|_{h} \leq C \quad \forall h<h_{0} .
$$


Prueba. Para cualquier $x_{h} \in V_{h}^{e}(\widehat{\Omega})$, podemos encontrar $\varsigma_{h}>0$, tan pequeño como queramos, que satisfaga $\left\|\left(\mathbf{A}-\mathbf{A}_{h}\right) x_{h}\right\|_{h} \leq \varsigma_{h}\left\|x_{h}\right\|_{h}$ ya que, por hipótesis, se satisface la propiedad P2. Además, en virtud del Lemma 3.3, para $z \in \rho(\boldsymbol{\Lambda})$, fijo, existe $C(z)$, una constante positiva independiente de $h$, tal que $\left\|(z-\mathbf{A}) x_{h}\right\|_{h} \geq C(z)\left\|x_{h}\right\|_{h}$. Luego, es inmediato que

$$
\left\|\left(z-\mathbf{A}_{h}\right) x_{h}\right\|_{h} \geq\left\|(z-\mathbf{A}) x_{h}\right\|_{h}-\left\|\left(\mathbf{A}-\mathbf{A}_{h}\right) x_{h}\right\|_{h} \geq \frac{C(z)}{2}\left\|x_{h}\right\|_{h} \quad \forall h \leq h_{0}
$$

donde $h_{0}$ queda determinado por la condición $\varsigma_{h} \leq C(z) / 2$. Este resultado nos permite probar que $z-\mathbf{A}_{h}: V_{h}^{e}(\widehat{\Omega}) \rightarrow V_{h}^{e}(\widehat{\Omega})$ es una aplicación uno a uno. Como el espacio $V_{h}^{e}(\widehat{\Omega})$ tiene dimensión finita, entoces la aplicación también resulta exhaustiva ${ }^{2}$. Luego, $z-\left.\mathbf{A}_{h}\right|_{V_{h}^{e}(\widehat{\Omega})}$ es una aplicación invertible. Finalmente,

$$
\left\|\left(z-\left.\mathbf{A}_{h}\right|_{V_{h}^{e}(\widehat{\Omega})}\right)^{-1}\right\|_{h}=\sup _{y_{h} \in V_{h}^{e}(\widehat{\Omega})} \frac{\left\|\left(z-\mathbf{A}_{h}\right)^{-1} y_{h}\right\|_{h}}{\left\|y_{h}\right\|_{h}}=\sup _{x_{h} \in V_{h}^{e}(\widehat{\Omega})} \frac{\left\|x_{h}\right\|_{h}}{\left\|\left(z-\mathbf{A}_{h}\right) x_{h}\right\|_{h}} \leq \frac{2}{C(z)},
$$

lo que nos permite completar la demostración.

A partir de ahora, con el fin de simplificar la notación, utilizaremos la siguiente definición $\boldsymbol{\Lambda}_{h}:=\left.\mathbf{A}_{h}\right|_{V_{h}^{e}(\widehat{\Omega})}$.

Corolario 3.7 Sea $\mathcal{G}$ un subconjunto cerrado de $\rho(\boldsymbol{\Lambda})$. Entonces, existen constantes positivas $C$ y $h_{0}$, independientes de $h$, tales que

$$
\left\|\left(z-\boldsymbol{\Lambda}_{h}\right)^{-1}\right\|_{h} \leq C \quad \forall z \in \mathcal{G}, \quad \forall h<h_{0} .
$$

Prueba. Como $\boldsymbol{\Lambda}_{h} \in \mathcal{L}(W(\widehat{\Omega}))$, pueden usarse los mismos argumentos empleados en la demostración del Corolario 3.4.

Teorema 3.8 Sea $\mathcal{O}$ un subconjunto compacto de $\mathbb{C}$, tal que $\mathcal{O} \cap \sigma(\boldsymbol{\Lambda})=\emptyset$. Existe una constante $h_{0}>0$, tal que, si $h<h_{0}$, entonces $\mathcal{O} \cap \sigma\left(\boldsymbol{\Lambda}_{h}\right)=\emptyset$.

Prueba. Como $\sigma(\boldsymbol{\Lambda})$ es el complemento en $\mathbb{C}$ de $\rho(\boldsymbol{\Lambda})$, el resultado es una consecuencia directa del lema anterior.

Observación 3.9 Podemos interpretar los resultados obtenidos hasta ahora del modo siguiente: para $h$ suficientemente pequeño, cualquier conjunto abierto del plano complejo que contenga a $\sigma(\boldsymbol{\Lambda})$ también va a contener a $\sigma\left(\boldsymbol{\Lambda}_{h}\right)$. Entonces, la aproximación $\mathbf{A}_{h}$ no introduce autovalores espurios.

El Teorema 3.8 asegura que $\gamma \subset \rho\left(\boldsymbol{\Lambda}_{h}\right)$ para $h$ suficientemente pequeño. Podemos definir entonces los proyectores espectrales $\mathbf{E}_{\lambda_{h}}: V_{h}^{e}(\widehat{\Omega}) \rightarrow V_{h}^{e}(\widehat{\Omega})$ asociados a los operadores $\boldsymbol{\Lambda}_{h}$ por medio de

$$
\mathbf{E}_{\lambda_{h}}=\frac{1}{2 \pi i} \int_{\gamma} R_{z}\left(\boldsymbol{\Lambda}_{h}\right) d z
$$

Observemos que:

- el operador $\mathbf{E}_{\lambda}$ proyecta $W(\widehat{\Omega})$ sobre el espacio propio de $\boldsymbol{\Lambda}$ asociado con $\lambda$,

- suponiendo que existan autovalores de $\boldsymbol{\Lambda}_{h}$ que yacen en el interior de $\gamma$, el operador $\mathbf{E}_{\lambda_{h}}$ proyectaría $V_{h}^{e}(\widehat{\Omega})$ sobre el subespacio invariante por $\boldsymbol{\Lambda}_{h}$ generado por los espacios propios de $\boldsymbol{\Lambda}_{h}$ asociados con aquellos autovalores de $\boldsymbol{\Lambda}_{h}$ encerrados por $\gamma$.

Sea $\Delta$ el dominio en el plano complejo que se encuentra limitado por la curva $\gamma$. Nuestro primer objetivo será probar que, para $h$ suficientemente chico, $\sigma\left(\boldsymbol{\Lambda}_{h}\right) \cap \Delta \neq \emptyset$.

\footnotetext{
${ }^{2} \operatorname{Si} \operatorname{dim}(V)=\operatorname{dim}(W)$, finita, el mapeo lineal $\mathcal{T}: V \rightarrow W$ es uno a uno si, y solo si, es suryectivo.
} 
Teorema 3.10 Supongamos que la propiedad $\mathbf{P 2}$ se satisface. Entonces, se verifica

$$
\lim _{h \rightarrow 0}\left\|\mathbf{E}_{\lambda_{h}}-\left.\mathbf{E}_{\lambda}\right|_{V_{h}^{e}(\widehat{\Omega})}\right\|_{h}=0 .
$$

Prueba. Sea $z \in \rho(\boldsymbol{\Lambda})$ y $x_{h}$ un elemento arbitrario de $V_{h}^{e}(\widehat{\Omega})$ con $\left\|x_{h}\right\|_{h}=1$. A partir de las definiciones de $\mathbf{E}_{\lambda}$ y $\mathbf{E}_{\lambda_{h}}$, tendremos

$$
\begin{aligned}
\left\|\left(\mathbf{E}_{\lambda}-\mathbf{E}_{\lambda_{h}}\right) x_{h}\right\|_{h} & =\frac{1}{2 \pi}\left\|\int_{\gamma}\left(R_{z}(\boldsymbol{\Lambda})-R_{z}\left(\boldsymbol{\Lambda}_{h}\right)\right) x_{h} d z\right\|_{h} \\
& \leq \frac{1}{2 \pi} \int_{\gamma}\left\|\left(R_{z}(\boldsymbol{\Lambda})-R_{z}\left(\boldsymbol{\Lambda}_{h}\right)\right) x_{h}\right\|_{h}|d z| \\
& =\frac{1}{2 \pi} \int_{\gamma} \|\left(R_{z}(\boldsymbol{\Lambda})\left(\boldsymbol{\Lambda}-\boldsymbol{\Lambda}_{h}\right) R_{z}\left(\boldsymbol{\Lambda}_{h}\right) x_{h} \|_{h}|d z| .\right.
\end{aligned}
$$

Ahora bien, dado que $\left\|x_{h}\right\|_{h}=1$,

$$
\|\left(R _ { z } ( \boldsymbol { \Lambda } ) ( \boldsymbol { \Lambda } - \boldsymbol { \Lambda } _ { h } ) R _ { z } ( \boldsymbol { \Lambda } _ { h } ) x _ { h } \| _ { h } \leq \| \left(R_{z}(\boldsymbol{\Lambda})\left\|_{\mathcal{L}(W(\widehat{\Omega}))}\right\| \boldsymbol{\Lambda}-\boldsymbol{\Lambda}_{h}\left\|_{\mathcal{L}\left(V_{h}^{e}(\widehat{\Omega}), W(\widehat{\Omega})\right)}\right\| R_{z}\left(\boldsymbol{\Lambda}_{h}\right) \|_{\mathcal{L}\left(V_{h}^{e}(\widehat{\Omega})\right)}\right.\right.
$$

y, por lo tanto,

$$
\left\|\left(\mathbf{E}_{\lambda}-\mathbf{E}_{\lambda_{h}}\right) x_{h}\right\|_{h} \leq \frac{|\gamma|}{2 \pi} \sup _{z \in \gamma}\left\|R_{z}(\boldsymbol{\Lambda})\right\|_{h}\left\|\left.\left(\mathbf{A}-\mathbf{A}_{h}\right)\right|_{V_{h}^{e}(\widehat{\Omega})}\right\|_{h} \sup _{z \in \gamma}\left\|R_{z}\left(\boldsymbol{\Lambda}_{h}\right)\right\|_{h} .
$$

A partir de este resultado, solo resta usar los Corolarios 3.4 y 3.7 y la propiedad P2 para concluir la prueba.

De acuerdo con el teorema anterior, $\left.\mathbf{E}_{\lambda_{h}}\right|_{V_{h}^{e}(\widehat{\Omega})}$ converge uniformemente a $\left.\mathbf{E}_{\lambda}\right|_{V_{h}^{e}(\widehat{\Omega})}$ cuando $h \rightarrow 0$

Teorema 3.11 Supongamos que las propiedades $\mathbf{P 1}$ y $\mathbf{P 2}$ se satisfacen. Entonces, para todo $x \in \mathbf{E}_{\lambda}(W(\widehat{\Omega}))$ se verifica

$$
\lim _{h \rightarrow 0} \delta\left(x, \mathbf{E}_{\lambda_{h}}\left(V_{h}^{e}(\widehat{\Omega})\right)\right)=0 .
$$

Prueba. Sea $x$ un elemento arbitrario de $\mathbf{E}_{\lambda}(W(\widehat{\Omega}))$. Necesitamos acotar la siguiente expresión

$$
\operatorname{dist}\left(x, \mathbf{E}_{\lambda_{h}}\left(V_{h}^{e}(\widehat{\Omega})\right)\right):=\inf _{y_{h} \in \mathbf{E}_{\lambda_{h}}\left(V_{h}^{e}(\widehat{\Omega})\right)}\left\|x-y_{h}\right\|_{h}=\inf _{y_{h} \in V_{h}^{e}(\widehat{\Omega})}\left\|x-\mathbf{E}_{\lambda_{h}} y_{h}\right\|_{h} .
$$

Por hipótesis, existe $x_{h} \in V_{h}^{e}(\widehat{\Omega})$ tal que $\left\|x-x_{h}\right\|_{h} \leq \eta_{1 h}\|x\|_{h}$, con $\eta_{1 h} \rightarrow 0$ cuando $h \rightarrow 0$. Teniendo en cuenta que $x=\mathbf{E}_{\lambda} x$, tendremos

$$
\begin{aligned}
\inf _{y_{h} \in V_{h}^{e}(\widehat{\Omega})}\left\|x-\mathbf{E}_{\lambda_{h}} y_{h}\right\|_{h} & \leq\left\|\mathbf{E}_{\lambda} x-\mathbf{E}_{\lambda_{h}} x_{h}\right\|_{h} \leq\left\|\mathbf{E}_{\lambda}\left(x-x_{h}\right)\right\|_{h}+\left\|\left(\mathbf{E}_{\lambda}-\mathbf{E}_{\lambda_{h}}\right) x_{h}\right\|_{h} \\
& \leq\left\|\mathbf{E}_{\lambda}\right\|_{\mathcal{L}(W(\widehat{\Omega}))}\left\|x-x_{h}\right\|_{h}+\left\|\mathbf{E}_{\lambda}-\mathbf{E}_{\lambda_{h}}\right\|_{\mathcal{L}\left(V_{h}^{e}(\widehat{\Omega}), W(\widehat{\Omega})\right)}\left\|x_{h}\right\|_{h} .
\end{aligned}
$$

A partir de esta desigualdad, podemos concluir

$$
\sup _{x \in \mathbf{E}_{\lambda}(W(\widehat{\Omega}))} \frac{\operatorname{dist}\left(x, \mathbf{E}_{\lambda_{h}}\left(V_{h}^{e}(\widehat{\Omega})\right)\right)}{\|x\|_{h}} \leq \eta_{1 h}\left\|\mathbf{E}_{\lambda}\right\|_{h}+\left(1+\eta_{1 h}\right)\left\|\mathbf{E}_{\lambda_{h}}-\left.\mathbf{E}_{\lambda}\right|_{\left.V_{h}^{e}(\widehat{\Omega})\right)}\right\|_{h} .
$$

Luego, el resultado es una consecuencia directa de la propiedad P1 y del Teorema 3.10.

De acuerdo con el teorema anterior,

- $\operatorname{dim} \mathbf{E}_{\lambda}(W(\widehat{\Omega})) \leq \operatorname{dim} \mathbf{E}_{\lambda_{h}}\left(V_{h}^{e}(\widehat{\Omega})\right)$ y, por lo tanto, $\sigma\left(\boldsymbol{\Lambda}_{h}\right) \cap \Delta \neq \emptyset$,

- cualquier elemento de $\mathbf{E}_{\lambda}(W(\widehat{\Omega}))$ puede ser aproximado por elementos de $\mathbf{E}_{\lambda_{h}}\left(V_{h}^{e}(\widehat{\Omega})\right)$. 
Observemos, sin embargo, que el número de vectores propios en $\mathbf{E}_{\lambda_{h}}\left(V_{h}^{e}(\widehat{\Omega})\right)$ podría ser demasiado grande. Es decir, las multiplicidades algebraicas podrían ser diferentes. En general, la falta de preservación de la multiplicidad algebraica, deteriora la convergencia de los vectores propios aproximados.

Teorema 3.12 Supongamos que la propiedad $\mathbf{P 2}$ se satisface. Entonces, para todo $x_{h} \in \mathbf{E}_{\lambda_{h}}\left(V_{h}^{e}(\widehat{\Omega})\right)$ se verifica

$$
\lim _{h \rightarrow 0} \delta\left(x_{h}, \mathbf{E}_{\lambda}(W(\widehat{\Omega}))\right)=0 .
$$

Prueba. Sea $x_{h}$ un elemento arbitrario de $\mathbf{E}_{\lambda_{h}}\left(V_{h}^{e}(\widehat{\Omega})\right)$. Necesitamos acotar la siguiente expresión

$$
\operatorname{dist}\left(x_{h}, \mathbf{E}_{\lambda}(W(\widehat{\Omega}))\right):=\inf _{y \in \mathbf{E}_{\lambda}(W(\widehat{\Omega}))}\left\|x_{h}-y\right\|_{h}=\inf _{y \in W(\widehat{\Omega})}\left\|x_{h}-\mathbf{E}_{\lambda} y\right\|_{h} .
$$

Teniendo en cuenta que $x_{h} \in W(\widehat{\Omega})$ y que $x_{h}=\mathbf{E}_{\lambda_{h}} x_{h}$, resulta

$$
\inf _{y \in W(\widehat{\Omega})}\left\|x_{h}-\mathbf{E}_{\lambda} y\right\|_{h} \leq\left\|x_{h}-\mathbf{E}_{\lambda} x_{h}\right\|_{h} \leq\left\|\left(\mathbf{E}_{\lambda_{h}}-\mathbf{E}_{\lambda}\right) x_{h}\right\|_{h} \leq\left\|\mathbf{E}_{\lambda_{h}}-\mathbf{E}_{\lambda}\right\|_{\mathcal{L}\left(V_{h}^{e}(\widehat{\Omega}), W(\widehat{\Omega})\right)}\left\|x_{h}\right\|_{h} .
$$

A partir de aquí, es inmediato que

$$
\sup _{x_{h} \in \mathbf{E}_{\lambda_{h}}\left(V_{h}^{e}(\widehat{\Omega})\right)} \frac{\operatorname{dist}\left(x_{h}, \mathbf{E}_{\lambda}(W(\widehat{\Omega}))\right)}{\left\|x_{h}\right\|_{h}} \leq\left\|\mathbf{E}_{\lambda_{h}}-\left.\mathbf{E}_{\lambda}\right|_{V_{h}^{e}(\widehat{\Omega})}\right\|_{h} .
$$

Luego, el resultado es una consecuencia directa del Teorema 3.10.

Teorema 3.13 Supongamos que las propiedades P1 y P2 se satisfacen. Entonces,

$$
\lim _{h \rightarrow 0} \widehat{\delta}\left(\mathbf{E}_{\lambda}(W(\widehat{\Omega})), \mathbf{E}_{\lambda_{h}}\left(V_{h}^{e}(\widehat{\Omega})\right)\right)=0 .
$$

Prueba. Inmediata a partir de los Teoremas 3.12 y 3.11 .

El teorema anterior nos permite asegurar que los espacios propios aproximados tienen la dimensión correcta. Explícitamente, para todo $h<h_{0}, \operatorname{dim} \mathbf{E}_{\lambda_{h}}\left(V_{h}^{e}(\widehat{\Omega})\right)=\operatorname{dim} \mathbf{E}_{\lambda}(W(\widehat{\Omega}))$. Luego, la aproximación $\mathbf{A}_{h}$ no introduce autovectores espurios.

\subsubsection{Convergencia de las autovalores}

En esta sección analizaremos la convergencia de los autovalores asociados con el operador $\boldsymbol{\Lambda}_{h}$ hacia los autovalores del operador $\boldsymbol{\Lambda}$.

Recordemos que $\Delta$ es el dominio en el plano complejo limitado por la curva $\gamma$. Claramente, tendremos $\sigma(\boldsymbol{\Lambda}) \cap \Delta=\{\lambda\}$.

Definición 3.14 El espectro de $\boldsymbol{\Lambda}_{h}$ en $\Delta$ converge a $\lambda$ si, y solo si,

$$
\lim _{h \rightarrow 0} \sigma\left(\boldsymbol{\Lambda}_{h}\right) \cap \Delta=\{\lambda\} .
$$

Es interesante destacar aquí que esta definición expresa la continuidad de $\sigma(\boldsymbol{\Lambda})$ en la vencindad $\Delta$ de $\lambda$ cuando el operador $\boldsymbol{\Lambda}$ está sujeto a la perturbación $\boldsymbol{\Lambda}-\boldsymbol{\Lambda}_{h}\left(\right.$ es decir, $\left.\boldsymbol{\Lambda}=\boldsymbol{\Lambda}_{h}+\left(\boldsymbol{\Lambda}-\boldsymbol{\Lambda}_{h}\right)\right)$ [84]. Esta continuidad implica que

- para $h$ suficientemente chico, existe al menos un punto de $\sigma\left(\boldsymbol{\Lambda}_{h}\right)$ en cualquier vecindad de $\lambda$; propiedad de semi-continuidad inferior, es decir,

$$
\{\lambda\} \subset \liminf _{h \rightarrow 0} \sigma\left(\boldsymbol{\Lambda}_{h}\right) \cap \Delta,
$$


- $\lambda$ es el único límite posible para cualquier sucesión de puntos en $\sigma\left(\boldsymbol{\Lambda}_{h}\right) \cap \Delta$; propiedad de semicontinuidad superior, es decir,

$$
\limsup _{h \rightarrow 0} \sigma\left(\boldsymbol{\Lambda}_{h}\right) \cap \Delta \subset\{\lambda\} .
$$

Como mostraremos a continuación, la convergencia de los autovalores aproximados es una consecuencia directa de los resultados que hemos obtenidos en las secciones anteriores. Para las pruebas, seguiremos los argumentos presentados en [83].

Proposición 3.15 El espectro de $\boldsymbol{\Lambda}_{h}$ en $\Delta$ converge a $\lambda$.

Prueba. Los Teoremas 3.10 y 3.11 nos permitieron asegurar que $\sigma\left(\boldsymbol{\Lambda}_{h}\right) \cap \Delta \neq \emptyset$, siempre que $h$ sea lo suficientemente chico. Para $z \in \Delta-\{\lambda\} \subset \rho(\boldsymbol{\Lambda})$, el Lema 3.6 nos asegura que $\boldsymbol{\Lambda}_{h}$ es estable en $z$; es decir, que existen constantes $h_{0}(z)$ y $C(z)$ tales que, para todo $h<h_{0}(z), z \in \rho\left(\boldsymbol{\Lambda}_{h}\right)$ y $\left\|R_{z}\left(\boldsymbol{\Lambda}_{h}\right)\right\|_{h} \leq C(z)$. En estas condiciones, elijamos otra curva cerrada $\widehat{\gamma}$ que encierre a $\lambda$ y de manera tal que quede contenida en el interior de $\gamma$. Por el Corolario 3.7, si $h$ es lo suficientemente pequeño, $\left\|R_{z}\left(\boldsymbol{\Lambda}_{h}\right)\right\|_{h}$ será uniformemente continua para todo $z$ en el domino anular comprendido entre las curvas $\gamma$ y $\widehat{\gamma}$. Por lo tanto, en ese dominio, no puede haber puntos pertenecientes a $\sigma\left(\boldsymbol{\Lambda}_{h}\right)$.

Definición 3.16 La aproximación $\boldsymbol{\Lambda}_{h}$, que es estable en el dominio $\Delta-\{\lambda\}$, se dice fuertemente estable en $\Delta-\{\lambda\}$ si existe una constante $h_{0}$ tal que

$$
\operatorname{dim} \mathbf{E}_{\lambda_{h}}\left(V_{h}^{e}(\widehat{\Omega})\right)=\operatorname{dim} \mathbf{E}_{\lambda}(W(\widehat{\Omega})) \quad \forall h<h_{0} .
$$

Es claro que, la estabilidad fuerte de $\boldsymbol{\Lambda}_{h}$ en $\Delta-\{\lambda\}$, es una consecuencia directa del Teorema 3.13.

Proposición 3.17 Para $h$ suficientemente chico, $\sigma\left(\boldsymbol{\Lambda}_{h}\right) \cap \Delta$ consiste exactamente en $m$ autovalores, no necesariamente distintos.

Prueba. Si $h$ es suficientemente chico, $\gamma$ separará a $\sigma\left(\boldsymbol{\Lambda}_{h}\right) \cap \Delta$ del resto del espectro de $\boldsymbol{\Lambda}_{h}$. Entonces, de acuerdo con Kato (Teorema 6.17 en [123]), $\sigma\left(\boldsymbol{\Lambda}_{h}\right) \cap \Delta$ será igual al espectro del operador $\boldsymbol{\Lambda}_{h}$ restringido al subespacio $\mathbf{E}_{\lambda_{h}}\left(V_{h}^{e}(\widehat{\Omega})\right)$, cuya imagen tiene dimensión igual a $m$ pues $\boldsymbol{\Lambda}_{h}$ es fuertemente estable en $\Delta-\{\lambda\}$. A partir de aquí, concluimos que el espectro de $\boldsymbol{\Lambda}_{h}$ contenido en $\Delta$ consistirá en un conjunto de autovalores con una multiplicidad algebraica total igual a $m$.

Como consecuencia de los resultados establecidos hasta ahora, podemos asegurar que partes aisladas del espectro de $\boldsymbol{\Lambda}$ son aproximadas por partes aisladas del espectro de $\boldsymbol{\Lambda}_{h}$. Más precisamente, para cualquier autovalor $\lambda$ de $\boldsymbol{\Lambda}$ con multiplicidad finita $m$, existen exactamente $m$ autovalores $\lambda_{1 h}, \cdots, \lambda_{m h}$ de $\boldsymbol{\Lambda}_{h}$, repetidos de acuerdo con sus respectivas multiplicidades, que convergen a $\lambda$ a medida que $h$ va a cero.

\subsubsection{Estimaciones del error para las autofunciones en la norma $\|\cdot\|_{h}$}

En esta sección obtendremos nuevas estimaciones para el error que nos permitirán determinar el orden con el que convergen las autofunciones aproximadas. Lograremos este objetivo extendiendo la teoría desarrollada en [95], establecida solo para métodos conformes.

Algunos de los resultados que se presentarán a continuación pueden probarse modificando ligeramente las pruebas de los correspondientes lemas o teoremas en [95]. A pesar de esto, hemos decidido incluir todas las pruebas en este trabajo con el fin de brindar un análisis completo de la teoría propuesta.

Comenzaremos por considerar el subespacio de $W(\widehat{\Omega})$ definido por

$$
W_{\lambda}(\widehat{\Omega}):=\mathbf{E}_{\lambda}(W(\widehat{\Omega}))+V_{h}^{e}(\widehat{\Omega})
$$

y supondremos que existe una norma $\|\cdot\|_{*}$ definida en $W_{\lambda}(\widehat{\Omega})$ tal que

$$
\text { - }\|u\|_{h} \leq\|u\|_{*} \quad \forall u \in W_{\lambda}(\widehat{\Omega}),
$$


- $\|u\|_{*} \simeq\|u\|_{h} \quad \forall u \in V_{h}^{e}(\widehat{\Omega})$,

- $\|u\|_{*} \leq C(\lambda)\|u\|_{h} \quad \forall u \in \mathbf{E}_{\lambda}(W(\widehat{\Omega}))$.

Para un operador lineal y continuo $\mathbf{L}: W_{\lambda}(\widehat{\Omega}) \rightarrow W_{\lambda}(\widehat{\Omega})$ quedan definidas las siguientes normas discretas

$$
\begin{aligned}
& \|\mathbf{L}\|_{* h}:=\sup _{y \in W_{\lambda}(\widehat{\Omega})} \frac{\|\mathbf{L} y\|_{h}}{\|y\|_{*}} \leq \sup _{y \in W_{\lambda}(\widehat{\Omega})} \frac{\|\mathbf{L} y\|_{h}}{\|y\|_{h}}=\|\mathbf{L}\|_{h}, \\
& \|\mathbf{L}\|_{h *}:=\sup _{y \in W_{\lambda}(\widehat{\Omega})} \frac{\|\mathbf{L} y\|_{*}}{\|y\|_{h}} \geq \sup _{y \in W_{\lambda}(\widehat{\Omega})} \frac{\|\mathbf{L} y\|_{h}}{\|y\|_{h}}=\|\mathbf{L}\|_{h}, \\
& \|\mathbf{L}\|_{*}:=\sup _{y \in W_{\lambda}(\widehat{\Omega})} \frac{\|\mathbf{L} y\|_{*}}{\|y\|_{*}} \leq \sup _{y \in W_{\lambda}(\widehat{\Omega})} \frac{\|\mathbf{L} y\|_{*}}{\|y\|_{h}}=\|\mathbf{L}\|_{h *} .
\end{aligned}
$$

A lo largo de toda esta sección asumiremos que:

- las formas $a(\cdot, \cdot), b(\cdot, \cdot)$ y sus respectivas aproximaciones, $a_{h}(\cdot, \cdot)$ y $b_{h}(\cdot, \cdot)$, son hermitianas,

- los dominios de definición de $a_{h}$ y $b_{h}$ pueden extenderse al espacio suma $\left(V+V_{h}\right)\left(\Omega_{h}\right)$ de manera tal que se verifiquen las siguientes condiciones:

a) la forma $a_{h}$ extendida resulta uniformemente continua en $W_{\lambda}(\widehat{\Omega})$ con respecto a la norma $\|\cdot\|_{*}$, es decir,

$$
\left|a_{h}\left(\left.v\right|_{\Omega_{h}},\left.w\right|_{\Omega_{h}}\right)\right| \leq C\|v\|_{*, \Omega_{h}}\|w\|_{*, \Omega_{h}} \leq C\|v\|_{*}\|w\|_{*} \quad \forall v, w \in W_{\lambda}(\widehat{\Omega})
$$

b) la forma $b_{h}$ extendida resulta uniformemente continua en $W(\widehat{\Omega})$ con la norma $\|\cdot\|_{h}$, es decir,

$$
\left|b_{h}\left(\left.v\right|_{\Omega_{h}},\left.w\right|_{\Omega_{h}}\right)\right| \leq C|v|_{X\left(\Omega_{h}\right)}|w|_{X\left(\Omega_{h}\right)} \leq C\|v\|_{h}\|w\|_{h} \quad \forall v, w \in W(\widehat{\Omega})
$$

c) las formas bilineales $a$ y $a_{h}$ pueden expresarse de la siguiente manera:

$$
\begin{aligned}
a(x, y) & =a_{1}\left(\left.x\right|_{\Omega \cap \Omega_{h}},\left.y\right|_{\Omega \cap \Omega_{h}}\right)+a_{2}\left(\left.x\right|_{\Omega \backslash \Omega_{h}},\left.y\right|_{\Omega \backslash \Omega_{h}}\right) \quad \forall x, y \in V(\widehat{\Omega}) \\
a_{h}(x, y) & =a_{1 h}\left(\left.x\right|_{\Omega \cap \Omega_{h}},\left.y\right|_{\Omega \cap \Omega_{h}}\right)+a_{2 h}\left(\left.x\right|_{\Omega_{h} \backslash \Omega},\left.y\right|_{\Omega_{h} \backslash \Omega}\right) \quad \forall x, y \in W(\widehat{\Omega})
\end{aligned}
$$

d) las formas $a_{1}$ y $a_{1 h}$ satisfacen la siguiente relación:

$$
a_{1}\left(\left.x\right|_{\Omega \cap \Omega_{h}},\left.y\right|_{\Omega \cap \Omega_{h}}\right) \quad=a_{1 h}\left(\left.x\right|_{\Omega \cap \Omega_{h}},\left.y\right|_{\Omega \cap \Omega_{h}}\right) \quad \forall x, y \in V(\widehat{\Omega})
$$

e) para las formas $a_{2}$ y $a_{2 h}$ se verifican las siguientes estimaciones:

$$
\begin{aligned}
\left|a_{2}\left(\left.x\right|_{\Omega \backslash \Omega_{h}},\left.y\right|_{\Omega \backslash \Omega_{h}}\right)\right| & \leq c_{1}\|x\|_{h, \Omega \backslash \Omega_{h}}\|y\|_{h, \Omega \backslash \Omega_{h}} \quad \forall x, y \in V(\widehat{\Omega}) \\
\left|a_{2 h}\left(\left.x\right|_{\Omega_{h} \backslash \Omega},\left.y\right|_{\Omega_{h} \backslash \Omega}\right)\right| & \leq c_{2}\|x\|_{*, \Omega_{h} \backslash \Omega}\|y\|_{*, \Omega_{h} \backslash \Omega} \quad \forall x, y \in V(\widehat{\Omega}),
\end{aligned}
$$

siendo $c_{1}$ y $c_{2}$ constantes positivas que no dependen de $h$.

La primera suposición tiene una consecuencia muy importante que aquí necesitaremos solo a efectos de simplificar la presentación. Asumiendo que las formas $a$ y $b$ son hermitianas simétricas, el operador $\left.\mathbf{T}\right|_{V(\Omega)}$ resultará autoadjunto con respecto a la forma $a^{3}$. Por lo tanto, sus autovalores serán todos reales y todos los autovectores generalizados serán autovectores.

Antes de continuar con nuestro análisis, necesitamos definir un operador de proyección sobre los espacios discretos, asociado con la forma bilineal $a_{h}$.

\footnotetext{
${ }^{3}$ Para operadores acotados en espacios de Hilbert, los conceptos simétrico y autoadjunto resultan equivalentes.
} 
Sea $\Pi_{h}: W_{\lambda}(\widehat{\Omega}) \rightarrow W_{\lambda}(\widehat{\Omega})$, el operador con rango en $V_{h}^{e}(\widehat{\Omega})$, definido por

$$
\left\{\begin{array}{l}
\Pi_{h} x=\mathbf{P}_{h} x_{h} \\
a_{h}\left(x-x_{h}, y_{h}\right)=0 \quad \forall y_{h} \in V_{h}\left(\Omega_{h}\right)
\end{array}\right.
$$

El operador $\Pi_{h}$, así definido, estará acotado en la norma $\|\cdot\|_{* h}$ uniformemente con respecto a $h$. En efecto, debido a que la forma bilineal $a_{h}$ es continua sobre $W_{\lambda}(\widehat{\Omega})$ (con respecto a la norma $\|\cdot\|_{*}$ ) y coerciva sobre $V_{h}\left(\Omega_{h}\right)$ (con respecto a la norma $\|\cdot\|_{h}$ ) y teniendo en cuenta que $\mathbf{P}_{h}$ es un operador acotado en $V_{h}\left(\Omega_{h}\right)$, valiendo todas las propiedades uniformemente en $h$, podemos escribir

$$
\begin{aligned}
\left\|\Pi_{h} x\right\|_{h}^{2} & =\left\|\mathbf{P}_{h} x_{h}\right\|_{h}^{2} \leq C\left\|x_{h}\right\|_{V_{h}\left(\Omega_{h}\right)}^{2} \leq C\left|a_{h}\left(x_{h}, x_{h}\right)\right|=C\left|a_{h}\left(x, x_{h}\right)\right| \\
& \leq C\|x\|_{*, \Omega_{h}}\left\|x_{h}\right\|_{*, \Omega_{h}} \leq C\|x\|_{*}\left\|x_{h}\right\|_{h} \leq C\|x\|_{*}\left\|\mathbf{P}_{h} x_{h}\right\|_{h}=C\|x\|_{*}\left\|\Pi_{h} x\right\|_{h},
\end{aligned}
$$

donde hemos usado estimaciones obvias, las propiedades de la norma $\|\cdot\|_{*}$ y la definición del espacio $W_{\lambda}(\widehat{\Omega})$ para obtener las últimas desigualdades.

Observemos también que $\Pi_{h}$ es un operador autoadjunto con respecto a la forma bilinear $a_{h}$ ya que hemos asumido que $a_{h}$ es simétrica.

Consideremos ahora el siguiente operador

$$
\mathbf{B}_{h}:=\mathbf{A}_{h} \Pi_{h}: W_{\lambda}(\widehat{\Omega}) \rightarrow V_{h}^{e}(\widehat{\Omega}) \subset W_{\lambda}(\widehat{\Omega}) .
$$

A partir de esta definición, tendremos

$$
\begin{gathered}
\left\|\mathbf{B}_{h} x\right\|_{h} \leq C\left\|\Pi_{h} x\right\|_{h} \leq C\|x\|_{*} \quad \forall x \in W_{\lambda}(\widehat{\Omega}), \\
\left\|\left(z-\mathbf{B}_{h}\right) x\right\|_{h} \leq|z|\|x\|_{h}+\left\|\mathbf{B}_{h} x\right\|_{h} \leq C(z)\|x\|_{*} \quad \forall z \in \mathbb{C}, \quad \forall x \in W_{\lambda}(\widehat{\Omega}) .
\end{gathered}
$$

Observación 3.18 A partir de los resultados anteriores, surge inmediadamente que los operadores $\Pi_{h}$, $\mathbf{B}_{h}$ y $z-\mathbf{B}_{h}$ también están uniformemente acotados en la norma $\|\cdot\|_{*}$. En efecto, como para cualquier $x \in W_{\lambda}(\widehat{\Omega}), \Pi_{h} x \in V_{h}^{e}(\widehat{\Omega})$, tendremos que para todo $x \in W_{\lambda}(\widehat{\Omega}),\left\|\Pi_{h} x\right\|_{*} \simeq\left\|\Pi_{h} x\right\|_{h}$. Luego, existirá una constante positiva, $C$, tal que

$$
\text { - }\left\|\Pi_{h} x\right\|_{*} \leq C\|x\|_{*} \text {. }
$$

Razonando de manera análoga, tendremos

$$
\text { - }\left\|\mathbf{B}_{h} x\right\|_{*} \leq C\|x\|_{*}
$$

$y$, por lo tanto,

$$
\text { - }\left\|\left(z-\mathbf{B}_{h}\right) x\right\|_{*} \leq C(z)\|x\|_{*} .
$$

Obviamente, los operadores $\mathbf{A}_{h}$ y $\mathbf{B}_{h}$ coinciden sobre $V_{h}^{e}(\widehat{\Omega})$ ya que, como $\Pi_{h}$ proyecta $W_{\lambda}(\widehat{\Omega})$ sobre $V_{h}^{e}(\widehat{\Omega}),\left(\Pi_{h}-\mathbf{I}\right) x_{h}=0$ para todo elemento $x_{h} \in V_{h}^{e}(\widehat{\Omega})$. Esto nos permite asegurar que

- $\sigma\left(\boldsymbol{\Lambda}_{h}\right)=\sigma\left(\mathbf{B}_{h}\right)$,

- para cualquier valor propio, distinto de cero, los correspondientes subespacios invariantes coinciden.

Además, el operador $\mathbf{B}_{h}$ es autoadjunto con respecto a la forma bilineal $a_{h}$. Esta propiedad es una consecuencia directa de la simetría de las formas bilineales $a_{h} \mathrm{y} b_{h}$. En efecto, dados $x, y$, elementos arbitrarios de $W_{\lambda}(\widehat{\Omega})$, tendremos

$$
a_{h}\left(\mathbf{B}_{h} x, y\right)=a_{h}\left(\mathbf{A}_{h} \Pi_{h} x, y\right)=\overline{a_{h}\left(y, \mathbf{A}_{h} \Pi_{h} x\right)}=\overline{a_{h}\left(\Pi_{h} y, \mathbf{A}_{h} \Pi_{h} x\right)}=a_{h}\left(\mathbf{A}_{h} \Pi_{h} x, \Pi_{h} y\right)=b_{h}\left(\Pi_{h} x, \Pi_{h} y\right),
$$

donde hemos usado los problemas (3.18) y (3.9) para establecer las últimas igualdades. De manera completamente análoga, se obtiene

$$
a_{h}\left(x, \mathbf{B}_{h} y\right)=\overline{b_{h}\left(\Pi_{h} y, \Pi_{h} x\right)} .
$$

El siguiente resultado muestra que $z-\mathbf{B}_{h}: W_{\lambda}(\widehat{\Omega}) \rightarrow W_{\lambda}(\widehat{\Omega})$ es una aplicación continua, invertible y con inversa uniformemente continua para todo $z \in \gamma$. 
Lema 3.19 Para todo $z \in \gamma$, existen constantes positivas $C_{1}(z), C_{2}(z)$ y $h_{0}$ tales que

$$
\begin{array}{cc}
\left\|\left(z-\mathbf{B}_{h}\right)^{-1}\right\|_{* h} \leq C_{1}(z) & \forall h \leq h_{0}, \\
\left\|\left(z-\mathbf{B}_{h}\right)^{-1}\right\|_{*} \leq C_{2}(z) & \forall h \leq h_{0} .
\end{array}
$$

Prueba. Sea $x$ un elemento arbitrario de $W_{\lambda}(\widehat{\Omega})$. A partir de la definición del operador $\mathbf{B}_{h}$, es inmediato que

$$
\mathbf{B}_{h}\left(\Pi_{h} x\right)=\mathbf{B}_{h} x
$$

Luego, si $z \in \gamma$, es $z \neq 0$ y podremos escribir

$$
\begin{aligned}
\left\|\left(\mathbf{I}-\Pi_{h}\right) x\right\|_{h} & =\frac{1}{z}\left\|\left(z-\mathbf{B}_{h}\right)\left(\mathbf{I}-\Pi_{h}\right) x\right\|_{h} \leq \frac{1}{z}\left(\left\|\left(z-\mathbf{B}_{h}\right) x\right\|_{h}+\left\|\left(z-\mathbf{B}_{h}\right) \Pi_{h} x\right\|_{h}\right) \\
& \leq \stackrel{C}{C}(z)\left(\left\|\left(z-\mathbf{B}_{h}\right) x\right\|_{h}+\left\|\Pi_{h}\left(z-\mathbf{B}_{h}\right) x\right\|_{h}\right) \leq C(z)\left\|\left(z-\mathbf{B}_{h}\right) x\right\|_{*} .
\end{aligned}
$$

Por otro lado, como $\Pi_{h} x \in V_{h}^{e}(\widehat{\Omega})$, de acuerdo con el Lema 3.6, existe una constante positiva $h_{0}$ tal que, si $h<h_{0}$, se verifica

$$
\left\|\Pi_{h} x\right\|_{h} \leq C(z)\left\|\left(z-\mathbf{A}_{h}\right) \Pi_{h} x\right\|_{h}=C(z)\left\|\Pi_{h}\left(\left(z-\mathbf{B}_{h}\right) x\right)\right\|_{h} \leq C(z)\left\|\left(z-\mathbf{B}_{h}\right) x\right\|_{*} .
$$

Concluimos entonces que la aplicación $z-\mathbf{B}_{h}: W_{\lambda}(\widehat{\Omega}) \rightarrow W_{\lambda}(\widehat{\Omega})$ es inyectiva pues

$$
\|x\|_{h} \leq\left\|\left(\mathbf{I}-\Pi_{h}\right) x\right\|_{h}+\left\|\Pi_{h} x\right\|_{h} \leq C(z)\left\|\left(z-\mathbf{B}_{h}\right) x\right\|_{*} \quad \forall h<h_{0}
$$

Como $\mathbf{B}_{h}$ es un operador compacto ${ }^{4}$, tendremos que $z-\mathbf{B}_{h}$ es también una aplicación exhaustiva ${ }^{5}$. Luego, $\left(z-\mathbf{B}_{h}\right)$ es invertible. Finalmente, como $\gamma$ es un conjunto compacto de $\mathbb{C}$, para todo $z \in \gamma$ se verifica

$$
\left\|\left(z-\mathbf{B}_{h}\right)^{-1}\right\|_{* h}=\sup _{y \in W_{\lambda}(\widehat{\Omega})} \frac{\left\|\left(z-\mathbf{B}_{h}\right)^{-1} y\right\|_{h}}{\|y\|_{*}}=\sup _{x \in W_{\lambda}(\widehat{\Omega})} \frac{\|x\|_{h}}{\left\|\left(z-\mathbf{B}_{h}\right) x\right\|_{*}} \leq \operatorname{máx}_{z \in \gamma} C(z) .
$$

Así concluimos la demostración de la primera desigualdad.

Razonando de la misma manera, con la $\|\cdot\|_{*}$ reemplazando a la $\|\cdot\|_{h}$, y teniendo en cuenta los resultados de la observación 3.18, es fácil concluir que, para todo $x \in W_{\lambda}(\widehat{\Omega})$,

$$
\|x\|_{*} \leq \widetilde{C}(z)\left\|\left(z-\mathbf{B}_{h}\right) x\right\|_{*} \quad \forall h<h_{0}
$$

Luego, para todo $z \in \gamma$, tendremos

$$
\left\|\left(z-\mathbf{B}_{h}\right)^{-1}\right\|_{*} \leq \operatorname{máx}_{z \in \gamma} \widetilde{C}(z)
$$

estimación que nos permite completar la prueba.

Sea $\mathbf{F}_{\lambda_{h}}: W_{\lambda}(\widehat{\Omega}) \rightarrow V_{h}^{e}(\widehat{\Omega})$ el proyector espectral asociado con el operador $\mathbf{B}_{h}$,

$$
\mathbf{F}_{\lambda_{h}}:=\frac{1}{2 \pi i} \int_{\gamma} R_{z}\left(\mathbf{B}_{h}\right) d z
$$

relativo a los autovalores $\lambda_{1 h}, \cdots, \lambda_{m h}$, los cuales convergen a $\lambda$ a medida que $h$ va a cero. Observemos que

- como $\mathbf{B}_{h}$ es autoadjunto con respecto a la forma bilineal $a_{h}, \mathbf{F}_{\lambda_{h}}$ resulta autoadjunto con respecto a $a_{h}$,

\footnotetext{
${ }^{4}$ Un operador lineal y continuo que transforma un espacio de Banach E en un subespacio de E con dimension finita es compacto.

${ }^{5}$ Sea $\mathcal{T}$ un operador compacto sobre un espacio de Banach y $z \in \mathbb{C} \backslash\{0\}$. El operador $z-\mathcal{T}$ es inyectivo si, y solo si, es suryectivo.
} 
- como $R_{z}\left(\mathbf{B}_{h}\right)$ está acotado para todo $z \in \gamma$ uniformemente en $h$ en las normas $\|\cdot\|_{* h} \mathrm{y}\|\cdot\|_{*}, \mathbf{F}_{\lambda_{h}}$ resulta acotado uniformemente en $h$ en las mismas normas,

- $\mathbf{F}_{\lambda_{h}}\left(W_{\lambda}(\widehat{\Omega})\right)$ coincide con el espacio propio $\mathbf{E}_{\lambda_{h}}\left(V_{h}^{e}(\widehat{\Omega})\right)$ del operador $\mathbf{A}_{h}$ asociado con los autovalores $\lambda_{1 h}, \cdots, \lambda_{m h}$.

Asumiremos que las siguientes propiedades se satisfacen:

P3: para $x \in \mathbf{E}_{\lambda}(W(\widehat{\Omega}))$, existe $x_{h} \in V_{h}^{e}(\widehat{\Omega})$ tal que $\left\|x-x_{h}\right\|_{*} \leq \eta_{2 h}\|x\|_{h}$, con $\eta_{2 h} \rightarrow 0$ para $h \rightarrow 0$,

P4: para todo $x \in \mathbf{E}_{\lambda}(W(\widehat{\Omega})),\left\|\left(\mathbf{A}-\mathbf{A}_{h}\right) x\right\|_{*} \leq \eta_{3 h}\|x\|_{h}$, con $\eta_{3 h} \rightarrow 0$ para $h \rightarrow 0$,

P5: para todo $x \in \mathbf{E}_{\lambda}(W(\widehat{\Omega})),\|x\|_{h, \Omega \backslash \Omega_{h}}+\|x\|_{h, \Omega_{h} \backslash \Omega}+\|x\|_{*, \Omega_{h} \backslash \Omega} \leq \eta_{4 h}\|x\|_{h}$, con $\eta_{4 h} \rightarrow 0$ para $h \rightarrow 0$.

Observación 3.20 A partir de la relación existente entre las normas $\|\cdot\|_{h} y\|\cdot\|_{*}$, es claro que la propiedad $\mathbf{P 3}$ implica la propiedad $\mathbf{P 1}$. Nótese, sin embargo, que no ha sido necesario introducir la norma $\|\cdot\|_{*}$ para analizar la convergencia del método de aproximación.

Es claro entonces que las siguientes cantidades

$$
\begin{aligned}
& \gamma_{1 h}:=\sup _{\substack{y \in \mathbf{E}_{\lambda}(W(\widehat{\Omega})) \\
\|y\|_{h}=1}} \inf _{\substack{x_{h} \in V_{h}^{e}(\widehat{\Omega}) \\
\gamma_{2 h}}}:=\sup _{\substack{y \in \mathbf{E}_{\lambda}(W(\widehat{\Omega})) \\
\|y\|_{h}=1}}\left(\|y\|_{h, \Omega \backslash \Omega_{h}}+\|y\|_{*, \Omega_{h} \backslash \Omega}\right) \\
& \gamma_{3 h}:=\left\|\left.\left(\mathbf{A}-\mathbf{A}_{h}\right)\right|_{\mathbf{E}_{\lambda}(W(\widehat{\Omega}))}\right\|_{h *}, \\
& \varrho_{h}:=\gamma_{1 h}+\gamma_{2 h}+\gamma_{3 h} .
\end{aligned}
$$

tienden a cero a medida que $h$ tiende a cero.

Lema 3.21 Existe una constante $C$, estrictamente positiva, tal que

$$
\left\|\left.\left(\mathbf{I}-\Pi_{h}\right)\right|_{\mathbf{E}_{\lambda}(W(\widehat{\Omega}))}\right\|_{h *} \leq C \gamma_{1 h} .
$$

Prueba. Sea $x \in \mathbf{E}_{\lambda}(W(\widehat{\Omega}))$, con $\|x\|_{h}=1$, y consideremos un elemento arbitrario $y_{h}$ de $V_{h}\left(\Omega_{h}\right)$. La desigualdad triangular, las propiedades del proyector $\Pi_{h}$ y la equivalencia entre las normas $\|\cdot\|_{*} \mathrm{y}\|\cdot\|_{h}$ en $V_{h}^{e}(\widehat{\Omega})$, nos permiten escribir

$$
\begin{aligned}
\left\|\left(\mathbf{I}-\Pi_{h}\right) x\right\|_{*} & \leq\left\|x-y_{h}^{e}\right\|_{*}+\left\|\Pi_{h}\left(y_{h}^{e}-x\right)\right\|_{*} \leq\left\|x-y_{h}^{e}\right\|_{*}+C\left\|\Pi_{h}\left(y_{h}^{e}-x\right)\right\|_{h} \\
& \leq\left\|x-y_{h}^{e}\right\|_{*}+C\left\|y_{h}^{e}-x\right\|_{*} \leq C \inf _{y_{h}^{e} \in V_{h}^{e}(\widehat{\Omega})}\left\|x-y_{h}^{e}\right\|_{*}
\end{aligned}
$$

de donde se obtiene fácilmente el resultado buscado.

Observación 3.22 Es claro que $\Pi_{h}$ converge en norma $\|\cdot\|_{h}$ al operador identidad en $\mathbf{E}_{\lambda}(W(\widehat{\Omega}))$. En efecto, en virtud de la propiedad $\mathbf{P 3}$, para cada $x \in \mathbf{E}_{\lambda}(W(\widehat{\Omega}))$, podemos encontrar $\eta_{2 h} \rightarrow 0$ con $h \rightarrow 0$ tal que $\inf _{y_{h}^{e} \in V_{h}^{e}(\widehat{\Omega})}\left\|x-y_{h}^{e}\right\|_{*} \leq \eta_{2 h}\|x\|_{h}$. Luego, tendremos

$$
\left\|\left(\mathbf{I}-\Pi_{h}\right) x\right\|_{h} \leq\left\|\left(\mathbf{I}-\Pi_{h}\right) x\right\|_{*} \leq \eta_{2 h}\|x\|_{h} \quad \text { para cada } \quad x \in \mathbf{E}_{\lambda}(W(\widehat{\Omega})) .
$$

Lema 3.23 Existe una constante $C$, estrictamente positiva, tal que

$$
\begin{gathered}
\left\|\left.\left(\mathbf{E}_{\lambda}-\mathbf{F}_{\lambda_{h}}\right)\right|_{\mathbf{E}_{\lambda}(W(\widehat{\Omega}))}\right\|_{h} \leq C\left\|\left.\left(\mathbf{A}-\mathbf{B}_{h}\right)\right|_{\mathbf{E}_{\lambda}(W(\widehat{\Omega}))}\right\|_{h *}, \\
\left.\left\|\left.\left(\mathbf{E}_{\lambda}-\mathbf{F}_{\lambda_{h}}\right)\right|_{\mathbf{E}_{\lambda}(W(\widehat{\Omega}))}\right\|\left\|_{h *} \leq C\right\|\left(\mathbf{A}-\mathbf{B}_{h}\right)\right|_{\mathbf{E}_{\lambda}(W(\widehat{\Omega}))}\|\|_{h *} .
\end{gathered}
$$


Prueba. Observemos que, dada la relación existente entre las normas $\|\cdot\|_{h} \mathrm{y}\|\cdot\|_{h *}$, solo será necesario probar la segunda desigualdad. Elijamos $x \in \mathbf{E}_{\lambda}(W(\widehat{\Omega}))$ de manera tal que $\|x\|_{h}=1$. La definición de los proyectores espectrales nos conduce a la siguiente expresión, válida si $h$ es suficientemente pequeño,

$$
\left\|\left(\mathbf{E}_{\lambda}-\mathbf{F}_{\lambda_{h}}\right) x\right\|_{*}=\frac{1}{2 \pi}\left\|\int_{\gamma}\left(R_{z}(\mathbf{A})-R_{z}\left(\mathbf{B}_{h}\right)\right) x d z\right\|_{*} \leq \frac{1}{2 \pi} \int_{\gamma}\left\|R_{z}\left(\mathbf{B}_{h}\right)\left(\mathbf{A}-\mathbf{B}_{h}\right) R_{z}(\mathbf{A}) x\right\|_{*}|d z| .
$$

Teniendo en cuenta que la aplicación $R_{z}(\mathbf{A})$ deja invariante al subespacio $\mathbf{E}_{\lambda}(W(\widehat{\Omega}))$, podemos escribir

$$
\left\|R_{z}\left(\mathbf{B}_{h}\right)\left(\mathbf{A}-\mathbf{B}_{h}\right) R_{z}(\mathbf{A}) x\right\|_{*} \leq\left\|R_{z}\left(\mathbf{B}_{h}\right)\right\|_{\mathcal{L}\left(W_{\lambda}(\widehat{\Omega})\right)}\left\|\mathbf{A}-\mathbf{B}_{h}\right\|_{\mathcal{L}\left(\mathbf{E}_{\lambda}(W(\widehat{\Omega})), W_{\lambda}(\widehat{\Omega})\right)}\left\|R_{z}(\mathbf{A})\right\|_{\mathcal{L}\left(\mathbf{E}_{\lambda}(W(\widehat{\Omega}))\right)} .
$$

Luego, tendremos

$$
\left\|\left(\mathbf{E}_{\lambda}-\mathbf{F}_{\lambda_{h}}\right) x\right\|_{h} \leq \frac{|\gamma|}{2 \pi} \sup _{z \in \gamma}\left\|R_{z}\left(\mathbf{B}_{h}\right)\right\|_{*}\left\|\left.\left(\mathbf{A}-\mathbf{B}_{h}\right)\right|_{\mathbf{E}_{\lambda}(W(\widehat{\Omega}))}\right\|\left\|_{h *} \sup _{z \in \gamma}\right\| R_{z}(\mathbf{A})\left\|_{h}\right\| x \|_{h} .
$$

Terminamos la prueba aplicando los resultados del Corolario 3.4 y Lema 3.19.

Lema 3.24 Existe una constante $C$, estrictamente positiva, tal que

$$
\left\|\left.\left(\mathbf{I}-\mathbf{F}_{\lambda_{h}}\right)\right|_{\mathbf{E}_{\lambda}(W(\widehat{\Omega}))}\right\| \|_{h *} \leq C\left(\left.\left\|\left.\left(\mathbf{I}-\Pi_{h}\right)\right|_{\mathbf{E}_{\lambda}(W(\widehat{\Omega}))}\right\|\left\|_{h *}+\right\|\left(\mathbf{A}-\mathbf{B}_{h}\right)\right|_{\mathbf{E}_{\lambda}(W(\widehat{\Omega}))} \|_{h *}\right) .
$$

Prueba. Elijamos $x \in \mathbf{E}_{\lambda}(W(\widehat{\Omega}))$ de manera tal que $\|x\|_{h}=1$. A partir de la desigualdad triangular y de las propiedades de la norma $\|\cdot\|_{*}$, tendremos

$$
\begin{aligned}
\left\|\left(\mathbf{I}-\mathbf{F}_{\lambda_{h}}\right) x\right\|_{*} & \leq\left\|\left(\mathbf{I}-\Pi_{h}\right) x\right\|_{*}+\left\|\left(\Pi_{h}-\mathbf{F}_{\lambda_{h}}\right) x\right\|_{*} \leq\left\|\left(\mathbf{I}-\Pi_{h}\right) x\right\|_{*}+C\left\|\left(\Pi_{h}-\mathbf{F}_{\lambda_{h}}\right) x\right\|_{h} \\
& \leq\left\|\left(\mathbf{I}-\Pi_{h}\right) x\right\|_{*}+C\left\|\Pi_{h}\left(\mathbf{I}-\mathbf{F}_{\lambda_{h}}\right) x\right\|_{h} \leq C\left(\left\|\left(\mathbf{I}-\Pi_{h}\right) x\right\|_{*}+\left\|\left(\mathbf{I}-\mathbf{F}_{\lambda_{h}}\right) x\right\|_{*}\right) \\
& \leq C\left(\left\|\left.\left(\mathbf{I}-\Pi_{h}\right)\right|_{\mathbf{E}_{\lambda}(W(\widehat{\Omega}))}\right\|_{h *}+\left\|\left.\left(\mathbf{E}_{\lambda}-\mathbf{F}_{\lambda_{h}}\right)\right|_{\mathbf{E}_{\lambda}(W(\widehat{\Omega}))}\right\|_{h *}\right)\|x\|_{h} .
\end{aligned}
$$

Terminamos la prueba aplicando el resultado del Lema 3.23.

Lema 3.25 Existe una constante $C$, estrictamente positiva, tal que

$$
\left\|\left.\left(\mathbf{A}-\mathbf{B}_{h}\right)\right|_{\mathbf{E}_{\lambda}(W(\widehat{\Omega}))}\right\|_{h *} \leq C\left(\gamma_{1 h}+\gamma_{3 h}\right) .
$$

Prueba. Elijamos $x \in \mathbf{E}_{\lambda}(W(\widehat{\Omega}))$ de manera tal que $\|x\|_{h}=1$. A partir de la desigualdad triangular y las propiedades de la norma $\|\cdot\|_{*}$, tendremos

$$
\begin{aligned}
\left\|\left(\mathbf{A}-\mathbf{B}_{h}\right) x\right\|_{*} & \leq\left\|\left(\mathbf{A}-\mathbf{A}_{h}\right) x\right\|_{*}+\left\|\mathbf{A}_{h}\left(\mathbf{I}-\Pi_{h}\right) x\right\|_{*} \leq\left\|\left(\mathbf{A}-\mathbf{A}_{h}\right) x\right\|_{*}+C\left\|\mathbf{A}_{h}\left(\mathbf{I}-\Pi_{h}\right) x\right\|_{h} \\
& \leq\left\|\left(\mathbf{A}-\mathbf{A}_{h}\right) x\right\|_{*}+C\left\|\mathbf{A}_{h}\right\|_{h}\left\|\left(\mathbf{I}-\Pi_{h}\right) y\right\|_{h} \leq\left\|\left(\mathbf{A}-\mathbf{A}_{h}\right) x\right\|_{*}+C\left\|\mathbf{A}_{h}\right\|_{h}\left\|\left(\mathbf{I}-\Pi_{h}\right) y\right\|_{*} \\
& \leq\left\|\left.\left(\mathbf{A}-\mathbf{A}_{h}\right)\right|_{\mathbf{E}_{\lambda}(W(\widehat{\Omega}))}\right\|_{h *}+C\left\|\mathbf{A}_{h}\right\|_{h}\left\|\left.\left(\mathbf{I}-\Pi_{h}\right)\right|_{\mathbf{E}_{\lambda}(W(\widehat{\Omega}))}\right\|_{h *} .
\end{aligned}
$$

La prueba puede concluirse teniendo en cuenta que $\left\|\mathbf{A}_{h}\right\|_{h}$ está acotada uniformemente en $h$ y aplicando los resultados del Lema 3.21.

El siguiente teorema da una cota superior para la distancia entre los subespacios $\mathbf{E}_{\lambda}(W(\widehat{\Omega}))$ y $\mathbf{F}_{\lambda_{h}}\left(W_{\lambda}(\widehat{\Omega})\right)$. Esta distancia coincide con la distancia existente entre los espacios propios $\mathbf{E}_{\lambda}(W(\widehat{\Omega}))$ y $\mathbf{E}_{\lambda_{h}}\left(V_{h}^{e}(\widehat{\Omega})\right)$ de los operadores $\boldsymbol{\Lambda}$ y $\boldsymbol{\Lambda}_{h}$, respectivamente.

Teorema 3.26 Existe una constante $C$, estrictamente positiva, tal que

$$
\widehat{\delta}\left(\mathbf{E}_{\lambda}(W(\widehat{\Omega})), \mathbf{F}_{\lambda_{h}}\left(W_{\lambda}(\widehat{\Omega})\right)\right) \leq C \varrho_{h} .
$$

Prueba. Comencemos observando que, para $h$ suficientemente pequeño, digamos para $h<h_{0}$, los subespacios $\mathbf{E}_{\lambda}(W(\widehat{\Omega}))$ y $\mathbf{F}_{\lambda_{h}}\left(W_{\lambda}(\widehat{\Omega})\right)$ tendrán la misma dimensión $m$, finita. En estas condiciones, tendremos

$$
\delta\left(\mathbf{F}_{\lambda_{h}}\left(W_{\lambda}(\widehat{\Omega})\right), \mathbf{E}_{\lambda}(W(\widehat{\Omega}))\right) \leq \frac{\delta\left(\mathbf{E}_{\lambda}(W(\widehat{\Omega})), \mathbf{F}_{\lambda_{h}}\left(W_{\lambda}(\widehat{\Omega})\right)\right)}{1-\delta\left(\mathbf{E}_{\lambda}(W(\widehat{\Omega})), \mathbf{F}_{\lambda_{h}}\left(W_{\lambda}(\widehat{\Omega})\right)\right.} .
$$


A partir de este resultado, solo nos resta probar que

$$
\delta\left(\mathbf{E}_{\lambda}(W(\widehat{\Omega})), \mathbf{F}_{\lambda_{h}}\left(W_{\lambda}(\widehat{\Omega})\right)\right) \leq C \varrho_{h},
$$

ya que, como $\lim _{h \rightarrow 0} \varrho_{h}=0$, existirá alguna constante $h_{+}$tal que, para todo $h \leq h_{+}$, será $\left(1-C \varrho_{h}\right)^{-1} \leq 2$.

Elijamos $x \in \mathbf{E}_{\lambda}(W(\widehat{\Omega}))$. Como $x=\mathbf{E}_{\lambda} x$ y $\mathbf{F}_{\lambda_{h}}\left(\mathbf{F}_{\lambda_{h}} y\right)=\mathbf{F}_{\lambda_{h}} y$, para todo $y \in W_{\lambda}(\widehat{\Omega})$, tendremos

$$
\inf _{y \in \mathbf{F}_{\lambda_{h}}\left(W_{\lambda}(\widehat{\Omega})\right)}\|x-y\|_{h}=\inf _{y \in W_{\lambda}(\widehat{\Omega})}\left\|\mathbf{E}_{\lambda} x-\mathbf{F}_{\lambda_{h}} y\right\|_{h} \leq\left\|\left(\mathbf{E}_{\lambda}-\mathbf{F}_{\lambda_{h}}\right) x\right\|_{h} .
$$

Luego, en virtud de los Lemas 3.23 y 3.25 ,

$$
\sup _{\substack{x \in \mathbf{E}_{\lambda}(W(\widehat{\Omega})) \\\|x\|_{h}=1}} \inf _{y \in \mathbf{F}_{\lambda_{h}}\left(W_{\lambda}(\widehat{\Omega})\right)}\|x-y\|_{h} \leq \sup _{\substack{x \in \mathbf{E}_{\lambda}(W(\widehat{\Omega})) \\\|x\|_{h}=1}}\left\|\left(\mathbf{E}_{\lambda}-\mathbf{F}_{\lambda_{h}}\right) x\right\|_{h} \leq C\left(\gamma_{1 h}+\gamma_{3 h}\right) .
$$

Luego, para todo $h<\operatorname{mín}\left\{h_{0}, h_{+}\right\}$, tendremos

$$
\widehat{\delta}\left(\mathbf{E}_{\lambda}(W(\widehat{\Omega})), \mathbf{F}_{\lambda_{h}}\left(W_{\lambda}(\widehat{\Omega})\right)\right) \leq C\left(\gamma_{1 h}+\gamma_{3 h}\right)
$$

que es el resultado buscado.

\subsubsection{Estimaciones del error para los autovalores}

Hasta aquí hemos probado que, para $h$ suficientemente pequeño, existen $m$ autovalores $\lambda_{1 h}, \cdots, \lambda_{m h}$ de $\boldsymbol{\Lambda}_{h}$ que convergen al autovalor $\lambda$ de $\boldsymbol{\Lambda}$ cuya multiplicidad algebraica es $m$. En otras palabras, si se satisfacen las propiedades $\mathbf{P} \mathbf{1}-\mathbf{P} \mathbf{2}$, hemos probado que

$$
\lim _{h \rightarrow 0} \lambda_{i h}=\lambda \quad \text { para } \quad i=1, \cdots, m .
$$

En esta sección analizaremos la rapidez con la que convergen los autovalores aproximados. Adoptaremos la linea argumental presentada en [150].

En lo que sigue, continuaremos usando la notación introducida en las secciones anteriores y sostendremos todas las hipótesis hechas previamente.

Comenzaremos definiendo la siguiente aplicación

$$
\boldsymbol{\Xi}_{h}:=\left.\mathbf{F}_{\lambda_{h}}\right|_{\mathbf{E}_{\lambda}(W(\widehat{\Omega}))}: \mathbf{E}_{\lambda}(W(\widehat{\Omega})) \rightarrow \mathbf{E}_{\lambda_{h}}\left(V_{h}^{e}(\widehat{\Omega})\right),
$$

i.e., el proyector $\mathbf{F}_{\lambda_{h}}$ como un operador restringido al subespacio $\mathbf{E}_{\lambda}(W(\widehat{\Omega}))$. Es posible demostrar que, para $h$ suficientemente pequeño, $\boldsymbol{\Xi}_{h}$ es una aplicación invertible y con inversa uniformemente acotada.

Lema 3.27 Existe una constante positiva $h_{0}$ tal que, si $h<h_{0},\left\|\boldsymbol{\Xi}_{h}^{-1}\right\|_{h}<2$.

Prueba. Elijamos $x \in \mathbf{E}_{\lambda}(W(\widehat{\Omega}))$ de manera tal que $\mathbf{F}_{\lambda_{h}} x=0$. Por la propiedad P3, sabemos que existe $x_{h} \in V_{h}^{e}(\widehat{\Omega})$ tal que

$$
\begin{aligned}
\|x\|_{h} & =\left\|\mathbf{E}_{\lambda} x\right\|_{h}=\left\|\left(\mathbf{E}_{\lambda}-\mathbf{F}_{\lambda_{h}}\right) x\right\|_{h} \leq\left\|\left(\mathbf{E}_{\lambda}-\mathbf{F}_{\lambda_{h}}\right)\left(x-x_{h}\right)\right\|_{h}+\left\|\left(\mathbf{E}_{\lambda}-\mathbf{E}_{\lambda_{h}}\right) x_{h}\right\|_{h} \\
& \leq \eta_{2 h}\left\|\mathbf{E}_{\lambda}-\mathbf{F}_{\lambda_{h}}\right\|_{* h}\|x\|_{h}+\left\|\left.\left(\mathbf{E}_{\lambda}-\mathbf{E}_{\lambda_{h}}\right)\right|_{V_{h}^{e}(\widehat{\Omega})}\right\| h\left\|x_{h}\right\|_{h} \\
& \leq \eta_{2 h}\left\|\mathbf{E}_{\lambda}-\mathbf{F}_{\lambda_{h}}\right\|_{* h}\|x\|_{h}+\left(1+\eta_{2 h}\right)\left\|\left.\left(\mathbf{E}_{\lambda}-\mathbf{E}_{\lambda_{h}}\right)\right|_{V_{h}^{e}(\widehat{\Omega})}\right\|_{h}\|x\|_{h} .
\end{aligned}
$$

Como las proyecciones $\mathbf{E}_{\lambda}$ y $\mathbf{F}_{\lambda_{h}}$ están uniformemente acotadas en la norma $\|\cdot\|_{* h}$ y $\eta_{2 h} \rightarrow 0$ cuando $h \rightarrow 0$, el Teorema 3.10 nos permite asegurar que, para todo $h$ menor que cierta constante $h_{0}$,

$$
\|x\|_{h}<\frac{1}{2}\|x\|_{h}
$$


Luego, la aplicación $\boldsymbol{\Xi}_{h}$ es uno a uno. Observemos que también es una aplicación onto ya que, por el Teorema 3.13,

$$
\operatorname{dim} \mathbf{E}_{\lambda}(W(\widehat{\Omega}))=\operatorname{dim} \mathbf{E}_{\lambda_{h}}\left(V_{h}^{e}(\widehat{\Omega})\right)=m .
$$

Concluimos entonces que $\mathbf{\Xi}_{h}^{-1}: \mathbf{E}_{\lambda_{h}}\left(V_{h}^{e}(\widehat{\Omega})\right) \rightarrow \mathbf{E}_{\lambda}(W(\widehat{\Omega}))$ está bien definida para $h$ chico. Más aún, si $h$ es chico, está acotada uniformemente en $h$. En efecto, para $x \in \mathbf{E}_{\lambda}(W(\widehat{\Omega}))$, tendremos

$$
\|x\|_{h}-\left\|\mathbf{F}_{\lambda_{h}} x\right\|_{h} \leq\left\|\left(\mathbf{E}_{\lambda}-\mathbf{F}_{\lambda_{h}}\right) x\right\|_{h} \leq \frac{1}{2}\|x\|_{h} .
$$

De aquí concluimos inmediatamente $\left\|\boldsymbol{\Xi}_{h}^{-1} y_{h}\right\|_{h} \leq 2\left\|y_{h}\right\|_{h}$ para todo $y_{h} \in \mathbf{E}_{\lambda_{h}}\left(V_{h}^{e}(\widehat{\Omega})\right)$.

Consideremos los siguientes operadores

$$
\begin{aligned}
& \widehat{\mathbf{A}}:=\left.\mathbf{A}\right|_{\mathbf{E}_{\lambda}(W(\widehat{\Omega}))}: \mathbf{E}_{\lambda}(W(\widehat{\Omega})) \rightarrow \mathbf{E}_{\lambda}(W(\widehat{\Omega})), \\
& \widehat{\mathbf{B}}_{h}:=\mathbf{\Xi}_{h}^{-1} \mathbf{B}_{h} \boldsymbol{\Xi}_{h}: \mathbf{E}_{\lambda}(W(\widehat{\Omega})) \rightarrow \mathbf{E}_{\lambda}(W(\widehat{\Omega})) .
\end{aligned}
$$

Elijamos $x \in \mathbf{E}_{\lambda}\left(W(\widehat{\Omega})\right.$. Usando el resultado del Lema 3.27 y teniendo en cuenta que $\left\|\mathbf{B}_{h}\right\|_{* h}$ está acotada uniformemente, tendremos

$$
\left\|\widehat{\mathbf{B}}_{h} x\right\|_{h}=\left\|\boldsymbol{\Xi}_{h}^{-1} \mathbf{B}_{h} \mathbf{\Xi}_{h} x\right\|_{h} \leq\left\|\boldsymbol{\Xi}_{h}^{-1}\right\|_{h}\left\|\mathbf{B}_{h} \boldsymbol{\Xi}_{h} x\right\|_{h} \leq 2\left\|\mathbf{B}_{h}\right\|_{* h}\left\|\boldsymbol{\Xi}_{h} x\right\|_{*} \leq C\left\|\boldsymbol{\Xi}_{h} x\right\|_{*}
$$

y, dado que $\boldsymbol{\Xi}_{h} x \in V_{h}^{e}\left(\Omega_{h}\right)$ y $\left\|\mathbf{F}_{\lambda_{h}}\right\|_{* h}$ está uniformemente acotada,

$$
\left\|\boldsymbol{\Xi}_{h} x\right\|_{*} \leq C\left\|\boldsymbol{\Xi}_{h} x\right\|_{h} \leq C\left\|\left.\mathbf{F}_{\lambda_{h}}\right|_{\mathbf{E}_{\lambda}(W(\widehat{\Omega}))}\right\|_{* h}\|x\|_{*} \leq C\|x\|_{h} .
$$

La combinación de las dos estimaciones anteriores nos permite afirmar que $\widehat{\mathbf{B}}_{h}$ está acotado uniformemente en la norma $\|\cdot\|_{h}$.

Además, como $\boldsymbol{\Xi}_{h}^{-1} \boldsymbol{\Xi}_{h}$ es igual a la identidad sobre $\mathbf{E}_{\lambda}(W(\widehat{\Omega}))$, tendremos

$$
\left\|\left(z-\widehat{\mathbf{B}}_{h}\right)^{-1} x\right\|_{h}=\left\|\left(\boldsymbol{\Xi}_{h}^{-1}\left(z-\mathbf{B}_{h}\right) \boldsymbol{\Xi}_{h}\right)^{-1} x\right\|_{h}=\left\|\boldsymbol{\Xi}_{h}^{-1}\left(z-\mathbf{B}_{h}\right)^{-1} \boldsymbol{\Xi}_{h} x\right\|_{h} \quad \forall x \in \mathbf{E}_{\lambda}(W(\widehat{\Omega})) .
$$

Luego, gracias a los Lemas 3.19, 3.27 y a la estimación (3.19), podremos asegurar que existe una constante positiva $C$ tal que, si $h$ es suficientemente pequeño,

$$
\left\|\left(z-\widehat{\mathbf{B}}_{h}\right)^{-1} x\right\|_{h} \leq\left\|\boldsymbol{\Xi}_{h}^{-1}\right\|_{h}\left\|\left(z-\mathbf{B}_{h}\right)^{-1}\right\|_{* h}\left\|\boldsymbol{\Xi}_{h} x\right\|_{*} \leq C\|x\|_{h} .
$$

Observemos también que

- como $\mathbf{E}_{\lambda}(W(\widehat{\Omega}))$ es A-invariante, $\sigma(\widehat{\mathbf{A}})=\lambda$,

- como $\mathbf{E}_{\lambda_{h}}\left(V_{h}^{e}(\widehat{\Omega})\right)$ es $\mathbf{B}_{h}$-invariante, $\sigma\left(\widehat{\mathbf{B}}_{h}\right)=\lambda_{1 h}, \cdots, \lambda_{m h}$.

El siguiente lema es clave para establecer las estimaciones que nos permitirán determinar el orden de convergencia de los autovalores aproximados.

Lema 3.28 Existen constantes $C$ y $h_{0}$, estrictamente positivas, tales que, para $h<h_{0}$,

$$
\left\|\widehat{\mathbf{A}}-\widehat{\mathbf{B}}_{h}\right\|_{h} \leq C\left(\varrho_{h}^{2}+M_{h}+N_{h}\right),
$$

siendo $M_{h}$ y $N_{h}$ términos de consistencia definidos por:

$$
\begin{gathered}
M_{h}=\sup _{\substack{x \in \mathbf{E}_{\lambda}(W(\widehat{\Omega})) \\
\|x\|_{h}=1}} \sup _{\substack{y \in \mathbf{E}_{\lambda}(W(\widehat{\Omega})) \\
\|y\|_{h}=1}}\left|a_{h}\left(\mathbf{A} x, \Pi_{h} y-y\right)-b_{h}\left(x, \Pi_{h} y-y\right)\right|, \\
N_{h}=\sup _{\substack{x \in \mathbf{E}_{\lambda}(W(\widehat{\Omega})) \\
\|x\|_{h}=1}} \sup _{\substack{y \in \mathbf{E}_{\lambda}(W(\widehat{\Omega})) \\
\|y\|_{h}=1}}\left|a_{h}(\mathbf{A} x, y)-b_{h}(x, y)\right| .
\end{gathered}
$$


Prueba. Tendremos

$$
\left\|\widehat{\mathbf{A}}-\widehat{\mathbf{B}}_{h}\right\|_{h} \leq \sup _{\substack{x \in \mathbf{E}_{\lambda}(W(\widehat{\Omega})) \\\|x\|_{h}=1}}\left\|\left(\widehat{\mathbf{A}}-\widehat{\mathbf{B}}_{h}\right) x\right\|_{h, \Omega}+\sup _{\substack{x \in \mathbf{E}_{\lambda}(W(\widehat{\Omega})) \\\|x\|_{h}=1}}\left\|\left(\widehat{\mathbf{A}}-\widehat{\mathbf{B}}_{h}\right) x\right\|_{h, \Omega_{h} \backslash \Omega}
$$

Observemos que $\left(\widehat{\mathbf{A}}-\widehat{\mathbf{B}}_{h}\right) x \in \mathbf{E}_{\lambda}(W(\widehat{\Omega}))$. Luego, debido a la propiedad P5,

$$
\left\|\left(\widehat{\mathbf{A}}-\widehat{\mathbf{B}}_{h}\right) x\right\|_{h, \Omega_{h} \backslash \Omega} \leq \eta_{4 h}\left\|\left(\widehat{\mathbf{A}}-\widehat{\mathbf{B}}_{h}\right) x\right\|_{h, \Omega} \leq \eta_{4 h}\left\|\widehat{\mathbf{A}}-\widehat{\mathbf{B}}_{h}\right\|_{h}\|x\|_{h},
$$

donde $\eta_{4 h} \rightarrow 0$ a medida que $h \rightarrow 0$. Entonces, para $h$ suficientemente chico, podremos escribir

$$
\sup _{\substack{x \in \mathbf{E}_{\lambda}(W(\widehat{\Omega})) \\\|x\|_{h}=1}}\left\|\left(\widehat{\mathbf{A}}-\widehat{\mathbf{B}}_{h}\right) x\right\|_{h, \Omega_{h} \backslash \Omega} \leq \frac{1}{2}\left\|\widehat{\mathbf{A}}-\widehat{\mathbf{B}}_{h}\right\|_{h} .
$$

Este resultado permite acotar fácilmente el segundo término de (3.21). Para el primer término, tendremos

$$
\begin{aligned}
& \sup _{x \in \mathbf{E}_{\lambda}(W(\widehat{\Omega}))}\left\|\left(\widehat{\mathbf{A}}-\widehat{\mathbf{B}}_{h}\right) x\right\|_{h, \Omega} \leq C \sup _{x \in \mathbf{E}_{\lambda}(W(\widehat{\Omega}))} \sup _{y \in W(\widehat{\Omega})} a\left(\left(\widehat{\mathbf{A}}-\widehat{\mathbf{B}}_{h}\right) x, y\right) \\
& \begin{array}{ccc}
\|x\|_{h}=1 & x \in \mathbf{E}_{\lambda}(W(\widehat{\Omega})) & y \in W(\widehat{\Omega}) \\
\|x\|_{h}=1 & \|y\|_{h}=1
\end{array} \\
& =C \sup _{x \in \mathbf{E}_{\lambda}(W(\widehat{\Omega}))} \sup _{y \in W(\widehat{\Omega})} a\left(\mathbf{E}_{\lambda}\left(\left(\widehat{\mathbf{A}}-\widehat{\mathbf{B}}_{h}\right) x\right), y\right) \\
& \begin{array}{cc}
x \in \mathbf{E}_{\lambda}(W(\widehat{\Omega})) & y \in W(\widehat{\Omega}) \\
\|x\|_{h}=1 & \|y\|_{h}=1
\end{array} \\
& =C \quad \sup \quad \sup a\left(\left(\widehat{\mathbf{A}}-\widehat{\mathbf{B}}_{h}\right) x, \mathbf{E}_{\lambda}(y)\right) \\
& \begin{array}{cc}
x \in \mathbf{E}_{\lambda}(W(\widehat{\Omega})) & y \in W(\widehat{\Omega}) \\
\|x\|_{h}=1 & \|y\|_{h}=1
\end{array} \\
& \leq C \sup _{x \in \mathbf{E}_{\lambda}(W(\widehat{\Omega}))} \sup _{y \in \mathbf{E}_{\lambda}(W(\widehat{\Omega}))} a\left(\left(\widehat{\mathbf{A}}-\widehat{\mathbf{B}}_{h}\right) x, y\right) .
\end{aligned}
$$

Dado que $\left(\widehat{\mathbf{A}}-\widehat{\mathbf{B}}_{h}\right) x$ e $y$ pertenecen al espacio $V(\widehat{\Omega})$, podemos usar (3.13), (3.14) y (3.15) para obtener

$$
\begin{aligned}
a\left(\left(\widehat{\mathbf{A}}-\widehat{\mathbf{B}}_{h}\right) x, y\right) & =a_{1 h}\left(\left.\left(\widehat{\mathbf{A}}-\widehat{\mathbf{B}}_{h}\right) x\right|_{\Omega \cap \Omega_{h}},\left.y\right|_{\Omega \cap \Omega_{h}}\right)+a_{2}\left(\left.\left(\widehat{\mathbf{A}}-\widehat{\mathbf{B}}_{h}\right) x\right|_{\Omega \backslash \Omega_{h}},\left.y\right|_{\Omega \backslash \Omega_{h}}\right) \\
& =a_{h}\left(\left(\widehat{\mathbf{A}}-\widehat{\mathbf{B}}_{h}\right) x, y\right)-a_{2 h}\left(\left.\left(\widehat{\mathbf{A}}-\widehat{\mathbf{B}}_{h}\right) x\right|_{\Omega_{h} \backslash \Omega},\left.y\right|_{\Omega_{h} \backslash \Omega}\right) \\
& +a_{2}\left(\left.\left(\widehat{\mathbf{A}}-\widehat{\mathbf{B}}_{h}\right) x\right|_{\Omega \backslash \Omega_{h}},\left.y\right|_{\Omega \backslash \Omega_{h}}\right) .
\end{aligned}
$$

Teniendo en cuenta que $\boldsymbol{\Xi}^{-1} \boldsymbol{\Xi}=\left.\mathbf{I}\right|_{\mathbf{E}_{\lambda}(W(\widehat{\Omega}))}$ y que $\mathbf{E}_{\lambda}(W(\widehat{\Omega}))$ es un subespacio invariante de $\mathbf{A}$, es inmediato que $\left.\left(\boldsymbol{\Xi}_{h}^{-1} \mathbf{F}_{\lambda_{h}}-\mathbf{I}\right) \mathbf{A}\right|_{\mathbf{E}_{\lambda}(W(\widehat{\Omega}))}=0$. Luego, como $\mathbf{B}_{h}$ commuta con su proyector espectral $\mathbf{F}_{\lambda_{h}}$, tendremos

$$
\widehat{\mathbf{A}}-\widehat{\mathbf{B}}_{h}=\left.\left(\mathbf{A}-\mathbf{B}_{h}\right)\right|_{\mathbf{E}_{\lambda}(W(\widehat{\Omega}))}+\left.\left(\boldsymbol{\Xi}_{h}^{-1} \mathbf{F}_{\lambda_{h}}-\mathbf{I}\right)\left(\mathbf{A}-\mathbf{B}_{h}\right)\right|_{\mathbf{E}_{\lambda}(W(\widehat{\Omega}))} .
$$

A continuación acotaremos cada término que resulta de reemplazar la expresión (3.24) en la ecuación (3.23) por separado.

Acotación del término $\left|a_{h}\left(\left(\mathbf{A}-\mathbf{B}_{h}\right) x, y\right)\right|$. Tendremos

$$
a_{h}\left(\left(\mathbf{A}-\mathbf{B}_{h}\right) x, y\right)=a_{h}\left(\left(\mathbf{A}-\mathbf{B}_{h}\right) x,\left(\mathbf{I}-\Pi_{h}\right) y\right)+a_{h}\left(\left(\mathbf{A}-\mathbf{B}_{h}\right) x, \Pi_{h} y\right) .
$$

Podemos usar los Lemas 3.21 y 3.25 para acotar el primer término en el lado derecho de esta ecuación. Obtendremos

$$
\begin{aligned}
\left|a_{h}\left(\left(\mathbf{A}-\mathbf{B}_{h}\right) x,\left(\mathbf{I}-\Pi_{h}\right) y\right)\right| & \leq C\left\|\left(\mathbf{A}-\mathbf{B}_{h}\right) x\right\|_{*}\left\|\left(\mathbf{I}-\Pi_{h}\right) x\right\|_{*} \\
& \leq C\left\|\left.\left(\mathbf{A}-\mathbf{B}_{h}\right)\right|_{\mathbf{E}_{\lambda}(W(\widehat{\Omega}))}\right\|_{h *}\left\|\left.\left(\mathbf{I}-\Pi_{h}\right)\right|_{\mathbf{E}_{\lambda}(W(\widehat{\Omega}))}\right\|_{h *} \\
& \leq C \gamma_{1 h} \gamma_{3 h} .
\end{aligned}
$$

Para el segundo término, escribimos

$$
a_{h}\left(\left(\mathbf{A}-\mathbf{B}_{h}\right) x, \Pi_{h} y\right)=a_{h}\left(\left(\mathbf{A}-\mathbf{A}_{h}\right) x, \Pi_{h} y\right)+a_{h}\left(\left(\mathbf{A}_{h}-\mathbf{B}_{h}\right) x, \Pi_{h} y\right) .
$$


Dado que $\left.\Pi_{h} y\right|_{\Omega_{h}} \in V_{h}\left(\Omega_{h}\right)$

$$
\begin{gathered}
\left|a_{h}\left(\left(\mathbf{A}-\mathbf{A}_{h}\right) x, \Pi_{h} y\right)\right|=\left|a_{h}\left(\mathbf{A} x, \Pi_{h} y\right)-b_{h}\left(x, \Pi_{h} y\right)\right| \leq M_{h}+N_{h}, \\
a_{h}\left(\left(\mathbf{A}_{h}-\mathbf{B}_{h}\right) x, \Pi_{h} y\right)=a_{h}\left(\mathbf{A}_{h}\left(\mathbf{I}-\Pi_{h}\right) x, \Pi_{h} y\right)=b_{h}\left(\left(\mathbf{I}-\Pi_{h}\right) x, \Pi_{h} y\right) .
\end{gathered}
$$

Es inmediato que

$$
b_{h}\left(\left(\mathbf{I}-\Pi_{h}\right) x, \Pi_{h} y\right)=b_{h}\left(\left(\mathbf{I}-\Pi_{h}\right) x, y\right)-b_{h}\left(\left(\mathbf{I}-\Pi_{h}\right) x,\left(\mathbf{I}-\Pi_{h}\right) y\right) .
$$

El primer término en el lado derecho de (3.30) puede escribirse

$$
b_{h}\left(\left(\mathbf{I}-\Pi_{h}\right) x, y\right)=a_{h}\left(\left(\Pi_{h}-\mathbf{I}\right) x, \mathbf{A} y\right)-b_{h}\left(\left(\Pi_{h}-\mathbf{I}\right) x, y\right)-a_{h}\left(\left(\Pi_{h}-\mathbf{I}\right) x,\left(\mathbf{I}-\Pi_{h}\right) \mathbf{A} y\right) .
$$

Observemos que los dos primeros términos de esta ecuación están acotados directamente por $M_{h}$. Para el término restante, tendremos

$$
\left|a_{h}\left(\left(\Pi_{h}-\mathbf{I}\right) x,\left(\mathbf{I}-\Pi_{h}\right) \mathbf{A} y\right)\right| \leq\left. C\left\|\left.\left(\Pi_{h}-\mathbf{I}\right)\right|_{\mathbf{E}_{\lambda}(W(\widehat{\Omega}))}\right\|\left\|_{h *}\right\|\left(\mathbf{I}-\Pi_{h}\right)\right|_{\mathbf{E}_{\lambda}(W(\widehat{\Omega}))} \|_{h *} .
$$

Luego, combinando esta estimación con el resultado del Lemma 3.21, obtenemos

$$
\left|b_{h}\left(\left(\mathbf{I}-\Pi_{h}\right) x, y\right)\right| \leq C\left(M_{h}+\gamma_{1 h}^{2}\right) .
$$

El segundo término en el lado derecho de la ecuación (3.30) puede acotarse fácilmente teniendo en cuenta la continuidad de la forma bilineal $b_{h}$ sobre $\left(V+V_{h}\right)\left(\Omega_{h}\right)$. Tendremos

$$
\left.\left|b_{h}\left(\left(\mathbf{I}-\Pi_{h}\right) x,\left(\mathbf{I}-\Pi_{h}\right) y\right)\right| \leq C\left\|\left(\mathbf{I}-\Pi_{h}\right) x\right\|_{h} \|\left(\mathbf{I}-\Pi_{h}\right) y\right) \|_{h} \leq C \gamma_{1 h}^{2} .
$$

Acotación del término $\left|a_{h}\left(\left(\boldsymbol{\Xi}_{h}^{-1} \mathbf{F}_{\lambda_{h}}-\mathbf{I}\right)\left(\mathbf{A}-\mathbf{B}_{h}\right) x, y\right)\right|$. Comencemos observando que $\mathbf{F}_{\lambda_{h}}\left(\boldsymbol{\Xi}_{h}^{-1} \mathbf{F}_{\lambda_{h}}-\right.$ $\mathbf{I})\left.\right|_{W_{\lambda}(\widehat{\Omega})}=0$. Luego, como $\mathbf{F}_{\lambda_{h}}$ es autoadjunto con respecto a la forma bilineal to $a_{h}$, tendremos

$$
\begin{aligned}
& \left|a_{h}\left(\left(\boldsymbol{\Xi}_{h}^{-1} \mathbf{F}_{\lambda_{h}}-\mathbf{I}\right)\left(\mathbf{A}-\mathbf{B}_{h}\right) x, y\right)\right| \\
& =\left|a_{h}\left(\left(\boldsymbol{\Xi}_{h}^{-1} \mathbf{F}_{\lambda_{h}}-\mathbf{I}\right)\left(\mathbf{A}-\mathbf{B}_{h}\right) x, y\right)-a_{h}\left(\mathbf{F}_{\lambda_{h}}\left(\boldsymbol{\Xi}_{h}^{-1} \mathbf{F}_{\lambda_{h}}-\mathbf{I}\right)\left(\mathbf{A}-\mathbf{B}_{h}\right) x, y\right)\right| \\
& =\left|a_{h}\left(\left(\boldsymbol{\Xi}_{h}^{-1} \mathbf{F}_{\lambda_{h}}-\mathbf{I}\right)\left(\mathbf{A}-\mathbf{B}_{h}\right) x, y\right)-a_{h}\left(\left(\boldsymbol{\Xi}_{h}^{-1} \mathbf{F}_{\lambda_{h}}-\mathbf{I}\right)\left(\mathbf{A}-\mathbf{B}_{h}\right) x, \mathbf{F}_{\lambda_{h}} y\right)\right| \\
& =\left|a_{h}\left(\left(\mathbf{\Xi}_{h}^{-1} \mathbf{F}_{\lambda_{h}}-\mathbf{I}\right)\left(\mathbf{A}-\mathbf{B}_{h}\right) x,\left(\mathbf{I}-\mathbf{F}_{\lambda_{h}}\right) y\right)\right| \\
& \leq C\left\|\left(\boldsymbol{\Xi}_{h}^{-1} \mathbf{F}_{\lambda_{h}}-\mathbf{I}\right)\left(\mathbf{A}-\mathbf{B}_{h}\right) x\right\|_{*}\left\|\left(\mathbf{I}-\mathbf{F}_{\lambda_{h}}\right) y\right\|_{*} \\
& \leq C\left(\left\|\left(\boldsymbol{\Xi}_{h}^{-1} \mathbf{F}_{\lambda_{h}}\left(\mathbf{A}-\mathbf{B}_{h}\right) x\left\|_{*}+\right\|\left(\mathbf{A}-\mathbf{B}_{h}\right) x \|_{*}\right)\right\|\left(\mathbf{I}-\mathbf{F}_{\lambda_{h}}\right) y \|_{*}\right. \\
& \leq C\left(\left\|\left(\boldsymbol{\Xi}_{h}^{-1} \mathbf{F}_{\lambda_{h}}\left(\mathbf{A}-\mathbf{B}_{h}\right) x\left\|_{h}+\right\|\left(\mathbf{A}-\mathbf{B}_{h}\right) x \|_{*}\right)\right\|\left(\mathbf{I}-\mathbf{F}_{\lambda_{h}}\right) y \|_{*}\right. \\
& \leq C\left(\left.\left\|\left(\left.\boldsymbol{\Xi}_{h}^{-1}\right|_{\mathbf{E}_{\lambda_{h}}\left(V_{h}^{e}(\widehat{\Omega})\right)}\|\|_{h}\left\|\left.\mathbf{F}_{\lambda_{h}}\right|_{W_{\lambda}(\widehat{\Omega})}\right\|_{* h}+1\right)\right\|\left(\mathbf{A}-\mathbf{B}_{h}\right)\right|_{\mathbf{E}_{\lambda}(W(\widehat{\Omega}))}\|\|_{h *}\left\|\left.\left(\mathbf{I}-\mathbf{F}_{\lambda_{h}}\right)\right|_{\mathbf{E}_{\lambda}(W(\widehat{\Omega}))}\right\|_{h *}\right. \\
& \leq C \gamma_{3 h}\left(\gamma_{1 h}+\gamma_{3 h}\right) .
\end{aligned}
$$

Para obtener las últimas desigualdades en (3.34) hemos usado que $a_{h}$ es uniformemente continua en el espacio $W_{\lambda}(\widehat{\Omega})$ con respecto a la norma $\|\cdot\|_{*}$, que los operadores $\boldsymbol{\Xi}_{h}^{-1}$ y $\mathbf{F}_{h}$ están uniformemente acotados en las normas indicadas y, finalmente, los resultados de los Lemas 3.21-3.25.

Acotación del término $\left|a_{2}\left(\left.\left(\widehat{\mathbf{A}}-\widehat{\mathbf{B}}_{h}\right) x\right|_{\Omega \backslash \Omega_{h}},\left.y\right|_{\Omega \backslash \Omega_{h}}\right)\right|$. Teniendo en cuenta la condición (3.16), que los operadores $\boldsymbol{\Xi}_{h}^{-1}$ y $\mathbf{F}_{h}$ están uniformemente acotados y el resultado del Lema 3.25 , tendremos

$$
\begin{aligned}
\left|a_{2}\left(\left.\left(\hat{\mathbf{A}}-\hat{\mathbf{B}}_{h}\right) x\right|_{\Omega \backslash \Omega_{h}},\left.y\right|_{\Omega \backslash \Omega_{h}}\right)\right| & \leq C\left\|\left(\widehat{\mathbf{A}}-\widehat{\mathbf{B}}_{h}\right) x\right\|_{h, \Omega \backslash \Omega_{h}}\|y\|_{h, \Omega \backslash \Omega_{h}} \\
& \leq C\left\|\mathbf{\Xi}^{-1} \mathbf{F}_{\lambda_{h}}\left(\left(\mathbf{A}-\mathbf{B}_{h}\right) x\right)\right\|_{h}\|y\|_{h, \Omega \backslash \Omega_{h}} \\
& \leq C\left\|\left.\left(\mathbf{A}-\mathbf{B}_{h}\right)\right|_{\mathbf{E}_{\lambda}(W(\widehat{\Omega}))}\right\| h *\|y\|_{h, \Omega \backslash \Omega_{h}} \\
& \leq C \gamma_{2 h} \gamma_{3 h} .
\end{aligned}
$$


Acotación del término $\left|a_{2 h}\left(\left.\left(\widehat{\mathbf{A}}-\widehat{\mathbf{B}}_{h}\right) x\right|_{\Omega_{h} \backslash \Omega},\left.y\right|_{\Omega_{h} \backslash \Omega}\right)\right|$. Teniendo en cuenta la condición (3.17), las propiedades de la norma $\|\cdot\|_{*}$, que los operadores $\boldsymbol{\Xi}_{h}^{-1}$ y $\mathbf{F}_{h}$ están uniformemente acotados y el resultado del Lema 3.25, tendremos

$$
\begin{aligned}
\left|a_{2 h}\left(\left.\left(\widehat{\mathbf{A}}-\widehat{\mathbf{B}}_{h}\right) x\right|_{\Omega_{h} \backslash \Omega},\left.y\right|_{\Omega_{h} \backslash \Omega}\right)\right| & \leq C\left\|\left(\widehat{\mathbf{A}}-\widehat{\mathbf{B}}_{h}\right) x\right\|_{*, \Omega_{h} \backslash \Omega}\|y\|_{*, \Omega_{h} \backslash \Omega} \\
& \leq C\left\|\left(\widehat{\mathbf{A}}-\widehat{\mathbf{B}}_{h}\right) x\right\|_{*}\|y\|_{*, \Omega_{h} \backslash \Omega} \\
& \leq C\left\|\mathbf{\Xi}^{-1} \mathbf{F}_{\lambda_{h}}\left(\left(\mathbf{A}-\mathbf{B}_{h}\right) x\right)\right\|_{h}\|y\|_{*, \Omega_{h} \backslash \Omega} \\
& \leq C\left\|\left.\left(\mathbf{A}-\mathbf{B}_{h}\right)\right|_{\mathbf{E}(V(\widehat{\Omega}))}\right\|\left\|_{h *}\right\| y \|_{*, \Omega_{h} \backslash \Omega} \\
& \leq C \gamma_{2 h} \gamma_{3 h} .
\end{aligned}
$$

Como consecuencia de las ecuaciones (3.21)-(3.36), obtenemos

$$
\sup _{\substack{x \in \mathbf{E}_{\lambda}(W(\widehat{\Omega})) \\\|x\|_{h}=1}}\left\|\left(\widehat{\mathbf{A}}-\widehat{\mathbf{B}}_{h}\right) x\right\|_{h, \Omega} \leq C\left(\varrho_{h}^{2}+M_{h}+N_{h}\right) .
$$

Así concluimos la prueba.

Si bien hemos establecido que cada uno de los autovalores aproximados $\lambda_{i h}, i=1, \cdots, m$, está muy cerca de $\lambda$ para $h$ suficientemente chico, la media aritmética es, en general, una representación más cercana del valor verdadero [49]. Definimos

$$
\widehat{\lambda}_{h}:=\frac{1}{m} \sum_{i=1}^{m} \lambda_{i h}
$$

El siguiente teorema establece estimaciones del error en la aproximación de los autovalores.

Teorema 3.29 Existen constantes $h_{0}$ y $C$, estrictamente positivas, tales que, para $h<h_{0}$,

$$
\left|\lambda-\widehat{\lambda}_{h}\right| \leq C\left(\varrho_{h}^{2}+M_{h}+N_{h}\right)
$$

ii) $\quad\left|\frac{1}{\lambda}-\frac{1}{m} \sum_{i=1}^{m} \frac{1}{\lambda_{i h}}\right| \leq C\left(\varrho_{h}^{2}+M_{h}+N_{h}\right)$,

iii)

$$
\operatorname{máx}_{i=1, \cdots, m}\left|\lambda-\lambda_{i h}\right| \leq C\left(\varrho_{h}^{2}+M_{h}+N_{h}\right) .
$$

Prueba. Denotaremos por $(\cdot, \cdot)$ al producto interno en $V(\widehat{\Omega})$ asociado con la forma bilineal $a(\cdot, \cdot)$. Sea $\zeta_{1}, \cdots, \zeta_{m}$ una base ortonormal para $\mathbf{E}_{\lambda}(\widehat{\Omega})$. Como señalamos al comienzo de esta sección, $\sigma(\widehat{\mathbf{A}})=\lambda \mathrm{y}$ $\sigma\left(\widehat{\mathbf{B}}_{h}\right)=\lambda_{1 h}, \cdots, \lambda_{m h}$.

i) Como los operadores $\widehat{\mathbf{A}}$ y $\widehat{\mathbf{B}}_{h}$ están definidos sobre el mismo espacio de funciones, podremos escribir

$$
\lambda-\widehat{\lambda}_{h}=\frac{1}{m}\left(\operatorname{tr}(\widehat{\mathbf{A}})-\operatorname{tr}\left(\widehat{\mathbf{B}}_{h}\right)\right)=\frac{1}{m} \operatorname{tr}\left(\widehat{\mathbf{A}}-\widehat{\mathbf{B}}_{h}\right)=\frac{1}{m} \sum_{k=1}^{m}\left(\left(\widehat{\mathbf{A}}-\widehat{\mathbf{B}}_{h}\right) \zeta_{k}, \zeta_{k}\right) .
$$

Luego, es inmediato que

$$
\left|\lambda-\widehat{\lambda}_{h}\right| \leq C\left\|\widehat{\mathbf{A}}-\widehat{\mathbf{B}}_{h}\right\|_{h}
$$

ii) Sea $f$ una función de $z$ holomorfa en una vecindad de $\lambda$. Por definición,

$$
\frac{1}{m} \operatorname{tr}\left(f(\widehat{\mathbf{A}})-f\left(\widehat{\mathbf{B}}_{h}\right)\right)=\frac{1}{m} \sum_{k=1}^{m} \frac{1}{2 \pi i}\left(\int_{\gamma_{\lambda}} f(z)\left(R_{z}(\widehat{\mathbf{A}})-R_{z}\left(\widehat{\mathbf{B}}_{h}\right)\right) \zeta_{k} d z, \zeta_{k}\right)=f(\lambda)-\frac{1}{m} \sum_{k=1}^{m} f\left(\lambda_{k h}\right) .
$$


Sea $x \in \mathbf{E}_{\lambda}(W(\widehat{\Omega}))$, con $\|x\|_{h}=1$. Entonces,

$$
\begin{aligned}
\left\|\left(f(\widehat{\mathbf{A}})-f\left(\widehat{\mathbf{B}}_{h}\right)\right) x\right\|_{h} & =\frac{1}{2 \pi}\left\|\int_{\gamma_{\lambda}} f(z)\left(R_{z}(\widehat{\mathbf{A}})-R_{z}\left(\widehat{\mathbf{B}}_{h}\right)\right) x d z\right\|_{h} \\
& =\frac{1}{2 \pi}\left\|\int_{\gamma_{\lambda}} f(z) R_{z}\left(\widehat{\mathbf{B}}_{h}\right)\left(\widehat{\mathbf{A}}-\widehat{\mathbf{B}}_{h}\right) R_{z}(\widehat{\mathbf{A}}) x d z\right\|_{h} \\
& \leq \frac{1}{2 \pi} \int_{\gamma_{\lambda}}|f(z)|\left\|R_{z}\left(\widehat{\mathbf{B}}_{h}\right)\left(\widehat{\mathbf{A}}-\widehat{\mathbf{B}}_{h}\right) R_{z}(\widehat{\mathbf{A}}) x\right\|_{h}|d z| .
\end{aligned}
$$

Teniendo en cuenta que $\widehat{\mathbf{A}}=\left.\mathbf{A}\right|_{\mathbf{E}_{\lambda}(W(\widehat{\Omega}))}$, que $\mathbf{E}_{\lambda}(W(\widehat{\Omega})$ ) es invariante por $\mathbf{A}$ (por lo tanto, también será invariante por $\left.R_{z}(\widehat{\mathbf{A}})\right)$ y que $\left.\left(\widehat{\mathbf{A}}-\widehat{\mathbf{B}}_{h}\right)\right|_{\mathbf{E}_{\lambda}(W(\widehat{\Omega}))} \subset \mathbf{E}_{\lambda}(W(\widehat{\Omega}))$, tendremos

$$
\begin{aligned}
\left\|\left(f(\widehat{\mathbf{A}})-f\left(\widehat{\mathbf{B}}_{h}\right)\right) x\right\|_{h} & \leq \frac{\left|\gamma_{\lambda}\right|}{2 \pi} \sup _{z \in \gamma_{\lambda}}|f(z)|\left\|R_{z}\left(\widehat{\mathbf{B}}_{h}\right)\right\|_{h}\left\|\left(\widehat{\mathbf{A}}-\widehat{\mathbf{B}}_{h}\right) R_{z}(\widehat{\mathbf{A}}) x\right\|_{h} \\
& \leq C \frac{\left|\gamma_{\lambda}\right|}{2 \pi} \sup _{z \in \gamma_{\lambda}}|f(z)|\left\|R_{z}\left(\widehat{\mathbf{B}}_{h}\right)\right\|_{h}\left\|\left.\left(\widehat{\mathbf{A}}-\widehat{\mathbf{B}}_{h}\right)\right|_{\mathbf{E}_{\lambda}(W(\widehat{\Omega}))}\right\|\left\|_{h}\right\| R_{z}(\widehat{\mathbf{A}}) \|_{h} .
\end{aligned}
$$

Usando la estimación (3.20) y el Corolario 3.4, concluimos que

$$
\left\|\left.\left(f(\widehat{\mathbf{A}})-f\left(\widehat{\mathbf{B}}_{h}\right)\right)\right|_{\mathbf{E}_{\lambda}(W(\widehat{\Omega}))}\right\|_{h} \leq C\left\|\left.\left(\widehat{\mathbf{A}}-\widehat{\mathbf{B}}_{h}\right)\right|_{\mathbf{E}_{\lambda}(W(\widehat{\Omega}))}\right\|_{h},
$$

siendo $C$ es una constante que depende de $f$ pero no de $h$. Luego, haciendo $f=\frac{1}{z}$,

$$
\left|\frac{1}{\lambda}-\frac{1}{m} \sum_{k=1}^{m} \frac{1}{\lambda_{k h}}\right| \leq C\left\|\left(\widehat{\mathbf{A}}-\widehat{\mathbf{B}}_{h}\right)\right\|_{h} .
$$

iii) Es una aplicación directa de resultados conocidos para matrices (ver, por ejemplo, [175], pp. 80-81).

A partir de estas estimaciones, el teorema es una consecuencia directa del Lema 3.28.

\subsubsection{Convergencia y estimaciones del error para las autofunciones en la norma $|\cdot|_{X(\widehat{\Omega})}$}

En las secciones previas, hemos estudiado el problema de aproximar el espectro del operador A usando métodos no-conformes y analizado la convergencia de las soluciones numéricas en la norma $\|\cdot\|_{h}$; que, usualmente, es equivalente a la norma de la energía. En muchas aplicaciones, interesa también investigar el comportamiento de la aproximación en la norma $|\cdot|_{X(\widehat{\Omega})}$.

Consideraremos nuevamente los operadores $\mathbf{A}$ y $\mathbf{A}_{h}$ (definidos en la sección 3.2) y las restricciones $\boldsymbol{\Lambda}=$ $\left.\mathbf{A}\right|_{W(\widehat{\Omega})}$ y $\boldsymbol{\Lambda}_{h}=\left.\mathbf{A}_{h}\right|_{V_{h}^{e}(\widehat{\Omega})}$ (introducidas en la sección 3.3). Siguiendo los argumentos utilizados en la prueba del Lema 3.3 y teniendo en cuenta la inclusión continua $W(\widehat{\Omega}) \hookrightarrow X(\widehat{\Omega})$, es claro que

$$
\left|(z-\boldsymbol{\Lambda})^{-1}\right|_{X(\widehat{\Omega})} \leq C \quad z \in \rho(\boldsymbol{\Lambda}), z \neq 0 .
$$

Como $\boldsymbol{\Lambda} \in \mathcal{L}(X(\widehat{\Omega}))$, los argumentos del Corolario 3.4 pueden usarse para probar que

$$
\left|(z-\boldsymbol{\Lambda})^{-1}\right|_{X(\widehat{\Omega})} \leq C \quad \forall z \in \mathcal{G}, \mathcal{G} \text { subconjunto cerrado de } \rho(\boldsymbol{\Lambda}) .
$$

Supongamos que se satisfacen las siguientes condiciones (versiones más débiles de las propiedades $\mathbf{P} 1$ y P2 anteriores):

$\mathcal{P} 1: \quad$ para $x \in \mathbf{E}_{\lambda}(W(\widehat{\Omega}))$, existe $x_{h} \in V_{h}^{e}(\widehat{\Omega})$ tal que $\left|x-x_{h}\right|_{X(\widehat{\Omega})} \leq \nu_{1 h}|x|_{X(\widehat{\Omega})}$, con $\nu_{1 h} \rightarrow 0$ para $h \rightarrow 0$,

$\mathcal{P} 2:\left.\quad \lim _{h \rightarrow 0}\left|\left(\mathbf{A}-\mathbf{A}_{h}\right)\right|_{V_{h}^{e}(\widehat{\Omega})}\right|_{X(\widehat{\Omega})}=0$. 
Podemos repetir los argumentos utilizados en las pruebas de los lemas, teoremas y corolarios de la sección 3.3.1 para obtener

$$
\lim _{h \rightarrow 0} \widehat{\delta}\left(\mathbf{E}_{\lambda}(W(\widehat{\Omega})), \mathbf{E}_{\lambda_{h}}\left(V_{h}^{e}(\widehat{\Omega})\right)\right)=0
$$

donde la distancia se mide ahora en la norma $|\cdot|_{X(\widehat{\Omega})}$.

Establecido entonces que las autofunciones del continuo, soluciones del problema (3.1), están bien aproximadas por las soluciones del problema discreto (3.8), pasamos a estimar el orden de convergencia en la norma $|\cdot|_{X(\widehat{\Omega})}$. Para esto, necesitaremos hacer hipótesis adicionales.

A continuación asumiremos que

- la forma bilineal $b_{h}$ es uniformemente coerciva con respecto a la norma $|\cdot|_{X\left(\Omega_{h}\right)}$ sobre el espacio $V_{h}\left(\Omega_{h}\right)$,

- el operador de extensión discreto $\mathbf{P}_{h}: V_{h}\left(\Omega_{h}\right) \rightarrow X(\widehat{\Omega})$ está uniformemente acotado en la norma $|\cdot|_{X}\left(\Omega_{h}\right) \cdot$

Observación 3.30 Cabe señalar aquí que estas condiciones no son tan restrictivas en la práctica. En la mayoría de las aplicaciones, el espacio $X\left(\Omega_{h}\right)$ puede identificarse con $L^{2}\left(\Omega_{h}\right)$ y la forma bilineal $b_{h}$ con el producto interno standard.

Sea ahora $\widehat{\Pi}_{h}: W_{\lambda}(\widehat{\Omega}) \rightarrow W_{\lambda}(\widehat{\Omega})$, el operador con rango en $V_{h}^{e}(\widehat{\Omega})$, definido por

$$
\left\{\begin{array}{l}
\widehat{\Pi}_{h} x=\mathbf{P}_{h} x_{h} \\
b_{h}\left(x-x_{h}, y_{h}\right)=0 \quad \forall y_{h} \in V_{h}\left(\Omega_{h}\right)
\end{array}\right.
$$

Con respecto a la norma $|\cdot|_{X(\widehat{\Omega})}$, la forma bilineal $b_{h}$ es continua sobre $W(\widehat{\Omega})$ y coerciva sobre $V_{h}\left(\Omega_{h}\right)$ y el operador $\mathbf{P}_{h}$ está uniformememte acotado en $h$. Luego, el proyector $\widehat{\Pi}_{h}$ resulta acotado uniformemente en $h$ con respecto a la misma norma.

Observemos que $\mathbf{A}_{h}\left(\widehat{\Pi}_{h}-\mathbf{I}\right) x=0$ para todo $x \in W_{\lambda}(\widehat{\Omega})$. En efecto, a partir de la definición del operador $\mathbf{A}_{h}$,

$$
a_{h}\left(\mathbf{A}_{h} \widehat{\Pi}_{h} x, v_{h}\right)=b_{h}\left(\widehat{\Pi}_{h} x, v_{h}\right)=b_{h}\left(x, v_{h}\right)=a_{h}\left(\mathbf{A}_{h} x, v_{h}\right) \quad \forall v_{h} \in V_{h}\left(\Omega_{h}\right) .
$$

Usando este resultado y argumentando como en la prueba del Lema 3.19, tendremos que, para todo $x \in W_{\lambda}(\widehat{\Omega})$ y $h$ suficientemente pequeño,

$$
|x|_{X(\widehat{\Omega})} \leq\left|\widehat{\Pi}_{h} x\right|_{X(\widehat{\Omega})}+\left|\left(\mathbf{I}-\widehat{\Pi}_{h}\right) x\right|_{X(\widehat{\Omega})} \leq C(z)\left|\left(z-\mathbf{A}_{h}\right) x\right|_{X(\widehat{\Omega})} \quad \forall z \in \gamma, \gamma \subset \rho\left(\boldsymbol{\Lambda}_{h}\right) .
$$

Como $\mathbf{A}_{h}$ es un operador compacto, la desigualdad anterior es suficiente para afirmar que el operador resolvente de $\mathbf{A}_{h}$ estará uniformemente acotado en la norma $|\cdot|_{X(\widehat{\Omega})}$ para todo $x \in W_{\lambda}(\widehat{\Omega})$. A partir de aquí, podremos extender la definición del proyector espectral correspondiente al operador $\mathbf{A}_{h}$.

Sea $\mathbf{E}_{\lambda_{h}}: W_{\lambda}(\widehat{\Omega}) \rightarrow V_{h}^{e}(\widehat{\Omega})$ definido por

$$
\mathbf{E}_{\lambda_{h}}:=\frac{1}{2 \pi i} \int_{\gamma} R_{z}\left(\left.\mathbf{A}_{h}\right|_{W_{\lambda}(\widehat{\Omega})}\right) d z .
$$

Obviamente, $\mathbf{E}_{\lambda_{h}}$, así definido, resulta acotado uniformemente en la norma $|\cdot|_{X(\widehat{\Omega})}$.

Supongamos ahora que la siguiente condición se satisface

$\mathcal{P} 3$ : para todo $x \in \mathbf{E}_{\lambda}(\widehat{\Omega}),\left|\left(\mathbf{A}-\mathbf{A}_{h}\right) x\right|_{X(\widehat{\Omega})} \leq \nu_{2 h}|x|_{X(\widehat{\Omega})}$, con $\nu_{2 h} \rightarrow 0$ para $h \rightarrow 0$.

Los mismos argumentos utilizados en las pruebas del Lema 3.23 y del Teorema 3.26, nos permiten establecer las siguientes estimaciones (válidas para $h$ chico):

$$
\begin{array}{r}
\left.\left|\left(\mathbf{E}_{\lambda}-\mathbf{E}_{\lambda_{h}}\right)\right|_{\mathbf{E}_{\lambda}(\widehat{\Omega})}\right|_{X(\widehat{\Omega})} \leq\left. C\left|\left(\mathbf{A}-\mathbf{A}_{h}\right)\right|_{\mathbf{E}_{\lambda}(\widehat{\Omega})}\right|_{X(\widehat{\Omega})}, \\
\widehat{\delta}\left(\mathbf{E}_{\lambda}(W(\widehat{\Omega})), \mathbf{E}_{\lambda_{h}}(W(\widehat{\Omega}))\right) \leq\left. C\left|\left(\mathbf{A}-\mathbf{A}_{h}\right)\right|_{\mathbf{E}_{\lambda}(\widehat{\Omega})}\right|_{X(\widehat{\Omega})} .
\end{array}
$$




\subsection{Problemas de autovalores en forma mixta}

Los resultados obtenidos en las secciones anteriores pueden adaptarse fácilmente para analizar las aproximaciones no-conformes de muchos problemas de autovalores escritos en forma mixta. En esta sección mostraremos como se realiza esta adaptación.

Comenzaremos definiendo un marco general en el cual plantear los problemas mixtos que vamos a analizar. Recordaremos también las condiciones suficientes que garantizan la existencia y unicidad de sus soluciones. Nos concentraremos primero en problemas tipo fuente ya que éstos nos permitirán definir operadores solución adecuados para los problemas de autovalores a considerar.

Igual que en las secciones previas, asumiremos que $\Omega$ es un dominio abierto y acotado en $\mathbb{R}^{n}(n=2$ o 3$)$, en general, no convexo, con un borde $\Gamma:=\partial \Omega$ Lipschitz-continuo.

Sean $X(\Omega), Y(\Omega), V(\Omega)$ y $Q(\Omega)$ espacios de Hilbert complejos equipados con las normas $|\cdot|_{X(\Omega)},|\cdot|_{Y(\Omega)}$, $\|\cdot\|_{V(\Omega)}$ y $\|\cdot\|_{Q(\Omega)}$, respectivamente. Asumiremos que $V(\Omega)$ y $Q(\Omega)$ son subespacios de $X(\Omega)$ e $Y(\Omega)$ y que las inclusiones $V(\Omega) \hookrightarrow X(\Omega)$ y $Q(\Omega) \hookrightarrow Y(\Omega)$ son continuas. Asumiremos también que las formas sesquilineales $a: V(\Omega) \times V(\Omega) \rightarrow \mathbb{C}, b: V(\Omega) \times Q(\Omega) \rightarrow \mathbb{C}, c: X(\Omega) \times X(\Omega) \rightarrow \mathbb{C}$ y $d: Y(\Omega) \times Y(\Omega) \rightarrow \mathbb{C}$ son continuas.

Consideremos el siguiente problema.

Dado el par $(\mathbf{f}, g) \in X(\Omega) \times Y(\Omega)$, encontrar $(\mathbf{u}, p) \in V(\Omega) \times Q(\Omega)$ solución de

$$
\left\{\begin{array}{l}
a(\mathbf{u}, \mathbf{v})+\overline{b(\mathbf{v}, p)}=c(\mathbf{f}, \mathbf{v}) \quad \forall \mathbf{v} \in V(\Omega), \\
b(\mathbf{u}, q)=d(g, q) \quad \forall q \in Q(\Omega) .
\end{array}\right.
$$

La existencia y unicidad de la solución del problema (3.38), como así también su dependencia continua con los datos, quedan garantizadas si se cumplen las siguientes condiciones sobre las formas bilineales $a$ y $b$ (conocidas como condiciones de Babuška-Brezzi [56]):

- existe una constante $\beta>0$ tal que

$$
\inf _{q \in Q(\Omega)} \sup _{\mathbf{v} \in V(\Omega)} \frac{|b(\mathbf{v}, q)|}{\|\mathbf{v}\|_{V(\Omega)}\|q\|_{Q(\Omega)}} \geq \beta
$$

- existe una constante $\alpha>0$ tal que

$$
\operatorname{Re} a(\mathbf{v}, \mathbf{v}) \geq \alpha\|\mathbf{v}\|_{V(\Omega)}^{2} \quad \forall \mathbf{v} \in K(\Omega),
$$

siendo $K(\Omega)$ el núcleo del operador asociado con la forma sesquilineal $b$; definido por

$$
K(\Omega):=\{\mathbf{v} \in V(\Omega): b(\mathbf{v}, q)=0, \forall q \in Q(\Omega)\} .
$$

Más aún, vale la siguiente estimación

$$
\|\mathbf{u}\|_{V(\Omega)}+\|p\|_{Q(\Omega)} \leq C\left(|\mathbf{f}|_{X(\Omega)}+|g|_{Y(\Omega)}\right) .
$$

Para definir una discretización del problema (3.38) necesitamos primero construir aproximaciones poligonales $\Omega_{h}$ del dominio curvo $\Omega$. A continuación asumiremos que los dominios discretos $\Omega_{h}$ satisfacen las propiedades enunciadas en la sección 3.1.

Debido a que, en general, $\Omega_{h} \not \subset \Omega$, será necesario definir una extensión de los datos f y $g$. Para ello, introducimos el dominio $\Omega \subset \mathbb{R}^{n}$ el cual verifica $\widehat{\Omega}:=\Omega \cup \Omega_{h} \subset \widetilde{\Omega}$, para todo $h$ suficientemente chico. Sobre $\widetilde{\Omega}$ consideramos las extensiones $\widetilde{\mathbf{f}} \in X(\widetilde{\Omega})$ y $\widetilde{g} \in Y(\widetilde{\Omega})$ definidas de modo que

$$
\left.\widetilde{\mathbf{f}}\right|_{\Omega}=\mathbf{f}, \quad|\widetilde{\mathbf{f}}|_{X(\widetilde{\Omega})} \leq C|\mathbf{f}|_{X(\Omega)},
$$




$$
\left.\widetilde{g}\right|_{\Omega}=g, \quad|\widetilde{g}|_{Y(\widetilde{\Omega})} \leq C|g|_{Y(\Omega)} .
$$

Sean $V_{h}\left(\Omega_{h}\right)$ y $Q_{h}\left(\Omega_{h}\right)$ espacios de dimensión finita definidos sobre cada aproximación poligonal $\Omega_{h}$ del dominio $\Omega$ y equipados con las normas $\|\cdot\|_{V_{h}\left(\Omega_{h}\right)}$ y $\|\cdot\|_{Q_{h}\left(\Omega_{h}\right)}$, respectivamente. Asumiremos en lo que sigue que estos espacios discretos y sus normas satisfacen las siguientes propiedades:

$$
\begin{aligned}
\|\mathbf{v}\|_{V\left(\Omega_{h}\right)} & =\|\mathbf{v}\|_{V_{h}\left(\Omega_{h}\right)} \quad \forall \mathbf{v} \in V\left(\Omega_{h}\right), \\
\|\eta\|_{Q\left(\Omega_{h}\right)} & =\|\eta\|_{Q_{h}\left(\Omega_{h}\right)} \quad \forall \eta \in Q\left(\Omega_{h}\right), \\
\left(V_{h}\left(\Omega_{h}\right),\|\cdot\|_{V_{h}\left(\Omega_{h}\right)}\right) & \hookrightarrow\left(X\left(\Omega_{h}\right),|\cdot|_{X\left(\Omega_{h}\right)}\right) \quad \text { uniformemente en } h, \\
\left(Q_{h}\left(\Omega_{h}\right),\|\cdot\|_{Q_{h}\left(\Omega_{h}\right)}\right) & \hookrightarrow\left(Y\left(\Omega_{h}\right),|\cdot|_{Y\left(\Omega_{h}\right)}\right) \quad \text { uniformemente en } h .
\end{aligned}
$$

Asumiremos también que las formas discretas $a_{h}: V_{h}\left(\Omega_{h}\right) \times V_{h}\left(\Omega_{h}\right) \rightarrow \mathbb{C}, b_{h}: V_{h}\left(\Omega_{h}\right) \times Q_{h}\left(\Omega_{h}\right) \rightarrow \mathbb{C}$, $c_{h}: X\left(\Omega_{h}\right) \times X\left(\Omega_{h}\right) \rightarrow \mathbb{C}$ y $d_{h}: Y\left(\Omega_{h}\right) \times Y\left(\Omega_{h}\right) \rightarrow \mathbb{C}$ son uniformemente continuas.

Consideremos entonces la siguiente discretización del problema fuente (3.38):

Dado $(\mathbf{f}, g) \in X(\Omega) \times Y(\Omega)$, encontrar $\left(\mathbf{u}_{h}, p_{h}\right) \in V_{h}\left(\Omega_{h}\right) \times Q_{h}\left(\Omega_{h}\right)$, solución de

$$
\left\{\begin{array}{l}
a_{h}\left(\mathbf{u}_{h}, \mathbf{v}_{h}\right)+\overline{b_{h}\left(\mathbf{v}_{h}, p_{h}\right)}=c_{h}\left(\left.\widetilde{\mathbf{f}}\right|_{\Omega_{h}}, \mathbf{v}_{h}\right) \quad \forall \mathbf{v}_{h} \in V_{h}\left(\Omega_{h}\right), \\
b_{h}\left(\mathbf{u}_{h}, q_{h}\right)=d_{h}\left(\left.\widetilde{g}\right|_{\Omega_{h}}, q_{h}\right) \quad \forall q_{h} \in Q_{h}\left(\Omega_{h}\right) .
\end{array}\right.
$$

Existen condiciones discretas, análogas a las condiciones (3.39)-(3.40), que garantizan que la aproximación $\left(\mathbf{u}_{h}, p_{h}\right)$ estará bien definida. Más precisamente, el problema (3.45) tendrá solución y será única si los espacios $V_{h}\left(\Omega_{h}\right)$ y $Q_{h}\left(\Omega_{h}\right)$ satisfacen las siguientes condiciones (ver [56] nuevamente):

- existe una constante $\widehat{\beta}>0$, independiente de $h$, tal que

$$
\inf _{q_{h} \in Q_{h}\left(\Omega_{h}\right)} \sup _{\mathbf{v}_{h} \in V_{h}\left(\Omega_{h}\right)} \frac{\left|b_{h}\left(\mathbf{v}_{h}, q_{h}\right)\right|}{\left\|\mathbf{v}_{h}\right\|_{V_{h}\left(\Omega_{h}\right)}\left\|q_{h}\right\|_{Q_{h}\left(\Omega_{h}\right)}}:=\beta_{h} \geq \widehat{\beta}
$$

- existe una constante $\widehat{\alpha}>0$, independiente de $h$, tal que

$$
\operatorname{Re} a_{h}\left(\mathbf{v}_{h}, \mathbf{v}_{h}\right) \geq \alpha\left\|\mathbf{v}_{h}\right\|_{V_{h}\left(\Omega_{h}\right)}^{2} \quad \forall \mathbf{v}_{h} \in K_{h}\left(\Omega_{h}\right),
$$

donde el núcleo discreto $K_{h}\left(\Omega_{h}\right)$ está definido por

$$
K_{h}\left(\Omega_{h}\right):=\left\{\mathbf{v}_{h} \in V_{h}\left(\Omega_{h}\right): b_{h}\left(\mathbf{v}_{h}, q_{h}\right)=0 \quad \forall q_{h} \in Q_{h}\left(\Omega_{h}\right)\right\} .
$$

Además, la aproximación $\left(\mathbf{u}_{h}, p_{h}\right)$ es estable; i.e., existe una constante $C$, independiente de $h$, tal que

$$
\left\|\mathbf{u}_{h}\right\|_{V_{h}\left(\Omega_{h}\right)}+\left\|p_{h}\right\|_{Q_{h}\left(\Omega_{h}\right)} \leq C\left(|\mathbf{f}|_{X(\Omega)}+|g|_{Y(\Omega)}\right) .
$$

Siguiendo a Mercier-Osborn-Rappaz-Raviart [138], consideraremos dos tipos de problemas de autovalores en forma mixta. El primer tipo corresponde a problemas de autovalores asociados con el sistema de ecuaciones (3.38) cuando $g=0$, razón por la cual también se los denomina problemas (f, 0$)$. El segundo tipo de problemas, o problemas $(\mathbf{0}, g)$, corresponde a problemas de autovalores asociados con el mismo sistema de ecuaciones pero cuando $\mathbf{f}=\mathbf{0}$.

En lo que sigue, asumiremos que las formas $a, c, d$ y sus respectivas aproximaciones, $a_{h}, c_{h}$ y $d_{h}$, son hermitianas. 


\subsubsection{Problemas del primer tipo o problemas $(\mathrm{f}, 0)$}

Consideremos el siguiente problema de autovalores:

Encontrar $\mu \in \mathbb{C},(\mathbf{u}, p) \in V(\Omega) \times Q(\Omega),(\mathbf{u}, p) \neq(\mathbf{0}, 0)$, tal que

$$
\left\{\begin{array}{l}
a(\mathbf{u}, \mathbf{v})+\overline{b(\mathbf{v}, p)}=\mu c(\mathbf{u}, \mathbf{v}) \quad \forall \mathbf{v} \in V(\Omega) \\
b(\mathbf{u}, q)=0 \quad \forall q \in Q(\Omega)
\end{array}\right.
$$

Introduciremos, ahora, al operador solución. En este contexto, es conveniente definirlo por componentes. Sean

$$
\begin{aligned}
\mathbf{T}: X(\Omega) & \rightarrow V(\Omega) \\
\mathbf{x} & \mapsto \mathbf{u}, \\
\mathbf{S}: X(\Omega) & \rightarrow Q(\Omega) \\
\mathbf{x} & \mapsto p,
\end{aligned}
$$

donde $(\mathbf{u}, p) \in V(\Omega) \times Q(\Omega)$ es la solución del siguiente problema fuente

$$
\left\{\begin{array}{l}
a(\mathbf{T x}, \mathbf{v})+\overline{b(\mathbf{v}, \mathbf{S x})}=c(\mathbf{x}, \mathbf{v}) \quad \forall \mathbf{v} \in V(\Omega) \\
b(\mathbf{T x}, q)=0 \quad \forall q \in Q(\Omega) .
\end{array}\right.
$$

Las condiciones (3.39)-(3.40) y la inclusión $V(\Omega) \hookrightarrow X(\Omega)$ nos permite asegurar que $\mathbf{T}$ es un operador lineal y acotado sobre $X(\Omega)$. Más aún, tendremos

$$
\|\mathbf{u}\|_{V(\Omega)} \leq C|\mathbf{x}|_{X(\Omega)} .
$$

El siguiente problema define la contrapartida discreta del problema mixto (3.48).

Encontrar $\mu_{h} \in \mathbb{C},\left(\mathbf{u}_{h}, p_{h}\right) \in V_{h}\left(\Omega_{h}\right) \times Q_{h}\left(\Omega_{h}\right),\left(\mathbf{u}_{h}, p_{h}\right) \neq(\mathbf{0}, 0)$, tal que

$$
\left\{\begin{array}{l}
a_{h}\left(\mathbf{u}_{h}, \mathbf{v}_{h}\right)+\overline{b_{h}\left(\mathbf{v}_{h}, p_{h}\right)}=\mu_{h} c_{h}\left(\mathbf{u}_{h}, \mathbf{v}_{h}\right) \quad \forall \mathbf{v}_{h} \in V_{h}\left(\Omega_{h}\right), \\
b_{h}\left(\mathbf{u}_{h}, q_{h}\right)=0 \quad \forall q_{h} \in Q_{h}\left(\Omega_{h}\right) .
\end{array}\right.
$$

Los operadores solución estarán definidos ahora por

$$
\begin{aligned}
\mathbf{T}_{h}: X\left(\Omega_{h}\right) & \rightarrow V_{h}\left(\Omega_{h}\right) \\
\mathbf{x} & \mapsto \mathbf{u}_{h}, \\
\mathbf{S}_{h}: X\left(\Omega_{h}\right) & \rightarrow Q_{h}\left(\Omega_{h}\right) \\
\mathbf{x} & \mapsto p_{h},
\end{aligned}
$$

donde $\left(\mathbf{u}_{h}, p_{h}\right) \in V_{h}\left(\Omega_{h}\right) \times Q_{h}\left(\Omega_{h}\right)$ es la solución del problema

$$
\left\{\begin{array}{l}
a_{h}\left(\mathbf{T}_{h} \mathbf{x}, \mathbf{v}_{h}\right)+\overline{b_{h}\left(\mathbf{v}_{h}, \mathbf{S}_{h} \mathbf{x}\right)}=c_{h}\left(\mathbf{x}, \mathbf{v}_{h}\right) \quad \forall \mathbf{v}_{h} \in V_{h}\left(\Omega_{h}\right), \\
b_{h}\left(\mathbf{T}_{h} \mathbf{x}, q_{h}\right)=0 \quad \forall q_{h} \in Q_{h}\left(\Omega_{h}\right) .
\end{array}\right.
$$

Como consecuencia de las condiciones (3.46)-(3.47), sabemos que existe una constante $C$, independiente de $h$, tal que

$$
\left\|\mathbf{u}_{h}\right\|_{V_{h}\left(\Omega_{h}\right)} \leq C|\mathbf{x}|_{X\left(\Omega_{h}\right)}
$$

y, por lo tanto, el operador $\mathbf{T}_{h}$ resulta acotado uniformemente en $h$.

De acuerdo con [138], los pares autovector/autovalor asociados al problema (3.48) pueden caracterizarse por las soluciones del siguiente problema:

Encontrar $\mu \in \mathbb{C}, \mathbf{u} \in K(\Omega), \mathbf{u} \neq \mathbf{0}$, tal que

$$
a(\mathbf{u}, \mathbf{v})=\mu c(\mathbf{u}, \mathbf{v}) \quad \forall \mathbf{v} \in K(\Omega) .
$$


Para ser más específicos, $(\mu, \mathbf{u})$ es un par propio correspondiente al problema $(3.53)$ si, y solo si, $(\mu,(\mathbf{u}, p))$, con $p=\mu \mathbf{S u}$, es un par propio correspondiente al problema (3.48).

De la misma manera, los pares autovector/autovalor asociados al problema (3.51) pueden caracterizarse por las soluciones del siguiente problema:

Encontrar $\mu_{h} \in \mathbb{C}, \mathbf{u}_{h} \in K_{h}\left(\Omega_{h}\right), \mathbf{u}_{h} \neq \mathbf{0}$, tal que

$$
a_{h}\left(\mathbf{u}_{h}, \mathbf{v}_{h}\right)=\mu_{h} c_{h}\left(\mathbf{u}_{h}, \mathbf{v}_{h}\right) \quad \forall \mathbf{v}_{h} \in K_{h}\left(\Omega_{h}\right) .
$$

En este caso, tendremos que $\left(\mu_{h}, \mathbf{u}_{h}\right)$ es un par propio correspondiente al problema (3.54) si, y solo si, $\left(\mu_{h},\left(\mathbf{u}_{h}, p_{h}\right)\right)$, con $p_{h}=\mu_{h} \mathbf{S}_{h} \mathbf{u}_{h}$, es un par propio correspondiente al problema (3.51).

Observación 3.31 En general, $K_{h}\left(\Omega_{h}\right) \not \subset K(\Omega)$; incluso en el caso en que $\Omega$ fuera un dominio poligonal y los espacios discretos estuvieran incluidos en los respectivos espacios continuos. Por esta razón, los métodos mixtos son considerados como casos particulares de aproximaciones no-conformes [138, 42].

Observación 3.32 El operador $\left.\mathbf{T}\right|_{K(\Omega)}$ resulta autoadjunto con respecto a la forma a; es consecuencia directa de la condición de hermiticidad impuesta sobre las formas sesquilineales. El mismo comentario es válido para el operador $\left.\mathbf{T}_{h}\right|_{\mathbf{K}_{h}\left(\Omega_{h}\right)}$.

A partir de estas observaciones, es claro que, reemplazando simplemente las restricciones indicadas sobre el espacio $V_{h}^{e}(\widehat{\Omega})$ por restricciones sobre

$$
K_{h}^{e}(\widehat{\Omega}):=\mathbf{P}_{h}\left(K_{h}\left(\Omega_{h}\right)\right) \subset V_{h}^{e}(\widehat{\Omega}),
$$

la teoría desarrollada en las secciones previas puede aplicarse para analizar la convergencia de los pares propios asociados a la componente $\mathbf{T}_{h}$ del operador $\left(\mathbf{T}_{h}, \mathbf{S}_{h}\right)$ hacia los pares propios de la componente $\mathbf{T}$ del operador $(\mathbf{T}, \mathbf{S})$.

Observemos además que podemos modificar la definición de la norma $\|\cdot\|_{h}$ de la siguiente manera

$$
\begin{aligned}
& \text { - }\|\mathbf{v}\|_{h, \Omega_{h}} \simeq\|\mathbf{v}\|_{V_{h}\left(\Omega_{h}\right)} \quad \text { si }\left.\quad \mathbf{v}\right|_{\Omega_{h}} \in K_{h}\left(\Omega_{h}\right) \\
& \text { - }\|\mathbf{v}\|_{h, \Omega} \simeq\|\mathbf{v}\|_{V(\Omega)} \quad \text { si }\left.\quad \mathbf{v}\right|_{\Omega} \in K(\Omega), \\
& \text { - }\|\mathbf{v}\|_{h} \simeq\|\mathbf{v}\|_{V(\widehat{\Omega})} \quad \text { si } \quad \mathbf{v} \in K^{e}(\widehat{\Omega}):=\mathbf{P}(K(\Omega)) \subset V^{e}(\widehat{\Omega}),
\end{aligned}
$$

que resultan condiciones menos restrictivas sobre los espacios.

En cuanto a las estimaciones para acotar el error asociado con la aproximación $\left(\lambda_{h}, \mathbf{u}_{h}\right)$, estas pueden obtenerse usando los mismos argumentos que los empleados en las secciones 3.3.3-3.3.4 e introduciendo la siguiente (obvia) modificación en la definición del operador de proyección $\Pi_{h}$ :

$$
\left\{\begin{array}{l}
\Pi_{h} \mathbf{x}=\mathbf{P}_{h} \mathbf{x}_{h} \\
a_{h}\left(\mathbf{x}-\mathbf{x}_{h}, \mathbf{y}_{h}\right)=0 \quad \forall \mathbf{y}_{h} \in K_{h}\left(\Omega_{h}\right) .
\end{array}\right.
$$

\subsubsection{Problemas del segundo tipo o problemas $(0, g)$}

Consideremos el siguiente problema de autovalores:

Encontrar $\mu \in \mathbb{C},(\mathbf{u}, \varphi) \in V(\Omega) \times Q(\Omega),(\mathbf{u}, \varphi) \neq(\mathbf{0}, 0)$, tal que

$$
\left\{\begin{array}{l}
a(\mathbf{u}, \mathbf{v})+\overline{b(\mathbf{v}, \varphi)}=0 \quad \forall \mathbf{v} \in V(\Omega), \\
-b(\mathbf{u}, \eta)=\mu d(\varphi, \eta) \quad \forall \eta \in Q(\Omega) .
\end{array}\right.
$$

Introduzcamos las componentes del operador solución correspondiente. Definimos

$$
\begin{aligned}
\mathbf{S}: Y(\Omega) & \rightarrow V(\Omega) \\
y & \mapsto \mathbf{u},
\end{aligned}
$$




$$
\begin{aligned}
\mathbf{T}: Y(\Omega) & \rightarrow Q(\Omega) \\
y & \mapsto \varphi,
\end{aligned}
$$

siendo $(\mathbf{u}, \varphi) \in V(\Omega) \times Q(\Omega)$ la solución del siguiente problema fuente

$$
\left\{\begin{array}{l}
a(\mathbf{S} y, \mathbf{v})+\overline{b(\mathbf{v}, \mathbf{T} y)}=0 \quad \forall \mathbf{v} \in V(\Omega) \\
-b(\mathbf{S} y, \eta)=d(y, \eta) \quad \forall \eta \in Q(\Omega)
\end{array}\right.
$$

Las condiciones (3.39)-(3.40) nos permiten concluir que el operador definido por el par (S, T) es un operador lineal y acotado sobre $Y(\Omega)$. Más aún, vale la siguiente estimación

$$
\|\mathbf{u}\|_{V(\Omega)}+\|\varphi\|_{Q(\Omega)} \leq C|y|_{Y(\Omega)} .
$$

Pasemos ahora a considerar la aproximación del problema (3.56) definida por los espacios $V_{h}\left(\Omega_{h}\right)$ y $Q_{h}\left(\Omega_{h}\right)$. Consideremos entonces el siguiente problema de autovalores discreto:

Encontrar $\mu_{h} \in \mathbb{C},\left(\mathbf{u}_{h}, \varphi_{h}\right) \in V_{h}\left(\Omega_{h}\right) \times Q_{h}\left(\Omega_{h}\right),\left(\mathbf{u}_{h}, \varphi_{h}\right) \neq(\mathbf{0}, 0)$, solución de

$$
\begin{cases}a_{h}\left(\mathbf{u}_{h}, \mathbf{v}_{h}\right)+\overline{b_{h}\left(\mathbf{v}_{h}, \varphi_{h}\right)}=0 & \forall \mathbf{v}_{h} \in V_{h}\left(\Omega_{h}\right), \\ -b_{h}\left(\mathbf{u}_{h}, \eta_{h}\right)=\mu_{h} d_{h}\left(\varphi_{h}, \eta_{h}\right) & \forall \eta_{h} \in Q_{h}\left(\Omega_{h}\right) .\end{cases}
$$

La contrapartida discreta del operador $(\mathbf{S}, \mathbf{T})$ se define como sigue:

$$
\begin{aligned}
\mathbf{S}_{h}: Y\left(\Omega_{h}\right) & \rightarrow V_{h}\left(\Omega_{h}\right) \\
y & \mapsto \mathbf{u}_{h}, \\
\mathbf{T}_{h}: Y\left(\Omega_{h}\right) & \rightarrow Q_{h}\left(\Omega_{h}\right) \\
y & \mapsto \varphi_{h},
\end{aligned}
$$

donde $\left(\mathbf{u}_{h}, \varphi_{h}\right) \in V_{h}\left(\Omega_{h}\right) \times Q_{h}\left(\Omega_{h}\right)$ es la solución del siguiente problema fuente:

$$
\left\{\begin{array}{l}
a_{h}\left(\mathbf{S}_{h} y, \mathbf{v}_{h}\right)+\overline{b_{h}\left(\mathbf{v}_{h}, \mathbf{T}_{h} y\right)}=0 \quad \forall \mathbf{v}_{h} \in V_{h}\left(\Omega_{h}\right), \\
-b_{h}\left(\mathbf{S}_{h} y, \eta_{h}\right)=d_{h}\left(y, \eta_{h}\right) \quad \forall \eta_{h} \in Q_{h}\left(\Omega_{h}\right) .
\end{array}\right.
$$

Las condiciones (3.46)-(3.47) nos permiten afirmar que los pares $\left(\mathbf{S}_{h}, \mathbf{T}_{h}\right)$ resultan operadores acotados uniformemente en $h$.

\section{Convergencia y estimaciones del error para el par solución}

$\mathrm{Al}$ igual que en el caso anterior, nuestro objetivo es analizar aproximaciones no-conformes del problema (3.56) aplicando la teoría desarrollada en las primeras secciones. Con esta idea de guía, comenzamos definiendo las siguientes formas sesquilineales:

$$
\begin{gathered}
A((\mathbf{u}, \varphi),(\mathbf{v}, \eta)):=a(\mathbf{u}, \mathbf{v})+\overline{b(\mathbf{v}, \varphi)}+b(\mathbf{u}, \eta) \quad \forall(\mathbf{u}, \varphi),(\mathbf{v}, \eta) \in V(\Omega) \times Q(\Omega), \\
B((\mathbf{f}, g),(\mathbf{v}, \eta)):=-d(g, \eta) \quad \forall(\mathbf{f}, g),(\mathbf{v}, \eta) \in X(\Omega) \times Y(\Omega) .
\end{gathered}
$$

Definamos también los espacios $G(\Omega):=V(\Omega) \times Q(\Omega)$ y $H(\Omega):=X(\Omega) \times Y(\Omega)$ equipados con las normas producto:

$$
\begin{gathered}
\|(\mathbf{u}, \varphi)\|_{G(\Omega)}^{2}=\|\mathbf{u}\|_{V(\Omega)}^{2}+\|\varphi\|_{Q(\Omega)}^{2}, \\
|(\mathbf{f}, g)|_{H(\Omega)}^{2}=|\mathbf{f}|_{X(\Omega)}^{2}+|g|_{Y(\Omega)}^{2} .
\end{gathered}
$$

A partir de la teoría clásica de Babuška [26] - Brezzi [53], sabemos que si las condiciones (3.39)-(3.40) se verifican, entonces la forma bilineal $A$ resulta débilmente coerciva sobre $G(\Omega)$. Es decir, existe una constante positiva $\varsigma$ tal que: 


$$
\begin{aligned}
& \sup _{(\mathbf{v}, \eta) \in G(\Omega)} \frac{|A((\mathbf{u}, \varphi),(\mathbf{v}, \eta))|}{\|(\mathbf{v}, \eta)\|_{G(\Omega)}} \geq \varsigma\|(\mathbf{u}, \varphi)\|_{G(\Omega)} \quad \forall(\mathbf{u}, \varphi) \in G(\Omega), \\
& \sup _{(\mathbf{u}, \varphi) \in G(\Omega)}|A((\mathbf{u}, \varphi),(\mathbf{v}, \eta))|>0 \quad \forall(\mathbf{v}, \eta) \in G(\Omega) \backslash\{(\mathbf{0}, 0)\} .
\end{aligned}
$$

Consideremos entonces el siguiente problema de autovalores:

Encontrar $\mu \in \mathbb{C},(\mathbf{u}, \varphi) \in G(\Omega),(\mathbf{u}, \varphi) \neq(\mathbf{0}, 0)$, tal que

$$
A((\mathbf{u}, \varphi),(\mathbf{v}, \eta))=\mu B((\mathbf{u}, \varphi),(\mathbf{v}, \eta)) \quad \forall(\mathbf{v}, \eta) \in G(\Omega) .
$$

El operador solución correspondiente a este problema estará definido por:

$$
\begin{aligned}
\Psi: H(\Omega) & \rightarrow G(\Omega) \hookrightarrow H(\Omega) \\
(\mathbf{x}, y) & \mapsto(\mathbf{u}, \varphi),
\end{aligned}
$$

donde $(\mathbf{u}, \varphi) \in G(\Omega)$ es la solución del siguiente problema fuente

$$
A(\mathbf{\Psi}(\mathbf{x}, y),(\mathbf{v}, \eta))=B((\mathbf{x}, y),(\mathbf{v}, \eta)) \quad \forall(\mathbf{v}, \eta) \in G(\Omega) .
$$

Las siguientes tres propiedades del operator $\boldsymbol{\Psi}$ son fundamentales.

- El operador $\boldsymbol{\Psi}$ es lineal, está bien definido y acotado.

Como consecuencia de la propiedad (3.61), la continuidad de la forma bilineal $d$ y la inclusión $G(\Omega) \hookrightarrow$ $H(\Omega)$, tendremos

$$
\begin{aligned}
\|\Psi(\mathbf{x}, y)\|_{G(\Omega)} & \leq \frac{1}{\varsigma} \sup _{(\mathbf{v}, \eta) \in G(\Omega)} \frac{|A(\mathbf{\Psi}(\mathbf{x}, y),(\mathbf{v}, \eta))|}{\|(\mathbf{v}, \eta)\|_{G(\Omega)}}=\frac{1}{\varsigma} \sup _{(\mathbf{v}, \eta) \in G(\Omega)} \frac{|B((\mathbf{x}, y),(\mathbf{v}, \eta))|}{\|(\mathbf{v}, \eta)\|_{G(\Omega)}} \\
& =\frac{1}{\varsigma} \sup _{(\mathbf{v}, \eta) \in G(\Omega)} \frac{|d(y, \eta)|}{\|(\mathbf{v}, \eta)\|_{G(\Omega)}} \leq \frac{c}{\varsigma} \sup _{(\mathbf{v}, \eta) \in G(\Omega)} \frac{|y|_{Y(\Omega)}|\eta|_{Y(\Omega)}}{\|(\mathbf{v}, \eta)\|_{G(\Omega)}} \\
& \leq C|(\mathbf{x}, y)|_{H(\Omega)} .
\end{aligned}
$$

A partir de este resultado, obtendremos inmediatamente

$$
|\Psi(\mathbf{x}, y)|_{H(\Omega)} \leq C|(\mathbf{x}, y)|_{H(\Omega)},
$$

y, si $(\mathbf{x}, y) \in G(\Omega)$, también será válida la siguiente acotación

$$
\|\mathbf{\Psi}(\mathbf{x}, y)\|_{G(\Omega)} \leq C\|(\mathbf{x}, y)\|_{G(\Omega)} .
$$

- Los pares propios del operador $\boldsymbol{\Psi}$ brindan información completa sobre las soluciones del problema espectral (3.56), las cuales están asociadas con los pares propios del operador $(\mathbf{S}, \mathbf{T})$.

Sea $\boldsymbol{\Psi}(\mathbf{x}, y)=(\mathbf{u}, \varphi)$ la solución del problema (3.63). Entonces, se satisface

$$
\left\{\begin{array}{l}
a(\mathbf{u}, \mathbf{v})+\overline{b(\mathbf{v}, \varphi)}=0 \quad \forall \mathbf{v} \in V(\Omega), \\
-b(\mathbf{u}, \eta)=d(y, \eta) \quad \forall \eta \in Q(\Omega) .
\end{array}\right.
$$

Teniendo en cuenta que el problema (3.57) es un problema bien definido, concluimos que $(\mathbf{u}, \varphi)=(\mathbf{S} y, \mathbf{T} y)$. Recíprocamente, sea (Sy, Ty) la solución del problema (3.57), entonces se satisface

$$
a(\mathbf{S} y, \mathbf{v})+\overline{b(\mathbf{v}, \mathbf{T} y)}+b(\mathbf{S} y, \eta)=-d(y, \eta) \quad \forall(\mathbf{v}, \eta) \in G(\Omega) .
$$

Luego, a partir de la definición del problema (3.63), podemos concluir que $(\mathbf{S} y, \mathbf{T} y)=\mathbf{\Psi}(\mathbf{x}, y)$.

- El operador $\left.\boldsymbol{\Psi}\right|_{G(\Omega)}$ es autoadjunto con respecto a la forma $A$. 
Es una consecuencia directa de la condición de hermiticidad impuesta sobre las formas sesquilineales.

Pasemos ahora a la discretización del problema espectral (3.62). Comenzaremos definiendo los espacios $H\left(\Omega_{h}\right):=X\left(\Omega_{h}\right) \times Y\left(\Omega_{h}\right)$ y $G_{h}\left(\Omega_{h}\right):=V_{h}\left(\Omega_{h}\right) \times Q_{h}\left(\Omega_{h}\right)$ y equipando al espacio $G_{h}\left(\Omega_{h}\right)$ con la siguiente norma

$$
\left\|\left(\mathbf{u}_{h}, \varphi_{h}\right)\right\|_{G_{h}\left(\Omega_{h}\right)}^{2}=\left\|\mathbf{u}_{h}\right\|_{V_{h}\left(\Omega_{h}\right)}^{2}+\left\|\varphi_{h}\right\|_{Q_{h}\left(\Omega_{h}\right)}^{2} \quad \forall\left(\mathbf{u}_{h}, \varphi_{h}\right) \in G_{h}\left(\Omega_{h}\right) .
$$

Como consecuencia directa de las propiedades (3.41)-(3.44), tendremos

$$
\begin{aligned}
\|(\mathbf{u}, \varphi)\|_{G\left(\Omega_{h}\right)} & =\|(\mathbf{u}, \varphi)\|_{G_{h}\left(\Omega_{h}\right)} \quad \forall(\mathbf{u}, \varphi) \in G\left(\Omega_{h}\right), \\
\left(G_{h}\left(\Omega_{h}\right),\|\cdot\|_{G_{h}\left(\Omega_{h}\right)}\right) & \hookrightarrow\left(H\left(\Omega_{h}\right),|\cdot|_{H\left(\Omega_{h}\right)}\right) \quad \text { uniformemente en } h .
\end{aligned}
$$

Las versiones discretas de las formas bilineales $A$ y $B$ quedarán definidas por:

$$
\begin{gathered}
A_{h}\left(\left(\mathbf{u}_{h}, \varphi_{h}\right),\left(\mathbf{v}_{h}, \eta_{h}\right)\right):=a_{h}\left(\mathbf{u}_{h}, v_{h}\right)+\overline{b_{h}\left(\mathbf{v}_{h}, \varphi_{h}\right)}+b_{h}\left(\mathbf{u}_{h}, \eta_{h}\right) \quad \forall\left(\mathbf{u}_{h}, \varphi_{h}\right),\left(\mathbf{v}_{h}, \eta_{h}\right) \in G_{h}\left(\Omega_{h}\right), \\
B_{h}\left((\mathbf{f}, g),\left(\mathbf{v}_{h}, \eta_{h}\right)\right):=-d_{h}\left(g, \eta_{h}\right) \quad \forall(\mathbf{f}, g),\left(\mathbf{v}_{h}, \eta_{h}\right) \in H\left(\Omega_{h}\right) .
\end{gathered}
$$

$\mathrm{Al}$ igual que en el caso continuo, si se verifican las condiciones (3.46)-(3.47), existirá una constante positiva $\widehat{\varsigma}$, independiente de $h$, tal que

$$
\begin{aligned}
& \sup _{\left(\mathbf{v}_{h}, \eta_{h}\right) \in G_{h}\left(\Omega_{h}\right)} \frac{\left|A_{h}\left(\left(\mathbf{u}_{h}, \varphi_{h}\right),\left(\mathbf{v}_{h}, \eta_{h}\right)\right)\right|}{\left\|\left(\mathbf{v}_{h}, \eta_{h}\right)\right\|_{G_{h}\left(\Omega_{h}\right)}} \geq \widehat{\varsigma}\left\|\left(\mathbf{u}_{h}, \varphi_{h}\right)\right\|_{G_{h}\left(\Omega_{h}\right)} \quad \forall\left(\mathbf{u}_{h}, \varphi_{h}\right) \in G_{h}\left(\Omega_{h}\right), \\
& \sup _{\left(\mathbf{u}_{h}, \varphi_{h}\right) \in G_{h}\left(\Omega_{h}\right)}\left|A_{h}\left(\left(\mathbf{u}_{h}, \varphi_{h}\right),\left(\mathbf{v}_{h}, \eta_{h}\right)\right)\right|
\end{aligned}
$$

Consideremos, entonces, el siguiente problema de autovalores:

Encontrar $\mu_{h} \in \mathbb{C},\left(\mathbf{u}_{h}, \varphi_{h}\right) \in G_{h}\left(\Omega_{h}\right),\left(\mathbf{u}_{h}, \varphi_{h}\right) \neq(\mathbf{0}, 0)$, tal que

$$
A_{h}\left(\left(\mathbf{u}_{h}, \varphi_{h}\right),\left(\mathbf{v}_{h}, \eta_{h}\right)\right)=\mu_{h} B_{h}\left(\left(\mathbf{u}_{h}, \varphi_{h}\right),\left(\mathbf{v}_{h}, \eta_{h}\right)\right) \quad \forall\left(\mathbf{v}_{h}, \eta_{h}\right) \in G_{h}\left(\Omega_{h}\right) .
$$

La versión discreta del operador $\boldsymbol{\Psi}$ estará dada por

$$
\begin{aligned}
\boldsymbol{\Psi}_{h}: H\left(\Omega_{h}\right) & \rightarrow G_{h}\left(\Omega_{h}\right) \hookrightarrow H\left(\Omega_{h}\right) \\
(\mathbf{x}, y) & \mapsto\left(\mathbf{u}_{h}, \varphi_{h}\right),
\end{aligned}
$$

siendo $\left(\mathbf{u}_{h}, \varphi_{h}\right) \in G_{h}\left(\Omega_{h}\right)$ la solución del siguiente problema:

$$
A_{h}\left(\mathbf{\Psi}_{h}(\mathbf{x}, y),\left(\mathbf{v}_{h}, \eta_{h}\right)\right)=B_{h}\left((\mathbf{x}, y),\left(\mathbf{v}_{h}, \eta_{h}\right)\right) \quad \forall\left(\mathbf{v}_{h}, \eta_{h}\right) \in G_{h}\left(\Omega_{h}\right) .
$$

Procediendo exactamente igual que en el caso continuo, tendremos

- $\boldsymbol{\Psi}_{h}$ es un operador lineal y acotado,

- $\mathbf{\Psi}_{h}(\mathbf{x}, y)=\left(\mathbf{S}_{h} y, \mathbf{T}_{h} y\right) \quad \forall(\mathbf{x}, y) \in H\left(\Omega_{h}\right)$,

- $\left.\boldsymbol{\Psi}_{h}\right|_{G_{h}\left(\Omega_{h}\right)}$ es un operador autoadjunto con respecto a la forma $A_{h}$.

En estas condiciones, es claro que podemos analizar la convergencia de los pares propios del operador $\boldsymbol{\Psi}_{h}$ hacia los pares propios del operador $\boldsymbol{\Psi}$ aplicando la teoría desarrollada en las primeras secciones sin modificaciones (simplemente, identificando la norma $\|\cdot\|_{h, \Omega_{h}}$ con la norma $\|\cdot\|_{G_{h}\left(\Omega_{h}\right)}$ ).

Para establecer estimaciones del error en las aproximaciones de los pares autovalor/autovector podemos utilizar los desarrollos de las secciones 3.3.3-3.3.4. En este contexto, el operador de proyección $\Pi_{h}$ admite la siguiente definición:

$$
\left\{\begin{array}{l}
\Pi_{h}(\mathbf{x}, \eta)=\mathbf{P}_{h}\left(\mathbf{x}_{h}, \eta_{h}\right) \\
A_{h}\left((\mathbf{x}, \eta)-\left(\mathbf{x}_{h}, \eta_{h}\right),\left(\mathbf{v}_{h}, \tau_{h}\right)\right)=0 \quad \forall\left(\mathbf{v}_{h}, \tau_{h}\right) \in G_{h}\left(\Omega_{h}\right) .
\end{array}\right.
$$


Observación 3.33 El operador $\Pi_{h}$ puede ser construido por componentes. En efecto, escribamos

$$
\left(\mathbf{x}_{h}, \eta_{h}\right)=\left(\Pi_{1 h} \mathbf{x}, \Pi_{2 h} \eta\right)
$$

y elijamos a $\Pi_{1 h}: V\left(\Omega_{h}\right) \rightarrow V_{h}\left(\Omega_{h}\right)$ como el operador de Fortin correspondiente al problema (3.60). Entonces, tendremos

$$
b_{h}\left(\mathbf{x}-\Pi_{1 h} \mathbf{x}, \eta_{h}\right)=0 \quad \forall \eta_{h} \in Q_{h}\left(\Omega_{h}\right) .
$$

Luego, para que (3.67) se verifique, el operador $\Pi_{2 h}: Q\left(\Omega_{h}\right) \rightarrow Q_{h}\left(\Omega_{h}\right)$ tendrá que satisfacer

$$
a_{h}\left(\mathbf{x}-\Pi_{1 h} \mathbf{x}, \mathbf{v}_{h}\right)+\overline{b_{h}\left(\mathbf{v}_{h}, \eta-\Pi_{2 h} \eta\right)}=0 \quad \forall \mathbf{v}_{h} \in V_{h}\left(\Omega_{h}\right)
$$

\section{Estimaciones del error para la segunda variable del par solución}

Hasta aquí hemos mostrado como se puede analizar la convergencia de las soluciones del problema (3.59) hacia las soluciones del problema (3.56) aplicando la teoría desarrollada en la sección 3.3 a los operadores $\Psi$ y $\boldsymbol{\Psi}_{h}$. Usualmente, este procedimiento nos permite obtener estimaciones óptimas del error en la aproximación de los pares propios del operador $\boldsymbol{\Psi}$ medido en la norma discreta asociada con el espacio producto $G_{h}(\widehat{\Omega})$. Sin embargo, si analizáramos el error en la aproximación de cada una de las componentes, esta estimación no resultará óptima en la mayoría de los casos. Esto se debe a la manera en la que las variables $(\mathbf{u}, \varphi)$ aparecen ligadas en las ecuaciones de error; particularidad que ya había sido señalada por Falk-Osborn [103] en el contexto de los problemas fuente.

Nuestro próximo objetivo será estimar el error en la approximación de la componente $\varphi$ en la norma $|\cdot|_{Y(\widehat{\Omega})}$ por separado.

Dado $\lambda \neq 0$, un autovalor aislado del operador $\left.\Psi\right|_{G(\Omega)}$ con multiplicidad finita $m$, sea $\Delta \subset \mathbb{C}$ un disco cerrado centrado en $\lambda$ tal que $0 \notin \Delta$ y $\Delta \cap \sigma(\Psi)=\{\lambda\}$. Asumiremos que ya hemos probado que el método numérico definido por los espacios $G_{h}\left(\Omega_{h}\right)$ produce aproximaciones que convergen correctamente usando la teoría abstracta presentada en la sección 3.3. Entonces, podremos asegurar que, para $h$ suficientemente chico, existen $m$ autovalores de $\Psi_{h}, \lambda_{1 h}, \lambda_{2 h}, \cdots, \lambda_{m h}$, contenidos en $\Delta$, y que $\lim _{h \rightarrow 0} \lambda_{i h}=\lambda$ para $i=1, \cdots, m$.

Consideremos al operador $\mathbf{T}: Y(\Omega) \rightarrow Q(\Omega) \hookrightarrow Y(\Omega)$, definido por las ecuaciones (3.57). Es claro que:

- es lineal y acotado en $Y(\Omega)$,

- para cualquier $z \in \partial \Delta, R_{z}(\mathbf{T})=(z-\mathbf{T})^{-1} \in \mathcal{L}(Y(\Omega))$,

- $\sigma(\mathbf{T})=\sigma(\mathbf{\Psi})$.

El operador $\mathbf{T}$ será aproximado por los operadores $\mathbf{T}_{h}: Y\left(\Omega_{h}\right) \rightarrow Q_{h}\left(\Omega_{h}\right) \hookrightarrow Y\left(\Omega_{h}\right)$, definidos por las ecuaciones (3.60). Como en el caso continuo, es claro que:

- es lineal y acotado en $Y\left(\Omega_{h}\right)$, uniformemente en $h$,

- $\sigma\left(\mathbf{T}_{h}\right)=\sigma\left(\mathbf{\Psi}_{h}\right)$

A continuación utilizaremos nuevamente la teoría desarrollada en las primeras secciones para estimar el orden con el que convergen las autofunciones del operador $\mathbf{T}_{h}$ hacia las autofunciones del operador $\mathbf{T}$.

Razonando como en la sección 3.2, definimos

* una norma discreta compatible

$$
\begin{aligned}
& \text { - }\|(\mathbf{u}, \varphi)\|_{h, \Omega_{h}} \simeq\|(\mathbf{u}, \varphi)\|_{G_{h}\left(\Omega_{h}\right)} \quad \text { si }\left.\quad(\mathbf{u}, \varphi)\right|_{\Omega_{h}} \in G_{h}\left(\Omega_{h}\right), \\
& \text { - }\|(\mathbf{u}, \varphi)\|_{h, \Omega} \simeq\|(\mathbf{u}, \varphi)\|_{G(\Omega)} \quad \text { si }\left.\quad(\mathbf{u}, \varphi)\right|_{\Omega} \in G(\Omega), \\
& \text { - }\|(\mathbf{u}, \varphi)\|_{h} \simeq\|(\mathbf{u}, \varphi)\|_{G(\widehat{\Omega})} \quad \text { si } \quad(\mathbf{u}, \varphi) \in G(\widehat{\Omega})
\end{aligned}
$$


* los operadores de extensión continuos

$$
\begin{aligned}
& \text { - } \mathbf{P}:=\left(\mathbf{P}_{1}, \mathbf{P}_{2}\right): G(\Omega) \rightarrow G(\widehat{\Omega}),(\mathbf{u}, \varphi) \mapsto\left(\mathbf{u}^{e}, \varphi^{e}\right), \\
& \text { - }\left.\left(\mathbf{u}^{e}, \varphi^{e}\right)\right|_{\Omega}=(\mathbf{u}, \varphi), \\
& \text { - }\left|\left(\mathbf{u}^{e}, \varphi^{e}\right)\right|_{H(\widehat{\Omega})} \leq C\left\|\left(\mathbf{u}^{e}, \varphi^{e}\right)\right\|_{h} \leq C\|(\mathbf{u}, \varphi)\|_{G(\Omega)}
\end{aligned}
$$

* los operadores de extensión discretos

$$
\begin{aligned}
& \text { - } \mathbf{P}_{h}:=\left(\mathbf{P}_{1 h}, \mathbf{P}_{2 h}\right): G_{h}\left(\Omega_{h}\right) \rightarrow H(\widehat{\Omega}),\left(\mathbf{u}_{h}, \varphi_{h}\right) \mapsto\left(\mathbf{u}_{h}^{e}, \varphi_{h}^{e}\right) \\
& \text { - }\left.\left(\mathbf{u}_{h}^{e}, \varphi_{h}^{e}\right)\right|_{\Omega_{h}}=\left(\mathbf{u}_{h}, \varphi_{h}\right), \\
& \text { - }\left|\left(\mathbf{u}_{h}^{e}, \varphi_{h}^{e}\right)\right|_{H(\widehat{\Omega})} \leq C\left\|\left(\mathbf{u}_{h}^{e}, \varphi_{h}^{e}\right)\right\|_{h} \leq C\left\|\left(\mathbf{u}_{h}, \varphi_{h}\right)\right\|_{G_{h}\left(\Omega_{h}\right)},
\end{aligned}
$$

* los espacios extendidos

$$
\begin{aligned}
Q^{e}(\widehat{\Omega}) & :=\mathbf{P}_{2}(Q(\Omega)) \subset Q(\widehat{\Omega}) \\
Q_{h}^{e}(\widehat{\Omega}) & :=\mathbf{P}_{2 h}\left(Q_{h}\left(\Omega_{h}\right)\right) \subset Y(\widehat{\Omega}) \\
Z(\widehat{\Omega}) & :=\left(Q^{e}+Q_{h}^{e}\right)(\widehat{\Omega})
\end{aligned}
$$

* la extensión del operador $\mathbf{T}$

$$
\begin{aligned}
\mathbf{A}: Y(\widehat{\Omega}) & \rightarrow Q^{e}(\widehat{\Omega}) \subset Z(\widehat{\Omega}) \hookrightarrow Y(\widehat{\Omega}) \\
\mathbf{x} & \mapsto \varphi^{e}=\mathbf{P}_{2} \circ \mathbf{T}\left(\left.\mathbf{x}\right|_{\Omega}\right),
\end{aligned}
$$

* la extensión del operador $\mathbf{T}_{h}$

$$
\begin{aligned}
\mathbf{A}_{h}: Y(\widehat{\Omega}) & \rightarrow Q_{h}^{e}(\widehat{\Omega}) \subset Z(\widehat{\Omega}) \hookrightarrow Y(\widehat{\Omega}) \\
\mathbf{x} & \mapsto \varphi_{h}^{e}=\mathbf{P}_{2 h} \circ \mathbf{T}_{h}\left(\left.\mathbf{x}\right|_{\Omega_{h}}\right),
\end{aligned}
$$

El paso siguiente sería probar que el operador $z-\Lambda: Z(\widehat{\Omega}) \rightarrow Z(\widehat{\Omega})$ define una aplicación con inversa acotada en la norma $|\cdot|_{Y(\widehat{\Omega})}$, uniformemente en $h$. Para obtener este resultado, seguimos exactamente los argumentos planteados en el Lema 3.3, reemplazando

- la norma $\|\cdot\|_{h}$ por la norma $|\cdot|_{Y(\widehat{\Omega})}$,

- el espacio $W(\widehat{\Omega})$ por el espacio $Z(\widehat{\Omega})$,

- el operador de extensión $\mathbf{P}$ por la componente $\mathbf{P}_{2}$ de este operador .

El siguiente lema implica que el operador resolvente $\left(z-\boldsymbol{\Lambda}_{h}\right)^{-1}$ es un operador continuo, bien definido para todo $z \in \partial \Delta$ y que está acotado en la norma $|\cdot|_{Y(\widehat{\Omega})}$, uniformemente en $h$.

Lema 3.34 Sea $z \in \partial \Delta$. Existen constantes positivas $C$ y $h_{0}$, que dependen solamente de $\Omega$ y $|z|$, tales que

$$
\left|\left(z-\mathbf{A}_{h}\right) \varphi\right|_{Y(\widehat{\Omega})} \geq C|\varphi|_{Y(\widehat{\Omega})} \quad \forall \varphi \in Q_{h}^{e}(\widehat{\Omega}), \quad \forall h<h_{0}
$$

Prueba. Sea $(u, \varphi) \in G_{h}^{e}(\widehat{\Omega})$. Usando la desigualdad triangular, tendremos

$$
\left|\left(z-\mathbf{A}_{h}\right) \varphi\right|_{Y(\widehat{\Omega})} \geq|(z-\mathbf{A}) \varphi|_{Y(\widehat{\Omega})}-\left|\left(\mathbf{A}-\mathbf{A}_{h}\right) \varphi\right|_{Y(\widehat{\Omega})} .
$$

Debido a que $z-\boldsymbol{\Lambda}$ admite inversa acotada como operador de $Y(\widehat{\Omega})$ a $Y(\widehat{\Omega})$, existe una constante positiva $C$, que depende solo de $|z|$ y de $\Omega$, tal que

$$
|(z-\mathbf{A}) \varphi|_{Y(\widehat{\Omega})} \geq C|\varphi|_{Y(\widehat{\Omega})} .
$$


Por otro lado, a partir de la definición de la norma discreta $\|\cdot\|_{h}$ y teniendo en cuenta la inclusión $Z(\widehat{\Omega}) \hookrightarrow Y(\widehat{\Omega})$, es inmediato que

$$
\left\|\left(\mathbf{P} \circ \boldsymbol{\Psi}-\mathbf{P}_{h} \circ \boldsymbol{\Psi}_{h}\right)(u, \varphi)\right\|_{h} \geq\left\|\left(\mathbf{A}-\mathbf{A}_{h}\right) \varphi\right\|_{Z(\widehat{\Omega})} \geq C_{1}\left|\left(\mathbf{A}-\mathbf{A}_{h}\right) \varphi\right|_{Y(\widehat{\Omega})},
$$

siendo $C_{1}$ una constante positiva independente de $h$. Ahora, la propiedad $\mathbf{P 2}{ }^{6}$ implica que, para $h$ suficientemente chico, podemos establecer

$$
\left\|\left(\mathbf{P} \circ \mathbf{\Psi}-\mathbf{P}_{h} \circ \boldsymbol{\Psi}_{h}\right)(u, \varphi)\right\|_{h}=\left\|\left(\mathbf{P} \circ(\mathbf{S}, \mathbf{T})-\mathbf{P}_{h} \circ\left(\mathbf{S}_{h}, \mathbf{T}_{h}\right)\right) \varphi\right\|_{h} \leq \xi_{h}\|\varphi\|_{Z(\widehat{\Omega})},
$$

con $\xi_{h} \rightarrow 0$ a medida que $h \rightarrow 0$. Entonces,

$$
-\left|\left(\mathbf{A}-\mathbf{A}_{h}\right) \varphi\right|_{Y(\widehat{\Omega})} \geq-\frac{\xi_{h}}{C_{1}}\|\varphi\|_{Z(\widehat{\Omega})} .
$$

Combinando todas estas desigualdades, tendremos

$$
\left|\left(z-\mathbf{A}_{h}\right) \varphi\right|_{Y(\widehat{\Omega})} \geq\left(C-\frac{\xi_{h}}{C_{1}} \frac{\|\varphi\|_{Z(\widehat{\Omega})}}{|\varphi|_{Y(\widehat{\Omega})}}\right)|\varphi|_{Y(\widehat{\Omega})} .
$$

Finalmente, observemos que, debido a la inclusión $Z(\widehat{\Omega}) \hookrightarrow Y(\widehat{\Omega})$, el número

$$
\alpha=\frac{1}{C_{1}} \frac{\|\varphi\|_{Z(\widehat{\Omega})}}{|\varphi|_{Y(\widehat{\Omega})}}
$$

es, en realidad, una constante positiva independiente de $\varphi$ y $h$. Luego, para $h$ suficientemente pequeño, podremos escribir $\xi_{h} \alpha \leq C / 2$; expresión que nos permite concluir la prueba.

Definimos entonces el proyector espectral $\widehat{\mathbf{E}}_{\lambda}: Y(\widehat{\Omega}) \rightarrow Y(\widehat{\Omega})$, asociado con el operador $\boldsymbol{\Lambda}$ relativo a $\lambda$,

$$
\widehat{\mathbf{E}}_{\lambda}=\frac{1}{2 \pi i} \int_{\partial \Delta} R_{z}(\boldsymbol{\Lambda}) d z
$$

y, para $h$ suficientemente chico, el proyector espectral $\widehat{\mathbf{E}}_{\lambda_{h}}: Q_{h}^{e}(\widehat{\Omega}) \rightarrow Q_{h}^{e}(\widehat{\Omega})$, asociado con el operador $\boldsymbol{\Lambda}_{h}$ relativo a los autovalores $\lambda_{1 h}, \lambda_{2 h}, \cdots, \lambda_{m h}$,

$$
\widehat{\mathbf{E}}_{\lambda_{h}}=\frac{1}{2 \pi i} \int_{\partial \Delta} R_{z}\left(\boldsymbol{\Lambda}_{h}\right) d z .
$$

A continuación asumiremos que

- la forma bilineal $d_{h}$ es uniformemente coerciva con respecto a la norma $|\cdot|_{Y\left(\Omega_{h}\right)}$ sobre el espacio $Q_{h}\left(\Omega_{h}\right)$

- el dominio de definición de la forma $d_{h}$ puede extenderse al espacio $Z(\widehat{\Omega})$ de manera tal que resulte uniformemente continua sobre el espacio suma,

- el operador de extensión $\mathbf{P}_{2 h}: Q_{h}\left(\Omega_{h}\right) \hookrightarrow Y\left(\Omega_{h}\right) \rightarrow Y(\widehat{\Omega})$, está uniformemente acotado en la norma $|\cdot|_{Y(\widehat{\Omega})} \cdot$

Sea ahora $\widehat{\Pi}_{h}: Z(\widehat{\Omega}) \rightarrow Z(\widehat{\Omega})$ el operador, con rango en $Q_{h}^{e}(\widehat{\Omega})$, definido por

$$
\left\{\begin{array}{l}
\widehat{\Pi}_{h} \eta=\mathbf{P}_{2 h} \eta_{h} \\
d_{h}\left(\eta-\eta_{h}, \tau_{h}\right)=0 \quad \forall \tau_{h} \in Q_{h}\left(\Omega_{h}\right)
\end{array}\right.
$$

\footnotetext{
${ }^{6}$ En este contexto, la propiedad $\mathbf{P 2}$ adopta la siguiente forma: $\lim _{h \rightarrow 0}\left\|\left.\left(\mathbf{P} \circ \mathbf{\Psi}-\mathbf{P}_{h} \circ \boldsymbol{\Psi}_{h}\right)\right|_{G_{h}^{e}(\widehat{\Omega})}\right\|_{h}=0$.
} 
Como, por hipótesis, la forma bilineal $d_{h}$ es continua sobre $Z(\widehat{\Omega})$ y coerciva sobre $Q_{h}\left(\Omega_{h}\right)$ y el operador $\mathbf{P}_{2 h}$ está acotado en la norma $|\cdot|_{Y(\widehat{\Omega})}$, todo uniformemente en $h$, el proyector $\widehat{\Pi}_{h}$ resulta acotado, uniformemente en $h$, con respecto a la misma norma.

Sea $\mathbf{B}_{h}:=\mathbf{A}_{h} \widehat{\Pi}_{h}: Z(\widehat{\Omega}) \rightarrow Q^{e}(\widehat{\Omega})$. Entonces, $\sigma\left(\mathbf{B}_{h}\right)=\sigma\left(\mathbf{A}_{h}\right)$ y, para cualquier autovalor distinto de cero, los subespacios invariantes correspondientes coinciden. Sea $\mathbf{F}_{\lambda_{h}}$ el proyector espectral asociado con el operador $\mathbf{B}_{h}$ relativo a los autovalores $\lambda_{1 h}, \lambda_{2 h}, \cdots, \lambda_{m h}$. Argumentando como en el Lema 3.19, puede probarse que existen constantes positivas, $h_{0}$ y $C$, tales que, para todo $z \in \partial \mathcal{D}$

$$
\sup _{\substack{\eta \in Z(\widehat{\Omega}) \\|\eta|_{Y(\widehat{\Omega})}=1}}\left|R_{z}\left(\mathbf{B}_{h}\right) \eta\right|_{Y(\widehat{\Omega})} \leq C \quad \forall h<h_{0} .
$$

Por lo tanto, si $h$ es suficientemente chico, los proyectores $\mathbf{F}_{\lambda_{h}}$ son operadores acotados, uniformemente en $h$, con respecto a la norma $|\cdot|_{Y(\widehat{\Omega})}$.

Asumiremos que la siguiente propiedad se satisface:

P6: $\quad$ para cada autovalor $\varphi \in \widehat{\mathbf{E}}_{\lambda}(Z(\widehat{\Omega})),\left|\left(\mathbf{A}-\mathbf{A}_{h}\right) \varphi\right|_{Y(\widehat{\Omega})} \leq \eta_{5 h}|\varphi|_{Y(\widehat{\Omega})}$, con $\eta_{5 h} \rightarrow 0$ para $h \rightarrow 0$.

Definimos

$$
\widehat{\varrho}_{h}:=\sup _{\substack{\varphi \in \widehat{\mathbf{E}}_{\lambda}(Z(\widehat{\Omega})) \\|\varphi|_{Y(\widehat{\Omega})}=1}}\left(\inf _{\substack{\eta_{h} \in Q_{h}^{e}(\widehat{\Omega})\\}}\left|\varphi-\eta_{h}\right|_{Y(\widehat{\Omega})}+\left|\left(\mathbf{A}-\mathbf{A}_{h}\right) \varphi\right|_{Y(\widehat{\Omega})}\right) .
$$

A partir de la propiedad $\mathbf{P} \mathbf{1}^{7}$, la definición de la norma discreta $\|\cdot\|_{h}$, la inclusión continua $Z(\widehat{\Omega}) \hookrightarrow Y(\widehat{\Omega})$ y la propiedad $\mathbf{P 6}$, tendremos que $\widehat{\varrho}_{h} \rightarrow 0$ a medida que $h \rightarrow 0$.

Lema 3.35 Existe una constante $C$, estrictamente positiva, tal que

$$
\left.\left|\left(\mathbf{I}-\widehat{\Pi}_{h}\right)\right|_{\widehat{\mathbf{E}}_{\lambda}(Z(\widehat{\Omega}))}\right|_{Y(\widehat{\Omega})} \leq C \widehat{\varrho}_{h} .
$$

Prueba. Sea $\eta \in \widehat{\mathbf{E}}_{\lambda}(Z(\widehat{\Omega}))$, con $|\eta|_{Y(\widehat{\Omega})}=1$, y consideremos un elemento arbitrario $\tau_{h}$ en el espacio $Q_{h}\left(\Omega_{h}\right)$. A partir de la definición de la proyección $\widehat{\Pi}_{h}$, sabemos que existe un único elemento $\eta_{h} \in Q_{h}\left(\Omega_{h}\right)$ tal que $\widehat{\Pi}_{h} \eta=\mathbf{P}_{2 h} \eta_{h}$. Luego, podemos escribir

$$
\left|\left(\mathbf{I}-\widehat{\Pi}_{h}\right) \eta\right|_{Y(\widehat{\Omega})} \leq\left|\eta-\mathbf{P}_{2 h} \tau_{h}\right|_{Y(\widehat{\Omega})}+\left|\mathbf{P}_{2 h}\left(\tau_{h}-\eta_{h}\right)\right|_{Y(\widehat{\Omega})} .
$$

Como $\mathbf{P}_{2 h}$ es un operador acotado en $Q_{h}\left(\Omega_{h}\right)$ y la forma $d_{h}$ es coerciva sobre $Q_{h}\left(\Omega_{h}\right)$, ambas propiedades valiendo uniformemente en $h$, tendremos

$$
\left|\mathbf{P}_{2 h}\left(\tau_{h}-\eta_{h}\right)\right|_{Y(\widehat{\Omega})}^{2} \leq C d_{h}\left(\tau_{h}-\eta_{h}, \tau_{h}-\eta_{h}\right)=C d_{h}\left(\tau_{h}-\eta_{h}, \tau_{h}-\eta\right),
$$

donde hemos usado la definición de $\widehat{\Pi}_{h}$ para establecer la última igualdad. Ahora, teniendo en cuenta que $d_{h}$ es uniformemente continua sobre $\left(Q+Q_{h}\right)\left(\Omega_{h}\right)$, obtendremos

$$
\left|\mathbf{P}_{2 h}\left(\tau_{h}-\eta_{h}\right)\right|_{Y(\widehat{\Omega})} \leq C \inf _{\tau_{h} \in Q_{h}\left(\Omega_{h}\right)}\left|\eta-\tau_{h}\right|_{Y\left(\Omega_{h}\right)} \leq C \inf _{\tau_{h}^{e} \in Q_{h}^{e}(\widehat{\Omega})}\left|\eta-\tau_{h}^{e}\right|_{Y(\widehat{\Omega})}
$$

Finalizamos la prueba, combinando esta estimación con (3.69).

Lema 3.36 Existen constantes positivas, $h_{0} y C$, tales que, si $h<h_{0}$, entonces

$$
\left.\left|\left(\widehat{\mathbf{E}}_{\lambda}-\mathbf{F}_{\lambda_{h}}\right)\right|_{\widehat{\mathbf{E}}_{\lambda}(Z(\widehat{\Omega}))}\right|_{Y(\widehat{\Omega})} \leq\left. C\left|\left(\mathbf{A}-\mathbf{B}_{h}\right)\right|_{\widehat{\mathbf{E}}_{\lambda}(Z(\widehat{\Omega}))}\right|_{Y(\widehat{\Omega})} \leq C \widehat{\varrho}_{h} .
$$

\footnotetext{
${ }^{7}$ En este contexto, la propiedad $\mathbf{P} \mathbf{1}$ adopta la siguiente forma: para $(\mathbf{u}, \varphi) \in \mathbf{E}_{\lambda}\left(G^{e}(\widehat{\Omega})\right)$, existe $\left(\mathbf{u}_{h}, \varphi_{h}\right) \in G_{h}^{e}(\widehat{\Omega})$ tal que $\left\|(\mathbf{u}, \varphi)-\left(\mathbf{u}_{h}, \varphi_{h}\right)\right\|_{h} \leq \eta_{1 h}\|(\mathbf{u}, \varphi)\|_{h}$, con $\eta_{1 h} \rightarrow 0$ para $h \rightarrow 0$.
} 
Prueba. Es esencialmente idéntica a las pruebas de los Lemas 3.23 y 3.25, reemplazando a las normas $\|\cdot\|_{h} \mathrm{y}\|\cdot\|_{h *}$ por la norma $|\cdot|_{Y(\widehat{\Omega})}$.

Teorema 3.37 Existen constantes estrictamente positivas, $C$ y $h_{0}$, tales que, si $h \leq h_{0}$, entonces

$$
\widehat{\delta}\left(\widehat{\mathbf{E}}_{\lambda}(Z(\widehat{\Omega})), \mathbf{F}_{\lambda_{h}}(Z(\widehat{\Omega}))\right) \leq C \widehat{\varrho}_{h},
$$

donde el gap entre los subespacios se evalúa en la norma $|\cdot|_{Y(\widehat{\Omega})}$.

Prueba. Es esencialmente idéntica a la prueba del Teorema 3.26, reemplazando la norma $\|\cdot\|_{h}$ por $|\cdot|_{Y(\widehat{\Omega})} \cdot$

\subsection{Problemas de autovalores no simétricos}

Indicaremos como extender la teoría desarrollada de manera que también pueda aplicarse en el análisis espectral de problemas cuya formulación variacional involucra formas bilineales no simétricas.

De lo expuesto en las secciones previas, es claro que la falta de simetría de las formas bilineales no afectará los resultados sobre la convergencia (libre de soluciones espurias) de todos aquellos métodos de aproximación que verifiquen las propiedades P1-P2.

En cambio, las estimaciones del orden con el que convergen los pares autovalor/autofunción aproximados fueron obtenidas bajo la hipótesis

- las formas sesquilineales $a(\cdot, \cdot)$ y $b(\cdot, \cdot)$ son hermitianas simétricas,

la cual fue introducida en la sección 3.3.3, y que, por supuesto, ya no será válida.

A continuación revisaremos la teoría desarrollada en las secciones 3.1-3.4 mostrando las modificaciones que se necesitan hacer para obtener la extensión buscada.

\subsubsection{El problema de autovalores dual}

La idea central, en el análisis que sigue, es la de asociar un problema de autovalores dual con el problema de autovalores no simétrico original (3.1).

Sea $V(\Omega)$ un espacio de Hilbert complejo, con producto interno $(\cdot, \cdot)_{V(\Omega)}$ y norma $\|\cdot\|_{V(\Omega)}$. Supongamos que $a: V(\Omega) \times V(\Omega) \rightarrow \mathbb{C}$ sea una forma sesquilinear continua. Gracias al Teorema de Riesz, existe la posibilidad de asociar con la forma a un mapeo lineal y continuo $\mathcal{A}: V(\Omega) \rightarrow V(\Omega)$ definido por

$$
a(u, v)=(\mathcal{A} u, v)_{V(\Omega)} \quad \forall u, v \in V(\Omega) .
$$

Si asumimos que la forma $a$ es $V(\Omega)$-elíptica, el Teorema de Lax-Milgram nos permitirá asegurar que $\mathcal{A}$ es un isomorfismo de $V(\Omega)$ sobre $V(\Omega)$. El mapeo adjunto $\mathcal{A}^{*}: V(\Omega) \rightarrow V(\Omega)$ será un mapeo lineal y acotado que verifica

$$
a(u, v)=\left(u, \mathcal{A}^{*} v\right)_{V(\Omega)} \quad \forall u, v \in V(\Omega) .
$$

Además, $\mathcal{A}^{*}$ es un isomorfismo si, y solo si, $\mathcal{A}$ es un isomorfismo.

Sea $X(\Omega)$ un espacio de Hilbert complejo, con producto interno $(\cdot, \cdot)_{X(\Omega)}$ y norma $|\cdot|_{X(\Omega)}$. Asumiremos que $V(\Omega) \subset X(\Omega)$ y que la inclusión es continua. Supongamos que $b: X(\Omega) \times X(\Omega) \rightarrow \mathbb{C}$ sea una forma sesquilineal continua.

Consideremos los siguientes operadores lineales

$$
\begin{aligned}
\mathbf{T}: X(\Omega) & \rightarrow V(\Omega) \hookrightarrow X(\Omega) \\
x & \mapsto u,
\end{aligned}
$$

con $u \in V(\Omega)$ solución del siguiente problema variacional

$$
a(u, y)=b(x, y) \quad \forall y \in V(\Omega)
$$




$$
\begin{aligned}
\mathbf{T}_{*}: X(\Omega) & \rightarrow V(\Omega) \hookrightarrow X(\Omega) \\
x & \mapsto u_{*},
\end{aligned}
$$

con $u_{*} \in V(\Omega)$ solución del siguiente problema variacional

$$
a\left(y, u_{*}\right)=b(y, x) \quad \forall y \in V(\Omega) .
$$

A partir de las definiciones anteriores, es inmediato que

- $a\left(u, \mathbf{T}_{*} v\right)=a(\mathbf{T} u, v) \quad \forall u, v \in V(\Omega)$,

- $\mathbf{T}_{*}=\left(\mathcal{A}^{*}\right)^{-1} \circ \mathbf{T}^{*} \circ \mathcal{A}^{*}$, siendo $\mathbf{T}^{*}$ el operador $V(\Omega)$-adjunto de $\mathbf{T}$ considerado como un operador lineal y continuo sobre $V(\Omega)$.

Como consecuencia de los resultados anteriores, tendremos que

- $\left\|\mathbf{T}_{*}\right\|_{\mathcal{L}(V(\Omega))} \leq\left\|\left(\mathcal{A}^{*}\right)^{-1}\right\|_{\mathcal{L}(V(\Omega))}\|\mathbf{T}\|_{\mathcal{L}(V(\Omega))}\left\|\mathcal{A}^{*}\right\|_{\mathcal{L}(V(\Omega))}$

- $\sigma\left(\mathbf{T}_{*}\right)=\sigma\left(\mathbf{T}^{*}\right)$,

- $(\bar{\lambda}, u)$ es un par propio de $\mathbf{T}_{*}$ si, y solo si, $\left(\frac{1}{\lambda}, u\right)$ es solución del siguiente problema de autovalores Encontrar $\mu_{*} \in \mathbb{C}, u_{*} \in V(\Omega), u_{*} \neq 0$, tal que

$$
a\left(v, u_{*}\right)=\mu_{*} b\left(v, u_{*}\right) \quad \forall v \in V(\Omega) .
$$

\subsubsection{Discretización de los problemas}

Considerando simultáneamente las versiones discretas del problema original (3.1) y del problema dual asociado (3.72), es posible obtener estimaciones a priori del error en la aproximación de los autovalores y las autofunciones. Como veremos más adelante, las estimaciones que hallaremos pueden considerarse como generalizaciones de los resultados presentados en [138] y [150].

Sea $\Omega_{h}$ una aproximación poligonal del dominio $\bar{\Omega}$ construida siguiendo todos los criterios introducidos en la sección 3.1. Asociada con la familia de dominios $\left\{\Omega_{h}\right\}_{0<h<1}$, seleccionaremos una familia de espacios de dimensión finita $\left\{V_{h}\left(\Omega_{h}\right)\right\}_{0<h<1}$. Asumiremos que cada espacio $V_{h}\left(\Omega_{h}\right)$ está equipado con la norma $\|\cdot\|_{V_{h}\left(\Omega_{h}\right)}$ y que se satisfacen las siguientes condiciones

$$
\begin{aligned}
& \|v\|_{V_{h}\left(\Omega_{h}\right)}=\|v\|_{V\left(\Omega_{h}\right)} \quad \forall v \in V\left(\Omega_{h}\right), \\
& \left(V_{h}\left(\Omega_{h}\right),\|\cdot\|_{V_{h}\left(\Omega_{h}\right)}\right) \hookrightarrow\left(X\left(\Omega_{h}\right),|\cdot|_{X\left(\Omega_{h}\right)}\right) \text { uniformemente en } h \text {. }
\end{aligned}
$$

Sean $a_{h}: V_{h}\left(\Omega_{h}\right) \times V_{h}\left(\Omega_{h}\right) \rightarrow \mathbb{C}$ y $b_{h}: X\left(\Omega_{h}\right) \times X\left(\Omega_{h}\right) \rightarrow \mathbb{C}$ las formas sesquilineales obtenidas mediante la discretización de las formas continuas $a$ y $b$, respectivamente. Asumiremos que las formas $a_{h} \mathrm{y} b_{h}$ verifican todas las propiedades enunciadas en la sección 3.1.

Introducimos los siguientes operadores acotados; análogos discretos de los operadores $\mathbf{T}$ y $\mathbf{T}_{*}$,

$$
\begin{aligned}
\mathbf{T}_{h}: X\left(\Omega_{h}\right) & \rightarrow V_{h}\left(\Omega_{h}\right) \hookrightarrow X_{h}\left(\Omega_{h}\right) \\
x & \mapsto u_{h},
\end{aligned}
$$

con $u_{h} \in V_{h}\left(\Omega_{h}\right)$ solución del siguiente problema variacional

$$
\begin{aligned}
& a_{h}\left(u_{h}, y\right)=b_{h}(x, y) \quad \forall y \in V_{h}\left(\Omega_{h}\right), \\
& \mathbf{T}_{* h}: X\left(\Omega_{h}\right) \rightarrow V_{h}\left(\Omega_{h}\right) \hookrightarrow X\left(\Omega_{h}\right) \\
& x \mapsto u_{* h},
\end{aligned}
$$

con $u_{* h} \in V_{h}\left(\Omega_{h}\right)$ solución del siguiente problema variacional

$$
a_{h}\left(y, u_{* h}\right)=b_{h}(y, x) \quad \forall y \in V_{h}\left(\Omega_{h}\right) .
$$




\subsubsection{Estimaciones del error}

Para poder continuar con el análisis, necesitaremos incorporar todos los problemas planteados (problemas (3.1), (3.72) y sus versiones discretas) dentro de un único marco teórico. Para ello, será necesario introducir operadores que estén asociados con estos problemas de autovalores y definidos sobre un mismo espacio funcional. Esta tarea no es trivial ya que, en general, $\Omega_{h} \not \subset \Omega$.

Siguiendo los argumentos introducidos en la sección 3.2, definiremos

- un dominio unión $\widehat{\Omega}:=\Omega \cup \Omega_{h}$,

- una norma discreta $\|\cdot\|_{h}$ para funciones con dominio $\widehat{\Omega}$,

- un operador de extensión para las funciones del continuo; $\mathbf{P}: V(\Omega) \rightarrow V(\widehat{\Omega})$,

- operadores de extensión para las funciones discretas; $\mathbf{P}_{h}: V_{h}\left(\Omega_{h}\right) \rightarrow X(\widehat{\Omega})$,

- espacios funcionales (convenientemente) extendidos

$$
\begin{aligned}
& V^{e}(\widehat{\Omega}):=\mathbf{P}(V(\Omega)) \subset V(\widehat{\Omega}), \\
& V_{h}^{e}(\widehat{\Omega}):=\mathbf{P}_{h}\left(V_{h}\left(\Omega_{h}\right)\right) \subset X(\widehat{\Omega}), \\
& W(\widehat{\Omega}):=\left(V^{e}+V_{h}^{e}\right)(\widehat{\Omega}) .
\end{aligned}
$$

Asumiremos, además, que se satisfacen todas las propiedades enunciadas en la sección 3.2 para la norma $\|\cdot\|_{h}$ y para los operadores $\mathbf{P}$ y $\mathbf{P}_{h}$. Entonces, equipando al espacio suma $W(\widehat{\Omega})$ con la norma $\|\cdot\|_{h}$, tendremos

$$
\begin{aligned}
\|v\|_{h} & \simeq\|v\|_{V(\widehat{\Omega})} \quad \forall v \in V(\widehat{\Omega}) \\
\left(W(\widehat{\Omega}),\|\cdot\|_{h}\right) & \hookrightarrow\left(X(\widehat{\Omega}),|\cdot|_{X(\widehat{\Omega})}\right) \quad \text { uniformemente en } h .
\end{aligned}
$$

Sean $\mathbf{A}$ y $\mathbf{A}_{h}$ los operadores, lineales y acotados, definidos por

$$
\begin{aligned}
\mathbf{A}: X(\widehat{\Omega}) & \rightarrow V^{e}(\widehat{\Omega}) \subset W(\widehat{\Omega}) \hookrightarrow X(\widehat{\Omega}) \\
x & \mapsto u^{e}=\mathbf{P} \circ \mathbf{T}\left(\left.x\right|_{\Omega}\right), \\
\mathbf{A}_{h}: X(\widehat{\Omega}) & \rightarrow V_{h}^{e}(\widehat{\Omega}) \subset W(\widehat{\Omega}) \hookrightarrow X(\widehat{\Omega}) \\
x & \mapsto u_{h}^{e}=\mathbf{P}_{h} \circ \mathbf{T}_{h}\left(\left.x\right|_{\Omega_{h}}\right) .
\end{aligned}
$$

Sea $\lambda$ un autovalor aislado de $\boldsymbol{\Lambda}:=\left.\mathbf{A}\right|_{W(\widehat{\Omega})}$, distinto de cero, con multiplicidad algebraica igual a $m$. Sea $\gamma$ un círculo en el plano complejo, centrado en $\lambda$ y contenido en $\rho(\boldsymbol{\Lambda})$, de manera tal que no incluya en su interior a ningún otro punto perteneciente a $\sigma(\boldsymbol{\Lambda})$.

Sean $\lambda_{1 h}, \cdots, \lambda_{q h}$, los autovalores de $\boldsymbol{\Lambda}_{h}:=\left.\mathbf{A}_{h}\right|_{V_{h}^{e}(\widehat{\Omega})}$ contenidos en el interior de $\gamma$ (repetidos de acuerdo con sus respectidas multiplicidades). Supongamos que se satisfacen las propiedades P1 y P2 enunciadas en la sección 3.3.1. Entonces, si $h$ es suficientemente chico, $q=m$ y además

$$
\lim _{h \rightarrow 0} \lambda_{i h}=\lambda \quad \text { para } i=1, \cdots, m
$$

Sean $\mathbf{A}_{*}$ y $\mathbf{A}_{* h}$ los operadores, lineales y acotados, definidos por

$$
\begin{aligned}
\mathbf{A}_{*}: X(\widehat{\Omega}) & \rightarrow V^{e}(\widehat{\Omega}) \subset W(\widehat{\Omega}) \hookrightarrow X(\widehat{\Omega}) \\
x & \mapsto u_{*}^{e}=\mathbf{P} \circ \mathbf{T}_{*}\left(\left.x\right|_{\Omega}\right),
\end{aligned}
$$




$$
\begin{aligned}
\mathbf{A}_{* h}: X(\widehat{\Omega}) & \rightarrow V_{h}^{e}(\widehat{\Omega}) \subset W(\widehat{\Omega}) \hookrightarrow X(\widehat{\Omega}) \\
x & \mapsto u_{* h}^{e}=\mathbf{P}_{h} \circ \mathbf{T}_{* h}\left(\left.x\right|_{\Omega_{h}}\right) .
\end{aligned}
$$

De lo expuesto anteriormente (sección 3.5.1), sabemos que $\bar{\lambda}$ será un autovalor aislado de $\boldsymbol{\Lambda}_{*}:=\left.\mathbf{A}_{*}\right|_{W(\widehat{\Omega})}$ con multiplicidad algebraica igual a $m$. Sea $\bar{\gamma}$ el círculo conjugado del círculo $\gamma$ (positivamente orientado). Entonces, si $h$ es suficientemente chico, el interior de $\bar{\gamma}$ contendrá a $\bar{\lambda}$ y al conjunto de puntos $\bar{\lambda}_{1 h}, \cdots, \bar{\lambda}_{m h}$. Sabemos, también, que los autovalores de $\boldsymbol{\Lambda}_{* h}:=\left.\mathbf{A}_{* h}\right|_{V_{h}^{e}(\widehat{\Omega})}$ encerrados por la curva $\bar{\gamma}$ serán, precisamente, $\bar{\lambda}_{1 h}, \cdots, \bar{\lambda}_{m h}{ }^{8}$. Luego, podremos definir los proyectores espectrales asociados con $\boldsymbol{\Lambda}_{*}$ (relativo a $\bar{\lambda}$ ) y con $\boldsymbol{\Lambda}_{* h}$ (relativo a $\bar{\lambda}_{1 h}, \cdots, \bar{\lambda}_{m h}$ ) por las expresiones

$$
\mathbf{E}_{* \bar{\lambda}}:=\frac{1}{2 \pi i} \int_{\bar{\gamma}} R_{z}\left(\boldsymbol{\Lambda}_{*}\right) d z \quad \text { y } \quad \mathbf{E}_{* \bar{\lambda}_{h}}:=\frac{1}{2 \pi i} \int_{\bar{\gamma}} R_{z}\left(\boldsymbol{\Lambda}_{* h}\right) d z,
$$

respectivamente. Es inmediato que

$$
\begin{aligned}
& \text { - } a\left(\left.\mathbf{E}_{\lambda} x\right|_{\Omega}, y\right)=a\left(x,\left.\mathbf{E}_{* \bar{\lambda}} y\right|_{\Omega}\right) \quad \forall x, y \in V(\Omega), \\
& \text { - } a_{h}\left(\left.\mathbf{E}_{\lambda_{h}} x\right|_{\Omega_{h}}, y\right)=a_{h}\left(x,\left.\mathbf{E}_{* \bar{\lambda}_{h}} y\right|_{\Omega_{h}}\right) \quad \forall x, y \in V_{h}\left(\Omega_{h}\right) .
\end{aligned}
$$

Asumiremos que las siguientes propiedades se satisfacen:

$\mathbf{P} 1_{*}:$ para $x \in \mathbf{E}_{* \bar{\lambda}}(W(\widehat{\Omega}))$, existe $x_{h} \in V_{h}^{e}(\widehat{\Omega})$ tal que $\left\|x-x_{h}\right\|_{h} \leq \eta_{1 * h}\|x\|_{h}$, con $\eta_{1 * h} \rightarrow 0$ para $h \rightarrow 0$,

$$
\mathbf{P 2} 2_{*}: \lim _{h \rightarrow 0}\left\|\left.\left(\mathbf{A}_{*}-\mathbf{A}_{* h}\right)\right|_{V_{h}^{e}(\widehat{\Omega})}\right\|_{h}=0 .
$$

Consideremos ahora el subespacio de $W(\widehat{\Omega})$ definido por

$$
W_{\bar{\lambda}}(\widehat{\Omega}):=\mathbf{E}_{* \bar{\lambda}}(W(\widehat{\Omega}))+V_{h}^{e}(\widehat{\Omega}) .
$$

Supondremos que la norma $\|\cdot\|_{*}$, introducida en la sección 3.3.3 para elementos de $W_{\lambda}(\widehat{\Omega})$, también está definida en $W_{\bar{\lambda}}(\widehat{\Omega})$ y se verfica

$$
\begin{aligned}
& \text { - }\|y\|_{h} \leq\|y\|_{*} \quad \forall y \in W_{\bar{\lambda}}(\widehat{\Omega}), \\
& \text { - }\|y\|_{*} \leq C(\bar{\lambda})\|y\|_{h} \quad \forall y \in \mathbf{E}_{* \bar{\lambda}}(W(\widehat{\Omega})) .
\end{aligned}
$$

En este contexto, asumiremos que la forma $a_{h}$ será uniformemente continua en $W_{\lambda}(\widehat{\Omega}) \times W_{\bar{\lambda}}(\widehat{\Omega})$ con respecto a la norma $\|\cdot\|_{*}$; es decir,

$$
\left|a_{h}\left(\left.v\right|_{\Omega_{h}},\left.w\right|_{\Omega_{h}}\right)\right| \leq C\|v\|_{*, \Omega_{h}}\|w\|_{*, \Omega_{h}} \leq C\|v\|_{*}\|w\|_{*} \quad v \in W_{\lambda}(\widehat{\Omega}), w \in W_{\bar{\lambda}}(\widehat{\Omega}) .
$$

Asumiremos también que las formas $a$ y $a_{h}$ verifican las propiedades (3.15)-(3.17), enunciadas en la sección 3.3.3.

Sea $\Pi_{* h}: W_{\bar{\lambda}}(\widehat{\Omega}) \rightarrow W_{\bar{\lambda}}(\widehat{\Omega})$ la proyección, con rango $V_{h}^{e}(\widehat{\Omega})$, definida por

$$
\left\{\begin{array}{l}
\Pi_{* h} y=\mathbf{P}_{h} y_{h} \\
a_{h}\left(x_{h}, y-y_{h}\right)=0 \quad \forall x_{h} \in V_{h}\left(\Omega_{h}\right)
\end{array}\right.
$$

Procediendo como en la sección 3.3.3, se puede probar fácilmente que $\left\|\Pi_{* h}\right\|_{* h}$ está acotada uniformemente en $h$. Definamos entonces el operador

$$
\mathbf{B}_{* h}:=\mathbf{A}_{* h} \Pi_{* h}: W_{\bar{\lambda}}(\widehat{\Omega}) \rightarrow V_{h}^{e}(\widehat{\Omega}) \subset W_{\bar{\lambda}}(\widehat{\Omega}) .
$$

Procediendo, nuevamente, como en la sección 3.3.3, se puede asegurar que

\footnotetext{
${ }^{8} a_{h}$ es una forma sesquilineal definida en un espacio de dimensión finita con producto interno.
} 
- $\left\|\mathbf{B}_{* h}\right\|_{* h}$ está acotada uniformemente en $h$,

- $\mathbf{A}_{* h}$ y $\mathbf{B}_{* h}$ coinciden sobre $V_{h}^{e}(\widehat{\Omega})$,

- $\sigma\left(\mathbf{A}_{* h}\right)=\sigma\left(\mathbf{B}_{* h}\right)$,

- para cualquier autovalor asociado, distinto de cero, los correspondientes subespacios invariantes coinciden.

Notemos que el operador $\mathbf{B}_{* h}$ es el operador adjunto de $\mathbf{B}_{h}$ con respecto de la forma $a_{h}$. En efecto, para cualesquiera $x \in W_{\lambda}(\widehat{\Omega})$ e $y \in W_{\bar{\lambda}}(\widehat{\Omega})$, sean $x_{h}$ e $y_{h}$ las soluciones de los problemas (3.18) y (3.79), respectivamente; entonces

$$
a_{h}\left(\left.\mathbf{B}_{h} x\right|_{\Omega_{h}}, y\right)=a_{h}\left(\left.\mathbf{A}_{h} \Pi_{h} x\right|_{\Omega_{h}}, y\right)=a_{h}\left(\mathbf{T}_{h} x_{h}, y\right)=a_{h}\left(\mathbf{T}_{h} x_{h},\left.\Pi_{* h} y\right|_{\Omega_{h}}\right)=a_{h}\left(\mathbf{T}_{h} x_{h}, y_{h}\right)=b_{h}\left(x_{h}, y_{h}\right)
$$

y, de manera análoga,

$a_{h}\left(x,\left.\mathbf{B}_{* h} y\right|_{\Omega_{h}}\right)=a_{h}\left(x,\left.\mathbf{A}_{* h} \Pi_{* h} y\right|_{\Omega_{h}}\right)=a_{h}\left(x, \mathbf{T}_{* h} y_{h}\right)=a_{h}\left(\left.\Pi_{h} x\right|_{\Omega_{h}}, \mathbf{T}_{* h} y_{h}\right)=a_{h}\left(x_{h}, \mathbf{T}_{* h} y_{h}\right)=b_{h}\left(x_{h}, y_{h}\right)$.

A partir de este resultado, es inmediato que $\left(\bar{z}-\mathbf{B}_{* h}\right)^{-1}$ es el adjunto de $\left(z-\mathbf{B}_{h}\right)^{-1}$ con respecto a la forma $a_{h}$. Luego, de acuerdo con el Lemma 3.19, existitán constantes $C$ y $h_{0}$ tales que

$$
\left\|\left(\bar{z}-\mathbf{B}_{* h}\right)^{-1}\right\|_{* h} \leq C \quad \forall h \leq h_{0} .
$$

Sea, entonces, $\mathbf{F}_{* \bar{\lambda}_{h}}: W_{\bar{\lambda}}(\widehat{\Omega}) \rightarrow W_{\bar{\lambda}}(\widehat{\Omega})$ el proyector espectral asociado con $\mathbf{B}_{* h}$ relativo a $\bar{\lambda}_{1 h}, \cdots, \bar{\lambda}_{m h}$; definido por

$$
\mathbf{F}_{* \bar{\lambda}_{h}}:=\frac{1}{2 \pi i} \int_{\bar{\gamma}} R_{z}\left(\mathbf{B}_{* h}\right) d z .
$$

Observemos que

- como $\mathbf{B}_{* h}$ es el adjunto de $\mathbf{B}_{h}$ con respecto a la forma $a_{h}$, entonces $\mathbf{F}_{* \bar{\lambda}_{h}}$ será el adjunto de $\mathbf{F}_{\lambda_{h}}$ con respecta a $a_{h}$,

- como $R_{z}\left(\mathbf{B}_{* h}\right)$ está acotado para todo $z \in \bar{\gamma}$ en la norma $\|\cdot\|_{* h}$, uniformemente en $h, \mathbf{F}_{* \bar{\lambda}_{h}}$ resultará acotado uniformemente en $h$ en la misma norma,

- $\mathbf{F}_{* \bar{\lambda}_{h}}\left(W_{\bar{\lambda}}\right)$ coincide con el espacio propio $\mathbf{E}_{* \bar{\lambda}_{h}}\left(V_{h}^{e}(\widehat{\Omega})\right)$ del operador $\mathbf{A}_{* h}$ asociado con los autovalores $\bar{\lambda}_{1 h}, \cdots, \bar{\lambda}_{m h}$.

Asumiremos que las siguientes propiedades se satisfacen:

$\mathbf{P 3}_{*}:$ para $x \in \mathbf{E}_{* \bar{\lambda}}(W(\widehat{\Omega}))$, existe $x_{h} \in V_{h}^{e}(\widehat{\Omega})$ tal que $\left\|x-x_{h}\right\|_{*} \leq \eta_{2 * h}\|x\|_{h}$, con $\eta_{2 * h} \rightarrow 0$ para $h \rightarrow 0$,

$\mathbf{P 4}_{*}$ : para todo $x \in \mathbf{E}_{* \bar{\lambda}}(W(\widehat{\Omega})),\left\|\left(\mathbf{A}_{*}-\mathbf{A}_{* h}\right) x\right\|_{*} \leq \eta_{3 * h}\|x\|_{h}$, con $\eta_{3 * h} \rightarrow 0$ para $h \rightarrow 0$.

$\mathbf{P 5}_{*}$ : $\quad$ para todo $x \in \mathbf{E}_{* \bar{\lambda}}(W(\widehat{\Omega})),\|x\|_{h, \Omega \backslash \Omega_{h}}+\|x\|_{h, \Omega_{h} \backslash \Omega}+\|x\|_{*, \Omega_{h} \backslash \Omega} \leq \eta_{4 * h}\|x\|_{h}$, con $\eta_{4 * h} \rightarrow 0$ para $h \rightarrow 0$.

Entonces las siguientes cantidades tenderán a cero a medida que $h$ tienda a cero.

$$
\begin{aligned}
\gamma_{1 * h} & :=\sup _{\substack{y \in \mathbf{E}_{* \bar{\lambda}}(W(\widehat{\Omega})) \\
\|y\|_{h}=1}} \inf _{\substack{x_{h} \in V_{h}^{e}(\widehat{\Omega}) \\
\gamma_{2 * h}}}:=\sup _{y \in \mathbf{E}_{* \bar{\lambda}}(W(\widehat{\Omega}))}\left(\|y\|_{h, \Omega \backslash \Omega_{h}}+\|y\|_{*, \Omega_{h} \backslash \Omega}\right), \\
\gamma_{3 * h} & :=\left\|\left.\left(\mathbf{A}_{*}-\mathbf{A}_{* h}\right)\right|_{\mathbf{E}_{* \bar{\lambda}}(W(\widehat{\Omega}))}\right\|_{h *}, \\
\varrho_{* h} & :=\gamma_{1 * h}+\gamma_{2 * h}+\gamma_{3 * h} .
\end{aligned}
$$

A partir de las definiciones anteriores y utilizando las propiedades enunciadas, podemos seguir los mismos argumentos que los usados en las pruebas de los Lemas 3.21-3.25 (con modificaciones obvias) para obtener los siguientes resultados. 
Lema 3.38 Existen constantes $C_{1}, C_{2}, C_{3}, C_{4}$, estrictamente positivas, tales que

$$
\begin{aligned}
& \left.\left\|\left.\left(\mathbf{I}-\Pi_{* h}\right)\right|_{\mathbf{E}_{* \bar{\lambda}}(W(\widehat{\Omega}))}\right\|\right|_{h *} \leq C_{1} \gamma_{1 * h}, \\
& \left\|\left.\left(\mathbf{E}_{* \bar{\lambda}}-\mathbf{F}_{* \bar{\lambda}_{h}}\right)\right|_{\mathbf{E}_{* \bar{\lambda}}(W(\widehat{\Omega}))}\right\|_{h *} \leq C_{2}\left\|\left.\left(\mathbf{A}_{*}-\mathbf{B}_{* h}\right)\right|_{\mathbf{E}_{* \bar{\lambda}}(W(\widehat{\Omega}))}\right\|_{h *}, \\
& \left\|\left.\left(\mathbf{E}_{* \bar{\lambda}}-\mathbf{F}_{* \bar{\lambda}_{h}}\right)\right|_{\mathbf{E}_{* \bar{\lambda}}(W(\widehat{\Omega}))}\right\|_{h} \leq C_{3}\left\|\left.\left(\mathbf{A}_{*}-\mathbf{B}_{* h}\right)\right|_{\mathbf{E}_{* \bar{\lambda}}(W(\widehat{\Omega}))}\right\|_{h *}, \\
& \left\|\left.\left(\mathbf{A}_{*}-\mathbf{B}_{* h}\right)\right|_{\mathbf{E}_{* \bar{\lambda}}(W(\widehat{\Omega}))}\right\|_{h *} \leq C_{4}\left(\gamma_{1 * h}+\gamma_{3 * h}\right) .
\end{aligned}
$$

La prueba del siguiente resultado es idéntica a la prueba del Teorema 3.26.

Teorema 3.39 Existe una constante $C$, estrictamente positiva, tal que

$$
\widehat{\delta}\left(\mathbf{E}_{\lambda}(W(\widehat{\Omega})), \mathbf{F}_{\lambda_{h}}\left(W_{\lambda}(\widehat{\Omega})\right)\right) \leq C \varrho_{h} .
$$

Consideremos ahora los operadores $\widehat{\mathbf{A}}$ y $\widehat{\mathbf{B}}_{h}$ introducidos en la sección 3.3.4. Con modificaciones obvias, la prueba del siguiente resultado usa los mismos argumentos que los utilizados para probar el Teorema 3.28 .

Teorema 3.40 Existen constantes $C$ y $h_{0}$, estrictamente positivas, tales que, para $h<h_{0}$,

$$
\left\|\widehat{\mathbf{A}}-\widehat{\mathbf{B}}_{h}\right\|_{h} \leq C\left(\varrho_{h} \varrho_{* h}+M_{h}+M_{* h}+N_{h}\right),
$$

siendo $M_{h}, M_{* h}$ y $N_{h}$ términos de consistencia definidos por:

$$
\begin{gathered}
M_{h}=\sup _{\substack{x \in \mathbf{E}_{\lambda}(W(\widehat{\Omega})) \\
\|x\|_{h}=1}} \sup _{\substack{\in \in \mathbf{E}_{* \bar{\lambda}}(W(\widehat{\Omega})) \\
\|y\|_{h}=1}}\left|a_{h}\left(\mathbf{A} x, \Pi_{* h} y-y\right)-b_{h}\left(x, \Pi_{* h} y-y\right)\right|, \\
M_{* h}=\sup _{\substack{x \in \mathbf{E}_{\lambda}(W(\widehat{\Omega})) \\
\|x\|_{h}=1}} \sup _{\substack{y \in \mathbf{E}_{* \bar{\lambda}}(W(\widehat{\Omega})) \\
\|y\|_{h}=1}}\left|a_{h}\left(\Pi_{h} x-x, \mathbf{A}_{*} y\right)-b_{h}\left(\Pi_{h} x-x, y\right)\right|, \\
N_{h}=\sup _{\substack{x \in \mathbf{E}_{\lambda}(W(\widehat{\Omega})) \\
\|x\|_{h}=1}} \sup _{\substack{y \in \mathbf{E}_{*}(W(\widehat{\Omega})) \\
\|y\|_{h}=1}}\left|a_{h}(\mathbf{A} x, y)-b_{h}(x, y)\right| .
\end{gathered}
$$

Los argumentos utilizados en la prueba del Teorema 3.29, nos conducen ahora al siguiente resultado.

Teorema 3.41 Sea $l$ el ascendente de $\lambda-\mathbf{T} ; 1 \leq l \leq m$. Sea $\widehat{\lambda}$ la media aritmética del conjunto $\lambda_{1 h}, \cdots, \lambda_{m h}$, autovalores de $\mathbf{T}_{h}$ que convergen a $\lambda$ cuando $h \rightarrow 0$. Exiten constantes, $h_{0}$ y $C$, estrictamente positivas, tales que, para $h<h_{0}$,

i)

$$
\left|\lambda-\widehat{\lambda}_{h}\right| \leq C\left(\varrho_{h} \varrho_{* h}+M_{h}+M_{* h}+N_{h}\right),
$$

ii)

$$
\left|\frac{1}{\lambda}-\frac{1}{m} \sum_{i=1}^{m} \frac{1}{\lambda_{i h}}\right| \leq C\left(\varrho_{h} \varrho_{* h}+M_{h}+M_{* h}+N_{h}\right)
$$

$$
\operatorname{máx}_{i=1, \cdots, m}\left|\lambda-\lambda_{i h}\right| \leq C\left(\varrho_{h} \varrho_{* h}+M_{h}+M_{* h}+N_{h}\right)^{1 / l} .
$$

Observación 3.42 Supongamos que el problema de valores propios estuviera planteado en un dominio poligonal (entonces serían $\gamma_{2 h}=0, \gamma_{2 * h}=0$ ). Si

- el método de aproximación fuera conforme (implicaría $\mathbf{A}_{h}=\Pi_{h} \mathbf{A}, \mathbf{A}_{* h}=\Pi_{* h} \mathbf{A}_{*}$ ), se obtendrían las estimaciones de Decloux-Nassif-Rappaz [95],

- el método de aproximación fuera no-conforme y su aplicación no modificara las formas bilineales, se obtendría el resultado de Rodríguez-Solomin [150]. 


\section{Aplicaciones}

\subsection{Dominios poligonales y espacios discretos no-conformes}

Supongamos que queremos estudiar las aproximaciones no-conformes de un problema espectral planteado sobre un dominio poligonal.

En esta situación, evidentemente, la aproximación poligonal del dominio coincidirá con el dominio mismo, i.e., $\Omega_{h}=\Omega$. Luego, tendremos que

- los operadores de extensión $\mathbf{P}$ y $\mathbf{P}_{h}$ se reducen a la identidad sobre $V(\Omega)$ y $V_{h}(\Omega)$, respectivamente,

- el espacio suma se simplifica a $W(\Omega)=\left(V+V_{h}\right)(\Omega)$,

- las condiciones sobre la norma discreta y los espacios de funciones se reducen a

$$
\begin{aligned}
\|v\|_{h} & \simeq\|v\|_{V(\Omega)} \quad \forall v \in V(\Omega) \\
\left(W(\Omega),\|\cdot\|_{h}\right) & \hookrightarrow\left(X(\Omega),|\cdot|_{X(\Omega)}\right) \quad \text { uniformemente en } h
\end{aligned}
$$

- las condiciones sobre las formas bilineales continuas y discretas se simplifican a

$$
\begin{aligned}
& a_{h}(u, v)=a(u, v) \quad \forall u, v \in V(\Omega), \\
& b_{h}(u, v)=b(u, v) \quad \forall u, v \in V(\Omega) .
\end{aligned}
$$

Para demostrar que el método de aproximación provee soluciones que convergen correctamente a las soluciones verdaderas, es suficiente que se satisfagan las propiedades P1 y P2. En este contexto, estas propiedades se leen de la siguiente manera

- P1: para $x \in \mathbf{E}_{\lambda}(V(\Omega))$, existe $x_{h} \in V_{h}(\Omega)$ tal que $\left\|x-x_{h}\right\|_{h} \leq \eta_{1 h}\|x\|_{h}$, con $\eta_{1 h} \rightarrow 0$ para $h \rightarrow 0$,

- P2: $\lim _{h \rightarrow 0}\left\|\left.\left(\mathbf{T}-\mathbf{T}_{h}\right)\right|_{V_{h}(\Omega)}\right\|_{h}=0$.

Las estimaciones del error en las aproximaciones se obtienen probando las propiedades P3 y P4 (la propiedad P5 se satisface trivialmente), que escribiremos

- P3 para $x \in \mathbf{E}_{\lambda}(V(\Omega))$, existe $x_{h} \in V_{h}(\Omega)$ tal que $\left\|x-x_{h}\right\|_{*} \leq \eta_{2 h}\|x\|_{h}$, con $\eta_{2 h} \rightarrow 0$ para $h \rightarrow 0$,

- P4: para todo $x \in \mathbf{E}_{\lambda}(W(\Omega)),\left\|\left(\mathbf{T}-\mathbf{T}_{h}\right) x\right\|_{*} \leq \eta_{3 h}\|x\|_{h}$, con $\eta_{3 h} \rightarrow 0$ para $h \rightarrow 0$, 
y estimando las cantidades $\varrho_{h}, M_{h}$ y $N_{h}$, las cuales se simplifican a

$$
\begin{aligned}
\varrho_{h} & =\left\|\left.\left(\mathbf{T}-\mathbf{T}_{h}\right)\right|_{\mathbf{E}_{\lambda}(W(\Omega))}\right\|_{h *}+\sup _{y \in \mathbf{E}_{\lambda}(W(\Omega))} \inf _{\substack{\|y\|_{h}=1 \\
V_{h} \in V_{h}(\Omega)}}\left\|y-x_{h}\right\|_{*}, \\
M_{h} & =\sup _{\substack{x \in \mathbf{E}_{\lambda}(W(\Omega)) \\
\|x\|_{h}=1}} \sup _{\substack{y \in \mathbf{E}_{\lambda}(W(\Omega)) \\
\|y\|_{h}=1}}\left|a_{h}\left(\mathbf{A} x, \Pi_{h} y\right)-b_{h}\left(x, \Pi_{h} y\right)\right|, \\
N_{h} & =0 .
\end{aligned}
$$

\subsubsection{El problema de autovalores de Steklov}

El problema de autovalores de Steklov, en el cual el autovalor aparece en la condición de borde, surge en numerosas aplicaciones. Mencionaremos aquí algunos ejemplos: estudio de las ondas de superficie [32], determinación de los modos de vibración de una estructura en contacto con un fluido incompresible [39], análisis de la estabilidad de osciladores mecánicos inmersos en medios viscosos [79] y estudio de la dinámica de líquidos contenidos en recipientes con movimiento [62], conocido como the sloshing problem.

Varios esquemas numéricos conformes, basados en el método de los elementos finitos o en el método de los elementos de borde, han sido utilizados para aproximar este problema. Entre los muchos y muy interesantes trabajos publicados en las últimas décadas, citaremos aquí a Bramble-Osborn [48], AndreevTodorov [10], Li-Yang [134] y Tang-Guan-Han [163].

Los estudios sobre aproximaciones no-conformes, en cambio, comenzaron a publicarse recien en el año 2009. Los primeros trabajos (aparecieron simultáneamente) se deben a Yang-Li-Li [176] y Alonso-Dello Russo [4].

\section{Planteo del problema y descripción del espectro continuo}

Asumiremos que $\Omega \subset \mathbb{R}^{2}$ es un dominio simplemente conexo con borde poligonal $\Gamma:=\partial \Omega$.

Denotaremos por $(\cdot, \cdot)$ al producto escalar en $L^{2}(\Omega)$ y por $\|\cdot\|_{0}$ a la norma en $L^{2}(\Omega)$. Usaremos la notación standard para los espacios de Sobolev $H^{s}(\Omega)$ de orden $s \geq 0$, con las normas $\|\cdot\|_{s}$ y seminormas $|\cdot|_{s}$ usuales.

Consideremos el siguiente problema de autovalores:

Encontrar $\mu \in \mathbb{R}, u \neq 0$ tal que

$$
\left\{\begin{aligned}
-\operatorname{div}(\alpha \nabla u)+\beta u & =0 \quad \text { en } \Omega, \\
\alpha \nabla u \cdot \mathbf{n} & =\mu u \quad \text { sobre } \Gamma,
\end{aligned}\right.
$$

donde $\mathbf{n}$ indica el vector unitario normal a $\Gamma$ orientado positivamente; i.e., hacia el exterior de $\Omega$. Asumiremos que los coeficientes $\alpha=\alpha(x)$ y $\beta=\beta(x)$ se encuentran acotados por constantes positivas y que, en particular, $\alpha \in C^{1}(\bar{\Omega})$.

Multiplicando por $v \in H^{1}(\Omega)$ a las ecuaciones en (4.5) e integrando por partes, obtenemos la siguiente formulación variacional del problema:

Encontrar $\mu \in \mathbb{R}, u \in H^{1}(\Omega), u \neq 0$, tal que

$$
\int_{\Omega} \alpha \nabla u \cdot \nabla v+\int_{\Omega} \beta u v=\mu \int_{\Gamma} u v \quad \forall v \in H^{1}(\Omega) .
$$

Sean $a^{*}$ y $b^{*}$ las formas bilineales (simétricas) definidas por

$$
a^{*}(u, v):=\int_{\Omega} \alpha \nabla u \cdot \nabla v+\int_{\Omega} \beta u v \quad \forall u, v \in H^{1}(\Omega),
$$




$$
b^{*}(u, v):=\int_{\Gamma} u v \quad \forall u, v \in H^{1}(\Omega) .
$$

Dado que $\alpha$ y $\beta$ se asumen acotadas positivamente en $\bar{\Omega}$, tendremos que $a^{*}$ es continua y coerciva sobre $H^{1}(\Omega)$. Luego, a partir de la teoría clásica para problemas elípticos $[9,29]$, podemos inferir que el problema (4.6) tiene un conjunto infinito numerable de autovalores, todos positivos y de multiplicidad finita, sin puntos de acumulación finitos. Escribiremos

$$
0<\mu_{1} \leq \mu_{2} \leq \cdots \leq \mu_{j} \cdots \rightarrow+\infty
$$

donde cada autovalor aparece tantas veces como lo indique su multiplicidad. Además, las autofunciones correspondientes $\left\{u_{j}\right\}_{j=1}^{\infty}$ son $L^{2}(\Gamma)$-ortogonales y proveen una base para $L^{2}(\bar{\Omega})$.

Consideremos los siguientes espacios de funciones:

$$
\begin{gathered}
X:=L^{2}(\Omega) \times L^{2}(\Gamma), \\
V:=\left\{(u, \xi) \in H^{1}(\Omega) \times H^{1 / 2}(\Gamma): \xi=\left.u\right|_{\Gamma}\right\},
\end{gathered}
$$

equipados con las siguientes normas

$$
\begin{gathered}
|(u, \xi)|^{2}:=\|u\|_{0}^{2}+\|\xi\|_{0, \Gamma}^{2}, \\
\|(u, \xi)\|^{2}:=\|u\|_{1}^{2}+\|\xi\|_{0, \Gamma}^{2} .
\end{gathered}
$$

Definimos las formas bilineales (simétricas) $a$ y $b$ por

$$
\begin{gathered}
a((u, \xi),(v, \eta)):=a^{*}(u, v)+b^{*}(\xi, \eta) \quad \forall(u, \xi),(v, \eta) \in V, \\
b((u, \xi),(v, \eta)):=b^{*}(\xi, \eta) \quad \forall(u, \xi),(v, \eta) \in X .
\end{gathered}
$$

Es claro que $a$ es continua y coerciva sobre $V$ y que $b$ es continua sobre $X$.

Consideremos entonces el siguiente problema espectral:

Encontrar $\mu \in \mathbb{R},(u, \xi) \in V, u \neq 0$, tal que

$$
a((u, \xi),(v, \eta))=(1+\mu) b((u, \xi),(v, \eta)) \quad \forall(v, \eta) \in V .
$$

Notemos que los problemas variacionales (4.6) y (4.7) son equivalentes al problema (4.5). En efecto, cualquier solución de (4.5) satisface a las ecuaciones (4.6) y (4.7). Recíprocamente, testeando estas ecuaciones con funciones adecuadas y suficientemente suaves, es fácil probar que cualquier solución de (4.6) o (4.7) es también solución de (4.5).

Definimos ahora el siguiente operador lineal:

$$
\begin{aligned}
\mathbf{T}: X & \rightarrow V \hookrightarrow X \\
(f, \tau) & \mapsto(u, \xi),
\end{aligned}
$$

siendo $(u, \xi)$ la solución del siguiente problema variacional

$$
a((u, \xi),(y, \zeta))=b((f, \tau),(y, \zeta)) \quad \forall(y, \zeta) \in V .
$$

Aplicando el Teorema de Lax-Milgram, tendremos

$$
\|(u, \xi)\| \leq C|(f, \tau)| .
$$

Luego, $\mathbf{T}$ es un operador acotado. Además, como $a$ y $b$ son simétricas, $\mathbf{T}$ es autoadjunto con respecto a la forma bilineal $a$. Más aún, $(\mu,(u, \xi))$ es una solución del problema $(4.7)$ si, y solo si, $\left(\frac{1}{1+\mu},(u, \xi)\right)$ es un par propio de $\mathbf{T}$.

El siguiente lema establece estimaciones a priori para las soluciones del problema (4.8) dependiendo de la regularidad de los datos. 
Lema 4.1 Sea $(u, \xi)$ una solución del problema (4.8). Existen constantes, $r \in(1 / 2,1]$ y $C>0$, tales que

- si $\tau \in L^{2}(\Gamma), u \in H^{1+r / 2}(\Omega), y$

$$
\|u\|_{1+r / 2} \leq C\|\tau\|_{0, \Gamma},
$$

- si $\tau \in H^{\epsilon}(\Gamma)$, con $\epsilon \in(0, r-1 / 2), u \in H^{3 / 2+\epsilon}(\Omega)$, y

$$
\|u\|_{3 / 2+\epsilon} \leq C\|\tau\|_{\epsilon, \Gamma},
$$

- si $\tau \in H^{1 / 2}(\Gamma), u \in H^{1+r}(\Omega), y$

$$
\|u\|_{1+r} \leq C\|\tau\|_{1 / 2, \Gamma} .
$$

Prueba. Se obtiene a partir de los resultados de regularidad clásicos para el problema de Neumann [88].

En la proposición anterior, $r=1$ si $\Omega$ es un dominio convexo. En caso contrario, $r<\frac{\pi}{\theta}$, siendo $\theta$ el mayor ángulo interior en $\Omega$ [111]. Como consecuencia de estos resultados, las autofunciones $\left(u_{j}, \xi_{j}\right)$ de $\mathbf{T}$ pertenecen a $H^{1+r}(\Omega) \times H^{1 / 2+r}(\Gamma)$ y satisfacen

$$
\left\|u_{j}\right\|_{1+r} \leq C\left\|\xi_{j}\right\|_{1 / 2, \Gamma} \leq C\left\|\left(u_{j}, \xi_{j}\right)\right\| .
$$

\section{Discretización del problema}

Sea $\left\{\mathcal{T}_{h}\right\}$ una familia de triangulaciones de $\Omega$ que satisface las siguientes condiciones:

- cualesquiera dos triángulos en $\mathcal{T}_{h}$ comparten a lo sumo un vértice o un lado,

- el mínimo ángulo interior de todos los triángulos en $\mathcal{T}_{h}$ está acotado por abajo por una constante positiva que no depende de $h$.

El índice $h$ denota, como es usual, al máximo diámetro de la grilla inducida por la partición $\mathcal{T}_{h}$, más precisamente, $h:=\operatorname{máx}_{T \in \mathcal{T}_{h}} h_{T}$, siendo $h_{T}$ el diámetro de $T$.

Sea $\mathcal{E}_{h}$ el conjunto de todos los lados de todos los triángulos en $\mathcal{T}_{h}$. Dividiremos a este conjunto como sigue: $\mathcal{E}_{h}=\mathcal{E}_{h}^{I} \cup \mathcal{E}_{h}^{\Gamma}$, siendo $\mathcal{E}_{h}^{I}:=\left\{\ell \in \mathcal{E}_{h}: \ell \not \subset \Gamma\right\}$, el conjunto de los lados internos, y $\mathcal{E}_{h}^{\Gamma}:=\left\{\ell \in \mathcal{E}_{h}: \ell \subset \Gamma\right\}$, el conjunto de los lados en el borde. Sea $\mathcal{N}_{h}$ el conjunto de todos los vértices de todos los elementos en $\mathcal{T}_{h}$.

Sobre cada triangulación $\mathcal{T}_{h}$, el espacio de Crouzeix-Raviart [81] está definido por

$$
C R_{h}(\Omega):=\left\{v_{h} \in L^{2}(\Omega):\left.v_{h}\right|_{T} \in \mathcal{P}_{1}(T) \forall T \in \mathcal{T}_{h} \text { y } \int_{\ell}\left[\left[v_{h}\right]\right]=0 \forall \ell \in \mathcal{E}_{h}^{I}\right\} .
$$

En la expresión anterior, $\left[\left[v_{h}\right]\right]$ denota el salto de $v_{h}$ a través del lado interno $\ell$.

A continuación, trabajaremos con los siguientes espacios no-conformes

$$
\begin{aligned}
\mathcal{L}_{h}(\Gamma) & :=\left\{\xi_{h} \in L^{2}(\Gamma):\left.\xi_{h}\right|_{\ell} \in \mathcal{P}_{1}(\ell), \forall \ell \subset \Gamma\right\}, \\
V_{h} & :=\left\{\left(v_{h}, \eta_{h}\right) \in C R_{h}(\Omega) \times \mathcal{L}_{h}(\Gamma):\left.\eta_{h}\right|_{\ell}=\left.v_{h}\right|_{\ell}, \forall \ell \subset \Gamma\right\}, \\
W(h) & :=V+V_{h} .
\end{aligned}
$$

Sean $a_{h}$ y $b_{h}$ las formas bilineales (simétricas) definidas por

$$
\begin{gathered}
a_{h}((u, \xi),(v, \eta)):=\sum_{T \in \mathcal{T}_{h}} \int_{T} \alpha \nabla u \cdot \nabla v+\int_{\Omega} \beta u v+\int_{\Gamma} \xi \eta \quad \forall(u, \xi),(v, \eta) \in W(h), \\
b_{h}((u, \xi),(v, \eta)):=b((u, \xi),(v, \eta)) \quad \forall(u, \xi),(v, \eta) \in X .
\end{gathered}
$$


Equipamos al espacio $W(h)$ con la siguiente norma:

$$
\left\|\left(v_{h}, \eta_{h}\right)\right\|_{h}^{2}=\left\|v_{h}\right\|_{0}^{2}+\sum_{T \in \mathcal{T}_{h}}\left|v_{h}\right|_{1, T}^{2}+\left\|\eta_{h}\right\|_{0, \Gamma}^{2}
$$

Observemos que se verifican las propiedades (4.1)-(4.4) y que la forma discreta $a_{h}$ resulta uniformemente continua y coerciva sobre $W(h)$.

Consideremos entonces la siguiente aproximación del problema espectral (4.7):

Encontrar $\mu_{h} \in \mathbb{R},\left(u_{h}, \xi_{h}\right) \in V_{h},\left(u_{h}, \xi_{h}\right) \neq(0,0)$, tal que

$$
a_{h}\left(\left(u_{h}, \xi_{h}\right),\left(v_{h}, \eta_{h}\right)\right)=\left(1+\mu_{h}\right) b\left(\left(u_{h}, \xi_{h}\right),\left(v_{h}, \eta_{h}\right)\right) \quad \forall\left(v_{h}, \eta_{h}\right) \in V_{h} .
$$

Observemos que este problema se reduce a un problema de autovalores generalizado que involucra matrices simétricas. La matriz asociada con la forma bilineal $a_{h}$ es definida positiva. La matriz asociada con la forma bilineal $b_{h}$ es definida semi-positiva con rango $N(h)=\operatorname{card}\left(\mathcal{E}_{h}^{\Gamma}\right)$. Luego, el problema (4.13) tendrá un número finito de pares propios $\left(\mu_{j h},\left(u_{j h}, \xi_{j h}\right)\right), 1 \leq j \leq N(h)$, con autovalores positivos. Escribiremos

$$
0<\mu_{1 h} \leq \mu_{2 h} \leq \cdots \leq \mu_{N(h) h}
$$

donde cada autovalor aparece repetido tantas veces como lo indique su multiplicidad.

La contrapartida discreta del operador $\mathbf{T}$ estará definida por

$$
\begin{aligned}
\mathbf{T}_{h}: X & \rightarrow V_{h} \hookrightarrow X \\
(f, \tau) & \mapsto\left(u_{h}, \xi_{h}\right),
\end{aligned}
$$

siendo $\left(u_{h}, \xi_{h}\right)$ la solución del siguiente problema variacional:

$$
a_{h}\left(\left(u_{h}, \xi_{h}\right),\left(v_{h}, \eta_{h}\right)\right)=b\left((f, \tau),\left(v_{h}, \eta_{h}\right)\right) \quad \forall\left(v_{h}, \eta_{h}\right) \in V_{h} .
$$

Como en el caso continuo, puede probarse que $\mathbf{T}_{h}$ es un operador uniformemente acotado, autoadjunto con respecto a $a_{h}$ y que sus pares propios están directamente relacionados con las soluciones del problema (4.13), i.e., $\left(\mu_{h},\left(u_{h}, \xi_{h}\right)\right)$ es solución de (4.13) si, y solo si, $\left(u_{h}, \xi_{h}\right) \neq(0,0)$ y

$$
\mathbf{T}_{h}\left(u_{h}, \xi_{h}\right)=\frac{1}{1+\mu_{h}}\left(u_{h}, \xi_{h}\right) .
$$

\section{Aproximación espectral}

De acuerdo con la teoría desarrollada en la sección 3.3, los resultados sobre la convergencia de los autovalores y autovectores aproximados dependerán únicamente de la validez de las propiedades P1 y P2 sobre los espacios discretos de Crouzeix-Raviart.

Sea $\lambda=\frac{1}{1+\mu}$ un autovalor fijo de $\mathbf{T}$, con multiplicidad algebraica igual a $m$, y sea $\mathbf{E}_{\lambda}(W(h))$ el espacio propio de $\mathbf{T}$ correspondiente a $\lambda$.

Teorema 4.2 (P1) Existe una constante positiva $C$ tal que

$$
\begin{gathered}
\inf _{\left(v_{h}, \eta_{h}\right) \in V_{h}}\left\|(u, \xi)-\left(v_{h}, \eta_{h}\right)\right\|_{0, \Gamma} \leq C h^{r+1 / 2}\|u\|_{1+r} \quad \forall(u, \xi) \in \mathbf{E}_{\lambda}(W(h)), \\
\inf _{\left(v_{h}, \eta_{h}\right) \in V_{h}}\left\|(u, \xi)-\left(v_{h}, \eta_{h}\right)\right\|_{h} \leq C h^{r}\|u\|_{1+r} \quad \forall(u, \xi) \in \mathbf{E}_{\lambda}(W(h)) .
\end{gathered}
$$

Prueba. Como $u \in H^{1+r}(\Omega), u \in \mathcal{C}^{0}(\Omega)$. Luego, la interpolada lineal de Lagrange de $u$, $u^{I}$, estará bien definida (ver [75], por ejemplo). Más aún, $u^{I} \in V_{h} \cap H^{1}(\Omega)$. Entonces, eligiendo $v_{h}=u^{I}$, usando un teorema de trazas local ${ }^{1}$ y resultados de interpolación standard ${ }^{2}$, obtendremos

$$
\ell \subset \Gamma,\left\|\xi-\eta_{h}\right\|_{0, \ell}=\left\|\left.u\right|_{\ell}-\left.v_{h}\right|_{\ell}\right\|_{0, \ell} \leq C\left(h_{T}^{-1 / 2}\left\|u-v_{h}\right\|_{0, T}+h_{T}^{1 / 2}\left|u-v_{h}\right|_{1, T}\right) \leq C h_{T}^{r+1 / 2}\|u\|_{1+r, T}
$$

\footnotetext{
${ }^{1}$ De aquí en adelante, cuando escribamos un teorema de trazas local estaremos refiriéndonos a la estimación (A.4).

${ }^{2}$ De aquí en adelante, cuando escribamos resultados de interpolación standard estaremos refiriéndonos al Lema A.3.
} 


$$
\left\|u-v_{h}\right\|_{1, T} \leq C h_{T}^{r}\|u\|_{1+r, T} .
$$

Combinando las estimaciones (4.15) y (4.16) con la definición de la norma $\|\cdot\|_{h}$, tendremos

$$
\begin{aligned}
& \left\|(u, \xi)-\left(v_{h}, \eta_{h}\right)\right\|_{0, \Gamma} \leq C h^{r+1 / 2}\|u\|_{1+r}, \\
& \left\|(u, \xi)-\left(v_{h}, \eta_{h}\right)\right\|_{h} \leq C h^{r}\|u\|_{1+r} .
\end{aligned}
$$

Concluimos la prueba tomando el ínfimo con respecto a $\left(v_{h}, \eta_{h}\right) \in V_{h}$.

Teorema 4.3 (P2) Existe una constante positiva $C$ tal que

$$
\left\|\left(\mathbf{T}-\mathbf{T}_{h}\right)(f, \tau)\right\|_{h} \leq C h^{r / 2}\|\tau\|_{0, \Gamma} \quad \forall(f, \tau) \in V_{h} .
$$

Prueba. Para cualquier $\operatorname{par}(f, \tau) \in V_{h}$, sean $(u, \eta):=\mathbf{T}(f, \tau)$ y $\left(u_{h}, \eta_{h}\right):=\mathbf{T}_{h}(f, \tau)$. La aplicación del segundo Lema de Strang [75], nos conduce a

$$
\begin{aligned}
\left\|(u, \xi)-\left(u_{h}, \xi_{h}\right)\right\|_{h} & \leq C\left(\inf _{\left(v_{h}, \eta_{h}\right) \in V_{h}}\left\|(u, \xi)-\left(v_{h}, \eta_{h}\right)\right\|_{h}\right. \\
& \left.+\sup _{\left(w_{h}, \chi_{h}\right) \in V_{h}} \frac{a_{h}\left((u, \xi),\left(w_{h}, \chi_{h}\right)\right)-b\left((f, \tau),\left(w_{h}, \chi_{h}\right)\right)}{\left\|\left(w_{h}, \chi_{h}\right)\right\|_{h}}\right) .
\end{aligned}
$$

Para acotar el primer término de esta desigualdad, procedemos como en la prueba del Teorema 4.2. Usando la estimación (4.9), obtendremos

$$
\inf _{\left(v_{h}, \eta_{h}\right) \in V_{h}}\left\|(u, \xi)-\left(v_{h}, \eta_{h}\right)\right\|_{h} \leq C h^{r / 2}\|\tau\|_{0, \Gamma} .
$$

Pasamos ahora a acotar el segundo término en (4.17). Testeando (4.14) con funciones adecuadas y suficientemente suaves, puede probarse que $u$ satisface el siguiente problema diferencial:

$$
\left\{\begin{aligned}
-\operatorname{div}(\alpha \nabla u)+\beta u & =0 & & \text { en } \Omega, \\
\alpha \frac{\partial u}{\partial \mathbf{n}}+\xi & =\tau & & \text { sobre } \Gamma,
\end{aligned}\right.
$$

siendo $\xi=\left.u\right|_{\Gamma}$. Multiplicando (4.18) por $w_{h} \in C R_{h}(\Omega)$ e integrando por partes, tendremos

$$
a_{h}\left((u, \xi),\left(w_{h}, \chi_{h}\right)\right)-b\left((f, \tau),\left(w_{h}, \chi_{h}\right)\right)=\sum_{T \in \mathcal{T}_{h}} \int_{\partial T} \alpha \frac{\partial u}{\partial \mathbf{n}} w_{h}=\sum_{\ell \in \mathcal{E}_{h}^{I}} \int_{\ell} \alpha \frac{\partial u}{\partial \mathbf{n}}\left[\left[w_{h}\right]\right]
$$

donde $\chi_{h}=\left.w_{h}\right|_{\Gamma}$.

Observación 4.4 Por hipótesis, $f \in C R_{h}(\Omega)$. Luego $\tau=\left.f\right|_{\Gamma} \in H^{\epsilon}(\Gamma), 0<\epsilon<1 / 2$. A partir de la estimación (4.10), tendremos $u \in H^{3 / 2+\epsilon}(\Omega)$; resultado que da significado a las integrales $\int_{\ell} \alpha \frac{\partial u}{\partial \mathbf{n}} w_{h}$ para todo $\ell \in \mathcal{E}_{h}^{I}$.

Sea $P_{\ell}$ la proyección $L_{\ell}^{2}$ de $H^{\epsilon}(\ell)$ sobre las constantes. Para $\ell \in \mathcal{E}_{h}$, indicaremos por $T_{1}$ y $T_{2}$ a los triángulos en $\mathcal{T}_{h}$ tales que $T_{1} \cap T_{2}=\ell$. Dado que $\left[\left[w_{h}\right]\right]$ es una función lineal que se anula en el punto medio de $\ell$, tendremos

$$
\begin{aligned}
\left|\int_{\ell} \alpha \frac{\partial u}{\partial \mathbf{n}}\left[\left[w_{h}\right]\right]\right| & =\left|\int_{\ell}\left(\alpha \frac{\partial u}{\partial \mathbf{n}}-P_{\ell}\left(\alpha \frac{\partial u}{\partial \mathbf{n}}\right)\right)\left[\left[w_{h}\right]\right]\right| \\
& \leq \sum_{i=1,2}\left|\int_{\ell}\left(\alpha \frac{\partial u}{\partial \mathbf{n}}-P_{\ell}\left(\alpha \frac{\partial u}{\partial \mathbf{n}}\right)\right)\left(\left.w_{h}\right|_{T_{i}}\right)\right| \\
& \leq \sum_{i=1,2}\left|\int_{\ell}\left(\alpha \frac{\partial u}{\partial \mathbf{n}}-P_{\ell}\left(\alpha \frac{\partial u}{\partial \mathbf{n}}\right)\right)\left[\left(\left.w_{h}\right|_{T_{i}}\right)-P_{\ell}\left(\left.w_{h}\right|_{T_{i}}\right)\right]\right| .
\end{aligned}
$$


Sea $P_{T}$ la proyección $L_{T}^{2}$ de $H^{1 / 2+\epsilon}(T)$ sobre las constantes. Usando un teorema de trazas local y estimaciones del error standard para la proyección ${ }^{3} P_{T}$, podemos escribir

$$
\begin{aligned}
\left|\int_{\ell} \alpha \frac{\partial u}{\partial \mathbf{n}}\left[\left[w_{h}\right]\right]\right| & \leq \sum_{i=1,2}\left\|\alpha \nabla u \cdot \mathbf{n}-P_{T}(\alpha \nabla u \cdot \mathbf{n})\right\|_{0, \ell}\left\|\left(\left.w_{h}\right|_{T_{i}}\right)-P_{T}\left(\left.w_{h}\right|_{T_{i}}\right)\right\|_{0, \ell} \\
& \leq C \sum_{i=1,2}\left(h_{T_{i}}^{r / 2-1 / 2}\|\nabla u\|_{r / 2, T_{i}}+h_{T_{i}}^{\epsilon}\|\nabla u\|_{1 / 2+\epsilon, T_{i}}\right)\left(h_{T_{i}}^{1 / 2}\left\|w_{h}\right\|_{1, T_{i}}\right) .
\end{aligned}
$$

Sumando sobre todos los lados $\ell \in \mathcal{E}_{h}^{I}$, usando el Lema 4.1 y una desigualdad inversa global (ver A.6), obtendremos

$$
\begin{aligned}
\sum_{\ell \in \mathcal{E}_{h}^{I}}\left|\int_{\ell} \alpha \frac{\partial u}{\partial \mathbf{n}}\left[\left[w_{h}\right]\right]\right| & \left.\leq C\left(h^{r / 2}\|u\|_{1+r / 2}+h^{1 / 2+\epsilon}\|u\|_{3 / 2+\epsilon}\right) \|\left(w_{h}, \chi_{h}\right)\right) \|_{h} \\
& \left.\leq C h^{r / 2}\left(\|\tau\|_{0, \Gamma}+h^{1 / 2-r / 2+\epsilon}\|\tau\|_{\epsilon, \Gamma}\right) \|\left(w_{h}, \chi_{h}\right)\right) \|_{h} \\
& \left.\leq C h^{r / 2}\left(1+h^{1 / 2-r / 2+\epsilon} h_{\text {min }}^{-\epsilon}\right)\|\tau\|_{0, \Gamma} \|\left(w_{h}, \chi_{h}\right)\right) \|_{h}
\end{aligned}
$$

donde, obviamente, $h_{\min }:=\min _{T \in \mathcal{T}_{h}} h_{T}$.

Observación 4.5 Si la grilla fuera localmente fuertemente refinada, la relación $h^{1 / 2-r / 2+\epsilon} / h_{\text {min }}^{\epsilon}$ podría ser muy grande y, por lo tanto, la acotación anterior volverse inútil. Para evitar este problema, suele hacerse la suposición adicional $h_{\text {min }} \sim h$; es decir, solo considerar grillas globalmente cuasi-uniformes, pero entonces los resultados obtenidos no serían apropiados para aplicarlos en el análisis de procedimientos adaptativos.

Introduciremos una restricción geométrica sobre la triangulación $\mathcal{T}_{h}$; específicamente, asumiremos que existen constantes uniformes $\varrho>0$ y $\nu \in(0,1)$ tales que

$$
h \leq \varrho h_{\text {min }}^{\nu}
$$

Sea, ahora, $\sigma \in[0, r / 4)$. Entonces, eligiendo

$$
\epsilon=\frac{(1-r+2 \sigma)}{2} \frac{\nu}{(1-\nu)}<\frac{1}{2},
$$

podremos escribir

$$
\left.\sum_{\ell \in \mathcal{E}_{h}^{I}}\left|\int_{\ell} \alpha \frac{\partial u}{\partial \mathbf{n}}\left[\left[w_{h}\right]\right]\right| \leq C h^{r / 2-\sigma}\left(1+\varrho^{\epsilon}\right)\|\tau\|_{0, \Gamma} \|\left(w_{h}, \chi_{h}\right)\right) \|_{h}
$$

Finalmente, tomando el límite $\sigma \rightarrow 0$, obtendremos

$$
\left|a_{h}\left((u, \xi),\left(w_{h}, \chi_{h}\right)\right)-b\left((f, \tau),\left(w_{h}, \chi_{h}\right)\right)\right| \leq C h^{r / 2}\|\tau\|_{0, \Gamma}\left\|\left(w_{h}, \chi_{h}\right)\right\|_{h},
$$

expresión que nos permite concluir la prueba.

Observación 4.6 La hipótesis (4.20) es menos restrictiva que la hipótesis de cuasi-uniformidad global. La experiencia numérica muestra que tanto las mallas óptimas para problemas con soluciones singulares, originadas por los ángulos re-entrantes en el dominio, como las mallas generadas por la mayoría de los esquemas adaptativos verifican esta hipótesis.

A partir de estos resultados, podemos establecer que el método numérico propuesto provee una aproximación espectralmente correcta del problema (4.7), en el sentido siguiente:

\footnotetext{
${ }^{3}$ De aquí en adelante, cuando nos refiramos a estimaciones de error standard para proyecciones $L^{2}$ estaremos invocando al Lema A.7/Lema A.8.
} 
- no aparecen autovalores espurios en el espectro discreto,

- no aparecen autofunciones espurias y la dimensión de los espacios propios discretos es igual a la dimensión de los correspondientes espacios propios continuos.

Para establecer el orden (óptimo) con el que convergen las autofunciones probaremos que se verifican las propiedades P3 y P4. Observemos que, en este caso, podemos elegir $\|\cdot\|_{*}=\|\cdot\|_{h}$. Luego, la propiedad P3 se convierte en la propiedad P1, necesaria para establecer la convergencia del método.

Teorema 4.7 (P4) Existe una constante positiva $C$ tal que

$$
\left\|\left.\left(\mathbf{T}-\mathbf{T}_{h}\right)\right|_{\mathbf{E}_{\lambda}(W(h))}\right\|_{h} \leq C h^{r} .
$$

Prueba. Notemos que para $(x, \zeta) \in \mathbf{E}_{\lambda}(W(h)),(u, \xi)=\mathbf{T}(x, \zeta) \in H^{1+r}(\Omega) \times H^{1 / 2+r}(\Gamma)$. Luego, podemos proceder exactamente igual que en la prueba del Teorema 4.3, sustituyendo $(f, \tau)$ por $(x, \zeta)$.

En virtud de los Teoremas 4.2 y 4.7 , es claro que existe una constante $C$, estrictamente positiva, tal que

$$
\varrho_{h}:=\left\|\left.\left(\mathbf{T}-\mathbf{T}_{h}\right)\right|_{\mathbf{E}_{\lambda}(W(h))}\right\|_{h}+\sup _{\substack{(u, \zeta) \in \mathbf{E}_{\lambda}(W(h)) \\\|(u, \zeta)\|_{h}=1}} \inf _{\left(v_{h}, \eta_{h}\right) \in V_{h}}\left\|(u, \zeta)-\left(v_{h}, \eta_{h}\right)\right\|_{h} \leq C h^{r} .
$$

De acuerdo con el Teorema 3.26, tendremos

- para cada $\left(u_{h}, \xi_{h}\right) \in \mathbf{E}_{\lambda_{h}}\left(V_{h}\right), \delta\left(\left(u_{h}, \xi_{h}\right), \mathbf{E}_{\lambda}(W(h))\right)$ es de orden $h^{r}$,

- para cada $(u, \xi) \in \mathbf{E}_{\lambda}(W(h)), \delta\left((u, \xi), \mathbf{E}_{\lambda_{h}}\left(V_{h}\right)\right)$ es de orden $h^{r}$.

Para establecer el orden con el que convergen los autovalores necesitamos estimar el siguiente término de consistencia

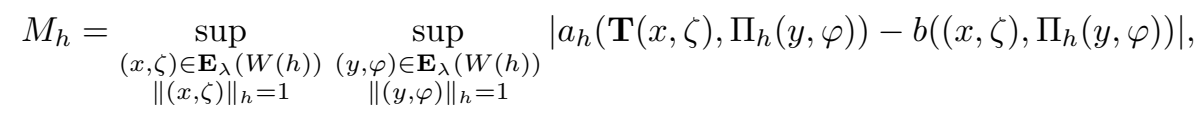

donde $\Pi_{h}: W(h) \rightarrow W(h)$ es el operador de proyección definido por

$$
a_{h}\left((x, \zeta)-\Pi_{h}(x, \zeta),\left(y_{h}, \varphi_{h}\right)\right)=0 \quad \forall\left(y_{h}, \varphi_{h}\right) \in V_{h}
$$

Lema 4.8 Existe una constante $C$, estrictamente positiva, tal que $M_{h} \leq C h^{2 r}$.

Prueba. Elijamos $(x, \zeta) \in \mathbf{E}_{\lambda}(W(h))$ tal que $\|(x, \zeta)\|_{h}=1$. A partir de $(4.11)$, sabemos que $(w, \chi):=$ $\mathbf{T}(x, \zeta) \in H^{1+r}(\Omega) \times H^{1 / 2+r}(\Gamma), r>1 / 2$, y que

$$
\|w\|_{1+r} \leq C\|(x, \zeta)\|_{h} \leq C .
$$

Procediendo como en la prueba del Teorema 4.3, podemos concluir que $(w, \chi)$ es solución del siguiente problema diferencial:

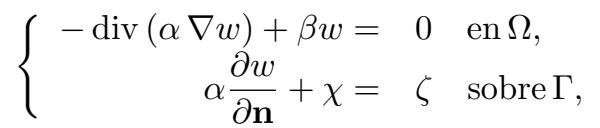

donde $\chi=\left.w\right|_{\Gamma}$. Consideremos ahora un elemento $(y, \varphi) \in \mathbf{E}_{\lambda}(W(h))$ con $\|(y, \varphi)\|_{h}=1$. Escribamos $\left(y_{h}, \varphi_{h}\right)=\Pi_{h}(y, \varphi)$. Multiplicando (4.21) por $y_{h}$ e integrando por partes, obtenemos fácilmente

$$
a_{h}\left((w, \chi), \Pi_{h}(y, \varphi)\right)-b_{h}\left((x, \zeta), \Pi_{h}(y, \varphi)\right)=\sum_{T \in \mathcal{T}_{h}} \int_{\partial T} \alpha \nabla w \cdot \mathbf{n} y_{h}
$$


Usaremos a continuación los mismos argumentos que los empleados en la prueba del Teorema 4.3. Teniendo en cuenta que $y_{h} \in C R_{h}(\Omega)$ y que $y \in \mathcal{C}^{0}(\Omega)$, llegamos a

$$
\begin{array}{r}
\left|a_{h}\left((w, \chi), \Pi_{h}(y, \varphi)\right)-b_{h}\left((x, \zeta), \Pi_{h}(y, \varphi)\right)\right| \leq \sum_{\ell \in \mathcal{E}_{h}^{I}}\left|\int_{\ell}\left(\alpha \frac{\partial w}{\partial \mathbf{n}}-P_{\ell}\left(\alpha \frac{\partial w}{\partial \mathbf{n}}\right)\right)\left[\left[y-y_{h}\right]\right]\right| \\
\leq C\left(\sum_{\ell \in \mathcal{E}_{h}^{I}} \sum_{i=1,2}\left\|\alpha \nabla w \cdot \mathbf{n}-P_{T}(\alpha \nabla w \cdot \mathbf{n})\right\|_{0, \ell}\left\|\left.\left(y-y_{h}\right)\right|_{T_{i}}-\left.P_{T}\left(y-y_{h}\right)\right|_{T_{i}}\right\|_{0, \ell}\right) .
\end{array}
$$

Usando un teorema de trazas local y estimaciones del error standard para la proyección $P_{T}$, tendremos

$$
\begin{gathered}
\left\|\alpha \nabla w \cdot \mathbf{n}-P_{T}(\alpha \nabla w \cdot \mathbf{n})\right\|_{0, \ell} \leq C h_{T}^{r-1 / 2}\|w\|_{1+r, T_{i}}, \\
\left\|\left(y_{h}-y\right)-P_{T}\left(y_{h}-y\right)\right\|_{0, \ell} \leq C h_{T}^{1 / 2}\left\|y_{h}-y\right\|_{1, T_{i}} .
\end{gathered}
$$

Luego, combinando las tres últimas estimaciones, sumando sobre todos los lados $\ell \in \mathcal{E}_{h}^{I}$, usando el Teorema 4.2 y la estimación (4.12), concluimos la prueba.

Estos resultados implican que la rapidez con la que convergen a $\lambda$ los $m$ autovalores del problema discreto $\lambda_{1 h}, \lambda_{2 h}, \cdots, \lambda_{m h}$ es exactamente el doble de la obtenida para las autofunciones. Más precisamente, de acuerdo con el Teorema 3.29, sabemos que existe una constante positiva $C$, tal que, si $h$ es suficientemente chico,

$$
\operatorname{máx}_{i=1, \cdots, m}\left|\lambda-\lambda_{i h}\right| \leq C h^{2 r} .
$$

\section{Estimaciones de orden superior}

El objetivo de esta sección es obtener estimaciones de orden superior para el error en la aproximación de los autovectores en la norma $\|\cdot\|_{0, \Gamma}$.

Comenzaremos definiendo el siguiente operador lineal

$$
\begin{aligned}
\mathcal{B}: L^{2}(\Gamma) & \rightarrow L^{2}(\Gamma) \\
\tau & \left.\mapsto u\right|_{\Gamma}
\end{aligned}
$$

donde $u$ es la solución (única) del problema variacional

$$
\int_{\Omega} \alpha \nabla u \cdot \nabla v+\int_{\Omega} \beta u v+\int_{\Gamma} u v=\int_{\Gamma} \tau v, \quad \forall v \in H^{1}(\Omega) .
$$

Así definido, $\mathcal{B}$ resulta un operador acotado. En efecto, a partir del Lema 4.1 y del Teorema de trazas [75], tendremos

$$
\|\boldsymbol{B} \tau\|_{0, \Gamma}=\|u\|_{0, \Gamma} \leq\|u\|_{(1+r) / 2, \Gamma} \leq C\|u\|_{1+r / 2, \Omega} \leq C\|\tau\|_{0, \Gamma} .
$$

La contrapartida discreta del operador $\mathcal{B}$ estará definida por

$$
\begin{aligned}
\mathcal{B}_{h}: L^{2}(\Gamma) & \rightarrow L^{2}(\Gamma) \\
\tau & \left.\mapsto u_{h}\right|_{\Gamma},
\end{aligned}
$$

siendo $u_{h} \in C R_{h}(\Omega)$ la solución (única) del problema variacional

$$
\int_{\Omega} \alpha \nabla u_{h} \cdot \nabla v_{h}+\int_{\Omega} \beta u_{h} v_{h}+\int_{\Gamma} u_{h} v_{h}=\int_{\Gamma} \tau v_{h}, \quad \forall v_{h} \in C R_{h}(\Omega) .
$$

Otra manera (equivalente) de definir a los operadores $\mathcal{B}$ y $\boldsymbol{B}_{h}$ es la siguiente: sea $\mathbf{C}$ el operador de filtrado definido por medio de

$$
\begin{aligned}
\mathbf{C}: X & \rightarrow L^{2}(\Gamma) \\
(u, \xi) & \mapsto \xi,
\end{aligned}
$$


entonces, tendremos

$$
\begin{gathered}
\mathcal{B} \tau=\mathbf{C} \circ \mathbf{T}(f, \tau) \quad \forall(f, \tau) \in X, \\
\mathcal{B}_{h} \tau=\mathbf{C} \circ \mathbf{T}_{h}(f, \tau) \quad \forall(f, \tau) \in X .
\end{gathered}
$$

La equivalencia entre ambas definiciones surge inmediatamente de la comparación de los problemas (4.8) y (4.22), utilizados para definir los operadores $\mathbf{T}$ y $\mathcal{B}$, respectivamente. De manera análoga, comparando los problemas (4.14) y (4.23), obtendremos la equivalencia de las definiciones para el caso discreto.

Lema 4.9 Existe una constante positiva $C$ tal que, para todo $(f, \tau) \in W(h)$,

$$
\left\|\left(\mathcal{B}-\mathcal{B}_{h}\right) \tau\right\|_{0, \Gamma} \leq C h^{r / 2}\|\tau\|_{0, \Gamma}
$$

Prueba. Es una consecuencia directa del Teorema 4.3 y las definiciones de los operadores $\mathcal{B}$ y $\mathcal{B}_{h}$.

El siguiente lema establece que el orden de convergencia es mayor cuando el término fuente pertenece al autoespacio asociado con $\lambda$.

Lema 4.10 Para $(f, \tau) \in \mathbf{E}_{\lambda}(W(h))$, vale la siguiente estimación

$$
\left\|\left(\mathcal{B}-\mathcal{B}_{h}\right) \tau\right\|_{0, \Gamma} \leq C h^{3 r / 2}\|\tau\|_{0, \Gamma} .
$$

Prueba. Para $(f, \tau) \in \mathbf{E}_{\lambda}(W(h))$, sean $(u, \xi)=\mathbf{T}(f, \tau)$ y $\left(u_{h}, \xi_{h}\right)=\mathbf{T}_{h}(f, \tau)$. Sean $e_{h}=u-u_{h}$ y $u^{I}$ la interpolada de Lagrange (lineal a trozos) de $u$. Es inmediato que

$$
\left\|e_{h}\right\|_{0, \Gamma} \leq\left\|u-u^{I}\right\|_{0, \Gamma}+\left\|u^{I}-u_{h}\right\|_{0, \Gamma} .
$$

Sea $\eta_{h}:=u^{I}-u_{h} \in V_{h}$. Usaremos un argumento de dualidad basado en la solución del siguiente problema:

$$
\left\{\begin{aligned}
-\operatorname{div}(\alpha \nabla \varphi)+\beta \varphi & =\mathbf{0} & & \text { in } \Omega \\
\alpha \frac{\partial \varphi}{\partial \mathbf{n}}+\varphi & =\eta_{h} & & \text { on } \Gamma .
\end{aligned}\right.
$$

Dado que $\left.\eta_{h}\right|_{\Gamma} \in H^{\epsilon}(\Gamma)$, con $\epsilon \in(0,1 / 2)$, los resultados del Lema 4.1 nos aseguran que $\varphi \in H^{3 / 2+\epsilon}(\Omega)$. Además,

$$
\begin{aligned}
& \|\varphi\|_{1+r / 2} \leq C\left\|\eta_{h}\right\|_{0, \Gamma}, \\
& \|\varphi\|_{3 / 2+\epsilon} \leq C\left\|\eta_{h}\right\|_{\epsilon, \Gamma} .
\end{aligned}
$$

Observemos también que la regularidad de $\varphi$ implica que $\left.\nabla \varphi\right|_{\Gamma} \cdot \mathbf{n}$ está bien definida como función de $L^{2}(\Gamma)$.

A partir del sistema de ecuaciones (4.24), tendremos

$$
\begin{aligned}
\int_{\Gamma} \eta_{h}^{2} & =\int_{\Gamma}\left(\alpha \frac{\partial \varphi}{\partial \mathbf{n}}+\varphi\right) \eta_{h}=\sum_{\ell \in \mathcal{E}_{h}^{\Gamma}} \int_{\ell}\left(\alpha \frac{\partial \varphi}{\partial \mathbf{n}}+\varphi\right) \eta_{h} \\
& =-\sum_{\ell \in \mathcal{E}_{h}^{I}} \int_{\ell} \alpha \frac{\partial \varphi}{\partial \mathbf{n}}\left[\left[\eta_{h}\right]\right]+\sum_{T \in \mathcal{T}_{h}}\left(\int_{T} \operatorname{div}(\alpha \nabla \varphi) \eta_{h}+\int_{T} \alpha \nabla \varphi \cdot \nabla \eta_{h}\right)+\int_{\Gamma} \varphi \eta_{h} \\
& =-\sum_{\ell \in \mathcal{E}_{h}^{I}} \int_{\ell} \alpha \frac{\partial \varphi}{\partial \mathbf{n}}\left[\left[\eta_{h}\right]\right]+\int_{\Omega} \beta \varphi \eta_{h}+\sum_{T \in \mathcal{T}_{h}} \int_{T} \alpha \nabla \varphi \cdot \nabla \eta_{h}+\int_{\Gamma} \varphi \eta_{h},
\end{aligned}
$$

donde $\left[\left[\eta_{h}\right]\right]$ denota el salto de $\eta_{h}$ a través del lado $\ell \in \mathcal{E}_{h}^{I}$. Para obtener la expresión anterior hemos usado integración por partes y la igualdad

$$
\sum_{T \in \mathcal{T}_{h}} \int_{\partial T} \alpha \frac{\partial \varphi}{\partial \mathbf{n}} \eta_{h}=\sum_{\ell \in \mathcal{E}_{h}^{\Gamma}} \int_{\ell} \alpha \frac{\partial \varphi}{\partial \mathbf{n}} \eta_{h}+\sum_{\ell \in \mathcal{E}_{h}^{I}} \int_{\ell} \alpha \frac{\partial \varphi}{\partial \mathbf{n}}\left[\left[\eta_{h}\right]\right] .
$$


Multiplicando las ecuaciones en (4.24) por $u-u^{I} \in H^{1}(\Omega)$ e integrando por partes, encontramos que

$$
\int_{\Omega} \alpha \nabla \varphi \cdot\left(u-u^{I}\right)+\int_{\omega} \beta \varphi\left(u-u^{I}\right)+\int_{\Gamma} \varphi\left(u-u^{I}\right)=\int_{\Gamma} \eta_{h}\left(u-u^{I}\right) .
$$

Insertando este resultado en la ecuación (4.26), tendremos

$$
\int_{\Gamma} \eta_{h}^{2}=-\sum_{\ell \in \mathcal{E}_{h}^{I}} \int_{\ell} \alpha \frac{\partial \varphi}{\partial \mathbf{n}}\left[\left[\eta_{h}\right]\right]+\int_{\Omega} \beta \varphi e_{h}+\sum_{T \in \mathcal{T}_{h}} \int_{T} \alpha \nabla \varphi \cdot \nabla e_{h}+\int_{\Gamma} \varphi e_{h}-\int_{\Gamma} \eta_{h}\left(u-u^{I}\right)
$$

Sea $\varphi^{I}$ la interpolada de Lagrange (lineal a trozos) de $\varphi$. Dado que $\varphi^{I} \in V \cap V_{h}$, podemos usarla como función test en los problemas (4.22) y (4.23) para obtener la siguiente ecuación de error

$$
\sum_{T \in \mathcal{T}_{h}} \int_{\partial T} \alpha \nabla e_{h} \cdot \nabla \varphi^{I}+\int_{\Omega} \beta e_{h} \varphi^{I}+\int_{\Gamma} e_{h} \varphi^{I}=0
$$

Entonces, restando las ecuaciones (4.27) y (4.28) y teniendo en cuenta que $\left[\left[\eta_{h}\right]\right]=\left[\left[e_{h}\right]\right]$, llegamos a

$\left\|\eta_{h}\right\|_{0, \Gamma}^{2}=\sum_{T \in \mathcal{T}_{h}} \int_{T} \alpha \nabla\left(\varphi-\varphi^{I}\right) \cdot \nabla e_{h}+\int_{\Omega} \beta\left(\varphi-\varphi^{I}\right) e_{h}+\int_{\Gamma}\left(\varphi-\varphi^{I}\right) e_{h}-\sum_{\ell \in \mathcal{E}_{h}^{I}} \int_{\ell} \alpha \frac{\partial \varphi}{\partial \mathbf{n}}\left[\left[e_{h}\right]\right]-\int_{\Gamma} \eta_{h}\left(u-u^{I}\right)$.

A continuación, vamos a estimar los términos que aparecen en la ecuación anterior por separado. Recordemos que los coeficientes $\alpha$ y $\beta$ están acotados, tanto por arriba como por abajo, por constantes positivas.

Acotación del primer término. Utilizando la desigualdad de Cauchy-Schwarz y estimaciones del error standard para la interpolada de Lagrange $\varphi^{I}$, obtendremos

$$
\left|\int_{T} \alpha \nabla\left(\varphi-\varphi^{I}\right) \cdot \nabla e_{h}\right| \leq\left\|\alpha \nabla\left(\varphi-\varphi^{I}\right)\right\|_{0, T}\left\|\nabla e_{h}\right\|_{0, T} \leq C h^{r / 2}\|\varphi\|_{1+r / 2, T}\left\|e_{h}\right\|_{1, T}
$$

Luego, sumando sobre todos los triángulos $T \in \mathcal{T}_{h}$, usando la estimación (4.25) y el Teorema 4.7, concluimos que

$$
\left|\sum_{T \in \mathcal{T}_{h}} \int_{T} \alpha \nabla\left(\varphi-\varphi^{I}\right) \cdot \nabla e_{h}\right| \leq C h^{3 r / 2}\left\|\eta_{h}\right\|_{0, \Gamma}\|\tau\|_{0, \Gamma}
$$

Acotación del segundo término. Procediendo como en la prueba de la estimación anterior, obtendremos

$$
\left|\sum_{T \in \mathcal{T}_{h}} \int_{T} \beta\left(\varphi-\varphi^{I}\right) e_{h}\right| \leq C h^{1+3 r / 2}\left\|\eta_{h}\right\|_{0, \Gamma}\|\tau\|_{0, \Gamma}
$$

Acotación del tercer término. Usando un teorema de trazas y estimaciones standard del error para la interpolada de Lagrange $\varphi^{I}$, tendremos

$$
\left\|\varphi-\varphi^{I}\right\|_{0, \ell} \leq C\left(h_{T}^{-1 / 2}\left\|\varphi-\varphi^{I}\right\|_{0, T}+h_{T}^{1 / 2}\left|\varphi-\varphi^{I}\right|_{1, T}\right) \leq C h_{T}^{(1+r) / 2}\|\varphi\|_{1+r / 2, T} .
$$

Sumando ahora sobre todos los lados $\ell \in \mathcal{E}_{h}^{\Gamma}$, usando la estimación (4.25) y el Teorema 4.7, podremos escribir

$$
\left|\int_{\Gamma}\left(\varphi-\varphi^{I}\right) e_{h}\right| \leq\left\|\varphi-\varphi^{I}\right\|_{0, \Gamma}\left\|e_{h}\right\|_{0, \Gamma} \leq C h^{(1+3 r) / 2}\left\|\eta_{h}\right\|_{0, \Gamma}\|\tau\|_{0, \Gamma} .
$$

Acotación del cuarto término. Sea $P_{\ell}$ la proyección $L_{\ell}^{2}$ de $H^{\epsilon}(\ell)$ sobre las constantes. Para un lado $\ell \in \mathcal{E}_{h}^{I}$, denotemos por $T_{1}$ y $T_{2}$ a los triángulos en $\mathcal{T}_{h}$ tales que $T_{1} \cap T_{2}=\ell$. Teniendo en cuenta que $u$ es continua en $\Omega$ y que $\left[\left[u_{h}\right]\right]$ es una función lineal que se anula en el punto medio de $\ell$, tendremos 


$$
\begin{aligned}
\left|\int_{\ell} \alpha \frac{\partial \varphi}{\partial \mathbf{n}}\left[\left[e_{h}\right]\right]\right| & =\left|\int_{\ell}\left(\alpha \frac{\partial \varphi}{\partial \mathbf{n}}-P_{\ell}\left(\alpha \frac{\partial u}{\partial \mathbf{n}}\right)\right)\left[\left[e_{h}\right]\right]\right| \\
& =\left|\int_{\ell}\left(\alpha \frac{\partial \varphi}{\partial \mathbf{n}}-P_{\ell}\left(\alpha \frac{\partial \varphi}{\partial \mathbf{n}}\right)\right)\left(\left.e_{h}\right|_{T_{1}}\right)-\int_{\ell}\left(\frac{\partial \varphi}{\partial \mathbf{n}}-P_{\ell}\left(\alpha \frac{\partial \varphi}{\partial \mathbf{n}}\right)\right)\left(\left.e_{h}\right|_{T_{2}}\right)\right| \\
& \leq \sum_{i=1,2}\left|\int_{\ell}\left(\alpha \frac{\partial \varphi}{\partial \mathbf{n}}-P_{\ell}\left(\alpha \frac{\partial \varphi}{\partial \mathbf{n}}\right)\right)\left(\left(\left.e_{h}\right|_{T_{i}}\right)-P_{\ell}\left(\left.e_{h}\right|_{T_{i}}\right)\right)\right| .
\end{aligned}
$$

Sea ahora $P_{T}$ la proyección $L_{T}^{2}$ de $H^{\epsilon+1 / 2}(T)$ sobre las constantes. Usando un teorema de trazas local y estimaciones del error standard para la proyección $P_{T}$, tendremos

$$
\begin{aligned}
\left|\int_{\ell} \alpha \frac{\partial \varphi}{\partial \mathbf{n}}\left[\left[e_{h}\right]\right]\right| & \leq \sum_{i=1,2}\left\|\alpha \nabla \varphi \cdot \mathbf{n}-P_{T}(\alpha \nabla \varphi \cdot \mathbf{n})\right\|_{0, \ell}\left\|\left(\left.e_{h}\right|_{T_{i}}\right)-P_{T}\left(\left.e_{h}\right|_{T_{i}}\right)\right\|_{0, \ell} \\
& \leq C \sum_{i=1,2}\left(h_{T_{i}}^{r / 2-1 / 2}\|\nabla \varphi\|_{r / 2, T_{i}}+h_{T_{i}}^{\epsilon}\|\nabla \varphi\|_{1 / 2+\epsilon, T_{i}}\right)\left(h_{T_{i}}^{1 / 2}\left\|e_{h}\right\|_{1, T_{i}}\right) .
\end{aligned}
$$

Sumando sobre todos los lados $\ell \in \mathcal{E}_{h}^{I}$ y procediendo exactamente igual que en la prueba del Teorema 4.3, usamos la estimación (4.25) y el Teorema 4.7 para obtener

$$
\left|\sum_{\ell \in \mathcal{E}_{h}^{I}} \int_{\ell} \alpha \frac{\partial \varphi}{\partial \mathbf{n}}\left[\left[e_{h}\right]\right]\right| \leq C h^{3 r / 2}\left\|\eta_{h}\right\|_{0, \Gamma}\|\tau\|_{0, \Gamma} .
$$

Acotación del quinto término. Utilizando la desigualdad de Cauchy-Schwarz y estimaciones del error standard para la interpolada de Lagrange $u^{I}$ (recordemos que, como $u$ es autofunción, $u \in H^{1+r}(\Omega)$ y, dado que $\xi=\left.u\right|_{\Gamma}$, será $\left.\|u\|_{1+r} \leq C\|(u, \xi)\| \leq C\|u\|_{1} \leq C\|\tau\|_{0, \Gamma}\right)$, obtendremos

$$
\left|\int_{\Gamma} \eta_{h}\left(u-u^{I}\right)\right| \leq\left\|\eta_{h}\right\|_{0, \Gamma}\left\|u-u^{I}\right\|_{0, \Gamma} \leq C h^{1 / 2+r}\left\|\eta_{h}\right\|_{0, \Gamma}\|u\|_{1+r} \leq C h^{r+1 / 2}\left\|\eta_{h}\right\|_{0, \Gamma}\|\tau\|_{0, \Gamma}
$$

La combinación de todas las estimaciones anteriores nos permite concluir la prueba.

Teorema 4.11 Sea $\mathcal{S}_{\lambda}$ el espacio propio del operador $\mathcal{B}$ asociado con el autovalor $\lambda$. Sea $\mathcal{S}_{\lambda_{h}}$ la suma directa de los espacios propios correspondientes a los autovalores $\lambda_{h 1}, \lambda_{h 2}, \cdots, \lambda_{h m}$ del operador $\boldsymbol{B}_{h}$, convergentes a $\lambda$. Existen constantes estrictamente positivas $C$ y $h_{0}$ tales que, si $h<h_{0}$, entonces

- para cada $u_{h} \in \mathcal{S}_{\lambda_{h}}$, con $\left\|u_{h}\right\|_{0, \Gamma}=1$, $\operatorname{dist}\left(u_{h}, \mathcal{S}_{\lambda}\right) \leq C h^{3 r / 2}$,

- para cada $u \in \mathcal{S}_{\lambda}$, con $\|u\|_{0, \Gamma}=1$, $\operatorname{dist}\left(u, \mathcal{S}_{\lambda_{h}}\right) \leq C h^{3 r / 2}$,

donde dist representa la distancia medida en la norma $\|\cdot\|_{0, \Gamma}$.

Prueba. Es claro que el problema espectral de Steklov también puede estudiarse utilizando el operador solución $\mathcal{B}$ y su correspondiente aproximación $\mathcal{B}_{h}$. En este contexto, los Lemas 4.9 y 4.10 confirman la validez de las propiedades $\mathbf{P 2}$ y $\mathbf{P 4}$, respectivamente, para los espacios discretos elegidos. Siguiendo entonces los mismos argumentos que los empleados en la sección 3.3, podemos asegurar convergencia (libre de modos espurios) de las soluciones aproximadas y un resultado análogo al Teorema 3.26 para la distancia entre los autoespacios continuo y discreto.

\subsubsection{Vibraciones en un medio elástico}

La determinación de los modos propios de vibración de un sólido elástico es un problema fundamental de la mecánica de los medios continuos por sus muchas y muy importantes aplicaciones.

Analizaremos este problema usando una formulación dual-mixta del problema lineal de la elasticidad. Esta formulación se deriva directamente del principio variacional de Hellinger-Reissner [142]. Comparados con los métodos clásicos (formulaciones en base a los desplazamientos), los métodos mixtos tienen las siguientes ventajas: 
- permiten obtener una aproximación directa del tensor de esfuerzos; usualmente, la variable de mayor interés,

- están libres de locking; fenómeno numérico que aparece con los métodos standard cuando el material es casi incompresible.

También presentan desventajas. Como ya fuera señalado por Brezzi-Fortin [56] y por muchos otros investigadores, la mayor dificultad con las formulaciones derivadas del principio de Hellinger-Reissner es que el tensor de esfuerzos tiene que ser simétrico y que esta condición de simetría no es fácil de imponer a nivel discreto.

Hasta hace pocos años, había esencialmente dos maneras de evitar esta dificultad. Una alternativa era usar principios variacionales modificados en los cuales la simetría del tensor de esfuerzos se impone solo débilmente (Amara-Thomas [8], Arnold-Brezzi-Douglas [18], Stenberg [155, 156, 157]) o es, directamente, abandonada (Arnold-Falk [21]). La segunda posibilidad era usar elementos compuestos (Watwood-Hartz [173], Johnson-Mercier [119], Arnold-Douglas-Gupta [20]).

En un trabajo pionero, Arnold-Winther [23] construyeron el primer elemento mixto estable, con tensores de esfuerzo simétricos, usando funciones de forma polinomiales y una sola triangulación para ambas variables (esfuerzos y desplazamientos). Posteriormente, los mismos autores [24] construyeron espacios de elementos finitos no-conformes relajando los requerimentos de continuidad de la componente normal de los tensores de esfuerzos sobre los lados internos de la triangulación. Estos métodos han sido extendidos para mallas rectangulares (Arnold-Awanou [15], Awanou [25]) y para tratar con problemas tri-dimensionales (Arnold-Awanou-Winther [16]). Más recientemente, Carstensen et al. [70,68] informaron sobre experimentos numéricos que confirmarían tanto las predicciones teóricas como la robustez de estos procedimientos al tratar con materiales casi incompresibles.

Varios métodos mixtos no-conformes, simétricos o débilmente simétricos, han surgido en los últimos años. En particular, citaremos aquí los trabajos de Cai-Ye [61], Kim [127], Yi [177] y Gopalakrishnan-Guzmán [114].

En todos los trabajos mencionados anteriormente, el análisis del método mixto propuesto quedó restringido al caso estático o problema fuente. De hecho, existen muy pocos estudios publicados sobre métodos mixtos no-conformes para problemas de autovalores. Una de las primeras contribuciones en este tema [92] se obtuvo mediante la aplicación de la teoría espectral presentada en la sección 3.4.

\section{Planteo del problema y descripción del espectro continuo}

Sea $\Omega$ un dominio acotado y simplemente conexo en $\mathbb{R}^{2}$ con borde Lipschitz-continuo $\Gamma$. El borde $\Gamma$ podría estar dividido en dos partes disjuntas $\Gamma_{D}$ y $\Gamma_{N}$ siempre que $\left|\Gamma_{D}\right| \neq 0$ y $\Gamma=\bar{\Gamma}_{D} \cup \bar{\Gamma}_{N}$.

Nuestro objetivo es determinar los modos de oscilación de un material elástico, homogéneo e isotrópico que ocupa la región plana $\Omega$. Asumiremos que el sólido elástico se encuentra fijo sobre $\Gamma_{D}$ y que es libre de moverse a lo largo de $\Gamma_{N}$.

Usaremos la siguiente notación para las magnitudes físicas:

- u: el campo de desplazamientos,

- $\rho_{S}$ : la densidad,

- $\lambda_{S}$ y $\mu_{S}$ : los coeficientes de Lamé, los cuales están definidos por

$$
\lambda_{S}:=\frac{\nu_{S} E_{S}}{\left(1-2 \nu_{S}\right)\left(1+\nu_{S}\right)}, \quad \mu_{S}:=\frac{E_{S}}{2\left(1+\nu_{S}\right)},
$$

donde $E_{S}>0$ es el módulo de Young y $\nu_{S} \in(0,1 / 2)$ es la razón de Poisson,

- $\varepsilon(\mathbf{u})$ : el tensor de deformaciones, definido por

$$
\varepsilon(\mathbf{u}):=\frac{1}{2}\left[\nabla \mathbf{u}+(\nabla \mathbf{u})^{t}\right],
$$


- $\boldsymbol{\sigma}(\mathbf{u})$ : el tensor de esfuerzos, relacionado con el tensor de deformaciones por medio de la Ley de Hooke:

$$
\boldsymbol{\sigma}(\mathbf{u})=\mathbb{C} \varepsilon(\mathbf{u}):=\lambda_{S} \operatorname{tr} \varepsilon(\mathbf{u}) \mathbf{I}+2 \mu_{S} \varepsilon(\mathbf{u}),
$$

donde $\operatorname{tr} \varepsilon(\mathbf{u})$ denota la traza de $\varepsilon(\mathbf{u})$, I es la $2 \times 2$ matriz identidad y $\mathbb{C}$ es el denominado tensor de rigidez.

Una manera equivalente de establecer la Ley de Hooke es

$$
\boldsymbol{\varepsilon}(\mathbf{u})=\mathbb{A} \boldsymbol{\sigma}(\mathbf{u}):=\frac{1}{2 \mu_{S}} \boldsymbol{\sigma}(\mathbf{u})-\frac{\lambda_{S}}{4 \mu_{S}\left(\lambda_{S}+\mu_{S}\right)} \operatorname{tr} \boldsymbol{\sigma}(\mathbf{u}) \mathbf{I} .
$$

Los materiales casi incompresibles se caracterizan por $\nu_{S} \rightarrow 1 / 2$, lo cual implica $\lambda_{S} \rightarrow \infty$. Observemos que, en este caso, $|\mathbb{C}|$ es grande mientras que $|\mathbb{A}|$ permanece acotado.

Asumiendo movimientos de pequeña amplitud, el modelo lineal nos conduce al siguiente problema de autovalores:

Encontrar $\omega>0$, un campo tensorial $\boldsymbol{\sigma} \neq \mathbf{0}$ y un campo vectorial $\mathbf{u} \neq \mathbf{0}$, tales que

$$
\begin{aligned}
\mathbb{A} \boldsymbol{\sigma} & =\boldsymbol{\varepsilon}(\mathbf{u}) & & \operatorname{in} \Omega, \\
-\operatorname{div} \boldsymbol{\sigma} & =\omega^{2} \rho_{S} \mathbf{u} & & \text { in } \Omega, \\
\boldsymbol{\sigma} \mathbf{n} & =\mathbf{0} & & \text { on } \Gamma_{N}, \\
\mathbf{u} & =\mathbf{0} & & \text { on } \Gamma_{D}
\end{aligned}
$$

siendo $\mathbf{n}$ el vector unitario normal al borde $\Gamma$, orientado hacia el exterior del dominio $\Omega$, y $\omega$ la frecuencia angular de las oscilaciones.

Usaremos la notación standard para los espacios de Sobolev, sus normas y seminormas. Para un dado espacio de Sobolev $H^{r}(\Omega), H^{r}\left(\Omega, \mathbb{R}^{2}\right)$ y $H^{r}\left(\Omega, \mathbb{S}^{2}\right)$ denotarán los espacios de vectores y tensores simétricos de orden 2 con entradas en $H^{r}(\Omega)$, respectivamente. En particular, denotaremos

$$
H_{0, \Gamma_{D}}^{1}\left(\Omega, \mathbb{R}^{2}\right):=\left\{\mathbf{v} \in H^{1}\left(\Omega, \mathbb{R}^{2}\right):\left.\mathbf{v}\right|_{\Gamma_{D}}=\mathbf{0}\right\} .
$$

Definimos además el espacio de tensores

$$
H\left(\operatorname{div}, \Omega, \mathbb{S}^{2}\right):=\left\{\boldsymbol{\tau} \in L^{2}\left(\Omega, \mathbb{S}^{2}\right): \operatorname{div} \boldsymbol{\tau} \in L^{2}\left(\Omega, \mathbb{R}^{2}\right)\right\}
$$

el cual es un espacio de Hilbert con el producto interno

$$
\langle\boldsymbol{\sigma}, \boldsymbol{\tau}\rangle:=\int_{\Omega} \boldsymbol{\sigma}: \boldsymbol{\tau}+\int_{\Omega} \operatorname{div} \boldsymbol{\sigma} \cdot \operatorname{div} \boldsymbol{\tau}
$$

y la norma inducida correspondiente

$$
\|\boldsymbol{\tau}\|_{H\left(\operatorname{div}, \Omega, \mathbb{S}^{2}\right)}^{2}=\|\boldsymbol{\tau}\|_{0, \Omega}^{2}+\|\operatorname{div} \boldsymbol{\tau}\|_{0, \Omega}^{2} .
$$

Por último, considerando el espacio

$$
H_{00}^{1 / 2}\left(\Gamma_{N}, \mathbb{R}^{2}\right):=\left\{\mathbf{v} \in H^{1 / 2}\left(\Gamma_{N}, \mathbb{R}^{2}\right): \widetilde{\mathbf{v}} \in H^{1 / 2}\left(\Gamma, \mathbb{R}^{2}\right)\right\}
$$

siendo $\widetilde{\mathbf{v}}$ la extensión de $\mathbf{v}$ a toda $\Gamma$ que es igual a cero fuera de $\Gamma_{N}$, definimos

$$
H_{0, \Gamma_{N}}\left(\operatorname{div}, \Omega, \mathbb{S}^{2}\right):=\left\{\boldsymbol{\tau} \in H\left(\operatorname{div}, \Omega, \mathbb{S}^{2}\right):<\boldsymbol{\tau} \mathbf{n}, \mathbf{v}>_{\Gamma_{N}}=0 \forall \mathbf{v} \in H_{00}^{1 / 2}\left(\Gamma_{N}, \mathbb{R}^{2}\right)\right\},
$$

el cual es un subespacio cerrado de $H\left(\operatorname{div}, \Omega, \mathbb{S}^{2}\right)$.

Basados en el principio de Hellinger-Reissner, podemos definir la siguiente formulación variacional (se denomina formulación dual-mixta o stress-desplazamiento) para el problema de autovalores (4.29): 
Encontrar $\omega \in \mathbb{R},(\boldsymbol{\sigma}, \mathbf{u}) \in H_{0, \Gamma_{N}}\left(\operatorname{div}, \Omega, \mathbb{S}^{2}\right) \times L^{2}\left(\Omega, \mathbb{R}^{2}\right),(\boldsymbol{\sigma}, \mathbf{u}) \neq(\mathbf{0}, \mathbf{0})$, tal que

$$
\begin{array}{ll}
\int_{\Omega} \mathbb{A} \boldsymbol{\sigma}: \boldsymbol{\tau}+\int_{\Omega} \operatorname{div} \boldsymbol{\tau} \cdot \mathbf{u}=0 & \forall \boldsymbol{\tau} \in H_{0, \Gamma_{N}}\left(\operatorname{div}, \Omega, \mathbb{S}^{2}\right) \\
-\int_{\Omega} \operatorname{div} \boldsymbol{\sigma} \cdot \mathbf{v}=\omega^{2} \int_{\Omega} \rho_{S} \mathbf{u} \cdot \mathbf{v} & \forall \mathbf{v} \in L^{2}\left(\Omega, \mathbb{R}^{2}\right) .
\end{array}
$$

Las formas bilineales

$$
\begin{gathered}
a(\boldsymbol{\sigma}, \boldsymbol{\tau}):=\int_{\Omega} \mathbb{A} \boldsymbol{\sigma}: \boldsymbol{\tau} \quad \boldsymbol{\sigma}, \boldsymbol{\tau} \in H\left(\operatorname{div}, \Omega, \mathbb{S}^{2}\right) \\
b(\boldsymbol{\tau}, \mathbf{u}):=\int_{\Omega} \operatorname{div} \boldsymbol{\tau} \cdot \mathbf{u} \quad \boldsymbol{\tau} \in H\left(\operatorname{div}, \Omega, \mathbb{S}^{2}\right), \mathbf{u} \in L^{2}\left(\Omega, \mathbb{R}^{2}\right) .
\end{gathered}
$$

satisfacen las propiedades que enunciaremos en el siguiente lema (las pruebas pueden encontrarse en [21]).

Lema 4.12 Denotemos por $\mathbf{K}$ al núcleo del operador asociado con la forma bilineal b. Existen constantes positivas $c_{a}$ y $c_{b}$, las cuales no depeden del coeficiente $\lambda_{S}$, tales que

$$
\begin{aligned}
& -a(\boldsymbol{\sigma}, \boldsymbol{\tau}) \leq \mu_{S}^{-1}\|\boldsymbol{\sigma}\|_{H\left(\operatorname{div}, \Omega, \mathbb{S}^{2}\right)}\|\boldsymbol{\tau}\|_{H\left(\operatorname{div}, \Omega, \mathbb{S}^{2}\right)} \quad \forall \boldsymbol{\sigma}, \boldsymbol{\tau} \in H\left(\operatorname{div}, \Omega, \mathbb{S}^{2}\right), \\
& -b(\boldsymbol{\sigma}, \mathbf{v}) \leq\|\boldsymbol{\sigma}\|_{H\left(\operatorname{div}, \Omega, \mathbb{S}^{2}\right)}\|\mathbf{v}\|_{0, \Omega} \quad \forall \boldsymbol{\sigma} \in H\left(\operatorname{div}, \Omega, \mathbb{S}^{2}\right), \mathbf{v} \in L^{2}\left(\Omega, \mathbb{R}^{2}\right), \\
& -a(\boldsymbol{\sigma}, \boldsymbol{\sigma}) \geq c_{a}\|\boldsymbol{\sigma}\|_{H\left(\operatorname{div}, \Omega, \mathbb{S}^{2}\right)}^{2} \quad \forall \boldsymbol{\sigma} \in \mathbf{K}, \\
& \sup _{-} \operatorname{siv}_{\boldsymbol{\sigma} \in H\left(\operatorname{div}, \mathbb{S}^{2}\right)} \frac{b(\boldsymbol{\sigma}, \mathbf{v})}{\|\boldsymbol{\sigma}\|_{H\left(\operatorname{div}, \Omega, \mathbb{S}^{2}\right)}} \geq c_{b}\|\mathbf{v}\|_{0, \Omega} \quad \forall \mathbf{v} \in L^{2}\left(\Omega, \mathbb{R}^{2}\right) .
\end{aligned}
$$

A partir de estos resultados y aplicando la teoría abstracta de Brezzi para métodos mixtos [56], se puede probar que el siguiente problema fuente está bien definido.

Dada $\mathbf{g} \in L^{2}\left(\Omega, \mathbb{R}^{2}\right)$, encontrar $(\boldsymbol{\sigma}, \mathbf{u}) \in H_{0, \Gamma_{N}}\left(\operatorname{div}, \Omega, \mathbb{S}^{2}\right) \times L^{2}\left(\Omega, \mathbb{R}^{2}\right)$ tal que

$$
\begin{aligned}
& \int_{\Omega} \mathbb{A} \boldsymbol{\sigma}: \boldsymbol{\tau}+\int_{\Omega} \operatorname{div} \boldsymbol{\tau} \cdot \mathbf{u}=0 \quad \forall \boldsymbol{\tau} \in H_{0, \Gamma_{N}}\left(\operatorname{div}, \Omega, \mathbb{S}^{2}\right) \\
& -\int_{\Omega} \operatorname{div} \boldsymbol{\sigma} \cdot \mathbf{v}=\int_{\Omega} \mathbf{g} \cdot \mathbf{v} \quad \forall \mathbf{v} \in L^{2}\left(\Omega, \mathbb{R}^{2}\right) .
\end{aligned}
$$

Más aún, vale la siguiente estimación:

$$
\|\boldsymbol{\sigma}\|_{H\left(\operatorname{div}, \Omega, \mathbb{S}^{2}\right)}+\|\mathbf{u}\|_{0, \Omega} \leq C\|\mathbf{g}\|_{0, \Omega},
$$

donde la constante $C$ es independiente del coeficiente de Lamé $\lambda_{S}$.

En estas condiciones, definimos el espacio producto:

$$
\boldsymbol{\Sigma} \times \mathbf{V}:=H_{0, \Gamma_{N}}\left(\operatorname{div}, \Omega, \mathbb{S}^{2}\right) \times L^{2}\left(\Omega, \mathbb{R}^{2}\right)
$$

y las formas bilineales continuas:

$$
\begin{gathered}
A((\boldsymbol{\sigma}, \mathbf{u}),(\boldsymbol{\tau}, \mathbf{v})):=\int_{\Omega} \mathbb{A} \boldsymbol{\sigma}: \boldsymbol{\tau}+\int_{\Omega} \operatorname{div} \boldsymbol{\tau} \cdot \mathbf{u}+\int_{\Omega} \operatorname{div} \boldsymbol{\sigma} \cdot \mathbf{v} \quad \forall(\boldsymbol{\sigma}, \mathbf{u}),(\boldsymbol{\tau}, \mathbf{v}) \in \boldsymbol{\Sigma} \times \mathbf{V} \\
B((\boldsymbol{\sigma}, \mathbf{u}),(\boldsymbol{\tau}, \mathbf{v})):=-\int_{\Omega} \mathbf{u} \cdot \mathbf{v} \quad \forall(\boldsymbol{\sigma}, \mathbf{u}),(\boldsymbol{\tau}, \mathbf{v}) \in \boldsymbol{\Sigma} \times \mathbf{V}
\end{gathered}
$$

El Lema 4.12 implica que la forma bilineal $A$ satisface la condición de estabilidad (3.61) con una constante independiente de $\lambda_{S}$. Así, el problema (4.31) puede ser re-escrito de la siguiente forma: 
$\operatorname{Dado}(\mathbf{f}, \mathbf{g}) \in L^{2}\left(\Omega, \mathbb{S}^{2}\right) \times L^{2}\left(\Omega, \mathbb{R}^{2}\right)$, encontrar $(\boldsymbol{\sigma}, \mathbf{u}) \in \mathbf{\Sigma} \times \mathbf{V}$ tal que

$$
A((\boldsymbol{\sigma}, \mathbf{u}),(\boldsymbol{\tau}, \mathbf{v}))=B((\mathbf{f}, \mathbf{g}),(\boldsymbol{\tau}, \mathbf{v})) \quad \forall(\boldsymbol{\tau}, \mathbf{v}) \in \mathbf{\Sigma} \times \mathbf{V}
$$

Denotemos por $\boldsymbol{\Psi}$ al operador lineal definido por

$$
\begin{aligned}
\mathbf{\Psi}: L^{2}\left(\Omega, \mathbb{S}^{2}\right) \times L^{2}\left(\Omega, \mathbb{R}^{2}\right) & \rightarrow \boldsymbol{\Sigma} \times \mathbf{V} \hookrightarrow L^{2}\left(\Omega, \mathbb{S}^{2}\right) \times L^{2}\left(\Omega, \mathbb{R}^{2}\right) \\
(\mathbf{f}, \mathbf{g}) & \mapsto(\boldsymbol{\sigma}, \mathbf{u}),
\end{aligned}
$$

siendo $(\boldsymbol{\sigma}, \mathbf{u})$ la solución (única) del problema (4.33).

Observemos que $\boldsymbol{\Psi}$ es un operador acotado, consecuencia directa de la estimación (4.32). Además, dado que las formas bilineales $A$ y $B$ son simétricas, $\boldsymbol{\Psi}$ es autoadjunto y definido positivo con respecto a $A$ y $B$. Por lo tanto, todos sus autovalores serán reales y positivos. Es fácil comprobar que $(\lambda,(\boldsymbol{\sigma}, \mathbf{u}))$ es un par propio de $\boldsymbol{\Psi}$ si, y solo si, $(\omega,(\boldsymbol{\sigma}, \mathbf{u}))$ es una solución del problema $(4.30)$ con $\omega=1 / \sqrt{\lambda}$.

Observemos también que, debido a (4.31), div $\boldsymbol{\sigma}=\mathbf{g} \in L^{2}\left(\Omega, \mathbb{R}^{2}\right)$. Luego, la componente $\boldsymbol{\sigma}$ del par solución no puede estar en un subconjunto compacto de $H\left(\operatorname{div}, \Omega, \mathbb{S}^{2}\right)$ ya que su divergencia es solamente un elemento de $L^{2}\left(\Omega, \mathbb{R}^{2}\right)$. Consecuentemente, $\boldsymbol{\Psi}: L^{2}\left(\Omega, \mathbb{S}^{2}\right) \times L^{2}\left(\Omega, \mathbb{R}^{2}\right) \rightarrow H_{0, \Gamma_{N}}\left(\operatorname{div}, \Omega, \mathbb{S}^{2}\right) \times L^{2}\left(\Omega, \mathbb{R}^{2}\right)$ no es un operador compacto.

Por otro lado, $\boldsymbol{\Psi}$ es un operador regularizante. El siguiente resultado de regularidad fue probado por Grisvard en $[111,112,113]$.

Lema 4.13 Supongamos que $\mathbf{g} \in L^{2}\left(\Omega, \mathbb{R}^{2}\right)$. Entonces, el problema (4.31) tiene una única solución $(\boldsymbol{\sigma}, \mathbf{u})$ que verifica $\boldsymbol{\sigma} \in H\left(\operatorname{div}, \Omega, \mathbb{S}^{2}\right) \cap H^{s}\left(\Omega, \mathbb{S}^{2}\right), \mathbf{u} \in H^{1+s}\left(\Omega, \mathbb{R}^{2}\right)$ y

$$
\|\boldsymbol{\sigma}\|_{s, \Omega}+\|\mathbf{u}\|_{1+s, \Omega} \leq C\|\mathbf{g}\|_{0, \Omega}
$$

donde $s \in(0,1-\alpha]$, siendo $\alpha$ un número positivo pequeño, y $C$ es una constante positiva que depende solamente de $\Omega, \Gamma_{N}$ y $\mathbb{A}$.

Podemos dar una caracterización completa del espectro del operador $\boldsymbol{\Psi}$ siguiendo un análisis similar al presentado en [29]. De hecho, los argumentos en la Section 11.2 de [29] pueden repetirse exactamente en nuestro caso y esto, junto con los resultados establecidos en la Section 10.1 de la misma referencia, permiten probar el siguiente teorema.

Teorema 4.14 El espectro de $\boldsymbol{\Psi}$ consiste en una sucesión $\lambda_{n}, n \in \mathbb{N}$, de autovalores estrictamente positivos, todos con multiplicidad finita, que convergen a 0.

Observación 4.15 En el mundo real, los cuerpos elásticos son siempre tri-dimensionales. Sin embargo, cuando el desplazamiento $\mathbf{u}$, el tensor de deformación $\varepsilon$ y el tensor de esfuerzos $\boldsymbol{\sigma}$ son (prácticamente) independientes de una de las coordenadas espaciales, el problema puede estudiarse mediante un modelo (aproximación) bi-dimensional. Los dos modelos típicos de la teoría de la elasticidad en el plano son:

- deformaciones en el plano; modela el comportamiento de estructuras cilíndricas (o prismáticas) bajo la acción de fuerzas que se ejercen en dirección paralela a la sección transversal del cilindro y asumiendo que no hay desplazamientos en la dirección del eje del cilindro,

- tensiones en el plano; modela el comportamiento de estructuras delgadas (una de las dimensiones de la estructura es muy pequeña comparada con las dimensiones restantes) bajo la acción de fuerzas que se ejercen en dirección paralela a las caras de la estructura y asumiendo que los esfuerzos están confinados al plano ocupado por la estructura.

Es interesante destacar que la única diferencia entre las ecuaciones que describen a ambos modelos está en la definición de las constantes elásticas; en efecto, para las tensiones en el plano

$$
\lambda_{S}=\frac{\nu_{S}}{1-\nu_{S}^{2}} E_{S}
$$




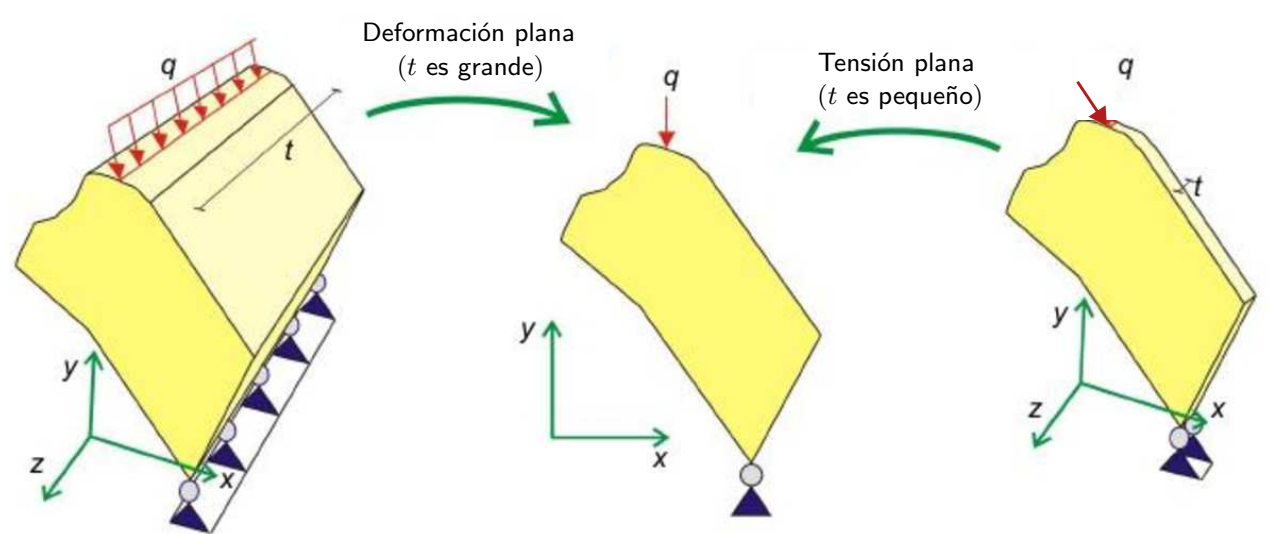

Figura 4.1: Problemas bi-dimensionales en Elasticidad lineal

\section{El método mixto no-conforme de Arnold-Winther}

En esta sección introduciremos los espacios de elementos finitos no-conformes de Arnold-Winther y recordaremos algunas de sus propiedades de aproximación.

Sea $\left\{\mathcal{T}_{h}\right\}_{h>0}$ una familia de particiones de $\Omega$, regular en el sentido descripto en la sección 4.1.1. Como es usual, $h$ representa el tamaño de la triangulación; es decir, el máximo de los diámetros de sus elementos. Sea $\mathcal{E}_{h}=\mathcal{E}_{h}^{I} \cup \mathcal{E}_{h}^{\Gamma}$ el conjunto de los lados de todos los triángulos $T \in \mathcal{T}_{h}$. Denotaremos por $\mathbf{n}_{\ell}$ y $\mathbf{t}_{\ell}$ a los vectores unitarios normal y tangente, respectivamente, a un lado $\ell \in \mathcal{E}_{h}$.

Para cada $T \in \mathcal{T}_{h}$, denotaremos por $\mathcal{P}_{k}(T)$ al espacio de los polinomios de grado menor o igual a $k$ restringidos a $T$. De manera análoga, para cada $\ell \in \mathcal{E}_{h}$, denotaremos por $\mathcal{P}_{k}(\ell)$ al espacio de los polinomios de grado menor o igual a $k$ restringidos a $\ell$. En este contexto, $\mathcal{P}_{k}\left(T, \mathbb{S}^{2}\right)=\left(\mathcal{P}_{k}(T)\right)^{3}$ y $\mathcal{P}_{k}\left(T, \mathbb{R}^{2}\right)=\left(\mathcal{P}_{k}(T)\right)^{2}$ representan a los espacios de tensores y vectores polinomiales, respectivamente.

Definimos:

$$
\begin{aligned}
& \boldsymbol{\Sigma}(T):=\left\{\boldsymbol{\tau}_{h} \in \mathcal{P}_{2}\left(T, \mathbb{S}^{2}\right): \boldsymbol{\tau}_{h} \mathbf{n}_{\ell} \cdot \mathbf{n}_{\ell} \in \mathcal{P}_{1}(\ell) \text { para cada } \ell \text { de } T\right\} \\
& \mathbf{V}(T):=\mathcal{P}_{1}\left(T, \mathbb{R}^{2}\right)
\end{aligned}
$$

Hay 15 grados de libertad para $\boldsymbol{\Sigma}(T)$ y 6 grados de libertad para $\mathbf{V}(T)$. Los grados de libertad de $\boldsymbol{\Sigma}(T)$ son

- los momentos de grado 0 y 1 de las dos componentes normales de $\tau_{h}$ sobre cada lado $\ell$ of $T$,

- los momentos de grado 0 de las tres componentes de $\boldsymbol{\tau}_{h}$ en $T$.
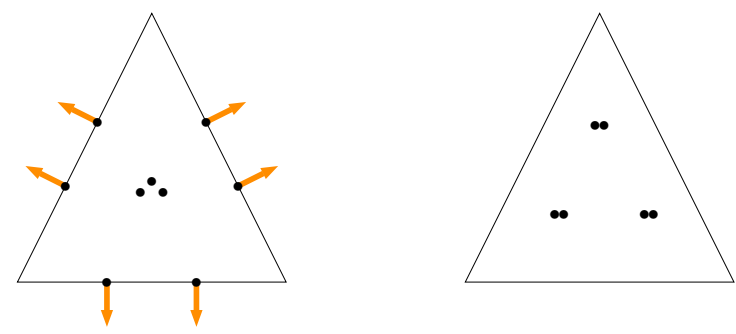

Figura 4.2: Grados de libertad para los espacios $\boldsymbol{\Sigma}(T)$ y $\mathbf{V}(T)$. 
Los grados de libertad de $\mathbf{V}(T)$ están dados por los valores que toman las dos componentes del vector en tres nodos interiores a $T$. De acuerdo con [24], estos grados de libertad permiten determinar a $\boldsymbol{\Sigma}(T)$ y a $\mathbf{V}(T)$ de manera única (ver Lemma 3.1 de esa misma referencia).

El espacio de elementos finitos mixto de Arnold-Winther (de orden cero) se define como sigue:

$$
\begin{aligned}
\boldsymbol{\Sigma}_{h}^{0}:= & \left\{\boldsymbol{\tau}_{h} \in L^{2}\left(\Omega, \mathbb{S}^{2}\right):\left.\boldsymbol{\tau}_{h}\right|_{T} \in \boldsymbol{\Sigma}(T) \forall T \in \mathcal{T}_{h}, \boldsymbol{\tau}_{h}\right. \text { es continua en los grados } \\
& \text { de libertad definidos sobre cada lado } \left.\ell \in \mathcal{E}_{h}\right\}, \\
\mathbf{V}_{h}:= & \left\{\mathbf{v}_{h} \in L^{2}\left(\Omega, \mathbb{R}^{2}\right):\left.\mathbf{v}_{h}\right|_{T} \in \mathbf{V}(T) \forall T \in \mathcal{T}_{h}\right\} .
\end{aligned}
$$

Observemos que para $\boldsymbol{\tau}_{h} \in \boldsymbol{\Sigma}_{h}^{0}$, las componentes $\boldsymbol{\tau}_{h} \mathbf{n}_{\ell} \cdot \mathbf{n}_{\ell}$ (esfuerzos por tracción) son continuas sobre cada lado interior $\ell \in \mathcal{E}_{h}$, mientras que $\boldsymbol{\tau}_{h} \mathbf{n}_{\ell} \cdot \mathbf{t}_{\ell}$ (esfuerzos de corte) son discontinuas. Como consecuencia, $\boldsymbol{\Sigma}_{h}^{0} \not \subset H\left(\operatorname{div}, \Omega, \mathbb{S}^{2}\right)$ y esta elección de espacios conduce a una aproximación no-conforme del problema.

La condición de borde impuesta sobre $\Gamma_{N}$ (condición de tracción) es una condición de borde esencial para la formulación mixta (4.31). Impondremos esta condición de manera débil, es decir, pediremos

$$
\int_{\ell} \boldsymbol{\tau}_{h} \mathbf{n}_{\ell} \cdot \mathbf{v}_{h}=\mathbf{0} \quad \forall \mathbf{v}_{h} \in \mathcal{P}_{1}\left(\ell, \mathbb{R}^{2}\right) \quad \forall \ell \subset \Gamma_{N}
$$

Luego, los espacios admisibles para los tensores discretos estarán dados por:

$$
\boldsymbol{\Sigma}_{h}:=\left\{\boldsymbol{\tau}_{h} \in \boldsymbol{\Sigma}_{h}^{0}: \boldsymbol{\tau}_{h} \text { satisface las condiciones }(4.35)\right\} .
$$

Denotemos por $\boldsymbol{\Pi}_{h}^{A W}: H^{1}\left(\Omega, \mathbb{S}^{2}\right) \rightarrow \boldsymbol{\Sigma}_{h}$ al operador de interpolación de Arnold-Winther. El operador $\Pi_{h}^{A W}$ está definido por:

$$
\begin{aligned}
& \int_{\ell}\left(\boldsymbol{\tau}-\Pi_{h}^{A W} \boldsymbol{\tau}\right) \mathbf{n}_{\ell} \cdot \mathbf{v}=0 \quad \forall \ell \in \mathcal{E}_{h}, \forall \mathbf{v} \in \mathcal{P}_{1}\left(\ell, \mathbb{R}^{2}\right), \\
& \int_{T}\left(\boldsymbol{\tau}-\Pi_{h}^{A W} \boldsymbol{\tau}\right)=0 \quad \forall T \in \mathcal{T}_{h} .
\end{aligned}
$$

Notemos que $\Pi_{h}^{A W}$ preserva la condición homogénea de borde impuesta sobre $\Gamma_{N}$ en el sentido débil dado por (4.35).

Para $\boldsymbol{\tau} \in H^{1}\left(\Omega, \mathbb{S}^{2}\right)$ y $\mathbf{v}_{h} \in \mathbf{V}(T)$ arbitrario, las condiciones (4.36)-(4.37) conducen al siguiente resultado

$$
\int_{T} \operatorname{div}\left(\boldsymbol{\tau}-\boldsymbol{\Pi}_{h}^{A W} \boldsymbol{\tau}\right) \cdot \mathbf{v}_{h}=-\int_{T}\left(\boldsymbol{\tau}-\boldsymbol{\Pi}_{h}^{A W} \boldsymbol{\tau}\right): \varepsilon\left(\mathbf{v}_{h}\right)+\int_{\partial T}\left(\boldsymbol{\tau}-\Pi_{h}^{A W} \boldsymbol{\tau}\right) \mathbf{n} \cdot \mathbf{v}_{h}=0 .
$$

Sean $\mathbf{P}_{h}: L^{2}\left(\Omega, \mathbb{R}^{2}\right) \rightarrow \mathbf{V}_{h}$ la proyección $L^{2}$-ortogonal sobre el espacio $\mathbf{V}_{h}$ y div $h$ la aplicación elemento por elemento del operador divergencia. Como $\left.\operatorname{div} \boldsymbol{\Pi}_{h}^{A W} \boldsymbol{\tau}\right|_{T} \in \mathbf{V}(T)$, el resultado anterior prueba que el operador de interpolación de Arnold-Winther verifica la propiedad del diagrama conmutativo; es decir,

$$
\operatorname{div}_{h} \boldsymbol{\Pi}_{h}^{A W} \boldsymbol{\tau}=\mathbf{P}_{h} \operatorname{div} . \boldsymbol{\tau}
$$

Como consecuencia, tendremos

$$
\operatorname{div}_{h} \boldsymbol{\Sigma}_{h}=\mathbf{V}_{h} .
$$

Arnold-Winther probaron en [24] que $\left(\boldsymbol{\Sigma}_{h}^{0}, \mathbf{V}_{h}\right)$ es un par de espacios estable para la discretización del problema de la elasticidad con condiciones homogéneas de borde para los desplazamientos únicamente $\left(\Gamma_{N}=\emptyset\right)$. Más aún, establecieron estimaciones del error para el operador de interpolación $\Pi_{h}^{A W}$ aplicado a funciones $H^{1}\left(\Omega, \mathbb{S}^{2}\right)$.

Usando los mismos argumentos que Arnold-Winther, estos resultados se pueden generalizar para el par de espacios $\left(\boldsymbol{\Sigma}_{h}, \mathbf{V}_{h}\right)$ y para tensores $\boldsymbol{\tau} \in H\left(\operatorname{div}, \Omega, \mathbb{S}^{2}\right) \cap H^{s}\left(\Omega, \mathbb{S}^{2}\right), s>1 / 2$. 
Lema 4.16 El operador de interpolación de Arnold-Winther se extiende a $H\left(\operatorname{div}, \Omega, \mathbb{S}^{2}\right) \cap H^{s}\left(\Omega, \mathbb{S}^{2}\right)$, $s>1 / 2$, univocamente, y vale la siguiente estimación de error

$$
\left\|\boldsymbol{\tau}-\Pi_{h}^{A W} \boldsymbol{\tau}\right\|_{0, \Omega} \leq C h^{s}\|\boldsymbol{\tau}\|_{s, \Omega},
$$

siendo $C$ una constante positiva independiente de $h$.

Prueba. Ver sección A.

Es inmediato, a partir de este lema, que:

$$
\left\|\boldsymbol{\Pi}_{h}^{A W} \boldsymbol{\tau}\right\|_{0, \Omega} \leq c\|\boldsymbol{\tau}\|_{s, \Omega} .
$$

Por otro lado, para el proyector $\mathbf{P}_{h}$ vale la siguiente estimación de error:

$$
\left\|\mathbf{v}-\mathbf{P}_{h} \mathbf{v}\right\|_{0, \Omega} \leq C h^{1+s}\|\mathbf{v}\|_{1+s, \Omega} \quad \forall \mathbf{v} \in H^{1+s}\left(\Omega, \mathbb{R}^{2}\right) .
$$

Los resultados anteriores implican que el par de espacios discretos $\left(\boldsymbol{\Sigma}_{h}, \mathbf{V}_{h}\right)$ satisface las condiciones de estabilidad de la teoría para métodos mixtos. Los argumentos que prueban esta aseveración son idénticos a los utilizados en [23] (ver también [103] para un abordaje más general).

\section{Discretización del problema}

A partir de las secciones previas, sabemos que las formas bilineales discretas

$$
\begin{gathered}
a_{h}\left(\boldsymbol{\sigma}_{h}, \boldsymbol{\tau}_{h}\right):=\int_{\Omega} \mathbb{A} \boldsymbol{\sigma}_{h}: \boldsymbol{\tau}_{h} \\
\boldsymbol{\sigma}_{h}, \boldsymbol{\tau}_{h} \in \boldsymbol{\Sigma}_{h}, \\
b_{h}\left(\boldsymbol{\tau}_{h}, \mathbf{u}_{h}\right):=\int_{\Omega} \operatorname{div}_{h} \boldsymbol{\tau}_{h} \cdot \mathbf{u}_{h} \quad \boldsymbol{\tau}_{h} \in \boldsymbol{\Sigma}_{h}, \mathbf{u}_{h} \in \mathbf{V}_{h} .
\end{gathered}
$$

satisfacen las condiciones de Brezzi para los espacios de elementos finitos $\left(\boldsymbol{\Sigma}_{h}, \mathbf{V}_{h}\right)$. Luego, aplicando la teoría clásica para métodos mixtos, podemos asegurar que existe una única solución del siguiente problema fuente:

Dada $\mathbf{g} \in L^{2}\left(\Omega, \mathbb{R}^{2}\right)$, encontrar $\left(\boldsymbol{\sigma}_{h}, \mathbf{u}_{h}\right) \in \boldsymbol{\Sigma}_{h} \times \mathbf{V}_{h}$ tal que:

$$
\begin{aligned}
& \int_{\Omega} \mathbb{A} \boldsymbol{\sigma}_{h}: \boldsymbol{\tau}_{h}+\int_{\Omega} \operatorname{div}_{h} \boldsymbol{\tau}_{h} \cdot \mathbf{u}_{h}=0 \quad \forall \boldsymbol{\tau}_{h} \in \boldsymbol{\Sigma}_{h}, \\
& -\int_{\Omega} \operatorname{div}_{h} \boldsymbol{\sigma}_{h} \cdot \mathbf{v}_{h}=\int_{\Omega} \mathbf{g} \cdot \mathbf{v}_{h} \quad \forall \mathbf{v}_{h} \in \mathbf{V}_{h} .
\end{aligned}
$$

Más aún, existe una constante $C$, independiente de $h$, tal que:

$$
\left\|\boldsymbol{\sigma}_{h}\right\|_{H\left(\operatorname{div}_{h}, \Omega, \mathbb{S}\right)}+\left\|\mathbf{u}_{h}\right\|_{0, \Omega} \leq C\|\mathbf{g}\|_{0, \Omega} .
$$

Sea $(\boldsymbol{\sigma}, \mathbf{u})$ la solución del problema variacional (4.31). Sabemos que esta solución tiene regularidad $H^{s}$; es decir, $\boldsymbol{\sigma} \in H\left(\operatorname{div}, \Omega, \mathbb{S}^{2}\right) \cap H^{s}\left(\Omega, \mathbb{S}^{2}\right)$ y $\mathbf{u} \in H^{1+s}\left(\Omega, \mathbb{R}^{2}\right)$ (ver Lema 4.13). Argumentos idénticos a los utilizados en [24], combinados con las estimaciones a priori (4.34), nos permiten escribir:

$$
\begin{gathered}
\left\|\boldsymbol{\sigma}-\boldsymbol{\sigma}_{h}\right\|_{0, \Omega}+\left\|\mathbf{u}-\mathbf{u}_{h}\right\|_{0, \Omega} \leq C h^{s}\|\mathbf{u}\|_{1+s, \Omega} \leq C h^{s}\|\mathbf{g}\|_{0, \Omega}, \\
\left\|\operatorname{div} \boldsymbol{\sigma}-\operatorname{div} \boldsymbol{\sigma}_{h}\right\|_{0, \Omega} \leq C h^{\operatorname{máx}(0, s-1)}\|\operatorname{div} \boldsymbol{\sigma}\|_{\operatorname{máx}(0, s-1), \Omega},
\end{gathered}
$$

donde $C$ es una constante positiva independiente de $h$.

Bajo las mismas condiciones, también tiene validez una estimación de orden superior para la norma $L^{2}$ del error en los desplazamientos. En efecto, procediendo (exactamente igual) como en la prueba del Teorema 3.8 en [114], podemos probar la siguiente estimación:

$$
\left\|\mathbf{u}-\mathbf{u}_{h}\right\|_{0, \Omega} \leq C h^{2 s}\|\mathbf{u}\|_{1+s, \Omega} .
$$


Señalamos aquí que este resultado completa el análisis del método no-conforme de Arnold-Winther que se presenta en [24].

Observemos ahora que las siguientes formas bilineales discretas

$$
\begin{gathered}
A_{h}((\boldsymbol{\sigma}, \mathbf{u}),(\boldsymbol{\tau}, \mathbf{v}))=\int_{\Omega} \mathbb{A} \boldsymbol{\sigma}: \boldsymbol{\tau}+\int_{\Omega} \operatorname{div}_{h} \boldsymbol{\tau} \cdot \mathbf{u}+\int_{\Omega} \operatorname{div}_{h} \boldsymbol{\sigma} \cdot \mathbf{v} \\
B_{h}((\boldsymbol{\sigma}, \mathbf{u}),(\boldsymbol{\tau}, \mathbf{v}))=-\int_{\Omega} \mathbf{u} \cdot \mathbf{v}
\end{gathered}
$$

están bien definidas sobre el espacio suma $\boldsymbol{\Sigma}+\boldsymbol{\Sigma}_{h} \times \mathbf{V}+\mathbf{V}_{h}$. Más aún, $A_{h}$ satisface la condición de estabilidad discreta (3.64) con una constante independiente de $h$. Luego, podemos re-escribir el problema (4.42) como sigue:

Dado $(\mathbf{f}, \mathbf{g}) \in L^{2}\left(\Omega, \mathbb{S}^{2}\right) \times L^{2}\left(\Omega, \mathbb{R}^{2}\right)$, encontrar $\left(\boldsymbol{\sigma}_{h}, \mathbf{u}_{h}\right) \in \boldsymbol{\Sigma}_{h} \times \mathbf{V}_{h}$ tal que

$$
A_{h}\left(\left(\boldsymbol{\sigma}_{h}, \mathbf{u}_{h}\right),\left(\boldsymbol{\tau}_{h}, \mathbf{v}_{h}\right)\right)=B_{h}\left((\mathbf{f}, \mathbf{g}),\left(\boldsymbol{\tau}_{h}, \mathbf{v}_{h}\right)\right) \quad \forall\left(\boldsymbol{\tau}_{h}, \mathbf{v}_{h}\right) \in \boldsymbol{\Sigma}_{h} \times \mathbf{V}_{h} .
$$

Denotemos por $\boldsymbol{\Psi}_{h}$ al operador lineal definido por

$$
\begin{aligned}
\mathbf{\Psi}_{h}: L^{2}\left(\Omega, \mathbb{S}^{2}\right) \times L^{2}\left(\Omega, \mathbb{R}^{2}\right) & \rightarrow \boldsymbol{\Sigma}_{h} \times \mathbf{V}_{h} \hookrightarrow L^{2}\left(\Omega, \mathbb{S}^{2}\right) \times L^{2}\left(\Omega, \mathbb{R}^{2}\right) \\
(\mathbf{f}, \mathbf{g}) & \mapsto\left(\boldsymbol{\sigma}_{h}, \mathbf{u}_{h}\right),
\end{aligned}
$$

donde $\left(\boldsymbol{\sigma}_{h}, \mathbf{u}_{h}\right)$ es solución del problema (4.44).

En la próxima sección probaremos que el espectro del operador $\boldsymbol{\Psi}_{h}$ brinda una buena aproximación del espectro del operador $\boldsymbol{\Psi}$. En otras palabras, aproximaremos las soluciones del problema (4.30) por medio de las soluciones del siguiente problema espectral:

Encontrar $\omega_{h} \in \mathbb{R},\left(\boldsymbol{\sigma}_{h}, \mathbf{u}_{h}\right) \in \boldsymbol{\Sigma}_{h} \times \mathbf{V}_{h},\left(\boldsymbol{\sigma}_{h}, \mathbf{u}_{h}\right) \neq(\mathbf{0}, \mathbf{0})$, tal que

$$
\begin{array}{ll}
\int_{\Omega} \mathbb{A} \boldsymbol{\sigma}_{h}: \boldsymbol{\tau}_{h}+\int_{\Omega} \operatorname{div}_{h} \boldsymbol{\tau}_{h} \cdot \mathbf{u}_{h}=0 & \forall \boldsymbol{\tau}_{h} \in \boldsymbol{\Sigma}_{h}, \\
-\int_{\Omega} \operatorname{div}_{h} \boldsymbol{\sigma}_{h} \cdot \mathbf{v}_{h}=\omega_{h}^{2} \int_{\Omega} \rho_{S} \mathbf{u}_{h} \cdot \mathbf{v}_{h} & \forall \mathbf{v}_{h} \in \mathbf{V}_{h} .
\end{array}
$$

Observemos que, como consecuencia de la estimación (4.43), el operador $\boldsymbol{\Psi}_{h}$ está acotado uniformemente en $h$. Además, el operador $\boldsymbol{\Psi}_{h}$ es autoadjunto y definido positivo con respecto a las formas $A_{h}$ y $B_{h}$. Luego, todos sus autovalores serán reales y positivos. Como en el caso continuo, $\omega_{h}^{2}$ es un autovalor del problema (4.45) si, y solo si, $\lambda_{h}=1 / \omega_{h}^{2}$ es un autovalor del operador $\boldsymbol{\Psi}_{h}$ y los correspondientes pares propios asociados coinciden.

\section{Análisis del error}

Usaremos la teoría desarrollada en la sección 3.1 (en la versión que se presenta en la sección 3.4.2) para probar que los autovalores y las autofunciones del problema (4.30) pueden ser aproximados correctamente por los autovalores y las autofunciones correspondientes al problema (4.45).

Comencemos observando que los problemas (4.30) y (4.45) son de la forma (3.62) y (3.65), respectivamente, con las siguientes identificaciones:

$$
\begin{array}{cr}
X(\Omega) \times Y(\Omega)=L^{2}\left(\Omega, \mathbb{S}^{2}\right) \times L^{2}\left(\Omega, \mathbb{R}^{2}\right) & V(\Omega) \times Q(\Omega)=H_{0, \Gamma_{N}}\left(\operatorname{div}, \Omega, \mathbb{S}^{2}\right) \times L^{2}\left(\Omega, \mathbb{R}^{2}\right) \\
(u, \varphi)=(\boldsymbol{\sigma}, \mathbf{u}) & (v, \eta)=(\boldsymbol{\tau}, \mathbf{v}) \\
A((u, \varphi),(v, \eta))=A((\boldsymbol{\sigma}, \mathbf{u}),(\boldsymbol{\tau}, \mathbf{v})) & B((u, \varphi),(v, \eta))=B((\boldsymbol{\sigma}, \mathbf{u}),(\boldsymbol{\tau}, \mathbf{v})) \\
V_{h} \times Q_{h}=\boldsymbol{\Sigma}_{h} \times \mathbf{V}_{h} & \|(u, \varphi)\|_{h}^{2}=\|\boldsymbol{\sigma}\|_{H\left(\operatorname{div}_{h}, \Omega, \mathbb{S}^{2}\right)}^{2}+\|\mathbf{u}\|_{0, \Omega}^{2}
\end{array}
$$

En lo que sigue, escribiremos $\mathbf{W}(h):=\left(\boldsymbol{\Sigma}+\boldsymbol{\Sigma}_{h}\right) \times\left(\mathbf{V}+\mathbf{V}_{h}\right)$ para simplificar la notación.

Sea $\lambda=1 / \omega^{2}$ un autovalor fijo de $\boldsymbol{\Psi}$, con multiplicidad algebraica igual a $m$, y sea $\mathbf{E}_{\lambda}(\mathbf{W}(h))$ el espacio propio de $\boldsymbol{\Psi}$ correspondiente a $\lambda$.

A continuación probaremos que se satisfacen las propiedades $\mathbf{P 1}$ y P2 para el método no-conforme de Arnold-Winther. 
Teorema 4.17 (P1) Existe una constante estrictamente positiva $C$ tal que

$$
\inf _{\left(\boldsymbol{\tau}_{h}, \mathbf{v}_{h}\right) \in \boldsymbol{\Sigma}_{h} \times \mathbf{V}_{h}}\left\|(\boldsymbol{\sigma}, \mathbf{u})-\left(\boldsymbol{\tau}_{h}, \mathbf{v}_{h}\right)\right\|_{h} \leq C h^{s}\left(\|\boldsymbol{\sigma}\|_{s, \Omega}+\|\mathbf{u}\|_{1+s, \Omega}\right) \quad \forall(\boldsymbol{\sigma}, \mathbf{u}) \in \mathbf{E}_{\lambda}(\mathbf{W}(h)) .
$$

Prueba. A partir de los resultados de regularidad para el problema de la elasticidad sobre dominios poligonales, sabemos que $\boldsymbol{\sigma} \in H^{s}\left(\Omega, \mathbb{S}^{2}\right)$ y $\mathbf{u} \in H^{1+s}\left(\Omega, \mathbb{R}^{2}\right)$. Más aún, a partir del problema de autovalores (4.30), concluimos que $\operatorname{div} \boldsymbol{\sigma} \in H^{1+s}\left(\Omega, \mathbb{R}^{2}\right)$ y

$$
\|\operatorname{div} \boldsymbol{\sigma}\|_{1+s, \Omega} \leq C\|\mathbf{u}\|_{1+s, \Omega}
$$

Ahora, como $\mathbf{u} \in H^{1+s}\left(\Omega, \mathbb{R}^{2}\right), \mathbf{u} \in \mathcal{C}^{0}\left(\Omega, \mathbb{R}^{2}\right)$. Por lo tanto, la interpolada lineal de Lagrange de $\mathbf{u}, \mathbf{u}^{I}$, está bien definida (ver [75], por ejemplo). Más aún, $\mathbf{u}^{I} \in \mathbf{V}_{h} \cap H^{1}\left(\Omega, \mathbb{R}^{2}\right)$. Entonces, eligiendo $\mathbf{v}_{h}=\mathbf{u}^{I} \mathrm{y}$ usando resultados de interpolación standard, tendremos

$$
\left\|\mathbf{u}-\mathbf{v}_{h}\right\|_{0, \Omega} \leq C h^{1+s}\|\mathbf{u}\|_{1+s, \Omega}
$$

Como $\boldsymbol{\sigma} \in H\left(\operatorname{div}, \Omega, \mathbb{S}^{2}\right) \cap H^{s}\left(\Omega, \mathbb{S}^{2}\right)$, la interpolada de Arnold-Winther de $\boldsymbol{\sigma}, \boldsymbol{\Pi}_{h}^{A W} \boldsymbol{\sigma}$, está bien definida. Eligiendo $\boldsymbol{\tau}_{h}=\boldsymbol{\Pi}_{h}^{A W} \boldsymbol{\sigma}$ y usando el Lema 4.16, obtendremos

$$
\left\|\boldsymbol{\sigma}-\boldsymbol{\tau}_{h}\right\|_{0, \Omega} \leq C h^{s}\|\boldsymbol{\sigma}\|_{s, \Omega}
$$

Ahora, a partir de la propiedad de conmutatividad (4.38), es claro que

$$
\int_{T}\left(\operatorname{div}\left(\boldsymbol{\sigma}-\boldsymbol{\tau}_{h}\right)\right)^{2}=\int_{T}\left(\operatorname{div}\left(\boldsymbol{\sigma}-\boldsymbol{\Pi}_{h}^{A W} \boldsymbol{\sigma}\right)\right)^{2}=\int_{T}\left(\operatorname{div} \boldsymbol{\sigma}-\mathbf{P}_{h} \operatorname{div} \boldsymbol{\sigma}\right)^{2} .
$$

Sumando sobre todos los triángulos $T \in \mathcal{T}_{h}$ y usando la estimación (4.41), tendremos

$$
\left\|\operatorname{div}_{h}\left(\boldsymbol{\sigma}-\boldsymbol{\tau}_{h}\right)\right\|_{0, \Omega} \leq C h^{1+s}\|\operatorname{div} \boldsymbol{\sigma}\|_{1+s, \Omega} \leq c h^{1+s}\|\mathbf{u}\|_{1+s, \Omega}
$$

Combinando los resultados $(4.46),(4.47)$ y (4.48) con la definición de la norma $\|\cdot\|_{h}$, podemos escribir

$$
\left\|(\boldsymbol{\sigma}, \mathbf{u})-\left(\boldsymbol{\tau}_{h}, \mathbf{v}_{h}\right)\right\|_{h} \leq C h^{s}\left(\|\boldsymbol{\sigma}\|_{s, \Omega}+\|\mathbf{u}\|_{1+s, \Omega}\right) .
$$

La prueba finaliza tomando el ínfimo con respecto a $\left(\boldsymbol{\tau}_{h}, \mathbf{v}_{h}\right) \in \boldsymbol{\Sigma}_{h} \times \mathbf{V}_{h}$.

Teorema 4.18 (P2) Existe una constante positiva $C$ tal que

$$
\left\|\left(\mathbf{\Psi}-\boldsymbol{\Psi}_{h}\right)(\mathbf{f}, \mathbf{g})\right\|_{h} \leq C h^{s}\|(\mathbf{f}, \mathbf{g})\|_{h} \quad \forall(\mathbf{f}, \mathbf{g}) \in \boldsymbol{\Sigma}_{h} \times \mathbf{V}_{h} .
$$

Prueba. Para $(\mathbf{f}, \mathbf{g}) \in \boldsymbol{\Sigma}_{h} \times \mathbf{V}_{h}$, arbitrario, sean $(\boldsymbol{\sigma}, \mathbf{u}):=\boldsymbol{\Psi}(\mathbf{f}, \mathbf{g})$ y $\left(\boldsymbol{\sigma}_{h}, \mathbf{u}_{h}\right):=\boldsymbol{\Psi}_{h}(\mathbf{f}, \mathbf{g})$. Siguiendo los argumentos de Brezzi-Fortin (ver Proposición 2.16 en [56]) y teniendo en cuenta que el par de espacios $\left(\boldsymbol{\Sigma}_{h}, \mathbf{V}_{h}\right)$ satisface las condiciones de estabilidad de Brezzi, es fácil mostrar que

$$
\left\|(\boldsymbol{\sigma}, \mathbf{u})-\left(\boldsymbol{\sigma}_{h}, \mathbf{u}_{h}\right)\right\|_{h} \leq C\left(\inf _{\boldsymbol{\tau}_{h} \in \boldsymbol{\Sigma}_{h}}\left\|\boldsymbol{\sigma}-\boldsymbol{\tau}_{h}\right\|_{H\left(\operatorname{div}_{h}, \Omega, \mathbb{S}^{2}\right)}+\inf _{\mathbf{v}_{h} \in \mathbf{V}_{h}}\left\|\mathbf{u}-\mathbf{v}_{h}\right\|_{0, \Omega}+N_{1 h}+N_{2 h}\right)
$$

donde

$$
N_{1 h}=\sup _{\boldsymbol{\tau}_{h} \in \boldsymbol{\Sigma}_{h}} \frac{\left|\int_{\Omega} \mathbb{A} \boldsymbol{\sigma}: \boldsymbol{\tau}_{h}+\int_{\Omega} \operatorname{div}{ }_{h} \boldsymbol{\tau}_{h} \cdot \mathbf{u}\right|}{\left.\left\|\boldsymbol{\tau}_{h}\right\|_{H\left(\operatorname{div}_{h}, \Omega, \mathbb{S}^{2}\right.}\right)}
$$

y

$$
N_{2 h}=\sup _{\mathbf{v}_{h} \in \mathbf{V}_{h}} \frac{\left|\int_{\Omega} \operatorname{div} \boldsymbol{\sigma} \cdot \mathbf{v}_{h}+\int_{\Omega} \mathbf{g} \cdot \mathbf{v}_{h}\right|}{\left\|\mathbf{v}_{h}\right\|_{0, \Omega}} .
$$


Para acotar los dos primeros términos del lado derecho de la desigualdad anterior podemos repetir los argumentos usados en la prueba del Teorema 4.17. Observemos primero que, como $\mathbf{g} \in \mathbf{V}_{h}$,

$$
\inf _{\boldsymbol{\tau}_{h} \in \boldsymbol{\Sigma}_{h}}\left\|\boldsymbol{\sigma}-\boldsymbol{\tau}_{h}\right\|_{H\left(\operatorname{div}_{h}, \Omega, \mathbb{S}^{2}\right)}=\inf _{\boldsymbol{\tau}_{h} \in \boldsymbol{\Sigma}_{h}}\left\|\boldsymbol{\sigma}-\boldsymbol{\tau}_{h}\right\|_{0, \Omega}
$$

Luego, usando la estimación (4.34), obtendremos

$$
\begin{gathered}
\inf _{\boldsymbol{\tau}_{h} \in \mathbf{\Sigma}_{h}}\left\|\boldsymbol{\sigma}-\boldsymbol{\tau}_{h}\right\|_{0, \Omega} \leq C h^{s}\|(\mathbf{f}, \mathbf{g})\|_{h}, \\
\inf _{\mathbf{v}_{h} \in \mathbf{V}_{h}}\left\|\mathbf{u}-\mathbf{v}_{h}\right\|_{0, \Omega} \leq C h^{1+s}\|(\mathbf{f}, \mathbf{g})\|_{h} .
\end{gathered}
$$

Ahora acotaremos el tercer término. A partir de la ecuación constitutiva para las deformaciones infinitesimales (ver problema (4.31)), podemos escribir

$$
\sum_{T \in \mathcal{T}_{h}}\left(\int_{T} \mathbb{A} \boldsymbol{\sigma}: \boldsymbol{\tau}_{h}-\int_{T} \boldsymbol{\varepsilon}(\mathbf{u}): \boldsymbol{\tau}_{h}\right)=0 \quad \forall \boldsymbol{\tau}_{h} \in \boldsymbol{\Sigma}_{h}
$$

Teniendo en cuenta la simetría del tensor $\boldsymbol{\tau}_{h}$ e integrando por partes,

$$
\int_{\Omega} \boldsymbol{\varepsilon}(\mathbf{u}): \boldsymbol{\tau}_{h}=\int_{\Omega} \nabla \mathbf{u}: \boldsymbol{\tau}_{h}=\sum_{T \in \mathcal{T}_{h}}\left(-\int_{T} \mathbf{u} \cdot \operatorname{div} \boldsymbol{\tau}_{h}+\int_{\partial T} \mathbf{u} \cdot \boldsymbol{\tau}_{h} \mathbf{n}\right) .
$$

Como $\mathbf{u}$ es continua en $\Omega$ y $\boldsymbol{\tau}_{h} \mathbf{n}_{\ell} \cdot \mathbf{n}_{\ell}$ es continua sobre cada lado interno $\ell \in \mathcal{E}_{h}$, podemos escribir

$$
\sum_{T \in \mathcal{T}_{h}} \int_{\partial T} \mathbf{u} \cdot \boldsymbol{\tau}_{h} \mathbf{n}=\sum_{\ell \in \mathcal{E}_{h}} \int_{\ell}\left(\mathbf{u} \cdot \mathbf{t}_{\ell}\right)\left[\left[\boldsymbol{\tau}_{h} \mathbf{n}_{\ell} \cdot \mathbf{t}_{\ell}\right]\right]
$$

donde $[[\cdot]]$ indica el salto del término $\boldsymbol{\tau}_{h} \mathbf{n}_{\ell} \cdot \mathbf{t}_{\ell}$ a través del lado $\ell$. Concluimos, entonces, que

$$
N_{1 h}=\sup _{\boldsymbol{\tau}_{h} \in \boldsymbol{\Sigma}_{h}} \frac{\left|\sum_{\ell \in \mathcal{E}_{h}} \int_{\ell}\left(\mathbf{u} \cdot \mathbf{t}_{\ell}\right)\left[\left[\boldsymbol{\tau}_{h} \mathbf{n}_{\ell} \cdot \mathbf{t}_{\ell}\right]\right]\right|}{\left\|\boldsymbol{\tau}_{h}\right\|_{H\left(\operatorname{div}_{h}, \Omega, \mathbb{S}^{2}\right)}} .
$$

Sea $P_{\ell}$ la proyección $L_{\ell}^{2}$ de $H^{\epsilon}(\ell)$ sobre las funciones lineales definidas sobre $\ell$. Para $\ell \in \mathcal{E}_{h}$, sean $T_{1}$ y $T_{2}$ los triángulos en $\mathcal{T}_{h}$ tales que $T_{1} \cap T_{2}=\ell$. Como $\left[\left[\boldsymbol{\tau}_{h} \mathbf{n}_{\ell} \cdot \mathbf{t}_{\ell}\right]\right]$ es ortogonal a cualquier polinomio de grado uno sobre $\ell$, tendremos

$$
\begin{aligned}
\left|\int_{\ell}\left(\mathbf{u} \cdot \mathbf{t}_{\ell}\right)\left[\left[\boldsymbol{\tau}_{h} \mathbf{n}_{\ell} \cdot \mathbf{t}_{\ell}\right]\right]\right| & =\left|\int_{\ell}\left(\mathbf{u} \cdot \mathbf{t}_{\ell}-P_{\ell}\left(\mathbf{u} \cdot \mathbf{t}_{\ell}\right)\right)\left[\left[\boldsymbol{\tau}_{h} \mathbf{n}_{\ell} \cdot \mathbf{t}_{\ell}\right]\right]\right| \\
& \leq \sum_{i=1,2}\left|\int_{\ell}\left(\mathbf{u} \cdot \mathbf{t}_{\ell}-P_{\ell}\left(\mathbf{u} \cdot \mathbf{t}_{\ell}\right)\right)\left(\left.\boldsymbol{\tau}_{h} \mathbf{n}_{\ell} \cdot \mathbf{t}_{\ell}\right|_{T_{i}}\right)\right|
\end{aligned}
$$

Ahora bien, $\boldsymbol{\tau}_{h} \mathbf{n}_{\ell} \cdot \mathbf{t}_{\ell} \in \mathcal{P}_{2}(\ell)$. Luego, un argumento clásico de scaling nos conduce a

$$
\left\|\left.\boldsymbol{\tau}_{h} \mathbf{n}_{\ell} \cdot \mathbf{t}_{\ell}\right|_{T_{i}}\right\|_{0, \ell} \leq C|\ell|^{-1 / 2}\left\|\boldsymbol{\tau}_{h}\right\|_{0, T_{i}}
$$

Sea $P_{T}$ la proyección $L_{T}^{2}$ de $H^{\epsilon+1 / 2}(T)$ sobre las funciones lineales definidas sobre $T$. Usando un teorema de trazas local, una estimación de error standard para la proyección $P_{T}$ y la acotación (4.51), obtendremos

$$
\begin{aligned}
\left|\int_{\ell}\left(\mathbf{u} \cdot \mathbf{t}_{\ell}\right)\left[\left[\boldsymbol{\tau}_{h} \mathbf{n}_{\ell} \cdot \mathbf{t}_{\ell}\right]\right]\right| & \leq \sum_{i=1,2}\left\|\mathbf{u} \cdot \mathbf{t}_{\ell}-P_{T}\left(\mathbf{u} \cdot \mathbf{t}_{\ell}\right)\right\|_{0, \ell}\left\|\left.\boldsymbol{\tau}_{h} \mathbf{n}_{\ell} \cdot \mathbf{t}_{\ell}\right|_{T_{i}}\right\|_{0, \ell} \\
& \leq C \sum_{i=1,2}\left(|\ell|^{s+1 / 2}\|\mathbf{u}\|_{1+s, T_{i}}\right)\left(|\ell|^{-1 / 2}\left\|\boldsymbol{\tau}_{h}\right\|_{0, T_{i}}\right) .
\end{aligned}
$$


Sumando sobre todos los lados $\ell \in \mathcal{E}_{h}$ y usando la estimación (4.34), podremos escribir

$$
N_{1 h} \leq C h^{s}\|(\mathbf{f}, \mathbf{g})\|_{h} .
$$

El término de consistencia restante $N_{2 h}$ es idénticamente cero. En efecto, a partir de la ecuación de equilibrio (ver problema (4.31)), obtendremos inmediatamente

$$
-\int_{\Omega} \operatorname{div} \boldsymbol{\sigma} \cdot \mathbf{v}_{h}=\int_{\Omega} \mathbf{g} \cdot \mathbf{v}_{h} \quad \forall \mathbf{v}_{h} \in \mathbf{V}_{h} .
$$

Así, concluimos la prueba.

Así, como se establece en la sección 3.1, los teoremas previos nos permiten afirmar que el espectro de los operadores $\boldsymbol{\Psi}_{h}$ proporciona una buena aproximación del espectro de $\boldsymbol{\Psi}$.

A continuación obtendremos los órdenes con los que convergen los autovectores y los autovalores aproximados. Observemos que podemos elegir $\|\cdot\|_{*}=\|\cdot\|_{h}$ y que, por lo tanto, la propiedad P3 se convierte en la propiedad $\mathbf{P} 1$.

El siguiente teorema nos permitirá determinar un orden óptimo de convergencia para los vectores propios aproximados.

Teorema 4.19 (P4) Existe una constante positiva $C$ tal que

$$
\left\|\left.\left(\mathbf{\Psi}-\mathbf{\Psi}_{h}\right)\right|_{\mathbf{E}_{\lambda}(\mathbf{W}(h))}\right\|_{h} \leq C h^{s} .
$$

Prueba. Para $(\boldsymbol{\xi}, \mathbf{z}) \in \mathbf{E}_{\lambda}(\mathbf{W}(h))$, la solución $(\boldsymbol{\sigma}, \mathbf{u})=\boldsymbol{\Psi}(\boldsymbol{\xi}, \mathbf{z}) \in H^{s}\left(\Omega, \mathbb{S}^{2}\right) \times H^{1+s}\left(\Omega, \mathbb{R}^{2}\right)$. Luego, procedemos exactamente igual que en la prueba del Teorema 4.18 , reemplazando $(\mathbf{f}, \mathbf{g})$ por $(\boldsymbol{\xi}, \mathbf{z})$.

De acuerdo con el Teorema 3.26,

- si $h$ es suficientemente pequeño, todos los autovectores del problema discreto son aproximados por autovectores del problema de continuo,

- cualquier autovector del problema continuo correspondiente a $\lambda$ puede ser aproximado con un error de orden $h^{s}$.

Estimaremos el orden con el que convergen los autovalores discretos vía Teorema 3.29. Para ello, necesitaremos acotar los términos de consistencia $M_{h}$.

\section{Lema 4.20 Sea}

$$
M_{h}=\sup _{\substack{(\boldsymbol{\tau}, \mathbf{y}) \in \mathbf{E}(\mathbf{W}(h)) \\\|(\boldsymbol{\tau}, \mathbf{y})\|_{h}=1}} \sup _{\substack{\boldsymbol{\xi}, \mathbf{z}) \in \mathbf{E}(\mathbf{W}(h)) \\\|(\boldsymbol{\xi}, \mathbf{z})\|_{h}=1}}\left|A_{h}\left(\boldsymbol{\Psi}(\boldsymbol{\tau}, \mathbf{y}), \boldsymbol{\Pi}_{h}(\boldsymbol{\xi}, \mathbf{z})\right)-B_{h}\left((\boldsymbol{\tau}, \mathbf{y}), \boldsymbol{\Pi}_{h}(\boldsymbol{\xi}, \mathbf{z})\right)\right|,
$$

siendo $\boldsymbol{\Pi}_{h}$ la proyección sobre $\boldsymbol{\Sigma}_{h} \times \mathbf{V}_{h}$ con respecto a la forma bilineal $A_{h}$ definida por la ecuación (3.67). Existe una constante positiva $C$ tal que

$$
M_{h} \leq C h^{2 s} .
$$

Prueba. Elijamos $(\boldsymbol{\tau}, \mathbf{y}) \in \mathbf{E}_{\lambda}(\mathbf{W}(h))$, con $\|(\boldsymbol{\tau}, \mathbf{y})\|_{h}=1$. Llamemos $(\boldsymbol{\eta}, \mathbf{w})=\boldsymbol{\Psi}(\boldsymbol{\tau}, \mathbf{y})$. Argumentando como en el Teorema 4.17, concluimos que $(\boldsymbol{\eta}, \mathbf{w}) \in H^{s}\left(\Omega, \mathbb{S}^{2}\right) \times H^{1+s}\left(\Omega, \mathbb{R}^{2}\right)$ y $\operatorname{div} \boldsymbol{\eta} \in H^{1+s}\left(\Omega, \mathbb{R}^{2}\right)$. Más aún, a partir de la estimación (4.34) y la definición de la norma $\|\cdot\|_{h}$, tendremos

$$
\|\boldsymbol{\eta}\|_{s, \Omega}+\|\operatorname{div} \boldsymbol{\eta}\|_{1+s, \Omega}+\|\mathbf{w}\|_{1+s, \Omega} \leq C\|(\boldsymbol{\tau}, \mathbf{y})\|_{h} \leq C .
$$

Testeando la siguiente ecuación

$$
\int_{\Omega} \mathbb{A} \boldsymbol{\eta}: \boldsymbol{\phi}+\int_{\Omega} \operatorname{div} \boldsymbol{\phi} \cdot \mathbf{w}-\int_{\Omega} \operatorname{div} \boldsymbol{\eta} \cdot \mathbf{x}=\int_{\Omega} \mathbf{y} \cdot \mathbf{x} \quad \forall(\boldsymbol{\phi}, \mathbf{x}) \in \mathbf{\Sigma} \times \mathbf{V}
$$


con funciones suficientemente suaves, puede probarse que el par $(\boldsymbol{\eta}, \mathbf{w})$ es solución (en el sentido de las distribuciones) del siguiente problema fuerte:

$$
\begin{aligned}
\mathbb{A} \boldsymbol{\eta}-\boldsymbol{\varepsilon}(\mathbf{w})=\mathbf{0} & \text { in } \Omega, \\
-\operatorname{div} \boldsymbol{\eta}=\mathbf{y} & \text { in } \Omega, \\
\mathbf{w}=\mathbf{0} & \text { on } \Gamma_{D} \\
\boldsymbol{\eta} \mathbf{n}=\mathbf{0} & \text { on } \Gamma_{N}
\end{aligned}
$$

Sea $(\boldsymbol{\xi}, \mathbf{z}) \in \mathbf{E}_{\lambda}(\mathbf{W}(h))$, un par arbitrario con $\|(\boldsymbol{\xi}, \mathbf{z})\|_{h}=1$. Llamemos $\left(\boldsymbol{\xi}_{h}, \mathbf{z}_{h}\right)=\boldsymbol{\Pi}_{h}(\boldsymbol{\xi}, \mathbf{z})$. A continuación, seguiremos el procedimiento usual para obtener la formulación stress-displazamiento del problema elástico en el plano (ver [56], por ejemplo): multiplicamos la primera y la segunda ecuación del sistema (4.54) por $\boldsymbol{\xi}_{h}$ y $\mathbf{z}_{h}$, respectivamente, e integramos por partes. Luego, usando los mismos argumentos que los empleados en la prueba del teorema anterior, obtendremos

$$
A_{h}\left((\boldsymbol{\eta}, \mathbf{w}),\left(\boldsymbol{\xi}_{h}, \mathbf{z}_{h}\right)\right)-B_{h}\left((\boldsymbol{\eta}, \mathbf{y}),\left(\boldsymbol{\xi}_{h}, \mathbf{z}_{h}\right)\right)=\sum_{\ell \in \mathcal{E}_{h}} \int_{\ell}\left(\mathbf{w} \cdot \mathbf{t}_{\ell}\right)\left[\left[\boldsymbol{\xi}_{h} \mathbf{n}_{\ell} \cdot \mathbf{t}_{\ell}\right]\right] .
$$

Para $\ell \in \mathcal{E}_{h}$, sean $T_{1}$ y $T_{2}$ los triángulos en $\mathcal{T}_{h}$ tales que $T_{1} \cap T_{2}=\ell$. Como $\left[\left[\boldsymbol{\xi}_{h} \mathbf{n}_{\ell} \cdot \mathbf{t}_{\ell}\right]\right]$ es ortogonal a cualquier polinomio de grado uno definido sobre $\ell$, tendremos

$$
\begin{aligned}
\left|\int_{\ell}\left(\mathbf{w} \cdot \mathbf{t}_{\ell}\right)\left[\left[\boldsymbol{\xi}_{h} \mathbf{n}_{\ell} \cdot \mathbf{t}_{\ell}\right]\right]\right| & =\left|\int_{\ell}\left(\mathbf{w} \cdot \mathbf{t}_{\ell}-P_{\ell}\left(\mathbf{w} \cdot \mathbf{t}_{\ell}\right)\right)\left[\left[\boldsymbol{\xi}_{h} \mathbf{n}_{\ell} \cdot \mathbf{t}_{\ell}\right]\right]\right| \\
& \leq \sum_{i=1,2}\left|\int_{\ell}\left(\mathbf{w} \cdot \mathbf{t}_{\ell}-P_{\ell}\left(\mathbf{w} \cdot \mathbf{t}_{\ell}\right)\right) \boldsymbol{\xi}_{h} \mathbf{n}_{\ell} \cdot \mathbf{t}_{\ell}\right|_{T_{i}} \mid \\
& \leq \sum_{i=1,2}\left|\int_{\ell}\left(\mathbf{w} \cdot \mathbf{t}_{\ell}-P_{\ell}\left(\mathbf{w} \cdot \mathbf{t}_{\ell}\right)\right)\left(\boldsymbol{\xi}_{h} \mathbf{n}_{\ell} \cdot \mathbf{t}_{\ell}-P_{\ell}\left(\boldsymbol{\xi}_{h} \mathbf{n}_{\ell} \cdot \mathbf{t}_{\ell}\right)\right)\right|_{T_{i}} \mid \\
& \leq \sum_{i=1,2}\left\|\mathbf{w} \cdot \mathbf{t}_{\ell}-P_{T}\left(\mathbf{w} \cdot \mathbf{t}_{\ell}\right)\right\|_{0, \ell}\left\|\left.\left(\boldsymbol{\xi}_{h} \mathbf{n}_{\ell} \cdot \mathbf{t}_{\ell}-P_{T}\left(\boldsymbol{\xi}_{h} \mathbf{n}_{\ell} \cdot \mathbf{t}_{\ell}\right)\right)\right|_{T_{i}}\right\|_{0, \ell} .
\end{aligned}
$$

Dado que $\mathbf{w} \in H^{1+s}\left(\Omega, \mathbb{R}^{2}\right)$, usando un teorema de trazas local y estimaciones del error standard para la proyección $P_{T}$, podremos escribir

$$
\left\|\mathbf{w} \cdot \mathbf{t}_{\ell}-P_{T}\left(\mathbf{w} \cdot \mathbf{t}_{\ell}\right)\right\|_{0, \ell} \leq C|\ell|^{s+1 / 2}\|\mathbf{w}\|_{1+s, T_{i}} .
$$

Por otro lado, usando un teorema de trazas local y una desigualdad inversa, obtendremos

$$
\left\|\left.\left(\boldsymbol{\xi}_{h} \mathbf{n}_{\ell} \cdot \mathbf{t}_{\ell}-P_{T}\left(\boldsymbol{\xi}_{h} \mathbf{n}_{\ell} \cdot \mathbf{t}_{\ell}\right)\right)\right|_{T_{i}}\right\|_{0, \ell} \leq C|\ell|^{-1 / 2}\left\|\boldsymbol{\xi}_{h}-P_{T} \boldsymbol{\xi}_{h}\right\|_{0, T_{i}}
$$

Además, es inmediato que

$$
\left\|\boldsymbol{\xi}_{h}-P_{T} \boldsymbol{\xi}_{h}\right\|_{0, T_{i}} \leq\left\|\left(\boldsymbol{\xi}_{h}-\boldsymbol{\xi}\right)-P_{T}\left(\boldsymbol{\xi}_{h}-\boldsymbol{\xi}\right)\right\|_{0, T_{i}}+\left\|\boldsymbol{\xi}-P_{T} \boldsymbol{\xi}\right\|_{0, T_{i}} \leq 2\left\|\boldsymbol{\xi}-\boldsymbol{\xi}_{h}\right\|_{0, T_{i}}+\left\|\boldsymbol{\xi}-P_{T} \boldsymbol{\xi}\right\|_{0, T_{i}} .
$$

Ahora, observemos que el operador de interpolación de Arnold-Winther puede identificarse con el operador de Fortin del problema (4.42). Elijamos entonces $\boldsymbol{\xi}_{h}=\boldsymbol{\Pi}_{h}^{A W} \boldsymbol{\xi}$. Usando el Lema 4.16 y estimaciones de error standard para la proyección $P_{T}$, tendremos

$$
\left|\int_{\ell}\left(\mathbf{w} \cdot \mathbf{t}_{\ell}\right)\left[\left[\boldsymbol{\xi}_{h} \mathbf{n}_{\ell} \cdot \mathbf{t}_{\ell}\right]\right]\right| \leq C h^{2 s} \sum_{i=1,2}\left(\|\boldsymbol{\xi}\|_{s, T_{i}}+|\boldsymbol{\xi}|_{s, T_{i}}\right)\|\mathbf{w}\|_{1+s, T_{i}} .
$$

Luego, sumando sobre todos $\operatorname{los} \operatorname{lados} \ell \in \mathcal{E}_{h}$ y usando la estimación (4.53) concluimos la prueba.

Teorema 4.21 Existe una constante positiva $C$ tal que

$$
\operatorname{máx}_{i=1, \cdots, m}\left|\lambda-\lambda_{i h}\right| \leq C h^{2 s} .
$$

Prueba. Es una consecuencia inmediata de las propiedades $\mathbf{P 1}$ y $\mathbf{P} 4$ y del lema anterior. 


\section{Estimaciones del error en norma $L^{2}\left(\Omega, \mathbb{R}^{2}\right)$}

Como hemos señalado previamente, los desplazamientos que definen las deformaciones en una estructura elástica sometida a la acción de una carga volumétrica $\mathbf{g}$ (ver problema (4.42)) pueden ser aproximados con orden $h^{2 s}$.

Nuestro próximo objetivo es probar que también se obtiene un doble orden de convergencia para el problema de vibraciones (4.45). Ya que los desplazamientos corresponden a la segunda componente de los pares propios del operador $\boldsymbol{\Psi}$, alcanzaremos este objetivo aplicando directamente los resultados presentados en la sección 3.4.2. De acuerdo con estos resultados, entonces, solo nos resta probar la propiedad P6.

En lo que sigue, consideraremos $(\mathbf{f}, \mathbf{g}) \in \mathbf{E}_{\lambda}(\mathbf{W}(h))$ fijo y denotaremos

$$
(\boldsymbol{\sigma}, \mathbf{u}):=\mathbf{\Psi}(\mathbf{f}, \mathbf{g}) \in \mathbf{E}_{\lambda}(\mathbf{W}(h)), \quad\left(\boldsymbol{\sigma}_{h}, \mathbf{u}_{h}\right):=\mathbf{\Psi}_{h}(\mathbf{f}, \mathbf{g}) \in \mathbf{E}_{\lambda}\left(\mathbf{W}_{h}\right), \quad \mathbf{e}:=\mathbf{u}-\mathbf{u}_{h} .
$$

Usaremos un argumento de dualidad basado en el siguiente problema auxiliar:

$$
\begin{aligned}
\mathbb{A} \boldsymbol{\eta}-\boldsymbol{\varepsilon}(\mathbf{w})=\mathbf{0} & \text { in } \Omega, \\
-\operatorname{div} \boldsymbol{\eta}=-\mathbf{e} & \text { in } \Omega, \\
\mathbf{w}=\mathbf{0} & \text { on } \Gamma .
\end{aligned}
$$

Observemos que es un problema de elasticidad en el plano, con fuente e $\in L^{2}\left(\Omega, \mathbb{R}^{2}\right)$ y condiciones homogéneas de borde en los desplazamientos. Luego, a partir de los resultados de regularidad clásicos (ver [112], por ejemplo), obtendremos la siguiente estimación a priori para su solución: $\boldsymbol{\eta} \in H^{s}\left(\Omega, \mathbb{S}^{2}\right)$, $\mathbf{w} \in H^{1+s}\left(\Omega, \mathbb{R}^{2}\right) \mathrm{y}$

$$
\|\boldsymbol{\eta}\|_{s, \Omega}+\|\operatorname{div} \boldsymbol{\eta}\|_{0, \Omega}+\|\mathbf{w}\|_{1+s, \Omega} \leq C\|\mathbf{e}\|_{0, \Omega} .
$$

Lema 4.22 Existe una constante positiva $C$ tal que

$$
\|\mathbf{e}\|_{0, \Omega} \leq C h^{2 s}\|\mathbf{g}\|_{0, \Omega} \quad \forall(\mathbf{f}, \mathbf{g}) \in \mathbf{E}_{\lambda}(\mathbf{W}(h)) .
$$

Prueba. A partir del sistema de ecuaciones (4.55), es inmediato que

$$
\|\mathbf{e}\|_{0, \Omega}^{2}=\int_{\Omega} \mathbf{e} \cdot \operatorname{div} \boldsymbol{\eta}=\int_{\Omega} \mathbf{e} \cdot \operatorname{div}_{h}\left(\boldsymbol{\eta}-\boldsymbol{\Pi}_{h}^{A W} \boldsymbol{\eta}\right)+\int_{\Omega} \mathbf{e} \cdot \operatorname{div}_{h}\left(\boldsymbol{\Pi}_{h}^{A W} \boldsymbol{\eta}\right) .
$$

Como $\operatorname{div}_{h}\left(\boldsymbol{\Pi}_{h}^{A W} \boldsymbol{\eta}\right)=\mathbf{P}_{h}(\operatorname{div} \boldsymbol{\eta})$, el primer término en el lado derecho de la ecuación anterior puede ser acotado como sigue

$$
\begin{aligned}
\left|\sum_{T \in \mathcal{T}_{h}} \int_{T} \mathbf{e} \cdot \operatorname{div}\left(\boldsymbol{\eta}-\mathbf{\Pi}_{h}^{A W} \boldsymbol{\eta}\right)\right| & =\left|\sum_{T \in \mathcal{T}_{h}} \int_{T}\left(\mathbf{u}-\mathbf{P}_{h} \mathbf{u}\right) \cdot\left(\operatorname{div} \boldsymbol{\eta}-\mathbf{P}_{h} \operatorname{div} \boldsymbol{\eta}\right)\right| \\
& \leq \sum_{T \in \mathcal{T}_{h}}\left\|\mathbf{u}-\mathbf{P}_{h} \mathbf{u}\right\|_{0, T}\left\|\operatorname{div} \boldsymbol{\eta}-\mathbf{P}_{h} \operatorname{div} \boldsymbol{\eta}\right\|_{0, T} \\
& \leq C h^{1+s}\|\mathbf{u}\|_{1+s, \Omega}\|\operatorname{div} \boldsymbol{\eta}\|_{0, \Omega},
\end{aligned}
$$

donde hemos usado la estimación (4.41) para obtener la última desigualdad.

Por otro lado, a partir de la definición del problema (4.42), es inmediato que

$$
\int_{\Omega} \mathbb{A} \boldsymbol{\sigma}_{h}:\left(\boldsymbol{\Pi}_{h}^{A W} \boldsymbol{\eta}\right)+\int_{\Omega} \mathbf{u}_{h} \cdot \operatorname{div}{ }_{h}\left(\boldsymbol{\Pi}_{h}^{A W} \boldsymbol{\eta}\right)=0
$$

mientras que, procediendo como en la prueba del Teorema 4.18, tendremos

$$
\int_{\Omega} \mathbb{A} \boldsymbol{\sigma}:\left(\boldsymbol{\Pi}_{h}^{A W} \boldsymbol{\eta}\right)+\int_{\Omega} \mathbf{u} \cdot \operatorname{div}{ }_{h}\left(\boldsymbol{\Pi}_{h}^{A W} \boldsymbol{\eta}\right)=\sum_{\ell \in \mathcal{E}_{h}} \int_{\ell}\left(\mathbf{u} \cdot \mathbf{t}_{\ell}\right)\left[\left[\left(\boldsymbol{\Pi}_{h}^{A W} \boldsymbol{\eta}\right) \mathbf{n}_{\ell} \cdot \mathbf{t}_{\ell}\right]\right] .
$$

Combinando las dos últimas ecuaciones, es claro que el segundo término de la ecuación de error puede escribirse como sigue

$$
\int_{\Omega} \mathbf{e} \cdot \operatorname{div}{ }_{h}\left(\boldsymbol{\Pi}_{h}^{A W} \boldsymbol{\eta}\right)=-\int_{\Omega} \mathbb{A}\left(\boldsymbol{\sigma}-\boldsymbol{\sigma}_{h}\right):\left(\boldsymbol{\Pi}_{h}^{A W} \boldsymbol{\eta}\right)+\sum_{\ell \in \mathcal{E}_{h}} \int_{\ell}\left(\mathbf{u} \cdot \mathbf{t}_{\ell}\right)\left[\left[\left(\boldsymbol{\Pi}_{h}^{A W} \boldsymbol{\eta}\right) \mathbf{n}_{\ell} \cdot \mathbf{t}_{\ell}\right]\right] .
$$


Vamos a acotar los términos que aparecen en el lado derecho de esta ecuación por separado.

Acotación del primer término. Es evidente que

$$
-\int_{\Omega} \mathbb{A}\left(\boldsymbol{\sigma}-\boldsymbol{\sigma}_{h}\right):\left(\boldsymbol{\Pi}_{h}^{A W} \boldsymbol{\eta}\right)=\int_{\Omega} \mathbb{A}\left(\boldsymbol{\sigma}-\boldsymbol{\sigma}_{h}\right):\left(\boldsymbol{\eta}-\boldsymbol{\Pi}_{h}^{A W} \boldsymbol{\eta}\right)-\int_{\Omega} \mathbb{A}\left(\boldsymbol{\sigma}-\boldsymbol{\sigma}_{h}\right): \boldsymbol{\eta} .
$$

El primero de estos términos se puede acotar fácilmente. En efecto, teniendo en cuenta que $\mathbb{A}$ está uniformemente acotada por arriba, usamos el Teorema 4.19 y el Lema 4.16 para obtener

$$
\left|\int_{\Omega} \mathbb{A}\left(\boldsymbol{\sigma}-\boldsymbol{\sigma}_{h}\right):\left(\boldsymbol{\eta}-\boldsymbol{\Pi}_{h}^{A W} \boldsymbol{\eta}\right)\right| \leq C\left\|\mathbb{A}\left(\boldsymbol{\sigma}-\boldsymbol{\sigma}_{h}\right)\right\|_{0, \Omega}\left\|\left(\boldsymbol{\eta}-\boldsymbol{\Pi}_{h}^{A W} \boldsymbol{\eta}\right)\right\|_{0, \Omega} \leq C h^{2 s}\|\mathbf{g}\|_{0, \Omega}\|\boldsymbol{\eta}\|_{s, \Omega} .
$$

A partir de las ecuaciones (4.55), el término restante puede escribirse como

$$
-\int_{\Omega} \mathbb{A}\left(\boldsymbol{\sigma}-\boldsymbol{\sigma}_{h}\right): \boldsymbol{\eta}=-\int_{\Omega} \mathbb{A} \boldsymbol{\eta}:\left(\boldsymbol{\sigma}-\boldsymbol{\sigma}_{h}\right)=-\int_{\Omega} \varepsilon(\mathbf{w}):\left(\boldsymbol{\sigma}-\boldsymbol{\sigma}_{h}\right) .
$$

Después de integrar por partes, tendremos

$$
\int_{\Omega} \boldsymbol{\varepsilon}(\mathbf{w}):\left(\boldsymbol{\sigma}-\boldsymbol{\sigma}_{h}\right)=-\int_{\Omega} \mathbf{w} \cdot \operatorname{div}{ }_{h}\left(\boldsymbol{\sigma}-\boldsymbol{\sigma}_{h}\right)+\sum_{\ell \in \mathcal{E}_{h}} \int_{\ell}\left(\mathbf{w} \cdot \mathbf{t}_{\ell}\right)\left[\left[\left(\boldsymbol{\sigma}-\boldsymbol{\sigma}_{h}\right) \mathbf{n}_{\ell} \cdot \mathbf{t}_{\ell}\right]\right] .
$$

Como $\operatorname{div}{ }_{h} \boldsymbol{\sigma}_{h}=\mathbf{P}_{h} \operatorname{div} \boldsymbol{\sigma}$,

$$
\int_{\Omega} \mathbf{w} \cdot \operatorname{div}{ }_{h}\left(\boldsymbol{\sigma}-\boldsymbol{\sigma}_{h}\right)=\int_{\Omega}\left(\mathbf{w}-\mathbf{P}_{h} \mathbf{w}\right) \cdot \operatorname{div}_{h}\left(\boldsymbol{\sigma}-\boldsymbol{\sigma}_{h}\right)
$$

Luego, usando la estimación (4.41) y el Teorema 4.19, obtendremos

$$
\left|\int_{\Omega} \mathbf{w} \cdot \operatorname{div}_{h}\left(\boldsymbol{\sigma}-\boldsymbol{\sigma}_{h}\right)\right| \leq C\left\|\mathbf{w}-\mathbf{P}_{h} \mathbf{w}\right\|_{0, \Omega}\left\|\boldsymbol{\sigma}-\boldsymbol{\sigma}_{h}\right\|_{H\left(\operatorname{div}_{h}, \Omega, \mathbb{S}^{2}\right)} \leq C h^{2 s+1}\|\mathbf{w}\|_{1+s, \Omega}\|\mathbf{g}\|_{0, \Omega} .
$$

Sea $P_{\ell}$ la proyección $L_{\ell}^{2}$ de $H^{\epsilon}(\ell)$ sobre las funciones lineales definidas en $\ell$. Para $\ell \in \mathcal{E}_{h}$, sean $T_{1}$ y $T_{2}$ los triángulos en $\mathcal{T}_{h}$ tales que $T_{1} \cap T_{2}=\ell$. Como $\boldsymbol{\sigma} \in H\left(\operatorname{div}, \Omega, \mathbb{S}^{2}\right)$ y $\boldsymbol{\sigma}_{h} \in \boldsymbol{\Sigma}_{h}$, [[( $\left.\left.\left.\boldsymbol{\sigma}-\boldsymbol{\sigma}_{h}\right) \mathbf{n}_{\ell} \cdot \mathbf{t}_{\ell}\right]\right]$ es ortogonal a cualquier polinomio de grado uno definido sobre $\ell$. Entonces

$$
\begin{aligned}
\left|\int_{\ell}\left(\mathbf{w} \cdot \mathbf{t}_{\ell}\right)\left[\left[\left(\boldsymbol{\sigma}-\boldsymbol{\sigma}_{h}\right) \mathbf{n}_{\ell} \cdot \mathbf{t}_{\ell}\right]\right]\right| & =\left|\int_{\ell}\left(\mathbf{w} \cdot \mathbf{t}_{\ell}-P_{\ell}\left(\mathbf{w} \cdot \mathbf{t}_{\ell}\right)\right)\left[\left[\left(\boldsymbol{\sigma}-\boldsymbol{\sigma}_{h}\right) \mathbf{n}_{\ell} \cdot \mathbf{t}_{\ell}\right]\right]\right| \\
& =\left|\int_{\ell}\left(\mathbf{w} \cdot \mathbf{t}_{\ell}-P_{\ell}\left(\mathbf{w} \cdot \mathbf{t}_{\ell}\right)\right)\left[\left[\boldsymbol{\sigma}_{h} \mathbf{n}_{\ell} \cdot \mathbf{t}_{\ell}\right]\right]\right| \\
& \leq \sum_{i=1,2}\left|\int_{\ell}\left(\mathbf{w} \cdot \mathbf{t}_{\ell}-P_{\ell}\left(\mathbf{w} \cdot \mathbf{t}_{\ell}\right)\right)\left(\boldsymbol{\sigma}_{h} \mathbf{n}_{\ell} \cdot \mathbf{t}_{\ell}-P_{\ell}\left(\boldsymbol{\sigma}_{h} \mathbf{n}_{\ell} \cdot \mathbf{t}_{\ell}\right)\right)\right| T_{i} \mid
\end{aligned}
$$

Siguiendo los argumentos empleados en la prueba del Lema 4.20, sustituyendo el tensor $\boldsymbol{\xi}_{h}$ por $\boldsymbol{\sigma}_{h}$, llegamos a

$$
\sum_{\ell \in \mathcal{E}_{h}} \sum_{i=1,2}\left|\int_{\ell}\left(\mathbf{w} \cdot \mathbf{t}_{\ell}-P_{\ell}\left(\mathbf{w} \cdot \mathbf{t}_{\ell}\right)\right)\left(\boldsymbol{\sigma}_{h} \mathbf{n}_{\ell} \cdot \mathbf{t}_{\ell}-P_{\ell}\left(\boldsymbol{\sigma}_{h} \mathbf{n}_{\ell} \cdot \mathbf{t}_{\ell}\right)\right)\right|_{T_{i}} \mid \leq C h^{2 s}\|\mathbf{w}\|_{1+s, \Omega}\|\boldsymbol{\sigma}\|_{s, \Omega} .
$$

Acotación del segundo término. Procediendo nuevamente como en la prueba del Lema 4.20 tendremos

$$
\sum_{\ell \in \mathcal{E}_{h}} \int_{\ell}\left(\mathbf{u} \cdot \mathbf{t}_{\ell}\right)\left[\left[\left(\boldsymbol{\Pi}_{h}^{A W} \boldsymbol{\eta}\right) \mathbf{n}_{\ell} \cdot \mathbf{t}_{\ell}\right]\right] \leq C h^{2 s}\|\boldsymbol{\eta}\|_{s, \Omega}\|\mathbf{u}\|_{1+s, \Omega}
$$

Combinando todas estas desigualdades con las estimaciones (4.34) y (4.56) y teniendo en cuenta que $2 s<s+1$, podemos concluir la prueba.

Asumiremos ahora que los operadores $\mathbf{T}$ y $\mathbf{T}_{h}$ están definidos como en la sección 3.4.2. Denotemos por $\mathbf{Z}(h)$ al espacio suma $\mathbf{V}+\mathbf{V}_{h}$ y por $\widehat{\mathbf{E}}_{\lambda}(\mathbf{Z}(h))$ al espacio propio del operador $\mathbf{T}$ relativo a $\lambda$. Como consecuencia del Lema 4.22, se establece el siguiente resultado. 
Teorema 4.23 (P6) Existen constantes positivas $C$ y $h_{0}$ tales que, si $h<h_{0}$,

$$
\left\|\left.\left(\mathbf{T}-\mathbf{T}_{h}\right)\right|_{\widehat{\mathbf{E}}_{\lambda}(\mathbf{Z}(h))}\right\|_{0, \Omega} \leq C h^{2 s} .
$$

Observemos que esta estimación de error es óptima desde el punto de vista de la mejor razón de convergencia posible y de la máxima regularidad de la solución exacta.

\subsection{Dominios curvos y espacios discretos conformes}

En esta sección consideraremos problemas de autovalores planteados en dominios con bordes curvos y métodos de aproximación conformes, en el siguiente sentido,

$$
V_{h}\left(\Omega_{h}\right) \subset V\left(\Omega_{h}\right) \not \subset V(\Omega) .
$$

En estas condiciones, es claro que podremos elegir

$$
\|\cdot\|_{*}=\|\cdot\|_{h} .
$$

Nótese que la propiedad P3 coincidirá entonces con la propiedad P1 y las estimaciones del error en la aproximación serán consecuencia de las propiedades $\mathbf{P} 4$ y P5 únicamente. En este contexto, escribiremos

- P4 para $x \in \mathbf{E}_{\lambda}(W(\widehat{\Omega}))$, existe $x_{h} \in V_{h}^{e}\left(\Omega_{h}\right)$ tal que $\left\|x-x_{h}\right\|_{h} \leq \eta_{3 h}\|x\|_{h}$, con $\eta_{3 h} \rightarrow 0$ para $h \rightarrow 0$,

- P5: para todo $x \in \mathbf{E}_{\lambda}(W(\widehat{\Omega})),\|x\|_{h, \Omega \backslash \Omega_{h}}+\|x\|_{h, \Omega_{h} \backslash \Omega} \leq \eta_{4 h}\|x\|_{h}$, con $\eta_{4 h} \rightarrow 0$ para $h \rightarrow 0$.

Como ya hemos señalado, la aproximación del problema comienza por la aproximación del conjunto $\bar{\Omega}$ mediante un dominio poliedral $\Omega_{h}$. Permitir que $\Omega$ sea un dominio curvo general implica que debemos tener cierto cuidado al construir las particiones de $\Omega_{h}$ sobre las cuales se formulará el método de aproximación. Terminaremos esta sección formulando un conjunto preciso de condiciones sobre cada partición de $\Omega_{h}$.

A continuación, usaremos varias definiciones y conceptos introducidos en [104] y en [3], adecuándolos para nuestro propósito.

Para un dado valor del parámetro de discretización $h \in\left(0, h_{0}\right)$, sea $\mathcal{T}_{h}$ una partición de $\Omega_{h}$ que consiste de triángulos cerrados $K$ tales que

- $\bar{\Omega}_{h}=\bigcup_{K \in \mathcal{T}_{h}} K$

- dos elementos cualesquiera $K$ y $K^{\prime}$ en $\mathcal{T}_{h}$ comparten a lo sumo un vértice o un lado.

Definamos $\Gamma_{h}:=\partial \Omega_{h}$ y denotemos por $\mathcal{V}_{h}$ y $\mathcal{E}_{h}$ los conjuntos de todos los vértices y todos los lados de la partición $\mathcal{T}_{h}$, respectivamente. Asumiremos que

- $\mathcal{V}_{h} \subset \bar{\Omega}$,

- $\mathcal{V}_{h} \cap \Gamma_{h} \subset \Gamma$,

- para todo $K \in \mathcal{T}_{h}$, a lo sumo un lado de $K$ yace sobre $\Gamma_{h}$,

- todos los puntos donde el borde $\Gamma$ deja de ser suave están contenidos en elementos de $\mathcal{E}_{h}$.

Consideremos los siguientes subconjuntos de $\mathcal{F}_{h}$ :

- $\mathcal{E}_{h}^{1}:=\left\{\ell \in \mathcal{E}_{h}: \ell=K \cap K^{\prime}\right.$ para distintos $\left.K, K^{\prime} \in \mathcal{T}_{h}\right\}$,

- $\mathcal{E}_{h}^{2}:=\left\{\ell \in \mathcal{E}_{h}: \ell \notin \mathcal{E}_{h}^{1}\right.$ y $\ell \subset \Gamma$ o $\left.\ell \subset \bar{\Omega}\right\}$, 


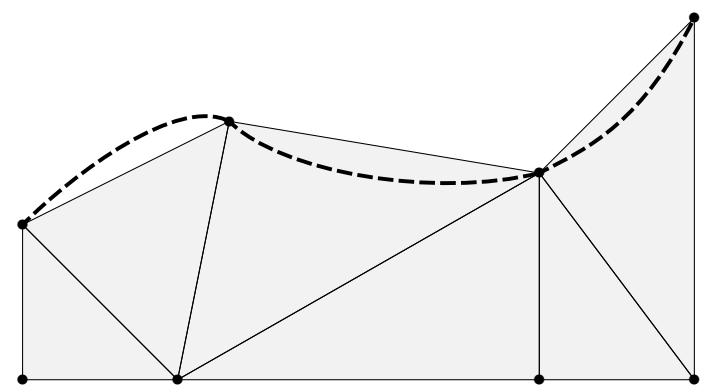

Figura 4.3: Triangulación en las inmediaciones del borde $\Gamma$.

- $\mathcal{E}_{h}^{3}:=\left\{\ell \in \mathcal{E}_{h}: \ell \notin \mathcal{E}_{h}^{1} \cup \mathcal{E}_{h}^{2}\right.$ y solo los vértices de $\ell$ pertenecen a $\left.\Gamma\right\}$.

Por simplicidad, asumiremos que la partición es tal que

$$
\mathcal{E}_{h}=\mathcal{E}_{h}^{1} \cup \mathcal{E}_{h}^{2} \cup \mathcal{E}_{h}^{3},
$$

lo que significa, simplemente, que el borde $\Gamma$ no cruzará ningún lado de los elementos de $\mathcal{T}_{h}$. Como consecuencia de esta clasificación, la partición $\mathcal{T}_{h}$ podrá separarse en los siguientes tres conjuntos disjuntos:

- $\mathcal{T}_{h}^{3}:=\left\{K \in \mathcal{T}_{h}: \partial K \cap \mathcal{E}_{h}^{3} \neq \emptyset\right\}$,

- $\mathcal{T}_{h}^{2}:=\left\{K \in \mathcal{T}_{h}: \partial K \cap \mathcal{E}_{h}^{2} \neq \emptyset\right\}$,

- $\mathcal{T}_{h}^{1}:=\mathcal{T}_{h} \backslash\left(\mathcal{T}_{h}^{1} \cup \mathcal{T}_{h}^{2}\right)$.

Basándonos en esta separación, definimos el siguiente subconjunto de $\mathcal{T}_{h}$ :

$$
\mathcal{B}_{h}:=\mathcal{T}_{h}^{2} \cup \mathcal{T}_{h}^{3}=\left\{K: \mathcal{T}_{h}: \partial K \cap \Gamma_{h} \neq \emptyset\right\},
$$

que contiene a aquellos elementos de la partición que forman parte del borde del dominio; los llamaremos, simplemente, elementos en el borde.

La diferencia entre los dominios $\Omega$ y $\Omega_{h}$ estará dada por

$$
\mathcal{D}_{h}:=\left(\Omega \backslash \Omega_{h}\right) \cup\left(\Omega_{h} \backslash \Omega\right)
$$

y, en general, será distinta del vacío. Obsérvese que el conjunto $\mathcal{D}_{h}$ puede pensarse como la unión de conjuntos $\omega_{K}$ disconexos. Cada $\omega_{K}$ estará asociado con un único $K \in \mathcal{T}_{h}$ que verifica $\partial \omega_{K} \cap \partial K=\ell_{K} \in \mathcal{E}_{h}$.

A cada elemento $K \in \mathcal{T}_{h}$ le asociaremos un triángulo $K^{i d}$ de la siguiente manera

$$
K^{i d}=\left\{\begin{array}{cc}
K & \text { si } K \in \mathcal{T}_{h}^{1}, \\
K \cup \omega_{K} & \text { si } K \in \mathcal{T}_{h}^{2}, \\
K \backslash \omega_{K} & \text { si } K \in \mathcal{T}_{h}^{3} .
\end{array}\right.
$$

A partir de esta asociación, podremos construir lo que llamaremos una partición ideal $\mathcal{T}_{h}^{i d}$, es decir,

$$
\bar{\Omega}=\bigcup_{K \in \mathcal{T}_{h}} K^{i d} .
$$

Obsérvese que los triángulos ideales $K^{i d}$ pueden tener a lo sumo un lado curvo. En lo que respecta a la suavidad de este lado curvo, asumiremos que localmente es la gráfica de una función $\mathcal{C}^{k}, k \geq 2$, definida sobre algún sistema de coordenadas.

Finalmente, asumiremos, como siempre, que la familia $\left\{\mathcal{T}_{h}\right\}_{h>0}$ es regular en el sentido del ángulo mínimo. 

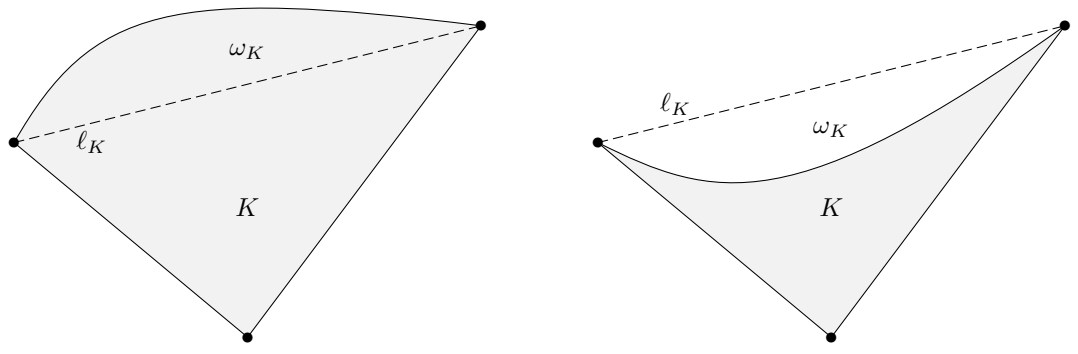

Figura 4.4: Triángulos ideales $K^{i d}$.

\subsubsection{Vibraciones en un sistema acoplado fluido-estructura}

El cálculo de los modos propios de vibración de un sistema acoplado fluido-estructura es un problema de gran relevancia en casi todos los campos de la ingeniería y en temas biomédicos. El conocimiento de estos modos vibracionales es fundamental para evitar el conocido fenómeno de resonancia. Tiene aplicaciones en la aeroelasticidad, en el diseño de contenedores de líquidos, en la definición de técnicas para la reducción de ruidos, en simulaciones biomecánicas y más.

Durante los últimos años, una enorme cantidad de trabajo ha sido destinada a estudiar este problema. Muchos de los resultados obtenidos pueden encontrarse en las monografías [79, 140, 143], donde también se presentan métodos numéricos apropiados para aproximar este problema y una extensa bibliografía.

Para determinar las vibraciones de un fluido confinado por paredes rígidas (problema espectral acústico), es usual describir el comportamiento del fluido eligiéndo como variable a la presión. Sin embargo, para problemas acoplados, tal elección conduce a un problema de autovalores no-simétrico; un gran inconveniente desde el punto de vista computacional. Para eludir esta dificultad, se consideraron distintas variables para el fluido (ver, por ejemplo, $[125,115,102,140,172]$ ).

Como el comportamiento de la estructura es descripto, casi siempre, en términos de los desplazamientos, elegir a la misma variable para describir al fluido presenta ciertas ventajas. En particular, las condiciones de compatibilidad/equilibrio que deben cumplirse sobre la interfaz se verificarán automáticamente. Además, este planteo conduce a un problema de autovalores generalizado con matrices simétricas de estructura banda. Sin embargo, la formulación variacional correspondiente es no elíptica, tiene un espectro esencial no vacío y el espacio de energía asociado no estará incluido en $L^{2}$ compactamente. Como consecuencia, algunas discretizaciones suelen presentar soluciones espurias. Por ejemplo, cuando se utilizan elementos lineales a trozos y continuos para aproximar a los desplazamientos en ambos medios, suelen aparecer soluciones relacionadas con movimientos de rotación pura en el fluido $[125,115]$. Estos movimientos de rotación son modos del problema continuo con frecuencia igual a cero y, por lo tanto, irrelevantes desde el punto de vista físico.

Una discretización alternativa para la formulación desplazamiento fue introducida en [38]: consiste en usar elementos de Raviart-Thomas de orden muy bajo para los desplazamientos en el fluido y elementos de Courant standard para los desplazamientos en el sólido; con un acoplamiento en la interfaz de tipo noconforme. Para problemas bi-dimensionales, se ha probado que el método converge libre de modos espurios y se han obtenido estimaciones óptimas del error [33, 150]. Este método también ha sido extendido para tratar con problemas tri-dimensionales [37, 36], fluidos incompresibles [34], interacción con estructuras delgadas [99] y medios disipativos [35].

En todas estas aplicaciones se asumió que el dominio ocupado por el sólido tenía bordes poligonales (o poliedrales en $\mathbb{R}^{3}$ ). Esto implica, obviamente, que la interacción fluido-sólido tendrá lugar a través de una interfaz poligonal; que aquí denotaremos $\Gamma_{\mathrm{I}}$. En consecuencia, cada interfaz discreta $\Gamma_{\mathrm{Ih}}$, inducida por particiones compatibles del dominio del sólido, satisfacerá la condición (conforme) $\Gamma_{\mathrm{I} h} \subset \Gamma_{\mathrm{I}}$. Claramente, la razón principal para sostener esta suposición es la de evitar los crímenes variacionales que se cometen cuando se aproximan bordes curvos por poligonales. 
En esta sección analizaremos la interacción entre un fluido compresible y una estructura lineal con bordes curvos [90].

\section{Definición del problema continuo}

Estamos interesados en determinar los modos propios de vibración de una estructura elástica lineal en interacción con un fluido ideal; es decir, un fluido no viscoso, incompresible y con densidad uniforme. El problema modelo que consideraremos consistirá en un recipiente elástico bi-dimensional lleno de fluido.

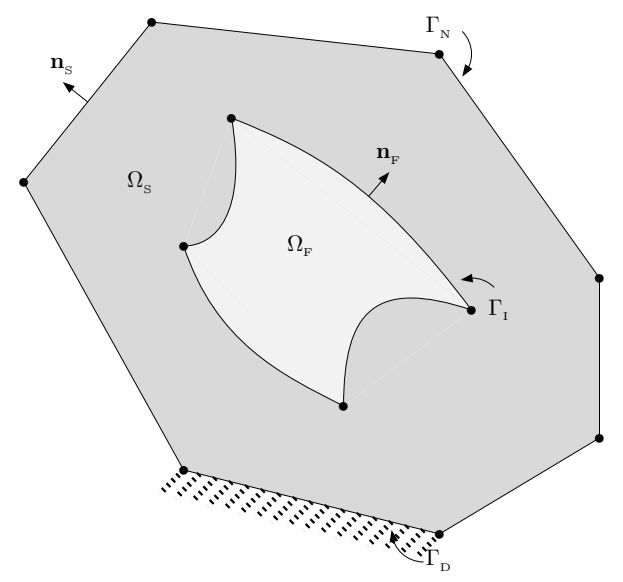

Figura 4.5: Dominios del fluido y del sólido.

Denotaremos por $\Omega_{\mathrm{F}}$ y $\Omega_{\mathrm{S}}$ los dominios ocupados por el fluido y el sólido, respectivamente, como se muestra en la Figura 1. Denotaremos por $\Gamma_{\mathrm{I}}$ a la interfaz entre ambos medios y por $\mathbf{n}$ al vector unitario en la dirección normal a $\Gamma_{\mathrm{I}}$ apuntando hacia $\Omega_{\mathrm{S}}$. Al borde exterior $\Gamma_{\mathrm{E}}$ del sólido lo pensaremos como la unión de las curvas $\Gamma_{\mathrm{D}}$ y $\Gamma_{\mathrm{N}} ; \Gamma_{\mathrm{D}} \cap \Gamma_{\mathrm{N}}=\emptyset$. A efectos de simplificar la presentación, asumiremos que $\Omega_{\mathrm{F}}$ es simplemente conexo (pero no necesariamente convexo), que $\Gamma_{\mathrm{E}}$ es poligonal y que $\left|\Gamma_{\mathrm{D}}\right|>0$.

Usaremos notación standard para los espacios de Sobolev y para sus normas y seminormas. Denotaremos por $H_{\Gamma_{\mathrm{D}}}^{1}\left(\Omega_{\mathrm{S}}\right)$ al espacio de las funciones en $H^{1}\left(\Omega_{\mathrm{S}}\right)$ con traza nula sobre $\Gamma_{\mathrm{D}} \mathrm{y}$, para los números positivos $s$ y $t$, definiremos

$$
H^{s, t}\left(\operatorname{div} ; \Omega_{\mathrm{F}}\right):=\left\{\mathbf{u} \in H^{s}\left(\Omega_{\mathrm{F}}\right)^{2}: \operatorname{div} \mathbf{u} \in H^{t}\left(\Omega_{\mathrm{F}}\right)\right\},
$$

que es un espacio de Hilbert equipado con la siguiente norma

$$
\|\mathbf{u}\|_{H^{s, t}\left(\operatorname{div} ; \Omega_{\mathrm{F}}\right)}^{2}:=\|\mathbf{u}\|_{s, \Omega_{\mathrm{F}}}^{2}+\|\operatorname{div} \mathbf{u}\|_{t, \Omega_{\mathrm{F}}}^{2} .
$$

Usaremos la siguiente notación para las cantidades físicas; en el fluido:

- u para el campo de desplazamientos,

- $p$ para la presión,

- $\rho_{\mathrm{F}}$ para la densidad,

- c para la velocidad acústica;

y en el sólido:

- w para el campo de desplazamientos,

- $\rho_{\mathrm{S}}$ para la densidad, 
- $\lambda_{S}$ y $\mu_{S}$ para los coeficientes de Lamé, definidos por

$$
\lambda_{S}:=\frac{\nu_{S} E_{S}}{\left(1-2 \nu_{S}\right)\left(1+\nu_{S}\right)} \quad \mu_{S}:=\frac{E_{S}}{2\left(1+\nu_{S}\right)}
$$

donde $E_{S}>0$ es el módulo de Young y $\nu_{S} \in(0,1 / 2)$ es la razón de Poisson,

- $\varepsilon(\mathbf{w})$ para el tensor de deformaciones, definido por

$$
\varepsilon_{i j}(\mathbf{w}):=\frac{1}{2}\left(\frac{\partial w_{i}}{\partial x_{j}}+\frac{\partial w_{j}}{\partial x_{i}}\right), \quad i, j=1,2,
$$

- $\boldsymbol{\sigma}(\mathbf{w})$ para el tensor de esfuerzos, relacionado con el tensor de deformaciones vía Ley de Hooke:

$$
\sigma_{i j}(\mathbf{w}):=\lambda_{S} \sum_{i=1}^{2} \varepsilon_{k k}(\mathbf{w}) \delta_{i j}+2 \mu_{S} \varepsilon_{i j}(\mathbf{w}), \quad i, j=1,2 .
$$

La aproximación clásica para movimientos de pequeña amplitud (en este caso, denominada aproximación elastoacústica) conduce al siguiente problema de autovalores para los modos de vibración del sistema acoplado y sus correspondientes frecuencias propias $\omega$ (ver, por ejemplo, [140]):

Problema 4.24 Encontrar $\omega>0, \mathbf{u} \in H\left(\operatorname{div} ; \Omega_{\mathrm{F}}\right), \mathbf{w} \in H^{1}\left(\Omega_{\mathrm{S}}\right)^{2}$ y $p \in H^{1}\left(\Omega_{\mathrm{F}}\right),(\mathbf{u}, \mathbf{w}, p) \neq(\mathbf{0}, \mathbf{0}, 0)$, tal que

$$
\begin{aligned}
\nabla p-\omega^{2} \rho_{\mathrm{F}} \mathbf{u}=\mathbf{0} & \text { en } \Omega_{\mathrm{F}}, \\
p+\rho_{\mathrm{F}} c^{2} \operatorname{div} \mathbf{u}=0 & \text { en } \Omega_{\mathrm{F}}, \\
\operatorname{div}[\boldsymbol{\sigma}(\mathbf{w})]+\omega^{2} \rho_{\mathrm{S}} \mathbf{w}=\mathbf{0} & \text { en } \Omega_{\mathrm{S}}, \\
\mathbf{u} \cdot \mathbf{n}-\mathbf{w} \cdot \mathbf{n}=0 & \text { sobre } \Gamma_{\mathrm{I}}, \\
\boldsymbol{\sigma}(\mathbf{w}) \mathbf{n}+p \mathbf{n}=\mathbf{0} & \text { sobre } \Gamma_{\mathrm{I}}, \\
\boldsymbol{\sigma}(\mathbf{w}) \mathbf{n}_{\mathrm{S}}=\mathbf{0} & \text { sobre } \Gamma_{\mathrm{N}}, \\
\mathbf{w}=\mathbf{0} & \text { sobre } \Gamma_{\mathrm{D}} .
\end{aligned}
$$

El acoplamiento entre el fluido y la estructura está dado por las ecuaciones (4.61) y (4.60). La primera de ellas, indica que el fluido y el sólido permanecen en contacto a través de la interfaz. La segunda, relaciona los esfuerzos generados en el sólido en la dirección normal a la interfaz con la presión en el fluido. Las ecuaciones (4.62) y (4.63) indican que la estructura estará libre de esfuerzos sobre $\Gamma_{\mathrm{N}}$ y permanecerá fija sobre $\Gamma_{\mathrm{D}}$, respectivamente.

Las ecuaciones (4.59)-(4.62) deben ser entendidas en el sentido de las distribuciones. En particular, las condiciones (4.61)-(4.60) son igualdades en $H^{-1 / 2}\left(\Gamma_{\mathrm{I}}\right)$. Sin embargo, como $p \in H^{1}\left(\Omega_{\mathrm{F}}\right)$ y w $\in H^{1}\left(\Omega_{\mathrm{S}}\right)^{2}$, estas condiciones serán válidas en $L^{2}\left(\Gamma_{\mathrm{I}}\right)$.

Desde el punto de vista computacional, las condiciones de compatibilidad en la interfaz pueden imponerse convenientemente por medio de un multiplicador de Lagrange. Esto nos conduce naturalmente a considerar una formulación híbrida, equivalente al problema espectral original, en la cual el multiplicador de Lagrange jugará el rol de una nueva variable: la presión en la interfaz fluido-sólido [34].

A fin de obtener esta formulación, introduciremos los siguientes espacios funcionales. En lo que sigue, escribiremos $\Omega:=\Omega_{\mathrm{F}} \cup \Omega_{\mathrm{S}}$. Sean

$$
\begin{gathered}
\mathcal{Q}(\Omega):=L^{2}\left(\Omega_{\mathrm{F}}\right)^{2} \times L^{2}\left(\Omega_{\mathrm{S}}\right)^{2} \\
\mathcal{X}(\Omega):=H\left(\operatorname{div} ; \Omega_{\mathrm{F}}\right) \times H_{\Gamma_{\mathrm{D}}}^{1}\left(\Omega_{\mathrm{S}}\right)^{2}, \\
\mathcal{V}(\Omega):=\left\{(\mathbf{u}, \mathbf{w}) \in \mathcal{X}(\Omega):\left.\mathbf{u} \cdot \mathbf{n}\right|_{\Gamma_{\mathrm{I}}} \in L^{2}\left(\Gamma_{\mathrm{I}}\right)\right\} .
\end{gathered}
$$

Sea $\|\cdot\|_{\mathcal{X}(\Omega)}$ la norma producto en $\mathcal{X}(\Omega)$. Observemos que $\mathcal{V}(\Omega)$, equipado con la siguiente norma

$$
\|(\mathbf{u}, \mathbf{w})\|_{\mathcal{V}(\Omega)}^{2}:=\|(\mathbf{u}, \mathbf{w})\|_{\mathcal{X}(\Omega)}^{2}+\|\mathbf{u} \cdot \mathbf{n}\|_{L^{2}\left(\Gamma_{\mathrm{I}}\right)}^{2},
$$


es un espacio cerrado de funciones y, por lo tanto, un espacio de Hilbert.

Integrando por partes, usando la ecuación (4.58) para eliminar a la presión $p$ en términos del campo de desplazamientos $\mathbf{u}$ y denotando por $\gamma$ a la presión en la interfaz fluido-sólido; es decir $\gamma:=\left.p\right|_{\Gamma_{\mathrm{I}}}$, obtendremos la siguiente formulación variacional (equivalente) del problema espectral (4.24):

Problema 4.25 Encontrar $\omega>0$ y $((\mathbf{u}, \mathbf{w}), \gamma) \in \mathcal{V}(\Omega) \times L^{2}\left(\Gamma_{\mathrm{I}}\right),((\mathbf{u}, \mathbf{w}), \gamma) \neq((\mathbf{0}, \mathbf{0}), 0)$, tal que:

$$
\begin{aligned}
\int_{\Omega_{\mathrm{F}}} \rho_{\mathrm{F}} c^{2} \operatorname{div} \mathbf{u} \operatorname{div} \mathbf{v} & +\int_{\Omega_{\mathrm{S}}} \boldsymbol{\sigma}(\mathbf{w}): \varepsilon(\mathbf{z})+\int_{\Gamma_{\mathrm{I}}} \gamma(\mathbf{v} \cdot \mathbf{n}-\mathbf{z} \cdot \mathbf{n}) \\
& =\omega^{2}\left(\int_{\Omega_{\mathrm{F}}} \rho_{\mathrm{F}} \mathbf{u} \cdot \mathbf{v}+\int_{\Omega_{\mathrm{S}}} \rho_{\mathrm{S}} \mathbf{w} \cdot \mathbf{z}\right) \quad \forall(\mathbf{v}, \mathbf{z}) \in \mathcal{V}(\Omega), \\
\int_{\Gamma_{\mathrm{I}}} \delta(\mathbf{u} \cdot \mathbf{n}-\mathbf{w} \cdot \mathbf{n}) & =0 \quad \forall \delta \in L^{2}\left(\Gamma_{\mathrm{I}}\right) .
\end{aligned}
$$

La siguiente caracterización espectral fue probada en [33].

Teorema 4.26 Sea $\mu=\omega^{2}$. El Problema 4.25 tiene dos clases de soluciones:

- $\mu_{0}=0$ correspondiente al autoespacio $\mathcal{H}(\Omega)$ definido por

$$
\mathcal{H}(\Omega):=\left\{(\mathbf{u}, \mathbf{0}) \in H\left(\operatorname{div} ; \Omega_{\mathrm{F}}\right) \times\{\mathbf{0}\}: \operatorname{div} \mathbf{u}=0 \text { in } \Omega_{\mathrm{F}} \text { and } \mathbf{u} \cdot \mathbf{n}=0 \text { on } \Gamma_{\mathrm{I}}\right\} ;
$$

- una sucesión de autovalores de multiplicidad finita $\mu_{n}>0, n \in \mathbb{N}$, convergentes a $+\infty$ y que corresponden a las autofunciones $\left(\mathbf{u}_{n}, \mathbf{w}_{n}\right) \in \mathcal{K}(\Omega)$ donde

$$
\mathcal{K}(\Omega):=\left\{(\mathbf{u}, \mathbf{w}) \in \mathcal{V}(\Omega): \int_{\Gamma_{\mathrm{I}}} \delta(\mathbf{u} \cdot \mathbf{n}-\mathbf{w} \cdot \mathbf{n})=0 \forall \delta \in L^{2}\left(\Gamma_{\mathrm{I}}\right)\right\} .
$$

El autoespacio de dimensión infinita $\mathcal{H}(\Omega)$ (asociado con $\mu_{0}=0$ ) admite también la siguiente caracterización:

$$
\mathcal{H}(\Omega):=\left\{(\operatorname{curl} \xi, \mathbf{0}): \xi \in H_{\Gamma_{\mathrm{I}}}^{1}\left(\Omega_{\mathrm{F}}\right)\right\},
$$

siendo $H_{\Gamma_{\mathrm{I}}}^{1}\left(\Omega_{\mathrm{F}}\right)$ el subespacio de las funciones en $H^{1}\left(\Omega_{\mathrm{F}}\right)$ que tienen traza igual a cero sobre $\Gamma_{\mathrm{I}}$. Esta caracterización muestra que $\mathcal{H}(\Omega)$ consiste en movimientos de rotación pura los cuales no inducen variaciones de la presión en el fluido ni vibraciones en la estructura. Estos modos de rotación son solución del problema (4.25) ya que no hemos incorporado en esta formulación la condición de irrotacionalidad sobre el campo de desplazamientos en el fluido.

El segundo conjunto de autofunciones (aquellas que corresponden a $\mu_{n}>0$ ) es un sistema ortogonal y completo de $\mathcal{G}(\Omega)$, subespacio de $\mathcal{K}(\Omega)$, que consiste en todos los campos de desplazamientos admisibles que son conservativos en el fluido; es decir,

$$
\mathcal{G}(\Omega):=\left\{(\mathbf{u}, \mathbf{w}) \in \mathcal{K}(\Omega): \mathbf{u}=\nabla \varphi, \varphi \in H^{1}\left(\Omega_{\mathrm{F}}\right)\right\} .
$$

Sean $\hat{a}, b$ y $d$ las formas bilineales continuas definidas por

$$
\begin{aligned}
& \hat{a}((\mathbf{u}, \mathbf{w}),(\boldsymbol{\eta}, \mathbf{z})):=\rho_{\mathrm{F}} c^{2} \int_{\Omega_{\mathrm{F}}} \operatorname{div} \mathbf{u} \operatorname{div} \boldsymbol{\eta}+\int_{\Omega_{\mathrm{S}}} \boldsymbol{\sigma}(\mathbf{w}): \boldsymbol{\varepsilon}(\mathbf{z}) \quad(\mathbf{u}, \mathbf{w}),(\boldsymbol{\eta}, \mathbf{z}) \in \mathcal{X}(\Omega), \\
& b((\mathbf{u}, \mathbf{w}), \gamma):=\int_{\Gamma_{\mathrm{I}}} \gamma(\mathbf{u} \cdot \mathbf{n}-\mathbf{w} \cdot \mathbf{n}) \quad(\mathbf{u}, \mathbf{w}) \in \mathcal{V}(\Omega), \gamma \in L^{2}\left(\Gamma_{\mathrm{I}}\right), \\
& d((\mathbf{u}, \mathbf{w}),(\boldsymbol{\eta}, \mathbf{z})):=\rho_{\mathrm{F}} \int_{\Omega_{\mathrm{F}}} \mathbf{u} \cdot \boldsymbol{\eta}+\rho_{\mathrm{S}} \int_{\Omega_{\mathrm{S}}} \mathbf{w} \cdot \mathbf{z} \quad(\mathbf{u}, \mathbf{w}),(\boldsymbol{\eta}, \mathbf{v}) \in \mathcal{Q}(\Omega) .
\end{aligned}
$$

La forma $\hat{a}$ es simétrica y positiva, en el sentido que $\hat{a}((\mathbf{u}, \mathbf{w}),(\mathbf{u}, \mathbf{w})) \geq 0$ para todos los $(\mathbf{u}, \mathbf{w}) \in \mathcal{X}(\Omega)$. La forma $d$ es simétrica y coerciva sobre $\mathcal{Q}(\Omega)$. Notemos que la forma $\hat{a}$ no es coerciva sobre $\mathcal{K}(\Omega)$. Sin embargo, $\hat{a}$ satisface una desigualdad tipo Gårding; es decir, $a:=\hat{a}+d$ sí es coerciva sobre $\mathcal{K}(\Omega)$.

Consideraremos entonces el siguiente problema de autovalores modificado: 
Problema 4.27 Encontrar $\mu \in \mathbb{R} y((\mathbf{u}, \mathbf{w}), \gamma) \in \mathcal{V}(\Omega) \times L^{2}\left(\Gamma_{\mathrm{I}}\right),((\mathbf{u}, \mathbf{w}), \gamma) \neq((\mathbf{0}, \mathbf{0}), 0)$, tal que:

$$
\begin{aligned}
a((\mathbf{u}, \mathbf{w}),(\boldsymbol{\eta}, \mathbf{z}))+b((\boldsymbol{\eta}, \mathbf{z}), \gamma) & =\mu d((\mathbf{u}, \mathbf{w}),(\boldsymbol{\eta}, \mathbf{z})) \quad \forall(\boldsymbol{\eta}, \mathbf{z}) \in \mathcal{V}(\Omega) \\
b((\mathbf{u}, \mathbf{w}), \delta) & =0 \quad \forall \delta \in L^{2}\left(\Gamma_{\mathrm{I}}\right) .
\end{aligned}
$$

Claramente, $\omega^{2}$ es un autovalor del problema $4.25 \mathrm{si}$, y solo si, $\mu=\left(1+\omega^{2}\right)$ es autovalor del problema 4.27 y las correspondientes autofunciones coinciden.

Las formas bilineales $a$ sobre $\mathcal{V}(\Omega) \times \mathcal{V}(\Omega)$ y $b$ sobre $\mathcal{V}(\Omega) \times L^{2}\left(\Gamma_{\mathrm{I}}\right)$ satisfacen las condiciones de Brezzi de la teoría clásica para problemas mixtos (ver Lema 8.1 en [34]). Entonces, el siguiente problema fuente estará bien definido.

Problema 4.28 Dado $(\mathbf{f}, \mathbf{g}) \in \mathcal{Q}(\Omega)$, encontrar $((\mathbf{u}, \mathbf{w}), \gamma) \in \mathcal{V}(\Omega) \times L^{2}\left(\Gamma_{\mathrm{I}}\right)$ tal que:

$$
\begin{aligned}
a((\mathbf{u}, \mathbf{w}),(\boldsymbol{\eta}, \mathbf{z}))+b((\boldsymbol{\eta}, \mathbf{z}), \gamma) & =d((\mathbf{f}, \mathbf{g}),(\boldsymbol{\eta}, \mathbf{z})) \quad \forall(\boldsymbol{\eta}, \mathbf{z}) \in \mathcal{V}(\Omega) \\
b((\mathbf{u}, \mathbf{w}), \delta) & =0 \quad \forall \delta \in L^{2}\left(\Gamma_{\mathrm{I}}\right) .
\end{aligned}
$$

Más aún, la solución (única) de este problema satisface [56]:

$$
\|(\mathbf{u}, \mathbf{w})\|_{\mathcal{V}(\Omega)}+\|\gamma\|_{L^{2}\left(\Gamma_{\mathrm{I}}\right)} \leq C\|(\mathbf{f}, \mathbf{g})\|_{\mathcal{Q}(\Omega)} .
$$

El operador solución correspondiente al problema acoplado quedará definido por

$$
\begin{aligned}
\mathbf{T}: \mathcal{X}(\Omega) & \rightarrow \mathcal{V}(\Omega) \hookrightarrow \mathcal{X}(\Omega) \\
(\mathbf{f}, \mathbf{g}) & \mapsto(\mathbf{u}, \mathbf{w}),
\end{aligned}
$$

donde $((\mathbf{u}, \mathbf{w}), \gamma) \in \mathcal{V}(\Omega) \times L^{2}\left(\Gamma_{\mathrm{I}}\right)$ es la solución del problema 4.28. Como las formas bilineales $a$ y $d$ son simétricas, $\mathbf{T}$ será autoadjunto con respecto a $d$. Gracias a (4.64), $\mathbf{T}$ será un operador acotado. Observemos que $\mathbf{T}$ no es un operador compacto. De hecho, $\left.\mathbf{T}\right|_{\mathcal{H}(\Omega)}$ es la identidad sobre el subespacio de dimensión infinita $\mathcal{H}(\Omega) \in \mathcal{V}(\Omega)$.

Es fácil probar que $(\lambda,(\mathbf{u}, \mathbf{v}))$ es un par propio del del operador $\mathbf{T}$ si, y solo si, existe $\gamma \in L^{2}\left(\Gamma_{\mathrm{I}}\right)$ tal que $\left(\frac{1}{\lambda},((\mathbf{u}, \mathbf{w}), \gamma)\right)$ es una solución del problema $(4.27)$ y, por lo tanto, $\left(\frac{1}{\lambda}-1,((\mathbf{u}, \mathbf{w}), \gamma)\right)$ es una solución del problema (4.25).

Además, valen las siguientes estimaciones a priori.

Teorema 4.29 Sea $((\mathbf{u}, \mathbf{w}), \gamma)$ la solución del problema (4.28). Existen constantes $C>0, s \in(1 / 2,1] y$ $t \in(0,1]$ tales que

$$
\begin{gathered}
\text { - si }(\mathbf{f}, \mathbf{g}) \in \mathcal{Q}(\Omega) \text {, entonces }(\mathbf{u}, \mathbf{w}) \in H^{0,1}\left(\operatorname{div}, \Omega_{\mathrm{F}}\right) \times H^{1+t}\left(\Omega_{\mathrm{S}}\right)^{2} y \\
\qquad \mathbf{u}\left\|_{0, \Omega_{\mathrm{F}}}+\right\| \mathbf{w}\left\|_{1+t, \Omega_{\mathrm{S}}}+\right\| \operatorname{div} \mathbf{u}\left\|_{1, \Omega_{\mathrm{F}}} \leq C\right\|(\mathbf{f}, \mathbf{g}) \|_{0, \Omega} \\
\text { - si }(\mathbf{f}, \mathbf{g}) \in\left\{(\nabla q, \mathbf{g}): q \in H^{1}\left(\Omega_{\mathrm{F}}\right), \mathbf{g} \in L^{2}\left(\Omega_{\mathrm{S}}\right)^{2}\right\} \text {, entonces }(\mathbf{u}, \mathbf{w}) \in H^{s, 1}\left(\operatorname{div}, \Omega_{\mathrm{F}}\right) \times H^{1+t}\left(\Omega_{\mathrm{S}}\right)^{2} y \\
\|\mathbf{u}\|_{s, \Omega_{\mathrm{F}}}+\|\mathbf{w}\|_{1+t, \Omega_{\mathrm{S}}}+\|\operatorname{div} \mathbf{u}\|_{1, \Omega_{\mathrm{F}}} \leq C\|(\mathbf{f}, \mathbf{g})\|_{0, \Omega} \\
\text { - si }(\mathbf{f}, \mathbf{g}) \in \mathcal{G}(\Omega), \text { entonces }(\mathbf{u}, \mathbf{w}) \in H^{s, 1+s}\left(\operatorname{div}, \Omega_{\mathrm{F}}\right) \times H^{1+t}\left(\Omega_{\mathrm{S}}\right)^{2} y \\
\|\mathbf{u}\|_{s, \Omega_{\mathrm{F}}}+\|\mathbf{w}\|_{1+t, \Omega_{\mathrm{S}}}+\|\operatorname{div} \mathbf{u}\|_{1+s, \Omega_{\mathrm{F}}} \leq C\|(\mathbf{f}, \mathbf{g})\|_{0, \Omega}
\end{gathered}
$$

Prueba. Es una extensión directa de la prueba del Teorema 2.6 en [33]. Aquí, $s=1$, si $\Omega_{\mathrm{F}}$ es convexo, o, en caso contrario, cualquier $s<\pi / \theta$, con $\theta$ el mayor ángulo re-entrante en $\Omega_{\mathrm{F}}$. Por otro lado, $t$ depende de los ángulos re-entrantes en $\Omega_{\mathrm{S}}$, de los ángulos entre $\Gamma_{\mathrm{D}}$ y $\Gamma_{\mathrm{N}}$, y de los coeficientes de Lamé (see [111]). 


\section{Discretización del problema}

Sean $\Omega_{\mathrm{F} h}$ y $\Omega_{\mathrm{S} h}$ los dominios poligonales que aproximan a los dominios con bordes curvos $\Omega_{\mathrm{F}}$ y $\Omega_{\mathrm{S}}$, respectivamente. Sean $\left\{\mathcal{T}_{h}^{\mathrm{F}}\right\}$ y $\left\{\mathcal{T}_{h}^{\mathrm{S}}\right\}$ dos familias de triangulaciones regulares de $\bar{\Omega}_{\mathrm{F} h}$ y $\bar{\Omega}_{\mathrm{Sh}}$, respectivamente. En este caso, definiremos al parámetro que caracteriza a la partición por $h:=\operatorname{máx}_{T \in \mathcal{T}_{h}^{\mathrm{F}} \cup \mathcal{T}_{h}^{\mathrm{S}}} h_{T}$, siendo $h_{T}$ el diámetro de $T$.

Asumiremos que, para todo $h$, las triangulaciones $\mathcal{T}_{h}^{\mathrm{F}}$ y $\mathcal{T}_{h}^{\mathrm{S}}$ tienen nodos coincidentes sobre el borde que tienen en común (interfaz sólido-fluido). Así, la poligonal que queda definida uniendo estos nodos, denotémosla $\Gamma_{I h}$, representará una aproximación compatible de la interfaz $\Gamma_{\mathrm{I}}$. También asumiremos que los puntos extremos del borde $\Gamma_{\mathrm{D}}$ coinciden con vértices de la partición $\mathcal{T}_{h}^{\mathrm{S}}$.

Asumiremos también que las triangulaciones $\mathcal{T}_{h}^{\mathrm{F}}$ y $\mathcal{T}_{h}^{\mathrm{s}}$ fueron construidas siguiendo las pautas establecidas en la sección 4.2. Continuando con la argumentación de esa sección, definiremos

- $\mathcal{T}_{h}^{\mathrm{F}, i d}$ y $\mathcal{T}_{h}^{\mathrm{S}, i d} ;$ las triangulaciones ideales asociadas a $\mathcal{T}_{h}^{\mathrm{F}}$ y $\mathcal{T}_{h}^{\mathrm{S}}$, respectivamente,

- $\mathcal{B}_{\mathrm{F} h}$ y $\mathcal{B}_{\mathrm{S} h} ;$ los conjuntos de triángulos en el borde $\Gamma_{\mathrm{I} h}$ pertenecientes a los dominios $\Omega_{\mathrm{F} h}$ y $\Omega_{\mathrm{S} h}$, respectivamente,

- $\mathcal{E}_{\mathrm{Fh}}$ y $\mathcal{E}_{\mathrm{Sh}} ;$ los conjuntos de lados de todos los triángulos en $\mathcal{T}_{h}^{\mathrm{F}}$ y $\mathcal{T}_{h}^{\mathrm{S}}$, respectivamente.

A continuación, listaremos algunas de las propiedades de las particiones consideradas (ver sección 4.2). Sea $\mathcal{N}_{h}$ el conjunto de todos los vértices en $\mathcal{T}_{h}^{\mathrm{F}} \cup \mathcal{T}_{h}^{\mathrm{S}}$. Se verifica

$-\mathcal{N}_{h} \in \bar{\Omega}_{\mathrm{F}} \cup \bar{\Omega}_{\mathrm{S}}$,

$-\mathcal{N}_{h} \cap \Gamma_{\text {Ih }} \subset \Gamma_{\text {I }}$,

- $\mathcal{N}_{h}$ contiene todos los puntos donde la interfaz $\Gamma_{\mathrm{I}}$ deja de ser $\mathcal{C}^{2}$,

- cada $T \in \mathcal{B}_{\mathrm{Fh}} \cup \mathcal{B}_{\mathrm{Sh}}$ tiene a lo sumo un lado sobre $\Gamma_{\mathrm{Ih}}$,

- para cada $T \in \mathcal{B}_{\mathrm{F} h} \cup \mathcal{B}_{\mathrm{S} h}$, existen solo dos posibilidades $T \subset T^{i d} \mathrm{o} T \supset T^{i d}$.

Para cada triangulación $\mathcal{T}_{h}:=\mathcal{T}_{h}^{\mathrm{F}} \cup \mathcal{T}_{h}^{\mathrm{S}}$, denotaremos

$$
\Omega_{h}=\Omega_{\mathrm{F} h} \cup \Omega_{\mathrm{S} h}, \quad \widehat{\Omega}_{\mathrm{F}}=\Omega_{\mathrm{F}} \cup \Omega_{\mathrm{F} h}, \quad \widehat{\Omega}_{\mathrm{S}}=\Omega_{\mathrm{S}} \cup \Omega_{\mathrm{S} h}, \quad \widehat{\Omega}=\widehat{\Omega}_{\mathrm{F}} \cup \widehat{\Omega}_{\mathrm{S}}
$$

y definiremos los siguientes conjuntos

$$
\Omega-\Omega_{h}:=\Omega_{\mathrm{F}} \backslash \Omega_{\mathrm{F} h} \cup \Omega_{\mathrm{S}} \backslash \Omega_{\mathrm{S} h}, \quad \Omega_{h}-\Omega:=\Omega_{\mathrm{F} h} \backslash \Omega_{\mathrm{F}} \cup \Omega_{\mathrm{S} h} \backslash \Omega_{\mathrm{S}} .
$$

Supongamos por el momento que $D$ representa a cualquiera de los dos siguientes dominios: $\Omega_{h}$ o $\widehat{\Omega}$. Introduciremos, entonces, los espacios funcionales $\mathcal{Q}(D), \mathcal{X}(D)$ y $\mathcal{V}(D)$ definidos como las extensiones naturales de los espacios $\mathcal{Q}(\Omega), \mathcal{X}(\Omega)$ y $\mathcal{V}(\Omega)$, respectivamente (en el sentido definido en la sección 3.1). Por ejemplo, si $D$ fuera $\widehat{\Omega}$, tendríamos

$$
\mathcal{V}(\widehat{\Omega}):=\left\{(\mathbf{u}, \mathbf{w}) \in H\left(\operatorname{div}, \widehat{\Omega}_{\mathrm{F}}\right) \times H_{\Gamma_{\mathrm{D}}}^{1}\left(\widehat{\Omega}_{\mathrm{S}}\right)^{2}:\left.\mathbf{u} \cdot \mathbf{n}\right|_{\Gamma_{\mathrm{I}}} \in L^{2}\left(\Gamma_{\mathrm{I}}\right)\right\} .
$$

Observemos que, como el borde exterior $\Gamma_{\mathrm{E}}$ se asumió poligonal, ambos dominios $\Omega_{h}$ y $\widehat{\Omega}$, coinciden exactamente con $\Omega$. Sin embargo, debe notarse que los espacios $\mathcal{V}(\Omega), \mathcal{V}\left(\Omega_{h}\right)$ y $\mathcal{V}(\widehat{\Omega})$ son todos distintos; en particular, los pares de funciones que pertenecen a estos espacios difieren significativamente en los alrededores de la interfaz. Esto se debe a que $\Omega_{h}$ es una aproximación externa de $\Omega$, significando en este caso, $\Omega_{\mathrm{F} h} \not \subset \Omega_{\mathrm{F}}$ y $\Omega_{\mathrm{S} h} \not \subset \Omega_{\mathrm{S}}$. Consideraciones similares son válidas para $\mathcal{K}\left(\Omega_{h}\right), \mathcal{H}\left(\Omega_{h}\right)$ y $\mathcal{G}\left(\Omega_{h}\right)$, subespacios de $\mathcal{V}\left(\Omega_{h}\right)$. 
Las formas bilineales discretas $a_{h}, b_{h}$ y $d_{h}$ estarán definidas por

$$
\begin{aligned}
& a_{h}((\mathbf{u}, \mathbf{w}),(\boldsymbol{\eta}, \mathbf{z})):=\rho_{\mathrm{F}} c^{2} \int_{\Omega_{\mathrm{F} h}} \operatorname{div} \mathbf{u} \operatorname{div} \boldsymbol{\eta}+\int_{\Omega_{\mathrm{S} h}} \boldsymbol{\sigma}(\mathbf{w}): \varepsilon(\mathbf{z}) \\
& +\rho_{\mathrm{F}} \int_{\Omega_{\mathrm{F} h}} \mathbf{u} \cdot \boldsymbol{\eta}+\rho_{\mathrm{S}} \int_{\Omega_{\mathrm{Sh}}} \mathbf{w} \cdot \mathbf{z} \quad(\mathbf{u}, \mathbf{w}),(\boldsymbol{\eta}, \mathbf{z}) \in \mathcal{X}\left(\Omega_{h}\right), \\
& b_{h}((\mathbf{u}, \mathbf{w}), \gamma):=\int_{\Gamma_{\mathrm{Ih}}} \gamma(\mathbf{u} \cdot \mathbf{n}-\mathbf{w} \cdot \mathbf{n}) \quad(\mathbf{u}, \mathbf{w}) \in \mathcal{V}\left(\Omega_{h}\right), \gamma \in L^{2}\left(\Gamma_{\mathrm{Ih}}\right), \\
& d_{h}((\mathbf{u}, \mathbf{w}),(\boldsymbol{\eta}, \mathbf{z})):=\rho_{\mathrm{F}} \int_{\Omega_{\mathrm{F} h}} \mathbf{u} \cdot \boldsymbol{\eta}+\rho_{\mathrm{S}} \int_{\Omega_{\mathrm{S} h}} \mathbf{w} \cdot \mathbf{z} \quad(\mathbf{u}, \mathbf{w}),(\boldsymbol{\eta}, \mathbf{v}) \in \mathcal{Q}\left(\Omega_{h}\right) .
\end{aligned}
$$

Cada componente de los desplazamientos en el sólido será aproximada usando el espacio de elementos lineales standard

$$
\mathcal{L}_{h}\left(\Omega_{\mathrm{Sh}}\right):=\left\{v_{h} \in H^{1}\left(\Omega_{\mathrm{Sh}}\right):\left.v_{h}\right|_{T} \in \mathcal{P}_{1}(T) \forall T \in \mathcal{T}_{h}^{\mathrm{s}}\right\} .
$$

Para el fluido, en cambio, usaremos el espacio de Raviart-Thomas ([147]) de orden más bajo

$$
\mathcal{R}_{h}\left(\Omega_{\mathrm{F} h}\right):=\left\{\mathbf{u}_{h} \in H\left(\operatorname{div} ; \Omega_{\mathrm{F} h}\right):\left.\mathbf{u}_{h}\right|_{T} \in \mathcal{R}_{0}(T) \forall T \in \mathcal{T}_{h}^{\mathrm{F}}\right\},
$$

donde

$$
\mathcal{R}_{0}(T):=\left\{\mathbf{u}_{h} \in \mathcal{P}_{1}(T)^{2}: \mathbf{u}_{h}(x, y)=(a+b x, c+b y), a, b, c \in \mathbb{R}\right\} .
$$

Los grados de libertad en $\mathcal{R}_{h}\left(\Omega_{\mathrm{F} h}\right)$ son los valores (constantes) de la componente normal de $\mathbf{u}_{h}$ sobre cada lado de la triangulación. Observemos que, cualquiera sea la función $\mathbf{u}_{h} \in \mathcal{R}_{h}\left(\Omega_{\mathrm{F} h}\right),\left.\mathbf{u}_{h} \cdot \mathbf{n}\right|_{\Gamma_{\mathrm{I} h}} \in L^{2}\left(\Gamma_{\mathrm{I} h}\right)$. Definiremos, entonces, la versión discreta del espacio $\mathcal{V}(\Omega)$ por

$$
\mathcal{V}_{h}\left(\Omega_{h}\right):=\left\{(\mathbf{u}, \mathbf{v}) \in \mathcal{R}_{h}\left(\Omega_{\mathrm{Fh}}\right) \times \mathcal{L}_{h}\left(\Omega_{\mathrm{Sh}}\right)^{2}:\left.\mathbf{v}_{h}\right|_{\Gamma_{\mathrm{D}}}=\mathbf{0}\right\} .
$$

Finalmente, para la presión en la interfaz usaremos el espacio de las funciones constantes a trozos,

$$
\mathcal{Y}_{h}\left(\Gamma_{\mathrm{I} h}\right):=\left\{\delta_{h} \in L^{2}\left(\Gamma_{\mathrm{I} h}\right):\left.\delta_{h}\right|_{\ell} \in \mathcal{P}_{0}(\ell) \forall \ell \subset \Gamma_{\mathrm{I} h}\right\} .
$$

Así, obtendremos la versión discreta del problema espectral (4.27):

Problema 4.30 Encontrar $\mu_{h} \in \mathbb{R} y\left(\left(\mathbf{u}_{h}, \mathbf{w}_{h}\right), \gamma_{h}\right) \in \mathcal{V}_{h}\left(\Omega_{h}\right) \times \mathcal{W}_{h}\left(\Gamma_{\text {Ih }}\right),\left(\left(\mathbf{u}_{h}, \mathbf{w}_{h}\right), \gamma_{h}\right) \neq((\mathbf{0}, \mathbf{0}), 0)$, tal que:

$$
\begin{aligned}
a_{h}\left(\left(\mathbf{u}_{h}, \mathbf{w}_{h}\right),\left(\boldsymbol{\eta}_{h}, \mathbf{z}_{h}\right)\right)+b_{h}\left(\left(\boldsymbol{\eta}_{h}, \mathbf{z}_{h}\right), \gamma_{h}\right) & =\mu_{h} d_{h}\left(\left(\mathbf{u}_{h}, \mathbf{w}_{h}\right),\left(\boldsymbol{\eta}_{h}, \mathbf{z}_{h}\right)\right) \quad \forall\left(\boldsymbol{\eta}_{h}, \mathbf{z}_{h}\right) \in \mathcal{V}_{h}\left(\Omega_{h}\right), \\
b_{h}\left(\left(\mathbf{u}_{h}, \mathbf{w}_{h}\right), \delta_{h}\right) & =0 \quad \forall \delta_{h} \in \mathcal{Y}_{h}\left(\Gamma_{\text {Ih }}\right) .
\end{aligned}
$$

El siguiente teorema es la versión discreta del Teorema 4.26 (la prueba puede encontrarse en [33]):

Teorema 4.31 El problema 4.30 tiene dos clases de soluciones:

- $\mu_{0}=0$ correspondiente al autoespacio $\mathcal{H}_{h}\left(\Omega_{h}\right)$, definido por

$$
\mathcal{H}_{h}\left(\Omega_{h}\right):=\left\{\left(\mathbf{u}_{h}, \mathbf{0}\right) \in \mathcal{R}_{h}\left(\Omega_{\mathrm{F} h}\right) \times\{\mathbf{0}\}: \operatorname{div} \mathbf{u}_{h}=0 \text { en } \Omega_{\mathrm{Fh}} \mathrm{y} \quad \mathbf{u}_{h} \cdot \mathbf{n}=0 \text { sobre } \Gamma_{\mathrm{Ih}}\right\} ;
$$

- un conjunto finito de autovalores positivos $\mu_{h}$ correspondientes a las autofunciones $\left(\mathbf{u}_{h}, \mathbf{w}_{h}\right) \in$ $\mathcal{G}_{h}\left(\Omega_{h}\right)$, siendo $\mathcal{G}_{h}\left(\Omega_{h}\right)$ el complemento ortogonal de $\mathcal{H}_{h}\left(\Omega_{h}\right)$ en $\mathcal{K}_{h}\left(\Omega_{h}\right)$, donde

$$
\mathcal{K}_{h}\left(\Omega_{h}\right):=\left\{\left(\mathbf{u}_{h}, \mathbf{w}_{h}\right) \in \mathcal{V}_{h}\left(\Omega_{h}\right): \int_{\ell}\left(\mathbf{u}_{h}-\mathbf{w}_{h}\right) \cdot \mathbf{n}=0, \forall \ell \subset \Gamma_{\mathrm{Ih}}\right\} .
$$

Procediendo como en [34], puede probarse que las formas bilineales $a_{h}$ y $b_{h}$ satisfacen las condiciones de Brezzi sobre los espacios discretos $\mathcal{V}_{h}\left(\Omega_{h}\right)$ y $\mathcal{Y}_{h}\left(\Omega_{h}\right)$, uniformemente en $h$ (ver Lema 8.2 en esa referencia). Como consecuencia, aplicando la teoría clásica sobre métodos mixtos [56], podremos asegurar que existe una solución única del siguiente problema: 
Problema 4.32 Dada $(\mathbf{f}, \mathbf{g}) \in \mathcal{Q}\left(\Omega_{h}\right)$, encontrar $\left(\left(\mathbf{u}_{h}, \mathbf{w}_{h}\right), \gamma_{h}\right) \in \mathcal{V}_{h}\left(\Omega_{h}\right) \times \mathcal{W}_{h}\left(\Gamma_{\text {I }}\right)$ tal que:

$$
\begin{aligned}
a_{h}\left(\left(\mathbf{u}_{h}, \mathbf{w}_{h}\right),\left(\boldsymbol{\eta}_{h}, \mathbf{z}_{h}\right)\right)+b_{h}\left(\left(\boldsymbol{\eta}_{h}, \mathbf{z}_{h}\right), \gamma_{h}\right) & =d_{h}\left((\mathbf{f}, \mathbf{g}),\left(\boldsymbol{\eta}_{h}, \mathbf{z}_{h}\right)\right) \quad \forall\left(\boldsymbol{\eta}_{h}, \mathbf{z}_{h}\right) \in \mathcal{V}_{h}\left(\Omega_{h}\right), \\
b_{h}\left(\left(\mathbf{u}_{h}, \mathbf{w}_{h}\right), \delta_{h}\right) & =0 \quad \forall \delta_{h} \in \mathcal{Y}_{h}\left(\Gamma_{\mathrm{I} h}\right) .
\end{aligned}
$$

Más aún, existe una constante positiva $C$, independiente de $h$, tal que

$$
\left\|\left(\mathbf{u}_{h}, \mathbf{w}_{h}\right)\right\|_{\mathcal{V}_{h}\left(\Omega_{h}\right)}+\left\|\gamma_{h}\right\|_{0, \Gamma_{\mathrm{I} h}} \leq C\|(\mathbf{f}, \mathbf{g})\|_{0, \Omega_{h}}
$$

Definimos el operador solución para el problema acoplado discreto por:

$$
\begin{aligned}
\mathbf{T}_{h}: \mathcal{X}\left(\Omega_{h}\right) & \rightarrow \mathcal{V}_{h}\left(\Omega_{h}\right) \hookrightarrow \mathcal{X}\left(\Omega_{h}\right) \\
(\mathbf{f}, \mathbf{g}) & \mapsto\left(\mathbf{u}_{h}, \mathbf{w}_{h}\right),
\end{aligned}
$$

donde $\left(\left(\mathbf{u}_{h}, \mathbf{w}_{h}\right), \gamma_{h}\right)$ es la solución del problema (4.32). Gracias a (4.65), los operadores $\mathbf{T}_{h}$ estarán acotados uniformemente en $h$. Como en el caso continuo, es simple probar que $\lambda_{h}$ es un autovalor del problema (4.30) si, y solo si, $1 / \lambda_{h}$ es un autovalor del operador $\mathbf{T}_{h}$ y los correspondientes autovectores están relacionados por $\left(\left(\mathbf{u}_{h}, \mathbf{w}_{h}\right), \gamma_{h}\right) \leftrightarrow\left(\mathbf{u}_{h}, \mathbf{w}_{h}\right)$.

\section{Aproximación espectral}

A fin de probar que los pares propios del operador $\mathbf{T}$ pueden ser aproximados de manera correcta por los pares propios del operador $\mathbf{T}_{h}$, vamos a usar la teoría desarrollada en la sección 3.2 en su versión adaptada para problemas mixtos (ver sección 3.4.1).

Nuestro primer paso será elegir una norma adecuada para funciones con dominio en $\widehat{\Omega}$.

Sea $\omega_{T}$ el dominio definido por $T^{i d} \backslash T$. Definimos

$$
\|(\mathbf{u}, \mathbf{v})\|_{h}^{2}=\|(\mathbf{u}, \mathbf{v})\|_{\mathcal{X}(\widehat{\Omega})}^{2}:=\|(\mathbf{u}, \mathbf{v})\|_{\mathcal{X}\left(\Omega_{h}\right)}^{2}+\sum_{\substack{T \in \mathcal{B}_{\mathrm{F} h} \\ T \subset T^{i d}}}\|\mathbf{u}\|_{H\left(\operatorname{div}, \omega_{T}\right)}^{2}+\sum_{\substack{T \in \mathcal{B}_{\mathrm{Sh}} \\ T \subset T^{i d}}}\|\mathbf{v}\|_{1, \omega_{T}}^{2} .
$$

Como es usual, $\|\cdot\|_{h, \mathcal{O}}$ denotará la restricción canónica de la norma $\|\cdot\|_{h}$ a cualquier conjunto abierto $\mathcal{O}$ contenido en $\widehat{\Omega}$. Por ejemplo, si $\mathcal{O}$ fuera $\Omega-\Omega_{h}$, tendríamos

$$
\|(\mathbf{u}, \mathbf{v})\|_{h, \Omega-\Omega_{h}}^{2}=\|\mathbf{u}\|_{H\left(\operatorname{div}, \Omega_{\mathrm{F}} \backslash \Omega_{\mathrm{F} h}\right)}^{2}+\|\mathbf{v}\|_{1, \Omega_{\mathrm{S}} \backslash \Omega_{\mathrm{S} h}}^{2} .
$$

Nuestro próximo paso será definir los operadores de extensión $\mathbf{P}$ y $\mathbf{P}_{h}$.

Construiremos primero el operador de extensión para las funciones del espacio continuo. Sea $(\mathbf{u}, \mathbf{w}) \in$ $\mathcal{V}(\Omega)$. Sea $\xi \in\left\{\zeta \in H^{1}\left(\Omega_{\mathrm{S}}\right): \int_{\Omega_{\mathrm{S}}} \zeta=0\right\}$ la solución (única) del siguiente problema de Neumann:

$$
\begin{aligned}
\Delta \xi & =\frac{1}{\left|\Omega_{\mathrm{S}}\right|} \int_{\Omega_{\mathrm{F}}} \operatorname{div} \mathbf{u} \text { en } \Omega_{\mathrm{S}}, \\
\frac{\partial \xi}{\partial \mathbf{n}_{\mathrm{S}}} & =\left\{\begin{array}{cl}
-\mathbf{u} \cdot \mathbf{n}_{\mathrm{S}} & \text { sobre } \Gamma_{\mathrm{I}}, \\
0 & \text { sobre } \Gamma_{\mathrm{E}},
\end{array}\right.
\end{aligned}
$$

donde $\mathbf{n}_{\mathrm{S}}$ denota al vector unitario normal a $\Gamma_{\mathrm{I}}$ en la dirección exterior a $\Omega_{\mathrm{S}}$. Observemos que el problema es compatible pues

$$
\int_{\Omega_{\mathrm{S}}} \Delta \xi=\int_{\Omega_{\mathrm{F}}} \operatorname{div} \mathbf{u}=\int_{\Gamma_{\mathrm{I}}} \mathbf{u} \cdot \mathbf{n}=-\int_{\Gamma_{\mathrm{I}}} \mathbf{u} \cdot \mathbf{n}_{\mathrm{S}}=\int_{\partial \Omega_{\mathrm{S}}} \frac{\partial \xi}{\partial \mathbf{n}_{\mathrm{S}}} .
$$

Luego, por el Teorema de Lax-Milgram, sabemos que existe $C>0$ tal que

$$
\|\nabla \xi\|_{0, \Omega_{\mathrm{S}}} \leq C\left({\frac{\left|\Omega_{\mathrm{F}}\right|^{1 / 2}}{\left|\Omega_{\mathrm{S}}\right|}}^{1 / 2} \operatorname{div} \mathbf{u}\left\|_{0, \Omega_{\mathrm{F}}}+\right\| \mathbf{u} \cdot \mathbf{n} \|_{0, \Gamma_{\mathrm{I}}}\right)
$$


Definiremos la extensión de $\mathbf{u}$ a $\widehat{\Omega}_{\mathrm{F}}$ por

$$
\mathbf{u}^{e}:= \begin{cases}\mathbf{u} & \text { en } \Omega_{\mathrm{F}} \\ \nabla \xi & \text { en } \omega_{T}: \omega_{T} \subset \Omega_{\mathrm{Fh}} \backslash \Omega_{\mathrm{F}} .\end{cases}
$$

Claramente, esta definición nos asegura que $\mathbf{u}^{e} \in H\left(\operatorname{div} ; \widehat{\Omega}_{\mathrm{F}}\right)$. Además, tendremos

$$
\left\|\mathbf{u}^{e}\right\|_{H\left(\operatorname{div} ; \widehat{\Omega}_{\mathrm{F}}\right)}^{2}=\|\mathbf{u}\|_{H\left(\operatorname{div} ; \Omega_{\mathrm{F}}\right)}^{2}+\sum_{\substack{T \in \mathcal{B}_{\mathrm{F} h} \\ T \supset T^{i d}}}\|\nabla \xi\|_{H\left(\operatorname{div} ; \omega_{T}\right)}^{2} \leq C\left(\|\mathbf{u}\|_{H\left(\operatorname{div} ; \Omega_{\mathrm{F}}\right)}^{2}+\|\mathbf{u} \cdot \mathbf{n}\|_{0, \Gamma_{\mathrm{I}}}^{2}\right) .
$$

Denotemos por $\mathbf{w}^{e}$ a la extensión del vector $\mathbf{w}$ a $\widehat{\Omega}_{\mathrm{S}}$ definida según el Teorema D.4. De acuerdo con este teorema, tendremos

$$
\left\|\left(\mathbf{u}^{e}, \mathbf{w}^{e}\right)\right\|_{h} \leq C\left(\|\mathbf{u}\|_{H\left(\operatorname{div} ; \Omega_{\mathrm{F}}\right)}+\|\mathbf{u} \cdot \mathbf{n}\|_{0, \Gamma_{\mathrm{I}}}+\left\|\mathbf{w}^{e}\right\|_{1, \widehat{\Omega}_{\mathrm{S}}}\right) \leq C\|(\mathbf{u}, \mathbf{w})\|_{\mathcal{V}(\Omega)} .
$$

Consideremos ahora las siguientes situaciones.

- Sea $(\mathbf{u}, \mathbf{w}) \in \mathcal{G}(\Omega)$. Gracias a las condiciones de compatibilidad en la interfaz, se cumplirá $\left.\mathbf{u} \cdot \mathbf{n}\right|_{\Gamma_{\mathrm{I}}}=$ $\left.\mathbf{w} \cdot \mathbf{n}\right|_{\Gamma_{\mathrm{I}}} \cdot$ Entonces,

$$
\left\|\mathbf{u}^{e}\right\|_{H\left(\operatorname{div} ; \widehat{\Omega}_{\mathrm{F}}\right)} \leq C\left(\|\mathbf{u}\|_{H\left(\operatorname{div} ; \Omega_{\mathrm{F}}\right)}+\|\mathbf{w}\|_{1, \Omega_{\mathrm{S}}}\right)
$$

y obtendremos $\left\|\left(\mathbf{u}^{e}, \mathbf{w}^{e}\right)\right\|_{h} \leq C\|(\mathbf{u}, \mathbf{w})\|_{h, \Omega}$.

- Sea $(\mathbf{u}, \mathbf{w}) \in \mathcal{H}(\Omega)$. Entonces, $\left.\operatorname{div} \mathbf{u}\right|_{\Omega_{\mathrm{F}}}=\left.0 \mathrm{y} \mathbf{w}\right|_{\Omega_{\mathrm{S}}}=\mathbf{0}$. De nuevo, gracias a las condiciones de compatibilidad en la interfaz, $\left.\mathbf{u} \cdot \mathbf{n}\right|_{\Gamma_{\mathrm{I}}}=0$. Por lo tanto, la única solución del problema (4.66) será $\xi=0$. En este caso, la extensión de la función $\mathbf{u}$ se simplifica a

$$
\mathbf{u}^{e}:= \begin{cases}\mathbf{u} & \text { en } \Omega_{\mathrm{F}} \\ \mathbf{0} & \text { en } \omega_{T}: \omega_{T} \subset \Omega_{\mathrm{F} h} \backslash \Omega_{\mathrm{F}},\end{cases}
$$

de donde, obtendremos $\left\|\left(\mathbf{u}^{e}, \mathbf{w}^{e}\right)\right\|_{h}=\|(\mathbf{u}, \mathbf{0})\|_{h, \Omega}$.

Observación 4.33 De acuerdo con las estimaciones a priori clásicas para la ecuación de Laplace con condiciones de borde de Neumann [111], la solución $\xi$ del problema (4.66) será más regular. Supongamos que $\left.\mathbf{u} \cdot \mathbf{n}\right|_{\tilde{\ell}_{j}} \in H^{1 / 2}\left(\tilde{\ell}_{j}\right)$, siendo $\tilde{\ell}_{j}, 1 \leq j \leq N$, los lados (rectos o curvos) cuya unión define a la interfaz $\Gamma_{\mathrm{I}}$, entonces existen constantes $C>0$ y $\alpha \in(1 / 2,1]$, ambas dependiendo sólamente de $\Omega_{\mathrm{S}}$, tales que $\xi \in H^{1+\alpha}\left(\Omega_{\mathrm{S}}\right) y$

$$
\|\xi\|_{1+\alpha, \Omega_{\mathrm{S}}}^{2} \leq C\left(\|\operatorname{div} \mathbf{u}\|_{0, \Omega_{\mathrm{F}}}^{2}+\sum_{j=1}^{N}\|\mathbf{u} \cdot \mathbf{n}\|_{1 / 2, \tilde{\ell}_{j}}^{2}\right)
$$

En general, para cualquier par $(\mathbf{u}, \mathbf{w}) \in \mathcal{V}(\Omega)$, solo podremos asegurar que $\left.\mathbf{u} \cdot \mathbf{n}\right|_{\Gamma_{\mathrm{I}}} \in L^{2}\left(\Gamma_{\mathrm{I}}\right)$. Consecuentemente, $\xi \in H^{1+\alpha / 2}\left(\Omega_{\mathrm{S}}\right)$ y además

$$
\|\xi\|_{1+\alpha / 2, \Omega_{\mathrm{S}}} \leq C\left(\|\operatorname{div} \mathbf{u}\|_{0, \Omega_{\mathrm{F}}}+\|\mathbf{u} \cdot \mathbf{n}\|_{0, \Gamma_{\mathrm{I}}}\right) .
$$

Construiremos ahora el operador de extensión para las funciones de los espacios discretos. Sea $\left(\mathbf{u}_{h}, \mathbf{w}_{h}\right) \in$ $\mathcal{V}_{h}\left(\Omega_{h}\right)$. Sea $T \in \mathcal{T}_{h}^{\mathrm{F}}$ un triángulo en el borde de $\Omega_{\mathrm{F} h}$ tal que $T \subset T^{\text {id. }}$. Sea $\omega_{T}$ el dominio definido por $T^{i d} \backslash T$. Definimos

$$
\mathbf{u}_{h}^{e}:= \begin{cases}\mathbf{u}_{h} & \text { en } \Omega_{\mathrm{Fh}}, \\ \breve{\mathbf{u}}_{h} & \text { en } \omega_{T}: \omega_{T} \subset \Omega_{\mathrm{F}} \backslash \Omega_{\mathrm{F} h},\end{cases}
$$

donde $\breve{\mathbf{u}}_{h}$ denota la extensión natural (por componentes) de la función lineal $\mathbf{u}_{h} \in \mathcal{P}_{1}(T)^{2}$ al conjunto más grande $T^{i d}$ (ver definición D.8). Luego, teniendo en cuenta que div $\left.\mathbf{u}_{h}\right|_{T}$ es constante para todo $T \in \mathcal{T}_{h}^{\mathrm{F}}$ y usando los Lemas D.5 y D.9, obtendremos

$$
\left\|\operatorname{div} \mathbf{u}_{h}^{e}\right\|_{0, \omega_{T}}=\left|\operatorname{div} \mathbf{u}_{h}\right|\left|\omega_{T}\right|^{1 / 2} \leq C h_{T}^{1 / 2}\left\|\operatorname{div} \mathbf{u}_{h}\right\|_{0, T},
$$




$$
\left\|\mathbf{u}_{h}^{e}\right\|_{0, \mathbf{T}^{i d}} \leq C\left\|\mathbf{u}_{h}\right\|_{0, T} .
$$

A partir de estos resultados, es inmediato que

$$
\left\|\mathbf{u}_{h}^{e}\right\|_{H\left(\operatorname{div} ; \widehat{\Omega}_{\mathrm{F}}\right)} \leq\left\|\mathbf{u}_{h}\right\|_{H\left(\operatorname{div}, \Omega_{\mathrm{F} h}\right)}
$$

Sea $T \in \mathcal{T}_{h}^{\mathrm{S}}$ un triángulo en el borde de $\Omega_{\mathrm{Sh}}$ tal que $T \subset T^{i d}$. Sea $\omega_{T}$ el dominio definido por $T^{i d} \backslash T$. Definimos

$$
\mathbf{w}_{h}^{e}:= \begin{cases}\mathbf{w}_{h} & \text { en } \Omega_{\mathrm{Sh}}, \\ \breve{\mathbf{w}}_{h} & \text { en } \omega_{T}: \omega_{T} \subset \Omega_{\mathrm{S}} \backslash \Omega_{\mathrm{S} h},\end{cases}
$$

donde $\breve{\mathbf{w}}_{h} \in \mathcal{P}_{1}\left(T^{i d}\right)^{2}$ denota la extensión natural (por componentes) de la función lineal $\left.\mathbf{w}_{h}\right|_{T} \in \mathcal{P}_{1}(T)^{2}$ al conjunto $T^{\text {id }}$. Aplicando el Lema D.9, tendremos

$$
\left\|\mathbf{w}_{h}^{e}\right\|_{1, \widehat{\Omega}_{\mathrm{S}}} \leq C\left\|\mathbf{w}_{h}\right\|_{1, \Omega_{\mathrm{Sh}}} .
$$

Entonces, el par $\left(\mathbf{u}_{h}^{e}, \mathbf{w}_{h}^{e}\right) \in \mathcal{V}(\widehat{\Omega})$ y se verifica

$$
\left\|\left(\mathbf{u}_{h}^{e}, \mathbf{w}_{h}^{e}\right)\right\|_{h} \leq C\left\|\left(\mathbf{u}_{h}, \mathbf{w}_{h}\right)\right\|_{\mathcal{V}\left(\Omega_{h}\right)} .
$$

Consideremos las siguientes situaciones.

- Sea $\left(\mathbf{u}_{h}, \mathbf{w}_{h}\right) \in \mathcal{G}_{h}\left(\Omega_{h}\right)$. A partir de la condición de compatibilidad en la interfaz, sabemos que

$$
\int_{\ell}\left(\mathbf{u}_{h}-\mathbf{w}_{h}\right) \cdot \mathbf{n}=0 \quad \forall \ell \subset \Gamma_{\mathrm{I} h} .
$$

Como $\left.\mathbf{u}_{h} \cdot \mathbf{n}\right|_{\ell}$ es constante y $\left.\mathbf{w}_{h} \cdot \mathbf{n}\right|_{\ell}$ es una función lineal, solo podremos asegurar que $\mathbf{u}_{h} \cdot \mathbf{n} \mathrm{y}$ $\mathbf{w}_{h} \cdot \mathbf{n}$ coinciden en el punto medio de cada lado $\ell \subset \Gamma_{\mathrm{I} h}$. Luego, tomando

$$
\left.\mathbf{u}_{h} \cdot \mathbf{n}\right|_{\ell}=\frac{1}{|\ell|} \int_{\ell} \mathbf{w}_{h} \cdot \mathbf{n}
$$

en el cálculo de la norma sobre $\Gamma_{\mathrm{I} h}$, tendremos

$$
\left\|\mathbf{u}_{h} \cdot \mathbf{n}\right\|_{0, \Gamma_{\mathrm{I} h}}^{2}=\sum_{\ell \subset \Gamma_{\mathrm{I} h}}|\ell|\left(\frac{1}{|\ell|} \int_{\ell} \mathbf{w}_{h} \cdot \mathbf{n}\right)^{2} \leq\left\|\mathbf{w}_{h} \cdot \mathbf{n}\right\|_{0, \Gamma_{\mathrm{I} h}}^{2} .
$$

Procediendo como en el Lema E.7, podemos asegurar que existe una constante $C$, independiente de $h$, tal que

$$
\left\|\mathbf{w}_{h} \cdot \mathbf{n}\right\|_{0, \Gamma_{\mathrm{I} h}}^{2} \leq C\left\|\mathbf{w}_{h}\right\|_{1, \Omega_{\mathrm{Sh}}}^{2} .
$$

Estos resultados nos conducen directamente a la estimación

$$
\left\|\left(\mathbf{u}_{h}^{e}, \mathbf{w}_{h}^{e}\right)\right\|_{h} \leq C\left\|\left(\mathbf{u}_{h}, \mathbf{w}_{h}\right)\right\|_{h, \Omega_{h}} .
$$

- Sea $\left(\mathbf{u}_{h}, \mathbf{w}_{h}\right) \in \mathcal{H}_{h}\left(\Omega_{h}\right)$. A partir de la condición de compatibilidad en la interfaz,

$$
\left.\int_{\ell} \mathbf{u}_{h} \cdot \mathbf{n}\right|_{\ell}=0 \quad \forall \ell \subset \Gamma_{\mathrm{Ih}},
$$

de donde obtendremos, nuevamente,

$$
\left\|\left(\mathbf{u}_{h}^{e}, \mathbf{w}_{h}^{e}\right)\right\|_{h} \leq C\left\|\left(\mathbf{u}_{h}, \mathbf{0}\right)\right\|_{h, \Omega_{h}} .
$$

Observación 4.34 Analizando cuidadosamente los cálculos realizados en la construcción de los operadores de extensión, surge claramente que 
- $\|\cdot\|_{\mathcal{V}(\Omega)} y\|\cdot\|_{h, \Omega}$ son normas equivalentes en $\mathcal{K}(\Omega)$,

- $\|\cdot\|_{\mathcal{V}\left(\Omega_{h}\right)} y\|\cdot\|_{h, \Omega_{h}}$ son normas equivalentes en $\mathcal{K}_{h}\left(\Omega_{h}\right)$.

Para cada partición $\Omega_{h}$ definimos

$$
\begin{array}{ll}
\mathcal{V}^{e}(\widehat{\Omega}) & :=\mathbf{P}(\mathcal{V}(\Omega)) \subset \mathcal{V}(\widehat{\Omega}), \\
\mathcal{K}^{e}(\widehat{\Omega}) & :=\left\{\left(\mathbf{u}^{e}, \mathbf{w}^{e}\right) \in \mathcal{V}^{e}(\widehat{\Omega}):\left.\left(\mathbf{u}^{e}-\mathbf{w}^{e}\right)\right|_{\Gamma_{\mathrm{I}}} \cdot \mathbf{n}=0\right\}, \\
\mathcal{V}_{h}^{e}(\widehat{\Omega}) & :=\mathbf{P}_{h}\left(\mathcal{V}_{h}\left(\Omega_{h}\right)\right) \subset \mathcal{X}(\widehat{\Omega}), \\
\mathcal{K}_{h}^{e}(\widehat{\Omega}) & :=\left\{\left(\mathbf{u}_{h}^{e}, \mathbf{w}_{h}^{e}\right) \in \mathcal{V}_{h}^{e}(\widehat{\Omega}): \int_{\Gamma_{\mathrm{I} h}} \delta_{h}\left(\mathbf{u}_{h}^{e}-\mathbf{w}_{h}^{e}\right) \cdot \mathbf{n}=0 \quad \forall \delta_{h} \in \mathcal{Y}_{h}\left(\Gamma_{\mathrm{I} h}\right)\right\}, \\
\mathcal{W}(\widehat{\Omega}) & :=\left(\mathcal{K}^{e}+\mathcal{K}_{h}^{e}\right)(\widehat{\Omega}) .
\end{array}
$$

Equipamos al espacio suma con la norma $\|\cdot\|_{h}$. Es claro que

$$
\|(\mathbf{u}, \mathbf{w})\|_{h} \simeq\|(\mathbf{u}, \mathbf{w})\|_{\mathcal{V}(\widehat{\Omega})} \quad \forall(\mathbf{u}, \mathbf{w}) \in \mathcal{K}^{e}(\widehat{\Omega}) .
$$

Introduciremos a continuación los operadores solución $\mathbf{A}$ y $\mathbf{A}_{h}$, los cuales verificarán las siguientes propiedades:

- estarán asociados a los problemas espectrales (4.27) y (4.30), respectivamente,

- estarán definidos sobre el mismo espacio de funciones,

- estarán acotados uniformemente en la norma $\|\cdot\|_{h}$.

Definimos al operador A por

$$
\begin{aligned}
\mathbf{A}: \mathcal{V}(\widehat{\Omega}) & \rightarrow \mathcal{K}^{e}(\widehat{\Omega}) \subset \mathcal{V}^{e}(\widehat{\Omega}) \subset \mathcal{V}(\widehat{\Omega}) \\
\left(\mathbf{x}_{1}, \mathbf{x}_{2}\right) & \mapsto\left(\mathbf{u}^{e}, \mathbf{w}^{e}\right)=\mathbf{P} \circ \mathbf{T}\left(\left.\left(\mathbf{x}_{1}, \mathbf{x}_{2}\right)\right|_{\Omega}\right) .
\end{aligned}
$$

Es obvio que $\left.\left(\mathbf{u}^{e}, \mathbf{w}^{e}\right)\right|_{\Omega}=(\mathbf{u}, \mathbf{w}) \in \mathcal{V}(\Omega)$, donde $(\mathbf{u}, \mathbf{w}) \leftrightarrow((\mathbf{u}, \mathbf{w}), \gamma)$, siendo $((\mathbf{u}, \mathbf{w}), \gamma)$ solución del problema (4.28).

Definimos al operador $\mathbf{A}_{h}$, análogo discreto del operador $\mathbf{A}$, por:

$$
\begin{aligned}
\mathbf{A}_{h}: \mathcal{V}(\widehat{\Omega}) & \rightarrow \mathcal{K}_{h}^{e}(\widehat{\Omega}) \subset \mathcal{V}_{h}^{e}(\widehat{\Omega}) \subset \mathcal{V}(\widehat{\Omega}) \\
\left(\mathbf{x}_{1}, \mathbf{x}_{2}\right) & \mapsto\left(\mathbf{u}_{h}^{e}, \mathbf{w}_{h}^{e}\right)=\mathbf{P}_{h} \circ \mathbf{T}_{h}\left(\left.\left(\mathbf{x}_{1}, \mathbf{x}_{2}\right)\right|_{\Omega_{h}}\right) .
\end{aligned}
$$

De la misma manera que en el caso continuo, $\left.\left(\mathbf{u}_{h}^{e}, \mathbf{w}_{h}^{e}\right)\right|_{\Omega_{h}}=\left(\mathbf{u}_{h}, \mathbf{w}_{h}\right) \in \mathcal{V}_{h}\left(\Omega_{h}\right)$, donde $\left(\mathbf{u}_{h}, \mathbf{w}_{h}\right) \leftrightarrow$ $\left(\left(\mathbf{u}_{h}, \mathbf{w}_{h}\right), \gamma_{h}\right)$, siendo $\left(\left(\mathbf{u}_{h}, \mathbf{w}_{h}\right), \gamma_{h}\right)$ solución del problema (4.32).

Como (por construcción) $\mathbf{P}: \mathcal{K}(\Omega) \rightarrow \mathcal{K}^{e}(\widehat{\Omega})$ y $\mathbf{P}_{h}: \mathcal{K}_{h}\left(\Omega_{h}\right) \rightarrow \mathcal{K}_{h}^{e}(\widehat{\Omega})$ definen operadores que están uniformemente acotados en la norma $\|\cdot\|_{h}$, los operadores lineales $\mathbf{A}$ y $\mathbf{A}_{h}$ también estarán acotados uniformemente en la norma $\|\cdot\|_{h}$.

\section{Análisis del error}

En esta sección probaremos que los autovalores y las autofunciones del problema (4.30) convergen a los autovalores y autofunciones del problema (4.27) sin introducir modos de vibración espurios. También analizaremos el orden de covergencia de las aproximaciones y probaremos que es (en cierto sentido) óptimo.

Antes de probar estos resultados, necesitaremos establecer varios lemas. En los siguientes lemas estimaremos los errores de consistencia del método (no conforme) que hemos elegido para discretizar el problema (4.30). Este método fue introducido y analizado en [33] pero solo para dominios poligonales.

De aquí en adelante, $r:=\operatorname{mín}\{s, t\}, r_{h}:=\operatorname{mín}\left\{\hat{s}_{h}, t\right\}$ y $\hat{r}_{h}:=\operatorname{mín}\left\{r_{h}, \alpha\right\}$, siendo $s, t, \alpha$ y $\hat{s}_{h}$ las constantes de regularidad definidas en el Teorema 4.29, la observación 4.33 y el Lema E.12, respectivamente.

Sea $(\mathbf{f}, \mathbf{g})$ un elemento arbitrario del espacio $\mathcal{G}^{e}(\widehat{\Omega}) \cup \widehat{\mathcal{G}}(\widehat{\Omega})$ (ver observación E.22). Consideremos las soluciones $\left(\mathbf{u}^{e}, \mathbf{w}^{e}\right):=\mathbf{A}(\mathbf{f}, \mathbf{g})$ y $\left(\mathbf{u}_{h}^{e}, \mathbf{w}_{h}^{e}\right):=\mathbf{A}_{h}(\mathbf{f}, \mathbf{g})$. 
Lema 4.35 Existe una constante positiva $C$ tal que

$$
\begin{aligned}
\left\|\left(\mathbf{u}^{e}, \mathbf{w}^{e}\right)-\left(\mathbf{u}_{h}^{e}, \mathbf{w}_{h}^{e}\right)\right\|_{h} \leq C & \inf _{\left(\boldsymbol{\xi}_{h}, \mathbf{v}_{h}\right) \in \mathcal{K}_{h}\left(\Omega_{h}\right)}\left\|\left(\hat{\mathbf{u}}-\boldsymbol{\xi}_{h}, \hat{\mathbf{w}}-\mathbf{v}_{h}\right)\right\|_{h, \Omega_{h}}+\left\|\mathbf{u}^{e}-\hat{\mathbf{u}}\right\|_{H\left(\operatorname{div} ; \Omega_{\mathrm{F} h}\right)} \\
& +\sup _{\left(\boldsymbol{\eta}_{h}, \mathbf{z}_{h}\right) \in \mathcal{K}_{h}\left(\Omega_{h}\right)} \frac{a_{h}\left(\left(\hat{\mathbf{u}}-\mathbf{u}_{h}, \hat{\mathbf{w}}-\mathbf{w}_{h}\right),\left(\boldsymbol{\eta}_{h}, \mathbf{z}_{h}\right)\right)}{\left\|\left(\boldsymbol{\eta}_{h}, \mathbf{z}_{h}\right)\right\|_{h, \Omega_{h}}} \\
& \left.+\left\|\mathbf{u}-\mathbf{u}_{h}^{e}\right\|_{H\left(\operatorname{div} ; \Omega_{\mathrm{F}} \backslash \Omega_{\mathrm{F} h}\right)}+\left\|\mathbf{w}-\mathbf{w}_{h}^{e}\right\|_{1, \Omega_{\mathrm{S}} \backslash \Omega_{\mathrm{S} h}}\right) .
\end{aligned}
$$

Prueba. Teniendo en cuenta la definición de la norma $\|\cdot\|_{h}$, es claro que

$$
\begin{aligned}
\left\|\left(\mathbf{u}^{e}, \mathbf{w}^{e}\right)-\left(\mathbf{u}_{h}^{e}, \mathbf{w}_{h}^{e}\right)\right\|_{h}^{2} & =\left\|\mathbf{u}^{e}-\mathbf{u}_{h}^{e}\right\|_{H\left(\operatorname{div} ; \widehat{\Omega}_{\mathrm{F}}\right)}^{2}+\left\|\mathbf{w}^{e}-\mathbf{w}_{h}^{e}\right\|_{1, \widehat{\Omega}_{\mathrm{S}}}^{2} \\
& =\left\|\mathbf{u}^{e}-\mathbf{u}_{h}\right\|_{H\left(\operatorname{div} ; \Omega_{\mathrm{F} h}\right)}^{2}+\left\|\mathbf{w}^{e}-\mathbf{w}_{h}\right\|_{1, \Omega_{\mathrm{S} h}}^{2} \\
& +\left\|\mathbf{u}-\mathbf{u}_{h}^{e}\right\|_{H\left(\operatorname{div} ; \Omega_{\mathrm{F}} \backslash \Omega_{\mathrm{F} h}\right)}^{2}+\left\|\mathbf{w}-\mathbf{w}_{h}^{e}\right\|_{1, \Omega_{\mathrm{S}} \backslash \Omega_{\mathrm{S} h}}^{2} .
\end{aligned}
$$

Sea $\left(\boldsymbol{\xi}_{h}, \mathbf{v}_{h}\right)$ un elemento arbitrario de $\mathcal{K}_{h}\left(\Omega_{h}\right)$. Eligiendo $(\hat{\mathbf{u}}, \hat{\mathbf{w}})=\left(\nabla \hat{q}, \mathbf{w}^{e}\right)$, como en la observación E.17. podremos escribir

$$
\left\|\hat{\mathbf{u}}-\mathbf{u}_{h}\right\|_{H\left(\operatorname{div} ; \Omega_{\mathrm{F} h}\right)}^{2}+\left\|\mathbf{w}^{e}-\mathbf{w}_{h}\right\|_{1, \Omega_{\mathrm{S} h}}^{2} \leq 2\left(\left\|\left(\hat{\mathbf{u}}-\boldsymbol{\xi}_{h}, \hat{\mathbf{w}}-\mathbf{v}_{h}\right)\right\|_{h, \Omega_{h}}^{2}+\left\|\left(\mathbf{u}_{h}-\boldsymbol{\xi}_{h}, \mathbf{w}_{h}-\mathbf{v}_{h}\right)\right\|_{h, \Omega_{h}}^{2}\right) .
$$

Ahora, dado que $a_{h}$ es continua y coerciva sobre $\mathcal{K}_{h}\left(\Omega_{h}\right)$ (uniformemente en $h$ ), tendremos

$$
\begin{aligned}
& C_{0}\left\|\left(\mathbf{u}_{h}-\boldsymbol{\xi}_{h}, \mathbf{w}_{h}-\mathbf{v}_{h}\right)\right\|_{h, \Omega_{h}}^{2} \leq a_{h}\left(\left(\mathbf{u}_{h}-\boldsymbol{\xi}_{h}, \mathbf{w}_{h}-\mathbf{v}_{h}\right),\left(\mathbf{u}_{h}-\boldsymbol{\xi}_{h}, \mathbf{w}_{h}-\mathbf{v}_{h}\right)\right) \\
& =a_{h}\left(\left(\hat{\mathbf{u}}-\boldsymbol{\xi}_{h}, \hat{\mathbf{w}}-\mathbf{v}_{h}\right),\left(\mathbf{u}_{h}-\boldsymbol{\xi}_{h}, \mathbf{w}_{h}-\mathbf{v}_{h}\right)\right) \\
& -a_{h}\left(\left(\hat{\mathbf{u}}-\mathbf{u}_{h}, \hat{\mathbf{w}}-\mathbf{w}_{h}\right),\left(\mathbf{u}_{h}-\boldsymbol{\xi}_{h}, \mathbf{w}_{h}-\mathbf{v}_{h}\right)\right) \\
& \leq C_{1}\left\|\hat{\mathbf{u}}-\boldsymbol{\xi}_{h}\right\|_{H\left(\operatorname{div} ; \Omega_{\mathrm{Fh}}\right)}\left\|\mathbf{u}_{h}-\boldsymbol{\xi}_{h}\right\|_{H\left(\operatorname{div} ; \Omega_{\mathrm{F} h}\right)} \\
& +C_{2}\left\|\hat{\mathbf{w}}-\mathbf{v}_{h}\right\|_{1, \Omega_{\mathrm{S} h}}\left\|\mathbf{w}_{h}-\mathbf{v}_{h}\right\|_{1, \Omega_{\mathrm{S} h}} \\
& -a_{h}\left(\left(\hat{\mathbf{u}}-\mathbf{u}_{h}, \hat{\mathbf{w}}-\mathbf{w}_{h}\right),\left(\mathbf{u}_{h}-\boldsymbol{\xi}_{h}, \mathbf{w}_{h}-\mathbf{v}_{h}\right)\right) \text {, }
\end{aligned}
$$

expresión a partir de la cual obtendremos

$$
\begin{aligned}
\left\|\left(\mathbf{u}_{h}-\boldsymbol{\xi}_{h}, \mathbf{w}_{h}-\mathbf{v}_{h}\right)\right\|_{h, \Omega_{h}} & \leq C\left(\left\|\hat{\mathbf{u}}-\boldsymbol{\xi}_{h}\right\|_{H\left(\operatorname{div} ; \Omega_{\mathrm{Fh}}\right)}+\left\|\hat{\mathbf{w}}-\mathbf{v}_{h}\right\|_{1, \Omega_{\mathrm{Sh}}}\right. \\
& \left.+\sup _{\left(\boldsymbol{\eta}_{h}, \mathbf{z}_{h}\right) \in \mathcal{K}_{h}\left(\Omega_{h}\right)} \frac{a_{h}\left(\left(\hat{\mathbf{u}}-\mathbf{u}_{h}, \hat{\mathbf{w}}-\mathbf{w}_{h}\right),\left(\boldsymbol{\eta}_{h}, \mathbf{z}_{h}\right)\right)}{\left\|\left(\boldsymbol{\eta}_{h}, \mathbf{z}_{h}\right)\right\|_{h, \Omega_{h}}}\right) .
\end{aligned}
$$

De esta manera, combinando las desigualdades halladas, concluimos la prueba.

A continuación, vamos a estimar cada uno de los términos que aparecen en el lado derecho de la desigualdad establecida en el Lema 4.35 por separado. Observemos que el primer término puede acotarse directamente usando el Lema E.16 y la observación E.17.

Lema 4.36 Existe una constante positiva $C$ tal que

$$
\sup _{\left(\boldsymbol{\eta}_{h}, \mathbf{z}_{h}\right) \in \mathcal{K}_{h}\left(\Omega_{h}\right)} \frac{a_{h}\left(\left(\hat{\mathbf{u}}-\mathbf{u}_{h}, \hat{\mathbf{w}}-\mathbf{w}_{h}\right),\left(\boldsymbol{\eta}_{h}, \mathbf{z}_{h}\right)\right)}{\left\|\left(\boldsymbol{\eta}_{h}, \mathbf{z}_{h}\right)\right\|_{h, \Omega_{h}}} \leq C h^{r_{h}}\|(\mathbf{f}, \mathbf{g})\|_{h} .
$$

Prueba. Recordemos primero que el núcleo discreto admite la siguiente descomposición (ver sección E.1)

$$
\mathcal{K}_{h}\left(\Omega_{h}\right)=\mathcal{H}_{h}\left(\Omega_{h}\right) \oplus \mathcal{G}_{h}\left(\Omega_{h}\right) .
$$

Sea entonces $\left(\boldsymbol{\eta}_{h}, \mathbf{z}_{h}\right)$ un elemento arbitrario de $\mathcal{G}_{h}\left(\Omega_{h}\right)$. Tendremos

$$
\begin{aligned}
& a_{h}\left(\left(\hat{\mathbf{u}}-\mathbf{u}_{h}, \hat{\mathbf{w}}-\mathbf{w}_{h}\right),\left(\boldsymbol{\eta}_{h}, \mathbf{z}_{h}\right)\right)=\rho_{\mathrm{F}} c^{2} \int_{\Omega_{\mathrm{F} h}} \operatorname{div}\left(\hat{\mathbf{u}}-\mathbf{u}_{h}\right) \operatorname{div} \boldsymbol{\eta}_{h}+\rho_{\mathrm{F}} \int_{\Omega_{\mathrm{F} h}}\left(\hat{\mathbf{u}}-\mathbf{u}_{h}\right) \cdot \boldsymbol{\eta}_{h} \\
& +\int_{\Omega_{\mathrm{S} h}} \boldsymbol{\sigma}\left(\hat{\mathbf{w}}-\mathbf{w}_{h}\right): \boldsymbol{\varepsilon}\left(\mathbf{z}_{h}\right)+\rho_{\mathrm{S}} \int_{\Omega_{\mathrm{S} h}}\left(\hat{\mathbf{w}}-\mathbf{w}_{h}\right) \cdot \mathbf{z}_{h} \\
& =\rho_{\mathrm{F}} c^{2} \int_{\Omega_{\mathrm{F} h}} \operatorname{div} \hat{\mathbf{u}} \operatorname{div} \boldsymbol{\eta}_{h}+\rho_{\mathrm{F}} \int_{\Omega_{\mathrm{F} h}}^{\mathrm{S} h} \cdot \boldsymbol{\eta}_{h}+\int_{\Omega_{\mathrm{S} h}} \boldsymbol{\sigma}(\hat{\mathbf{w}}): \boldsymbol{\varepsilon}\left(\mathbf{z}_{h}\right) \\
& +\rho_{\mathrm{S}} \int_{\Omega_{\mathrm{S} h}} \hat{\mathbf{w}} \cdot \mathbf{z}_{h}-\rho_{\mathrm{F}} \int_{\Omega_{\mathrm{F} h}} \mathbf{f} \cdot \boldsymbol{\eta}_{h}-\rho_{\mathrm{S}} \int_{\Omega_{\mathrm{S} h}} \mathbf{g} \cdot \mathbf{z}_{h} \text {. }
\end{aligned}
$$


Los primeros cuatro términos en el lado derecho de la expresión anterior, pueden ser escritos de la siguiente manera

$$
\begin{aligned}
& \rho_{\mathrm{F}} c^{2} \int_{\Omega_{\mathrm{F} h}} \operatorname{div} \hat{\mathbf{u}} \operatorname{div} \boldsymbol{\eta}_{h}+\rho_{\mathrm{F}} \int_{\Omega_{\mathrm{F} h}} \hat{\mathbf{u}} \cdot \boldsymbol{\eta}_{h}=\rho_{\mathrm{F}} c^{2} \int_{\Omega_{\mathrm{F}}} \operatorname{div} \hat{\mathbf{u}} \operatorname{div} \boldsymbol{\eta}_{h}^{e}+\rho_{\mathrm{F}} \int_{\Omega_{\mathrm{F}}} \hat{\mathbf{u}} \cdot \boldsymbol{\eta}_{h}^{e} \\
& +\rho_{\mathrm{F}} c^{2} \int_{\Omega_{\mathrm{F} h} \backslash \Omega_{\mathrm{F}}} \operatorname{div} \hat{\mathbf{u}} \operatorname{div} \boldsymbol{\eta}_{h}+\rho_{\mathrm{F}} \int_{\Omega_{\mathrm{F} h} \backslash \Omega_{\mathrm{F}}} \hat{\mathbf{u}} \cdot \boldsymbol{\eta}_{h} \\
& -\rho_{\mathrm{F}} c^{2} \int_{\Omega_{\mathrm{F}} \backslash \Omega_{\mathrm{F} h}}^{\mathrm{F} h} \operatorname{div} \hat{\mathbf{u}} \operatorname{div} \boldsymbol{\eta}_{h}^{e}-\rho_{\mathrm{F}} \int_{\Omega_{\mathrm{F}} \backslash \Omega_{\mathrm{F} h}}^{\mathrm{F} h} \cdot \boldsymbol{\eta}_{h}^{e}, \\
& \int_{\Omega_{\mathrm{S} h}} \boldsymbol{\sigma}(\hat{\mathbf{w}}): \varepsilon\left(\mathbf{z}_{h}\right)+\rho_{\mathrm{S}} \int_{\Omega_{\mathrm{S} h}} \hat{\mathbf{w}} \cdot \mathbf{z}_{h}=\int_{\Omega_{\mathrm{S}}} \boldsymbol{\sigma}(\hat{\mathbf{w}}): \varepsilon\left(\mathbf{z}_{h}^{e}\right)+\rho_{\mathrm{S}} \int_{\Omega_{\mathrm{S}}} \hat{\mathbf{w}} \cdot \mathbf{z}_{h}^{e} \\
& +\int_{\Omega_{\mathrm{Sh}} \backslash \Omega_{\mathrm{S}}} \boldsymbol{\sigma}(\hat{\mathbf{w}}): \varepsilon\left(\mathbf{z}_{h}\right)+\rho_{\mathrm{S}} \int_{\Omega_{\mathrm{S} h} \backslash \Omega_{\mathrm{S}}} \hat{\mathbf{w}} \cdot \mathbf{z}_{h} \\
& -\int_{\Omega_{\mathrm{S}} \backslash \Omega_{\mathrm{S} h}} \boldsymbol{\sigma}(\hat{\mathbf{w}}): \varepsilon\left(\mathbf{z}_{h}^{e}\right)-\rho_{\mathrm{S}} \int_{\Omega_{\mathrm{S}} \backslash \Omega_{\mathrm{S} h}}^{\mathrm{w}} \cdot \mathbf{z}_{h}^{e} .
\end{aligned}
$$

Como el par $\left.\left(\boldsymbol{\eta}_{h}^{e}, \mathbf{z}_{h}^{e}\right)\right|_{\Omega} \in \mathcal{V}(\Omega)$, podríamos usarlo como función test en el problema 4.28. Haciendo esto y teniendo en cuenta la definición de $\hat{\mathbf{u}}$, obtendremos

$$
\begin{aligned}
& \rho_{\mathrm{F}} c^{2} \int_{\Omega_{\mathrm{F}}} \operatorname{div} \hat{\mathbf{u}} \operatorname{div} \boldsymbol{\eta}_{h}^{e}+\rho_{\mathrm{F}} \int_{\Omega_{\mathrm{F}}} \hat{\mathbf{u}} \cdot \boldsymbol{\eta}_{h}^{e}+\int_{\Omega_{\mathrm{S}}} \boldsymbol{\sigma}(\hat{\mathbf{w}}): \varepsilon\left(\mathbf{z}_{h}^{e}\right)+\rho_{\mathrm{S}} \int_{\Omega_{\mathrm{S}}} \hat{\mathbf{w}} \cdot \mathbf{z}_{h}^{e} \\
& \quad=\rho_{\mathrm{F}} \int_{\Omega_{\mathrm{F}}}\left(c^{2}(\operatorname{div} \mathbf{u}+\hat{c}) \operatorname{div} \boldsymbol{\eta}_{h}^{e}+\hat{\mathbf{u}} \cdot \boldsymbol{\eta}_{h}^{e}\right)+\int_{\Omega_{\mathrm{S}}}\left(\boldsymbol{\sigma}(\mathbf{w}): \varepsilon\left(\mathbf{z}_{h}^{e}\right)+\rho_{\mathrm{S}} \mathbf{w} \cdot \mathbf{z}_{h}^{e}\right) \\
& \quad=\rho_{\mathrm{F}} \int_{\Omega_{\mathrm{F}}}\left(c^{2} \hat{c} \operatorname{div} \boldsymbol{\eta}_{h}^{e}+(\hat{\mathbf{u}}-\mathbf{u}) \cdot \boldsymbol{\eta}_{h}^{e}+\mathbf{f} \cdot \boldsymbol{\eta}_{h}^{e}\right)+\rho_{\mathrm{S}} \int_{\Omega_{\mathrm{S}}} \mathbf{g} \cdot \mathbf{z}_{h}^{e}-\int_{\Gamma_{\mathrm{I}}} \gamma\left(\boldsymbol{\eta}_{h}^{e}-\mathbf{z}_{h}^{e}\right) \cdot \mathbf{n} .
\end{aligned}
$$

Reemplazando las ecuaciones (4.73)-(4.75) en (4.72), resultará

$$
\begin{aligned}
& a_{h}\left(\left(\hat{\mathbf{u}}-\mathbf{u}_{h}, \hat{\mathbf{w}}-\mathbf{w}_{h}\right),\left(\boldsymbol{\eta}_{h}, \mathbf{z}_{h}\right)\right) \\
& =\rho_{\mathrm{F}} \int_{\Omega_{\mathrm{F}} \backslash \Omega_{\mathrm{F} h}} \mathbf{f} \cdot \boldsymbol{\eta}_{h}^{e}-\rho_{\mathrm{F}} \int_{\Omega_{\mathrm{F} h} \backslash \Omega_{\mathrm{F}}} \mathbf{f} \cdot \boldsymbol{\eta}_{h}+\rho_{\mathrm{S}} \int_{\Omega_{\mathrm{S}} \backslash \Omega_{\mathrm{Sh}}} \mathbf{g} \cdot \mathbf{z}_{h}^{e}-\rho_{\mathrm{S}} \int_{\Omega_{\mathrm{S} h} \backslash \Omega_{\mathrm{S}}} \mathbf{g} \cdot \mathbf{z}_{h} \\
& +\rho_{\mathrm{F}} \int_{\Omega_{\mathrm{F}}}\left(c^{2} \hat{c} \operatorname{div} \boldsymbol{\eta}_{h}^{e}+(\hat{\mathbf{u}}-\mathbf{u}) \cdot \boldsymbol{\eta}_{h}^{e}\right)-\int_{\Gamma_{\mathrm{I}}} \gamma\left(\boldsymbol{\eta}_{h}^{e} \cdot \mathbf{n}-\mathbf{z}_{h}^{e} \cdot \mathbf{n}\right) \\
& +\rho_{\mathrm{F}} \int_{\Omega_{\mathrm{F} h} \backslash \Omega_{\mathrm{F}}}\left(c^{2} \operatorname{div} \hat{\mathbf{u}} \operatorname{div} \boldsymbol{\eta}_{h}+\hat{\mathbf{u}} \cdot \boldsymbol{\eta}_{h}\right)-\rho_{\mathrm{F}} \int_{\Omega_{\mathrm{F}} \backslash \Omega_{\mathrm{F} h}}\left(c^{2} \operatorname{div} \hat{\mathbf{u}} \operatorname{div} \boldsymbol{\eta}_{h}^{e}-\hat{\mathbf{u}} \cdot \boldsymbol{\eta}_{h}^{e}\right) \\
& +\int_{\Omega_{\mathrm{S} h} \backslash \Omega_{\mathrm{S}}}\left(\boldsymbol{\sigma}(\hat{\mathbf{w}}): \varepsilon\left(\mathbf{z}_{h}\right)+\rho_{\mathrm{S}} \hat{\mathbf{w}} \cdot \mathbf{z}_{h}\right)-\int_{\Omega_{\mathrm{S}} \backslash \Omega_{\mathrm{Sh}}}\left(\boldsymbol{\sigma}(\mathbf{w}): \varepsilon\left(\mathbf{z}_{h}^{e}\right)+\rho_{\mathrm{S}} \mathbf{w} \cdot \mathbf{z}_{h}^{e}\right) .
\end{aligned}
$$

Todos los términos en el lado derecho de la expresión anterior pueden acotarse usando los Lemas E.24E.31. Así, obtendremos

$$
\sup _{\left(\boldsymbol{\eta}_{h}, \mathbf{z}_{h}\right) \in \mathcal{G}_{h}\left(\Omega_{h}\right)} \frac{a_{h}\left(\left(\hat{\mathbf{u}}-\mathbf{u}_{h}, \hat{\mathbf{w}}-\mathbf{w}_{h}\right),\left(\boldsymbol{\eta}_{h}, \mathbf{z}_{h}\right)\right)}{\left\|\left(\boldsymbol{\eta}_{h}, \mathbf{z}_{h}\right)\right\|_{h, \Omega_{h}}} \leq C h^{r_{h}}\|(\mathbf{f}, \mathbf{g})\|_{h} .
$$

Elijamos ahora un elemento $\left(\boldsymbol{\eta}_{h}, \mathbf{0}\right)$ en $\mathcal{H}_{h}\left(\Omega_{h}\right)$. Tendremos

$$
\begin{aligned}
a_{h}\left(\left(\hat{\mathbf{u}}-\mathbf{u}_{h}, \hat{\mathbf{w}}-\mathbf{w}_{h}\right),\left(\boldsymbol{\eta}_{h}, \mathbf{0}\right)\right) & =\rho_{\mathrm{F}} \int_{\Omega_{\mathrm{F} h}}\left(\hat{\mathbf{u}}-\mathbf{u}_{h}\right) \cdot \boldsymbol{\eta}_{h}=\rho_{\mathrm{F}} \int_{\widehat{\Omega_{\mathrm{F}}}} \hat{\mathbf{u}} \cdot \boldsymbol{\eta}_{h}^{e}-\rho_{\mathrm{F}} \int_{\Omega_{\mathrm{F} h}} \mathbf{f} \cdot \boldsymbol{\eta}_{h} \\
& =\rho_{\mathrm{F}} \int_{\Omega_{\mathrm{F}}}(\hat{\mathbf{u}}-\mathbf{u}) \cdot \boldsymbol{\eta}_{h}^{e}+\rho_{\mathrm{F}} \int_{\Omega_{\mathrm{F} h} \backslash \Omega_{\mathrm{F}}} \hat{\mathbf{u}} \cdot \boldsymbol{\eta}_{h}+\rho_{\mathrm{F}} \int_{\Omega_{\mathrm{F}}} \mathbf{u} \cdot \boldsymbol{\eta}_{h}^{e} \\
& -\rho_{\mathrm{F}} \int_{\Omega_{\mathrm{F} h}}^{\mathbf{f} \cdot \boldsymbol{\eta}_{h} .}
\end{aligned}
$$

A partir de la descomposición (discreta) de Helmholtz, sabemos que

$$
\boldsymbol{\eta}_{h}=\operatorname{curl} p_{h}, \quad p_{h} \in \mathcal{Z}_{h}\left(\Omega_{\mathrm{Fh}}\right) .
$$


Sea $\hat{p}$ la función asociada a $p_{h}$ (ver definición D.10). Sea $\hat{\boldsymbol{\eta}}=\operatorname{curl} \hat{p}$. Es fácil verificar que $(\hat{\boldsymbol{\eta}}, \mathbf{0}) \in \mathcal{V}(\Omega)$. Luego, podemos elegirlo como función test en el problem 4.28. De esta manera, obtendremos

$$
\rho_{\mathrm{F}} \int_{\Omega_{\mathrm{F}}} \mathbf{u} \cdot \hat{\boldsymbol{\eta}}=\rho_{\mathrm{F}} \int_{\Omega_{\mathrm{F}}} \mathbf{f} \cdot \hat{\boldsymbol{\eta}} .
$$

Reemplazando este resultado en la ecuación (4.78), tendremos

$$
\begin{aligned}
& a_{h}\left(\left(\hat{\mathbf{u}}-\mathbf{u}_{h}, \hat{\mathbf{w}}-\mathbf{w}_{h}\right),\left(\boldsymbol{\eta}_{h}, \mathbf{0}\right)\right)=\rho_{\mathrm{F}} \int_{\Omega_{\mathrm{F}}}(\hat{\mathbf{u}}-\mathbf{u}) \cdot \boldsymbol{\eta}_{h}^{e} \\
& +\rho_{\mathrm{F}} \int_{\Omega_{\mathrm{F} h} \backslash \Omega_{\mathrm{F}}} \hat{\mathbf{u}} \cdot \boldsymbol{\eta}_{h}+\rho_{\mathrm{F}} \int_{\Omega_{\mathrm{F}}} \mathbf{u} \cdot\left(\boldsymbol{\eta}_{h}^{e}-\hat{\boldsymbol{\eta}}\right) \\
& -\rho_{\mathrm{F}} \int_{\Omega_{\mathrm{F}}} \mathbf{f} \cdot\left(\boldsymbol{\eta}_{h}^{e}-\hat{\boldsymbol{\eta}}\right)-\rho_{\mathrm{F}} \int_{\Omega_{\mathrm{F} h} \backslash \Omega_{\mathrm{F}}} \mathbf{f} \cdot \boldsymbol{\eta}_{h} .
\end{aligned}
$$

Los términos que aparecen en el lado derecho de la expresión anterior pueden acotarse usando los Lemas E.32 - E.35. Haciendo esto, llegamos a

$$
\sup _{\left(\boldsymbol{\eta}_{h}, \mathbf{0}\right) \in \mathcal{H}_{h}\left(\Omega_{h}\right)} \frac{a_{h}\left(\left(\hat{\mathbf{u}}-\mathbf{u}_{h}, \hat{\mathbf{w}}-\mathbf{w}_{h}\right),\left(\boldsymbol{\eta}_{h}, \mathbf{0}\right)\right)}{\left\|\left(\boldsymbol{\eta}_{h}, \mathbf{0}\right)\right\|_{h, \Omega_{h}}} \leq C h^{r_{h}}\|(\mathbf{f}, \mathbf{g})\|_{h} .
$$

Así, las desigualdades (4.77) y (4.80), nos permiten concluir la prueba.

Lema 4.37 Existe una constante positiva $C$ tal que

$$
\left\|\mathbf{u}^{e}-\hat{\mathbf{u}}\right\|_{H\left(\operatorname{div} ; \Omega_{\mathrm{Fh}}\right)} \leq h^{\hat{r}_{h}}\|(\mathbf{f}, \mathbf{g})\|_{0, \Omega}
$$

Prueba. Tendremos

$$
\left\|\mathbf{u}^{e}-\hat{\mathbf{u}}\right\|_{H\left(\operatorname{div} ; \Omega_{\mathrm{Fh}}\right)} \leq\|\mathbf{u}-\hat{\mathbf{u}}\|_{H\left(\operatorname{div} ; \Omega_{\mathrm{F}} \cap \Omega_{\mathrm{F} h}\right)}+\left\|\mathbf{u}^{e}-\hat{\mathbf{u}}\right\|_{H\left(\operatorname{div} ; \Omega_{\mathrm{F} h} \backslash \Omega_{\mathrm{F}}\right) .} .
$$

Gracias al Lema E.12,

$$
\|\mathbf{u}-\hat{\mathbf{u}}\|_{H\left(\operatorname{div} ; \Omega_{\mathrm{F}} \cap \Omega_{\mathrm{F} h}\right)} \leq\|\mathbf{u}-\hat{\mathbf{u}}\|_{H\left(\operatorname{div} ; \Omega_{\mathrm{F}}\right)} \leq C h^{\left(r+\hat{s}_{h}\right) / 2}\|(\mathbf{f}, \mathbf{g})\|_{0, \Omega} .
$$

Como $\hat{\mathbf{u}} \in H^{s_{h}}\left(\operatorname{div} ; \widehat{\Omega}_{\mathrm{F}}\right)$, aplicando el Lema D.6 y usando la estimación (E.11),

$$
\begin{aligned}
\left\|\mathbf{u}^{e}-\hat{\mathbf{u}}\right\|_{H\left(\operatorname{div} ; \Omega_{\mathrm{F} h} \backslash \Omega_{\mathrm{F}}\right)} & \leq C\left(\left\|\mathbf{u}^{e}\right\|_{H\left(\operatorname{div} ; \Omega_{\mathrm{F} h} \backslash \Omega_{\mathrm{F}}\right)}+\|\hat{\mathbf{u}}\|_{H\left(\operatorname{div} ; \Omega_{\mathrm{F} h} \backslash \Omega_{\mathrm{F}}\right)}\right) \\
& \leq C\left(\left\|\mathbf{u}^{e}\right\|_{H\left(\operatorname{div} ; \Omega_{\mathrm{F} h} \backslash \Omega_{\mathrm{F}}\right)}+h^{s_{h}}\|\hat{\mathbf{u}}\|_{s_{h}, \Omega_{\mathrm{F} h}}\right) \\
& \leq C\left(\left\|\mathbf{u}^{e}\right\|_{H\left(\operatorname{div} ; \Omega_{\mathrm{F} h} \backslash \Omega_{\mathrm{F}}\right)}+h^{s_{h}}\|(\mathbf{f}, \mathbf{g})\|_{0, \Omega}\right) .
\end{aligned}
$$

Sea $T \in \mathcal{T}_{h}^{\mathrm{F}}$ un triángulo en el borde del dominio $\Omega_{\mathrm{F}}$ tal que $T \supset T^{i d}$. Sea $\omega_{T}$ el conjunto definido por $T \backslash T^{i d}$. A partir de la definición de la extensión $\mathbf{u}^{e}$, tendremos

$$
\left\|\mathbf{u}^{e}\right\|_{H\left(\operatorname{div} ; \Omega_{\mathrm{F} h} \backslash \Omega_{\mathrm{F}}\right)}^{2}=\sum_{\substack{T \in \mathcal{B}_{\mathrm{F} h} \\ T \supset T^{i d}}}\|\nabla \xi\|_{0, \omega_{T}}^{2}=\|\nabla \xi\|_{0, \Omega_{\mathrm{S}} \backslash \Omega_{\mathrm{Sh}}}^{2} .
$$

Procediendo como lo hicimos en la prueba de la Propiedad E.19 (ver Pasos 1 y 2 en esa demostración), usando la estimación (4.67), la condición de compatibilidad sobre la interfaz $\Gamma_{\mathrm{I}}$ y el Teorema 4.29, llegaremos a

$$
\|\nabla \xi\|_{0, \Omega_{\mathrm{S}} \backslash \Omega_{\mathrm{Sh}}} \leq C h^{\alpha}\|\xi\|_{1+\alpha, \Omega_{\mathrm{S}}} \leq C h^{\alpha}\|(\mathbf{f}, \mathbf{g})\|_{0, \Omega} .
$$

De esta manera, el lema es una consecuencia de las estimaciones halladas.

Lema 4.38 Existe una constante positiva $C$ tal que

$$
\left\|\mathbf{w}-\mathbf{w}_{h}^{e}\right\|_{1, \Omega_{\mathrm{S}} \backslash \Omega_{\mathrm{Sh}}} \leq C h^{r_{h}}\|(\mathbf{f}, \mathbf{g})\|_{0, \widehat{\Omega}} .
$$


Prueba. Sabemos que

$$
\left\|\mathbf{w}-\mathbf{w}_{h}^{e}\right\|_{1, \Omega_{\mathrm{S}} \backslash \Omega_{\mathrm{S} h}} \leq\left\|\mathbf{w}-\mathbf{w}_{h}^{e}\right\|_{0, \Omega_{\mathrm{S}} \backslash \Omega_{\mathrm{S} h}}+\left\|\nabla\left(\mathbf{w}-\mathbf{w}_{h}^{e}\right)\right\|_{0, \Omega_{\mathrm{S}} \backslash \Omega_{\mathrm{S} h}} .
$$

Gracias a los Lemas D.6 y D.9, el Teorema 4.29 y la estimación (4.65), podremos obtener

$$
\left\|\mathbf{w}-\mathbf{w}_{h}^{e}\right\|_{0, \Omega_{\mathrm{S}} \backslash \Omega_{\mathrm{S} h}} \leq C\left(\|\mathbf{w}\|_{0, \Omega_{\mathrm{S}} \backslash \Omega_{\mathrm{S} h}}+\left\|\mathbf{w}_{h}^{e}\right\|_{0, \Omega_{\mathrm{S}} \backslash \Omega_{\mathrm{S} h}}\right) \leq C h\left(\|\mathbf{w}\|_{1, \Omega_{\mathrm{S}}}+\left\|\mathbf{w}_{h}^{e}\right\|_{1, \Omega_{\mathrm{S}}}\right) \leq C h\|(\mathbf{f}, \mathbf{g})\|_{0, \widehat{\Omega}} .
$$

Sea $T \in \mathcal{T}_{h}^{\mathrm{s}}$ un triángulo en el borde del dominio $\Omega_{\mathrm{S}}$ tal que $T \subset T^{i d}$. Sea $\omega_{T}$ el conjunto definido por $T \backslash T^{i d}$. Gracias a los Lemas D.7 y D.9, obtendremos ahora

$$
\begin{aligned}
\left\|\nabla\left(\mathbf{w}-\mathbf{w}_{h}^{e}\right)\right\|_{0, \Omega_{\mathrm{S}} \backslash \Omega_{\mathrm{S} h}}^{2} & =\sum_{\substack{T \in \mathcal{B}_{\mathrm{Sh}} \\
T \subset T^{i d}}}\left\|\nabla\left(\mathbf{w}-\mathbf{w}_{h}^{e}\right)\right\|_{0, \omega_{T}}^{2} \\
\leq & C \sum_{\substack{T \in \mathcal{B}_{\mathrm{Sh}} \\
T \subset T^{i d}}}\left(\left\|\nabla\left(\mathbf{w}-\mathbf{w}_{h}\right)\right\|_{0, T}+h^{2 t}\left\|\nabla\left(\mathbf{w}-\mathbf{w}_{h}^{e}\right)\right\|_{t, T^{i d}}\right)^{2} \\
\leq & C\left(\left\|\nabla\left(\mathbf{w}^{e}-\mathbf{w}_{h}\right)\right\|_{0, \Omega_{\mathrm{S} h}}^{2}+h^{4 t}\left(\|\mathbf{w}\|_{1+t, \Omega_{\mathrm{S}}}^{2}+\left\|\mathbf{w}_{h}^{e}\right\|_{1, \Omega_{\mathrm{S}}}^{2}\right)\right),
\end{aligned}
$$

donde hemos usado que $\left\|\nabla \mathbf{w}_{h}^{e}\right\|_{t, T^{i d}}=\left\|\nabla \mathbf{w}_{h}^{e}\right\|_{0, T^{i d}}$, ya que $\left.\mathbf{w}_{h}^{e}\right|_{T} \in \mathcal{P}_{1}\left(T^{i d}\right)^{2}$. El término $\| \nabla\left(\mathbf{w}^{e}-\right.$ $\left.\mathbf{w}_{h}\right) \|_{0, \Omega_{\mathrm{S} h}}$ puede ser acotado combinando las estimaciones (4.70) y (4.71) en la prueba del Lema $4.35 \mathrm{y}$ usando los Lemas 4.36 y E.16 junto con la observación E.17. Los términos $\|\mathbf{w}\|_{1+t, \Omega_{\mathrm{S}}}^{2} \mathrm{y}\left\|\mathbf{w}_{h}^{e}\right\|_{1, \Omega_{\mathrm{S}}}^{2}$ pueden ser acotados directamente usando el Teorema 4.29, el Lema D.9 y la estimación (4.65). Haciendo esto, obtendremos

$$
\left\|\mathbf{w}^{e}-\mathbf{w}_{h}\right\|_{1, \Omega_{\mathrm{Sh}}} \leq C h^{r_{h}}\|(\mathbf{f}, \mathbf{g})\|_{0, \widehat{\Omega}} .
$$

Así, el lema es una consecuencia de las estimaciones halladas.

Lema 4.39 Exite una constante positiva $C$ tal que

$$
\left\|\mathbf{u}-\mathbf{u}_{h}^{e}\right\|_{H\left(\operatorname{div} ; \Omega_{\mathrm{F}} \backslash \Omega_{\mathrm{F} h}\right)} \leq h^{r_{h}}\|(\mathbf{f}, \mathbf{g})\|_{0, \widehat{\Omega}} .
$$

Prueba. Sea $T \in \mathcal{T}_{h}^{\mathrm{F}}$ un triángulo en el borde de $\Omega_{\mathrm{F}}$ tal que $T \subset T^{i d}$. Sea $\omega_{T}$ el conjunto definido por $T^{i d} \backslash T$. Dado que $\left.\mathbf{u}_{h}^{e}\right|_{T^{i d}} \in \mathcal{P}\left(T^{i d}\right)^{2},\left.\mathbf{u}_{h}^{e}\right|_{T^{i d}} \in H^{1}\left(T^{i d}\right)^{2}$. Dado que $\left.\mathbf{u}_{h}\right|_{T} \in \mathcal{R}_{0}(T),\left\|\nabla \mathbf{u}_{h}^{e}\right\|_{0, T^{i d}}=$ $\frac{1}{\sqrt{2}}\left\|\operatorname{div} \mathbf{u}_{h}^{e}\right\|_{0, T^{i d}}$. Luego, para todo $s \in(1 / 2,1]$, tendremos

$$
\left\|\mathbf{u}_{h}^{e}\right\|_{s, T^{i d}} \leq C\left\|\mathbf{u}_{h}^{e}\right\|_{1, T^{i d}} \leq C\left(\left\|\mathbf{u}_{h}^{e}\right\|_{0, T^{i d}}^{2}+\left\|\nabla \mathbf{u}_{h}^{e}\right\|_{0, T^{i d}}^{2}\right)^{1 / 2} \leq C\left(\left\|\mathbf{u}_{h}^{e}\right\|_{0, T^{i d}}^{2}+\left\|\operatorname{div} \mathbf{u}_{h}^{e}\right\|_{0, T^{i d}}^{2}\right)^{1 / 2} .
$$

Teniendo en cuenta ahora las propiedades de la extensión discreta, podremos escribir

$$
\begin{gathered}
\left\|\mathbf{u}_{h}^{e}\right\|_{s, T^{i d}} \leq C\left\|\mathbf{u}_{h}\right\|_{H(\operatorname{div}, T)}, \\
\left\|\operatorname{div} \mathbf{u}_{h}^{e}\right\|_{s, T^{i d}}=\left\|\operatorname{div} \mathbf{u}_{h}^{e}\right\|_{0, T^{i d}} \leq C\left\|\operatorname{div} \mathbf{u}_{h}^{e}\right\|_{0, T} .
\end{gathered}
$$

Aplicando los Lemas D.7 y D.9, obtendremos

$$
\begin{aligned}
\left\|\mathbf{u}-\mathbf{u}_{h}^{e}\right\|_{0, \omega_{T}}^{2} & =\left(\left\|\mathbf{u}-\mathbf{u}_{h}\right\|_{0, T}+h^{2 s}\left\|\mathbf{u}-\mathbf{u}_{h}^{e}\right\|_{s, T^{i d}}\right)^{2} \\
& \leq C\left(\left\|\mathbf{u}-\mathbf{u}_{h}\right\|_{0, T}^{2}+h^{4 s}\left(\|\mathbf{u}\|_{s, T^{i d}}^{2}+\left\|\mathbf{u}_{h}^{e}\right\|_{s, T^{i d}}^{2}\right)\right) \\
& \leq C\left(\left\|\mathbf{u}-\mathbf{u}_{h}\right\|_{0, T}^{2}+h^{4 s}\left(\|\mathbf{u}\|_{s, T^{i d}}^{2}+\left\|\mathbf{u}_{h}\right\|_{H(\operatorname{div}, T)}^{2}\right)\right), \\
\left\|\operatorname{div}\left(\mathbf{u}-\mathbf{u}_{h}^{e}\right)\right\|_{0, \omega_{T}}^{2} & =\left(\left\|\operatorname{div}\left(\mathbf{u}-\mathbf{u}_{h}\right)\right\|_{0, T}+h^{2 s}\left\|\operatorname{div}\left(\mathbf{u}-\mathbf{u}_{h}^{e}\right)\right\|_{s, T^{i d}}\right)^{2} \\
\leq & C\left(\left\|\operatorname{div}\left(\mathbf{u}-\mathbf{u}_{h}\right)\right\|_{0, T}^{2}+h^{4 s}\left(\|\operatorname{div} \mathbf{u}\|_{s, T^{i d}}^{2}+\left\|\operatorname{div} \mathbf{u}_{h}\right\|_{0, T^{i d}}^{2}\right)\right) \\
\leq & C\left(\left\|\operatorname{div}\left(\mathbf{u}-\mathbf{u}_{h}\right)\right\|_{0, T}^{2}+h^{4 s}\left(\|\operatorname{div} \mathbf{u}\|_{s, T^{i d}}^{2}+\left\|\operatorname{div} \mathbf{u}_{h}\right\|_{0, T}^{2}\right)\right) .
\end{aligned}
$$


Combinando las dos expresiones anteriores y usando los mismos argumentos que los empleados en la prueba del Lema 4.38, llegaremos a

$$
\begin{aligned}
\left\|\mathbf{u}-\mathbf{u}_{h}^{e}\right\|_{H\left(\operatorname{div}, \Omega_{\mathrm{F}} \backslash \Omega_{\mathrm{F} h}\right)}^{2} & =\sum_{T \in \mathcal{B}_{\mathrm{F} h}}\left\|\mathbf{u}-\mathbf{u}_{h}^{e}\right\|_{0, \omega_{T}}^{2}+\left\|\operatorname{div}\left(\mathbf{u}-\mathbf{u}_{h}^{e}\right)\right\|_{0, \omega_{T}}^{2} \\
& \leq C\left(\| T^{i d}\right. \\
\leq & C\left(\left\|\mathbf{u}^{e}-\mathbf{u}_{h}\right\|_{H\left(\operatorname{div}, \Omega_{\mathrm{F} h}\right)}^{2}+h^{4 s}\left[\|\mathbf{u}\|_{H^{s}\left(\operatorname{div}, \Omega_{\mathrm{F}}\right)}^{2}+\left\|\mathbf{u}_{h}\right\|_{H\left(\operatorname{div}, \Omega_{\mathrm{F} h}\right)}^{2}\right]\right) \\
\leq & C h^{r_{h}}\|(\mathbf{f}, \mathbf{g})\|_{0, \widehat{\Omega}} .
\end{aligned}
$$

De esta manera, concluimos la prueba.

El siguiente resultado es una consecuencia directa de los Lemas 4.35-4.39.

Lema 4.40 Para todo elemento $(\mathbf{f}, \mathbf{g})$ en $\mathcal{G}^{e}(\widehat{\Omega}) \cup \widehat{\mathcal{G}}(\widehat{\Omega})$, existe una constante positica $C$ tal que

$$
\left\|\left(\mathbf{A}-\mathbf{A}_{h}\right)(\mathbf{f}, \mathbf{g})\right\|_{h} \leq C h^{\hat{r}_{h}}\|(\mathbf{f}, \mathbf{g})\|_{0, \widehat{\Omega}} .
$$

Ahora estamos en condiciones de probar las propiedades P1 y P2 que nos permitirán establecer la convergencia de las aproximaciones.

Sea $\lambda>0$ un valor propio de $\mathbf{A}$, con multiplicidad algebraica igual a $m$. Sea $\mathbf{E}_{\lambda}(\mathcal{W}(\widehat{\Omega}))$ el espacio propio asociado con $\lambda$.

Teorema 4.41 (P1) Para cada autofunción $\left(\mathbf{u}^{e}, \mathbf{w}^{e}\right)$ de $\mathbf{A}$ asociada a $\lambda$, existe una constante $C$, estrictamente positiva, tal que

$$
\inf _{\left(\mathbf{z}_{h}^{e}, \mathbf{v}_{h}^{e}\right) \in \mathcal{K}_{h}^{e}(\widehat{\Omega})}\left\|\left(\mathbf{u}^{e}, \mathbf{w}^{e}\right)-\left(\mathbf{z}_{h}^{e}, \mathbf{v}_{h}^{e}\right)\right\|_{h} \leq C h^{\hat{r}_{h}}\left\|\left(\mathbf{u}^{e}, \mathbf{w}^{e}\right)\right\|_{h}
$$

Prueba. Como $\mathbf{A}=\mathbf{P} \circ \mathbf{T}$ y $\mathbf{T}(\mathbf{u}, \mathbf{w})=\lambda(\mathbf{u}, \mathbf{w})$, aplicando el Teorema 4.29 , tendremos

$$
\|\mathbf{u}\|_{s, \Omega_{\mathrm{F}}}+\|\mathbf{w}\|_{1+t, \Omega_{\mathrm{S}}}+\|\operatorname{div} \mathbf{u}\|_{1+s, \Omega_{\mathrm{F}}} \leq C\|(\mathbf{u}, \mathbf{w})\|_{0, \Omega}
$$

Sean ( $\hat{\mathbf{u}}, \hat{\mathbf{w}})$, como en la observación E.17, y $\left(\mathbf{z}_{h}, \mathbf{v}_{h}\right)$ un elemento arbitrario de $\mathcal{K}_{h}\left(\Omega_{h}\right)$. Usando la desigualdad triangular, obtendremos

$$
\begin{aligned}
\left\|\left(\mathbf{u}^{e}, \mathbf{w}^{e}\right)-\left(\mathbf{z}_{h}^{e}, \mathbf{v}_{h}^{e}\right)\right\|_{h} & \leq\left\|\mathbf{u}^{e}-\hat{\mathbf{u}}\right\|_{H\left(\operatorname{div} ; \widehat{\Omega}_{\mathrm{F}}\right)}+\left\|(\hat{\mathbf{u}}, \hat{\mathbf{w}})-\left(\mathbf{z}_{h}, \mathbf{v}_{h}\right)\right\|_{\Omega_{h}}+\left\|\hat{\mathbf{u}}-\mathbf{z}_{h}^{e}\right\|_{H\left(\operatorname{div} ; \Omega_{\mathrm{F}} \backslash \Omega_{\mathrm{F} h}\right)} \\
& +\left\|\mathbf{w}-\mathbf{v}_{h}^{e}\right\|_{1, \Omega_{\mathrm{S}} \backslash \Omega_{\mathrm{S} h}} .
\end{aligned}
$$

El primer término en el lado derecho de la desigualdad anterior, puede acotarse fácilmente usando los Lemas E.12 y 4.37 y teniendo en cuenta la estimación (4.83). En efecto,

$$
\left\|\mathbf{u}^{e}-\hat{\mathbf{u}}\right\|_{H\left(\operatorname{div}, \widehat{\Omega}_{\mathrm{F}}\right)} \leq\|\mathbf{u}-\hat{\mathbf{u}}\|_{H\left(\operatorname{div}, \Omega_{\mathrm{F}}\right)}+\left\|\mathbf{u}^{e}-\hat{\mathbf{u}}\right\|_{H\left(\operatorname{div}, \Omega_{\mathrm{F} h} \backslash \Omega_{\mathrm{F}}\right)} \leq C h^{\hat{r}_{h}}\|(\mathbf{u}, \mathbf{w})\|_{0, \Omega}
$$

Pasaremos ahora a acotar los otros tres términos. Para ello, observemos primero que $\mathbf{I}_{h}(\hat{\mathbf{u}}, \hat{\mathbf{w}}) \in \mathcal{K}_{h}\left(\Omega_{h}\right)$, siendo $\mathbf{I}_{h}$ el operador de interpolación definido en la sección E.4. Luego, nada impide que elijamos $\left(\mathbf{z}_{h}, \mathbf{v}_{h}\right)=\mathbf{I}_{h}(\hat{\mathbf{u}}, \hat{\mathbf{w}})$. A partir de la observación E.17 y usando la estimación (4.83), tendremos

$$
\left\|(\hat{\mathbf{u}}, \hat{\mathbf{w}})-\left(\mathbf{z}_{h}, \mathbf{v}_{h}\right)\right\|_{h, \Omega_{h}} \leq C h^{r_{h}}\|(\mathbf{u}, \mathbf{w})\|_{0, \Omega} .
$$

Procediendo como lo hicimos en la prueba del Lema 4.39, teniendo en cuenta los resultados de regularidad (E.11)-(E.12) y usando las estimaciones (4.83)-(4.84), tendremos

$$
\begin{aligned}
\left\|\hat{\mathbf{u}}-\mathbf{z}_{h}^{e}\right\|_{H\left(\operatorname{div} ; \Omega_{\mathrm{F}} \backslash \Omega_{\mathrm{F} h}\right)}^{2} & \leq C\left(\left\|\hat{\mathbf{u}}-\mathbf{z}_{h}\right\|_{H\left(\operatorname{div}, \Omega_{\mathrm{F} h}\right)}^{2}+h^{4 s_{h}}\|\hat{\mathbf{u}}\|_{H^{s_{h}\left(\operatorname{div}, \Omega_{\mathrm{F}}\right)}}^{2}+h^{4 s_{h}}\left\|\mathbf{z}_{h}\right\|_{H\left(\operatorname{div}, \Omega_{\mathrm{F} h}\right)}^{2}\right) \\
& \leq\left(C h^{r_{h}}\|(\mathbf{u}, \mathbf{w})\|_{0, \Omega}+\left\|\hat{\mathbf{u}}-\mathbf{z}_{h}\right\|_{H\left(\operatorname{div}, \Omega_{\mathrm{F} h}\right)}+\|\hat{\mathbf{u}}\|_{H\left(\operatorname{div}, \Omega_{\mathrm{F}}\right)}\right) \\
& \leq C h^{r_{h}}\|(\mathbf{u}, \mathbf{w})\|_{0, \Omega} .
\end{aligned}
$$


Procediendo ahora como en la prueba del Lema 4.38, podremos escribir

$$
\left\|\mathbf{w}-\mathbf{v}_{h}^{e}\right\|_{1, \Omega_{\mathrm{S}} \backslash \Omega_{\mathrm{S} h}}^{2} \leq\left\|\mathbf{w}-\mathbf{v}_{h}^{e}\right\|_{0, \Omega_{\mathrm{S}} \backslash \Omega_{\mathrm{S} h}}^{2}+C\left(\left\|\nabla\left(\hat{\mathbf{w}}-\mathbf{v}_{h}\right)\right\|_{0, \Omega_{\mathrm{S} h}}^{2}+h^{4 t}\left(\|\mathbf{w}\|_{1+t, \Omega_{\mathrm{S}}}^{2}+\left\|\mathbf{v}_{h}^{e}\right\|_{1, \Omega_{\mathrm{S}}}^{2}\right)\right) .
$$

Luego, usando el Lema D.9 y el Teorema (D.4), tendremos

$$
\begin{aligned}
&\left\|\mathbf{w}-\mathbf{v}_{h}^{e}\right\|_{0, \Omega_{\mathrm{S}} \backslash \Omega_{\mathrm{S} h}} \leq C h\left(\|\mathbf{w}\|_{1+t, \Omega_{\mathrm{S}}}+\left\|\mathbf{v}_{h}^{e}\right\|_{1, \Omega_{\mathrm{S}}}\right) \leq C h\left(\|\mathbf{w}\|_{1+t, \Omega_{\mathrm{S}}}+\left\|\mathbf{v}_{h}\right\|_{1, \Omega_{\mathrm{S} h}}\right) \\
& \leq C h\left(\|\mathbf{w}\|_{1+t, \Omega_{\mathrm{S}}}+\left\|\hat{\mathbf{w}}-\mathbf{v}_{h}\right\|_{1, \Omega_{\mathrm{S} h}}+\|\hat{\mathbf{w}}\|_{1, \Omega_{\mathrm{S} h}}\right) \\
& \leq C h\left(\|\mathbf{w}\|_{1+t, \Omega_{\mathrm{S}}}+\left\|\hat{\mathbf{w}}-\mathbf{v}_{h}\right\|_{1, \Omega_{\mathrm{S} h}}\right) \\
&\left\|\mathbf{v}_{h}^{e}\right\|_{1, \Omega_{\mathrm{S}}} \leq C\left\|\mathbf{v}_{h}\right\|_{1, \Omega_{\mathrm{S} h}} \leq C\left(\left\|\hat{\mathbf{w}}-\mathbf{v}_{h}\right\|_{1, \Omega_{\mathrm{S} h}}+\|\hat{\mathbf{w}}\|_{1, \Omega_{\mathrm{S} h}}\right) \leq C\left(\left\|\hat{\mathbf{w}}-\mathbf{v}_{h}\right\|_{1, \Omega_{\mathrm{S} h}}+\|\mathbf{w}\|_{1+t, \Omega_{\mathrm{S}}}\right) .
\end{aligned}
$$

Usando nuevamente las estimaciones (4.83) y (4.84), llegaremos a

$$
\left\|\mathbf{w}-\mathbf{v}_{h}^{e}\right\|_{1, \Omega_{\mathrm{S}} \backslash \Omega_{\mathrm{S} h}} \leq C h^{r_{h}}\|(\mathbf{u}, \mathbf{w})\|_{0, \Omega} .
$$

Finalmente, combinando las estimaciones halladas, podremos concluir la prueba

Teorema 4.42 (P2) Existe una constante positiva $C$ tal que

$$
\left\|\left(\mathbf{A}-\mathbf{A}_{h}\right)\left(\mathbf{f}_{h}^{e}, \mathbf{g}_{h}^{e}\right)\right\|_{h} \leq C h^{\min \{1 / 2, t\}}\left\|\left(\mathbf{f}_{h}^{e}, \mathbf{g}_{h}^{e}\right)\right\|_{h} \quad \forall\left(\mathbf{f}_{h}^{e}, \mathbf{g}_{h}^{e}\right) \in \mathcal{K}_{h}^{e}(\widehat{\Omega})
$$

Prueba. Sabemos que todo elemento $\left(\mathbf{f}_{h}^{e}, \mathbf{g}_{h}^{e}\right) \in \mathcal{K}_{h}^{e}(\widehat{\Omega})$ admite la siguiente descomposición ortogonal

$$
\left.\left(\mathbf{f}_{h}^{e}, \mathbf{g}_{h}^{e}\right)\right|_{\Omega_{h}}=\left(\mathbf{f}_{1 h}, \mathbf{0}\right) \oplus\left(\mathbf{f}_{2 h}, \mathbf{g}_{h}\right),
$$

con $\left(\mathbf{f}_{1 h}, \mathbf{0}\right) \in \mathcal{H}_{h}\left(\Omega_{h}\right)$ y $\left(\mathbf{f}_{2 h}, \mathbf{g}_{h}\right) \in \mathcal{G}_{h}\left(\Omega_{h}\right)$. Sea $(\mathbf{f}, \mathbf{g}) \in \widehat{\mathcal{G}}(\widehat{\Omega})$ (ver observación E.22). Entonces, usando la desigualdad triangular, podremos escribir

$$
\begin{aligned}
\left\|\left(\mathbf{A}-\mathbf{A}_{h}\right)\left(\mathbf{f}_{h}^{e}, \mathbf{g}_{h}^{e}\right)\right\|_{h} & \leq\left\|\left(\mathbf{A}-\mathbf{A}_{h}\right)\left(\mathbf{f}_{1 h}^{e}, \mathbf{0}\right)\right\|_{h}+\left\|\left(\mathbf{A}-\mathbf{A}_{h}\right)\left(\mathbf{f}_{2 h}^{e}, \mathbf{g}_{h}^{e}\right)\right\|_{h} \\
& \leq\left\|\left(\mathbf{A}-\mathbf{A}_{h}\right)\left(\mathbf{f}_{1 h}^{e}, \mathbf{0}\right)\right\|_{h}+\left\|\left(\mathbf{A}-\mathbf{A}_{h}\right)(\mathbf{f}, \mathbf{g})\right\|_{h} \\
& +\left\|\left(\mathbf{A}-\mathbf{A}_{h}\right)\left(\left(\mathbf{f}_{2 h}^{e}, \mathbf{g}_{h}^{e}\right)-(\mathbf{f}, \mathbf{g})\right)\right\|_{h} .
\end{aligned}
$$

Para acotar el tercer término en el lado derecho de la desigualdad (4.85), tendremos en cuenta que el operador $\mathbf{A}-\mathbf{A}_{h} \in \mathcal{L}(\mathcal{X}(\widehat{\Omega}), \mathcal{W}(\widehat{\Omega})$ ) (ver sección 3.2). Entonces, usando la Propiedad E.19, tendremos

$$
\left\|\left(\mathbf{A}-\mathbf{A}_{h}\right)\left(\left(\mathbf{f}_{2 h}^{e}, \mathbf{g}_{h}^{e}\right)-(\mathbf{f}, \mathbf{g})\right)\right\|_{h} \leq C\left\|\left(\mathbf{f}_{2 h}^{e}, \mathbf{g}_{h}^{e}\right)-(\mathbf{f}, \mathbf{g})\right\|_{h} \leq C h^{1 / 2}\left\|\left(\mathbf{f}_{2 h}^{e}, \mathbf{g}_{h}^{e}\right)\right\|_{h} .
$$

El segundo término puede acotarse usando el Lema 4.40 y la Propiedad E.19 de la siguiente manera:

$$
\begin{aligned}
\left\|\left(\mathbf{A}-\mathbf{A}_{h}\right)(\mathbf{f}, \mathbf{g})\right\|_{h} & \leq C h^{r_{h}}\|(\mathbf{f}, \mathbf{g})\|_{h} \leq C h^{r_{h}}\left(\left\|(\mathbf{f}, \mathbf{g})-\left(\mathbf{f}_{2 h}^{e}, \mathbf{g}_{h}^{e}\right)\right\|_{h}+\left\|\left(\mathbf{f}_{2 h}^{e}, \mathbf{g}_{h}^{e}\right)\right\|_{h}\right) \\
& \leq C h^{r_{h}}\left(h^{1 / 2}+1\right)\left\|\left(\mathbf{f}_{2 h}^{e}, \mathbf{g}_{h}^{e}\right)\right\|_{h} .
\end{aligned}
$$

A continuación acotaremos el primer término. Para ganar en claridad, definiremos

$$
\left(\mathbf{u}^{e}, \mathbf{w}^{e}\right):=\mathbf{A}\left(\mathbf{f}_{h}^{e}, \mathbf{0}\right) \quad \text { y } \quad\left(\mathbf{u}_{h}^{e}, \mathbf{w}_{h}^{e}\right):=\mathbf{A}_{h}\left(\mathbf{f}_{h}^{e}, \mathbf{0}\right) .
$$

Probaremos primero que $\left(\mathbf{u}_{h}^{e}, \mathbf{w}_{h}^{e}\right)=\left(\mathbf{f}_{h}^{e}, \mathbf{0}\right)$. Como $\left(\mathbf{f}_{h}, \mathbf{0}\right) \in \mathcal{H}_{h}\left(\Omega_{h}\right)$, será $\mathbf{f}_{h}=\operatorname{curl} p_{h}, \operatorname{con} p_{h} \in \mathcal{Z}_{h}\left(\Omega_{\mathrm{F} h}\right)$. Entonces, un cálculo directo muestra que

$$
a_{h}\left(\left(\mathbf{f}_{h}, \mathbf{0}\right),\left(\boldsymbol{\eta}_{h}, \mathbf{z}_{h}\right)\right)=\rho_{\mathrm{F}} \int_{\Omega_{h}} \mathbf{f}_{h} \cdot \boldsymbol{\eta}_{h}=d_{h}\left(\left(\mathbf{f}_{h}, \mathbf{0}\right),\left(\boldsymbol{\eta}_{h}, \mathbf{z}_{h}\right)\right) \quad \forall\left(\boldsymbol{\eta}_{h}, \mathbf{z}_{h}\right) \in \mathcal{V}_{h}\left(\Omega_{h}\right) .
$$

Luego, $\left(\left(\mathbf{u}_{h}^{e}, \mathbf{w}_{h}^{e}\right), \gamma_{h}\right)=\left(\left(\mathbf{f}_{h}^{e}, \mathbf{0}\right), 0\right)$ es una solución del problema $4.32 \mathrm{y}$, como este problema está bien planteado, esta solución es única. Consecuentemente, $\mathbf{A}_{h}\left(\mathbf{f}_{h}^{e}, \mathbf{0}\right)=\left(\mathbf{f}_{h}^{e}, \mathbf{0}\right)$. 
Sea ahora $\hat{p}$ la función associada a $p_{h}$ (según la definición D.10). Dado que $\hat{p} \in H_{\Gamma_{\mathrm{I}}}^{1}\left(\Omega_{\mathrm{F}}\right), \hat{\mathbf{f}}=\operatorname{curl} \hat{p} \in$ $H\left(\operatorname{div} ; \Omega_{\mathrm{F}}\right)$ y $\left.\hat{\mathbf{f}} \cdot \mathbf{n}\right|_{\Gamma_{\mathrm{I}}}=0$. Luego, $(\hat{\mathbf{f}}, \mathbf{0}) \in \mathcal{H}(\Omega)$. Usando la desigualdad triangular,

$$
\begin{aligned}
\left\|\left(\mathbf{A}-\mathbf{A}_{h}\right)\left(\mathbf{f}_{h}^{e}, \mathbf{0}\right)\right\|_{h} & =\left\|\left(\mathbf{u}^{e}, \mathbf{w}^{e}\right)-\left(\mathbf{f}_{h}^{e}, \mathbf{0}\right)\right\|_{h} \leq\left\|\left(\mathbf{u}^{e}-\hat{\mathbf{f}}^{e}, \mathbf{w}^{e}\right)\right\|_{h}+\left\|\left(\hat{\mathbf{f}}^{e}-\mathbf{f}_{h}^{e}, \mathbf{0}\right)\right\|_{h} \\
& \leq\|(\mathbf{u}-\hat{\mathbf{f}}, \mathbf{w})\|_{h, \Omega}+\left\|\left(\mathbf{u}^{e}, \mathbf{w}^{e}\right)\right\|_{h, \Omega_{h} \backslash \Omega}+\left\|\hat{\mathbf{f}}^{e}-\mathbf{f}_{h}^{e}\right\|_{H\left(\operatorname{div} ; \widehat{\Omega}_{\mathrm{F}}\right)}
\end{aligned}
$$

Gracias a la Propiedad E.21, tendremos

$$
\left\|\hat{\mathbf{f}}^{e}-\mathbf{f}_{h}^{e}\right\|_{H\left(\operatorname{div} ; \widehat{\Omega}_{\mathrm{F}}\right)} \leq C h^{1 / 2}\left\|\mathbf{f}_{h}\right\|_{0, \Omega_{\mathrm{F} h}} .
$$

Por otro lado, como $\left(\left.\mathbf{f}_{h}^{e}\right|_{\Omega_{\mathrm{F}}}, \mathbf{0}\right) \in \mathcal{Q}(\Omega)$, el Teorema 4.29 establece que $\mathbf{w} \in H^{1+t}\left(\Omega_{\mathrm{S}}\right)$. Procediendo como en la prueba de la Propiedad E.19 (ver Pasos 1 y 2 en esa demostración), usando el Lema D.6 y el Teorema 4.29 ,

$$
\begin{aligned}
\left\|\left(\mathbf{u}^{e}, \mathbf{w}^{e}\right)\right\|_{h, \Omega_{h} \backslash \Omega}^{2} & =\|\nabla \xi\|_{H\left(\operatorname{div}, \Omega_{\mathrm{S}} \backslash \Omega_{\mathrm{Sh}}\right)}^{2}+\left\|\mathbf{w}^{e}\right\|_{1, \Omega_{\mathrm{S} h} \backslash \Omega_{\mathrm{S}}}^{2} \leq C\left(h^{2 \alpha}\left(\|(\mathbf{u}, \mathbf{w})\|_{h, \Omega}^{2}+h^{2 t}\|\mathbf{w}\|_{1+t ; \Omega_{\mathrm{S}}}^{2}\right)\right. \\
& \leq C h^{2 \min \{\alpha, t\}}\left\|\left(\mathbf{f}_{h}^{e}, \mathbf{0}\right)\right\|_{0 ; \widehat{\Omega}}^{2} .
\end{aligned}
$$

Por último, acotaremos el primer término en el lado derecho de la expresión (4.87). Sea $(\boldsymbol{\eta}, \mathbf{z})$ un elemento arbitrario de $\mathcal{K}(\Omega)$. A partir de la definición del problema 4.28, tendremos

$$
\begin{aligned}
a((\mathbf{u}-\hat{\mathbf{f}}, \mathbf{w}),(\boldsymbol{\eta}, \mathbf{z})) & =\rho_{\mathrm{F}} \int_{\Omega_{\mathrm{F}}}\left(\mathbf{f}_{h}^{e}-\hat{\mathbf{f}}\right) \cdot \boldsymbol{\eta}=\rho_{\mathrm{F}} \int_{\Omega_{\mathrm{F}} \cap \Omega_{\mathrm{F}}}\left(\mathbf{f}_{h}-\hat{\mathbf{f}}\right) \cdot \boldsymbol{\eta} \\
& =\rho_{\mathrm{F}} \int_{\Omega_{\mathrm{F}} \cap \Omega_{\mathrm{F} h}} \mathbf{f}_{h} \cdot \boldsymbol{\eta}-\sum_{\substack{T \in \mathcal{T}_{h}^{\mathrm{F}} \\
T \subset T^{i d}}} \rho_{\mathrm{F}} \int_{T} \mathbf{f}_{h} \cdot \boldsymbol{\eta}-\sum_{\substack{T \in \mathcal{B}_{\mathrm{F} h} \\
T^{i d} \subset T}} \rho_{\mathrm{F}} \int_{T^{i d}} \hat{\mathbf{f}} \cdot \boldsymbol{\eta} \\
& =-\sum_{\substack{T \in \mathcal{B}_{\mathrm{F} h} \\
T \supset T^{i d}}} \rho_{\mathrm{F}} \int_{T^{i d}}\left(\hat{\mathbf{f}}-\mathbf{f}_{h}\right) \cdot \boldsymbol{\eta} .
\end{aligned}
$$

Como $(\mathbf{u}-\hat{\mathbf{f}}, \mathbf{w}) \in \mathcal{K}(\Omega)$ y a es coerciva sobre $\mathcal{K}(\Omega)$, elegimos $(\boldsymbol{\eta}, \mathbf{z})=(\mathbf{u}-\hat{\mathbf{f}}, \mathbf{w})$ para obtener

$$
\|(\mathbf{u}-\hat{\mathbf{f}}, \mathbf{w})\|_{h, \Omega} \leq C\left(\sum_{\substack{T \in \mathcal{B}_{\mathrm{F} h} \\ T \supset T^{i d}}}\left\|\hat{\mathbf{f}}-\mathbf{f}_{h}\right\|_{0, T^{i d}}^{2}\right)^{1 / 2}
$$

Reemplazando las estimaciones (4.88)-(4.90) en (4.87), llegaremos a

$$
\left\|\left(\mathbf{u}^{e}, \mathbf{w}^{e}\right)-\left(\mathbf{u}_{h}^{e}, \mathbf{w}_{h}^{e}\right)\right\| \leq C h^{\min \{1 / 2, t\}}\left\|\left(\mathbf{f}_{h}^{e}, \mathbf{0}\right)\right\|_{0 ; \widehat{\Omega}},
$$

resultado que nos permite concluir la prueba.

Según los resultados de la sección 3.3.1 y en virtud de los teoremas previos, hemos probado que partes aisladas de $\sigma(\mathbf{A})$ son aproximadas por partes aisladas de $\sigma\left(\mathbf{A}_{h}\right)$. Como consecuencia, podemos asegurar que este método numérico no introduce modos de vibración espurios.

A continuación probaremos que los espacios propios asociados a $\mathbf{A}_{h}$ convergen con orden óptimo.

Teorema 4.43 (P4) Existe una constante positiva $C$ tal que

$$
\left\|\left(\mathbf{A}-\mathbf{A}_{h}\right)(\mathbf{u}, \mathbf{w})\right\|_{h} \leq C h^{\hat{r}_{h}}\|(\mathbf{u}, \mathbf{w})\|_{h} \quad \forall(\mathbf{u}, \mathbf{w}) \in \mathbf{E}_{\lambda}(\mathcal{W}(\widehat{\Omega})) .
$$

Prueba. Es una consecuencia directa del Lema 4.40.

Sean $\lambda_{1 h}, \cdots, \lambda_{m h}$ los $m$ autovalores de $\mathbf{A}_{h}$ que convergen a $\lambda$ (repetidos de acuerdo con sus respectivas multiplicidades). Sea $\mathbf{E}_{\lambda_{h}}(\mathcal{W}(\widehat{\Omega}))$ la suma directa de los espacios propios correspondientes. De acuerdo con los resultados obtenidos en la sección 3.3.3, los teoremas previos nos permiten asegurar que 
- para cada $\left(\mathbf{u}_{h}, \mathbf{w}_{h}\right) \in \mathbf{E}_{\lambda_{h}}(\mathcal{W}(\widehat{\Omega})), \operatorname{dist}\left(\left(\mathbf{u}_{h}, \mathbf{w}_{h}\right), \mathbf{E}_{\lambda}(\mathcal{W}(\widehat{\Omega})) \leq C h^{\hat{r}_{h}}\left\|\left(\mathbf{u}_{h}, \mathbf{w}_{h}\right)\right\|_{h, \Omega_{h}}\right.$,

- para cada $(\mathbf{u}, \mathbf{w}) \in \mathbf{E}_{\lambda}(\mathcal{W}(\widehat{\Omega})), \operatorname{dist}\left((\mathbf{u}, \mathbf{w}), \mathbf{E}_{\lambda_{h}}(\mathcal{W}(\widehat{\Omega})) \leq C h^{\hat{r}_{h}}\|(\mathbf{u}, \mathbf{w})\|_{h, \Omega}\right.$,

con la distancia siendo medida en la norma $\|\cdot\|_{h}$.

A continuación probaremos que los autovalores aproximados convergen con doble orden. Para ello, tendremos que verificar que se satisface la Propiedad P5 y estimar los errores de consistencia definidos en la sección 3.3.4.

Teorema 4.44 (P5) Para cada autofunción $\left(\mathbf{u}^{e}, \mathbf{w}^{e}\right)$ de $\mathbf{A}$ asociada a $\lambda$, existe una constante positiva C tal que

$$
\|(\mathbf{u}, \mathbf{w})\|_{h, \Omega-\Omega_{h}}+\left\|\left(\mathbf{u}^{e}, \mathbf{w}^{e}\right)\right\|_{h, \Omega_{h}-\Omega} \leq C h^{\min \{r, \alpha\}}\|(\mathbf{u}, \mathbf{w})\|_{h, \Omega} .
$$

Prueba. Observemos que, como consecuencia de la regularidad de las autofunciones (u, w) del operador T (ver, en particular, la estimación (4.83)) y del Lema D.6, vale la siguiente estimación

$$
\|(\mathbf{u}, \mathbf{w})\|_{h, \Omega-\Omega_{h}} \leq C h^{r}\|(\mathbf{u}, \mathbf{w})\|_{h, \Omega} .
$$

Por otro lado, procediendo como en la prueba del Lema 4.37 y usando el Lema D.6 y el Teorema D.4, tendremos

$$
\left\|\left(\mathbf{u}^{e}, \mathbf{w}^{e}\right)\right\|_{h, \Omega_{h}-\Omega} \leq C\left(\left\|\mathbf{u}^{e}\right\|_{H\left(\operatorname{div} ; \Omega_{\mathrm{F} h} \backslash \Omega_{\mathrm{F}}\right)}+\left\|\mathbf{w}^{e}\right\|_{1, \Omega_{\mathrm{S} h} \backslash \Omega_{\mathrm{S}}}\right) \leq C h^{\operatorname{mín}\{\alpha, t\}}\|(\mathbf{u}, \mathbf{w})\|_{h, \Omega} .
$$

Así, el resultado buscado es inmediato a partir de las dos estimaciones anteriores.

Lema 4.45 Sea

$$
M_{h}=\sup _{\substack{(\mathbf{x}, \mathbf{y}) \in \mathbf{E}(\mathcal{V}(\widehat{\Omega})) \\\|(\mathbf{x}, \mathbf{y})\|_{h}=1}} \sup _{\substack{\boldsymbol{\eta}, \boldsymbol{\varphi}) \in \mathbf{E}(\mathcal{V}(\widehat{\Omega})) \\\|(\boldsymbol{\eta}, \boldsymbol{\varphi})\|_{h}=1}}\left|a_{h}\left(\mathbf{A}(\mathbf{x}, \mathbf{y}),\left(\Pi_{h}-\mathbf{I}\right)(\boldsymbol{\eta}, \boldsymbol{\varphi})\right)-d_{h}\left((\mathbf{x}, \mathbf{y}),\left(\Pi_{h}-\mathbf{I}\right)(\boldsymbol{\eta}, \boldsymbol{\varphi})\right)\right|,
$$

siendo $\Pi_{h}$ la proyección sobre $\mathcal{V}(\widehat{\Omega})$, con respecto a la forma bilineal $a_{h}$, definida por la ecuación (3.55). Existe una constante positiva $C$ tal que

$$
M_{h} \leq C h^{r+\hat{r}_{h}}
$$

Prueba. Sea $(\mathbf{x}, \mathbf{y}) \in \mathbf{E}(\mathcal{V}(\widehat{\Omega}))$, con $\|(\mathbf{x}, \mathbf{y})\|_{h}=1$, y pongamos $(\mathbf{u}, \mathbf{v})=\mathbf{A}(\mathbf{x}, \mathbf{y})$. De acuerdo con la definición del operador $\mathbf{A}$, tendremos $(\mathbf{u}, \mathbf{v})=\left.\mathbf{P} \circ \mathbf{T}(\mathbf{x}, \mathbf{y})\right|_{\Omega}$. Luego, a partir del Teorema 4.29, sabemos que $\left.(\mathbf{u}, \mathbf{v})\right|_{\Omega} \in H^{s, 1+s}\left(\operatorname{div} ; \Omega_{\mathrm{F}}\right) \times H^{1+t}\left(\Omega_{\mathrm{S}}\right)$ y que

$$
\|\mathbf{u}\|_{s, \Omega_{\mathrm{F}}}+\|\mathbf{v}\|_{1+t, \Omega_{\mathrm{S}}}+\|\operatorname{div} \mathbf{u}\|_{1+s, \Omega_{\mathrm{F}}} \leq C\|(\mathbf{x}, \mathbf{y})\|_{0, \Omega} \leq C .
$$

Tomando alternativamente $(\boldsymbol{\xi}, \mathbf{0})$, con $\boldsymbol{\xi} \in \mathcal{D}\left(\Omega_{\mathrm{F}}\right)$ y $(\mathbf{0}, \boldsymbol{\psi})$, con $\boldsymbol{\psi} \in \mathcal{D}\left(\Omega_{\mathrm{S}}\right)$, como función test en el problema (4.28) y procediendo de manera standard, puede probarse que $\left.(\mathbf{u}, \mathbf{v})\right|_{\Omega}$ es también solución del siguiente problema fuerte:

$$
\begin{aligned}
\rho_{\mathrm{F}} c^{2} \nabla(\operatorname{div} \mathbf{u})+\rho_{\mathrm{F}} \mathbf{u}=\rho_{\mathrm{F}} \mathbf{x} & \text { en } \Omega_{\mathrm{F}}, \\
\operatorname{div}[\boldsymbol{\sigma}(\mathbf{v})]+\rho_{\mathrm{S}} \mathbf{v}=\rho_{\mathrm{S}} \mathbf{y} & \text { en } \Omega_{\mathrm{S}}, \\
(\mathbf{u}-\mathbf{v}) \cdot \mathbf{n}=0 & \text { sobre } \Gamma_{\mathrm{I}}, \\
\boldsymbol{\sigma}(\mathbf{v}) \mathbf{n}-\left(c^{2} \rho_{\mathrm{F}} \operatorname{div} \mathbf{u}\right) \mathbf{n}=\mathbf{0} & \text { sobre } \Gamma_{\mathrm{I}}, \\
\boldsymbol{\sigma}(\mathbf{v}) \mathbf{n}=\mathbf{0} & \text { sobre } \Gamma_{\mathrm{N}}, \\
\mathbf{v}=\mathbf{0} & \text { sobre } \Gamma_{\mathrm{D}} .
\end{aligned}
$$

La condición de borde sobre $\Gamma_{\mathrm{D}}$ corresponde a la definición de los espacios variacionales. La condición de compatibilidad cinemática (4.93) puede ser entendida en el sentido de $L^{2}\left(\Gamma_{\mathrm{I}}\right)$. La condición natural de borde sobre $\Gamma_{\mathrm{N}}$ y la condición de compatibilidad cinética (4.94) se obtienen después de integrar por partes y tienen que ser entendidas en el sentido de $H^{-1 / 2}\left(\Gamma_{\mathrm{I}}\right)$ (ver la prueba del Teorema 2.1 en [33] para más detalles). 
Sea $(\boldsymbol{\eta}, \boldsymbol{\varphi}) \in \mathbf{E}(\mathcal{V}(\widehat{\Omega}))$, con $\|(\boldsymbol{\eta}, \boldsymbol{\varphi})\|_{h}=1$, y pongamos $(\mathbf{z}, \mathbf{w})=\left(\Pi_{h}-\mathbf{I}\right)(\boldsymbol{\eta}, \boldsymbol{\varphi}) \in \mathcal{X}(\widehat{\Omega})$. De la definición de $\Pi_{h}$, sabemos que $\Pi_{h}(\boldsymbol{\eta}, \boldsymbol{\varphi}) \in \mathcal{K}_{h}^{e}(\widehat{\Omega})$ (ver sección 3.4.1). Luego, gracias al Lema 3.21 y al Teorema 4.41, la siguiente estimación será válida

$$
\|(\mathbf{z}, \mathbf{w})\|_{h} \leq C h^{\hat{r}_{h}}
$$

Multiplicando las ecuaciones (4.91) y (4.92) por $\boldsymbol{\eta}$ y $\boldsymbol{\varphi}$, respectivamente, e integrando por partes, tendremos

$$
\begin{aligned}
& a_{h}((\mathbf{u}, \mathbf{v}),(\mathbf{z}, \mathbf{w}))-d_{h}((\mathbf{x}, \mathbf{y}),(\mathbf{z}, \mathbf{w}))= \\
& -\rho_{\mathrm{F}} c^{2} \int_{\Omega_{\mathrm{F} h} \backslash \Omega_{\mathrm{F}}} \operatorname{div} \mathbf{u} \operatorname{div} \mathbf{z}-\rho_{\mathrm{F}} \int_{\Omega_{\mathrm{F} h} \backslash \Omega_{\mathrm{F}}} \mathbf{u} \cdot \mathbf{z}-\int_{\Omega_{\mathrm{S} h} \backslash \Omega_{\mathrm{S}}} \boldsymbol{\sigma}(\mathbf{v}): \boldsymbol{\varepsilon}(\mathbf{w})-\rho_{\mathrm{S}} \int_{\Omega_{\mathrm{S} h} \backslash \Omega_{\mathrm{S}}} \mathbf{v} \cdot \mathbf{w}
\end{aligned}
$$

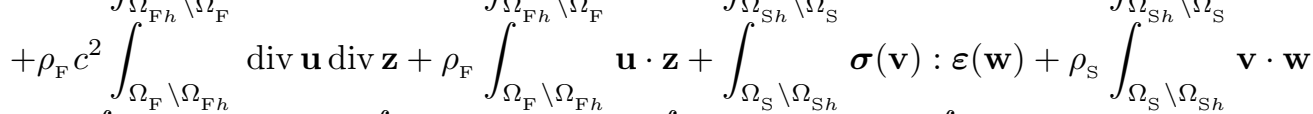

$$
\begin{aligned}
& +\rho_{\mathrm{F}} \int_{\Omega_{\mathrm{F}} \backslash \Omega_{\mathrm{F} h}} \mathbf{x} \cdot \mathbf{z}+\rho_{\mathrm{S}} \int_{\Omega_{\mathrm{S}} \backslash \Omega_{\mathrm{S} h}} \mathbf{y} \cdot \mathbf{w}-\rho_{\mathrm{F}} \int_{\Omega_{\mathrm{F} h} \backslash \Omega_{\mathrm{F}}} \mathbf{x} \cdot \mathbf{z}-\rho_{\mathrm{S}} \int_{\Omega_{\mathrm{S}} \backslash \Omega_{\mathrm{S} h}} \mathbf{y} \cdot \mathbf{w} \\
& +\rho_{\mathrm{F}} c^{2} \int_{\Gamma_{\mathrm{I}}} \operatorname{div} \mathbf{u} \mathbf{z} \cdot \mathbf{n}-\int_{\Gamma_{\mathrm{I}}}^{\boldsymbol{\sigma}} \boldsymbol{\sigma}(\mathbf{v}) \mathbf{w} \cdot \mathbf{n} .
\end{aligned}
$$

Los primeros términos en el lado derecho de la expresión anterior pueden acotarse fácilmente. En efecto, teniendo en cuenta la regularidad de los elementos de $\mathbf{E}_{\lambda}(\mathcal{W}(\widehat{\Omega}))$ y usando la desigualdad de CauchySchwarz, el Lema D.6, el Teorema 4.44 y la estimación (4.97), puede probarse que

$$
\begin{gathered}
\left|\rho_{\mathrm{F}} \int_{\Omega_{\mathrm{F}} \backslash \Omega_{\mathrm{F} h}} c^{2} \operatorname{div} \mathbf{u} \operatorname{div} \mathbf{z}+(\mathbf{u}-\mathbf{x}) \cdot \mathbf{z}\right|+\left|\int_{\Omega_{\mathrm{S}} \backslash \Omega_{\mathrm{S} h}} \boldsymbol{\sigma}(\mathbf{v}): \boldsymbol{\varepsilon}(\mathbf{w})+\rho_{\mathrm{S}}(\mathbf{v}-\mathbf{y}) \cdot \mathbf{w}\right| \leq C h^{r+\hat{r}_{h}}, \\
\left|\rho_{\mathrm{F}} \int_{\Omega_{\mathrm{F} h} \backslash \Omega_{\mathrm{F}}} c^{2} \operatorname{div} \mathbf{u} \operatorname{div} \mathbf{z}+(\mathbf{u}-\mathbf{x}) \cdot \mathbf{z}\right|+\left|\int_{\Omega_{\mathrm{S} h} \backslash \Omega_{\mathrm{S}}} \boldsymbol{\sigma}(\mathbf{v}): \boldsymbol{\varepsilon}(\mathbf{w})+\rho_{\mathrm{S}}(\mathbf{v}-\mathbf{y}) \cdot \mathbf{w}\right| \leq C h^{2 \hat{r}_{h}} .
\end{gathered}
$$

Consideraremos ahora los términos restantes. En lo que sigue, será $\left(\boldsymbol{\eta}_{h}, \boldsymbol{\varphi}_{h}\right)=\Pi_{h}(\boldsymbol{\eta}, \boldsymbol{\varphi})$. Dado que $(\boldsymbol{\eta}, \boldsymbol{\varphi}) \in \mathbf{E}(\mathcal{V}(\widehat{\Omega}))$, tendremos

$$
\left.(\mathbf{z}-\mathbf{w}) \cdot \mathbf{n}\right|_{\Gamma_{\mathrm{I}}}=\left.\left(\boldsymbol{\eta}_{h}-\boldsymbol{\varphi}_{h}\right) \cdot \mathbf{n}\right|_{\Gamma_{\mathrm{I}}}
$$

Usando la ecuación (4.94) para eliminar la componente normal del tensor de esfuerzos sobre la interfaz en términos de la presión ejercida por el fluido y teniendo en cuenta la condición de borde (4.93), obtendremos

$$
\rho_{\mathrm{F}} c^{2} \int_{\Gamma_{\mathrm{I}}} \operatorname{div} \mathbf{u} \mathbf{z} \cdot \mathbf{n}-\int_{\Gamma_{\mathrm{I}}} \boldsymbol{\sigma}(\mathbf{v}) \mathbf{w} \cdot \mathbf{n}=\rho_{\mathrm{F}} c^{2} \int_{\Gamma_{\mathrm{I}}} \operatorname{div} \mathbf{u}\left(\boldsymbol{\eta}_{h}-\boldsymbol{\varphi}_{h}\right) \cdot \mathbf{n} .
$$

Sea $\widetilde{\operatorname{div} \mathbf{u}}$ la extensión de la función $\left.\operatorname{div} \mathbf{u}\right|_{\Omega_{\mathrm{F}}} \in H^{1+s}\left(\Omega_{\mathrm{F}}\right)$ a $\mathbb{R}^{2}$ como en el Teorema D.4. Sea $T$ un triángulo en el borde del dominio $\Omega_{\mathrm{F}}$ y sea $\omega_{T}$ el conjunto definido por $T \backslash T^{i d}$ o $T^{i d} \backslash T$, según corresponda. Un cálculo sencillo nos conducirá a

$$
\int_{\Gamma_{\mathrm{I}}} \operatorname{div} \mathbf{u}\left(\boldsymbol{\eta}_{h}-\boldsymbol{\varphi}_{h}\right) \cdot \mathbf{n}=\sum_{T \in \mathcal{B}_{\mathrm{F} h}} \epsilon(T) \int_{\partial \omega_{T}} \widetilde{\operatorname{div} \mathbf{u}}\left(\boldsymbol{\eta}_{h}-\boldsymbol{\varphi}_{h}\right) \cdot \mathbf{n}+\int_{\Gamma_{\mathrm{I} h}} \widetilde{\operatorname{div} \mathbf{u}}\left(\boldsymbol{\eta}_{h}-\boldsymbol{\varphi}_{h}\right) \cdot \mathbf{n},
$$

donde el coeficiente $\epsilon(T)$ está definido por

$$
\epsilon(T):=\left\{\begin{aligned}
1 & \text { si } T \subset T^{i d} \\
-1 & \text { si } T^{i d} \subset T
\end{aligned}\right.
$$

A continuación, acotaremos el primer término en el lado derecho de la expresión anterior. En virtud de la definición de $\Pi_{h},\left(\boldsymbol{\eta}_{h}-\varphi_{h}\right) \in H\left(\operatorname{div} ; \omega_{T}\right)$. Por lo tanto,

$$
\int_{\partial \omega_{T}} \widetilde{\operatorname{div} \mathbf{u}}\left(\boldsymbol{\eta}_{h}-\boldsymbol{\varphi}_{h}\right) \cdot \mathbf{n}=\int_{\omega_{T}}\left(\boldsymbol{\eta}_{h}-\boldsymbol{\varphi}_{h}\right) \cdot \widetilde{\operatorname{div} \mathbf{u}}+\int_{\omega_{T}} \operatorname{div}\left(\boldsymbol{\eta}_{h}-\boldsymbol{\varphi}_{h}\right) \widetilde{\operatorname{div} \mathbf{u}}
$$


Sumando sobre todos los triángulos en $\mathcal{B}_{\mathrm{F} h}$, usando la desigualdad de Cauchy-Schwarz, el Lema D.6 y el Teorema D.4, obtendremos

$$
\sum_{T \in \mathcal{B}_{\mathrm{F} h}}\left|\int_{\partial \omega_{T}} \widetilde{\operatorname{div} \mathbf{u}}\left(\boldsymbol{\eta}_{h}-\boldsymbol{\varphi}_{h}\right) \cdot \mathbf{n}\right| \leq C h^{s}\|\operatorname{div} \mathbf{u}\|_{1+s, \Omega_{\mathrm{F}}}\left\|\left(\boldsymbol{\eta}_{h}, \boldsymbol{\varphi}_{h}\right)\right\|_{h, \Omega_{\mathrm{F}} \backslash \Omega_{\mathrm{F} h} \cup \Omega_{\mathrm{F} h} \backslash \Omega_{\mathrm{F}}} .
$$

Usando ahora el Teorema 4.44 y la estimación (4.97), tendremos

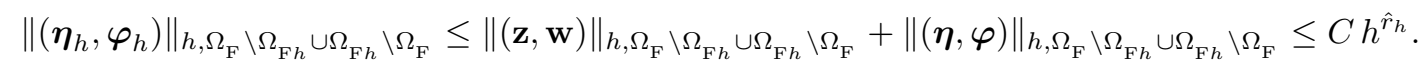

Acotaremos ahora el término restante. Sea $P_{\Gamma_{\mathrm{Ih}}}$ la proyección $L^{2}\left(\Gamma_{\mathrm{I} h}\right)$-ortogonal sobre las funciones constantes a trozos definidas en $\Gamma_{\mathrm{I} h}$. Dado que $\Pi_{h}(\boldsymbol{\eta}, \boldsymbol{\varphi}) \in \mathcal{K}_{h}^{e}(\widehat{\Omega})$, la condición de compatibilidad sobre $\Gamma_{\mathrm{I} h}$ implica que $\boldsymbol{\eta}_{h} \cdot \mathbf{n}=P_{\Gamma_{\mathrm{I} h}}\left(\boldsymbol{\varphi}_{h} \cdot \mathbf{n}\right)$. Luego,

$$
\begin{aligned}
\left|\int_{\Gamma_{\mathrm{I} h}} \widetilde{\operatorname{div} \mathbf{u}}\left(\boldsymbol{\eta}_{h}-\boldsymbol{\varphi}_{h}\right) \cdot \mathbf{n}\right| & =\left|\int_{\Gamma_{\mathrm{I} h}}\left(\mathbf{I}-P_{\Gamma_{\mathrm{I} h}}\right) \widetilde{\operatorname{div} \mathbf{u}}\left(P_{\Gamma_{\mathrm{I} h}}-\mathbf{I}\right)\left(\boldsymbol{\varphi}_{h} \cdot \mathbf{n}\right)\right| \\
& \leq\left\|\left(\mathbf{I}-P_{\Gamma_{\mathrm{I} h}}\right) \widetilde{\operatorname{div} \mathbf{u}}\right\|_{0, \Gamma_{\mathrm{I} h}}\left\|\left(P_{\Gamma_{\mathrm{I} h}}-\mathbf{I}\right)\left(\boldsymbol{\varphi}_{h} \cdot \mathbf{n}\right)\right\|_{0, \Gamma_{\mathrm{I} h}} .
\end{aligned}
$$

Además, es claro que

$$
\begin{aligned}
\left\|\left(P_{\Gamma_{\mathrm{I} h}}-\mathbf{I}\right)\left(\boldsymbol{\varphi}_{h} \cdot \mathbf{n}\right)\right\|_{0, \Gamma_{\mathrm{I} h}} & \leq\left\|\left(P_{\Gamma_{\mathrm{I} h}}-\mathbf{I}\right)(\boldsymbol{\varphi} \cdot \mathbf{n})\right\|_{0, \Gamma_{\mathrm{I} h}}+\left\|\left(P_{\Gamma_{\mathrm{I} h}}-\mathbf{I}\right)\left(\boldsymbol{\varphi}_{h} \cdot \mathbf{n}-\boldsymbol{\varphi} \cdot \mathbf{n}\right)\right\|_{0, \Gamma_{\mathrm{I} h}} \\
& \leq\left\|\left(P_{\Gamma_{\mathrm{I} h}}-\mathbf{I}\right)(\boldsymbol{\varphi} \cdot \mathbf{n})\right\|_{0, \Gamma_{\mathrm{I} h}}+\left\|\left(\boldsymbol{\varphi}_{h}-\boldsymbol{\varphi}\right) \cdot \mathbf{n}\right\|_{0, \Gamma_{\mathrm{I} h}} .
\end{aligned}
$$

Como $\widetilde{\operatorname{div} \mathbf{u}} \in H^{1+s}\left(\widehat{\Omega}_{\mathrm{F}}\right), s \in(1 / 2,1], \mathrm{y} \varphi \in H^{1+t}\left(\widehat{\Omega}_{\mathrm{S}}\right)^{2}, t \in(0,1]$, tendremos, de acuerdo con el Lema E.6,

$$
\begin{gathered}
\left\|\left(\mathbf{I}-P_{\Gamma_{\mathrm{I} h}}\right) \widetilde{\operatorname{div} \mathbf{u}}\right\|_{0, \Gamma_{\mathrm{I} h}} \leq C h\|\operatorname{div} \mathbf{u}\|_{1+s, \Omega_{\mathrm{F}}} \leq C h, \\
\left\|\left(P_{\Gamma_{\mathrm{I} h}}-\mathbf{I}\right)(\boldsymbol{\varphi} \cdot \mathbf{n})\right\|_{0, \Gamma_{\mathrm{I} h}} \leq C h^{\operatorname{mín}\{1,1 / 2+t\}}\|\boldsymbol{\varphi}\|_{1+t, \Omega_{\mathrm{S}}} \leq C h^{\operatorname{mín}\{1,1 / 2+t\}}
\end{gathered}
$$

y, finalmente, usando el Lema E.7 y la estimación (4.97),

$$
\left\|\left(\boldsymbol{\varphi}_{h}-\boldsymbol{\varphi}\right) \cdot \mathbf{n}\right\|_{0, \Gamma_{\mathrm{I} h}} \leq C\left\|\boldsymbol{\varphi}_{h}-\boldsymbol{\varphi}\right\|_{1, \Omega_{\mathrm{S}}} \leq C\|(\mathbf{z}, \mathbf{w})\|_{h} \leq C h^{\hat{r}_{h}} .
$$

De esta manera, combinando las estimaciones halladas, podremos concluir la prueba.

Lema $4.46 S e a$

$$
N_{h}=\sup _{\substack{(\mathbf{x}, \mathbf{y}) \in \mathbf{E}(\mathcal{V}(\widehat{\Omega})) \\\|(\mathbf{x}, \mathbf{y})\|_{h}=1}} \sup _{\substack{\boldsymbol{\eta}, \boldsymbol{\varphi}) \in \mathbf{E}(\mathcal{V}(\widehat{\Omega})) \\\|(\boldsymbol{\eta}, \boldsymbol{\varphi})\|_{h}=1}}\left|a_{h}(\mathbf{A}(\mathbf{x}, \mathbf{y}),(\boldsymbol{\eta}, \boldsymbol{\varphi}))-d_{h}((\mathbf{x}, \mathbf{y}),(\boldsymbol{\eta}, \boldsymbol{\varphi}))\right| .
$$

Existe una constante positiva $C$ tal que

$$
N_{h} \leq C h^{r+\hat{r}_{h}}
$$

Prueba. Igual a la prueba del lema previo, sustituyendo $\left(\Pi_{h}-\mathbf{I}\right)(\boldsymbol{\eta}, \boldsymbol{\varphi})$ por $(\boldsymbol{\eta}, \boldsymbol{\varphi})$ y teniendo en cuenta que, en este caso,

$$
\rho_{\mathrm{F}} c^{2} \int_{\Gamma_{\mathrm{I}}} \operatorname{div} \mathbf{u}(\boldsymbol{\eta}-\boldsymbol{\varphi}) \cdot \mathbf{n}=0
$$

De acuerdo con los resultados de la sección 3.3.4 (ver Teorema 3.29), podemos asegurar que los autovalores aproximados convergen con orden óptimo. Estableceremos este resultado en el siguiente teorema.

Teorema 4.47 Existe una constante positiva $C$ tal que

$$
\operatorname{máx}_{i=1, \cdots, m}\left|\lambda-\lambda_{i h}\right| \leq C h^{r+\hat{r}_{h}} .
$$


Prueba. Es una consecuencia directa de los Teoremas 4.41-4.44 y de los lemas previos.

Observación 4.48 Denotemos por $\theta$ y $\theta_{h}$ a los máximos ángulos re-entrantes presentes en los dominios $\Omega_{\mathrm{F}} y \Omega_{\mathrm{F} h}$, respectivamente. Dado que $\Gamma_{\mathrm{I}}$ se asumió $\mathcal{C}^{2}$ a trozos, tendremos que $\theta_{h} \rightarrow \theta$ a medida que $h \rightarrow 0$. Cosecuentemente, $s_{h}$ y $\hat{s}_{h}$ serán asintóticamente iguales a s y, por lo tanto, $r_{h}$ será asintóticamente igual a r. Concluimos entonces que

$$
\lim _{h \rightarrow 0} \hat{r}_{h}=\min \{r, \alpha\} .
$$

Por otro lado, teniendo en cuenta que los desplazamientos en la estructura son solución del siguiente problema de elasticidad lineal

$$
\begin{aligned}
-\operatorname{div}[\boldsymbol{\sigma}(\mathbf{w})]+\rho_{\mathrm{S}} \mathbf{w} & =\rho_{\mathrm{S}} \mathbf{g} & & \text { en } \Omega_{\mathrm{S}}, \\
\boldsymbol{\sigma}(\mathbf{w}) \mathbf{n} & =\left(\rho_{\mathrm{F}} c^{2} \operatorname{div} \mathbf{u}\right) \mathbf{n} & & \text { sobre } \Gamma_{\mathrm{I}}, \\
\boldsymbol{\sigma}(\mathbf{w}) \mathbf{n}_{\mathrm{S}} & =\mathbf{0} & & \text { sobre } \Gamma_{\mathrm{N}}, \\
\mathbf{w} & =\mathbf{0} & & \text { sobre } \Gamma_{\mathrm{D}}
\end{aligned}
$$

tendremos que, para $\mathbf{g} \in L^{2}\left(\Omega_{\mathrm{S}}\right)$ y $\left(\rho_{\mathrm{F}} c^{2} \operatorname{div} \mathbf{u}\right) \mathbf{n} \in L^{2}\left(\Gamma_{\mathrm{I}}\right)$, será $\alpha \geq t$ [111].

\section{Approximación de la presión en la interfaz}

En esta sección probaremos que la presión aproximada sobre la interfaz converge con el mismo orden que los desplazamientos.

Sea $\gamma_{h}$ la aproximación de la presión ejercida por el fluido sobre la interfaz discreta $\Gamma_{\mathrm{I} h}$. Denotaremos por $\gamma_{h}^{e}$ a la extensión (continua) de $\gamma_{h}$ a $\Omega_{\mathrm{F}} \backslash \Omega_{\mathrm{F} h} \cup \Omega_{\mathrm{F} h} \backslash \Omega_{\mathrm{F}}$ definida de manera que sea constante sobre direcciones normales a $\Gamma_{\mathrm{I} h}$. Como $\gamma_{h}$ es constante a trozos, tendremos que $\gamma_{h}^{e}$ será constante a trozos sobre $\Gamma_{\mathrm{I}}$.

Teorema 4.49 Sean $(\lambda,((\mathbf{u}, \mathbf{w}), \gamma))$ y $\left(\lambda_{h},\left(\left(\mathbf{u}_{h}, \mathbf{w}_{h}\right), \gamma_{h}\right)\right)$ pares propios correspondientes a los problemas 4.27 y 4.30, respectivamente, tales que $\lambda_{h} \rightarrow \lambda$ cuando $h \rightarrow 0$ y $\|\left(\mathbf{u}^{e}, \mathbf{w}^{e}\right)-\left(\mathbf{u}_{h}^{e}, \mathbf{w}_{h}^{e}\left\|_{h} \leq C h^{\hat{r}_{h}}\right\|(\mathbf{u}, \mathbf{w}) \|_{h, \Omega}\right.$. Entonces,

$$
\left\|\gamma-\gamma_{h}^{e}\right\|_{0, \Gamma_{\mathrm{I}}} \leq C h^{\hat{r}_{h}}\|(\mathbf{u}, \mathbf{w})\|_{h, \Omega} .
$$

Prueba. Sea $\widetilde{\operatorname{div} \mathbf{u}}$ la extensión de la función $\operatorname{div} \mathbf{u} \in H^{1+s}\left(\Omega_{\mathrm{F}}\right)$ a $\mathbb{R}^{2}$ definida como en el Teorema D.4. Sea $\gamma^{e}$ la restricción de la función $-\rho_{\mathrm{F}} c^{2} \widehat{\operatorname{div} \mathbf{u}}$ sobre la interfaz $\Gamma_{\mathrm{I} h}$. Denotaremos por $\zeta$ a la diferencia $\gamma-\left.\gamma_{h}^{e}\right|_{\Gamma_{\mathrm{I}}}$. Sean $\bar{\zeta}$ y $\bar{\gamma}$ las extensiones de $\zeta$ y $\gamma$ a $\Gamma_{\mathrm{I} h}$, respectivamente, obtenidas según la construcción dada en [47] (ver sección E.2). Es fácil comprobar que $\bar{\zeta}=\bar{\gamma}-\gamma_{h}$. Luego, usando los Lemas E.1-E.2, tendremos

$$
\|\zeta\|_{0, \Gamma_{\mathrm{I}}} \leq C\|\bar{\zeta}\|_{0, \Gamma_{\mathrm{I} h}} \leq C\left(\left\|\bar{\gamma}-\gamma^{e}\right\|_{0, \Gamma_{\mathrm{I} h}}+\left\|\gamma^{e}-\gamma_{h}\right\|_{0, \Gamma_{\mathrm{I} h}}\right) \leq C\left(h^{s}\|\operatorname{div} \mathbf{u}\|_{s, \Omega_{\mathrm{F}}}+\left\|\gamma^{e}-\gamma_{h}\right\|_{0, \Gamma_{\mathrm{I} h}}\right) .
$$

La prueba se completa combinando los argumentos presentados en [34] (ver la prueba del Teorema 8.1 en esa referencia) con las técnicas empleadas en la prueba del Lemma E.31 y la estimación anterior.

\subsection{Dominios curvos y espacios discretos no conformes}

\subsubsection{El problema de autovalores de Laplace con condiciones de borde de Neumann}

En esta sección, analizaremos el problema de autovalores del operador de Laplace definido sobre un dominio curvo, con condición de borde de Neumann, y su aproximación por un método discontinuo de Galerkin.

El problema espectral de Laplace definido sobre dominios curvos ya ha sido analizado en varios trabajos anteriores pero solo usando métodos conformes. Por ejemplo, en [133, 165, 166, 167] se considera este problema con condiciones de borde de Dirichlet, en [116], con condiciones de borde de Neumann y en [1], con condiciones de borde mixtas. 
En la actualidad, no se conocen resultados similares utilizando un método discontinuo de Galerkin.

En 1973, Reed-Hill [148] introdujeron los métodos discontinuos de Galerkin (DG) como métodos numéricos adecuados para aproximar ecuaciones de tipo hiperbólico. También en los años 70, pero de manera totalmente independiente, se propusieron varios métodos de Galerkin basados en elementos finitos discontinuos para aproximar problemas elípticos y parabólicos [14, 30, 96, 174]. A estos esquemas numéricos se los denominó originalmente interior penalty methods (IP) y su desarrollo permaneció por muchos años independiente del desarrollo de los métodos DG. Actualmente, se cuenta con una teoría unificada que permite el análisis de una clase muy amplia de métodos DG para problemas elípticos de segundo orden [19].

Los métodos DG también han sido utilizados para aproximar los espectros del operador de Laplace [12] y de Maxwell [60]. En ambos trabajos, se asumieron dominios poligonales y condiciones de frontera de Dirichlet.

\section{Definición del problema continuo}

Sea $\Omega \subset \mathbb{R}^{2}$ un dominio acotado, simplemente conexo, no necesariamente convexo, con un borde $\Gamma:=\partial \Omega$ Lipschitz y suave a trozos. Más aún, supondremos que $\Gamma$ es de clase $\mathcal{C}^{2}$ a trozos. Indicaremos con $\mathbf{n}$ al vector unitario en la dirección normal al borde $\Gamma$, positivamente orientado.

Consideremos el siguiente problema de autovalores:

Encontrar $u \neq 0$ y $\mu \in \mathbb{R}$ tal que

$$
\begin{aligned}
-\triangle u+u & =\mu u & & \text { en } \Omega, \\
\frac{\partial u}{\partial \mathbf{n}} & =0 & & \text { sobre } \Gamma .
\end{aligned}
$$

Sean $X \Omega):=L^{2}(\Omega)$ y $V(\Omega):=H^{1}(\Omega)$, equipados con las normas usuales. Sean $a$ y $b$ las formas bilineales definidas por

$$
\begin{gathered}
a(u, v):=\int_{\Omega} \nabla u \cdot \nabla v+\int_{\Omega} u v \quad \forall u, v \in V(\Omega), \\
b(u, v):=\int_{\Omega} u v \quad \forall u, v \in X(\Omega) .
\end{gathered}
$$

Claramente, $a$ es continua y coerciva sobre $V(\Omega)$ y $b$ es continua sobre $X(\Omega)$. No es difícil comprobar que el problema (4.99) puede ser re-escrito de la siguiente manera:

Encontrar $\mu \in \mathbb{R}$ y $u \in V(\Omega), u \neq 0$, tal que

$$
a(u, v)=\mu b(u, v) \quad \forall v \in V(\Omega) .
$$

Es bien sabido que este problema tiene un conjunto infinito numerable de autovalores, todos positivos y de multiplicidad finita, sin puntos de acumulación finitos. Escribiremos

$$
1=\mu_{1}<\mu_{2} \leq \mu_{3} \leq \cdots \leq \mu_{k} \leq \cdots \rightarrow+\infty
$$

donde cada autovalor aparece tantas veces como lo indique su multiplicidad. Además, las funciones propias correspondientes, $\left\{u_{k}\right\}_{k=1}^{\infty}$, son $L^{2}(\Omega)$-ortogonales y forman una base para $H^{1}(\Omega)$.

El operador solución correspondiente al problema de autovalores (4.100) será

$$
\begin{aligned}
\mathbf{T}: X(\Omega) & \rightarrow V(\Omega) \hookrightarrow X(\Omega) \\
f & \mapsto u,
\end{aligned}
$$

donde $u$ es la solución del siguiente problema fuente:

$$
a(u, v)=b(f, v) \quad \forall v \in V(\Omega) .
$$


El Teorema de Lax-Milgran garantiza que el operador T estará bien definido y será acotado. En efecto, las propiedades de las formas bilineales aseguran la existencia de una única solución del problema (4.101) y se verifica

$$
\|u\|_{V(\Omega)} \leq C\|f\|_{0, \Omega} .
$$

A causa de la simetría de las formas bilineales, $\mathbf{T}$ es un operador autoadjunto. Además, gracias a la inclusión compacta $V(\Omega) \hookrightarrow X(\Omega)$, $\mathbf{T}$ es un operador compacto. Observemos también que $(\mu, u)$ es un par propio del operador T si, y solo si, $\left(\frac{1}{\mu}, u\right)$ es solución del problema (4.100).

Las soluciones del problema fuente (4.101) satisfacen la propiedad de regularidad elíptica; es decir, la solución $u \in H^{1+s}(\Omega)$, para algún $s>0$, y vale la siguiente estimación

$$
\|u\|_{1+s, \Omega} \leq C\|f\|_{0, \Omega} .
$$

De acuerdo con [111], los valores posibles de $s$ solo dependen de la geometría de $\Omega ; s=1$ si $\Omega$ es convexo o, en caso contrario, $s=\frac{\pi}{\theta}$, siendo $\theta$ el máximo ángulo re-entrante en $\Omega$. Como consecuencia de estos resultados, las autofunciones $u_{k}$, del operador $\mathbf{T}$ pertenecen al espacio $H^{1+s}(\Omega)$ y satisfacen

$$
\left\|u_{k}\right\|_{1+s, \Omega} \leq C\left\|u_{k}\right\|_{0, \Omega} .
$$

\section{Discretización del problema}

Sean $\Omega_{h}$ una aproximación poligonal del dominio $\Omega$ y $\left\{\mathcal{T}_{h}\right\}_{h>0}$ una familia de particiones de $\bar{\Omega}_{h}$ en triángulos. Asumiremos que la familia de particiones satisface todas las propiedades formuladas en la sección 4.2 .

Para una dada partición $\mathcal{T}_{h}$ de $\bar{\Omega}_{h}$, sea $\mathcal{E}_{h}$ el conjunto de todos los lados en $\mathcal{T}_{h}$. Dividiremos a este conjunto como sigue: $\mathcal{E}_{h}=\mathcal{E}_{h}^{I} \cup \mathcal{E}_{h}^{\Gamma}$, siendo $\mathcal{E}_{h}^{I}:=\left\{\ell \in \mathcal{E}_{h}: \ell \not \subset \Gamma\right\}$, el conjunto de los lados internos, y $\mathcal{E}_{h}^{\Gamma}:=\left\{\ell \in \mathcal{E}_{h}: \ell \subset \Gamma\right\}$, el conjunto de los lados en el borde.

Como es usual en el análisis de los métodos DG, introduciremos los espacios de Sobolev quebrados $H^{m}\left(\mathcal{T}_{h}\right)$, $m \geq 1$,

$$
H^{m}\left(\mathcal{T}_{h}\right):=\left\{u \in L^{2}\left(\Omega_{h}\right):\left.u\right|_{T} \in H^{m}(T) \forall T \in \mathcal{T}_{h}\right\} .
$$

En este contexto, consideraremos que $T$ es un conjunto abierto para asegurar así que las funciones en $H^{m}\left(\mathcal{T}_{h}\right)$ sean monovaluadas. Los operadores de trazas [[ $\left.\cdot\right]$ y $\{\cdot\}$, introducidos en la sección 5.1 , serán utilizados para definir los valores asignados a las funciones en $H^{m}\left(\mathcal{T}_{h}\right)$ sobre los lados $\ell \in \mathcal{E}_{h}$.

Sea $\mathcal{P}_{k}(T)$ el espacio de los polinomios de grado menor o igual que $k$ definidos sobre $T$. Introducimos el espacio de elementos finitos discontinuos

$$
V_{h}\left(\Omega_{h}\right):=\left\{v_{h} \in X\left(\Omega_{h}\right):\left.v_{h}\right|_{T} \in \mathcal{P}_{1}(T) \forall T \in \mathcal{T}_{h}\right\} \subset H^{1}\left(\mathcal{T}_{h}\right) .
$$

y lo equipamos con la siguiente norma discreta

$$
\left\|u_{h}\right\|_{V_{h}\left(\Omega_{h}\right)}^{2}:=\left\|u_{h}\right\|_{0, \Omega_{h}}^{2}+\sum_{T \in \mathcal{T}_{h}}\left|u_{h}\right|_{1, T}^{2}+\sum_{\ell \in \mathcal{E}_{h}^{I}} h_{\ell}^{-1}\left\|\left[\left[u_{h}\right]\right]\right\|_{0, \ell}^{2} .
$$

A continuación definiremos un método DG para la discretización del problema (4.100). Comenzaremos por introducir las siguientes formas bilineales $a_{h}: V_{h}\left(\Omega_{h}\right) \times V_{h}\left(\Omega_{h}\right) \rightarrow \mathbb{R}$ y $b_{h}: X\left(\Omega_{h}\right) \times X\left(\Omega_{h}\right) \rightarrow \mathbb{R}$,

$$
\begin{aligned}
a_{h}\left(u_{h}, v_{h}\right) & :=\sum_{T \in \mathcal{T}_{h}} \int_{T} \nabla u_{h} \cdot \nabla v_{h}+\int_{\Omega_{h}} u_{h} v_{h}-\sum_{\ell \in \mathcal{E}_{h}^{I}} \int_{\ell}\left(\left\{\nabla u_{h}\right\} \cdot\left[\left[v_{h}\right]\right]+\left\{\nabla v_{h}\right\} \cdot\left[\left[u_{h}\right]\right]\right) \\
& +\alpha \sum_{\ell \in \mathcal{E}_{h}^{I}} h_{\ell}^{-1} \int_{\ell}\left[\left[u_{h}\right]\right] \cdot\left[\left[v_{h}\right]\right] \quad u_{h}, v_{h} \in V_{h}\left(\Omega_{h}\right), \\
b_{h}\left(u_{h}, v_{h}\right) & :=\int_{\Omega_{h}} u_{h} v_{h} \quad u_{h}, v_{h} \in X\left(\Omega_{h}\right) .
\end{aligned}
$$

En las expresiones anteriores, $h_{\ell}=|\ell|$ y $\alpha$ es un parámetro positivo (interior penalty parameter). En relación a la continuidad y la coercividad de la forma $a_{h}$ puede probarse el siguiente resultado. 
Lema 4.50 Existen constantes positivas $c_{1}, c_{2} y \alpha_{0}$, tales que

$$
\begin{aligned}
& \text { - }\left|a_{h}\left(u_{h}, v_{h}\right)\right| \leq c_{1}\left\|u_{h}\right\|_{V_{h}\left(\Omega_{h}\right)}\left\|v_{h}\right\|_{V_{h}\left(\Omega_{h}\right)} \quad \forall u_{h}, v_{h} \in V_{h}\left(\Omega_{h}\right), \\
& \text { - } a_{h}\left(u_{h}, u_{h}\right) \geq c_{2}\left\|u_{h}\right\|_{V_{h}\left(\Omega_{h}\right)}^{2} \quad \forall \alpha \geq \alpha_{0}, \forall u_{h} \in V_{h}\left(\Omega_{h}\right) .
\end{aligned}
$$

Prueba. Se obtiene siguiendo los argumentos en [19]. Las constantes solo dependen de los parámetros que determinan la regularidad de la partición.

La aproximación del problema (4.100) se define entonces como sigue:

Encontrar $\mu_{h} \in \mathbb{R} y u_{h} \in V_{h}\left(\Omega_{h}\right), u_{h} \neq 0$, tal que

$$
a_{h}\left(u_{h}, v_{h}\right)=\mu_{h} b_{h}\left(u_{h}, v_{h}\right) \quad \forall v_{h} \in V_{h}\left(\Omega_{h}\right) .
$$

Observemos que este problema se reduce a un problema de autovalores generalizado que involucra matrices simétricas y definidas positivas. Luego, el problema (4.105) tendrá un número finito de pares propios $\left(\mu_{j h}, u_{j h}\right), 1 \leq j \leq M(h), M(h)=\operatorname{dim} V_{h}\left(\Omega_{h}\right)$, todos con autovalores positivos. Escribiremos

$$
1=\mu_{1 h}<\mu_{2 h} \leq \mu_{3 h} \leq \cdots \leq \mu_{M(h) h},
$$

donde cada autovalor aparece repetido tantas veces como lo indique su multiplicidad.

La versión discreta del operador solución $\mathbf{T}$ estará dada por

$$
\begin{aligned}
\mathbf{T}_{h}: X\left(\Omega_{h}\right) & \rightarrow X\left(\Omega_{h}\right) \\
f & \mapsto u_{h},
\end{aligned}
$$

donde $u_{h}$ es la solución del problema

$$
a_{h}\left(u_{h}, v_{h}\right)=b_{h}\left(f, v_{h}\right) \quad \forall v_{h} \in V_{h}\left(\Omega_{h}\right) .
$$

Argumentando como en el caso continuo, podremos asegurar que el problema (4.106) tiene solución única y que

$$
\left\|u_{h}\right\|_{V_{h}\left(\Omega_{h}\right)} \leq C\|f\|_{0, \Omega_{h}}
$$

Consecuentemente, el operador $\mathbf{T}_{h}$ estará bien definido y estará acotado uniformemente en $h$. Una vez más, como en el caso continuo, $\left(\mu_{h}, u_{h}\right) \in \mathbb{R} \times V_{h}\left(\Omega_{h}\right)$ es un par propio del operador $\mathbf{T}_{h}$ si, y solo si, $\left(\frac{1}{\mu_{h}}, u_{h}\right)$ es solución del problema $(4.105)$.

\section{Aproximación espectral}

Para analizar la convergencia de las soluciones del problema (4.105), seguiremos la teoría expuesta en la sección 3.2 .

Nuestro primer objetivo será elegir una norma discreta adecuada para funciones con dominio en $\widehat{\Omega}$. Definimos:

$$
\|\cdot\|_{h}^{2}:=\sum_{\substack{T \in \mathcal{T}_{h} \\ T \notin \mathcal{B}_{h}}}\|\cdot\|_{1, T}^{2}+\sum_{\substack{T \in \mathcal{B}_{h} \\ T^{i d} \subset T}}\|\cdot\|_{1, T}^{2}+\sum_{\substack{T \in \mathcal{B}_{h} \\ T \subset T^{i d}}}\|\cdot\|_{1, T^{i d}}^{2}+\sum_{\ell \in \mathcal{E}_{h}^{I}} h_{\ell}^{-1}\|[[\cdot]]\|_{0, \ell}^{2} .
$$

Observación 4.51 Las siguientes son expresiones equivalentes para la norma $\|\cdot\|_{h}$. En estas expresiones, $\omega_{T}$ indicará el dominio definido por la diferencia $T \backslash T^{i d}$ o $T^{i d} \backslash T$, según corresponda. Tendremos

$$
\begin{aligned}
& \|\cdot\|_{h}^{2}=\|\cdot\|_{h, \Omega_{h}}^{2}+\sum_{\substack{T \in \mathcal{B}_{h} \\
T \subset T^{i d}}}\|\cdot\|_{1, \omega_{T}}^{2}:=\sum_{T \in \mathcal{T}_{h}}\|\cdot\|_{1, T}^{2}+\sum_{\ell \in \mathcal{E}_{h}^{I}} h_{\ell}^{-1}\|[[\cdot]]\|_{0, \ell}^{2}+\sum_{\substack{T \in \mathcal{B}_{h} \\
T \subset T^{i d}}}\|\cdot\|_{1, \omega_{T}}^{2}, \\
& \|\cdot\|_{h}^{2}=\|\cdot\|_{h, \Omega}^{2}+\sum_{\substack{T \in \mathcal{B}_{h} \\
T^{i d} \subset T}}\|\cdot\|_{1, \omega_{T}}^{2}:=\sum_{T \in \mathcal{T}_{h}}\|\cdot\|_{1, T^{i d}}^{2}+\sum_{\ell \in \mathcal{E}_{h}^{I}} h_{\ell}^{-1}\|[[\cdot]]\|_{0, \ell}^{2}+\sum_{\substack{T \in \mathcal{B}_{h} \\
T^{i d} \subset T}}\|\cdot\|_{1, \omega_{T}}^{2} .
\end{aligned}
$$


Por su definición, la norma $\|\cdot\|_{h}$ satisface todos las propiedades requeridas. En particular,

$$
\text { si }\left.v\right|_{\Omega} \in V(\Omega) \quad \text { entonces } \quad\|v\|_{h, \Omega}=\|v\|_{V(\Omega)} \text {. }
$$

Nuestro próximo objetivo será definir los operadores de extensión $\mathbf{P}$ y $\mathbf{P}_{h}$.

Para las funciones en los espacios del continuo, consideraremos una extensión que preserve la regularidad de las funciones originales, como la definida en la sección D; es decir, dentificaremos al operador $\mathbf{P}$ con el operador de extensión de Stein. Luego, en virtud del Teorema (D.4), tendremos

$$
\begin{aligned}
& \text { - } \mathbf{P}: V(\Omega) \rightarrow V(\widehat{\Omega}), u \mapsto u^{e}, \\
& \text { - }\left.u^{e}\right|_{\Omega}=u \\
& \text { - }\left\|u^{e}\right\|_{0, \widehat{\Omega}} \leq\left\|u^{e}\right\|_{h} \leq C\|u\|_{V(\Omega)} .
\end{aligned}
$$

Para las funciones en los espacios discretos, consideraremos la extensión natural definida en D.8. Definiremos a $\mathbf{P}_{h}$ como el operador que realiza esta extensión. A partir del Lema D.9, es claro que

$$
\begin{aligned}
& \text { - } \mathbf{P}_{h}: V_{h}\left(\Omega_{h}\right) \rightarrow X(\widehat{\Omega}), u_{h} \mapsto u_{h}^{e}, \\
& \text { - }\left.u_{h}^{e}\right|_{\Omega_{h}}=u_{h}, \\
& \text { - }\left\|u_{h}^{e}\right\|_{0, \widehat{\Omega}} \leq\left\|u_{h}^{e}\right\|_{h} \leq C\left\|u_{h}\right\|_{V_{h}\left(\Omega_{h}\right)} .
\end{aligned}
$$

Para cada aproximación $\Omega_{h}$, definimos los espacios

$$
\begin{aligned}
V^{e}(\widehat{\Omega}) & :=\mathbf{P}(V(\Omega)) \subset V(\widehat{\Omega}), \\
V_{h}^{e}(\widehat{\Omega}) & :=\mathbf{P}_{h}\left(V_{h}\left(\Omega_{h}\right)\right) \subset X(\widehat{\Omega}), \\
W(\widehat{\Omega}) & :=\left(V^{e}+V_{h}^{e}\right)(\widehat{\Omega}) .
\end{aligned}
$$

Equipamos al espacio suma $W(\widehat{\Omega})$ con la norma $\|\cdot\|_{h}$. Es claro que,

$$
\begin{aligned}
\|v\|_{h} & \simeq\|v\|_{V(\widehat{\Omega})} \quad \forall v \in V(\widehat{\Omega}), \\
\left(W(\widehat{\Omega}),\|\cdot\|_{h}\right) & \hookrightarrow\left(X(\widehat{\Omega}),|\cdot|_{X(\widehat{\Omega})}\right) \quad \text { uniformemente en } h .
\end{aligned}
$$

Definiremos a continuación los operadores que usaremos para analizar la convergencia de las soluciones del problema (4.105) hacia las soluciones del problema continuo (4.100).

Procediendo como en la sección 3.2, definimos

$$
\begin{aligned}
\mathbf{A}: X(\widehat{\Omega}) & \rightarrow V^{e}(\widehat{\Omega}) \subset W(\widehat{\Omega}) \hookrightarrow X(\widehat{\Omega}) \\
x & \mapsto u^{e}=\mathbf{P} \circ \mathbf{T}\left(\left.x\right|_{\Omega}\right), \\
\mathbf{A}_{h}: X(\widehat{\Omega}) & \rightarrow V_{h}^{e}(\widehat{\Omega}) \subset W(\widehat{\Omega}) \hookrightarrow X(\widehat{\Omega}) \\
x & \mapsto u_{h}^{e}=\mathbf{P}_{h} \circ \mathbf{T}_{h}\left(\left.x\right|_{\Omega_{h}}\right) .
\end{aligned}
$$

De acuerdo con los resultados de la sección 3.2, ambos operadores son lineales y están acotados uniformemente en la norma $\|\cdot\|_{h}$.

El próximo paso será probar que se satisfacen las propiedades P1 y P2 sobre los espacios discontinuos $V_{h}\left(\Omega_{h}\right)$.

Sea $\lambda=\frac{1}{\mu}$ un autovalor fijo de $\mathbf{A}$, con multiplicidad algebraica igual a $m$. Sea $\mathbf{E}_{\lambda}(\mathbf{W}(\widehat{\Omega}))$ el espacio propio de $\mathbf{A}$ correspondiente a $\lambda$. 
Teorema 4.52 (P1) Para cada autofunción $u^{e}$ de $\mathbf{A}$ asociada a $\lambda$, existe una constante $C$, estrictamente positiva, tal que

$$
\begin{gathered}
\inf _{v_{h} \in V_{h}\left(\Omega_{h}\right)}\left|u^{e}-v_{h}^{e}\right|_{X(\widehat{\Omega})} \leq C h^{1+s}\|u\|_{0, \Omega}, \\
\inf _{v_{h} \in V_{h}\left(\Omega_{h}\right)}\left\|u^{e}-v_{h}^{e}\right\|_{h} \leq C h^{s}\|u\|_{0, \Omega} .
\end{gathered}
$$

Prueba. Como $u^{e} \in H^{1+s}(\widehat{\Omega}), u^{e} \in \mathcal{C}^{0}(\widehat{\Omega})$. Luego, la interpolada lineal de Lagrange de $\left.u^{e}\right|_{\Omega_{h}}, u_{I}$, estará bien definida. Más aún, $u_{I} \in V_{h}\left(\Omega_{h}\right) \cap H^{1}\left(\Omega_{h}\right)$. Sea $u_{I}^{e}=\mathbf{P}_{h} u_{I}$. Denotando $\omega_{T}$ al conjunto $T^{i d} \backslash T$, tendremos

$$
\left\|u^{e}-u_{I}^{e}\right\|_{0, \widehat{\Omega}}^{2}=\left\|u^{e}-u_{I}\right\|_{0, \Omega_{h}}^{2}+\sum_{\substack{T \in \mathcal{B}_{h} \\ T \subset T^{i d}}}\left\|u-u_{I}^{e}\right\|_{0, \omega_{T}}^{2} .
$$

Para $h$ suficientemente chico, el segundo término en el lado derecho de la expresión anterior estará acotado por el primer término más un término de orden superior. En efecto, usando el Lema D.7 (caso $T \subset T^{i d}$ ) y el Lema D.9 (ya que $u_{I}^{e}$ es lineal a trozos) tendremos

$$
\begin{aligned}
\left\|u-u_{I}^{e}\right\|_{0, \omega_{T}}^{2} & \leq C\left(\left\|u-u_{I}\right\|_{0, T}+h_{T}^{2}\left\|u-u_{I}^{e}\right\|_{1, T^{i d}}\right)^{2} \\
& \leq C\left(\left\|u-u_{I}\right\|_{0, T}^{2}+h_{T}^{4}\left\|u-u_{I}^{e}\right\|_{0, \omega_{T}}^{2}+h_{T}^{4}|u|_{1, T^{i d}}^{2}+h_{T}^{4}\left|u_{I}^{e}\right|_{1, T^{i d}}^{2}\right) \\
& \leq C\left(\left\|u-u_{I}\right\|_{0, T}^{2}+h_{T}^{4}\left\|u-u_{I}^{e}\right\|_{0, \omega_{T}}^{2}+h_{T}^{4}|u|_{1, T^{i d}}^{2}+h_{T}^{4}\left|u_{I}\right|_{1, T}^{2}\right) \\
& \leq C\left(\left\|u-u_{I}\right\|_{0, T}^{2}+h_{T}^{4}\left\|u-u_{I}^{e}\right\|_{0, \omega_{T}}^{2}+h_{T}^{4}|u|_{1, T^{i d}}^{2}+h_{T}^{4}\left(\left|u-u_{I}\right|_{1, T}^{2}+|u|_{1, T}^{2}\right)\right) .
\end{aligned}
$$

Es claro que, para $h_{T}$ suficientemente chico, se obtiene

$$
\left\|u-u_{I}^{e}\right\|_{0, \omega_{T}}^{2} \leq C\left(\left\|u-u_{I}\right\|_{0, T}^{2}+h_{T}^{4}\left|u-u_{I}\right|_{1, T}^{2}+h_{T}^{4}|u|_{1, T^{i d}}^{2}\right) .
$$

Sumando sobre todos los triángulos en el borde tales que $T \subset T^{i d}$ y usando resultados de interpolación standard, concluimos que

$$
\left\|u^{e}-u_{I}^{e}\right\|_{0, \widehat{\Omega}}^{2} \leq C h^{1+s}\|u\|_{1+s, \Omega} .
$$

Pasaremos ahora a analizar la aproximación en la norma $\|\cdot\|_{h}$. Observemos que, gracias al resultado recién obtenido para la norma $\|\cdot\|_{0, \widehat{\Omega}}$ y a la definición de la norma $\|\cdot\|_{h}$, solo nos resta acotar los siguientes términos:

$$
\left|u^{e}-u_{I}\right|_{1, \Omega_{h}}^{2}+\sum_{\substack{T \in \mathcal{B}_{h} \\ T \subset T^{i d}}}\left|u-u_{I}^{e}\right|_{1, \omega_{T}}^{2} .
$$

Procediendo como en el caso anterior; es decir, usando los Lemas D.7 (caso $T \subset T^{\text {id }}$ ) y D.9, obtendremos

$$
\begin{aligned}
\left|u-u_{I}^{e}\right|_{1, \omega_{T}}^{2} & \leq C\left(\left\|\nabla\left(u-u_{I}\right)\right\|_{0, T}+h_{T}^{2 s}\left\|\nabla\left(u-u_{I}^{e}\right)\right\|_{s, T^{i d}}\right)^{2} \\
& \leq C\left(\left\|\nabla\left(u-u_{I}\right)\right\|_{0, T}^{2}+h_{T}^{4 s}\left\|\nabla\left(u-u_{I}^{e}\right)\right\|_{s, T^{i d}}^{2}\right) \\
& \leq C\left(\left\|\nabla\left(u-u_{I}\right)\right\|_{0, T}^{2}+h_{T}^{4 s}\left(\|\nabla u\|_{s, T^{i d}}^{2}+\left\|\nabla u_{I}\right\|_{0, T}^{2}\right)\right) \\
& \leq C\left(\left\|\nabla\left(u-u_{I}\right)\right\|_{0, T}^{2}+h_{T}^{4 s}\left(\|\nabla u\|_{s, T^{i d}}^{2}+\left\|\nabla\left(u-u_{I}\right)\right\|_{0, T}^{2}+\|\nabla u\|_{0, T}^{2}\right)\right) .
\end{aligned}
$$

Luego, combinando estas desigualdades, podremos escribir

$$
\left|u-u_{I}^{e}\right|_{1, \omega_{T}}^{2} \leq C\left(\left|u-u_{I}\right|_{1, T}^{2}+h_{T}^{4 s}\|\nabla u\|_{s, T^{i d}}^{2}\right) .
$$

Reemplazando esta expresión en la ecuación (4.108), sumando sobre todos los triángulos en el borde tales que $T \subset T^{\text {id }}$ y usando resultados de interpolación standard, tendremos

$$
\left\|u^{e}-u_{I}^{e}\right\|_{h}^{2} \leq C\left(\left\|u^{e}-u_{I}^{e}\right\|_{0, \widehat{\Omega}}^{2}+\left|u^{e}-u_{I}\right|_{1, \Omega_{h}}^{2}+h^{4 s}\|u\|_{1+s, \Omega}^{2}\right) \leq C h^{2 s}\|u\|_{1+s, \Omega}^{2} .
$$

Terminamos la prueba, invocando la estimación (4.104), eligiendo $v_{h}=u_{I}$ y tomando el ínfimo con respecto a $v_{h} \in V_{h}\left(\Omega_{h}\right)$. 
Teorema 4.53 (P2) Para todo $f \in V_{h}\left(\Omega_{h}\right)$, existe una constante positiva $C$, tal que

$$
\left\|\left(\mathbf{A}-\mathbf{A}_{h}\right) f^{e}\right\|_{h} \leq C h^{s}\|f\|_{0, \widehat{\Omega}}
$$

Prueba. Dada $f \in V_{h}\left(\Omega_{h}\right)$, sean $u^{e}=\mathbf{A} f^{e}$ y $u_{h}^{e}=\mathbf{A}_{h} f^{e}$. Entonces,

$$
\left\|u^{e}-u_{h}^{e}\right\|_{h}^{2}=\left\|u^{e}-u_{h}\right\|_{h, \Omega_{h}}^{2}+\sum_{\substack{T \in \mathcal{B}_{h} \\ T \subset T^{i d}}}\left\|u-u_{h}^{e}\right\|_{1, \omega_{T}}^{2}
$$

Procediendo exactamente igual a como procedimos en la prueba del teorema anterior, obtendremos la siguiente estimación, válida para $h$ suficientemente chico,

$$
\sum_{\substack{T \in \mathcal{B}_{h} \\ T \subset T^{i d}}}\left\|u-u_{h}^{e}\right\|_{1, \omega_{T}}^{2} \leq C\left(\left\|u^{e}-u_{h}\right\|_{h, \Omega_{h}}^{2}+h^{4 s}\|u\|_{1+s, \Omega}^{2}\right) .
$$

Sea $u_{I}$ la interpolada lineal de Lagrange de $\left.u^{e}\right|_{\Omega_{h}}$. A partir del Lema 4.50, tendremos

$$
c_{2}\left\|u_{I}-u_{h}\right\|_{h, \Omega_{h}}^{2} \leq a_{h}\left(u_{I}-u_{h}, u_{I}-u_{h}\right)=a_{h}\left(u_{I}-u^{e}, u_{I}-u_{h}\right)+a_{h}\left(u^{e}-u_{h}, u_{I}-u_{h}\right) .
$$

Ahora, como $u_{I}-u^{e}$ es una función continua sobre $\Omega_{h}$, la definición de $a_{h}$ nos conduce directamente a

$$
\left|a_{h}\left(u_{I}-u^{e}, u_{I}-u_{h}\right)\right| \leq \sum_{T \in \mathcal{T}_{h}}\left\|u_{I}-u^{e}\right\|_{1, T}\left\|u_{I}-u_{h}\right\|_{1, T}+\left|\sum_{\ell \in \mathcal{E}_{h}^{I}} \int_{\ell}\left\{\nabla\left(u_{I}-u^{e}\right)\right\} \cdot\left[\left[u_{I}-u_{h}\right]\right]\right| .
$$

Teniendo en cuenta que $u_{I}-\left.u^{e}\right|_{T} \in H^{1+s}(T)$, podremos aplicar una desigualdad de trazas local para obtener

$$
\left\|\nabla\left(u_{I}-u^{e}\right)\right\|_{0, \ell} \leq C\left(h_{\ell}^{-1}\left\|\nabla\left(u_{I}-u^{e}\right)\right\|_{0, T}^{2}+h_{\ell}^{2 s-1}\left|\nabla\left(u_{I}-u^{e}\right)\right|_{s, T}^{2}\right)^{1 / 2} .
$$

A partir de esta estimación, tendremos

$$
\left|\sum_{\ell \in \mathcal{E}_{h}^{I}} \int_{\ell} \nabla\left(u_{I}-u^{e}\right) \cdot\left[\left[u_{I}-u_{h}\right]\right]\right| \leq C\left(\left\|\nabla\left(u_{I}-u^{e}\right)\right\|_{0, \Omega_{h}}+h^{s}\|u\|_{1+s, \Omega}\right)\left(\sum_{\ell \in \mathcal{E}_{h}^{I}} h_{\ell}^{-1}\left\|\left[\left[u_{I}-u_{h}\right]\right]\right\|_{0, \ell}^{2}\right)^{1 / 2} .
$$

Luego, usando resultados de interpolación standard,

$$
\left|a_{h}\left(u_{I}-u^{e}, u_{I}-u_{h}\right)\right| \leq C h^{s}\|u\|_{1+s, \Omega}\left\|u_{I}-u_{h}\right\|_{h, \Omega_{h}} .
$$

Combinando todas las estimaciones anteriores y usando la estimación (4.103), obtendremos finalmente

$$
\left\|u_{I}-u_{h}\right\|_{h, \Omega_{h}} \leq C\left(h^{s}\left\|f^{e}\right\|_{0, \Omega}+\sup _{w_{h} \in V_{h}\left(\Omega_{h}\right)} \frac{a_{h}\left(u^{e}-u_{h}, w_{h}\right)}{\left\|w_{h}\right\|_{h, \Omega_{h}}}\right)
$$

En lo que sigue, acotaremos el término de consistencia restante. Observemos que este término aparece únicamente debido a la discrepancia entre $\Omega$ y $\Omega_{h}$ (ver, e.g., [57]).

Observemos primero que $u$, solución del problema variacional (4.106), también es solución del siguiente problema fuerte:

$$
\begin{array}{rlrl}
-\Delta u+u & =f^{e} & \text { en } \Omega, \\
\frac{\partial u}{\partial \mathbf{n}} & =0 & & \text { sobre } \Gamma .
\end{array}
$$

Sea $\omega_{T}$ el conjunto definido por $T \backslash T^{i d}$ o $T^{i d} \backslash T$, según corresponda. Usando la definición de $a_{h}$, la definición del problema discreto (4.106) e integrando por partes, tendremos 


$$
\begin{aligned}
& a_{h}\left(u^{e}-u_{h}, w_{h}\right)=a_{h}\left(u^{e}, w_{h}\right)-a_{h}\left(u_{h}, w_{h}\right) \\
& =\sum_{T \in \mathcal{T}_{h}} \int_{T} \nabla u^{e} \cdot \nabla w_{h}+\sum_{T \in \mathcal{T}_{h}} \int_{T} u^{e} w_{h}-\sum_{\ell \in \mathcal{E}_{h}^{I}} \int_{\ell}\{\nabla u\} \cdot\left[\left[w_{h}\right]\right]-\int_{\Omega_{h}} f w_{h} \\
& =\sum_{T \in \mathcal{T}_{h}} \int_{T^{i d}} \nabla u \cdot \nabla w_{h}^{e}+\sum_{T \in \mathcal{T}_{h}} \int_{T^{i d}} u w_{h}^{e}-\sum_{\ell \in \mathcal{E}_{h}^{I}} \int_{\ell}\{\nabla u\} \cdot\left[\left[w_{h}\right]\right]-\int_{\Omega_{h}} f w_{h} \\
& +\sum_{\substack{T \in \mathcal{B}_{h} \\
T^{i d} \subset T}} \int_{\omega_{T}}\left(\nabla u^{e} \cdot \nabla w_{h}+u^{e} w_{h}\right)-\sum_{\substack{T \in \mathcal{B}_{h} \\
T \subset T^{i d}}} \int_{\omega_{T}}\left(\nabla u \cdot \nabla w_{h}^{e}+u w_{h}^{e}\right) \\
& =\sum_{T \in \mathcal{T}_{h}}^{T^{i d} \subset T}\left(\int_{T^{i d}}(-\triangle u+u) w_{h}^{e}+\int_{\partial T^{i d}} \frac{T \subset T^{i d}}{\partial \mathbf{n}} w_{h}^{e}\right)-\sum_{\ell \in \mathcal{E}_{h}^{I}} \int_{\ell}\{\nabla u\} \cdot\left[\left[w_{h}\right]\right]-\int_{\Omega_{h}} f w_{h} \\
& +\sum_{\substack{T \in \mathcal{B}_{h} \\
T^{i d} \subset T}} \int_{\omega_{T}}\left(\nabla u^{e} \cdot \nabla w_{h}+u^{e} w_{h}\right)-\sum_{\substack{T \in \mathcal{B}_{h} \\
T \subset T^{i d}}} \int_{\omega_{T}}\left(\nabla u \cdot \nabla w_{h}^{e}+u w_{h}^{e}\right) \\
& =\int_{\Omega}^{T^{i d} \subset} f^{e} w_{h}^{e}+\sum_{T \in \mathcal{T}_{h}} \int_{\partial T^{i d}} \frac{\partial u}{\partial \mathbf{n}} w_{h}^{e}-\sum_{\ell \in \mathcal{E}_{h}^{I}}^{T \subset T^{i d}} \int_{\ell}\{\nabla u\} \cdot\left[\left[w_{h}\right]\right]-\int_{\Omega_{h}} f w_{h} \\
& +\sum_{\substack{T \in \mathcal{B}_{h} \\
T^{i d} \subset T}} \int_{\omega_{T}}\left(\nabla u^{e} \cdot \nabla w_{h}+u^{e} w_{h}\right)-\sum_{\substack{T \in \mathcal{B}_{h} \\
T \subset T^{i d}}} \int_{\omega_{T}}\left(\nabla u \cdot \nabla w_{h}^{e}+u w_{h}^{e}\right),
\end{aligned}
$$

No es difícil probar (seguiremos aquí los argumentos en [57]) que

$$
\sum_{T \in \mathcal{T}_{h}} \int_{\partial T^{i d}} \frac{\partial u}{\partial \mathbf{n}} w_{h}^{e}-\sum_{\ell \in \mathcal{E}_{h}^{I}} \int_{\ell}\{\nabla u\} \cdot\left[\left[w_{h}\right]\right]=0 .
$$

En efecto, la regularidad de $u\left(u \in H^{1}(\Omega)\right.$ y $\left.\triangle u \in L^{2}(\Omega)\right)$ implica que $\nabla u \cdot \mathbf{n}$ es continua a través de cada lado interior de la partición. Además, $\nabla u \cdot \mathbf{n}=0$ sobre cada lado curvo incluido en $\Gamma$. Dado $\ell \in \mathcal{E}_{h}^{I}$, denotaremos por $T_{1}^{\ell}$ y $T_{2}^{\ell}$ a los elementos de la partición que comparten a $\ell$; es decir, $\ell=\partial T_{1}^{\ell} \cap \partial T_{2}^{\ell}$. Un cálculo directo nos conduce a

$$
\sum_{T \in \mathcal{T}_{h}} \int_{\partial T^{i d}} \frac{\partial u}{\partial \mathbf{n}} w_{h}^{e}=\sum_{\ell \in \mathcal{E}_{h}^{I}} \int_{\ell} \nabla u \cdot \mathbf{n}\left(\left.w_{h}\right|_{T_{2}^{\ell}}-\left.w_{h}\right|_{T_{1}^{\ell}}\right)=\sum_{\ell \in \mathcal{E}_{h}^{I}} \int_{\ell}\{\nabla u\} \cdot\left[\left[w_{h}\right]\right] .
$$

Como consecuencia de estos resultados, solo faltará acotar los siguientes términos para completar la prueba del teorema:

$$
\sum_{\substack{T \in \mathcal{B}_{h} \\ T^{i d} \subset T}} \int_{\omega_{T}}\left(\nabla u^{e} \cdot \nabla w_{h}+u^{e} w_{h}-f w_{h}\right)-\sum_{\substack{T \in \mathcal{B}_{h} \\ T \subset T^{i d}}} \int_{\omega_{T}}\left(\nabla u \cdot \nabla w_{h}^{e}+u w_{h}^{e}-f^{e} w_{h}^{e}\right) .
$$

A partir de la desigualdad de Cauchy-Schwarz, la definición de la norma $\|\cdot\|_{h}$ y usando el Lema D.9, tendremos

$$
\begin{aligned}
& \mathrm{I}=\left|\sum_{\substack{T \in \mathcal{B}_{h} \\
T^{i d} \subset T}} \int_{\omega_{T}} \nabla u^{e} \cdot \nabla w_{h}-\sum_{\substack{T \in \mathcal{B}_{h} \\
T \subset T^{i d}}} \int_{\omega_{T}} \nabla u \cdot \nabla w_{h}^{e}\right| \leq\left(\left\|\nabla u^{e}\right\|_{0, \Omega_{h} \backslash \Omega}+\|\nabla u\|_{0, \Omega \backslash \Omega_{h}}\right)\left\|w_{h}\right\|_{h, \Omega_{h}}, \\
& \mathrm{II}=\left|\sum_{\substack{T \in \mathcal{B}_{h} \\
T^{i d} \subset T}} \int_{\omega_{T}} u^{e} w_{h}-\sum_{\substack{T \in \mathcal{B}_{h} \\
T \subset T^{d}}} \int_{\omega_{T}} u w_{h}^{e}\right| \leq\left(\left\|u^{e}\right\|_{0, \Omega_{h} \backslash \Omega}+\|u\|_{0, \Omega \backslash \Omega_{h}}\right)\left\|w_{h}\right\|_{0, \Omega_{h}},
\end{aligned}
$$


$\mathrm{III}=\left|\sum_{\substack{T \in \mathcal{B}_{h} \\ T^{i d} \subset T}} \int_{\omega_{T}} f w_{h}-\sum_{\substack{T \in \mathcal{B}_{h} \\ T \subset T^{i d}}} \int_{\omega_{T}} f^{e} w_{h}^{e}\right| \leq\|f\|_{0, \Omega_{h}}\left(\left\|w_{h}\right\|_{0, \Omega_{h} \backslash \Omega}+\left\|w_{h}^{e}\right\|_{0, \Omega \backslash \Omega_{h}}\right)$.

Usando el Lemma D.6 y las estimaciones (D.4) y (4.103), obtendremos

$$
I+I I \leq C h^{s}\|u\|_{1+s, \Omega}\left\|w_{h}\right\|_{h, \Omega_{h}} \leq C h^{s}\left\|f^{e}\right\|_{0, \Omega}\left\|w_{h}\right\|_{h, \Omega_{h}} .
$$

Para acotar el último término, usaremos el operador de post-procesamiento (definido en la sección 5.1) $\mathbf{R}_{h}: V_{h}\left(\Omega_{h}\right) \rightarrow V_{h}^{c}\left(\Omega_{h}\right) \subset H^{1}\left(\Omega_{h}\right)$. Sea $z_{h}=\mathbf{R}_{h} w_{h}$. Entonces, usando los Lemas D.6 y 5.2 , tendremos

$$
\left\|w_{h}\right\|_{0, \Omega_{h} \backslash \Omega}^{2} \leq 2\left(\left\|w_{h}-z_{h}\right\|_{0, \Omega_{h} \backslash \Omega}^{2}+\left\|z_{h}\right\|_{0, \Omega_{h} \backslash \Omega}^{2}\right) \leq C\left(\left\|w_{h}-z_{h}\right\|_{0, \Omega_{h}}^{2}+h^{2}\left\|z_{h}\right\|_{1, \Omega_{h}}^{2}\right) \leq C h^{2}\left\|w_{h}\right\|_{h, \Omega_{h}}^{2} .
$$

De manera análoga, usando los Lemas D.6, D.9 y 5.2,

$$
\begin{aligned}
\left\|w_{h}^{e}\right\|_{0, \Omega \backslash \Omega_{h}}^{2} & \leq 2\left(\left\|w_{h}^{e}-z_{h}^{e}\right\|_{0, \Omega \backslash \Omega_{h}}^{2}+\left\|z_{h}^{e}\right\|_{0, \Omega \backslash \Omega_{h}}^{2}\right) \leq C\left(\left\|w_{h}^{e}-z_{h}^{e}\right\|_{0, \Omega}^{2}+h^{2}\left\|z_{h}^{e}\right\|_{1, \Omega}^{2}\right) \\
& \leq C\left(\left\|w_{h}-z_{h}\right\|_{0, \Omega_{h}}^{2}+h^{2}\left\|z_{h}\right\|_{1, \Omega_{h}}^{2}\right) \leq C h^{2}\left\|w_{h}\right\|_{h, \Omega_{h}}^{2} .
\end{aligned}
$$

Así, llegamos al siguiente resultado:

$$
I I I \leq C h\|f\|_{0, \Omega_{h}}\left\|w_{h}\right\|_{h, \Omega_{h}} .
$$

Finalmente, colectando las estimaciones anteriores, tendremos

$$
\frac{\left|a_{h}\left(u^{e}-u_{h}, w_{h}\right)\right|}{\left\|w_{h}\right\|_{h, \Omega_{h}}} \leq C h^{s}\|f\|_{0, \Omega_{h}}
$$

resultado que nos permite concluir la prueba.

De acuerdo con los resultados de las secciones 3.3.1-3.3.2, podemos asegurar que para cada autovalor aislado $\lambda$ de $\mathbf{A}$, con multiplicidad igual a $m$, existen exactamente $m$ autovalores $\lambda_{1 h}, \cdots, \lambda_{m h}$ (repetidos tantas veces lo indiquen sus respectivas multiplicidades) de $\mathbf{A}_{h}$ que convergen a $\lambda$ cuando $h$ tiende a cero. Más aún, el método converge libre de modos espurios.

Para establecer el orden con el que convergen los pares autofunción/autovalor del problema aproximado, seguiremos la linea argumental de la sección 3.3.3.

Consideremos el subespacio de $W(\widehat{\Omega})$ definido por

$$
W_{\lambda}(\widehat{\Omega}):=\mathbf{E}_{\lambda}(W(\widehat{\Omega}))+V_{h}^{e}(\widehat{\Omega})
$$

y lo equipamos con la siguiente norma

$$
\|\cdot\|_{*}^{2}:=\|\cdot\|_{h}^{2}+\sum_{\substack{T \in \mathcal{T}_{h} \\ T \notin \mathcal{B}_{h}}} h_{T}^{2 s}|\cdot|_{1+s, T}^{2}+\sum_{\substack{T \in \mathcal{B}_{h} \\ T^{i d} \subset T}} h_{T}^{2 s}|\cdot|_{1+s, T}^{2}+\sum_{\substack{T \in \mathcal{B}_{h} \\ T \subset T^{i d}}} h_{T}^{2 s}|\cdot|_{1+s, T^{i d}}^{2} .
$$

Restringida a funciones $v_{h} \in V_{h}^{e}\left(\Omega_{h}\right)$, esta norma es equivalente a la norma $\|\cdot\|_{h}$ gracias a las propiedades del operador $\mathbf{P}_{h}$. Lo mismo sucede para funciones $v \in \mathbf{E}_{\lambda}(W(\widehat{\Omega}))$. Observemos que, en este caso, las propiedades de $\mathbf{P}$ están basadas en la estimación (4.104).

Una propiedad importante de la norma $\|\cdot\|_{*}$ (puede probarse siguiendo los argumentos en [19]) es la siguiente:

$$
a_{h}\left(\left.u\right|_{\Omega_{h}},\left.v\right|_{\Omega_{h}}\right) \leq C\|u\|_{*, \Omega_{h}}\|v\|_{*, \Omega_{h}} \quad \forall u, v \in W_{\lambda}(\widehat{\Omega}),
$$

donde $C$ es una constante positiva que solo depende de la regularidad de $\mathcal{T}_{h}$.

Teorema 4.54 (P3) Para cada autofunción $u^{e}$ de $\mathbf{A}$ asociada a $\lambda$, existe una constante $C$, estrictamente positiva, tal que

$$
\inf _{v_{h} \in V_{h}\left(\Omega_{h}\right)}\left\|u^{e}-v_{h}^{e}\right\|_{*} \leq C h^{s}\|u\|_{0, \Omega} .
$$


Prueba. Argumentando como en la prueba del Teorema 4.52, sabemos que $u_{I}$, la interpolada lineal de Lagrange de $\left.u^{e}\right|_{\Omega_{h}}$, está bien definida. Sea $\omega_{T}=T^{i d} \backslash T$. A partir de la definición de la norma $\|\cdot\|_{*}$, teniendo en cuenta que $\left.\nabla u_{I}\right|_{T} \in \mathcal{P}_{0}(T)$ y las propiedades del operador $\mathbf{P}_{h}$,

$$
\begin{aligned}
\left\|u^{e}-u_{I}^{e}\right\|_{*}^{2} & =\left\|u^{e}-u_{I}^{e}\right\|_{h}^{2}+\sum_{T \in \mathcal{T}_{h}} h_{T}^{2 s}\left|u^{e}-u_{I}\right|_{1+s, T}^{2}+\sum_{\substack{T \in \mathcal{B}_{h} \\
T \subset T^{i d}}} h_{T}^{2 s}\left|u-u_{I}^{e}\right|_{1+s, \omega_{T}}^{2} \\
& \leq C\left(\left\|u^{e}-u_{I}^{e}\right\|_{h}^{2}+h^{2 s}\left|u^{e}\right|_{1+s, \widehat{\Omega}}^{2}\right) .
\end{aligned}
$$

Terminamos la prueba aplicando los Teoremas 4.52 y D.4 y la estimación (4.104).

Teorema 4.55 (P4) Existe una constante positiva $C$ tal que

$$
\left\|\left(\mathbf{A}-\mathbf{A}_{h}\right) u^{e}\right\|_{*} \leq C h^{s}\|u\|_{0, \Omega} \quad \forall u^{e} \in \mathbf{E}_{\lambda}(W(\widehat{\Omega})) .
$$

Prueba. Para $u^{e} \in \mathbf{E}_{\lambda}(W(\widehat{\Omega}))$, la solución $w^{e}=\mathbf{A} u^{e} \in H^{1+s}(\widehat{\Omega})$. Por lo tanto, podemos proceder como en la prueba del Teorema 4.53, con $f^{e}$ sustituida por $u^{e}$, para obtener

$$
\left\|\left(\mathbf{A}-\mathbf{A}_{h}\right) u^{e}\right\|_{h} \leq C h^{s}\|u\|_{0, \Omega}
$$

Sea $\omega_{T}$ el conjunto $T^{i d} \backslash T$. A partir de la definición de la norma $\|\cdot\|_{*}$, tendremos

$$
\left\|\left(\mathbf{A}-\mathbf{A}_{h}\right) u^{e}\right\|_{*}^{2}=\left\|\left(\mathbf{A}-\mathbf{A}_{h}\right) u^{e}\right\|_{h}^{2}+\sum_{T \in \mathcal{T}_{h}} h_{T}^{2 s}\left|\left(\mathbf{A}-\mathbf{A}_{h}\right) u^{e}\right|_{1+s, T}^{2}+\sum_{\substack{T \in \mathcal{B}_{h} \\ T \subset T^{i d}}} h_{T}^{2 s}\left|\left(\mathbf{A}-\mathbf{A}_{h}\right) u^{e}\right|_{1+s, \omega_{T}}^{2} .
$$

Teniendo en cuenta que $\mathbf{A}_{h} u^{e} \in V_{h}^{e}\left(\Omega_{h}\right)$, los mismos argumentos que los utilizados en la prueba del Teorema 4.54 nos conducen directamente a las siguientes estimaciones

$$
\begin{gathered}
\sum_{T \in \mathcal{T}_{h}}\left|\left(\mathbf{A}-\mathbf{A}_{h}\right) u^{e}\right|_{1+s, T}^{2}+\sum_{\substack{T \in \mathcal{B}_{h} \\
T \subset T^{i d}}}\left|\left(\mathbf{A}-\mathbf{A}_{h}\right) u^{e}\right|_{1+s, \omega_{T}}^{2} \leq\left|\mathbf{A} u^{e}\right|_{1+s, \widehat{\Omega}}^{2}, \\
\left|\mathbf{A} u^{e}\right|_{1+s, \widehat{\Omega}} \leq C\|\mathbf{A} u\|_{1+s, \Omega} \leq C(\lambda)\|u\|_{0, \Omega} .
\end{gathered}
$$

Concluiremos la prueba combinando las estimaciones anteriores.

Teorema 4.56 (P5) Para toda autofunción u de $\mathbf{A}$ asociada a $\lambda$ existe una constante $C$, estrictamente positiva, tal que

$$
\|u\|_{h, \Omega \backslash \Omega_{h}}+\|u\|_{h, \Omega_{h} \backslash \Omega}+\|u\|_{*, \Omega_{h} \backslash \Omega} \leq C h^{s}\|u\|_{0, \Omega} .
$$

Prueba. Es consecuencia directa de la regularidad de las autofunciones del operador A. En efecto, a partir del Lema D.6 y de la estimación (4.104), tendremos

$$
\|u\|_{h, \Omega \backslash \Omega_{h}}=\|u\|_{1, \Omega \backslash \Omega_{h}} \leq C h^{s}\|u\|_{1+s, \Omega} \leq C h^{s}\|u\|_{0, \Omega} .
$$

Procediendo de manera análoga, usando el Teorema D.4 y la estimación (4.104),

$$
\|u\|_{h, \Omega_{h} \backslash \Omega} \leq C h^{s}\|u\|_{0, \Omega} .
$$

Finalmente, sea $\omega_{T}=T \backslash T^{i d}$. Usando nuevamente las propiedades de la extensión (ver Teorema D.4), es inmediato que

$$
\left.\left|\sum_{\substack{T \in \mathcal{B}_{h} \\ T^{i d} \subset T}} h_{T}^{2 s}\right| u\right|_{1+s, \omega_{T}} ^{2} \mid \leq C h^{2 s}\|u\|_{1+s, \Omega}^{2} .
$$

A partir de estos resultados y de la definición de la norma $\|\cdot\|_{*}$, es claro que

$$
\|u\|_{*, \Omega_{h} \backslash \Omega} \leq C h^{s}\|u\|_{0, \Omega}
$$

Así, concluimos la prueba.

De acuerdo con los resultados de la sección 3.3.3, podemos asegurar que existen constantes positivas $C$ y $h_{0}$ tales que, si $h<h_{0}$, entonces 


$$
\begin{aligned}
& \text { para cada } u_{h} \in \mathbf{E}_{\lambda_{h}}\left(V_{h}^{e}(\widehat{\Omega})\right), \operatorname{dist}\left(u_{h}, \mathbf{E}_{\lambda}(W(\widehat{\Omega}))\right) \leq C h^{s}\left\|u_{h}\right\|_{h}, \\
& \text { para cada } u \in \mathbf{E}_{\lambda}(W(\widehat{\Omega})), \operatorname{dist}\left(u, \mathbf{E}_{\lambda_{h}}\left(V_{h}^{e}(\widehat{\Omega})\right)\right) \leq C h^{s}\|u\|_{h},
\end{aligned}
$$

con la distancia siendo medida en la norma $\|\cdot\|_{h}$.

Para establecer el orden con el que convergen los autovalores necesitamos estimar los siguientes términos de consistencia

$$
\begin{gathered}
M_{h}=\sup _{\substack{\left.x \in \mathbf{E}_{\lambda}(W(\widehat{\Omega}))\right) \\
\|x\|_{h}=1}} \sup _{\substack{y \in \mathbf{E}_{\lambda}(W(\widehat{\Omega})) \\
\|y\|_{h}=1}}\left|a_{h}\left(\mathbf{A} x, \Pi_{h} y-y\right)-b_{h}\left(x, \Pi_{h} y-y\right)\right|, \\
N_{h}=\sup _{\substack{x \in \mathbf{E}_{\lambda}(W(\widehat{\Omega})) \\
\|x\|_{h}=1}} \sup _{\substack{y \in \mathbf{E}_{\lambda}(W(\widehat{\Omega})) \\
\|y\|_{h}=1}}\left|a_{h}(\mathbf{A} x, y)-b_{h}(x, y)\right|,
\end{gathered}
$$

donde $\Pi_{h}$ es el operador de proyección definido por

$$
\left\{\begin{array}{l}
\Pi_{h} x=\mathbf{P}_{h} x_{h} \\
a_{h}\left(x-x_{h}, y_{h}\right)=0 \quad \forall y_{h} \in V_{h}^{e}\left(\Omega_{h}\right) .
\end{array}\right.
$$

Lema 4.57 Existe una constante $C$, estrictamente positiva, tal que

$$
M_{h} \leq C h^{2 s} \text {. }
$$

Prueba. Sea $x \in \mathbf{E}_{\lambda}(W(\widehat{\Omega}))$ con $\|x\|_{h}=1$. Sea $u=\mathbf{T} x$. Sabemos que $u \in H^{1+s}(\Omega)$ y, además,

$$
\left\|u^{e}\right\|_{1+s, \widehat{\Omega}} \leq C\|u\|_{1+s, \Omega} \leq C\|x\|_{0, \Omega} \leq C .
$$

Testeando (4.101) con funciones suficientemente suaves, concluiremos que $u$ es solución del siguiente problema fuerte:

$$
\begin{array}{rlrl}
-\triangle u+u & =x & \text { en } \Omega, \\
\frac{\partial u}{\partial \mathbf{n}} & =0 & & \text { sobre } \Gamma .
\end{array}
$$

Sea $y \in \mathbf{E}_{\lambda}(W(\widehat{\Omega}))$ con $\|y\|_{h}=1$. Sea $y_{h}=\Pi_{h} y$. Claramente, $\widehat{y}=y-y_{h} \in W_{\lambda}(\widehat{\Omega})$. Multiplicando la ecuación (4.110) por $\widehat{y}$ e integrando por partes, tendremos

$$
\sum_{T \in \mathcal{T}_{h}}\left(\int_{T^{i d}} \nabla u \cdot \nabla \widehat{y}-\int_{\partial T^{i d}} \frac{\partial u}{\partial \mathbf{n}} \widehat{y}\right)+\int_{\Omega} u \widehat{y}=\int_{\Omega} x \widehat{y}
$$

Denotemos por $\omega_{T}$ al conjunto $T^{i d} \backslash T$ o $T \backslash T^{i d}$, según corresponda. Usando (4.109) y teniendo en cuenta que $u$ es una función continua sobre $\Omega$, la expresión anterior también puede escribirse de la siguiente manera

$$
a_{h}\left(u^{e}, \widehat{y}\right)+\sum_{\substack{T \in \mathcal{B}_{h} \\ T \subset T^{d i}}} \int_{\omega_{T}}(\nabla u \cdot \nabla \widehat{y}+u \widehat{y})-\sum_{\substack{T \in \mathcal{B}_{h} \\ T^{i d} \subset T}} \int_{\omega_{T}}\left(\nabla u^{e} \cdot \nabla \widehat{y}+u^{e} \widehat{y}\right)=\int_{\Omega} x \widehat{y} .
$$

A partir de la ecuación anterior, es inmediato que

$$
a_{h}\left(u^{e}, \widehat{y}\right)-b_{h}(x, \widehat{y})=\sum_{\substack{T \in \mathcal{B}_{h} \\ T \subset T^{i d}}} \int_{\omega_{T}}(x \widehat{y}-\nabla u \cdot \nabla \widehat{y}-u \widehat{y})-\sum_{\substack{T \in \mathcal{B}_{h} \\ T^{i d} \subset T}} \int_{\omega_{T}}\left(x \widehat{y}-\nabla u^{e} \cdot \nabla \widehat{y}-u^{e} \widehat{y}\right) .
$$

Usando la desigualdad de Cauchy-Schwarz, las propiedades de la extensión (ver Teorema D.4) y que $x \in \mathbf{E}_{\lambda}(W(\widehat{\Omega}))$, tendremos

$$
\begin{aligned}
& \left|\int_{\Omega \backslash \Omega_{h}} x \widehat{y}-\int_{\Omega_{h} \backslash \Omega} x \widehat{y}\right| \leq C h^{1+s}\|x\|_{1+s, \widehat{\Omega}}\|\widehat{y}\|_{0, \widehat{\Omega}} \leq C h^{1+s}\|x\|_{0, \widehat{\Omega}}\|\widehat{y}\|_{0, \widehat{\Omega}}, \\
& \left|\int_{\Omega \backslash \Omega_{h}} u \widehat{y}-\int_{\Omega_{h} \backslash \Omega} u^{e} \widehat{y}\right| \leq C h^{1+s}\left\|u^{e}\right\|_{1+s, \widehat{\Omega}}\|\widehat{y}\|_{0, \widehat{\Omega}} \leq C h^{1+s}\|x\|_{0, \widehat{\Omega}}\|\widehat{y}\|_{0, \widehat{\Omega}},
\end{aligned}
$$




$$
\left|\sum_{\substack{T \in \mathcal{B}_{h} \\ T \subset T^{i d}}} \int_{\omega_{T}} \nabla u \cdot \nabla \widehat{y}-\sum_{\substack{T \in \mathcal{B}_{h} \\ T^{i d} \subset T}} \int_{\omega_{T}} \nabla u^{e} \cdot \nabla \widehat{y}\right| \leq C h^{s}\left\|u^{e}\right\|_{1+s, \widehat{\Omega}}\|\widehat{y}\|_{h} .
$$

Finalmente, del Lema 3.21,

$$
\|\widehat{y}\|_{h}=\left\|\left(\mathbf{I}-\Pi_{h}\right) y\right\|_{h} \leq\left\|\left(\mathbf{I}-\Pi_{h}\right) y\right\|_{*} \leq C \gamma_{1 h}\|y\|_{h} \leq C h^{s},
$$

estimación que nos permite concluir la prueba.

Lema 4.58 Existe una constante $C$, estrictamente positiva, tal que

$$
N_{h} \leq C h^{2 s} .
$$

Prueba. Es igual a la prueba del lema anterior, cambiando $\widehat{y}$ por $y \in H^{1+s}(\widehat{\Omega})$.

De acuerdo con los resultados de la sección 3.3.4, podemos asegurar que existen constantes positivas $C$ y $h_{0}$ tales que, si $h<h_{0}$,

$$
\left|\lambda-\lambda_{i h}\right| \leq C h^{2 s} \quad i=1, \cdots, m .
$$

\section{Estimaciones de orden superior}

En esta sección probaremos estimaciones del error en la aproximación DG de las funciones propias del operador A en la norma $|\cdot|_{X(\widehat{\Omega})}$.

Observemos que, como consecuencia del Teorema 4.53 y de la inclusión continua $W(\widehat{\Omega}) \hookrightarrow X(\widehat{\Omega})$, vale la siguiente estimación

$$
\left|\left(\mathbf{A}-\mathbf{A}_{h}\right) f^{e}\right|_{X(\widehat{\Omega})} \leq C h^{s}\|f\|_{0, \Omega_{h}} \quad \forall f \in V_{h}\left(\Omega_{h}\right) .
$$

El siguiente lema prueba que el orden de convergencia es mayor cuando el término fuente pertenece al autoespacio asociado con $\lambda$.

Lema 4.59 Para $f \in \mathbf{E}_{\lambda}(W(\widehat{\Omega}))$ vale la siguiente estimación

$$
\left|\left(\mathbf{A}-\mathbf{A}_{h}\right) f\right|_{X(\widehat{\Omega})} \leq C h^{2 s}\|f\|_{0, \widehat{\Omega}} .
$$

Prueba. Dada $f \in \mathbf{E}_{\lambda}(W(\widehat{\Omega}))$, sean $u^{e}=\mathbf{A} f, u_{h}^{e}=\mathbf{A}_{h} f$ y $e_{h}:=u^{e}-u_{h}^{e} \in X(\widehat{\Omega})$. Como es usual en los argumentos de dualidad, consideraremos el siguiente problema auxiliar

$$
\begin{aligned}
-\Delta w+w & =e_{h} & & \text { en } \Omega, \\
\frac{\partial w}{\partial \mathbf{n}} & =0 & & \text { sobre } \Gamma .
\end{aligned}
$$

Sabemos que la solución $w \in H^{1+s}(\Omega)$ y, además,

$$
\|w\|_{1+s, \Omega} \leq C\left\|e_{h}\right\|_{0, \Omega} .
$$

Sea $w_{I} \in V_{h}^{c}\left(\Omega_{h}\right)$ la solución del problema discreto conforme

$$
\int_{\Omega_{h}} \nabla w_{I} \cdot \nabla v_{h}+\int_{\Omega_{h}} w_{I} v_{h}=\int_{\Omega_{h}} e_{h} v_{h} \quad \forall v_{h} \in V_{h}^{c}\left(\Omega_{h}\right) .
$$

En este caso, tendremos

$$
\left\|w-w_{I}^{e}\right\|_{1, \Omega} \leq C h^{s}\left\|e_{h}\right\|_{0, \Omega} .
$$

La estimación anterior puede obtenerse siguiendo los mismos argumentos que los usados en la prueba del Teorema 4.53 o, directamente, del Lema 3.6 en [116]. 
Sea $\omega_{T}$ el conjunto $T \backslash T^{i d}$ o $T^{i d} \backslash T$, según corresponda. A partir del problema (4.111) e integrando por partes

$$
\begin{aligned}
\left\|e_{h}\right\|_{0, \Omega}^{2}= & \sum_{T \in \mathcal{T}_{h}}\left(\int_{T^{i d}} \nabla w \cdot \nabla e_{h}-\int_{\partial T^{i d}} \frac{\partial w}{\partial \mathbf{n}} e_{h}\right)+\int_{\Omega} w e_{h} \\
= & \sum_{T \in \mathcal{T}_{h}}\left(\int_{T} \nabla\left(w^{e}-w_{I}\right) \cdot \nabla e_{h}-\int_{\partial T} \frac{\partial w}{\partial \mathbf{n}} e_{h}\right)+\int_{\Omega_{h}}\left(w^{e}-w_{I}\right) e_{h} \\
& +\sum_{T \in \mathcal{B}_{h}} \int_{\omega_{T}}\left(\nabla w \cdot \nabla e_{h}+w e_{h}\right)-\sum_{\substack{T \in \mathcal{B}_{h} \\
T^{i d} \subset T}} \int_{\omega_{T}}\left(\nabla w^{e} \cdot \nabla e_{h}+w^{e} e_{h}\right) \\
& +\sum_{T \subset T^{i d}} \int_{T} \nabla w_{I} \cdot \nabla e_{h}+\int_{\Omega_{h}} w_{I} e_{h} .
\end{aligned}
$$

Como $u$ y $u_{h}$ son solución de los problemas (4.101) y (4.106), respectivamente, y teniendo en cuenta que $w_{I} \in V_{h}\left(\Omega_{h}\right) \cap H^{1}\left(\Omega_{h}\right)$, tendremos

$$
\begin{gathered}
\int_{\Omega} \nabla u \cdot \nabla w_{I}^{e}+\int_{\Omega} u w_{I}^{e}=\int_{\Omega} f w_{I}^{e} \\
\sum_{T \in \mathcal{T}_{h}} \int_{T} \nabla u_{h} \cdot \nabla w_{I}+\int_{\Omega_{h}} u w_{I}+\sum_{\ell \in \mathcal{E}_{h}^{I}} \int_{\ell}\left\{\nabla w_{I}\right\}\left[\left[u_{h}\right]\right]=\int_{\Omega_{h}} f w_{I} .
\end{gathered}
$$

Combinando las dos ecuaciones anteriores, llegaremos a

$$
\begin{aligned}
\sum_{T \in \mathcal{T}_{h}} \int_{T} \nabla w_{I} \cdot \nabla e_{h}+\int_{\Omega_{h}} w_{I} e_{h} & =\int_{\Omega} f w_{I}^{e}-\int_{\Omega_{h}} f w_{I}-\sum_{\ell \in \mathcal{E}_{h}^{I}} \int_{\ell}\left\{\nabla w_{I}\right\}\left[\left[u_{h}\right]\right] \\
& -\sum_{\substack{T \in \mathcal{B}_{h} \\
T \subset T^{i d}}} \int_{\omega_{T}}\left(\nabla u \cdot \nabla w_{I}^{e}+u w_{I}^{e}\right) \\
& +\sum_{\substack{T \in \mathcal{B}_{h} \\
T^{i d} \subset T}} \int_{\omega_{T}}\left(\nabla u^{e} \cdot \nabla w_{I}+u^{e} w_{I}\right) .
\end{aligned}
$$

Procediendo como en la prueba del Lema 4.53, encontramos

$$
\sum_{T \in \mathcal{T}_{h}} \int_{\partial T} \frac{\partial w}{\partial \mathbf{n}} e_{h}=\sum_{\ell \in \mathcal{E}_{h}^{I}} \int_{\ell}\{\nabla w\}\left[\left[u-u_{h}\right]\right] .
$$

Luego, reemplazando las dos últimas expresiones en la ecuación del error, obtendremos

$$
\begin{aligned}
\left\|e_{h}\right\|_{0, \Omega}^{2} & =\sum_{T \in \mathcal{T}_{h}} \int_{T}\left(\nabla\left(w^{e}-w_{I}\right) \cdot \nabla e_{h}+\left(w^{e}-w_{I}\right) e_{h}\right)+\sum_{\ell \in \mathcal{E}_{h}^{I}} \int_{\ell}\left\{\nabla\left(w-w_{I}\right)\right\}\left[\left[e_{h}\right]\right] \\
& +\sum_{\substack{T \in \mathcal{B}_{h} \\
T \subset T^{i d}}} \int_{\omega_{T}}\left(\nabla w \cdot \nabla e_{h}+w e_{h}\right)-\sum_{\substack{T \in \mathcal{B}_{h} \\
T^{i d} \subset T}} \int_{\omega_{T}}\left(\nabla w^{e} \cdot \nabla e_{h}+w^{e} e_{h}\right) \\
& -\int_{\Omega \backslash \Omega_{h}}\left(\nabla u \cdot \nabla w_{I}^{e}+u w_{I}^{e}\right)+\int_{\Omega_{h} \backslash \Omega}\left(\nabla u^{e} \cdot \nabla w_{I}+u^{e} w_{I}\right)+\int_{\Omega \backslash \Omega_{h}} f w_{I}^{e}-\int_{\Omega_{h} \backslash \Omega} f w_{I} .
\end{aligned}
$$

A continuación, acotaremos los términos que aparecen en el lado derecho de la ecuación anterior por separado.

Usando la desigualdad de Cauchy-Schwarz y la definición de la norma $\|\cdot\|_{h}$, es inmediato que

$$
\left|\sum_{T \in \mathcal{T}_{h}} \int_{T}\left(\nabla\left(w^{e}-w_{I}\right) \cdot \nabla e_{h}+\left(w^{e}-w_{I}\right) e_{h}\right)\right| \leq\left\|w^{e}-w_{I}\right\|_{1, \Omega_{h}}\left\|e_{h}\right\|_{h, \Omega_{h}} .
$$


Argumentando como en la prueba del Teorema 4.53, obtendremos

$$
\begin{aligned}
\left|\sum_{\ell \in \mathcal{E}_{h}^{I}} \int_{\ell}\left\{\nabla\left(w-w_{I}\right)\right\}\left[\left[e_{h}\right]\right]\right| & \leq C\left(\left\|\nabla\left(w^{e}-w_{I}\right)\right\|_{0, \Omega_{h}}+h^{s}\|w\|_{1+s, \Omega}\right)\left(\sum_{\ell \in \mathcal{E}_{h}^{I}} h_{\ell}^{-1}\left\|\left[\left[e_{h}\right]\right]\right\|_{0, \ell}^{2}\right)^{1 / 2} \\
& \leq C\left(\left\|w^{e}-w_{I}\right\|_{1, \Omega_{h}}+h^{s}\|w\|_{1+s, \Omega}\right)\left\|e_{h}\right\|_{h} .
\end{aligned}
$$

Usando la desigualdad de Cauchy-Schwarz y el Lema D.6, tendremos

$$
\begin{aligned}
& \left|\int_{\Omega \backslash \Omega_{h}} f w_{I}^{e}\right|=\left|\int_{\Omega \backslash \Omega_{h}} f\left(w_{I}^{e}-w\right)+\int_{\Omega \backslash \Omega_{h}} f w\right| \leq\|f\|_{0, \Omega \backslash \Omega_{h}}\left(\left\|w-w_{I}^{e}\right\|_{0, \Omega \backslash \Omega_{h}}+\|w\|_{0, \Omega \backslash \Omega_{h}}\right) \\
& \leq C h\|f\|_{1+s, \Omega}\left(\left\|w-w_{I}^{e}\right\|_{0, \Omega}+h\|w\|_{1+s, \Omega}\right) \text {, } \\
& \left|\int_{\Omega_{h} \backslash \Omega} f w_{I}\right| \leq C h\|f\|_{1+s, \widehat{\Omega}}\left(\left\|w^{e}-w_{I}^{e}\right\|_{0, \widehat{\Omega}}+h\|w\|_{1+s, \Omega}\right), \\
& \left|\int_{\Omega \backslash \Omega_{h}}\left(\nabla u \cdot \nabla w_{I}^{e}+u w_{I}^{e}\right)\right|=\left|\int_{\Omega \backslash \Omega_{h}}\left(\nabla u \cdot \nabla\left(w_{I}^{e}-w\right)+u\left(w_{I}^{e}-w\right)+\nabla u \cdot \nabla w+u w\right)\right| \\
& \leq\|u\|_{1, \Omega \backslash \Omega_{h}}\left(\left\|w-w_{I}^{e}\right\|_{1, \Omega \backslash \Omega_{h}}+\|w\|_{1, \Omega \backslash \Omega_{h}}\right) \\
& \leq C h^{s}\|u\|_{1+s, \Omega}\left(\left\|w-w_{I}^{e}\right\|_{1, \Omega}+h^{s}\|w\|_{1+s, \Omega}\right) \text {, } \\
& \left|\int_{\Omega_{h} \backslash \Omega}\left(\nabla u^{e} \cdot \nabla w_{I}+u^{e} w_{I}\right)\right| \leq C h^{s}\|u\|_{1+s, \Omega}\left(\left\|w^{e}-w_{I}^{e}\right\|_{1, \widehat{\Omega}}+h^{s}\|w\|_{1+s, \Omega}\right), \\
& \left|\sum_{\substack{T \in \mathcal{B}_{h} \\
T \subset T^{i d}}} \int_{\omega_{T}}\left(\nabla w \cdot \nabla e_{h}+w e_{h}\right)\right| \leq\|w\|_{1, \Omega \backslash \Omega_{h}}\left\|u^{e}-u_{h}^{e}\right\|_{h, \widehat{\Omega}} \leq C h^{s}\|w\|_{1+s, \Omega}\left\|u^{e}-u_{h}^{e}\right\|_{h, \widehat{\Omega}}, \\
& \left|\sum_{\substack{T \in \mathcal{B}_{h} \\
T^{i d} \subset T}} \int_{\omega_{T}}\left(\nabla w^{e} \cdot \nabla e_{h}+w^{e} e_{h}\right)\right| \leq\left\|w^{e}\right\|_{1, \Omega_{h} \backslash \Omega}\left\|u^{e}-u_{h}^{e}\right\|_{h, \widehat{\Omega}} \leq C h^{s}\|w\|_{1+s, \Omega}\left\|u^{e}-u_{h}^{e}\right\|_{h, \widehat{\Omega}} .
\end{aligned}
$$

Combinando las estimaciones anteriores con el Teorema 4.55, las estimaciones (4.112), (4.113), (4.103) y teniendo en cuenta que $f$ pertenece al espacio propio asociado con $\lambda$ (vale ls estimación (4.104)), podremos escribir

$$
\left\|e_{h}\right\|_{0, \Omega}^{2} \leq C\left(h^{2 s}\left\|e_{h}\right\|_{0, \Omega}+h^{s}\left\|w^{e}-w_{I}\right\|_{0, \Omega_{h} \backslash \Omega}+h^{s}\left\|\nabla\left(w^{e}-w_{I}\right)\right\|_{0, \widehat{\Omega}}\right)\|f\|_{0, \Omega}
$$

Ahora, utilizando los Lemas D.6 y D.9 y las estimaciones (4.112)-(4.113), tendremos

$$
\begin{aligned}
\left\|w^{e}-w_{I}\right\|_{0, \Omega_{h} \backslash \Omega}^{2} & \leq 2\left(\left\|w^{e}\right\|_{0, \Omega_{h} \backslash \Omega}^{2}+\left\|w_{I}\right\|_{0, \Omega_{h} \backslash \Omega}^{2}\right) \leq C\left(h^{2(1+s)}\|w\|_{1+s, \Omega}^{2}+h^{2}\left\|w_{I}\right\|_{1, \Omega_{h}}^{2}\right) \\
& \leq C\left(h^{2(1+s)}\|w\|_{1+s, \Omega}^{2}+h^{2}\left\|w_{I}^{e}\right\|_{1, \Omega}^{2}\right) \leq C h^{2}\left(\|w\|_{1+s, \Omega}^{2}+\left\|w-w_{I}^{e}\right\|_{1, \Omega}^{2}\right) \\
& \leq C h^{2}\left\|e_{h}\right\|_{0, \Omega}^{2} .
\end{aligned}
$$

A partir del Lema D.7 (caso $T^{i d} \subset T$ ), llegamos a

$$
\begin{aligned}
\left\|\nabla\left(w^{e}-w_{I}\right)\right\|_{0, \omega_{T}}^{2} & \leq C\left(\left\|\nabla\left(w-w_{I}^{e}\right)\right\|_{0, T^{i d}}+h_{T}^{2 s}\left\|\nabla\left(w^{e}-w_{I}\right)\right\|_{s, T}\right)^{2} \\
& \leq C\left(\left\|\nabla\left(w-w_{I}^{e}\right)\right\|_{0, T^{i d}}^{2}+h_{T}^{4 s}\left\|\nabla\left(w^{e}-w_{I}\right)\right\|_{0, T}^{2}+h_{T}^{4 s}\left|w^{e}\right|_{s, T}^{2}\right) .
\end{aligned}
$$


Sumando sobre todos los triángulos en el borde, tales que $T^{i d} \subset T$, y teniendo en cuenta las propiedades de la extensión,

$$
\left\|\nabla\left(w^{e}-w_{I}\right)\right\|_{0, \Omega_{h} \backslash \Omega}^{2} \leq C\left(\left\|\nabla\left(w-w_{I}^{e}\right)\right\|_{0, \Omega}^{2}+h^{4 s}\left\|\nabla\left(w^{e}-w_{I}^{e}\right)\right\|_{0, \widehat{\Omega}}^{2}+h^{4 s}\|w\|_{1+s, \Omega}^{2}\right) .
$$

A partir de este resultado y considerando $h$ suficientemente chico, las estimaciones (4.112)-(4.113) nos conducen a

$$
\left\|\nabla\left(w^{e}-w_{I}^{e}\right)\right\|_{0, \widehat{\Omega}}^{2} \leq C\left(\left\|\nabla\left(w-w_{I}^{e}\right)\right\|_{0, \Omega}^{2}+h^{4 s}\|w\|_{1+s, \Omega}^{2}\right) \leq C h^{2 s}\left\|e_{h}\right\|_{0, \Omega}^{2} .
$$

Reemplazando todo en la expresión (4.114), obtendremos

$$
\left\|e_{h}\right\|_{0, \Omega} \leq C h^{2 s}\|f\|_{0, \Omega} .
$$

Finalmente, teniendo en cuenta que

$$
\left\|e_{h}\right\|_{0, \widehat{\Omega}}^{2}=\left\|e_{h}\right\|_{0, \Omega}^{2}+\left\|e_{h}\right\|_{0, \Omega_{h} \backslash \Omega}^{2}=\left\|e_{h}\right\|_{0, \Omega}^{2}+\sum_{\substack{T \in \mathcal{B}_{h} \\ T^{i d} \subset T}}\left\|e_{h}\right\|_{0, \omega_{T}}^{2}
$$

y, usando el Lema D.7 (caso $T^{i d} \subset T$ ) para acotar los términos en la suma

$$
\sum_{\substack{T \in \mathcal{B}_{h} \\ T^{i d} \subset T}}\left\|e_{h}\right\|_{0, \omega_{T}}^{2} \leq C \sum_{\substack{T \in \mathcal{B}_{h} \\ T^{i d} \subset T}}\left(\left\|e_{h}\right\|_{0, T^{i d}}+h_{T}^{2}\left\|e_{h}\right\|_{1, T}\right)^{2} \leq C\left(\left\|e_{h}\right\|_{0, \Omega}^{2}+h^{4 s}\left\|e_{h}\right\|_{h}^{2}\right),
$$

obtendremos, después de aplicar el Teorema 4.55, el resultado buscado; es decir,

$$
\left\|e_{h}\right\|_{0, \widehat{\Omega}} \leq C h^{2 s}\|f\|_{0, \Omega} .
$$

Así concluimos la prueba. 
CAPÍTULO 5

\section{Estimaciones a posteriori del error}

Desde el punto de vista de las aplicaciones, es muy importante tener la capacidad de diseñar mallas correctamente refinadas de manera tal que los errores en la aproximación se reduzcan tanto como sea posible y con el menor costo computacional.

La manera standard de alcanzar este objetivo es calcular, sobre una malla inicial $\mathcal{T}_{h}$, una aproximación del par autovector/autovalor de interés y, posteriormente, usar esta aproximación para construir indicadores del error para cada elemento $T \in \mathcal{T}_{h}$ (es decir, alguna cantidad equivalente al error medido en alguna norma apropiada) a fin de saber cuales de todos estos elementos deberán ser nuevamente refinados. Esta es la idea base en los procesos de refinamiento adaptativo de mallas [149].

El análisis a posteriori del error para métodos de elementos finitos no-standard, tales como los métodos mixtos o no-conformes, ha experimentado un notable desarrollo durante los últimos años. Sin embargo, la mayoría de los estudios publicados trata únicamente con problemas fuente. Entre las muchas y muy importantes contribuciones, citaremos aquí los siguientes trabajos $[100,86,118,154,2,65,71,72,73]$ (formulación primaria del problema/aproximación no-conforme) y [46, 64, 137, 97] (formulación mixta del problema/aproximación conforme).

Debido a las características no lineales de los problemas de autovalores, la extensión de las técnicas desarrolladas originalmente para problemas fuente no es obvia ni directa.

El primer avance en esta dirección se debe a Durán-Gastaldi-Padra [98], quienes obtuvieron un estimador del error a posteriori para la aproximación con métodos mixtos del espectro del operador de Laplace usando la equivalencia entre el método de Raviart-Thomás de orden cero y el método lineal de CrouzeixRaviart. Estas técnicas fueron adaptadas luego para definir indicadores locales del error para métodos $H(\operatorname{div}, \Omega)$-conformes $[7,5]$.

Muy recientemente, aparecieron las primeras publicaciones sobre estimaciones a posteriori para métodos no-conformes y problemas de autovalores; se deben a Dari-Durán-Padra [87] y a Dello Russo-Alonso [91].

En esta sección presentaremos el análisis a posteriori correspondiente a las aproximaciones no-conformes de los siguientes dos problemas de autovalores:

- de Steklov (formulación primaria y espacios de Crouzeix-Raviart),

- de las vibraciones en un sólido elástico (formulación mixta y espacios de Arnold-Winther).

Las estimaciones del error se obtendrán a partir de una descomposición adecuada del error e introduciendo una técnica de post-procesamiento. 


\subsection{Preliminares}

Introduciremos algunas nociones generales y supuestos que utilizaremos a lo largo de este capítulo. Aquí y en las secciones que siguen, preservaremos la notación empleada en las secciones 3.1-3.3.

A continuación, nos concentraremos en un par propio $(\mu, u)$ en particular (solución del problema continuo (3.1)) y su correspondiente aproximación por elementos finitos $\left(\mu_{h}, u_{h}\right)$ (solución del problema discreto (3.8)). Por simplicidad, asumiremos que $\mu$ es un autovalor de multiplicidad 1 y (para evitar complicaciones intrínsecas vinculadas a la aproximación del domino de definición) asumiremos que $\Omega$ es poligonal.

Al trabajar con métodos no-standard, es frecuente que la norma adecuada para el análisis a posteriori del error $e_{h}=u-u_{h}$ no coincida con la norma original; es decir, con la norma que se utilizó para establecer las estimaciones a priori. Para describir esta situación apropiadamente, comenzaremos por introducir nuevos espacios funcionales: $U(\Omega)$, con norma $\|\cdot\|_{U(\Omega)}$, para las funciones del continuo y $U_{h}(\Omega)$ para las funciones discretas. Exigiremos que

- si $v$ es solución del problema continuo, entonces $v \in U(\Omega)$,

- si $v_{h} \in V_{h}(\Omega)$, entonces $v_{h} \in U_{h}(\Omega)$.

Sea $\|\cdot\|_{1, h}$ una norma discreta definida sobre el espacio suma $\left(U+U_{h}\right)(\Omega)$ que verifique las siguientes condiciones:

- $\|v\|_{1, h} \sim\|v\|_{U(\Omega)} \quad \forall v \in U(\Omega)$,

- si $\left\|e_{h}\right\|_{h} \rightarrow 0$, entonces $\left\|e_{h}\right\|_{1, h} \rightarrow 0$, al menos con la misma rapidez.

Sea $\left\{\mathcal{T}_{h}\right\}_{h>0}$ una familia de triangulaciones del dominio $\Omega$. Asumiremos que $\mathcal{T}_{h}$ es regular; es decir, satisface las condiciones establecidas en la sección 4.1.1.

Buscamos indicadores del error $\eta_{T}$, a definir en cada elemento $T \in \mathcal{T}_{h}$, que satisfagan las siguientes propiedades:

1. Confiabilidad: deben proveer una cota superior para el error global:

$$
\left\|u-u_{h}\right\|_{1, h} \leq C\left(\sum_{T \in \mathcal{T}_{h}} \eta_{T}^{2}\right)^{1 / 2}+\text { h.o.t. }
$$

donde h.o.t. denota términos de orden superior, i.e., términos que, cuando $h$ tiende a cero, son muy pequeños en comparación con los otros términos en la estimación.

2. Eficiencia: deben proveer cotas inferiores para los errores locales, tan locales como sea posible, a fin de indicar cuáles son los elementos de la partición que deben ser refinados:

$$
\eta_{T} \leq C\left\|u-u_{h}\right\|_{1, h, \theta_{T}}+\text { h.o.t. }
$$

donde $\theta_{T}$ denota la unión de $T$ y algunos pocos elementos vecinos.

3. Bajo costo computacional: el cálculo efectivo de $\eta_{T}$ debe ser muy poco costoso en comparación con el cálculo global del par $\left(\mu_{h}, u_{h}\right)$.

\section{Notación}

Utilizaremos la notación de la sección 4.1.1 en lo que respecta a las triangulaciones del dominio $\Omega$ y los correspondientes conjuntos de nodos, conjuntos de lados internos y de borde.

Para cada $T \in \mathcal{T}_{h}$, denotemos por $\varrho_{T}$ al máximo diámetro de todos los discos contenidos en $T$ y, para cada $\ell \in \mathcal{E}_{h}$, denotemos por $h_{\ell}$ o por $|\ell|$, indistintamente, a la medida de $\ell$. La condición de regularidad asumida 
para las triangulaciones $\mathcal{T}_{h}$ de $\Omega$ implica que existen constantes positivas $c_{0}$ y $c_{1}$, que no dependen del triángulo $T$, tales que

$$
\begin{aligned}
h_{T} / \varrho_{T} \leq c_{0} \quad \forall T \in \mathcal{T}_{h}, \\
h_{T} / h_{\ell} \leq c_{1} \quad \forall \ell \subset \partial T .
\end{aligned}
$$

En los cálculos con funciones discontinuas, es frecuente el empleo de conceptos tales como salto o promedio de las funciones sobre los lados de la partición $\mathcal{T}_{h}$.

Sea $\ell \in \mathcal{E}_{h}^{I}$, un lado interior que es compartido por los elementos $T_{1}$ y $T_{2}$; i.e., $\ell=\partial T_{1} \cap \partial T_{2}$. Sea $\mathbf{n}_{\ell}$ el vector unitario normal que apunta hacia afuera de $T_{2}$. Para una función vectorial $\tau_{h}$, suave a trozos sobre $\mathcal{T}_{h}$, definimos

$$
\begin{aligned}
\left\{\boldsymbol{\tau}_{h}\right\} & :=\frac{1}{2}\left(\left.\left(\left.\boldsymbol{\tau}_{h}\right|_{T_{1}}\right)\right|_{\ell}+\left.\left(\left.\boldsymbol{\tau}_{h}\right|_{T_{2}}\right)\right|_{\ell}\right), \\
{\left[\left[\boldsymbol{\tau}_{h}\right]\right]_{\mathbf{n}} } & :=\left.\boldsymbol{\tau}_{h}\right|_{T_{2}} \cdot \mathbf{n}_{\ell}-\left.\boldsymbol{\tau}_{h}\right|_{T_{1}} \cdot \mathbf{n}_{\ell}, \\
{\left[\left[\boldsymbol{\tau}_{h}\right]\right]_{\mathbf{t}} } & :=\left.\boldsymbol{\tau}_{h}\right|_{T_{2}} \times \mathbf{n}_{\ell}-\left.\boldsymbol{\tau}_{h}\right|_{T_{1}} \times \mathbf{n}_{\ell},
\end{aligned}
$$

que corresponden al promedio y a los saltos en la componente normal y tangencial de $\tau_{h}$ sobre $\ell$, respectivamente. Observemos que estos valores son independientes de la elección de la dirección del vector normal $\mathbf{n}_{\ell}$. Más aún, si $\mathbf{n}_{\ell}=\left(n_{\ell}^{1}, n_{\ell}^{2}\right)$, definiremos la dirección tangente por $\mathbf{t}_{\ell}=\left(-n_{\ell}^{2}, n_{\ell}^{1}\right)$ y escribiremos

$$
\left[\left[\boldsymbol{\tau}_{h}\right]\right]_{\mathbf{t}}:=\left.\boldsymbol{\tau}_{h}\right|_{T_{2}} \cdot \mathbf{t}_{\ell}-\left.\boldsymbol{\tau}_{h}\right|_{T_{1}} \cdot \mathbf{t}_{\ell}
$$

Para una función escalar $v_{h}$, suave a trozos sobre $\mathcal{T}_{h}$, definimos

$$
\begin{aligned}
\left\{v_{h}\right\} & :=\frac{1}{2}\left(\left.\left(\left.v_{h}\right|_{T_{1}}\right)\right|_{\ell}+\left.\left(\left.v_{h}\right|_{T_{2}}\right)\right|_{\ell}\right), \\
{\left.\left[\left[v_{h}\right]\right]\right]_{\ell} } & :=\left.\left(\left.v_{h}\right|_{T_{2}}\right)\right|_{\ell}-\left.\left(\left.v_{h}\right|_{T_{1}}\right)\right|_{\ell}, \\
{\left[\left[v_{h}\right]\right] } & :=\left(\left.\left(\left.v_{h}\right|_{T_{2}}\right)\right|_{\ell}-\left.\left(\left.v_{h}\right|_{T_{1}}\right)\right|_{\ell}\right) \mathbf{n}_{\ell}
\end{aligned}
$$

Observemos que $\left[\left[v_{h}\right]\right]_{\ell}=0$ si $v_{h} \in H^{t}\left(T_{1} \cup T_{2}\right)$, para todo $t \geq 1$.

Para un lado $\ell$ en el borde de $\Omega$, extenderemos las definiciones anteriores de la siguiente manera

$$
\begin{gathered}
\left\{v_{h}\right\}:=\left.v_{h}\right|_{\ell}, \quad\left[\left[v_{h}\right]_{\ell}:=\left.v_{h}\right|_{\ell}, \quad\left[\left[v_{h}\right]\right]:=\left(\left.v_{h}\right|_{\ell}\right) \mathbf{n}_{\ell} \quad \ell \in \mathcal{E}_{h}^{\Gamma},\right. \\
\left\{\boldsymbol{\tau}_{h}\right\}:=\left.\boldsymbol{\tau}_{h}\right|_{\ell}, \quad\left[\left[\boldsymbol{\tau}_{h}\right]\right]_{\mathbf{n}}:=\left.\boldsymbol{\tau}_{h}\right|_{\ell} \cdot \mathbf{n}_{\ell}, \quad\left[\left[\boldsymbol{\tau}_{h}\right]_{\mathbf{t}}:=\left.\boldsymbol{\tau}_{h}\right|_{\ell} \cdot \mathbf{t}_{\ell} \quad \ell \in \mathcal{E}_{h}^{\Gamma} .\right.
\end{gathered}
$$

A cada $T \in \mathcal{T}_{h}$, le asociaremos los siguientes conjuntos:

$$
\begin{aligned}
\theta_{\ell} & :=\bigcup\left\{\widetilde{T} \in \mathcal{T}_{h}: \widetilde{T} \text { comparte un lado con } T\right\} \\
\theta_{T} & :=\bigcup\left\{\widetilde{T} \in \mathcal{T}_{h}: \widetilde{T} \text { comparte al menos un vértice con } T\right\} .
\end{aligned}
$$

\section{Operador de post-procesamiento}

Vamos a introducir un procedimiento que permite suavizar las soluciones discontinuas que se obtienen cuando se utiliza un método no-conforme. Para ello, seguiremos las ideas presentadas por Schieweck [153] y Karakashian-Pascal [121].

Para ubicarnos en el caso más general, supondremos que el borde $\Gamma$ del dominio $\Omega$ puede partirse de la siguiente manera $\Gamma=\Gamma_{D} \cup \Gamma_{N}$, con $\Gamma_{D} \cap \Gamma_{N}=\emptyset$. Supondremos además que la solución buscada satisface condiciones homogéneas de borde, tipo Dirichlet sobre $\Gamma_{D}$ y tipo Neumann sobre $\Gamma_{N}$.

Asumiremos también que la triangulación $\mathcal{T}_{h}$ induce sobre $\Gamma$ una partición compatible con las condiciones de borde; es decir, si $\ell \in \mathcal{E}_{h}^{\Gamma}$, entonces $\ell$ se encuentra enteramente contenido en $\Gamma_{D}$ o en $\Gamma_{N}$. Denotaremos por $\mathcal{E}_{h}^{D}:=\left\{\ell \in \mathcal{E}_{h}^{\Gamma}: \ell \cap \Gamma_{D} \neq \emptyset\right\}$ al conjunto de lados tipo Dirichlet y por $\mathcal{E}_{h}^{N}:=\left\{\ell \in \mathcal{E}_{h}^{\Gamma}: \ell \cap \Gamma_{N} \neq \emptyset\right\}$ al conjunto de lados tipo Neumann.

Para $k \geq 1$, definimos los espacios discretos

$$
U_{h}(\Omega):=\left\{v_{h} \in L^{2}(\Omega):\left.v_{h}\right|_{T} \in \mathcal{P}_{k}(T), \forall T \in \mathcal{T}_{h}\right\}
$$




$$
U_{h}^{c}(\Omega):=\left\{v_{h} \in \mathcal{C}^{0}(\bar{\Omega}):\left.v_{h}\right|_{T} \in \mathcal{P}_{k}(T), \forall T \in \mathcal{T}_{h}\right\}=U_{h}(\Omega) \cap H^{1}(\Omega)
$$

Observemos que $U_{h}^{c}(\Omega)$ es el espacio de elementos finitos de Lagrange de grado $k$. Sea $\left\{\psi_{\nu}: \nu \in \mathcal{M}_{h}\right\}$ la base canónica correspondiente a este espacio. Una función $\psi_{\nu}$ de esta base, es una función polinomial de grado $k$ a trozos y continua, que toma el valor 1 en el nodo $\mathbf{x}_{\nu}$ y se anula en cualquier otro nodo $\mathbf{x}_{\iota} \neq \mathbf{x}_{\nu}$, $\iota \in \mathcal{M}_{h}$. Para cada $\nu \in \mathcal{M}_{h}$, sea $\theta_{\nu}:=\left\{T \in \mathcal{T}_{h}: \mathbf{x}_{\nu} \in T\right\}$. Claramente, $\bigcup_{T \in \theta_{\nu}} T=\operatorname{supp} \psi_{\nu}$. Denotaremos por $\left|\theta_{\nu}\right|$ al cardinal de $\theta_{\nu}$.

Para $v_{h} \in U_{h}(\Omega)$, definimos el operador de post-procesamiento $\mathbf{R}_{h}: U_{h}(\Omega) \rightarrow U_{h}^{c}(\Omega)$ por

$$
\mathbf{R}_{h} v_{h}:=\sum_{\nu \in \mathcal{M}_{h}} \alpha_{\nu} \psi_{\nu}
$$

siendo $\alpha_{\nu}$ el promedio de los valores que $v_{h}$ toma en el nodo $\mathbf{x}_{\nu}$ dado por

$$
\alpha_{\nu}:= \begin{cases}\frac{1}{\left|\theta_{\nu}\right|} \sum_{T \in \theta_{\nu}}\left(\left.v_{h}\right|_{T}\right)\left(\mathbf{x}_{\nu}\right) & \text { si } \mathbf{x}_{\nu} \in \bar{\Omega} \backslash \bar{\Gamma}_{D}, \\ 0 & \text { si } \mathbf{x}_{\nu} \in \bar{\Gamma}_{D} .\end{cases}
$$

Es claro que, así definido, $\mathbf{R}_{h}$ es un operador lineal. Más aún, vale el siguiente resultado:

Lema 5.1 El operador de post-procesamiento $\mathbf{R}_{h}$ satisface

- para todo $v_{h} \in U_{h}^{c}(\Omega), \quad \mathbf{R}_{h} v_{h}=v_{h}$,

- para cada $T \in \mathcal{T}_{h}$, existe una constante $C$, independiente de $h$, tal que

$$
\left\|\mathbf{R}_{h} v_{h}\right\|_{1, T} \leq C\left(\sum_{T \subset \theta_{T}}\left\|v_{h}\right\|_{1, T}^{2}\right)^{1 / 2} \quad \forall v_{h} \in U_{h}(\Omega) .
$$

Prueba. Está esencialmente contenida en la prueba de los Lemas 2, 3 y 7 en [153].

Lema 5.2 Para toda $u_{h} \in U_{h}(\Omega)$, existe una constante positiva $C$, que depende solamente de $k$ y de la regularidad de $\mathcal{T}_{h}$, tal que

$$
\begin{aligned}
& -\sum_{T \in \mathcal{T}_{h}}\left\|u_{h}-\mathbf{R}_{h} u_{h}\right\|_{0, T}^{2} \leq C \sum_{\ell \in \mathcal{E}_{h}^{I}}|\ell|\left\|\mid\left[\left[u_{h}\right]\right]_{\ell}\right\|_{0, \ell}^{2}, \\
& -\sum_{T \in \mathcal{T}_{h}}\left\|u_{h}-\mathbf{R}_{h} u_{h}\right\|_{1, T}^{2} \leq C\left\{\begin{array}{l}
\sum_{\ell \in \mathcal{E}_{h}^{I}}|\ell|^{-1}\left\|\left[\left[u_{h}\right]\right]_{\ell}\right\|_{0, \ell}^{2} \text { si }\left|\Gamma_{D}\right|=0, \\
\sum_{\ell \in \mathcal{E}_{h}^{I}}|\ell|^{-1}\left\|\left[\left[u_{h}\right]\right]_{\ell}\right\|_{0, \ell}^{2}+\sum_{\ell \in \mathcal{E}_{h}^{D}}|\ell|^{-1}\left\|u_{h}\right\|_{0, \ell}^{2} \text { si }\left|\Gamma_{D}\right| \neq 0 .
\end{array}\right.
\end{aligned}
$$

Prueba. Está esencialmente contenida en la prueba del Teorema 2.1 en [122] (ver también [121] y las referencias allí citadas)

En lo que sigue, $C$ denotará una constante genérica independiente del parámetro $h$ y de la solución $u$. Esta constante será también independiente del autovalor discreto $\mu_{h}$, a menos que se especifique lo contrario.

\subsection{El problema de autovalores de Steklov}

Supongamos que $(\mu,(u, \xi))$ es una solución de $(4.7)$, con $\|(u, \xi)\|_{0, \Gamma}=1$. A partir de los resultados obtenidos en la sección 4.1.1, sabemos que existe $\left(\mu_{h},\left(u_{h}, \xi_{h}\right)\right)$ solución de $(4.13)$, con $\left\|\left(u_{h}, \xi_{h}\right)\right\|_{0, \Gamma}=1$, tal que

$$
\begin{aligned}
\left\|(u, \xi)-\left(u_{h}, \xi_{h}\right)\right\|_{h} & \leq C h^{r} \\
\left\|u-u_{h}\right\|_{0, \Gamma} & \leq C h^{3 r / 2} \\
\left|\mu-\mu_{h}\right| & \leq C h^{2 r}
\end{aligned}
$$


donde $r$ es el coeficiente de regularidad definido en el Lema 4.1 (aplicar el Lema B.1, reemplazando $u$ por $(u, \xi)$ e identificando $\|\cdot\|_{*}$ con $\left.\|\cdot\|_{0, \Gamma}\right)$.

El primer paso para establecer estimaciones a posteriori del error en la aproximación de la autofunción $u_{h}$, consiste en elegir una norma adecuada para medir el error. Para ello, introduciremos los espacios de funciones

$$
\begin{aligned}
U & :=H^{1}(\Omega), \\
U_{h} & :=C R_{h}(\Omega), \\
U_{h}^{c} & :=\left\{v_{h} \in H^{1}(\Omega):\left.v_{h}\right|_{T} \in \mathcal{P}_{1}(T), \forall T \in \mathcal{T}_{h}\right\} .
\end{aligned}
$$

A partir de estas definiciones, es inmediato que, si $(v, \eta) \in W(h)$ entonces $v \in U+U_{h}$. Sobre el espacio suma $U+U_{h}$ definimos la siguiente norma discreta

$$
\|v\|_{1, h}^{2}:=\|v\|_{0, \Omega}^{2}+\sum_{T \in \mathcal{T}_{h}}|v|_{1, T}^{2},
$$

la cual, claramente, satisface todas las propiedades listadas en la sección 5.1.

\subsubsection{Indicadores locales del error. Confiabilidad de las estimaciones del error}

Consideremos un par propio $(\mu, u)$ correspondiente al problema de autovalores de Steklov, fijo, y su aproximación $\left(\mu_{h}, u_{h}\right)$ obtenida mediante el método no-conforme de Crouzeix-Raviart. Denotaremos por $e_{h}=u-u_{h}$ al error en la aproximación.

Utilizando la desigualdad triangular, es inmediato que

$$
\left\|e_{h}\right\|_{1, h} \leq\left\|u-\mathbf{R}_{h} u_{h}\right\|_{1, h}+\left\|u_{h}-\mathbf{R}_{h} u_{h}\right\|_{1, h},
$$

siendo $\mathbf{R}_{h} u_{h} \in U_{h}^{c}$ la aproximación post-procesada asociada a la solución no-conforme $u_{h}$, como se definió en la sección anterior.

A partir de la definición de la norma discreta $\|\cdot\|_{1, h}$, se sigue que las formas bilineales $a_{h}^{*}$ son uniformemente continuas y coercivas sobre $U+U_{h}$ con respecto a esta norma. Luego, existirán constantes positivas $c_{1}$ y $c_{2}$ tales que

$$
\begin{aligned}
& c_{1}\left\|u-\mathbf{R}_{h} u_{h}\right\|_{1, h} \\
& \quad \leq \sup _{v \in U} \frac{a_{h}^{*}\left(u-\mathbf{R}_{h} u_{h}, v\right)}{\|v\|_{1, \Omega}} \leq \sup _{v \in U} \frac{a_{h}^{*}\left(e_{h}, v\right)}{\|v\|_{1, \Omega}}+\sup _{v \in U} \frac{a_{h}^{*}\left(u_{h}-\mathbf{R}_{h} u_{h}, v\right)}{\|v\|_{1, \Omega}}, \\
& \frac{a_{h}^{*}\left(u_{h}-\mathbf{R}_{h} u_{h}, v\right)}{\|v\|_{1, \Omega}} \leq c_{2}\left\|u_{h}-\mathbf{R}_{h} u_{h}\right\|_{1, h} .
\end{aligned}
$$

Las constantes $c_{1}$ y $c_{2}$ son directamente las constantes de coercividad y de continuidad. Observemos que dependen solamente de los coeficientes variables $\alpha$ y $\beta$ (ver la definición del problema (4.6)). En lo que sigue, por razones de simplicidad, nos restringiremos al caso $\alpha=\beta=1$. El caso general, requiere solo de modificaciones técnicas. Obtendremos entonces

$$
\left\|e_{h}\right\|_{1, h} \leq \sup _{v \in U} \frac{a_{h}^{*}\left(e_{h}, v\right)}{\|v\|_{1, \Omega}}+2\left\|u_{h}-\mathbf{R}_{h} u_{h}\right\|_{1, h} .
$$

A continuación introduciremos estimadores que nos permitirán acotar los dos términos que aparecen en el lado derecho de la ecuación (5.7) por separado.

Para cada lado $\ell \in \mathcal{E}_{h}$, sea

$$
J_{\ell, \mathbf{t}}:= \begin{cases}{\left[\left[\nabla u_{h}\right]\right]_{\mathbf{t}}} & \text { si } \ell \in \mathcal{E}_{h}^{I} \\ 0 & \text { si } \ell \in \mathcal{E}_{h}^{\Gamma}\end{cases}
$$

Definimos

$$
\eta_{1, T}:=\left(\frac{1}{24} \sum_{\ell \subset \partial T}|\ell|\left\|J_{\ell, \mathbf{t}}\right\|_{0, \ell}^{2}\right)^{1 / 2}
$$

y

$$
\eta_{1}=\left(\sum_{T \in \mathcal{T}_{h}} \eta_{1, T}^{2}\right)^{1 / 2}
$$


Lema 5.3 Existe una constante positiva $C$, que solo depende de la regularidad de $\mathcal{T}_{h}$, tal que

$$
\left\|u_{h}-\mathbf{R}_{h} u_{h}\right\|_{1, h} \leq C \eta_{1} .
$$

Prueba. Denotemos por $T^{-}$y $T^{+}$a dos triángulos adyacentes en $\mathcal{T}_{h}$ y por $\hat{\mathbf{x}}$ al punto medio correspondiente al lado $\ell$ que comparten. Denotemos por $P_{i}=\left(x_{i}, y_{i}\right), i=1,2$ a los puntos finales de $\ell$. Entonces,

$$
x(\xi)=\frac{x_{1}+x_{2}}{2}+\frac{x_{2}-x_{1}}{2} \xi, \quad y(\xi)=\frac{y_{1}+y_{2}}{2}+\frac{y_{2}-y_{1}}{2} \xi, \quad-1 \leq \xi \leq 1
$$

es una representación paramétrica de $\ell$. Consideremos las extensiones naturales de las funciones lineales $\left.u_{h}\right|_{T^{-}}$y $\left.u_{h}\right|_{T^{+}}$al conjunto más grande $T^{-} \cup T^{+}$. Por conveniencia en la notación, seguiremos llamando $\left.u_{h}\right|_{T^{-}}$y $\left.u_{h}\right|_{T^{+}}$a las funciones extendidas. En esta situación,

$$
\left.u_{h}\right|_{T^{ \pm}}(\xi)=\left.u_{h}\right|_{T^{ \pm}}\left(\hat{\mathbf{x}}_{\ell}\right)+\frac{|\ell|}{2}\left(\left.\nabla u_{h}\right|_{T^{ \pm}} \cdot \mathbf{t}_{\ell}\right) \xi, \quad-1 \leq \xi \leq 1 .
$$

Como $\left.u_{h}\right|_{T^{-}}$and $\left.u_{h}\right|_{T^{+}}$coinciden en el punto $\hat{\mathbf{x}}$,

$$
\left[\left[u_{h}\right]\right]_{\ell}(\xi)=\frac{|\ell|}{2}\left(\left.\nabla u_{h}\right|_{T^{+}} \cdot \mathbf{t}_{\ell}-\left.\nabla u_{h}\right|_{T^{-}} \cdot \mathbf{t}_{\ell}\right) \xi, \quad-1 \leq \xi \leq 1 .
$$

Es inmediato entonces que

$$
|\ell|^{-1}\left\|\left[\left[u_{h}\right]\right]_{\ell}\right\|_{0, \ell}^{2}=\frac{|\ell|}{12}\left\|\left[\left[\nabla u_{h}\right]\right]_{\mathrm{t}}\right\|_{\ell}^{2} .
$$

Concluimos la prueba sumando sobre todos los lados $\ell \in \mathcal{E}_{h}^{I}$ y usando el Lema 5.2.

Para cada $T \in \mathcal{T}_{h}$, sea

$$
J_{\ell, \mathbf{n}}:= \begin{cases}{\left[\left[\nabla u_{h}\right]\right]_{\mathbf{n}}} & \text { si } \ell \in \mathcal{E}_{h}^{I}, \\ \left.2\left(\nabla u_{h} \cdot \mathbf{n}_{\ell}-\mu_{h} u_{h}\right)\right|_{\ell} & \text { si } \ell \in \mathcal{E}_{h}^{\Gamma} .\end{cases}
$$

Definimos

$$
\begin{gathered}
\eta_{2, T}:=\left(|T|\left\|u_{h}\right\|_{0, T}^{2}+\frac{1}{2} \sum_{\ell \subset \partial T}|\ell|\left\|J_{\ell, \mathbf{n}}\right\|_{0, \ell}^{2}\right)^{1 / 2}, \\
\eta_{2}=\left(\sum_{T \in \mathcal{T}_{h}} \eta_{2, T}^{2}\right)^{1 / 2} .
\end{gathered}
$$

Lema 5.4 Existe una constante positiva $C$, que solo depende de la regularidad de $\mathcal{T}_{h}$, tal que

$$
\sup _{v \in U} \frac{a_{h}^{*}\left(e_{h}, v\right)}{\|v\|_{1, \Omega}} \leq C\left(\eta_{2}+\left\|\mu u-\mu_{h} u_{h}\right\|_{0, \Gamma}\right),
$$

Prueba. Sea $v \in U$, arbitrario. Integrando por partes y usando la ecuación (4.7), obtendremos

$$
\begin{aligned}
a_{h}^{*}\left(e_{h}, v\right) & =\mu \int_{\Gamma} u v-\sum_{T \in \mathcal{T}_{h}}\left(\int_{\partial T} \nabla u_{h} \cdot \mathbf{n} v+\int_{T} u_{h} v\right) \\
& =\mu \int_{\Gamma} u v-\sum_{T \in \mathcal{T}_{h}} \int_{T} u_{h} v-\sum_{\ell \in \mathcal{E}_{h}^{I}} \int_{\ell}\left[\left[\nabla u_{h}\right]\right]_{\mathbf{n}} v-\sum_{\ell \in \mathcal{E}_{h}^{\Gamma}} \int_{\ell}\left(\nabla u_{h} \cdot \mathbf{n}-\mu_{h} u_{h}\right) v-\mu_{h} \int_{\Gamma} u_{h} v \\
& =\int_{\Gamma}\left(\mu u-\mu_{h} u_{h}\right) v-\sum_{T \in \mathcal{T}_{h}}\left(\int_{T} u_{h} v-\frac{1}{2} \sum_{\ell \subset \partial T} \int_{\ell} J_{\ell, \mathbf{n}} v\right) .
\end{aligned}
$$

Sea ahora $v^{I}$ una aproximación, lineal a trozos y continua, de $v$ tal que

$$
\left\|v-v^{I}\right\|_{0, T} \leq C|v|_{1, \theta_{T}}|T|^{1 / 2}, \forall T \in \mathcal{T}_{h},
$$




$$
\left\|v-v^{I}\right\|_{0, \ell} \leq C|v|_{1, \theta_{T}}|\ell|^{1 / 2}, \forall \ell \subset \partial T,
$$

siendo $\theta_{T}$ la unión de $T$ y unos pocos elementos vecinos (ver [77], ver también [152]). Como $v^{I} \in U \cap U_{h}$, podemos usar (4.7) y (4.13) para obtener

$$
a_{h}^{*}\left(u-u_{h}, v^{I}\right)=\mu \int_{\Gamma} u v^{I}-\mu_{h} \int_{\Gamma} u_{h} v^{I}
$$

Después de algunos cálculos directos, llegamos a

$$
\begin{aligned}
a_{h}^{*}\left(e_{h}, v\right) & =\int_{\Gamma}\left(\mu u-\mu_{h} u_{h}\right) v^{I}+a_{h}^{*}\left(e_{h}, v-v^{I}\right) \\
& =\int_{\Gamma}\left(\mu u-\mu_{h} u_{h}\right) v-\int_{\Omega} u_{h}\left(v-v^{I}\right)-\frac{1}{2} \sum_{T \in \mathcal{T}_{h}} \sum_{\ell \subset \partial T} \int_{\ell} J_{\ell, \mathbf{n}}\left(v-v^{I}\right) .
\end{aligned}
$$

Finalmente, usando la desigualdad de Cauchy-Schwarz, las estimaciones para el error en la interpolación (5.11) y (5.12) y la definición del estimador $\eta_{2}$, tendremos

$$
a_{h}^{*}\left(e_{h}, v\right) \leq\left\|\mu u-\mu_{h} u_{h}\right\|_{0, \Gamma}\|v\|_{0, \Gamma}+C \eta_{2}|v|_{1, \Omega},
$$

a partir de la cual podemos concluir la prueba.

Guiados por los resultados anteriores, proponemos el siguiente estimador local del error en la aproximación no-conforme

$$
\eta_{T}^{2}:=\eta_{1, T}^{2}+\eta_{2, T}^{2}
$$

y definimos

$$
\eta=\left(\sum_{T \in \mathcal{T}_{h}} \eta_{T}^{2}\right)^{1 / 2}
$$

El siguiente teorema establece una cota superior para el error global en términos de $\eta$.

Teorema 5.5 La siguiente estimación es válida:

$$
\left\|u-u_{h}\right\|_{1, h} \leq C\left(\eta+\left\|\mu u-\mu_{h} u_{h}\right\|_{0, \Gamma}\right) .
$$

Prueba. Es una consecuencia inmediata de los Lemas 5.3 y 5.4 y de la definición de $\eta$.

Observación 5.6 El Teorema previo muestra que el estimador global provee una cota superior para la norma (discreta) de la energía del error, salvo términos que son de orden superior comparados con la estimación del error establecida en el Lema B.1. En efecto, a partir de la estimación (5.4) y teniendo en cuenta que $\left\|u_{h}\right\|_{0, \Gamma}=1$, es fácil probar que existen constantes estrictamente positivas $C$ y $h_{0}$ tales que, para todo $h<h_{0}$,

$$
\left\|\mu u-\mu_{h} u_{h}\right\|_{0, \Gamma} \leq\left|\mu-\mu_{h}\right|+C \mu h^{3 r / 2} .
$$

\subsubsection{Eficiencia de los indicadores de error}

Nuestro próximo objetivo es mostrar que los estimadores locales $\eta_{T}$ proveen una cota inferior para la norma de la energía del error en una vecindad de $T$.

Teorema 5.7 Para todo $T \in \mathcal{T}_{h}$, existe una constante positiva $C$, que depende solamente de la regularidad de los elementos en $\theta_{\ell}$, tal que

$$
\eta_{1, T} \leq C\left\|u-u_{h}\right\|_{1, h, \theta_{\ell}}
$$


Prueba. A partir de la ecuación (5.9), bastará con probar que para cada lado $\ell \in \mathcal{E}_{h}^{I}$, tal que $\ell=T_{1} \cap T_{2}$, se verifica

$$
|\ell|^{-1 / 2}\left\|\left[\left[u_{h}\right]\right]_{\ell}\right\|_{0, \ell} \leq C \sum_{i=1}^{2}\left|u-u_{h}\right|_{1, T_{i}} .
$$

Sabemos, gracias al Lema 4.1, que la solución $u$ es continua en $\Omega$. Consecuentemente, podremos escribir

$$
\int_{\ell}\left[\left[u_{h}\right]\right]_{\ell}^{2}=\int_{\ell}\left[\left[u-u_{h}\right]\right]_{\ell}\left[\left[u_{h}\right]\right]_{\ell}=\left.\int_{\ell}\left(u-u_{h}\right)\right|_{T_{2}}\left[\left[u_{h}\right]\right]_{\ell}-\left.\int_{\ell}\left(u-u_{h}\right)\right|_{T_{1}}\left[\left[u_{h}\right]\right]_{\ell} .
$$

Denotemos por $P_{\ell}$ a la proyección $L_{\ell}^{2}$ de $H^{1 / 2}(\ell)$ sobre las constantes. Como $\left[\left[u_{h}\right]\right]_{\ell}$ es una función lineal que se anula en el punto medio de $\ell$, tendremos, para cada $T_{i=1,2}$,

$$
\left.\int_{\ell}\left(u-u_{h}\right)\right|_{T_{i}}\left[\left[u_{h}\right]\right]_{\ell}=\left.\int_{\ell}\left(I-P_{\ell}\right)\left(u-u_{h}\right)\right|_{T_{i}}\left[\left[u_{h}\right]\right]_{\ell} \leq C|\ell|^{1 / 2}\left\|\nabla\left(u-u_{h}\right)\right\|_{0, T_{i}}\left\|\left[\left[u_{h}\right]\right]_{\ell}\right\|_{0, \ell},
$$

donde la última desigualdad se obtuvo usando estimaciones standard para $P_{\ell}$.

En los siguientes lemas obtendremos estimaciones (cotas superiores) para cada uno de los términos que aparecen en la definición de $\eta_{2, T}$.

Lema 5.8 Para cada elemento $T \in \mathcal{T}_{h}$,

$$
|T|^{1 / 2}\left\|u_{h}\right\|_{0, T} \leq C\left(|T|^{1 / 2}\left\|u-u_{h}\right\|_{0, T}+\left|u-u_{h}\right|_{1, T}\right)
$$

Prueba. Sea $\varphi_{T}:=u_{h} b_{T}$, siendo $b_{T}$ una burbuja cúbica, escaleada de manera que se satisfaga la siguiente igualdad

$$
\int_{T} u_{h} \varphi_{T}=\int_{T}\left(u_{h}\right)^{2} b_{T}=\left\|u_{h}\right\|_{0, T}^{2}|T| .
$$

Argumentos clásicos de scaling conducen a:

$$
\begin{array}{ll}
\left\|\varphi_{T}\right\|_{0, T} & \leq C\left\|u_{h}\right\|_{0, T}|T|, \\
\left\|\nabla \varphi_{T}\right\|_{0, T} & \leq C\left\|u_{h}\right\|_{0, T}|T|^{1 / 2} .
\end{array}
$$

Como $\varphi_{T}$ se anula sobre el borde de $T$, tendremos

$$
\int_{T} \nabla u_{h} \cdot \nabla \varphi_{T}=\int_{\partial T} \nabla u_{h} \cdot \mathbf{n} \varphi_{T}=0
$$

Consideremos ahora la extensión de $\varphi_{T}$ por cero fuera de $T$, denotándola $\widetilde{\varphi}$. Claramente, $\widetilde{\varphi} \in U$. Luego, tomado a $\widetilde{\varphi}$ como función de prueba en (4.6), obtendremos

$$
\int_{T} \nabla u \cdot \nabla \varphi_{T}+\int_{T} u \varphi_{T}=0
$$

Luego, como consecuencia de todo esto, tendremos

$$
\begin{aligned}
\left\|u_{h}\right\|_{0, T}^{2}|T| & =\int_{T} u_{h} \varphi_{T}-\int_{T} u \varphi_{T}-\int_{T} \nabla u \cdot \nabla \varphi_{T}=-\int_{T}\left(u-u_{h}\right) \varphi_{T}-\int_{T} \nabla\left(u-u_{h}\right) \cdot \nabla \varphi_{T} \\
& \leq\left\|u-u_{h}\right\|_{0, T}\left\|\varphi_{T}\right\|_{0, T}+\left\|\nabla\left(u-u_{h}\right)\right\|_{0, T}\left\|\nabla \varphi_{T}\right\|_{0, T} \\
& \leq C\left(\left\|u-u_{h}\right\|_{0, T}|T|+\left\|\nabla\left(u-u_{h}\right)\right\|_{0, T}|T|^{1 / 2}\right)\left\|u_{h}\right\|_{0, T}
\end{aligned}
$$

estimación que nos permite concluir la prueba.

Lema 5.9 Para cada lado $\ell \in \mathcal{E}_{h}^{I}$ tal que $\ell=T_{1} \cap T_{2}$, with $T_{1}, T_{2} \in \mathcal{T}_{h}$, se verifica

$$
|\ell|^{1 / 2}\left\|J_{\ell, \mathbf{n}}\right\|_{0, \ell} \leq C \sum_{i=1}^{2}\left(\left|T_{i}\right|^{1 / 2}\left\|u-u_{h}\right\|_{0, T_{i}}+\left|u-u_{h}\right|_{1, T_{i}}\right) .
$$


Prueba. Para cada $J_{\ell, \mathbf{n}}=\left[\left[\nabla u_{h}\right]\right]_{\mathbf{n}}$, sea $\varphi_{\ell} \in H_{0}^{1}\left(T_{1} \cup T_{2}\right)$ tal que

$$
\begin{aligned}
\int_{\ell} J_{\ell, \mathbf{n}} \varphi_{\ell} & =|\ell|\left\|J_{\ell, \mathbf{n}}\right\|_{0, \ell}^{2}, \\
\int_{T_{i}} \psi \varphi_{\ell} & =0 \quad \forall \psi \in \mathcal{P}_{1}\left(T_{i}\right), \quad i=1,2 .
\end{aligned}
$$

La función $\varphi_{\ell}$ puede construirse utilizando polinomios cuadráticos a trozos, continuos sobre $\ell$, enriquecidos con burbujas locales de grado cuatro. Argumentos clásicos de scaling conducen a:

$$
\begin{aligned}
& \left\|\varphi_{\ell}\right\|_{0, T_{i}} \leq C|\ell|^{1 / 2}\left\|J_{\ell, \mathbf{n}}\right\|_{0, \ell}\left|T_{i}\right|^{1 / 2}, \quad i=1,2 \\
& \left\|\nabla \varphi_{\ell}\right\|_{0, T_{i}} \leq C|\ell|^{1 / 2}\left\|J_{\ell, \mathbf{n}}\right\|_{0, \ell}, \quad i=1,2 .
\end{aligned}
$$

Extendiendo a $\varphi_{\ell}$ por cero fuera de $T_{1} \cup T_{2}$, denotándo a esta extensión $\widetilde{\varphi}$, y usando las ecuaciones en (4.6) con $\widetilde{\varphi} \in U$, obtendremos

$$
\int_{T_{1} \cup T_{2}} \nabla u \cdot \nabla \varphi_{\ell}+\int_{T_{1} \cup T_{2}} u \varphi_{\ell}=0 .
$$

Por otro lado, integrando por partes sobre cada triángulo, tendremos

$$
\sum_{i=1}^{2}\left(\int_{T_{i}} \nabla u_{h} \cdot \nabla \varphi_{\ell}+\int_{T_{i}} u_{h} \varphi_{\ell}\right)=\sum_{i=1}^{2} \int_{\ell} \nabla u_{h} \cdot \mathbf{n}_{i} \varphi_{\ell}=\int_{\ell} J_{\ell, \mathbf{n}} \varphi_{\ell} .
$$

Luego,

$$
\begin{aligned}
|\ell|\left\|J_{\ell, \mathbf{n}}\right\|_{0, \ell}^{2} & =-\sum_{i=1}^{2}\left(\int_{T_{i}} \nabla\left(u-u_{h}\right) \cdot \nabla \varphi_{\ell}+\int_{T_{i}}\left(u-u_{h}\right) \varphi_{\ell}\right) \\
& \leq \sum_{i=1}^{2}\left(\left\|\nabla\left(u-u_{h}\right)\right\|_{0, T_{i}}\left\|\nabla \varphi_{\ell}\right\|_{0, T_{i}}+\left\|u-u_{h}\right\|_{0, T_{i}}\left\|\varphi_{\ell}\right\|_{0, T_{i}}\right) \\
& \leq C \sum_{i=1}^{2}\left(\left\|\nabla\left(u-u_{h}\right)\right\|_{0, T_{i}}+\left|T_{i}\right|^{1 / 2}\left\|u-u_{h}\right\|_{0, T_{i}}\right)|\ell|^{1 / 2}\left\|J_{\ell, \mathbf{n}}\right\|_{0, \ell} .
\end{aligned}
$$

Así, concluimos la prueba.

Lema 5.10 Para cada $\ell \in \mathcal{E}_{h}^{\Gamma}$, sea $T \in \mathcal{T}_{h}$ tal que $\partial T \cap \ell \neq \emptyset$. Entonces, se verifica

$$
|\ell|^{1 / 2}\left\|J_{\ell, \mathbf{n}}\right\|_{0, \ell} \leq C\left(\left\|u-u_{h}\right\|_{1, T}+|\ell|^{1 / 2}\left\|\lambda u-\lambda_{h} u_{h}\right\|_{0, \ell}\right) .
$$

Prueba. Busquemos una función $\varphi_{T} \in \mathcal{P}_{3}(T)$ que satisfaga

$$
\begin{array}{ll}
\left.\varphi_{T}\right|_{\ell^{\prime}}=0 & \forall \ell^{\prime} \neq \ell, \\
\int_{\ell}^{\varphi_{T} \psi}=-2 \int_{\ell} J_{\ell, \mathbf{n}} \psi & \forall \psi \in \mathcal{P}_{1}(\ell), \\
\int_{T} \varphi_{T}=0 . &
\end{array}
$$

Es simple mostrar que $\varphi_{T}$ existe y es única. Más aún, argumentos cásicos de scaling nos permiten obtener:

$$
\begin{array}{ll}
\left\|\varphi_{T}\right\|_{0, T} & \leq C|\ell|^{1 / 2}\left\|J_{\ell, \mathbf{n}}\right\|_{0, \ell}, \\
\left\|\nabla \varphi_{T}\right\|_{0, T} & \leq C|\ell|^{-1 / 2}\left\|J_{\ell, \mathbf{n}}\right\|_{0, \ell}
\end{array}
$$

Procediendo como antes, extendemos a $\varphi_{T}$ por cero fuera de $T$ para obtener una función $\widetilde{\varphi} \in H^{1}(\Omega)$ y usamos a esta función extendida para evaluar la ecuación residual (5.10). Haciendo esto, y eligiendo $\psi=J_{\ell, \mathbf{n}} \in \mathcal{P}_{1}(\ell)$, obtendremos

$$
\begin{aligned}
\left\|J_{\ell, \mathbf{n}}\right\|_{0, \ell}^{2} & =\int_{\ell}\left(\mu_{h} u_{h}-\nabla u_{h} \cdot \mathbf{n}\right) \varphi_{T} \\
& =\int_{T} \nabla\left(u-u_{h}\right) \cdot \nabla \varphi_{T}+\int_{T}\left(u-u_{h}\right) \varphi_{T}-\int_{\ell}\left(\mu u-\mu_{h} u_{h}\right) \varphi_{T}+\int_{T} u_{h} \varphi_{T} \\
& \leq\left\|\nabla\left(u-u_{h}\right)\right\|_{0, T}\left\|\nabla \varphi_{T}\right\|_{0, T}+\left\|u-u_{h}\right\|_{0, T}\left\|\varphi_{T}\right\|_{0, T}+\left\|\mu u-\mu_{h} u_{h}\right\|_{0, \ell}\left\|\varphi_{T}\right\|_{0, \ell}+\left\|u_{h}\right\|_{0, T}\left\|\varphi_{T}\right\|_{0, T} \\
& \leq C\left(|\ell|^{-1 / 2}\left|u-u_{h}\right|_{1, T}+|\ell|^{1 / 2}\left\|u-u_{h}\right\|_{0, T}+\left\|\mu u-\mu_{h} u_{h}\right\|_{0, \ell}+|\ell|^{1 / 2}\left\|u_{h}\right\|_{0, T}\right)\left\|J_{\ell, \mathbf{n}}\right\|_{0, \ell}
\end{aligned}
$$


donde hemos usado un teorema de trazas local para estimar $\left\|\varphi_{T}\right\|_{0, \ell}$. Observemos que $|\ell| \sim|T|^{1 / 2}$ pues hemos asumido que la familia $\left\{\mathcal{T}_{h}\right\}_{h>0}$ es regular. Luego, podremos escribir

$$
|\ell|^{1 / 2}\left\|J_{\ell, \mathbf{n}}\right\|_{0, \ell} \leq C\left(\left\|u-u_{h}\right\|_{1, T}+|\ell|^{1 / 2}\left\|\mu u-\mu_{h} u_{h}\right\|_{0, \ell}+|T|^{1 / 2}\left\|u_{h}\right\|_{0, T}\right) .
$$

Concluimos la prueba aplicando el Lema 5.8.

El siguiente lema muestra que el término $\left\|\mu u-\mu_{h} u_{h}\right\|_{0, \ell}|\ell|^{1 / 2}$ puede separarse en dos: uno de esos términos está acotado por la 1-norma del error local; el otro es de orden superior si lo comparamos con el indicador local del error.

Lema 5.11 Para cada $\ell$ de un triángulo $T \in \mathcal{T}_{h}$ tal que $\ell \subset \Gamma$,

$$
|\ell|^{1 / 2}\left\|\mu u-\mu_{h} u_{h}\right\|_{0, \ell} \leq C\left(\mu\left\|u-u_{h}\right\|_{1, T}+h^{\sigma} \eta_{2, T}\right),
$$

$\operatorname{con} \sigma>0$

Prueba. Usando la desigualdad triangular, podremos escribir

$$
\left\|\mu u-\mu_{h} u_{h}\right\|_{0, \ell}|\ell|^{1 / 2} \leq \mu\left\|u-u_{h}\right\|_{0, \ell}|\ell|^{1 / 2}+\left|\mu-\mu_{h}\right|\left\|u_{h}\right\|_{0, \ell}|\ell|^{1 / 2} .
$$

Como $u-u_{h} \in H^{1}(T)$, podemos usar una desigualdad de trazas local para obtener

$$
\left\|u-u_{h}\right\|_{0, \ell} \leq C\left(|\ell|^{-1 / 2}\left\|u-u_{h}\right\|_{0, T}+|\ell|^{1 / 2}\left|u-u_{h}\right|_{1, T}\right) .
$$

Luego, el primer término en el lado derecho de la desigualdad (5.14) resulta acotado por

$$
\left\|u-u_{h}\right\|_{0, \ell}|\ell|^{1 / 2} \leq C\left(\left\|u-u_{h}\right\|_{0, T}+\left|\ell \| u-u_{h}\right|_{1, T}\right) \leq C\left\|u-u_{h}\right\|_{1, T} .
$$

Para acotar el término restante, usaremos nuevamente una desigualdad de trazas local y una estimación inversa. Haciendo esto, tendremos

$$
\left\|u_{h}\right\|_{0, \ell} \leq C\left(|\ell|^{-1 / 2}\left\|u_{h}\right\|_{0, T}+|\ell|^{1 / 2}\left|u_{h}\right|_{1, T}\right) \leq C|\ell|^{-1 / 2}\left\|u_{h}\right\|_{0, T} .
$$

Entonces, a partir la estimación (5.5) y la definición de $\eta_{2, T}$, llegamos a

$$
\left|\mu-\mu_{h}\right|\left\|u_{h}\right\|_{0, \ell}|\ell|^{1 / 2} \leq C\left|\mu-\mu_{h}\right|\left\|u_{h}\right\|_{0, T} \leq C h^{2 r-1} \eta_{2, T} .
$$

Concluimos la prueba combinando las estimaciones anteriores y teniendo en cuenta que $r \in(1 / 2,1]$.

El siguiente Teorema es una consecuencia directa de los lemas previos.

Teorema 5.12 Para $T \in \mathcal{T}_{h}$, existe una constante positiva $C$, que depende solamente de la regularidad de los elementos en $\theta_{\ell}$, tal que

- si todos los lados de T son internos, entonces

$$
\eta_{2, T} \leq C\left\|u-u_{h}\right\|_{1, h, \theta_{\ell}},
$$

- si $T$ tiene un lado sobre $\Gamma$, entonces

$$
\eta_{2, T} \leq C(1+\mu)\left\|u-u_{h}\right\|_{1, h, \theta_{\ell}}+\mathcal{O}\left(h^{\sigma}\right) \eta_{2, T}
$$

Observación 5.13 El primer estimador a posteriori del error en la aproximación de un problema de Poisson (problema de valores de contorno elíptico de segundo orden) mediante el método de CrouzeixRaviart fue introducido por Dari et al. en [86]. La técnica usada en ese trabajo para la construcción del estimador está basada en una descomposición tipo Helmholtz del gradiente del error, en combinación con ciertas relaciones de ortogonalidad que son válidas solo para problemas fuentes. Las mismas técnicas fueron generalizadas por Carstensen et al. en [65] para analizar una clase más amplia de métodos noconformes. Señalaremos aquí que no se conoce ninguna extensión adecuada de estas técnicas para tratar con el problema de autovalores de Steklov. 
Observación 5.14 La idea de derivar estimadores locales del error basándose en un post-procesamiento de la solución discreta fue introducida en [100] para el análisis a posteriori de las aproximaciones noconformes de un problema no-lineal. Más tarde, esta idea fue generalizada en [156, 2]. A diferencia de los resultados que se presentan en los trabajos antes mencionados, para obtener los estimadores aquí propuestos, no es necesario calcular la solución post-procesada $\mathbf{R}_{h} u_{h}$.

\subsubsection{Otros dos estimadores locales del error}

Llamaremos residuos de borde a la norma $\|\cdot\|_{0, \ell}$ de aquellos términos en el estimador que involucran a los saltos a través de cada lado interior $\ell$ de la triangulación de la derivada normal (y/o tangencial) de la solución aproximada. Los términos restantes, que aparecen en la definición de $\eta_{T}$, serán llamados parte volumétrica del residuo o, simplemente, residuo volumétrico.

Para problemas fuente y métodos lineales conformes, es un hecho conocido que los residuos de borde dominan a todo el error (ver [27, 74], por ejemplo). Este resultado fue extendido para problemas de autovalores y aproximaciones conformes en [101, 13].

El objetivo de esta sección es obtener estimaciones del error para la aproximación de Crouzeix-Raviart del problema de autovalores de Steklov que estén dominadas por los saltos, a través de cada lado interno de la triangulación, de la derivada tangencial de la solución discreta. Más precisamente, probaremos que

- la parte volumétrica del residuo está dominada en cada $T$ por el residuo de borde,

- la componente normal del salto, a través de cada lado interno de la triangulación, del gradiente discreto está dominada por la parte volumétrica del residuo, salvo términos de orden superior.

Estos resultados nos permitirán, en particular, definir indicadores locales del error más simples (comparados con los cálculos necesarios para hallar $\eta_{T}$ ) y que también resultan equivalentes a la norma discreta de la energía del error, salvo términos de orden superior. Resultados similares a estos, pero para problemas fuente únicamente, fueron presentados por primera vez en [71].

En lo que sigue, designaremos por $\left\{\varphi_{\ell}: \ell \in \mathcal{E}_{h}\right\}$ al conjunto base (natural) del espacio de CrouzeixRaviart asociado con los lados $\ell$, i.e., funciones lineales a trozos que toman el valor 1 en el punto medio de $\ell$ y se anulan sobre los puntos medios de cualquier otro lado $\tilde{\ell} \neq \ell$.

Lema 5.15 Para $T \in \mathcal{T}_{h}$, tal que $\partial T \cap \Gamma=\emptyset$, se verifica

$$
\frac{1}{3}|T|\left\|u_{h}\right\|_{0, T}^{2} \leq \sum_{\ell \subset \partial T}|\ell|\left\|J_{\ell, \mathbf{n}}\right\|_{0, \ell}^{2} \leq \frac{4}{3}|T|\left\|u_{h}\right\|_{0, T}^{2}+\frac{2}{3} \sum_{\widetilde{T} \subset \theta_{\ell}}|\widetilde{T}|\left\|u_{h}\right\|_{0, \widetilde{T}}^{2} .
$$

Prueba. Elijamos un elemento interior $T \in \mathcal{T}_{h}$. Denotemos por $T_{i}, i=1,2,3$ a los triángulos vecinos y llamemos $\ell$ al lado de $T$ tal que $\ell=T \cap T_{i}$. Entonces, a partir de (4.13) tendremos

$$
\begin{aligned}
-\int_{T \cup T_{i}} u_{h} \varphi_{\ell} & =\int_{T} \nabla u_{h} \cdot \nabla \varphi_{\ell}+\int_{T_{i}} \nabla u_{h} \cdot \nabla \varphi_{\ell}=\int_{\partial T} \nabla u_{h} \cdot \mathbf{n}_{\ell} \varphi_{\ell}+\int_{\partial T_{i}} \nabla u_{h} \cdot \mathbf{n}_{\ell} \varphi_{\ell} \\
& =\left[\left[\nabla u_{h}\right]\right]_{\mathbf{n}}|\ell|=J_{\ell, \mathbf{n}}|\ell| .
\end{aligned}
$$

Ahora, como $\left\|\varphi_{\ell}\right\|_{0, T_{i}}^{2}=\frac{\left|T_{i}\right|}{3}$,

$$
\left|\int_{T \cup T_{i}} u_{h} \varphi_{\ell}\right| \leq \frac{1}{\sqrt{3}}\left(\left\|u_{h}\right\|_{0, T}|T|^{1 / 2}+\left\|u_{h}\right\|_{0, T_{i}}\left|T_{i}\right|^{1 / 2}\right)
$$

y, por lo tanto,

$$
\sum_{\ell \subset \partial T}|\ell|\left\|J_{\ell, \mathbf{n}}\right\|_{0, \ell}^{2} \leq 2|T|\left\|u_{h}\right\|_{0, T}^{2}+\frac{2}{3} \sum_{i=1}^{3}\left|T_{i}\right|\left\|u_{h}\right\|_{0, T_{i}}^{2} .
$$

Por otro lado, un cálculo directo conduce a

$$
\int_{T \cup T_{i}} u_{h} \varphi_{\ell}=\frac{|T|+\left|T_{i}\right|}{3} u_{h}\left(\hat{\mathbf{x}}_{\ell}\right)
$$




$$
\int_{T} u_{h}^{2}=\frac{|T|}{3} \sum_{\ell \subset \partial T} u_{h}^{2}\left(\hat{\mathbf{x}}_{\ell}\right)
$$

siendo $\hat{\mathbf{x}}_{\ell}$ el punto medio correspondiente al lado $\ell$. Luego,

$$
\sum_{\ell \subset \partial T}|\ell|\left\|J_{\ell, \mathbf{n}}\right\|_{0, \ell}^{2}=\sum_{i=1}^{3} \frac{\left(|T|+\left|T_{i}\right|\right)^{2}}{9} u_{h}^{2}\left(\hat{\mathbf{x}}_{\ell_{i}}\right) \geq \frac{|T|^{2}}{9} \sum_{\ell \subset \partial T} u_{h}^{2}\left(\hat{\mathbf{x}}_{\ell}\right)=\frac{|T|}{3}\left\|u_{h}\right\|_{0, T}^{2} .
$$

Así, combinando las desigualdades anteriores, concluimos la prueba.

Lema 5.16 Para $T \in \mathcal{T}_{h}$, tal que $\partial T \cap \Gamma=\ell$, se verifica

$$
\begin{gathered}
\frac{4}{3}|T|\left\|u_{h}\right\|_{0, T}^{2} \leq \sum_{\tilde{\ell} \subset \partial T}|\tilde{\ell}|\left\|J_{\tilde{\ell}, \mathbf{n}}\right\|_{0, \tilde{\ell}}^{2}, \\
|\ell|\left\|J_{\ell, \mathbf{n}}\right\|_{0, \ell}^{2} \leq \frac{4}{3}|T|\left\|u_{h}\right\|_{0, T}^{2}+\frac{\mu_{h}^{2}}{12}|\ell|^{2}\left\|\nabla u_{h} \cdot \mathbf{t}_{\ell}\right\|_{0, \ell}^{2} .
\end{gathered}
$$

Prueba. Llamemos $\ell$ al lado de $T$ tal que $\ell=\partial T \cap \Gamma$. A partir de (4.13) tendremos

$$
\begin{aligned}
\int_{T} u_{h} \varphi_{\ell} & =\int_{\ell} \mu_{h} u_{h} \varphi_{\ell}-\int_{T} \nabla u_{h} \cdot \nabla \varphi_{\ell}=\int_{\ell}\left(\mu_{h} u_{h}-\nabla u_{h} \cdot \mathbf{n}_{\ell}\right) \varphi_{\ell} \\
& \leq\left\|\mu_{h} u_{h}-\nabla u_{h} \cdot \mathbf{n}_{\ell}\right\|_{0, \ell}\left\|\varphi_{\ell}\right\|_{0, \ell}=\frac{1}{2}\left\|J_{\ell, \mathbf{n}}\right\|_{0, \ell}\left\|\varphi_{\ell}\right\|_{0, \ell} .
\end{aligned}
$$

Entonces, como $\left\|\varphi_{\ell}\right\|_{0, \ell}^{2}=|\ell|$ y $\int_{T} u_{h} \varphi_{\ell}=\frac{|T|}{3} u_{h}\left(\hat{\mathbf{x}}_{\ell}\right)$,

$$
\frac{|T|}{3} u_{h}\left(\hat{\mathbf{x}}_{\ell}\right) \leq\left\|J_{\ell, \mathbf{n}}\right\|_{0, \ell}|\ell|^{1 / 2}
$$

Denotemos ahora por $T_{i}, i=1,2$, a los dos triángulos que comparten un lado con $T$. Llamemos $\ell_{i}, i=1,2$, a este lado en común. Procediendo como en la prueba del lema anterior, podremos escribir

$$
\left|\ell_{i}\right|\left\|J_{\ell, \mathbf{n}}\right\|_{0, \ell_{i}}^{2}=\frac{\left(|T|+\left|T_{i}\right|\right)^{2}}{9} u_{h}^{2}\left(\hat{\mathbf{x}}_{\ell_{i}}\right) \geq \frac{|T|^{2}}{9} u_{h}^{2}\left(\mathbf{x}_{\ell_{i}}\right),
$$

siendo $\hat{\mathbf{x}}_{\ell_{i}}$ el punto medio del lado $\ell_{i}$. Consequentemente,

$$
\frac{|T|}{3}\left\|u_{h}\right\|_{0, T}^{2}=\frac{|T|^{2}}{9} \sum_{\hat{\ell} \subset \partial T} u_{h}^{2}\left(\hat{\mathbf{x}}_{\hat{\ell}}\right) \leq \sum_{\hat{\ell} \subset \partial T}|\hat{\ell}|\left\|J_{\hat{\ell}, \mathbf{n}}\right\|_{0, \hat{\ell}}^{2} .
$$

Este resultado nos permite establecer la primera estimación del lema. Para obtener la segunda estimación, consideremos $\ell \in \mathcal{E}_{h}^{\Gamma}$ y calculemos

$$
\begin{gathered}
\int_{\ell}\left(\mu_{h} u_{h}-\nabla u_{h} \cdot \mathbf{n}_{\ell}\right)^{2}=\mu_{h}^{2} \int_{\ell} u_{h}^{2}-\left(2 \mu_{h} u_{h}\left(\hat{\mathbf{x}}_{\ell}\right) \nabla u_{h} \cdot \mathbf{n}_{\ell}-\left(\nabla u_{h} \cdot \mathbf{n}_{\ell}\right)^{2}\right)|\ell|, \\
\int_{\ell} u_{h}^{2}=u_{h}^{2}\left(\hat{\mathbf{x}}_{\ell}\right)|\ell|+\frac{1}{12}\left(\nabla u_{h} \cdot \mathbf{t}_{\ell}\right)^{2}|\ell|^{3} .
\end{gathered}
$$

Luego, a partir de la definición de $J_{\ell, \mathbf{n}}$, tendremos

$$
\left\|J_{\ell, \mathbf{n}}\right\|_{0, \ell}^{2}=4\left(\left|\mu_{h} u_{h}\left(\hat{\mathbf{x}}_{\ell}\right)-\nabla u_{h} \cdot \mathbf{n}_{\ell}\right|^{2}|\ell|+\frac{1}{12}\left\|\nabla u_{h} \cdot \mathbf{t}_{\ell}\right\|_{0, \ell}^{2}|\ell|^{2}\right) .
$$

Por otro lado, como $\varphi_{\ell}=1$ sobre $\ell \mathrm{y}\left\|\varphi_{\ell}\right\|_{0, T}^{2}=\frac{|T|}{3}$,

$$
\int_{T} u_{h} \varphi_{\ell}=\int_{\ell}\left(\mu_{h} u_{h}-\nabla u_{h} \cdot \mathbf{n}_{\ell}\right) \varphi_{\ell}=\left(\mu_{h} u_{h}\left(\hat{\mathbf{x}}_{\ell}\right)-\nabla u_{h} \cdot \mathbf{n}_{\ell}\right)|\ell|,
$$




$$
\left|\int_{T} u_{h} \varphi_{\ell}\right| \leq \sqrt{\frac{|T|}{3}}\left\|u_{h}\right\|_{0, T}
$$

Combinando las estimaciones anteriores, obtendremos

$$
\frac{|T|}{3}\left\|u_{h}\right\|_{0, T}^{2} \geq\left|\mu_{h} u_{h}\left(\hat{\mathbf{x}}_{\ell}\right)-\nabla u_{h} \cdot \mathbf{n}_{\ell}\right|^{2}|\ell|=\frac{1}{4}\left\|J_{\ell, \mathbf{n}}\right\|_{0, \ell}^{2}-\frac{1}{12}\left(\nabla u_{h} \cdot \mathbf{t}_{\ell}\right)^{2}|\ell|^{3} .
$$

Así, concluimos la prueba.

Los Lemas 5.15 y 5.16 nos permiten omitir la contribución volumétrica en la expresión de $\eta_{2, T}$ y definir un estimador de error más simple, basado solamente en los saltos de las derivadas normal y tangencial de la solución aproximada en cada $T$.

Definimos

$$
\widehat{\eta}_{2, T}^{2}:=\frac{1}{2} \sum_{\ell \subset \partial T}|\ell|\left\|J_{\ell, \mathbf{n}}\right\|_{0, \ell}^{2}
$$

y, correspondientemente,

$$
\widehat{\eta}:=\left(\sum_{T \in \mathcal{T}_{h}} \eta_{1, T}^{2}+\widehat{\eta}_{2, T}^{2}\right)^{1 / 2} .
$$

Los siguientes teoremas muestran que este estimador es globalmente confiable y localmente eficiente, salvo términos de orden superior.

Teorema 5.17 Existe una constante positiva $C$, que depende solamente de la regularidad de $\mathcal{T}_{h}$, tal que

$$
\left\|u-u_{h}\right\|_{1, h} \leq C\left(\widehat{\eta}+\left\|\mu u-\mu_{h} u_{h}\right\|_{0, \Gamma}\right) .
$$

Prueba. Los resultados de los Lemas 5.15 y 5.16 implican directamente $\eta_{T} \leq C \widehat{\eta}_{T}$ para todo $T \in \mathcal{T}_{h}$, siendo $C$ una constante que solo depende de la regularidad de la grilla. Luego, el resultado sigue a partir del Teorema 5.5.

Teorema 5.18 Para cada $T \in \mathcal{T}_{h}$, existe una constante positiva $C$, que depende solamente de la regularidad de los elementos en $\theta_{\ell}$, tal que

- si todos los lados de T son internos, entonces

$$
\widehat{\eta}_{T} \leq C\left\|u-u_{h}\right\|_{1, h, \theta_{\ell}},
$$

- si $T$ tiene un lado sobre $\Gamma$, entonces

$$
\widehat{\eta}_{T} \leq C(1+\mu)\left\|u-u_{h}\right\|_{1, h, \theta_{\ell}}+\mathcal{O}\left(h^{\sigma}\right) \widehat{\eta}_{2, T} .
$$

Prueba. Es obvio que $\widehat{\eta}_{T} \leq \eta_{T}$ para todo $T \in \mathcal{T}_{h}$. Luego, los resultados se obtienen directamente, aplicando los Teoremas 5.7 y 5.12 y el Lema 5.16.

En virtud de los Lemas 5.15 y 5.16, podríamos definir otro estimador de error. En efecto, a partir de estos resultados, es claro que los términos correspondientes a los saltos de la derivada normal de la solución discreta a través de los bordes elementales están dominados por los residuos volumétricos, salvo términos que, como probaremos más adelante, resultarán ser de orden superior.

Definimos

$$
\widetilde{\eta}_{2, T}^{2}:=|T||| u_{h} \|_{0, T}^{2}
$$

y, el correspondiente estimador global,

$$
\widetilde{\eta}:=\left(\sum_{T \in \mathcal{T}_{h}} \eta_{1, T}^{2}+\widetilde{\eta}_{2, T}^{2}\right)^{1 / 2} .
$$

El siguiente teorema muestra que este estimador brinda una cota superior global para el error medido en la norma $\|\cdot\|_{1, h}$. 
Teorema 5.19 Existe una constante positiva $C$, que depende solamente de la regularidad de $\mathcal{T}_{h}$, tal que

$$
\left\|u-u_{h}\right\|_{1, h}^{2} \leq C\left(\widetilde{\eta}^{2}+\left\|\mu u-\mu_{h} u_{h}\right\|_{0, \Gamma}^{2}+\mu_{h}^{2} \sum_{\ell \in \mathcal{E}_{h}^{\Gamma}}|\ell|\left\|u_{h}-u_{h}\left(\mathbf{x}_{\ell}\right)\right\|_{0, \ell}^{2}\right),
$$

siendo $\mathbf{x}_{\ell}$ el punto medio correspondiente al lado $\ell$. Más aún, para $h$ suficientemente pequeño, se verifica

$$
\mu_{h}^{2} \sum_{\ell \in \mathcal{E}_{h}^{\Gamma}}|\ell|\left\|u_{h}-u_{h}\left(\mathbf{x}_{\ell}\right)\right\|_{0, \ell}^{2} \leq C h\left(\left\|\mu u-\mu_{h} u_{h}\right\|_{0, \Gamma}^{2}+\mu^{4} h^{2}\right),
$$

con $r$ como en el Lema 4.1.

Prueba. Para cada $T \in \mathcal{T}_{h}$, tal que $\partial T \cap \Gamma=\emptyset$, la aplicación directa del Lema 5.15 nos permite escribir

$$
\eta_{T} \leq C \widetilde{\eta}_{T}
$$

Para cada $\ell \in \mathcal{E}_{h}^{\Gamma}$, sea $T$ el triángulo en $\mathcal{T}_{h}$ tal que $\ell \subset \partial T$. Gracias al Lema 5.16 y a la expresión (5.8), tendremos

$$
\eta_{T}^{2} \leq C \widetilde{\eta}_{T}^{2}+\frac{\mu_{h}^{2}}{12}|\ell|^{2}\left\|\nabla u_{h} \cdot \mathbf{t}_{\ell}\right\|_{0, \ell}^{2}=C \widetilde{\eta}_{T}^{2}+\mu_{h}^{2}|\ell|\left\|u_{h}-u_{h}\left(\mathbf{x}_{\ell}\right)\right\|_{0, \ell}^{2}
$$

Así, sumando sobre todos los triángulos $T \in \mathcal{T}_{h}$ y usando el Teorema 5.5, completamos la prueba de la primera estimación.

Sea ahora $\ell$ un lado sobre el borde $\Gamma$ y denotemos por $\mathbf{x}_{\ell}$ al punto medio de $\ell$. Sea $P_{\Gamma}$ la proyección $L^{2}$-ortogonal del espacio $L^{2}(\Gamma)$ sobre el espacio discreto

$$
\mathcal{C}_{h}:=\left\{c_{h} \in L^{2}(\Gamma):\left.c_{h}\right|_{\ell} \in \mathcal{P}_{0}(\ell) \quad \forall \ell \in \mathcal{E}_{h}^{\Gamma}\right\}
$$

Como $\left.u_{h}\right|_{\ell}$ es una función lineal, es claro que $\left.P_{\Gamma} u_{h}\right|_{\ell}=u_{h}\left(\mathbf{x}_{\ell}\right)$. Luego, podremos escribir

$$
\left.\mu_{h}\left(u_{h}-u_{h}\left(\mathbf{x}_{\ell}\right)\right)\right|_{\ell}=\left.\left(\mu_{h} u_{h}-\mu u\right)\right|_{\ell}+\left.\mu\left(u-P_{\Gamma} u\right)\right|_{\ell}+\left.P_{\Gamma}\left(\mu u-\mu_{h} u_{h}\right)\right|_{\ell},
$$

a partir de la cual obtendremos

$$
\mu_{h}\left\|u_{h}-u_{h}\left(\mathbf{x}_{\ell}\right)\right\|_{0, \ell} \leq C\left(\left\|\mu_{h} u_{h}-\mu u\right\|_{0, \ell}+\mu\left\|u-P_{\Gamma} u\right\|_{0, \ell}\right) .
$$

Luego, sumando sobre todos los lados $\ell \in \mathcal{E}_{h}^{\Gamma}$,

$$
\mu_{h}^{2} \sum_{\ell \in \mathcal{E}_{h}^{\Gamma}}|\ell|\left\|u_{h}-u_{h}\left(\mathbf{x}_{\ell}\right)\right\|_{0, \ell}^{2} \leq C h\left(\left\|\mu u-\mu_{h} u_{h}\right\|_{0, \Gamma}^{2}+\mu^{2}\left\|u-P_{\Gamma} u\right\|_{0, \Gamma}^{2}\right),
$$

Usando resultados de aproximación standard para $P_{\Gamma}$ y aplicando el teorema de trazas tendremos

$$
\left\|u-P_{\Gamma} u\right\|_{0, \Gamma} \leq C h^{\min \left\{1, \frac{1}{2}+r\right\}}\|u\|_{1+r, \Omega},
$$

donde hemos tenido en cuenta que $u$ es solución del problema (4.6) y hemos usado los resultados del Lema 4.1. Luego, combinando las estimaciones anteriores, y recordando que

$$
\|u\|_{1+r, \Omega} \leq C \mu\|u\|_{0, \Gamma} \leq C \mu,
$$

completamos la prueba de la segunda estimación.

Teorema 5.20 Para cada $T \in \mathcal{T}_{h}$, existe una constante positiva $C$, que depende solamente de la regularidad de los elementos en $\theta_{\ell}$, tal que

$$
\widetilde{\eta}_{T} \leq C\left\|u-u_{h}\right\|_{1, h, \theta_{\ell}} .
$$

Prueba. La prueba es inmediata a partir del Teorema 5.7 y del Lema 5.8.

Observación 5.21 The final form of estimator $\widetilde{\eta}$ resembles those derived in [86] and [71] for the CrouzeixRaviart approximation of source problems. 


\subsection{El problema de las vibraciones en un medio elástico}

Actualmente, se conocen varios estimadores de error a posteriori para las aproximaciones del problema de la elasticidad obtenidas usando métodos mixtos débilmente simétricos; es decir, métodos en los cuales la condición de simetría sobre el tensor de esfuerzos se impone débilmente. Los primeros resultados fueron obtenidos por Braess et al. [45] y, posteriormente, por Carstensen et al. [66, 67] y Lonsing-Verfürth [135]. En todos estos trabajos, los métodos analizados son $H(\operatorname{div}, \Omega)$-conformes, los estimadores propuestos son de tipo residual y se derivan, básicamente, a partir de una descomposición tipo Helmholtz del tensor de esfuerzos.

Una manera alternativa de obtener los mismos estimadores fue propuesta recientemente por Kim [128]. Los argumentos de Kim se basan también en una descomposición del error en el tensor de esfuerzos, matemáticamente equivalente a la descomposición de Helmholtz, pero que no impone requerimientos adicionales sobre la regularidad de las soluciones o la geometría de los dominios.

Otro estimador de error para este problema podría derivarse directamente de los resultados presentados por Lovadina-Stenberg [137]. El trabajo de estos autores comienza por mostrar que la solución de un método mixto puede post-procesarse localmente para obtener una aproximación de los desplazamientos con mayor exactitud y, por lo tanto, apropiada para establecer estimaciones a posteriori.

Combinando los argumentos de Lovadina-Stenberg con la descomposición propuesta por Kim para el error, hemos podido establecer estimaciones a posteriori del error en las aproximaciones de los modos de vibración de un sólido elástico obtenidas usando el método mixto no-conforme de Arnold-Winther [93]. Hasta donde sabemos, estos resultados son nuevos.

El estimador así obtenido resulta equivalente, salvo términos de orden superior, al error en la aproximación medido en una norma discreta, convenientemente pesada. Las constantes que realizan la equivalencia son independientes del correspondiente autovalor y del coeficiente de Lamé $\lambda_{S}$. Más aún, salvo términos de orden superior, el estimador provee una cota superior global e inferior local del error.

En lo que resta de esta sección utilizaremos la notación introducida en la sección 4.1.2.

\subsubsection{Normas discretas}

Supongamos que $(\omega,(\boldsymbol{\sigma}, \mathbf{u}))$ es una solución de $(4.30)$, con $\|\mathbf{u}\|_{0, \Omega}=1$. A partir de los resultados obtenidos en la sección 4.1.2, sabemos que existe $\left(\omega_{h},\left(\boldsymbol{\sigma}_{h}, \mathbf{u}_{h}\right)\right)$ solución de $(4.45)$, con $\left\|\mathbf{u}_{h}\right\|_{0, \Omega}=1$, tal que

$$
\begin{aligned}
\left\|(\boldsymbol{\sigma}, \mathbf{u})-\left(\boldsymbol{\sigma}_{h}, \mathbf{u}_{h}\right)\right\|_{h} & \leq C h^{s} \\
\left\|\mathbf{u}-\mathbf{u}_{h}\right\|_{0, \Omega} & \leq C h^{2 s}, \\
\left|\omega^{2}-\omega_{h}^{2}\right| & \leq C h^{2 s}
\end{aligned}
$$

donde $s$ es el coeficiente de regularidad definido en el Lema 4.13 (aplicar el Lema B.1, reemplazando $u$ por $(\boldsymbol{\sigma}, \mathbf{u})$ e identificando $\|(\boldsymbol{\sigma}, \mathbf{u})\|_{*}$ con $\left.\|\mathbf{u}\|_{0, \Omega}\right)$.

Para $\boldsymbol{\tau} \in L^{2}\left(\Omega, \mathbb{S}^{2}\right)$, definimos las siguientes normas pesadas:

$$
\|\boldsymbol{\tau}\|_{\mathbb{A}, \Omega}^{2}:=\int_{\Omega} \mathbb{A} \boldsymbol{\tau}: \boldsymbol{\tau} \quad \text { y } \quad\|\boldsymbol{\tau}\|_{\mathbb{C}, \Omega}^{2}:=\int_{\Omega} \mathbb{C} \boldsymbol{\tau}: \boldsymbol{\tau},
$$

donde $\mathbb{C}=\mathbb{A}^{-1}$ es el tensor de rigidez. A partir de la definición de $\mathbb{C}$, tendremos

$$
\begin{gathered}
\|\boldsymbol{\tau}\|_{0, \Omega} \leq \frac{1}{\sqrt{2 \mu_{S}}}\|\boldsymbol{\tau}\|_{\mathbb{C}, \Omega}, \\
\|\boldsymbol{\tau}\|_{\mathbb{A}, \Omega} \leq \operatorname{máx}\left\{\frac{1}{\sqrt{2 \mu_{S}}}, \frac{2}{\lambda_{S}+\mu_{S}}\right\}\|\boldsymbol{\tau}\|_{0, \Omega} .
\end{gathered}
$$

Para $\mathbf{v} \in \mathbf{V}(h):=H_{0, \Gamma_{D}}^{1}\left(\Omega, \mathbb{R}^{2}\right)+\mathbf{V}_{h} \subset L^{2}\left(\Omega, \mathbb{R}^{2}\right)$, definimos la siguiente seminorma discreta:

$$
\left\|\left|\mathbf{v}\left\|\left.\right|_{E, h} ^{2}:=\sum_{T \in \mathcal{T}_{h}}\right\| \varepsilon(\mathbf{v})\left\|_{0, T}^{2}+\sum_{\ell \in \mathcal{E}_{h}} h_{\ell}^{-1}\right\|[[\mathbf{v}]]_{\ell} \|_{0, \ell}^{2},\right.\right.
$$


donde $\varepsilon(\cdot)$ denota al operador gradiente simétrico. Observemos que $\left\||\cdot \||_{E, h}\right.$ es, en realidad, una norma sobre $\mathbf{V}(h)$. En efecto, supongamos que existe $\mathbf{v} \in \mathbf{V}(h)$ tal que \|\| $\mathbf{v}\|\|_{E, h}=0$. Esto implica que $[[\mathbf{v}]]_{\ell}=0$ para todo $\ell \in \mathcal{E}_{h}$. Luego, aplicando la primera desigualdad de Korn, ${ }^{1}$ concluimos que debe ser $\mathbf{v}=0$.

Consideremos ahora los siguientes espacios de funciones:

$$
\begin{aligned}
\mathbf{U} & :=H_{0, \Gamma_{N}}\left(\operatorname{div}, \Omega, \mathbb{S}^{2}\right) \times H_{0, \Gamma_{D}}^{1}\left(\Omega, \mathbb{R}^{2}\right) \\
\mathbf{U}_{h} & :=\boldsymbol{\Sigma}_{h} \times\left\{\mathbf{v}_{h} \in L^{2}\left(\Omega, \mathbb{R}^{2}\right):\left.\mathbf{v}_{h}\right|_{T} \in\left(\mathcal{P}_{3}(T)\right)^{2} \forall T \in \mathcal{T}_{h}\right\}, \\
\mathbf{U}_{h}^{c} & :=\boldsymbol{\Sigma}_{h} \times\left\{\mathbf{v}_{h} \in H_{0, \Gamma_{D}}^{1}\left(\Omega, \mathbb{R}^{2}\right):\left.\mathbf{v}_{h}\right|_{T} \in\left(\mathcal{P}_{3}(T)\right)^{2} \forall T \in \mathcal{T}_{h}\right\} .
\end{aligned}
$$

A partir de estas definiciones, es claro que

- $\boldsymbol{\Sigma}_{h} \times \mathbf{V}_{h} \subset \mathbf{U}_{h}$,

- si $\left(\boldsymbol{\tau}_{h}, \mathbf{v}_{h}\right) \in \mathbf{U}_{h}^{c}$, entonces $\left(\boldsymbol{\tau}_{h}, \mathbf{v}_{h}\right) \in \mathbf{U}_{h}$.

Para $(\boldsymbol{\tau}, \mathbf{v}) \in \mathbf{U}+\mathbf{U}_{h}$ definimos la siguiente norma discreta

$$
\|(\boldsymbol{\tau}, \mathbf{v})\|_{1, h}^{2}:=\|\boldsymbol{\tau}\|_{\mathbb{A}, \Omega}^{2}+2 \mu_{S}\|\mid \mathbf{v}\|_{E, h}^{2} .
$$

El peso en la norma $L^{2}\left(\Omega, \mathbb{S}^{2}\right)$ permitirá controlar el error en las aproximaciones de los tensores, incluso cuando los materiales sean casi incompresibles; es decir, permitirá obtener estimaciones con constantes independientes del coeficiente de Lamé $\lambda_{S}$. El factor $\sqrt{2 \mu_{S}}$ en la norma para los desplazamientos fue elegido para lograr un balance dimensional entre las magnitudes físicas intervinientes.

El siguiente resultado es fundamental en nuestro análisis.

Teorema 5.22 El espacio de Arnold-Winther es estable con respecto a la norma $\|\cdot\|_{1, h}$. Más aún, existe una constante $C$, estrictamente positiva, tal que

$$
\left\|(\boldsymbol{\sigma}, \mathbf{u})-\left(\boldsymbol{\sigma}_{h}, \mathbf{u}_{h}\right)\right\|_{1, h} \leq C h^{s} .
$$

Prueba. Ver sección C.

\subsubsection{Método de post-procesamiento}

Arnold-Brezzi mostraron que la solucióm obtenida usando un método mixto puede ser localmente postprocesada para construir una aproximación del campo de desplazamientos de mayor exactitud que la original [17]. Otros métodos de post-procesamientos fueron introducidos en los trabajos [55, 54, 50, 158, 159].

En general, estas técnicas de post-procesamiento proponen que, en cada $T \in \mathcal{T}_{h}$, las componentes del desplazamiento sean aproximadas por un polinomio de un grado mayor que el correspondiente a las entradas del tensor discreto. Si llamamos $\mathbf{u}_{h}^{*}$ al desplazamiento así obtenido, entonces

$$
\operatorname{gr} \varepsilon\left(\left.\mathbf{u}_{h}^{*}\right|_{T}\right)=\left.\operatorname{gr} \mathbb{A} \boldsymbol{\sigma}_{h}\right|_{T}
$$

y, por lo tanto, a nivel discreto, quedaría establecida una relación más exacta entre las dos variables. Esto induce a pensar que para realizar un análisis a posteriori del error, sería razonable reemplazar el campo de desplazamientos obtenido a partir del propio método por el campo de desplazamientos post-procesado.

A continuación, definimos el proceso de post-procesamiento que utilizaremos para mejorar al campo u; seguiremos el método introducido en [159]. Sea

$$
\mathbf{V}_{h}^{*}:=\left\{\mathbf{v}_{h} \in L^{2}\left(\Omega, \mathbb{R}^{2}\right):\left.\mathbf{v}_{h}\right|_{T} \in\left(\mathcal{P}_{3}(T)\right)^{2} \forall T \in \mathcal{T}_{h}\right\}
$$

\footnotetext{
${ }^{1}$ Supongamos que $\Omega \subset \mathbb{R}^{n}$ es un dominio acotado, con borde Lipschitz $\Gamma=\Gamma_{D} \cup \Gamma_{N}$, y sea $\mathbf{v}: \Omega \rightarrow \mathbb{R}^{n}$. Entonces, existe $c^{+}>0$ tal que para todo $\mathbf{v} \in H_{0, \Gamma_{D}}^{1}\left(\Omega, \mathbb{R}^{2}\right),\|\varepsilon(\mathbf{v})\|_{0, \Omega} \geq c^{+}\|\mathbf{v}\|_{1, \Omega}$. La constante $c^{+}$depende de la geometría del domino $\Omega$ y del borde $\Gamma$.
} 
Definimos $\mathbf{u}_{h}^{*} \in \mathbf{V}_{h}^{*}$ de manera tal que $\left.\mathbf{u}_{h}^{*}\right|_{T}$ es solución del siguiente sistema:

$$
\begin{aligned}
\int_{T} \varepsilon\left(\mathbf{u}_{h}^{*}\right): \nabla \mathbf{v}_{h} & =\int_{T} \mathbb{A} \boldsymbol{\sigma}_{h}: \nabla \mathbf{v}_{h} \quad \forall \mathbf{v}_{h} \in\left(\mathbf{I}-\left.\mathbf{P}_{h}\right|_{T}\right)\left(\mathcal{P}_{3}(T)\right)^{2}, \\
\left.\mathbf{P}_{h}\right|_{T} \mathbf{u}_{h}^{*} & =\mathbf{u}_{h} .
\end{aligned}
$$

Para esta nueva aproximación de $\mathbf{u}$ obtenemos el siguiente resultado.

Teorema 5.23 Existe una constante $C$, estrictamente positiva, tal que

$$
\left\|(\boldsymbol{\sigma}, \mathbf{u})-\left(\boldsymbol{\sigma}_{h}, \mathbf{u}_{h}^{*}\right)\right\|_{1, h} \leq C h^{s} .
$$

Prueba. Ver sección $C$.

\subsubsection{Indicadores locales del error para los tensores}

La primera desigualdad de Korn y el Teorema de Lax-Milgram permiten asegurar que el problema variacional

$$
\int_{\Omega} \mathbb{C} \varepsilon(\psi): \varepsilon(\mathbf{v})=\int_{\Omega} \boldsymbol{\sigma}_{h}: \varepsilon(\mathbf{v}) \quad \forall \mathbf{v} \in H_{0, \Gamma_{D}}^{1}\left(\Omega, \mathbb{R}^{2}\right)
$$

tiene una única solución $\boldsymbol{\psi} \in H_{0, \Gamma_{D}}^{1}\left(\Omega, \mathbb{R}^{2}\right)$. Este resultado induce a considerar la siguiente descomposición del error en la aproximación de los tensores:

$$
\boldsymbol{\sigma}-\boldsymbol{\sigma}_{h}=\mathbb{C} \varepsilon(\mathbf{u}-\boldsymbol{\psi})+\left(\mathbb{C} \varepsilon(\boldsymbol{\psi})-\boldsymbol{\sigma}_{h}\right),
$$

donde $\boldsymbol{\psi} \in H_{0, \Gamma_{D}}^{1}\left(\Omega, \mathbb{R}^{2}\right)$ es la solución del problema (5.19). A partir de aquí, es inmediato que

$$
\left\|\boldsymbol{\sigma}-\boldsymbol{\sigma}_{h}\right\|_{\mathbb{A}, \Omega}^{2}=\|\varepsilon(\mathbf{u}-\boldsymbol{\psi})\|_{\mathbb{C}, \Omega}^{2}+\left\|\varepsilon(\psi)-\mathbb{A} \boldsymbol{\sigma}_{h}\right\|_{\mathbb{C}, \Omega}^{2}
$$

La importancia de esta expresión es evidente: el problema de encontrar un estimador para la $\mathbb{A}$-norma del error $\mathbf{e}_{h}:=\boldsymbol{\sigma}-\boldsymbol{\sigma}_{h}$ se reduce al problema de estimar cada término de la descomposición por separado.

Estimación del término $\|\varepsilon(\mathbf{u}-\psi)\|_{\mathbb{C}, \Omega}$

Para cada $\ell \in \mathcal{E}_{h}$, definimos los términos de salto

$$
J_{\mathbf{n}_{\ell}}:=\left\{\begin{array}{cl}
{\left[\left[\boldsymbol{\sigma}_{h} \mathbf{n}_{\ell}\right]\right]} & \text { si } \ell \in \mathcal{E}_{h}^{I}, \\
-2 \boldsymbol{\sigma}_{h} \mathbf{n}_{\ell} & \text { si } \ell \in \mathcal{E}_{h}^{N}, \\
0 & \text { si } \ell \in \mathcal{E}_{h}^{D} .
\end{array}\right.
$$

Para cada $T \in \mathcal{T}_{h}$, definimos los indicadores locales

$$
\eta_{1, T}^{2}:=\frac{1}{2} \sum_{\ell \subset \partial T}|\ell|\left\|J_{\mathbf{n}_{\ell}}\right\|_{0, \ell}^{2}
$$

y, finalmente, proponemos el estimador global

$$
\eta_{1}:=\frac{1}{\sqrt{2 \mu_{S}}}\left(\sum_{T \in \mathcal{T}_{h}} \eta_{1, T}^{2}\right)^{1 / 2} .
$$

Teorema 5.24 Existe una constante positiva $C$, que depende solamente del dominio $\Omega$, de la constante de Korn $c^{+}$y de la regularidad de $\mathcal{T}_{h}$, tal que

$$
\|\varepsilon(\mathbf{u}-\boldsymbol{\psi})\|_{\mathbb{C}, \Omega} \leq C\left(\eta_{1}+\omega^{2} \frac{\rho_{S}}{\sqrt{2 \mu_{S}}}\left\|\mathbf{u}-\mathbf{u}_{h}\right\|_{0, \Omega}+\frac{\rho_{S}}{\sqrt{2 \mu_{S}}}\left|\omega^{2}-\omega_{h}^{2}\right|\right) .
$$


Prueba. Sea $\mathbf{v} \in H_{0, \Gamma_{D}}^{1}\left(\Omega, \mathbb{R}^{2}\right)$. Entonces, por (4.30), (5.19), (4.45) y una integración por partes, se tendrá

$$
\int_{\Omega} \mathbb{C} \varepsilon(\mathbf{u}-\boldsymbol{\psi}): \varepsilon(\mathbf{v})=\omega^{2} \rho_{S} \int_{\Omega}\left(\mathbf{u}-\mathbf{u}_{h}\right) \cdot \mathbf{v}+\left(\omega^{2}-\omega_{h}^{2}\right) \rho_{S} \int_{\Omega} \mathbf{u}_{h} \cdot \mathbf{v}-\sum_{T \in \mathcal{T}_{h}} \int_{\partial T} \boldsymbol{\sigma}_{h} \mathbf{n}_{T} \cdot \mathbf{v} .
$$

A partir de la definición de $J_{\mathbf{n}_{\ell}}$ y teniendo en cuenta que

- $\boldsymbol{\sigma}_{h} \mathbf{n}_{\ell} \cdot \mathbf{n}_{\ell}$ es continua sobre cada lado interno $\ell \in \mathcal{E}_{h}$,

- $\boldsymbol{\sigma}_{h} \mathbf{n}_{\ell} \cdot \mathbf{n}_{\ell}=0$ para todo $\ell \in \mathcal{E}_{\ell}^{N}$,

el último término de la expresión anterior puede escribirse como

$$
-\sum_{T \in \mathcal{T}_{h}} \int_{\partial T} \boldsymbol{\sigma}_{h} \mathbf{n}_{T} \cdot \mathbf{v}=\frac{1}{2} \sum_{T \in \mathcal{T}_{h}} \sum_{\ell \subset \partial T} \int_{\ell} J_{\mathbf{n}_{\ell}} \cdot \mathbf{v}=\frac{1}{2} \sum_{T \in \mathcal{T}_{h}} \sum_{\ell \subset \partial T} \int_{\ell}\left(J_{\mathbf{n}_{\ell}} \cdot \mathbf{t}_{\ell}\right)\left(\mathbf{v} \cdot \mathbf{t}_{\ell}\right) .
$$

Sea $P_{\ell}$ la proyección $L_{\ell}^{2}$ de $H^{1 / 2}(\ell)$ sobre las constantes. Para $\ell \in \mathcal{E}_{h}^{I}$, sean $T_{1}$ y $T_{2}$ los triángulos en $\mathcal{T}_{h}$ tales que $T_{1} \cap T_{2}=\ell$. Como $J_{\mathbf{n}_{\ell}} \cdot \mathbf{t}_{\ell}$ tiene valor medio igual a cero sobre cada $\ell \in \mathcal{E}_{h}^{I}$,

$$
\int_{\ell}\left(J_{\mathbf{n}_{\ell}} \cdot \mathbf{t}_{\ell}\right)\left(\mathbf{v} \cdot \mathbf{t}_{\ell}\right) \leq\left\|J_{\mathbf{n}_{\ell}}\right\|_{0, \ell}\left\|\mathbf{v} \cdot \mathbf{t}_{\ell}-P_{\ell}\left(\mathbf{v} \cdot \mathbf{t}_{\ell}\right)\right\|_{0, \ell} \leq C|\ell|^{1 / 2}\left\|J_{\mathbf{n}_{\ell}}\right\|_{0, \ell}\|\mathbf{v}\|_{1, T_{1} \cup T_{2}} .
$$

$J_{\mathbf{n}_{\ell}}$ también tiene valor medio igual a cero sobre cada $\ell \in \mathcal{E}_{h}^{N}$. Esto se debe a la condición que satisfacen sobre $\Gamma_{N}$ los tensores pertenecientes al espacio $\boldsymbol{\Sigma}_{h}$. Sea $T$ el único triángulo en $\mathcal{T}_{h}$ tal que $\partial T \cap \Gamma_{N}=\ell$. Razonando de manera análoga al caso anterior, tendremos

$$
\int_{\ell}\left(J_{\mathbf{n}_{\ell}} \cdot \mathbf{t}_{\ell}\right)\left(\mathbf{v} \cdot \mathbf{t}_{\ell}\right) \leq C|\ell|^{1 / 2}\left\|J_{\mathbf{n}_{\ell}}\right\|_{0, \ell}\|\mathbf{v}\|_{1, T}
$$

Por otro lado, para $\mathbf{v} \in H_{0, \Gamma_{D}}^{1}\left(\Omega, \mathbb{R}^{2}\right)$ son válidas las siguientes desigualdades

$$
\|\mathbf{v}\|_{0, \Omega} \leq C(\Omega)|\nabla \mathbf{v}|_{0, \Omega} \leq \frac{C(\Omega)}{c^{+}}\|\varepsilon(\mathbf{v})\|_{0, \Omega} \leq \frac{C(\Omega)}{\sqrt{2 \mu_{S}} c^{+}}\|\varepsilon(\mathbf{v})\|_{\mathbb{C}, \Omega} .
$$

Luego, combinando todas las estimaciones anteriores con la definición de $\eta_{1}$ y teniendo en cuenta que $\left\|\mathbf{u}_{h}\right\|_{0, \Omega}=1$, obtendremos

$$
\int_{\Omega} \mathbb{C} \varepsilon(\mathbf{u}-\boldsymbol{\psi}): \varepsilon(\mathbf{v}) \leq C\left(\eta_{1}+\omega^{2} \frac{\rho_{S}}{\sqrt{2 \mu_{S}}}\left\|\mathbf{u}-\mathbf{u}_{h}\right\|_{0, \Omega}+\frac{\rho_{S}}{\sqrt{2 \mu_{S}}}\left|\omega^{2}-\omega_{h}^{2}\right|\right)\|\varepsilon(\mathbf{v})\|_{\mathbb{C}, \Omega},
$$

resultado que nos permite concluir la prueba.

El teorema anterior muestra que, salvo términos de orden superior, el estimador $\eta_{1}$ acota superiormente a la parte conforme del error en la variable tensorial. A continuación probaremos que $\eta_{1, T}$ provee una cota inferior local para el error en la aproximación de los tensores, salvo términos de orden superior.

Lema 5.25 i) Para cada $\ell \in \mathcal{E}_{h}^{I}$, sean $T_{1}$ y $T_{2}$ los triángulos en $\mathcal{T}_{h}$ tales que $\ell=T_{1} \cap T_{2}$. Entonces, se verifica

$$
|\ell|^{1 / 2}\left\|J_{\mathbf{n}_{\ell}}\right\|_{0, \ell} \leq C\left(\rho_{S}\left\|\omega^{2} \mathbf{u}-\omega_{h}^{2} \mathbf{u}_{h}\right\|_{0, T_{1} \cup T_{2}}|\ell|+\left\|\boldsymbol{\sigma}-\boldsymbol{\sigma}_{h}\right\|_{0, T_{1} \cup T_{2}}\right) .
$$

ii) Para cada $\ell \in \mathcal{E}_{h}^{N}$, sea $T \in \mathcal{T}_{h}$ tal que $\ell \subset \partial T$. Entonces, se verifica

$$
|\ell|^{1 / 2}\left\|J_{\mathbf{n}_{\ell}}\right\|_{0, \ell} \leq C\left(\rho_{S}\left\|\omega^{2} \mathbf{u}-\omega_{h}^{2} \mathbf{u}_{h}\right\|_{0, T}|\ell|+\left\|\boldsymbol{\sigma}-\boldsymbol{\sigma}_{h}\right\|_{0, T}\right) .
$$

Prueba. Probaremos solamente el enunciado $i$ ) ya que, con obvias modificaciones, ii) se prueba de manera similar.

A partir de los resultados obtenidos por Verfürth [170], sabemos que existe un operador de extensión $\boldsymbol{\epsilon}: \mathcal{C}^{0}(\ell) \rightarrow \mathcal{C}^{0}(T), T \in \mathcal{T}_{h}$, el cual extiende polinomios de grado $k$ sobre $\ell$ a polinomios de grado $k$ sobre 
$T$, de manera tal que, si $p \in \mathcal{P}_{k}(\ell),\left.\boldsymbol{\epsilon} p\right|_{\ell}=p$. Eligiendo $k=2$, denotemos por $\widetilde{J}_{\mathbf{n}_{\ell}}$ a la extensión de $J_{\mathbf{n}_{\ell}}$ a $T_{1} \cup T_{2}$ definida por

$$
\left.\widetilde{J}_{\mathbf{n}_{\ell}}\right|_{T_{i}}:=\boldsymbol{\epsilon}\left(J_{\mathbf{n}_{\ell}} \cdot \mathbf{t}_{\ell}\right), \quad i=1,2 .
$$

Sea $\beta_{\ell}$ la función cuadrática a trozos definida sobre $T_{1} \cup T_{2}$ de manera tal que tome el valor 1 sobre el punto medio de $\ell$ y se anule sobre el borde de $T_{1} \cup T_{2}$. Definimos

$$
\boldsymbol{\varphi}_{\ell}(\mathbf{x}):=\left\{\begin{array}{cl}
c_{\ell} \widetilde{J}_{\mathbf{n}_{\ell} \beta_{\ell}(\mathbf{x})} & \mathbf{x} \in \overline{T_{1} \cup T_{2}}, \\
0 & \mathbf{x} \in \Omega \backslash \overline{T_{1} \cup T_{2}}
\end{array}\right.
$$

donde el coeficiente $c_{\ell}$ está dado por

$$
c_{\ell}:=\frac{\int_{\ell}\left(\widetilde{J}_{\mathbf{n}_{\ell}}\right)^{2}}{\int_{\ell}\left(\widetilde{J}_{\mathbf{n}_{\ell}}\right)^{2} \beta_{\ell}} .
$$

Observemos que, así definida, $\varphi_{\ell} \in H_{0, \Gamma_{N}}^{1}\left(\Omega, \mathbb{R}^{2}\right)$ y verifica

$$
\int_{\ell} J_{\mathbf{n}_{\ell}} \cdot \varphi_{\ell}=\int_{\ell} \widetilde{J}_{\mathbf{n}_{\ell}} \cdot \varphi_{\ell}=\left\|\widetilde{J}_{\mathbf{n}_{\ell}}\right\|_{0, \ell}^{2}
$$

Argumentos standard basados en la equivalencia de normas en el espacio $\mathcal{P}_{2}(\widehat{\ell})(\widehat{\ell}$ segmento de referencia), muestran que existe una constante positiva $C$ tal que $c_{\ell} \leq C$. Entonces, usando argumentos clásicos de scaling, tendremos

$$
\begin{gathered}
\left\|\boldsymbol{\varphi}_{\ell}\right\|_{0, T_{i}} \leq C|\ell|^{1 / 2}\left\|\widetilde{J}_{\mathbf{n}_{\ell}}\right\|_{0, \ell}, \\
\left\|\nabla \boldsymbol{\varphi}_{\ell}\right\|_{0, T_{i}} \leq C|\ell|^{-1 / 2}\left\|\widetilde{J}_{\mathbf{n}_{\ell}}\right\|_{0, \ell}
\end{gathered}
$$

Luego, integrando por partes, usando las ecuaciones (4.29) y (4.45) y recordando (4.39), llegamos a

$$
\begin{aligned}
\sum_{1 \leq i \leq 2} \int_{T_{i}}\left(\boldsymbol{\sigma}-\boldsymbol{\sigma}_{h}\right): \nabla \boldsymbol{\varphi}_{\ell} & =\sum_{1 \leq i \leq 2}\left(-\int_{T_{i}} \operatorname{div}\left(\boldsymbol{\sigma}-\boldsymbol{\sigma}_{h}\right) \cdot \boldsymbol{\varphi}_{\ell}+\int_{\partial T_{i}}\left(\boldsymbol{\sigma}-\boldsymbol{\sigma}_{h}\right) \mathbf{n} \cdot \boldsymbol{\varphi}_{\ell}\right) \\
& =-\sum_{1 \leq i \leq 2} \int_{T_{i}} \rho_{S}\left(\omega^{2} \mathbf{u}-\omega_{h}^{2} \mathbf{u}_{h}\right) \cdot \boldsymbol{\varphi}_{\ell}+\int_{\ell} J_{\mathbf{n}_{\ell}} \cdot \boldsymbol{\varphi}_{\ell}
\end{aligned}
$$

de donde obtendremos

$$
|\ell|^{1 / 2}\left\|J_{\mathbf{n}_{\ell}}\right\|_{0, \ell} \leq C\left(\rho_{S}\left\|\omega^{2} \mathbf{u}-\omega_{h}^{2} \mathbf{u}_{h}\right\|_{0, T_{1} \cup T_{2}}|\ell|+\left\|\boldsymbol{\sigma}-\boldsymbol{\sigma}_{h}\right\|_{0, T_{1} \cup T_{2}}\right) .
$$

El siguiente teorema es una consecuencia directa del resultado anterior.

Teorema 5.26 Existe una constante positiva $C$ tal que,

$$
\eta_{1, T} \leq C\left(\rho_{S}\left\|\omega^{2} \mathbf{u}-\omega_{h}^{2} \mathbf{u}_{h}\right\|_{0, \theta_{\ell}}|\ell|+\left\|\boldsymbol{\sigma}-\boldsymbol{\sigma}_{h}\right\|_{0, \theta_{\ell}}\right)
$$

Estimación del término $\left\|\varepsilon(\psi)-\mathbb{A} \sigma_{h}\right\|_{\mathbb{C}, \Omega}$

Teorema 5.27 Sean $\boldsymbol{\psi}$ la solución del problema variacional (5.19) y $\gamma$ la constante inf-sup asociada al problema de Stokes en $\Omega$. Entonces,

$$
\left\|\varepsilon(\psi)-\mathbb{A} \boldsymbol{\sigma}_{h}\right\|_{\mathbb{C}, \Omega} \leq \sqrt{2 \mu_{S}}\left(1+\frac{1}{\gamma}\right) \inf _{\mathbf{v} \in H_{0, \Gamma_{D}}^{1}\left(\Omega, \mathbb{R}^{2}\right)}\left\|\varepsilon(\mathbf{v})-\mathbb{A} \boldsymbol{\sigma}_{h}\right\|_{0, \Omega}
$$


Prueba. Sea $\mathbf{v} \in H_{0, \Gamma_{D}}^{1}\left(\Omega, \mathbb{R}^{2}\right)$, arbitrario. Entonces, si $\boldsymbol{\psi}$ es solución de (5.19), tendremos

$$
\begin{aligned}
\left\|\varepsilon(\boldsymbol{\psi})-\mathbb{A} \boldsymbol{\sigma}_{h}\right\|_{\mathbb{C}, \Omega}^{2} & =\left(\mathbb{C} \varepsilon(\boldsymbol{\psi})-\boldsymbol{\sigma}_{h}, \boldsymbol{\varepsilon}(\boldsymbol{\psi})-\mathbb{A} \boldsymbol{\sigma}_{h}\right)=\left(\mathbb{C} \varepsilon(\boldsymbol{\psi})-\boldsymbol{\sigma}_{h}, \boldsymbol{\varepsilon}(\mathbf{v})-\mathbb{A} \boldsymbol{\sigma}_{h}\right) \\
& \leq\left\|\mathbb{C} \varepsilon(\boldsymbol{\psi})-\boldsymbol{\sigma}_{h}\right\|_{0, \Omega}\left\|\varepsilon(\mathbf{v})-\mathbb{A} \boldsymbol{\sigma}_{h}\right\|_{0, \Omega}
\end{aligned}
$$

La prueba quedará completa si podemos mostrar que el cociente

$$
\left\|\mathbb{C} \varepsilon(\psi)-\sigma_{h}\right\|_{0, \Omega} /\left\|\varepsilon(\psi)-\mathbb{A} \boldsymbol{\sigma}_{h}\right\|_{\mathbb{C}, \Omega}
$$

está acotado por constantes que no dependen de $h$ ni del coeficiente de Lamé $\lambda_{S}$. El primer paso para lograr este objetivo será observar que

$$
\mathbb{C} \varepsilon(\boldsymbol{\psi})-\boldsymbol{\sigma}_{h}=\mathbb{C}\left(\varepsilon(\boldsymbol{\psi})-\mathbb{A} \boldsymbol{\sigma}_{h}\right)=2 \mu_{S}\left(\varepsilon(\boldsymbol{\psi})-\mathbb{A} \boldsymbol{\sigma}_{h}\right)+\lambda_{S} \operatorname{tr}\left(\varepsilon(\boldsymbol{\psi})-\mathbb{A} \boldsymbol{\sigma}_{h}\right) \mathbf{I}
$$

y que, como $\boldsymbol{\psi}$ es solución de (5.19), se verifica

$$
2 \mu_{S} \int_{\Omega}\left(\varepsilon(\psi)-\mathbb{A} \boldsymbol{\sigma}_{h}\right): \varepsilon(\mathbf{w})+\lambda_{S} \int_{\Omega} \operatorname{tr}\left(\varepsilon(\psi)-\mathbb{A} \boldsymbol{\sigma}_{h}\right) \mathbf{I}: \varepsilon(\mathbf{w})=0 \quad \forall \mathbf{w} \in H_{0, \Gamma_{D}}^{1}\left(\Omega, \mathbb{R}^{2}\right) .
$$

El segundo paso es comprobar que $\operatorname{tr}\left(\varepsilon(\psi)-\mathbb{A} \boldsymbol{\sigma}_{h}\right) \in L^{2}(\Omega, \mathbb{R})$. Luego, podremos usar el siguiente resultado (derivado del problema de Stokes en el plano): existe una constante positiva $\gamma>0$, que solo depende de $\Omega$, tal que ${ }^{2}$

$$
\sup _{\mathbf{w} \in H_{0, \Gamma_{D}}^{1}\left(\Omega, \mathbb{R}^{2}\right)} \frac{(\operatorname{div} \mathbf{w}, q)}{\|\nabla \mathbf{w}\|_{0, \Omega}} \geq \gamma\|q\|_{0, \Omega} \quad \forall q \in L^{2}(\Omega, \mathbb{R}) .
$$

Entonces, teniendo en cuenta que $\boldsymbol{\varepsilon}(\boldsymbol{\psi})-\mathbb{A} \boldsymbol{\sigma}_{h}$ es un tensor simétrico,

$$
\begin{aligned}
\lambda_{S}\left\|\operatorname{tr}\left(\varepsilon(\boldsymbol{\psi})-\mathbb{A} \boldsymbol{\sigma}_{h}\right)\right\|_{0, \Omega} & \leq \frac{1}{\gamma} \sup _{\mathbf{w} \in H_{0, \Gamma_{D}}^{1}\left(\Omega, \mathbb{R}^{2}\right)} \frac{\left(\operatorname{tr} \nabla \mathbf{w}, \lambda_{S} \operatorname{tr}\left(\varepsilon(\boldsymbol{\psi})-\mathbb{A} \boldsymbol{\sigma}_{h}\right)\right)}{\|\nabla \mathbf{w}\|_{0, \Omega}} \\
& =\frac{1}{\gamma} \sup _{\mathbf{w} \in H_{0, \Gamma_{D}}^{1}\left(\Omega, \mathbb{R}^{2}\right)} \frac{\left(\nabla \mathbf{w}, \lambda_{S} \operatorname{tr}\left(\varepsilon(\boldsymbol{\psi})-\mathbb{A} \boldsymbol{\sigma}_{h}\right) \mathbf{I}\right)}{\|\nabla \mathbf{w}\|_{0, \Omega}} \\
& =\frac{1}{\gamma} \sup _{\mathbf{w} \in H_{0, \Gamma_{D}}^{1}\left(\Omega, \mathbb{R}^{2}\right)} \frac{\left(\varepsilon(\mathbf{w}), \lambda_{S} \operatorname{tr}\left(\varepsilon(\boldsymbol{\psi})-\mathbb{A} \boldsymbol{\sigma}_{h}\right) \mathbf{I}\right)}{\|\nabla \mathbf{w}\|_{0, \Omega}} \\
& =\frac{1}{\gamma} \sup _{\mathbf{w} \in H_{0, \Gamma_{D}}^{1}\left(\Omega, \mathbb{R}^{2}\right)} \frac{\left(\varepsilon(\mathbf{w}),-2 \mu_{S}\left(\varepsilon(\boldsymbol{\psi})-\mathbb{A} \boldsymbol{\sigma}_{h}\right)\right.}{\|\nabla \mathbf{w}\|_{0, \Omega}} \\
& =\frac{1}{\gamma} \sup _{\mathbf{w} \in H_{0, \Gamma_{D}}^{1}\left(\Omega, \mathbb{R}^{2}\right)} \frac{\left(\nabla \mathbf{w},-2 \mu_{S}\left(\varepsilon(\boldsymbol{\psi})-\mathbb{A} \boldsymbol{\sigma}_{h}\right)\right.}{\|\nabla \mathbf{w}\|_{0, \Omega}} \\
& \leq \frac{2 \mu_{S}}{\gamma}\left\|\varepsilon(\boldsymbol{\psi})-\mathbb{A} \boldsymbol{\sigma}_{h}\right\|_{0, \Omega} .
\end{aligned}
$$

Luego, combinando la estimación anterior con la ecuación (5.20),

$$
\left\|\mathbb{C} \boldsymbol{\varepsilon}(\boldsymbol{\psi})-\boldsymbol{\sigma}_{h}\right\|_{0, \Omega} \leq 2 \mu_{S}\left(1+\frac{1}{\gamma}\right)\left\|\boldsymbol{\varepsilon}(\boldsymbol{\psi})-\mathbb{A} \boldsymbol{\sigma}_{h}\right\|_{0, \Omega} \leq \sqrt{2 \mu_{S}}\left(1+\frac{1}{\gamma}\right)\left\|\varepsilon(\boldsymbol{\psi})-\mathbb{A} \boldsymbol{\sigma}_{h}\right\|_{\mathbb{C}, \Omega}
$$

de donde se obtiene

$$
\left\|\varepsilon(\boldsymbol{\psi})-\mathbb{A} \boldsymbol{\sigma}_{h}\right\|_{0, \Omega} /\left\|\varepsilon(\boldsymbol{\psi})-\mathbb{A} \boldsymbol{\sigma}_{h}\right\|_{\mathbb{C}, \Omega} \leq \sqrt{2 \mu_{S}}\left(1+\frac{1}{\gamma}\right) .
$$

Así, concluimos la prueba.

Observación 5.28 La constante $\gamma$ puede acotarse aproximadamente resolviendo problemas (discretos) de autovalores asociados con las ecuaciones de Stokes [56]. Por ejemplo, para $\Omega$ un L-dominio y $\Gamma_{D}=\partial \Omega$, Stoyan encontró que $\gamma \approx 0.3101$ utilizando el método de los elementos finitos de Crouzeix-Raviart sobre una grilla de triángulos uniforme [161].

\footnotetext{
${ }^{2}$ Para toda $q \in L^{2}(\Omega, \mathbb{R})$, existe $\mathbf{w} \in H_{0, \Gamma_{D}}^{1}\left(\Omega, \mathbb{R}^{2}\right)$ tal que $\operatorname{div} \mathbf{w}=q \mathrm{y}\|\mathbf{w}\|_{1, \Omega} \lesssim\|q\|_{0, \Omega}$. Consecuentemente, la condición inf-sup se satisface.
} 
El teorema anterior nos permite calcular una cota superior para la parte no-conforme del error en la variable tensorial; es decir, el término de error $\left\|\boldsymbol{\varepsilon}(\boldsymbol{\psi})-\mathbb{A} \boldsymbol{\sigma}_{h}\right\|_{\mathbb{C}, \Omega}$, eligiendo simplemente una función en $H_{0, \Gamma_{D}}^{1}\left(\Omega, \mathbb{R}^{2}\right)$ de manera adecuada. Una elección posible sería la siguiente:

$$
\widetilde{\mathbf{u}}_{h}:=\mathbf{R}_{h} \mathbf{u}_{h}^{*}
$$

donde $\mathbf{u}_{h}^{*} \in L^{2}\left(\Omega, \mathbb{R}^{2}\right)$ es la solución del problema (5.18) y $\mathbf{R}_{h}$ es el operador de post-procesamiento definido en la sección 5.1. En este caso, haciendo uso de la desigualdad triangular y del Lema 5.2,

$$
\begin{aligned}
\left\|\varepsilon\left(\widetilde{\mathbf{u}}_{h}\right)-\mathbb{A} \boldsymbol{\sigma}_{h}\right\|_{0, \Omega} & \leq\left\|\varepsilon\left(\mathbf{u}_{h}^{*}\right)-\mathbb{A} \boldsymbol{\sigma}_{h}\right\|_{0, \Omega}+\left\|\varepsilon\left(\widetilde{\mathbf{u}}_{h}-\mathbf{u}_{h}^{*}\right)\right\|_{0, \Omega} \\
& \leq\left\|\varepsilon\left(\mathbf{u}_{h}^{*}\right)-\mathbb{A} \boldsymbol{\sigma}_{h}\right\|_{0, \Omega}+\left\|\nabla\left(\widetilde{\mathbf{u}}_{h}-\mathbf{u}_{h}^{*}\right)\right\|_{0, \Omega} \\
& \leq\left\|\varepsilon\left(\mathbf{u}_{h}^{*}\right)-\mathbb{A} \boldsymbol{\sigma}_{h}\right\|_{0, \Omega}+\left(\sum_{\ell \in \mathcal{E}_{h}^{I}}|\ell|^{-1}\left\|\left[\left[\mathbf{u}_{h}^{*}\right]\right]_{\ell}\right\|_{0, \ell}^{2}+\sum_{\ell \in \mathcal{E}_{h}^{D}}|\ell|^{-1}\left\|\mathbf{u}_{h}^{*}\right\|_{0, \ell}^{2}\right)^{1 / 2} .
\end{aligned}
$$

El resultado anterior nos induce a definir los siguientes indicadores locales

$$
\begin{aligned}
\eta_{2, T} & :=\left\|\varepsilon\left(\mathbf{u}_{h}^{*}\right)-\mathbb{A} \boldsymbol{\sigma}_{h}\right\|_{0, T}, \\
\eta_{3, \ell}^{2} & :=\left\{\begin{array}{cl}
|\ell|^{-1}\left\|\left[\left[\mathbf{u}_{h}^{*}\right]\right]_{\ell}\right\|_{0, \ell}^{2} & \text { si } \ell \in \mathcal{E}_{h}^{I}, \\
2|\ell|^{-1}\left\|\mathbf{u}_{h}^{*}\right\|_{0, \ell}^{2} & \text { si } \ell \in \mathcal{E}_{h}^{D} \\
0 & \text { si } \ell \in \mathcal{E}_{h}^{N},
\end{array}\right. \\
\eta_{3, T} & :=\left(\frac{1}{2} \sum_{\ell \subset \partial T} \eta_{3, \ell}^{2}\right)^{1 / 2}
\end{aligned}
$$

y los correspondientes estimadores globales del error

$$
\eta_{2}:=\left(\sum_{T \in \mathcal{T}_{h}} \eta_{2, T}^{2}\right)^{1 / 2} \quad \text { y } \quad \eta_{3}:=\left(\sum_{T \in \mathcal{T}_{h}} \eta_{3, T}^{2}\right)^{1 / 2}
$$

Teorema 5.29 Existe una constante positiva $C$, que depende solamente del dominio $\Omega$, tal que

$$
\left\|\varepsilon(\psi)-\mathbb{A} \boldsymbol{\sigma}_{h}\right\|_{\mathbb{C}, \Omega} \leq \sqrt{2 \mu_{S}} C\left(\eta_{2}+\eta_{3}\right) .
$$

Prueba. Inmediata a partir del Teorema 5.27 y la definición de los estimadores $\eta_{2}$ y $\eta_{3}$.

Los estimadores $\eta_{2, T}$ y $\eta_{3, \ell}$ también proporcionan cotas inferiores para el error local medido en la norma $\|\cdot\|_{1, h}$. De la definición de $\eta_{2, T}$,

$$
\begin{aligned}
\left\|\varepsilon\left(\mathbf{u}_{h}^{*}\right)-\mathbb{A} \boldsymbol{\sigma}_{h}\right\|_{0, T}^{2} & =\int_{T} \varepsilon\left(\mathbf{u}_{h}^{*}-\mathbf{u}\right):\left(\varepsilon\left(\mathbf{u}_{h}^{*}\right)-\mathbb{A} \boldsymbol{\sigma}_{h}\right)+\int_{T} \mathbb{A}\left(\boldsymbol{\sigma}-\boldsymbol{\sigma}_{h}\right):\left(\varepsilon\left(\mathbf{u}_{h}^{*}\right)-\mathbb{A} \boldsymbol{\sigma}_{h}\right) \\
& \leq\left(\left\|\varepsilon\left(\mathbf{u}_{h}^{*}-\mathbf{u}\right)\right\|_{0, T}+\frac{1}{\sqrt{2 \mu_{S}}}\left\|\boldsymbol{\sigma}-\boldsymbol{\sigma}_{h}\right\|_{\mathbb{A}, T}\right)\left\|\varepsilon\left(\mathbf{u}_{h}^{*}\right)-\mathbb{A} \boldsymbol{\sigma}_{h}\right\|_{0, T}
\end{aligned}
$$

de donde obtendremos

$$
\eta_{2, T} \leq\left\|\varepsilon\left(\mathbf{u}-\mathbf{u}_{h}^{*}\right)\right\|_{0, T}+\frac{1}{\sqrt{2 \mu_{S}}}\left\|\boldsymbol{\sigma}-\boldsymbol{\sigma}_{h}\right\|_{\mathbb{A}, T}
$$

Por otro lado, como $\mathbf{u} \in H^{1+s}\left(\Omega, \mathbb{R}^{2}\right) \cap H_{0, \Gamma_{D}}^{1}\left(\Omega, \mathbb{R}^{2}\right)$, $\mathbf{u}$ es continua en $\Omega$ y $\left.\mathbf{u}\right|_{\Gamma_{D}}=0$. Entonces, es directo que

$$
\eta_{3, \ell}=|\ell|^{-1 / 2}\left\|\left[\left[\mathbf{u}_{h}^{*}\right]\right]_{\ell}\right\|_{0, \ell}=|\ell|^{-1 / 2}\left\|\left[\left[\mathbf{u}-\mathbf{u}_{h}^{*}\right]\right]_{\ell}\right\|_{0, \ell} .
$$




\subsubsection{Indicadores locales del error para los desplazamientos}

En esta sección mostraremos que los estimadores propuestos también acotan al error en la aproximación post-procesada de los desplazamientos medido en la norma discreta $\|\mid \cdot\| \|_{E, h}$.

Primero, teniendo en cuenta que $\mathbf{u}$ es continua sobre $\Omega$, tendremos inmediatamente

$$
2 \mu_{S} \sum_{\ell \in \mathcal{E}_{h}}|\ell|^{-1}\left\|\left[\left[\left(\mathbf{u}-\mathbf{u}_{h}^{*}\right)\right]\right]_{\ell}\right\|_{0, \ell}^{2}=2 \mu_{S} \sum_{\ell \in \mathcal{E}_{h}}|\ell|^{-1}\left\|\left[\left[\mathbf{u}_{h}^{*}\right]\right]_{\ell}\right\|_{0, \ell}^{2}=2 \mu_{S} \eta_{3}^{2} .
$$

Luego, procediendo como en la sección anterior, podremos escribir

$$
\begin{aligned}
\left\|\varepsilon\left(\mathbf{u}-\mathbf{u}_{h}^{*}\right)\right\|_{0, T}^{2} & =\int_{T} \mathbb{A}\left(\boldsymbol{\sigma}-\boldsymbol{\sigma}_{h}\right): \boldsymbol{\varepsilon}\left(\mathbf{u}-\mathbf{u}_{h}^{*}\right)+\int_{T}\left(\mathbb{A} \boldsymbol{\sigma}_{h}-\boldsymbol{\varepsilon}\left(\mathbf{u}_{h}^{*}\right)\right): \boldsymbol{\varepsilon}\left(\mathbf{u}-\mathbf{u}_{h}^{*}\right) \\
& \leq\left(\frac{1}{\sqrt{2 \mu_{S}}}\left\|\boldsymbol{\sigma}-\boldsymbol{\sigma}_{h}\right\|_{\mathbb{A}, T}+\left\|\mathbb{A} \boldsymbol{\sigma}_{h}-\boldsymbol{\varepsilon}\left(\mathbf{u}_{h}^{*}\right)\right\|_{0, T}\right)\left\|\boldsymbol{\varepsilon}\left(\mathbf{u}-\mathbf{u}_{h}^{*}\right)\right\|_{0, T},
\end{aligned}
$$

de donde obtendremos

$$
2 \mu_{S} \sum_{T \in \mathcal{T}_{h}}\left\|\varepsilon\left(\mathbf{u}-\mathbf{u}_{h}^{*}\right)\right\|_{0, T}^{2} \leq 2\left(\left\|\boldsymbol{\sigma}-\boldsymbol{\sigma}_{h}\right\|_{\mathbb{A}, \Omega}^{2}+2 \mu_{S} \eta_{2}^{2}\right) .
$$

Finalmente, de las estimaciones anteriores se deduce

$$
\sqrt{2 \mu_{S}}\left\|\left|\mathbf{u}-\mathbf{u}_{h}^{*} \|\right|_{E, h} \leq \sqrt{2}\left(\left\|\boldsymbol{\sigma}-\boldsymbol{\sigma}_{h}\right\|_{\mathbb{A}, \Omega}+\sqrt{2 \mu_{S}}\left(\eta_{2}+\eta_{3}\right)\right) .\right.
$$

\subsubsection{Estimador del error para los pares tensor/desplazamiento. Confiabilidad y eficiencia de los indicadores del error.}

Usando los estimadores definidos en las secciones previas, podemos construir un estimador del error en la aproximación de los modos propios de vibración de un sólido elástico obtenida por medio del método no-conforme de Arnold-Winther.

Definimos

$$
\eta_{T}^{2}:=\frac{1}{2 \mu_{S}} \eta_{1, T}^{2}+2 \mu_{S}\left(\eta_{2, T}^{2}+\eta_{3, T}^{2}\right)
$$

y

$$
\eta:=\left(\sum_{T \in \mathcal{T}_{h}} \eta_{T}^{2}\right)^{1 / 2}
$$

Los siguientes resultados confirman que $\eta$ determina una estimación confiable para el error en la aproximación y que los indicadores locales $\eta_{T}$ son eficientes.

Teorema 5.30 Existe una constante $C$, estrictamente positiva y que solo depende del dominio $\Omega$, de la constante de Korn $c^{+}$y de la regularidad de $\mathcal{T}_{h}$, tal que

$$
\left\|(\boldsymbol{\sigma}, \mathbf{u})-\left(\boldsymbol{\sigma}_{h}, \mathbf{u}_{h}^{*}\right)\right\|_{1, h} \leq C\left(\eta+\omega^{2} \frac{\rho_{S}}{\sqrt{2 \mu_{S}}}\left\|\mathbf{u}-\mathbf{u}_{h}\right\|_{0, \Omega}+\frac{\rho_{S}}{\sqrt{2 \mu_{S}}}\left|\omega^{2}-\omega_{h}^{2}\right|\right) .
$$

Prueba. Es una consecuencia inmediata de los Teoremas 5.24, 5.27 y 5.29 y de la estimación (5.23).

Teorema 5.31 Existe una constante positiva $C$, que depende solamente de la regularidad de los elementos en $\theta_{\ell}$, tal que

$$
\eta_{T} \leq C\left(\left\|(\boldsymbol{\sigma}, \mathbf{u})-\left(\boldsymbol{\sigma}_{h}, \mathbf{u}_{h}^{*}\right)\right\|_{1, h, T}+\frac{1}{\sqrt{2 \mu_{S}}}\left\|\boldsymbol{\sigma}-\boldsymbol{\sigma}_{h}\right\|_{0, \theta_{\ell}}+\frac{\rho_{S}}{\sqrt{2 \mu_{S}}}|\ell|\left\|\omega^{2} \mathbf{u}-\omega_{h}^{2} \mathbf{u}_{h}\right\|_{0, \theta_{\ell}}\right) .
$$

Prueba. Es una consecuencia inmediata del Teorema 5.26 y de las estimaciones (5.21)-(5.22). 


\section{APÉNDICE A}

\section{Resultados sobre la aproximación por elementos finitos.}

Enunciaremos a continuación algunos resultados sobre las propiedades de aproximación del método de los elementos finitos. Estos resultados son clásicos y sus demostraciones pueden encontrarse en varios textos (fundamentales), tales como [75, 109, 52]. Seguiremos aquí la presentación propuesta en las monografías [51] y [162].

Sea $\Omega \subset \mathbb{R}^{d}, 1 \leq d \leq 3$, un dominio poligonal. Sea $\mathcal{T}_{h}$ una descomposición de $\Omega$ en subdominios elementales $K$. El diámetro de un elemento $K$ se denotará por $h_{K}$; quedando definido el tamaño de la partición por $h:=\operatorname{máx}_{K \in \mathcal{T}_{h}} h_{K}$. Los elementos $K \in \mathcal{T}_{h}$ se asumen de forma regular en el sentido usual; es decir, si existe una constante positiva $C$ tal que

$$
\gamma(K):=\frac{h_{K}}{\rho_{K}} \leq C \quad \forall K \in \mathcal{T}_{h}
$$

siendo $\rho_{K}$ el diámetro de la bola más grande contenida en $\bar{K}$. Observemos que esta suposición incluye el caso de grillas localmente fuertemente refinadas para las cuales la razón $h / h_{K}$ podría ser muy grande para los elementos $K$ en alguna subregión crítica de $\Omega$.

Finalmente, para un triángulo o tetraedro $K$, una cota inferior para los ángulos internos de $K$ conduce a una cota superior para el cociente $\gamma(K)$ y viceversa. Luego, la condición de regularidad de un elemento triangular o tetraedral es equivalente a la siguiente condición de ángulo mínimo: existe una constante positiva $\alpha_{0}$ tal que

$$
\alpha(K):=\text { menor ángulo interior en } K \geq \alpha_{0} \quad \forall K \in \mathcal{T}_{h} \text {. }
$$

\section{A.1 Interpolaciones clásicas}

Definición A.1 Supongamos que

i) $K \subset \mathbb{R}^{d}$ es un conjunto abierto simplemente conexo cuyo borde es suave a trozos,

ii) $\mathcal{P}_{K}$ es un espacio de funciones definidas sobre $K$ (conjunto de las funciones base o funciones de forma) de dimensión finita,

iii) $\mathcal{N}_{K}$ es una base para el espacio $\left(\mathcal{P}_{K}\right)^{*}$, dual algebraico del espacio lineal $\mathcal{P}_{K}$ (conjunto de las variables nodales o grados de libertad).

Entonces, $\left(K, \mathcal{P}_{K}, \mathcal{N}_{K}\right)$ define un elemento finito.

Denotaremos a las variables nodales en $\mathcal{N}_{K}$ por $N_{1}, N_{2}, \cdots, N_{n}, n=\operatorname{dim}\left(\mathcal{P}_{K}\right)$, y a la base de $\mathcal{P}_{K}$, dual de $\mathcal{N}_{K}$, por $\phi_{1}, \phi_{2}, \cdots, \phi_{n}$; es decir

$$
N_{i}\left(\phi_{j}\right)=\delta_{i j}
$$


Definición A.2 Sean $\left(\widehat{K}, \mathcal{P}_{\widehat{K}}, \mathcal{N}_{\widehat{K}}\right)$, un elemento finito, y $F(\widehat{\mathbf{x}})$, un mapeo afin de la forma $F(\widehat{\mathbf{x}})=$ $\mathbf{A} \widehat{\mathbf{x}}+\mathbf{b}$ donde $\mathbf{A}$ es una $d \times d$-matríz no-singular, $\widehat{\mathbf{x}} y \mathbf{b}$ son vectores columna con d-componentes. El elemento finito $\left(K, \mathcal{P}_{K}, \mathcal{N}_{K}\right)$ es afin equivalente a $\left(\widehat{K}, \mathcal{P}_{\widehat{K}}, \mathcal{N}_{\widehat{K}}\right)$ si

i) $F(\widehat{K})=K$,

ii) $v \in \mathcal{P}_{K}$ si, y solo si, $v \circ F \in \mathcal{P}_{\widehat{K}}$,

iii) $N \in \mathcal{N}_{K}$ si, y solo si, la funcional lineal $\widehat{v} \mapsto N\left(\widehat{v} \circ F^{-1}\right)$ en $\mathcal{P}_{\widehat{K}}$ pertenece a $\mathcal{N}_{\widehat{K}}$.

Asumiremos que el mapeo $\zeta \rightarrow N_{i}(\zeta)$ está bien definido para $\zeta \in H^{s}(K)$, siendo $s$ un número positivo lo suficientemente grande. Entonces, podemos definir el operador de interpolación local $\mathcal{I}_{K}: H^{s}(K) \rightarrow \mathcal{P}_{K}$ por medio de

$$
\mathcal{I}_{K} \zeta:=\sum_{j=1}^{n} N_{j}(\zeta) \phi_{j}
$$

Observemos que esta definición implica que

$$
\mathcal{I}_{K} v=v \quad \forall v \in \mathcal{P}_{K}
$$

El error de interpolación para un elemento $\left(K, \mathcal{P}_{K}, \mathcal{N}_{K}\right)$ triangular o cuadrangular convexo en $\mathbb{R}^{2}$ (respectivamente, tetraedro o hexaedro en $\mathbb{R}^{3}$ ) puede ser controlado en términos de la regularidad de $K$.

Lema A.3 Supongamos que

i) el interpolador local $\mathcal{I}_{K}$ está bien definido sobre $H^{s}(K)$,

ii) $\left(K, \mathcal{P}_{K}, \mathcal{N}_{K}\right)$ es afin equivalente al elemento de referencia $\left(\widehat{K}, \mathcal{P}_{\widehat{K}}, \mathcal{N}_{\widehat{K}}\right)$,

iii) $\mathcal{P}_{m}(K) \subset \mathcal{P}_{K}$,

iv) $m$ es el entero más grande tal que $m<s$.

Entonces, $\mathcal{I}_{K}$ satisface la estimación

$$
\sum_{j=0}^{m} h_{K}^{j}\left|\zeta-\mathcal{I}_{K} \zeta\right|_{j, K} \leq C h_{K}^{s}|\zeta|_{s, K},
$$

donde la constante $C$ depende solamente de s y del supremo del cociente $\gamma(K)$.

Supongamos que las sucesivas descomposiciones de $\Omega$ forman una familia $\left\{\mathcal{T}_{h}\right\}_{h>0}$ de triangulaciones regulares; es decir, existe una constante positiva $\sigma$ tal que

$$
\gamma(K) \leq \sigma \quad \forall K \in \mathcal{T}_{h}, \quad \forall h>0
$$

Sea $l$ el orden de la derivada parcial más alta involucrada en la definición de las variables nodales. Si $\zeta \in H^{s}(\Omega), s-l-d / 2>0$, la interpolada global $\mathcal{I}_{h} v$ está bien definida en $\bar{\Omega}$ por

$$
\left.\mathcal{I}_{h} \zeta\right|_{K_{i}}=\mathcal{I}_{K_{i}} \zeta \quad \forall K_{i} \in \mathcal{T}_{h}
$$

y vale la siguiente estimación

$$
\left|\zeta-\mathcal{I}_{h} \zeta\right|_{j, \Omega} \leq C h^{s-j}|\zeta|_{s, \Omega} \quad 0 \leq j \leq s .
$$




\section{A.2 Interpolación de Arnold-Winther}

Para acotar el error en la aproximación del par tensor/desplazamiento utilizando el espacio no-conforme de Arnold-Winther, necesitaremos conocer las propiedades de aproximación de la interpolación $\boldsymbol{\Pi}_{h}^{A W}$, definida en la sección 4.1.2. Seguiremos aquí la metodología standard para obtener este tipo de resultados. Preservaremos la notación introducida en la sección 4.1.2.

Sea $\widehat{T}$ un triángulo de referencia y sea $F_{T}: \widehat{T} \rightarrow T$ un isomorfismo afin de la forma $F_{T} \hat{x}=B_{T} \hat{x}+b_{T}$. Para $\widehat{\boldsymbol{\tau}} \in \mathbf{L}^{2}\left(\widehat{T}, \mathbb{S}^{2}\right)$, se define $\boldsymbol{\tau} \in \mathbf{L}^{2}\left(T, \mathbb{S}^{2}\right)$ mediante la transformación

$$
\boldsymbol{\tau}(x)=B_{T} \widehat{\boldsymbol{\tau}}(\hat{x}) B_{T}^{t},
$$

donde $x=F_{T}(\hat{x})$. Un cálculo directo muestra que

$$
\operatorname{div} \boldsymbol{\tau}(x)=B_{T} \operatorname{div} \widehat{\boldsymbol{\tau}}(\hat{x})
$$

y, por lo tanto, tendremos que $\boldsymbol{\tau} \in H\left(\operatorname{div}, T, \mathbb{S}^{2}\right)$ si, y solo si, $\widehat{\boldsymbol{\tau}} \in H\left(\operatorname{div}, \widehat{T}, \mathbb{S}^{2}\right)$.

Observemos ahora que el operador $\boldsymbol{\Pi}_{h}^{A W}: H_{0, \Gamma_{N}}\left(\operatorname{div}, \Omega, \mathbb{S}^{2}\right) \cap H^{s}\left(\Omega, \mathbb{S}^{2}\right) \rightarrow \boldsymbol{\Sigma}_{h}$ está bien definido por medio de

$$
\begin{aligned}
& -\int_{\ell}\left(\boldsymbol{\tau}-\boldsymbol{\Pi}_{h}^{A W} \boldsymbol{\tau}\right) \mathbf{n}_{\ell} \cdot \mathbf{v}=0 \quad \forall \ell \in \mathcal{E}_{h}, \quad \forall \mathbf{v} \in \mathcal{P}_{1}\left(\ell, \mathbb{R}^{2}\right), \\
& -\int_{T}\left(\boldsymbol{\tau}-\boldsymbol{\Pi}_{h}^{A W} \boldsymbol{\tau}\right)=0 \quad \forall T \in \mathcal{T}_{h},
\end{aligned}
$$

cuando $s>1 / 2$.

Lema A.4 Para $\boldsymbol{\tau} \in H\left(\operatorname{div}, T, \mathbb{S}^{2}\right) \cap H^{s}\left(T, \mathbb{S}^{2}\right), s>1 / 2$, tendremos

$$
\left.\boldsymbol{\Pi}_{h}^{A W}\right|_{T} \boldsymbol{\tau}(x)=\left.B_{T} \boldsymbol{\Pi}_{h}^{A W}\right|_{\widehat{T}} \widehat{\boldsymbol{\tau}}(\hat{x}) B_{T}^{t} .
$$

Prueba. Es idéntica a la prueba presentada en [23].

Lema A.5 Para todo $\tau \in H_{0, \Gamma_{N}}\left(\operatorname{div}, \Omega, \mathbb{S}^{2}\right) \cap H^{s}\left(\Omega, \mathbb{S}^{2}\right), 1 / 2<s \leq 1$, existe una constante positiva, independiente de $h_{T}$, tal que

$$
\left\|\boldsymbol{\Pi}_{h}^{A W} \boldsymbol{\tau}\right\|_{0, T} \leq C\left(\|\boldsymbol{\tau}\|_{0, T}+h_{T}^{s}\|\boldsymbol{\tau}\|_{s, T}\right) \quad \forall T \in \mathcal{T}_{h}
$$

Prueba. Comenzaremos probando la estimación en el triángulo de referencia $\widehat{T}$. Sea $\left\{\boldsymbol{\eta}_{k}\right\}_{1 \leq k \leq 3}$ una base para el espacio $\mathcal{P}_{0}\left(\widehat{T}, \mathbb{S}^{2}\right)$. Para cada $\widehat{\ell}_{i}$ de $\widehat{T}$, sea $\left\{\mathbf{p}_{i, j}\right\}_{1 \leq j \leq 4}$ una base para el espacio $\mathcal{P}_{1}\left(\widehat{\ell}, \mathbb{R}^{2}\right)$. Asociadas con estas bases, introducimos las bases de Arnold-Winther $\left\{\left(\boldsymbol{\varphi}_{i, j}, \boldsymbol{\zeta}_{k}\right)\right\}$ definidas por

$$
\begin{aligned}
& -\int_{\widehat{\ell}_{i}} \boldsymbol{\varphi}_{i, j} \mathbf{n}_{\widehat{\ell}_{i}} \cdot \mathbf{p}_{r, s}=\delta_{i r} \delta_{j s} \quad 1 \leq i, r \leq 3, \quad 1 \leq j, s \leq 4, \\
& -\int_{\widehat{T}} \boldsymbol{\varphi}_{i, j}: \boldsymbol{\eta}_{k}=0 \quad 1 \leq i \leq 3, \quad 1 \leq j \leq 4, \quad 1 \leq k \leq 3, \\
& -\int_{\widehat{T}} \boldsymbol{\zeta}_{k}: \boldsymbol{\eta}_{m}=\delta_{k m} \quad 1 \leq k, m \leq 3, \\
& -\int_{\widehat{\ell}_{i}} \boldsymbol{\zeta}_{k} \mathbf{n}_{\widehat{\ell}_{i}} \cdot \mathbf{p}_{r, s}=0 \quad 1 \leq k \leq 3, \quad 1 \leq r \leq 3, \quad 1 \leq s \leq 4 .
\end{aligned}
$$

Entonces, podremos escribir

$$
\left.\boldsymbol{\Pi}_{h}^{A W} \widehat{\boldsymbol{\tau}}(\hat{x})\right|_{\widehat{T}}=\sum_{1 \leq i \leq 3} \sum_{1 \leq j \leq 4}\left(\int_{\widehat{\ell}_{i}} \widehat{\boldsymbol{\tau}}(\hat{x}) \mathbf{n}_{\widehat{\ell}_{i}} \cdot \mathbf{p}_{i, j}(\hat{x})\right) \boldsymbol{\varphi}_{i, j}(\hat{x})+\sum_{1 \leq k \leq 3}\left(\int_{\widehat{T}} \widehat{\boldsymbol{\tau}}(\hat{x}): \boldsymbol{\eta}_{k}(\hat{x})\right) \boldsymbol{\zeta}_{k}(\hat{x}) .
$$


Por la desigualdad de Cauchy-Schwarz, un teorema de trazas local y cálculos directos, llegamos a

$$
\begin{gathered}
\left|\int_{\widehat{T}} \widehat{\boldsymbol{\tau}}(\hat{x}): \boldsymbol{\eta}_{k}(\hat{x})\right| \leq\|\widehat{\boldsymbol{\tau}}\|_{0, \widehat{T}}\left\|\boldsymbol{\eta}_{k}\right\|_{0, \widehat{T}} \leq C(\widehat{T})\|\widehat{\boldsymbol{\tau}}\|_{0, \widehat{T}}, \\
\left|\int_{\widehat{\ell}_{i}} \widehat{\boldsymbol{\tau}}(\hat{x}) \mathbf{n}_{\widehat{\ell}_{i}} \cdot \mathbf{p}_{i, j}(\hat{x})\right| \leq C\left(\left|\widehat{\ell}_{i}\right|^{-1 / 2}\|\widehat{\boldsymbol{\tau}}\|_{0, \widehat{T}}+\left|\widehat{\ell}_{i}\right|^{s-1 / 2}|\widehat{\boldsymbol{\tau}}|_{s, \widehat{T}}\right)\left\|\mathbf{p}_{i, j}\right\|_{0, \widehat{\ell_{i}}} \leq C(\widehat{T})\|\widehat{\boldsymbol{\tau}}\|_{s, \widehat{T}} .
\end{gathered}
$$

Teniendo en cuenta que las normas $\left\|\boldsymbol{\varphi}_{i, j}\right\|_{0, \widehat{T}} \mathrm{y}\left\|\boldsymbol{\zeta}_{k}\right\|_{0, \widehat{T}}$ están acotadas por constantes que solo dependen de $\widehat{T}$, obtendremos finalmente

$$
\left\|\left.\boldsymbol{\Pi}_{h}^{A W}\right|_{\widehat{T}} \widehat{\boldsymbol{\tau}}\right\|_{0, \widehat{T}} \leq C\|\widehat{\boldsymbol{\tau}}\|_{s, \widehat{T}} .
$$

Ahora, en virtud del Lema A.4,

$$
\begin{aligned}
\left.\int_{T}\left|\boldsymbol{\Pi}_{h}^{A W}\right|_{T} \boldsymbol{\tau}(x)\right|^{2} d x & =\left.\int_{\widehat{T}}\left|B_{T} \boldsymbol{\Pi}_{h}^{A W}\right|_{\widehat{T}} \widehat{\boldsymbol{\tau}}(\hat{x}) B_{T}^{t}\right|^{2}\left|\operatorname{det} B_{T}\right| d \hat{x} \\
& \leq\left.\left|\operatorname{det} B_{T}\right|\left\|B_{T}\right\|^{2}\left\|B_{T}^{t}\right\|^{2} \int_{\widehat{T}}\left|\boldsymbol{\Pi}_{h}^{A W}\right|_{\widehat{T}} \widehat{\boldsymbol{\tau}}(\hat{x})\right|^{2} d \hat{x} \\
& \leq C(\widehat{T})\left|\operatorname{det} B_{T}\right|\left\|B_{T}\right\|^{2}\left\|B_{T}^{t}\right\|^{2}\|\widehat{\boldsymbol{\tau}}\|_{s, \widehat{T}}^{2}
\end{aligned}
$$

De la definición para la seminorma $|\cdot|_{s, \widehat{T}}$,

$$
\begin{aligned}
|\widehat{\boldsymbol{\tau}}|_{s, \widehat{T}}^{2}=\int_{\widehat{T}} \int_{\widehat{T}} \frac{|\widehat{\boldsymbol{\tau}}(\hat{x})-\widehat{\boldsymbol{\tau}}(\hat{y})|^{2}}{|\hat{x}-\hat{y}|^{2+2 s}} d \hat{x} d \hat{y} & =\int_{T} \int_{T} \frac{\left|B_{T}^{-1}(\boldsymbol{\tau}(x)-\boldsymbol{\tau}(y)) B_{T}^{-t}\right|^{2}}{\left|B_{T}^{-1}(x-y)\right|^{2+2 s}}\left|\operatorname{det} B_{T}^{-1}\right|^{2} d x d y \\
& \leq\left|\operatorname{det} B_{T}^{-1}\right|^{2}\left\|B_{T}^{-1}\right\|^{2}\left\|B_{T}^{-t}\right\|^{2}\left\|B_{T}\right\|^{2 s+2}|\boldsymbol{\tau}|_{s, T}^{2} .
\end{aligned}
$$

Combinando las dos expresiones anteriores con las siguientes acotaciones (ver, por ejemplo, [75])

$$
\left|\operatorname{det} B_{T}\right|=\frac{|T|}{|\widehat{T}|}, \quad\left\|B_{T}\right\| \leq \frac{h_{T}}{\rho_{\widehat{T}}}, \quad\left\|B_{T}^{-1}\right\| \leq \frac{h_{\widehat{T}}}{\rho_{T}},
$$

podremos establecer que

$$
\left\|\boldsymbol{\Pi}_{h}^{A W} \boldsymbol{\tau}\right\|_{0, T} \leq C\left(\|\boldsymbol{\tau}\|_{0, T}+\frac{h_{T}^{s}}{\rho_{\widehat{T}}^{s}}|\boldsymbol{\tau}|_{s, T}\right),
$$

estimación que nos permite completar la prueba.

Teorema A.6 Para todo $\tau \in H_{0, \Gamma_{N}}\left(\operatorname{div}, \Omega, \mathbb{S}^{2}\right) \cap H^{s}\left(\Omega, \mathbb{S}^{2}\right), 1 / 2<s \leq 1$, existe una constante estrictamente positiva $C$, independiente de $h_{T}$, tal que

$$
\left\|\boldsymbol{\tau}-\boldsymbol{\Pi}_{h}^{A W} \boldsymbol{\tau}\right\|_{0, T} \leq C h_{T}^{s}\|\boldsymbol{\tau}\|_{s, T} .
$$

Prueba. Sabemos que $\boldsymbol{\Pi}_{h}^{A W}$ está definida localmente con respecto a la triangulación $\mathcal{T}_{h}$ y que localmente preserva tensores lineales a trozos; es decir, si $\boldsymbol{\eta} \in \mathcal{P}_{1}\left(T, \mathbb{S}^{2}\right)$, tendremos $\left.\boldsymbol{\Pi}_{h}^{A W} \boldsymbol{\eta}\right|_{T}=\boldsymbol{\eta}$. Entonces, aplicando el lema anterior

$$
\left\|\boldsymbol{\tau}-\boldsymbol{\Pi}_{h}^{A W} \boldsymbol{\tau}\right\|_{0, T}=\left\|(\boldsymbol{\tau}-\boldsymbol{\eta})-\boldsymbol{\Pi}_{h}^{A W}(\boldsymbol{\tau}-\boldsymbol{\eta})\right\|_{0, T} \leq C\left(\|\boldsymbol{\tau}-\boldsymbol{\eta}\|_{0, T}+h_{T}^{s}|\boldsymbol{\tau}-\boldsymbol{\eta}|_{s, T}\right) .
$$

Concluimos la prueba eligiendo $\boldsymbol{\eta}$ igual a la proyección $L_{T}^{2}$-ortogonal de $\boldsymbol{\tau}$ sobre el espacio de los tensores constantes definidos en $T$ y aplicando el Lema A.7.

\section{A.3 Proyecciones $L^{2}$-ortogonales}

Sea $\zeta \in L^{2}(\Omega)$ y $m \geq 0$ un entero. Definimos

$$
\left.\mathbf{P}_{h} \zeta\right|_{K} \in \mathcal{P}_{m}(K), \quad \int_{K}\left(\zeta-\mathbf{P}_{h} \zeta\right) v=0 \quad \forall v \in \mathcal{P}_{m}(K) \quad \forall K \in \mathcal{T}_{h}
$$

como la proyección $L^{2}$-ortogonal de $\zeta$ sobre el espacio discreto

$$
V_{h}:=\left\{v \in L^{2}(\Omega):\left.v\right|_{K} \in \mathcal{P}_{m}(K) \quad \forall K \in \mathcal{T}_{h}\right\}
$$

El siguiente resultado puede encontrarse en [109]. 
Lema A.7 Sea $\zeta \in H^{s}(\Omega)$ para algún real $s \in[0, m+1]$. La proyección $\mathbf{P}_{h}$ satisface la estimación de error

$$
\left\|\zeta-\mathbf{P}_{h} \zeta\right\|_{0, \Omega} \leq C h^{s}|\zeta|_{s, \Omega},
$$

donde la constante, estrictamente positiva, $C$ es independiente de $h$ y de $\zeta$.

Para funciones más regulares, también puede establecerse el siguiente resultado.

Lema A.8 Sea $\zeta \in H^{1+s}(\Omega)$ para algún real $s>0$. Si $m \geq 1$, la proyección $\mathbf{P}_{h}$ satisface la estimación de error

$$
\left|\zeta-\mathbf{P}_{h} \zeta\right|_{1, \Omega} \leq C h^{s}|\zeta|_{1+s, \Omega},
$$

donde la constante, estrictamente positiva, $C$ es independiente de $h$ y de $\zeta$.

Prueba. Como $\zeta \in H^{1+s}(\Omega), \zeta \in \mathcal{C}^{0}(\Omega)$. Por lo tanto, $\mathcal{I}_{h} \zeta$, la interpolada lineal de Lagrange de $\zeta$, está bien definida en $\Omega$. Gracias a la desigualdad triangular,

$$
\left|\zeta-\mathbf{P}_{h} \zeta\right|_{1, K} \leq\left|\zeta-\mathcal{I}_{h} \zeta\right|_{1, K}+\left|\mathcal{I}_{h} \zeta-\mathbf{P}_{h} \zeta\right|_{1, K}
$$

Como $\mathcal{I}_{h} \zeta-\mathbf{P}_{h} \zeta$ es un polinomio, usaremos una desigualdad inversa (A.5) para escribir

$$
\left|\mathcal{I}_{h} \zeta-\mathbf{P}_{h} \zeta\right|_{1, K} \leq C h_{K}^{-1}\left\|\mathcal{I}_{h} \zeta-\mathbf{P}_{h} \zeta\right\|_{0, K} \leq C h_{K}^{-1}\left(\left\|\mathcal{I}_{h} \zeta-\zeta\right\|_{0, K}+\left\|\zeta-\mathbf{P}_{h} \zeta\right\|_{0, K}\right) .
$$

Finalmente, usando el Lema A.7 y la estimación A.1, tendremos

$$
\left|\zeta-\mathbf{P}_{h} \zeta\right|_{1, K} \leq C h^{s}|\zeta|_{1+s, K} .
$$

A partir de la estimación anterior, se infiere fácilmente el enunciado.

\section{A.4 Desigualdades de traza e inversa}

En las pruebas que se incluyen en este trabajo, utilizaremos varias veces las siguientes desigualdades locales:

- Sea $K$ un elemento en $\mathcal{T}_{h}$ y $\ell$ uno de sus lados. Existe una constante positiva $C$, que solo depende del ángulo mínimo en $K$, tal que, si $\zeta \in H^{\nu}(K)$, con $\nu \in(1 / 2,1]$, entonces

$$
\|\zeta\|_{0, \ell} \leq C\left(|\ell|^{-1 / 2}\|\zeta\|_{0, K}+|\ell|^{\nu-1 / 2}|\zeta|_{\nu, K}\right) .
$$

La prueba de este resultado puede encontrarse en [6] (ver el Lema 5.2 en esa referencia).

- Sea $\left(K, \mathcal{P}_{K}, \mathcal{N}_{K}\right)$, un elemento finito tal que $\mathcal{P}_{K} \subset H^{m}(K)$. Como cualquier seminorma definida sobre un espacio de dimensión finita es continua con respecto a una norma, argumentos clásicos de scaling nos conducen a la siguiente estimación inversa

$$
|\zeta|_{m, K} \leq C h_{K}^{l-m}\|\zeta\|_{l, K} \quad \forall \zeta \in \mathcal{P}_{K}, 0 \leq l \leq m
$$

donde $C$ es una constante positiva que depende del cociente $\gamma(K)$ y del espacio $\mathcal{P}_{K}$.

Las estimaciones inversas clásicas (como las estimaciones de error a priori) son usualmente globales. Si la triangulación satisface las condiciones usuales de regularidad (ver comentarios en la introducción de la sección A), la estimación inversa establece

$$
\left\|u_{h}\right\|_{H^{s}(\Omega)} \lesssim h_{\min }^{-s}\left\|u_{h}\right\|_{0, \Omega},
$$

para un rango adecuado de $s>0$ y para todas las funciones $u_{h} \in H^{s}(\Omega)$ que son polinomios a trozos (de grado $m \geq 1$ ) con respecto a la grilla utilizada en la discretización. La cantidad $h_{\min }$ representa al mínimo global de todos los diámetros en la triangulación; es decir,

$$
h_{\min }:=\min _{T \in \mathcal{T}_{h}} h_{T} .
$$

Cuando la grilla es globalmente cuasi-uniforme $\left(h \lesssim h_{\min }\right)$, las estimaciones inversas pueden ser usadas para determinar la razón de convergencia de varias cantidades aproximadas en potencias de $h$ y en varias normas. 


\section{APÉNDICE B}

\section{Cambios en la normalización de los autovectores y orden de convergencia de la aproximación.}

Examinando las pruebas de los lemas y teoremas de la sección 3.3, surge claramente que los resultados de convergencia obtenidos son válidos si los autovectores continuos y discretos están normalizados con respecto a la norma $\|\cdot\|_{h}$, i.e., cuando

$$
u:=\frac{\hat{u}}{\|\hat{u}\|_{h}} \quad y \quad u_{h}:=\frac{\hat{u}_{h}}{\left\|\hat{u}_{h}\right\|_{h}} .
$$

Supongamos entonces que $\lambda_{h}$ es un autovalor de $\mathbf{T}_{h}$, convergente a $\lambda$, y que $u_{h}$ es un vector propio (unitario) de $\mathbf{T}_{h}$ correspondiente a $\lambda_{h}$. Del Teorema 3.26 se deduce inmediatamente que existe un vector propio (unitario) $u$ de $\mathbf{T}$, correspondiente a $\lambda$, tal que

$$
\left\|u-u_{h}\right\|_{h} \leq C h^{r}
$$

A continuación probaremos que se obtienen los mismos órdenes de convergencia para la aproximación de los autovectores cuando se los normaliza de manera diferente. Sea $\|\cdot\|_{*}$ otra norma en $W(h)$. Supongamos que

$$
\|\hat{u}\|_{*}=1 \quad \mathrm{y} \quad\left\|\hat{u}_{h}\right\|_{*}=1 .
$$

Lema B.1 Existen constantes positivas $\widehat{C} y h_{0}$, tales que, si $h \leq h_{0}$, entonces

$$
\left\|\hat{u}-\hat{u}_{h}\right\|_{h} \leq \widehat{C} h^{r} \text {. }
$$

Prueba. Asumiendo (B.2), un cálculo directo nos conduce a la siguiente expresión

$$
\left\|\hat{u}-\hat{u}_{h}\right\|_{h} \leq\|\hat{u}\|_{h}\left\|u-u_{h}\right\|_{h}+\left\|u_{h}\right\|_{h}\left|\|\hat{u}\|_{h}-\left\|\hat{u}_{h}\right\|_{h}\right|=\|\hat{u}\|_{h}\left(\left\|u-u_{h}\right\|_{h}+\left|1-\frac{\left\|\hat{u}_{h}\right\|_{h}}{\|\hat{u}\|_{h}}\right|\right) .
$$

Dado que $\|u\|_{*}=1 /\|\hat{u}\|_{h} \mathrm{y}\left\|u_{h}\right\|_{*}=1 /\left\|\hat{u}_{h}\right\|_{h}$, podremos escribir

$$
\left|1-\frac{\left\|\hat{u}_{h}\right\|_{h}}{\|\hat{u}\|_{h}}\right|=\left|\frac{\left\|u_{h}\right\|_{*}-\|u\|_{*}}{\left\|u_{h}\right\|_{*}}\right| \leq\left\|\hat{u}_{h}\right\|_{h}\left\|u-u_{h}\right\|_{*} \leq\left(\left\|\hat{u}-\hat{u}_{h}\right\|_{h}+\|\hat{u}\|_{h}\right)\left\|u-u_{h}\right\|_{h} .
$$

Entonces,

$$
\left\|\hat{u}-\hat{u}_{h}\right\|_{h} \leq\|\hat{u}\|_{h}\left(1+\|\hat{u}\|_{h}\right)\left\|u-u_{h}\right\|_{h}+\|\hat{u}\|_{h}\left\|\hat{u}-\hat{u}_{h}\right\|_{h}\left\|u-u_{h}\right\|_{h} .
$$

A partir de (B.1), es claro que podremos elegir $h_{0}$ tal que $C\|\hat{u}\|_{h} h_{0}^{r}<1 / 2$. Haciendo esta elección, tendremos

$$
\left\|\hat{u}-\hat{u}_{h}\right\|_{h} \leq 2\left(1+\|\hat{u}\|_{h}\right)\|\hat{u}\|_{h}\left\|u-u_{h}\right\|_{h} \leq \widehat{C} h^{r}, \quad \forall h<h_{0} .
$$

Así, concluimos la prueba. 


\section{APÉNDICE C}

\section{Otras estimaciones a priori para el método de Arnold-Winther}

Casi todas las pruebas que daremos en este capítulo siguen la línea argumental presentada por LovadinaStenberg en [137]. Sin embargo, debido a que

- el problema aquí analizado es de autovalores,

- el método de aproximación elegido es no-conforme,

aparecen, en los cálculos para la estimación del error, términos adicionales relacionados con la discontinuidad de los tensores sobre los lados que determinan la partición del dominio y con la ausencia de la ortogonalidad de Galerkin, típica de los problemas fuente. Estas suma de dificultades impide que los resultados en [137] se puedan extender directamente para cubrir nuestro caso.

En todo este capítulo, utilizaremos la notación introducida en la sección 4.1.2 y y las definiciones de la sección 5.1 .

\section{C.1 Convergencia del método en la norma de la energía}

A continuación probaremos que el espacio de Arnold-Winther es estable con respecto a la norma $\|\cdot\|_{1, h}$.

El claro que el problema de autovalores discreto (4.45) puede escribirse también de la siguiente manera: Encontrar $\omega_{h} \in \mathbb{R},\left(\boldsymbol{\sigma}_{h}, \mathbf{u}_{h}\right) \in \mathbf{\Sigma}_{h} \times \mathbf{V}_{h},\left(\boldsymbol{\sigma}_{h}, \mathbf{u}_{h}\right) \neq(\mathbf{0}, \mathbf{0})$, tal que

$$
\mathcal{A}_{h}\left(\left(\boldsymbol{\sigma}_{h}, \mathbf{u}_{h}\right),\left(\boldsymbol{\tau}_{h}, \mathbf{v}_{h}\right)\right)=-\omega_{h}^{2} \int_{\Omega} \mathbf{u}_{h} \cdot \mathbf{v}_{h} \quad \forall\left(\boldsymbol{\tau}_{h}, \mathbf{v}_{h}\right) \in \boldsymbol{\Sigma}_{h} \times \mathbf{V}_{h}
$$

donde la forma bilineal $\mathcal{A}_{h}$ queda definida en el espacio unión $\mathbf{W}(h)$ por

$$
\mathcal{A}_{h}((\boldsymbol{\sigma}, \mathbf{u}),(\boldsymbol{\tau}, \mathbf{v})):=\int_{\Omega} \mathbb{A} \boldsymbol{\sigma}: \boldsymbol{\tau}+\int_{\Omega} \operatorname{div}_{h} \boldsymbol{\tau} \cdot \mathbf{u}+\int_{\Omega} \operatorname{div}_{h} \boldsymbol{\sigma} \cdot \mathbf{v} .
$$

Debemos probar que $\mathcal{A}_{h}$ es continua y débilmente coerciva sobre $\boldsymbol{\Sigma}_{h} \times \mathbf{V}_{h}$ con respecto a la norma $\|\cdot\|_{1, h}$, uniformenente en $h$. Observemos que será suficiente con probar que existen constantes positivas $c_{1}$ y $c_{2}$, independientes de $h$, tales que

i) $\int_{\Omega} \operatorname{div}_{h} \boldsymbol{\sigma} \cdot \mathbf{v} \leq c_{1}\|\boldsymbol{\sigma}\|_{0, \Omega}\|\mid \mathbf{v}\| \|_{E, h} \quad \forall(\boldsymbol{\sigma}, \mathbf{v}) \in \boldsymbol{\Sigma}_{h} \times \mathbf{V}_{h}$

ii) $\sup _{\boldsymbol{\tau} \in \boldsymbol{\Sigma}_{h}} \frac{\int_{\Omega} \operatorname{div}_{h} \boldsymbol{\sigma} \cdot \mathbf{v}}{\|\boldsymbol{\tau}\|_{0, \Omega}} \geq c_{2}\|\mid \mathbf{v}\| \|_{E, h} \quad \forall \mathbf{v} \in \mathbf{V}_{h}$. 
Integrando por partes, teniendo en cuenta que $\boldsymbol{\sigma}$ es un tensor simétrico, los grados de libertad que definen a los elementos de Arnold-Winther y la condición de borde (4.35) sobre $\Gamma_{N}$, obtendremos

$$
\begin{aligned}
\int_{\Omega} \operatorname{div}_{h} \boldsymbol{\sigma} \cdot \mathbf{v} & =\sum_{T \in \mathcal{T}_{h}}\left(-\int_{T} \boldsymbol{\sigma}: \nabla \mathbf{v}+\int_{\partial T} \boldsymbol{\sigma} \mathbf{n} \cdot \mathbf{v}\right) \\
& =-\sum_{T \in \mathcal{T}_{h}} \int_{T} \boldsymbol{\sigma}: \boldsymbol{\varepsilon}(\mathbf{v})+\sum_{\ell \in \mathcal{E}_{h}}\left(\int_{\ell}\{\boldsymbol{\sigma} \mathbf{n}\} \cdot[[\mathbf{v}]]+\int_{\ell}[[\boldsymbol{\sigma} \mathbf{n}]] \cdot\{\mathbf{v}\}\right) \\
& \leq\|\boldsymbol{\sigma}\|_{0, \Omega}\left(\sum_{T \in \mathcal{T}_{h}}\|\varepsilon(\mathbf{v})\|_{0, T}^{2}\right)^{1 / 2}+\left(\sum_{\ell \in \mathcal{E}_{h}}|\ell|\|\{\boldsymbol{\sigma} \mathbf{n}\}\|_{0, \ell}^{2}\right)^{1 / 2}\left(\sum_{\ell \in \mathcal{E}_{h}}|\ell|^{-1}\|[[\mathbf{v}]]\|_{0, \ell}\right)^{1 / 2} .
\end{aligned}
$$

Sean $T_{1}$ y $T_{2}$ los triángulos en $\mathcal{T}_{h}$ tales que $T_{1} \cap T_{2}=\ell$. Gracias a la regularidad de $\mathcal{T}_{h}$ y a un argumento de scaling,

$$
|\ell|^{1 / 2}\|\{\boldsymbol{\sigma} \mathbf{n}\}\|_{0, \ell} \leq C\|\boldsymbol{\sigma}\|_{0, T_{1} \cup T_{2}} .
$$

Combinando esta estimación con la obtenida previamente, podemos concluir la prueba del primer ítem. Para $\mathbf{v} \in \mathbf{V}_{h}$, existe un único $\boldsymbol{\sigma} \in \boldsymbol{\Sigma}_{h}$ tal que

$$
\begin{aligned}
& \int_{\ell} \boldsymbol{\sigma} \mathbf{n} \cdot \mathbf{w} \quad=|\ell|^{-1} \int_{\ell}[[\mathbf{v}]] \cdot \mathbf{w} \quad \forall \mathbf{w} \in \mathcal{P}_{1}\left(\ell, \mathbb{R}^{2}\right) \quad \ell \in \mathcal{E}_{h}^{I} \cup \mathcal{E}_{h}^{D} \\
& \int_{\ell}^{\boldsymbol{\sigma} \mathbf{n} \cdot \mathbf{w}}=0 \quad \forall \mathbf{w} \in \mathcal{P}_{1}\left(\ell, \mathbb{R}^{2}\right) \quad \ell \in \mathcal{E}_{h}^{N} \\
& \int_{T}^{\boldsymbol{\sigma}_{i, j}} \quad=\int_{T} \boldsymbol{\varepsilon}(\mathbf{v})_{i, j} \quad 1 \leq i, j \leq 2, \quad T \in \mathcal{T}_{h} .
\end{aligned}
$$

Teniendo en cuenta que $[[\mathbf{v} \mid \ell]] \in \mathcal{P}_{1}\left(\ell, \mathbb{R}^{2}\right)$ y $\varepsilon\left(\left.\mathbf{v}\right|_{T}\right) \in \mathcal{P}_{0}\left(T, \mathbb{S}^{2}\right)$, las condiciones anteriores permiten escribir

$$
\begin{aligned}
& \int_{\ell} \boldsymbol{\sigma} \mathbf{n} \cdot[[\mathbf{v}]]=|\ell|^{-1}\|[[\mathbf{v}]]\|_{0, \ell}^{2} \quad \ell \in \mathcal{E}_{h}^{I} \cup \mathcal{E}_{h}^{D}, \\
& \int_{\ell} \boldsymbol{\sigma} \mathbf{n} \cdot[[\mathbf{v}]]=0 \quad \ell \in \mathcal{E}_{h}^{N}, \\
& \int_{T} \boldsymbol{\sigma}: \boldsymbol{\varepsilon}(\mathbf{v})=-\|\varepsilon(\mathbf{v})\|_{0, T}^{2} .
\end{aligned}
$$

A partir de estas condiciones y usando argumentos de scaling, obtendremos

$$
\|\boldsymbol{\sigma}\|_{0, \Omega} \leq C\left(\sum_{T \in \mathcal{T}_{h}}\|\varepsilon(\mathbf{v})\|_{0, T}^{2}+\sum_{\ell \in \mathcal{E}_{h}^{I} \cup \mathcal{E}_{h}^{D}}|\ell|^{-1}\|[[\mathbf{v}]]\|_{0, \ell}^{2}\right)^{1 / 2} \leq C\|\| \mathbf{v}\|\|_{E, h} .
$$

Entonces,

$$
\begin{aligned}
\int_{\Omega} \operatorname{div}_{h} \boldsymbol{\sigma} \cdot \mathbf{v} & =\sum_{T \in \mathcal{T}_{h}} \int_{T} \operatorname{div} \boldsymbol{\sigma} \cdot \mathbf{v}=\sum_{T \in \mathcal{T}_{h}}-\int_{T} \boldsymbol{\sigma}: \nabla \mathbf{v}+\int_{\partial T} \boldsymbol{\sigma} \mathbf{n} \cdot \mathbf{v} \\
& =-\sum_{T \in \mathcal{T}_{h}} \int_{T} \boldsymbol{\sigma}: \boldsymbol{\varepsilon}(\mathbf{v})+\sum_{\ell \in \mathcal{E}_{h}} \int_{\ell}\{\boldsymbol{\sigma} \mathbf{n}\} \cdot[[\mathbf{v}]] \\
& =\sum_{T \in \mathcal{T}_{h}}\|\varepsilon(\mathbf{v})\|_{0, T}^{2}+\sum_{\ell \in \mathcal{E}_{h}}|\ell|^{-1}\|[[\mathbf{v}]]\|_{0, \ell}^{2} .
\end{aligned}
$$

Combinando las dos últimas desigualdades, podemos concluir la prueba del segundo ítem.

Ahora estamos en condiciones de probar que el espacio de Arnold-Winther es estable con respecto a la norma $\|\cdot\|_{1, h}$. El resultado se infiere del siguiente lema.

Lema C.1 Existe una constante positiva $\varsigma$, independiente de $h$, tal que

$$
\sup _{(\boldsymbol{\tau}, \mathbf{v}) \in \boldsymbol{\Sigma}_{h} \times \mathbf{V}_{h}} \frac{\mathcal{A}_{h}((\boldsymbol{\sigma}, \mathbf{u}),(\boldsymbol{\tau}, \mathbf{v}))}{\|\boldsymbol{\tau}\|_{0, \Omega}+\|\mathbf{v}\| \|_{E, h}} \geq \varsigma\left(\|\boldsymbol{\sigma}\|_{0, \Omega}+\|\| \mathbf{u}\|\|_{E, h}\right) \quad \forall(\boldsymbol{\sigma}, \mathbf{u}) \in \boldsymbol{\Sigma} \times \mathbf{V}_{h}
$$

Prueba. Es una consecuencia directa de la definición de $\mathcal{A}_{h}$, de la equivalencia entre las normas tensoriales $\|\cdot\|_{\mathbb{A}, \Omega}$ y $\|\cdot\|_{0, \Omega}$ y de los resultados anteriores. 
Teorema C.2 Sean $(\omega,(\boldsymbol{\sigma}, \mathbf{u}))$ y $\left(\omega_{h},\left(\boldsymbol{\sigma}_{h}, \mathbf{u}_{h}\right)\right)$ las soluciones de los problemas de autovalores (4.30) y (4.45), respectivamente. Existe una constante positiva $C$ tal que

$$
\left\|(\boldsymbol{\sigma}, \mathbf{u})-\left(\boldsymbol{\sigma}_{h}, \mathbf{u}_{h}\right)\right\|_{1, h} \leq C h^{s} .
$$

Prueba. A partir del Lema C.1, sabemos que existe un par $\left(\boldsymbol{\tau}_{h}, \mathbf{v}_{h}\right) \in \boldsymbol{\Sigma}_{h} \times \mathbf{V}_{h}$, con norma $\left\|\boldsymbol{\tau}_{h}\right\|_{0, \Omega}+$ $\left\|\mid \mathbf{v}_{h}\right\| \|_{E, h}=1$, tal que

$$
\left\|\boldsymbol{\sigma}_{h}-\boldsymbol{\Pi}_{h}^{A W} \boldsymbol{\sigma}\right\|_{0, \Omega}+\left\|\left|\mathbf{u}_{h}-\mathbf{P}_{h} \mathbf{u} \|\right|_{E, h} \leq \frac{1}{\varsigma} \mathcal{A}_{h}\left(\left(\boldsymbol{\sigma}_{h}-\boldsymbol{\Pi}_{h}^{A W} \boldsymbol{\sigma}, \mathbf{u}_{h}-\mathbf{P}_{h} \mathbf{u}\right),\left(\boldsymbol{\tau}_{h}, \mathbf{v}_{h}\right)\right)\right.
$$

Teniendo en cuenta que $\operatorname{div}_{h} \boldsymbol{\tau}_{h} \in \mathbf{V}_{h}$, tendremos

$$
\int_{\Omega} \operatorname{div}_{h} \boldsymbol{\tau}_{h} \cdot\left(\mathbf{u}_{h}-\mathbf{P}_{h} \mathbf{u}\right)=-\int_{\Omega} \operatorname{div}_{h} \boldsymbol{\tau}_{h} \cdot\left(\mathbf{u}-\mathbf{u}_{h}\right) \quad \forall \boldsymbol{\tau}_{h} \in \boldsymbol{\Sigma}_{h}
$$

y, dado que $\left(\boldsymbol{\sigma}_{h}, \mathbf{u}_{h}\right)$ es solución de $(4.45)$, se verifica

$$
\int_{\Omega} \mathbb{A} \boldsymbol{\sigma}_{h}: \boldsymbol{\tau}_{h}+\int_{\Omega} \operatorname{div}_{h} \boldsymbol{\tau}_{h} \cdot \mathbf{u}_{h}=0
$$

Entonces, procediendo exactamente igual a como procedimos para acotar el término de consistencia $N_{1 h}$, ecuación (4.49), llegamos a

$$
\int_{\Omega} \operatorname{div}_{h} \boldsymbol{\tau}_{h} \cdot\left(\mathbf{u}_{h}-\mathbf{P}_{h} \mathbf{u}\right)=\int_{\Omega} \mathbb{A}\left(\boldsymbol{\sigma}-\boldsymbol{\sigma}_{h}\right): \boldsymbol{\tau}_{h}-\sum_{T \in \mathcal{T}_{h}} \int_{\partial T} \mathbf{u} \cdot \boldsymbol{\tau}_{h} \mathbf{n} .
$$

Ahora, como $\boldsymbol{\sigma}_{h}-\boldsymbol{\Pi}_{h}^{A W} \boldsymbol{\sigma} \in \boldsymbol{\Sigma}_{h}$, podemos proceder de manera idéndica a como procedimos para hallar la estimación (C.1). Haciendo esto, obtendremos

$$
\int_{\Omega} \operatorname{div}_{h}\left(\boldsymbol{\sigma}_{h}-\boldsymbol{\Pi}_{h}^{A W} \boldsymbol{\sigma}\right) \cdot \mathbf{v}_{h} \leq C\left\|\boldsymbol{\sigma}_{h}-\boldsymbol{\Pi}_{h}^{A W} \boldsymbol{\sigma}\right\|_{0, \Omega}\left\|\mid \mathbf{v}_{h}\right\| \|_{E, h}
$$

Entonces, de la definición de $\mathcal{A}_{h}$ y las estimaciones anteriores, tendremos

$$
\left\|\boldsymbol{\sigma}_{h}-\boldsymbol{\Pi}_{h}^{A W} \boldsymbol{\sigma}\right\|_{0, \Omega}+\left\|\mid \mathbf{u}_{h}-\mathbf{P}_{h} \mathbf{u}\right\| \|_{E, h} \leq C\left(\left\|\boldsymbol{\sigma}_{h}-\boldsymbol{\Pi}_{h}^{A W} \boldsymbol{\sigma}\right\|_{0, \Omega}+\left\|\boldsymbol{\sigma}-\boldsymbol{\sigma}_{h}\right\|_{\mathbb{A}, \Omega}+\left|\sum_{T \in \mathcal{T}_{h}} \int_{\partial T} \mathbf{u} \cdot \boldsymbol{\tau}_{h} \mathbf{n}\right|\right) .
$$

A partir de este resultado y haciendo uso de la desigualdad triangular, llegamos a

$$
\begin{aligned}
\left\|\boldsymbol{\sigma}-\boldsymbol{\sigma}_{h}\right\|_{0, \Omega}+\left.\left\|\mathbf{u}-\mathbf{u}_{h}\right\|\right|_{E, h} \leq C & \left(\left\|\boldsymbol{\sigma}-\boldsymbol{\Pi}_{h}^{A W} \boldsymbol{\sigma}\right\|_{0, \Omega}+\left\|\mid \mathbf{u}-\mathbf{P}_{h} \mathbf{u}\right\|\left\|_{E, h}+\right\| \boldsymbol{\sigma}-\boldsymbol{\sigma}_{h} \|_{\mathbb{A}, \Omega}\right. \\
& \left.+\left|\sum_{\ell \in \mathcal{E}_{h}} \int_{\ell}\left(\mathbf{u} \cdot \mathbf{t}_{\ell}\right)\left[\left[\boldsymbol{\tau}_{h} \mathbf{n}_{\ell} \cdot \mathbf{t}_{\ell}\right]\right]\right|\right) .
\end{aligned}
$$

Observemos que, gracias al Teorema A.6, al Lema A.8 y a las estimaciones (4.52) y (5.15), solo nos falta acotar los términos de salto que aparecen en la definición de la norma $\left\||\cdot \||_{E, h}\right.$. Sean $T_{1}$ y $T_{2}$ los triángulos en $\mathcal{T}_{h}$ tales que $T_{1} \cap T_{2}=\ell$. Aplicando un teorema de trazas local a la función $\mathbf{z}=\mathbf{u}-\left.\mathbf{P}_{h} \mathbf{u}\right|_{T} \in H^{1}\left(T_{i}\right)$, $i=1,2$, tendremos

$$
\|\mathbf{z}\|_{0, \ell} \leq C\left(|\ell|^{-1 / 2}\|\mathbf{z}\|_{0, T_{i}}+|\ell|^{1 / 2}\|\nabla \mathbf{z}\|_{0, T_{i}}\right), \quad i=1,2
$$

$\mathrm{y}$, como $\mathbf{z}$ tiene valor medio igual a cero sobre $T_{i}$,

$$
\|\mathbf{z}\|_{0, T_{i}} \leq C h_{T_{i}}\|\nabla \mathbf{z}\|_{0, T_{i}}, \quad i=1,2 .
$$

Luego, para cada $\ell \in \mathcal{E}_{h}$,

$$
|\ell|^{-1 / 2}\|[[\mathbf{z}]]\|_{0, \ell} \leq C\|\nabla \mathbf{z}\|_{0, T_{1} \cup T_{2}}
$$

y, finalmente,

$$
\left.\|\mathbf{z}\|\right|_{E, h} ^{2} \leq C \sum_{T \in \mathcal{T}_{h}}\|\nabla \mathbf{z}\|_{0, T}^{2}
$$


Luego, combinando las expresiones anteriores con los Lemas 4.16 y A.8, las estimaciones (4.52) y (5.15) y la acotación (4.34) (con $\left.\mathbf{g}=\omega^{2} \mathbf{u},\|\mathbf{u}\|_{0, \Omega}=1\right)$, llegamos a

$$
\left\|\boldsymbol{\sigma}-\boldsymbol{\sigma}_{h}\right\|_{0, \Omega}+\left\|\left|\mathbf{u}-\mathbf{u}_{h} \|\right|_{E, h} \leq C h^{s},\right.
$$

de donde obtendremos fácilmente,

$$
\left\|\boldsymbol{\sigma}-\boldsymbol{\sigma}_{h}\right\|_{\mathbb{A}, \Omega}+\sqrt{2 \mu_{S}}\left\|\left|\mathbf{u}-\mathbf{u}_{h} \|\right|_{E, h} \leq C h^{s}\right.
$$

que es el resultado buscado.

Observación C.3 Se puede comprobar fácilmente, siguiendo los argumentos de la prueba anterior, que el Teorema A.6, las estimaciones (4.52), (5.15) y la acotación (4.34) nos hubieran conducido a la siguiente estimación

$$
\left\|\mid \mathbf{u}_{h}-\mathbf{P}_{h} \mathbf{u}\right\| \|_{E, h} \leq C h^{s} .
$$

El interés en este tipo de resultados radica en el hecho de que son el punto de partida para construir aproximaciones de $\mathbf{u}$ que converjan con un orden mayor que el que le corresponde a $\mathbf{u}_{h}$; la solución discreta original (ver [159]). Esta idea subyace en el análisis a posteriori del error presentado en la sección 5.3.

\section{C.2 Estimaciones del error para la solución post-procesada}

Probaremos a continuación que el campo de desplazamientos post-procesado $\mathbf{u}_{h}^{*}$ preserva el orden de convergencia del método original; es decir, se satisface la estimación (a priori) del error

$$
\left\|(\boldsymbol{\sigma}, \mathbf{u})-\left(\boldsymbol{\sigma}_{h}, \mathbf{u}_{h}^{*}\right)\right\|_{1, h} \leq C h^{s} .
$$

Recordemos que $\mathbf{u}_{h}^{*} \in \mathbf{V}_{h}^{*}$ y que $\left.\mathbf{u}_{h}^{*}\right|_{T}$ es solución del siguiente sistema:

$$
\begin{aligned}
\int_{T} \varepsilon\left(\mathbf{u}_{h}^{*}\right): \nabla \mathbf{v}_{h} & =\int_{T} \mathbb{A} \boldsymbol{\sigma}_{h}: \nabla \mathbf{v}_{h} \quad \forall \mathbf{v}_{h} \in\left(\mathbf{I}-\left.\mathbf{P}_{h}\right|_{T}\right)\left(\mathcal{P}_{3}(T)\right)^{2}, \\
\left.\mathbf{P}_{h}\right|_{T} \mathbf{u}_{h}^{*} & =\mathbf{u}_{h} .
\end{aligned}
$$

Sea $\mathcal{B}_{h}$ la forma bilineal discreta definida sobre los elementos de $\mathbf{U}_{h}$ por medio de

$$
\begin{aligned}
\mathcal{B}_{h}\left(\left(\boldsymbol{\sigma}_{h}, \mathbf{u}_{h}^{*}\right),\left(\boldsymbol{\tau}_{h}, \mathbf{v}_{h}^{*}\right)\right):= & \int_{\Omega} \mathbb{A} \boldsymbol{\sigma}_{h}: \boldsymbol{\tau}_{h}+\int_{\Omega} \operatorname{div}_{h} \boldsymbol{\tau}_{h} \cdot \mathbf{u}_{h}^{*}+\int_{\Omega} \operatorname{div}_{h} \boldsymbol{\sigma}_{h} \cdot \mathbf{v}_{h}^{*} \\
& +\sum_{T \in \mathcal{T}_{h}} \int_{T}\left(\varepsilon\left(\mathbf{u}_{h}^{*}\right)-\mathbb{A} \boldsymbol{\sigma}_{h}\right): \nabla\left(\mathbf{I}-\mathbf{P}_{h}\right) \mathbf{v}_{h}^{*} .
\end{aligned}
$$

Asociado al problema (C.2), se define el siguiente problema: Encontrar $\left(\boldsymbol{\sigma}_{h}, \mathbf{u}_{h}^{*}\right) \in \mathbf{U}_{h}$ tal que

$$
\mathcal{B}_{h}\left(\left(\boldsymbol{\sigma}_{h}, \mathbf{u}_{h}^{*}\right),\left(\boldsymbol{\tau}_{h}, \mathbf{v}_{h}^{*}\right)\right)=-\omega_{h}^{2} \rho_{S} \int_{\Omega} \mathbf{u}_{h} \cdot \mathbf{v}_{h}^{*} \quad \forall\left(\boldsymbol{\tau}_{h}, \mathbf{v}_{h}^{*}\right) \in \mathbf{U}_{h} .
$$

Los siguientes resultados probarán la equivalencia entre las soluciones del problema de autovalores discreto (4.45) y el problema fuente (C.3). Para esto, necesitaremos el siguiente lema.

Lema C.4 Para todo $\mathbf{v} \in \mathbf{V}_{h}^{*},\|\mid \mathbf{v}\|_{E, h}$ y la norma discreta de la energía, definida por

$$
|\mathbf{v}|_{h}^{2}=\sum_{T \in \mathcal{T}_{h}}\|\nabla \mathbf{v}\|_{0, T}^{2}+\sum_{\ell \in \mathcal{E}_{h}}|\ell|^{-1 / 2}\|[[\mathbf{v}]]\|_{0, \ell}^{2},
$$

son equivalentes. 
Prueba. Sea $\mathbf{v}$ un elemento arbitrario en $\mathbf{V}_{h}^{*}$. De la definición de $\varepsilon(\mathbf{v})$, se obtiene muy fácilmente $\|\varepsilon(\mathbf{v})\|_{0, T} \leq\|\nabla \mathbf{v}\|_{0, T}$. Luego, $\|\mathbf{v}\| \|_{E, h} \leq|\mathbf{v}|_{h}$.

Sea $\widetilde{\mathbf{v}}:=\mathbf{R}_{h} \mathbf{v} \in H_{0, \Gamma_{D}}^{1}$. Entonces, de la desigualdad triangular, la desigualdad de Korn y el Lema 5.2, se tendrá

$$
\begin{aligned}
\sum_{T \in \mathcal{T}_{h}}\|\nabla \mathbf{v}\|_{0, T}^{2} & \leq 2\left(\sum_{T \in \mathcal{T}_{h}}\|\nabla(\mathbf{v}-\widetilde{\mathbf{v}})\|_{0, T}^{2}+\|\nabla \widetilde{\mathbf{v}}\|_{0, \Omega}^{2}\right) \leq C\left(\|\varepsilon(\widetilde{\mathbf{v}})\|_{0, \Omega}^{2}+\sum_{\ell \in \mathcal{E}_{h}}|\ell|^{-1}\|[[\mathbf{v}]]\|_{0, \ell}^{2}\right) \\
& \leq C\left(\sum_{T \in \mathcal{T}_{h}}\|\varepsilon(\widetilde{\mathbf{v}}-\mathbf{v})\|_{0, T}^{2}+\sum_{T \in \mathcal{T}_{h}}\|\varepsilon(\mathbf{v})\|_{0, T}^{2}+\sum_{\ell \in \mathcal{E}_{h}}|\ell|^{-1}\|[[\mathbf{v}]]\|_{0, \ell}^{2}\right) \\
& \leq C\left(\sum_{T \in \mathcal{T}_{h}}\|\nabla(\widetilde{\mathbf{v}}-\mathbf{v})\|_{0, T}^{2}+\|\mathbf{v}\|_{E, h}^{2}\right) \leq C\left\||\mathbf{v} \||_{E, h}^{2},\right.
\end{aligned}
$$

resultado que nos permite establecer $|\mathbf{v}|_{h} \leq C\left\||\mathbf{v} \||_{E, h}\right.$.

Lema C.5 Sea $\left(\boldsymbol{\sigma}_{h}, \mathbf{u}_{h}^{*}\right)$ solución del problema (C.3) y definamos $\mathbf{u}_{h}=\mathbf{P}_{h} \mathbf{u}_{h}^{*}$. Entonces, $\left(\omega_{h},\left(\boldsymbol{\sigma}_{h}, \mathbf{u}_{h}\right)\right)$ es solución del problema de autovalores (4.45).

Prueba. Reemplazando $\left(\boldsymbol{\tau}_{h}, \mathbf{0}\right) \in \mathbf{U}_{h}$ en (C.3), encontramos

$$
\int_{\Omega} \mathbb{A} \boldsymbol{\sigma}_{h}: \boldsymbol{\tau}_{h}+\int_{\Omega} \operatorname{div}_{h} \boldsymbol{\tau}_{h} \cdot \mathbf{u}_{h}^{*}=0 \quad \forall \boldsymbol{\tau}_{h} \in \boldsymbol{\Sigma}_{h}
$$

Teniendo en cuenta que $\operatorname{div}_{h} \boldsymbol{\tau}_{h} \in \mathbf{V}_{h}$,

$$
\int_{\Omega} \operatorname{div}_{h} \boldsymbol{\tau}_{h} \cdot \mathbf{u}_{h}^{*}=\int_{\Omega} \operatorname{div}_{h} \boldsymbol{\tau}_{h} \cdot \mathbf{P}_{h} \mathbf{u}_{h}^{*}=\int_{\Omega} \operatorname{div}_{h} \boldsymbol{\tau}_{h} \cdot \mathbf{u}_{h}
$$

y, por lo tanto,

$$
\int_{\Omega} \mathbb{A} \boldsymbol{\sigma}_{h}: \boldsymbol{\tau}_{h}+\int_{\Omega} \operatorname{div}{ }_{h} \boldsymbol{\tau}_{h} \cdot \mathbf{u}_{h}=0 \quad \forall \boldsymbol{\tau}_{h} \in \boldsymbol{\Sigma}_{h}
$$

Reemplazando $\left(0, \mathbf{v}_{h}^{*}\right) \in \mathbf{U}_{h}$ en (C.3), encontramos

$$
\int_{\Omega} \operatorname{div}{ }_{h} \boldsymbol{\tau}_{h} \cdot \mathbf{v}_{h}^{*}=-\omega_{h}^{2} \rho_{S} \int_{\Omega} \mathbf{u}_{h} \cdot \mathbf{v}_{h}^{*} \quad \forall \mathbf{v}_{h}^{*} \in \mathbf{V}_{h}^{*},
$$

pues $\mathbf{u}_{h}^{*}$ es solución de (C.2). Observemos que, cualquiera sea $\mathbf{v}_{h}^{*} \in \mathbf{V}_{h}^{*}, \mathbf{v}_{h}=\mathbf{P}_{h} \mathbf{v}_{h}^{*} \in \mathbf{V}_{h}$. Concluimos que $\mathbf{V}_{h}=\mathbf{P}_{h} \mathbf{V}_{h}^{*}$. Luego,

$$
\int_{\Omega} \operatorname{div}{ }_{h} \boldsymbol{\tau}_{h} \cdot \mathbf{v}_{h}=-\omega_{h}^{2} \rho_{S} \int_{\Omega} \mathbf{u}_{h} \cdot \mathbf{v}_{h} \quad \forall \mathbf{v}_{h} \in \mathbf{V}_{h} .
$$

Así, $\left(\omega_{h},\left(\boldsymbol{\sigma}_{h}, \mathbf{u}_{h}\right)\right)$ es solución de (4.45).

Lema C.6 Sea $\left(\omega_{h},\left(\boldsymbol{\sigma}_{h}, \mathbf{u}_{h}\right)\right)$ solución del problema de autovalores (4.45) y sea $\mathbf{u}_{h}^{*}$ la solución del poblema (C.2). Entonces, $\left(\boldsymbol{\sigma}_{h}, \mathbf{u}_{h}^{*}\right)$ es solución del problema (C.3).

Prueba. Sea $\mathbf{v}_{h}^{*} \in \mathbf{V}_{h}^{*}$ arbitrario. Es ovbio que, $\mathbf{v}_{h}^{*}=\mathbf{P}_{h} \mathbf{v}_{h}^{*}+\left(\mathbf{I}-\mathbf{P}_{h}\right) \mathbf{v}_{h}^{*}$. Reemplazando el par $\left(\boldsymbol{\tau}_{h}, \mathbf{P}_{h} \mathbf{v}_{h}^{*}+\right.$ $\left.\left(\mathbf{I}-\mathbf{P}_{h}\right) \mathbf{v}_{h}^{*}\right)$ en la expresión de $\mathcal{B}_{h}$, encontramos

$$
\mathcal{B}_{h}\left(\left(\boldsymbol{\sigma}_{h}, \mathbf{u}_{h}^{*}\right),\left(\boldsymbol{\tau}_{h}, \mathbf{v}_{h}^{*}\right)\right)=\int_{\Omega} \mathbb{A} \boldsymbol{\sigma}_{h}: \boldsymbol{\tau}_{h}+\int_{\Omega} \operatorname{div}_{h} \boldsymbol{\tau}_{h} \cdot \mathbf{u}_{h}^{*}+\int_{\Omega} \operatorname{div}_{h} \boldsymbol{\sigma}_{h} \cdot \mathbf{v}_{h}^{*},
$$

pues, $\left(\mathbf{I}-\mathbf{P}_{h}\right) \mathbf{P}_{h} \mathbf{v}_{h}^{*}=0$ para todo $\mathbf{v}_{h}^{*},\left(\mathbf{I}-\mathbf{P}_{h}\right)$ es un operador idempotente y $\mathbf{u}_{h}^{*}$ es solución de (C.2). Luego, si $\left(\omega_{h},\left(\boldsymbol{\sigma}_{h}, \mathbf{u}_{h}\right)\right)$ es solución de (4.45),

$$
\int_{\Omega} \mathbb{A} \boldsymbol{\sigma}_{h}: \boldsymbol{\tau}_{h}+\int_{\Omega} \operatorname{div}{ }_{h} \boldsymbol{\tau}_{h} \cdot \mathbf{P}_{h} \mathbf{u}_{h}^{*}=\int_{\Omega} \mathbb{A} \boldsymbol{\sigma}_{h}: \boldsymbol{\tau}_{h}+\int_{\Omega} \operatorname{div}{ }_{h} \boldsymbol{\tau}_{h} \cdot \mathbf{u}_{h}=0
$$

y

$$
\int_{\Omega} \operatorname{div}_{h} \boldsymbol{\sigma}_{h} \cdot \mathbf{P}_{h} \mathbf{v}_{h}^{*}=\int_{\Omega} \operatorname{div}_{h} \boldsymbol{\sigma}_{h} \cdot \mathbf{v}_{h}^{*}=-\omega_{h}^{2} \rho_{S} \int_{\Omega} \mathbf{u}_{h} \cdot \mathbf{v}_{h}^{*}
$$

de donde concluimos que $\left(\boldsymbol{\sigma}_{h}, \mathbf{u}_{h}^{*}\right)$ es solución de (C.3). 
Lema C.7 Existe una constante $\varsigma$, estrictamente positiva e independiente de $h$, tal que

$$
\sup _{\left(\boldsymbol{\tau}_{h}, \mathbf{w}_{h}^{*}\right) \in \mathbf{U}_{h}} \frac{\mathcal{B}_{h}\left(\left(\boldsymbol{\sigma}_{h}, \mathbf{u}_{h}^{*}\right),\left(\boldsymbol{\tau}_{h}, \mathbf{w}_{h}^{*}\right)\right)}{\left\|\left(\boldsymbol{\tau}_{h}, \mathbf{w}_{h}^{*}\right)\right\|_{1, h}} \geq \varsigma\left\|\left(\boldsymbol{\sigma}_{h}, \mathbf{u}_{h}^{*}\right)\right\|_{1, h} \quad \forall\left(\boldsymbol{\sigma}_{h}, \mathbf{u}_{h}^{*}\right) \in \mathbf{U}_{h} .
$$

Prueba. Sea $\left(\boldsymbol{\sigma}_{h}, \mathbf{u}_{h}^{*}\right) \in \mathbf{U}_{h}$, arbitrario. Observemos que, si $\left(\boldsymbol{\tau}_{h}, \mathbf{v}_{h}\right) \in \boldsymbol{\Sigma}_{h} \times \mathbf{V}_{h}$, entonces $\left(\boldsymbol{\tau}_{h}, \mathbf{v}_{h}\right) \in \mathbf{U}_{h}$. Luego,

$$
\begin{aligned}
\mathcal{B}_{h}\left(\left(\boldsymbol{\sigma}_{h}, \mathbf{u}_{h}^{*}\right),\left(\boldsymbol{\tau}_{h}, \mathbf{v}_{h}\right)\right) & =\int_{\Omega} \mathbb{A} \boldsymbol{\sigma}_{h}: \boldsymbol{\tau}_{h}+\int_{\Omega} \operatorname{div}{ }_{h} \boldsymbol{\tau}_{h} \cdot \mathbf{u}_{h}^{*}+\int_{\Omega} \operatorname{div}{ }_{h} \boldsymbol{\sigma}_{h} \cdot \mathbf{v}_{h} \\
& =\int_{\Omega} \mathbb{A} \boldsymbol{\sigma}_{h}: \boldsymbol{\tau}_{h}+\int_{\Omega} \operatorname{div}{ }_{h} \boldsymbol{\tau}_{h} \cdot \mathbf{P}_{h} \mathbf{u}_{h}^{*}+\int_{\Omega} \operatorname{div}{ }_{h} \boldsymbol{\sigma}_{h} \cdot \mathbf{v}_{h} \\
& =\mathcal{A}_{h}\left(\left(\boldsymbol{\sigma}_{h}, \mathbf{P}_{h} \mathbf{u}_{h}^{*}\right),\left(\boldsymbol{\tau}_{h}, \mathbf{v}_{h}\right)\right) .
\end{aligned}
$$

Gracias al Lema C.1, sabemos que existe $\left(\boldsymbol{\tau}_{h}, \mathbf{v}_{h}\right) \in \boldsymbol{\Sigma}_{h} \times \mathbf{V}_{h}$ tal que

$$
\begin{aligned}
\mathcal{B}_{h}\left(\left(\boldsymbol{\sigma}_{h}, \mathbf{P}_{h} \mathbf{u}_{h}^{*}\right),\left(\boldsymbol{\tau}_{h}, \mathbf{v}_{h}\right)\right) & \geq\left\|\left(\boldsymbol{\sigma}_{h}\left\|_{0, \Omega}+\right\| \mid \mathbf{P}_{h} \mathbf{u}_{h}^{*}\right)\right\| \|_{E, h} \\
\left\|\boldsymbol{\tau}_{h}\right\|_{0, \Omega}+\left\|\mid \mathbf{v}_{h}\right\| \|_{E, h} & \leq c_{1}\left(\left\|\boldsymbol{\sigma}_{h}\right\|_{0, \Omega}+\left\|\mid \mathbf{P}_{h} \mathbf{u}_{h}^{*}\right\| \|_{E, h}\right) .
\end{aligned}
$$

Sea ahora $\mathbf{w}_{h}^{*}=\mathbf{v}_{h}+\delta \mathbf{z}_{h}^{*}$, con $\mathbf{z}_{h}^{*}=\left(\mathbf{I}-\mathbf{P}_{h}\right) \mathbf{u}_{h}^{*} \mathrm{y} \delta \in \mathbb{R}^{+}$a determinar. Gracias a la equivalencia de las normas discretas, probada en el Lema C.4, podemos aplicar el Lema 2.5 de [137] para estimar la norma del par $\left(\boldsymbol{\tau}_{h}, \mathbf{w}_{h}^{*}\right)$. En efecto,

$$
\begin{aligned}
\left\|\boldsymbol{\tau}_{h}\right\|_{0, \Omega}+\left\|\left|\mathbf{w}_{h}^{*} \|\right|_{E, h}\right. & \leq\left\|\boldsymbol{\tau}_{h}\right\|_{0, \Omega}+\left\|\left|\mathbf{v}_{h}\left\|\left.\right|_{E, h}+\delta\right\|\left\|\mathbf{z}_{h}^{*}\right\|\right|_{E, h}\right. \\
& \leq c_{1}\left(\left\|\boldsymbol{\sigma}_{h}\right\|_{0, \Omega}+\left\|\mathbf{P}_{h} \mathbf{u}_{h}^{*}\right\| \|_{E, h}\right)+\left.\delta\left\|\mathbf{z}_{h}^{*}\right\|\right|_{E, h} \\
& \leq c_{2}\left(\left\|\boldsymbol{\sigma}_{h}\right\|_{0, \Omega}+\left\|\mathbf{P}_{h} \mathbf{u}_{h}^{*}\right\|\left\|_{E, h}+\right\|\left(\mathbf{I}-\mathbf{P}_{h}\right) \mathbf{u}_{h}^{*} \|\left.\right|_{E, h}\right) \\
& \leq c_{3}\left(\left\|\boldsymbol{\sigma}_{h}\right\|_{0, \Omega}+\left\|\left|\left\|\mathbf{u}_{h}^{*}\right\|\right|_{E, h}\right)\right.
\end{aligned}
$$

de donde obtendremos

$$
\left(\left\|\boldsymbol{\tau}_{h}\right\|_{0, \Omega}^{2}+\left\|\mathbf{w}_{h}^{*}\right\|_{E, h}^{2}\right)^{1 / 2} \leq c_{3} \sqrt{2}\left(\left\|\boldsymbol{\sigma}_{h}\right\|_{0, \Omega}^{2}+\left\|\left|\mathbf{u}_{h}^{*} \|\right|_{E, h}^{2}\right)^{1 / 2} .\right.
$$

Comencemos con el par $\left(0, \mathbf{z}_{h}^{*}\right)$. Tendremos

$$
\mathcal{B}_{h}\left(\left(\boldsymbol{\sigma}_{h}, \mathbf{P}_{h} \mathbf{u}_{h}^{*}\right),\left(0, \mathbf{z}_{h}^{*}\right)\right)=\int_{\Omega} \operatorname{div}{ }_{h} \boldsymbol{\sigma}_{h} \cdot \mathbf{z}_{h}^{*}+\sum_{T \in \mathcal{T}_{h}} \int_{T}\left(\varepsilon\left(\mathbf{u}_{h}^{*}\right)-\mathbb{A} \boldsymbol{\sigma}_{h}\right): \nabla \mathbf{z}_{h}^{*} .
$$

Acotaremos cada término que aparece en el lado derecho de esta expresión por separado.

Como $\operatorname{div}_{h} \boldsymbol{\sigma}_{h} \in \mathbf{V}_{h}$, tendremos

$$
\int_{\Omega} \operatorname{div}{ }_{h} \boldsymbol{\sigma}_{h} \cdot \mathbf{z}_{h}^{*}=0
$$

Debido a la simetría del tensor $\varepsilon(\cdot)$,

$$
\begin{aligned}
\sum_{T \in \mathcal{T}_{h}} \int_{T} \boldsymbol{\varepsilon}\left(\mathbf{u}_{h}^{*}\right): \nabla \mathbf{z}_{h}^{*} & =\sum_{T \in \mathcal{T}_{h}}\left(\int_{T} \boldsymbol{\varepsilon}\left(\mathbf{z}_{h}^{*}\right): \nabla \mathbf{z}_{h}^{*}+\int_{T} \boldsymbol{\varepsilon}\left(\mathbf{P}_{h} \mathbf{u}_{h}^{*}\right): \boldsymbol{\varepsilon}\left(\mathbf{z}_{h}^{*}\right)\right) \\
& \leq \sum_{T \in \mathcal{T}_{h}}\left\|\nabla \mathbf{z}_{h}^{*}\right\|_{0, T}^{2}+\left.\left\|\mathbf{P}_{h} \mathbf{u}_{h}^{*}\right\|\right|_{E, h}\left\|\mathbf{z}_{h}^{*}\right\| \|_{E, h} .
\end{aligned}
$$

Debido a la simetría del tensor $\mathbb{A} \boldsymbol{\sigma}_{h}$,

$$
\sum_{T \in \mathcal{T}_{h}} \int_{T} \mathbb{A} \boldsymbol{\sigma}_{h}: \nabla \mathbf{z}_{h}^{*}=\sum_{T \in \mathcal{T}_{h}} \int_{T} \mathbb{A} \boldsymbol{\sigma}_{h}: \varepsilon\left(\mathbf{z}_{h}^{*}\right) \leq\left\|\mathbb{A} \boldsymbol{\sigma}_{h}\right\|_{0, \Omega}\left\|\left|\mathbf{z}_{h}^{*} \|\right|_{E, h}\right.
$$

Luego, para $\alpha \in \mathbb{R}^{+}$, podemos escribir

$$
\begin{aligned}
\mathcal{B}_{h}\left(\left(\boldsymbol{\sigma}_{h}, \mathbf{P}_{h} \mathbf{u}_{h}^{*}\right),\left(0, \mathbf{z}_{h}^{*}\right)\right) & \geq \sum_{T \in \mathcal{T}_{h}}\left\|\nabla \mathbf{z}_{h}^{*}\right\|_{0, T}^{2}-c_{1}\left(\left\|\boldsymbol{\sigma}_{h}\right\|_{0, \Omega}+\left\|\left|\mathbf{P}_{h} \mathbf{u}_{h}^{*} \|\right|_{E, h}\right)\left\|\left|\mathbf{z}_{h}^{*} \|\right|_{E, h}\right.\right. \\
& \geq \sum_{T \in \mathcal{T}_{h}}\left\|\nabla \mathbf{z}_{h}^{*}\right\|_{0, T}^{2}-\frac{c_{1}}{2 \alpha}\left(\left\|\boldsymbol{\sigma}_{h}\right\|_{0, \Omega}+\left\|\left|\mathbf{P}_{h} \mathbf{u}_{h}^{*} \|\right|_{E, h}\right)^{2}-\left.\frac{\alpha}{2}\left\|\mathbf{z}_{h}^{*}\right\|\right|_{E, h} ^{2} .\right.
\end{aligned}
$$


Como $\mathbf{z}_{h}^{*}$ tiene valor medio igual a cero sobre $\ell$, podemos usar los mismos argumentos que utilizamos en el Teorema C.2 para obtener

$$
\left\|\left|\mathbf{z}_{h}^{*}\left\|\left.\right|_{E, h} ^{2} \leq c_{4} \sum_{T \in \mathcal{T}_{h}}\right\| \nabla \mathbf{z}_{h}^{*} \|_{0, T}^{2}\right.\right.
$$

Volviendo al cálculo anterior, tendremos

$$
\mathcal{B}_{h}\left(\left(\boldsymbol{\sigma}_{h}, \mathbf{P}_{h} \mathbf{u}_{h}^{*}\right),\left(0, \mathbf{z}_{h}^{*}\right)\right) \geq \sum_{T \in \mathcal{T}_{h}}\left(1-\frac{c_{4} \alpha}{2}\right)\left\|\nabla \mathbf{z}_{h}^{*}\right\|_{0, T}^{2}-\frac{c_{1}}{2 \alpha}\left(\left\|\boldsymbol{\sigma}_{h}\right\|_{0, \Omega}+\left\|\left|\mathbf{P}_{h} \mathbf{u}_{h}^{*} \|\right|_{E, h}\right)^{2}\right.
$$

y, eligiendo $\alpha$ suficientemente chico,

$$
\mathcal{B}_{h}\left(\left(\boldsymbol{\sigma}_{h}, \mathbf{P}_{h} \mathbf{u}_{h}^{*}\right),\left(0, \mathbf{z}_{h}^{*}\right)\right) \geq c_{5}\left(\sum_{T \in \mathcal{T}_{h}}\left\|\nabla \mathbf{z}_{h}^{*}\right\|_{0, T}^{2}-\left\|\boldsymbol{\sigma}_{h}\right\|_{0, \Omega}^{2}-\left.\left\|\mathbf{P}_{h} \mathbf{u}_{h}^{*}\right\|\right|_{E, h} ^{2}\right) .
$$

A partir de las estimaciones previas, es claro que

$$
\mathcal{B}_{h}\left(\left(\boldsymbol{\sigma}_{h}, \mathbf{P}_{h} \mathbf{u}_{h}^{*}\right),\left(\boldsymbol{\tau}_{h}, \mathbf{w}_{h}^{*}\right)\right) \geq\left(1-\delta c_{5}\right)\left(\left\|\boldsymbol{\sigma}_{h}\right\|_{0, \Omega}^{2}+\left\|\left|\mathbf{P}_{h} \mathbf{u}_{h}^{*} \|\right|_{E, h}^{2}\right)+\delta c_{5} \sum_{T \in \mathcal{T}_{h}}\left\|\nabla \mathbf{z}_{h}^{*}\right\|_{0, T}^{2} .\right.
$$

De la desigualdad triangular, la definición de $\mathbf{z}_{h}^{*}$ y la estimación (C.4), obtendremos

$$
\left.\left\|\left|\mathbf{u}_{h}^{*}\left\|\left.\right|_{E, h} ^{2} \leq\right\|\right| \mathbf{P}_{h} \mathbf{u}_{h}^{*}\right\|\right|_{E, h} ^{2}+\left.\left\|\left|\mathbf{z}_{h}^{*}\left\|\left.\right|_{E, h} ^{2} \leq\right\|\right| \mathbf{P}_{h} \mathbf{u}_{h}^{*}\right\|\right|_{E, h} ^{2}+\left(1+c_{4}\right) \sum_{T \in \mathcal{T}_{h}}\left\|\nabla \mathbf{z}_{h}^{*}\right\|_{0, T}^{2} .
$$

Utilizando la estimación anterior, llegamos a

$$
\mathcal{B}_{h}\left(\left(\boldsymbol{\sigma}_{h}, \mathbf{P}_{h} \mathbf{u}_{h}^{*}\right),\left(\boldsymbol{\tau}_{h}, \mathbf{w}_{h}^{*}\right)\right) \geq\left(1-\delta c_{5}\right)\left\|\boldsymbol{\sigma}_{h}\right\|_{0, \Omega}^{2}+\left.\left(1-2 \delta c_{5}\right)\left\|\left|\mathbf{P}_{h} \mathbf{u}_{h}^{*}\left\|\left.\right|_{E, h} ^{2}+\delta c_{5} c_{6}\right\|\right| \mathbf{u}_{h}^{*}\right\|\right|_{E, h} ^{2}
$$

$\mathrm{y}$, eligiendo $\delta=1 / 2 c_{5}$, tendremos

$$
\mathcal{B}_{h}\left(\left(\boldsymbol{\sigma}_{h}, \mathbf{P}_{h} \mathbf{u}_{h}^{*}\right),\left(\boldsymbol{\tau}_{h}, \mathbf{w}_{h}^{*}\right)\right) \geq \frac{1}{2}\left\|\boldsymbol{\sigma}_{h}\right\|_{0, \Omega}^{2}+\frac{c_{6}}{2}\left\|\mathbf{u}_{h}^{*}\right\|_{E, h}^{2} .
$$

Por último, dividiendo por la norma del par $\left(\boldsymbol{\tau}_{h}, \mathbf{w}_{h}^{*}\right)$,

$$
\frac{\mathcal{B}_{h}\left(\left(\boldsymbol{\sigma}_{h}, \mathbf{P}_{h} \mathbf{u}_{h}^{*}\right),\left(\boldsymbol{\tau}_{h}, \mathbf{w}_{h}^{*}\right)\right)}{\left\|\left(\boldsymbol{\tau}_{h}, \mathbf{w}_{h}^{*}\right)\right\|_{1, h}} \geq \frac{c_{7}}{c_{3} \sqrt{2}}\left(\left\|\boldsymbol{\sigma}_{h}\right\|_{0, \Omega}^{2}+\left\|\left|\mathbf{u}_{h}^{*} \|\right|_{E, h}^{2}\right)^{1 / 2} .\right.
$$

Terminamos la prueba eligiendo $\varsigma=\frac{c_{7}}{c_{3} \sqrt{2}}$.

Teorema C.8 Sean $(\omega,(\boldsymbol{\sigma}, \mathbf{u}))$ y $\left(\boldsymbol{\sigma}_{h}, \mathbf{u}_{h}^{*}\right)$ las soluciones de los problemas (4.30) y (C.3), respectivamente. Existe una constante positiva $C$ tal que

$$
\left\|(\boldsymbol{\sigma}, \mathbf{u})-\left(\boldsymbol{\sigma}_{h}, \mathbf{u}_{h}^{*}\right)\right\|_{1, h} \leq C h^{s} .
$$

Prueba. Vamos a comenzar recordando que el par propio $(\boldsymbol{\sigma}, \mathbf{u}) \in H^{s}\left(\Omega, \mathbb{S}^{2}\right) \times H^{1+s}\left(\Omega, \mathbb{R}^{2}\right)$ y que $\operatorname{div} \boldsymbol{\sigma} \in H^{1+s}\left(\Omega, \mathbb{R}^{2}\right)$. A partir de la estimación (4.34), sustituyendo $\mathbf{g}$ por $\omega^{2} \mathbf{u}$, con $\|\mathbf{u}\|_{0, \Omega}=1$, tendremos

$$
\|\boldsymbol{\sigma}\|_{s, \Omega}+\|\operatorname{div} \boldsymbol{\sigma}\|_{1+s, \Omega}+\|\mathbf{u}\|_{1+s, \Omega} \leq C \omega^{2}\|\mathbf{u}\|_{0, \Omega} \leq C(\omega)
$$

Gracias al Lema C.7, podemos elegir un par $\left(\boldsymbol{\tau}_{h}, \mathbf{w}_{h}^{*}\right) \in \mathbf{U}_{h}$, con $\left\|\left(\boldsymbol{\tau}_{h}, \mathbf{w}_{h}^{*}\right)\right\|_{1, h}=1$, tal que

$$
\left\|\boldsymbol{\sigma}_{h}-\boldsymbol{\Pi}_{h}^{A W} \boldsymbol{\sigma}\right\|_{0, \Omega}+\left\|\left|\mathbf{u}_{h}^{*}-\mathbf{v}_{h}^{*} \|\right|_{E, h} \leq \frac{1}{\varsigma} \mathcal{B}_{h}\left(\left(\boldsymbol{\sigma}_{h}-\boldsymbol{\Pi}_{h}^{A W} \boldsymbol{\sigma}, \mathbf{u}_{h}^{*}-\mathbf{v}_{h}^{*}\right),\left(\boldsymbol{\tau}_{h}, \mathbf{w}_{h}^{*}\right)\right) .\right.
$$

De la definición de $\mathcal{B}_{h}$ y del planteo del problema (C.3), tendremos

$$
\begin{aligned}
\mathcal{B}_{h}\left(\left(\boldsymbol{\sigma}_{h}-\mathbf{\Pi}_{h}^{A W} \boldsymbol{\sigma}, \mathbf{u}_{h}^{*}-\mathbf{v}_{h}^{*}\right),\left(\boldsymbol{\tau}_{h}, \mathbf{w}_{h}^{*}\right)\right)= & -\rho_{S} \omega_{h}^{2} \int_{\Omega} \mathbf{u}_{h} \cdot \mathbf{w}_{h}^{*}-\int_{\Omega} \mathbb{A} \boldsymbol{\Pi}_{h}^{A W} \boldsymbol{\sigma}: \boldsymbol{\tau}_{h} \\
& -\int_{\Omega} \operatorname{div}_{h} \boldsymbol{\tau}_{h} \cdot \mathbf{v}_{h}^{*}-\int_{\Omega} \operatorname{div}_{h} \boldsymbol{\Pi}_{h}^{A W} \boldsymbol{\sigma}_{h} \cdot \mathbf{w}_{h}^{*} \\
& -\sum_{T \in \mathcal{T}_{h}} \int_{T}\left(\varepsilon\left(\mathbf{v}_{h}^{*}\right)-\mathbb{A} \boldsymbol{\Pi}_{h}^{A W} \boldsymbol{\sigma}\right): \nabla\left(\mathbf{I}-\mathbf{P}_{h}\right) \mathbf{w}_{h}^{*} .
\end{aligned}
$$


A partir del problema espectral (4.30) y razonando como lo hicimos en la sección 4.1.2, es fácil probar que

$$
\int_{\Omega} \mathbb{A} \boldsymbol{\sigma}: \boldsymbol{\tau}_{h}+\int_{\Omega} \operatorname{div}_{h} \boldsymbol{\tau}_{h} \cdot \mathbf{u}+\int_{\Omega} \operatorname{div}_{h} \boldsymbol{\sigma} \cdot \mathbf{w}_{h}^{*}-\sum_{\ell \in \mathcal{E}_{h}} \int_{\ell}\left(\mathbf{u} \cdot \mathbf{t}_{\ell}\right) \cdot\left[\left[\boldsymbol{\tau}_{h} \mathbf{n}_{\ell} \cdot \mathbf{t}_{\ell}\right]\right]+\rho_{S} \omega^{2} \int_{\Omega} \mathbf{u} \cdot \mathbf{w}_{h}^{*}=0 .
$$

Combinando las dos expresiones anteriores, es claro que el resultado que buscamos probar es consecuencia directa de la acotación de los siguientes términos:

$$
\begin{aligned}
& -\int_{\Omega}\left(\omega^{2} \mathbf{u}-\omega_{h}^{2} \mathbf{u}_{h}\right) \cdot \mathbf{w}_{h}^{*}, \\
& -\int_{\Omega} \mathbb{A}\left(\boldsymbol{\sigma}-\boldsymbol{\Pi}_{h}^{A W} \boldsymbol{\sigma}\right): \boldsymbol{\tau}_{h}, \\
& -\int_{\Omega} \operatorname{div}_{h} \boldsymbol{\tau}_{h} \cdot\left(\mathbf{u}-\mathbf{v}_{h}^{*}\right), \\
& \text { - } \int_{\Omega} \operatorname{div}_{h}\left(\boldsymbol{\sigma}-\mathbf{\Pi}_{h}^{A W} \boldsymbol{\sigma}\right) \cdot \mathbf{w}_{h}^{*}, \\
& \text { - } \sum_{T \in \mathcal{T}_{h}} \int_{T}\left(\varepsilon\left(\mathbf{v}_{h}^{*}\right)-\mathbb{A} \boldsymbol{\Pi}_{h}^{A W} \boldsymbol{\sigma}\right): \nabla\left(\mathbf{I}-\mathbf{P}_{h}\right) \mathbf{w}_{h}^{*}, \\
& -\sum_{\ell \in \mathcal{E}_{h}} \int_{\ell}\left(\mathbf{u} \cdot \mathbf{t}_{\ell}\right) \cdot\left[\left[\boldsymbol{\tau}_{h} \mathbf{n}_{\ell} \cdot \mathbf{t}_{\ell}\right]\right] .
\end{aligned}
$$

Acotación del primer término. Sea $P_{T}$ la proyección $L_{T}^{2}$ de $H^{1}(T)$ sobre las constantes. Entonces, $P_{T} \mathbf{w}_{h}^{*} \in$ $\mathbf{V}(T)$. Tendremos

$$
\rho_{S} \int_{\Omega}\left(\omega^{2} \mathbf{u}-\omega_{h}^{2} \mathbf{u}_{h}\right) \cdot \mathbf{w}_{h}^{*}=\rho_{S} \sum_{T \in \mathcal{T}_{h}}\left(\int_{T}\left(\omega^{2} \mathbf{u}-\omega_{h}^{2} \mathbf{u}_{h}\right) \cdot\left(\mathbf{w}_{h}^{*}-P_{T} \mathbf{w}_{h}^{*}\right)+\int_{T}\left(\omega^{2} \mathbf{u}-\omega_{h}^{2} \mathbf{u}_{h}\right) \cdot P_{T} \mathbf{w}_{h}^{*}\right) .
$$

El primer término del lado derecho de esta expresión se acota fácilmente. De hecho, usando la desigualdad de Cauchy-Schwarz, estimaciones del error standard para la proyección $P_{T}$ y el Lema C.4, obtendremos

$$
\begin{aligned}
\left|\sum_{T \in \mathcal{T}_{h}} \int_{T}\left(\omega^{2} \mathbf{u}-\omega_{h}^{2} \mathbf{u}_{h}\right) \cdot\left(\mathbf{w}_{h}^{*}-P_{T} \mathbf{w}_{h}^{*}\right)\right| & \leq C\left\|\omega^{2} \mathbf{u}-\omega_{h}^{2} \mathbf{u}_{h}\right\|_{0, \Omega}\left(\sum_{T \in \mathcal{T}_{h}} h_{T}^{2}\left\|\nabla \mathbf{w}_{h}^{*}\right\|_{0, T}^{2}\right)^{1 / 2} \\
& \leq C\left\|\omega^{2} \mathbf{u}-\omega_{h}^{2} \mathbf{u}_{h}\right\|_{0, \Omega}\left\|\left|\mathbf{w}_{h}^{*} \|\right|_{E, h}\right. \\
& \leq C\left(\left|\omega^{2}-\omega_{h}^{2}\right|\|\mathbf{u}\|_{0, \Omega}+\omega_{h}^{2}\left\|\mathbf{u}-\mathbf{u}_{h}\right\|_{0, \Omega}\right)\left\|\mid \mathbf{w}_{h}^{*}\right\| \|_{E, h} .
\end{aligned}
$$

Analicemos el término restante. Usando la definición de los problemas (4.30) y (4.45) y la propiedad del diagrama conmutativo (4.38), podremos escribir

$$
\rho_{S} \int_{T}\left(\omega^{2} \mathbf{u}-\omega_{h}^{2} \mathbf{u}_{h}\right) \cdot P_{T} \mathbf{w}_{h}^{*}=\int_{T}\left(\operatorname{div} \boldsymbol{\sigma}-\operatorname{div} \boldsymbol{\sigma}_{h}\right) \cdot P_{T} \mathbf{w}_{h}^{*}=\int_{T} \operatorname{div}\left(\boldsymbol{\Pi}_{h}^{A W} \boldsymbol{\sigma}-\boldsymbol{\sigma}_{h}\right) \cdot P_{T} \mathbf{w}_{h}^{*} .
$$

Como $\boldsymbol{\Pi}_{h}^{A W} \boldsymbol{\sigma}-\boldsymbol{\sigma}_{h} \in \boldsymbol{\Sigma}_{h}$, podemos proceder exactamente igual a como procedimos para hallar la estimación (C.1). Haciendo esto, la expresión anterior queda acotada por

$$
\left|\sum_{T \in \mathcal{T}_{h}} \int_{T} \operatorname{div}\left(\boldsymbol{\Pi}_{h}^{A W} \boldsymbol{\sigma}-\boldsymbol{\sigma}_{h}\right) \cdot P_{T} \mathbf{w}_{h}^{*}\right| \leq\left\|\boldsymbol{\Pi}_{h}^{A W} \boldsymbol{\sigma}-\boldsymbol{\sigma}_{h}\right\|_{0, \Omega}\left(\sum_{\ell \in \mathcal{E}_{h}}|\ell|^{-1}\left\|\left[\left[P_{T} \mathbf{w}_{h}^{*}\right]\right]\right\|_{0, \ell}^{2}\right)^{1 / 2} .
$$

Sean ahora $T_{1}$ y $T_{2}$ triángulos en $\mathcal{T}_{h}$ tales que $T_{1} \cap T_{2}=\ell$. Usando un teorema de trazas local, estimaciones del error standard para la proyección $P_{T}$ y el Lema C.4,

$$
\begin{aligned}
|\ell|^{-1}\left\|\left[\left[P_{T} \mathbf{w}_{h}^{*}\right]\right]\right\|_{0, \ell}^{2} & \leq 2|\ell|^{-1}\left(\left\|\left[\left[\mathbf{w}_{h}^{*}\right]\right]\right\|_{0, \ell}^{2}+\left\|\left[\left[\mathbf{w}_{h}^{*}-P_{T} \mathbf{w}_{h}^{*}\right]\right]\right\|_{0, \ell}^{2}\right) \\
& \leq C\left(|\ell|^{-1}\left\|\left[\left[\mathbf{w}_{h}^{*}\right]\right]\right\|_{0, \ell}^{2}+\left\|\nabla \mathbf{w}_{h}^{*}\right\|_{0, T_{1} \cup T_{2}}^{2}\right) \\
& \leq\left. C\left\|\left|\mathbf{w}_{h}^{*}\right|_{T_{1} \cup T_{2}}\right\|\right|_{E, h} ^{2} .
\end{aligned}
$$


Finalmente, sumando sobre todos los lados $\ell \in \mathcal{E}_{h}$, tendremos

$$
\begin{aligned}
\left|\rho_{S} \sum_{T \in \mathcal{T}_{h}} \int_{T}\left(\omega^{2} \mathbf{u}-\omega_{h}^{2} \mathbf{u}_{h}\right) \cdot P_{T} \mathbf{w}_{h}^{*}\right| & \leq C\left\|\boldsymbol{\Pi}_{h}^{A W} \boldsymbol{\sigma}-\boldsymbol{\sigma}_{h}\right\|_{0, \Omega}\left\|\left|\mathbf{w}_{h}^{*} \|\right|_{E, h}\right. \\
& \leq C\left(\left\|\boldsymbol{\sigma}-\boldsymbol{\Pi}_{h}^{A W} \boldsymbol{\sigma}\right\|_{0, \Omega}+\left\|\boldsymbol{\sigma}-\boldsymbol{\sigma}_{h}\right\|_{0, \Omega}\right)\left\|\mid \mathbf{w}_{h}^{*}\right\| \|_{E, h} .
\end{aligned}
$$

Luego, en virtud de los Teoremas 4.19, 4.21, A.6 y de la estimación (C.5), concluimos

$$
\left|\rho_{S} \int_{\Omega}\left(\omega^{2} \mathbf{u}-\omega_{h}^{2} \mathbf{u}_{h}\right) \cdot \mathbf{w}_{h}^{*}\right| \leq C h^{s}\left\|\left|\mathbf{w}_{h}^{*} \|\right|_{E, h} .\right.
$$

Acotación del segundo término. Directamente, por la desigualdad de Cauchy-Schwarz, el Lema 4.16 y la estimación (C.5),

$$
\left|\int_{\Omega} \mathbb{A}\left(\boldsymbol{\sigma}-\boldsymbol{\Pi}_{h}^{A W} \boldsymbol{\sigma}\right): \boldsymbol{\tau}_{h}\right| \leq \frac{1}{\sqrt{2 \mu_{S}}}\left\|\boldsymbol{\sigma}-\boldsymbol{\Pi}_{h}^{A W} \boldsymbol{\sigma}\right\|_{0 \Omega}\left\|\boldsymbol{\tau}_{h}\right\|_{0, \Omega} \leq C h^{s}\left\|\boldsymbol{\tau}_{h}\right\|_{0, \Omega} .
$$

Acotación del tercer término. Elijamos $\mathbf{v}_{h}^{*} \in \mathbf{V}_{h}^{*} \cap H^{1}(\Omega)$. Después de integrar por partes, usar la desigualdad de Cauchy-Schwarz, un teorema de trazas local y la estimación (4.51), obtendremos

$$
\begin{aligned}
\int_{\Omega} \operatorname{div}_{h} \boldsymbol{\tau}_{h} \cdot\left(\mathbf{u}-\mathbf{v}_{h}^{*}\right) & =\sum_{T \in \mathcal{T}_{h}}\left(-\int_{T} \nabla\left(\mathbf{u}-\mathbf{v}_{h}^{*}\right): \boldsymbol{\tau}_{h}+\int_{\partial T}\left(\mathbf{u}-\mathbf{v}_{h}^{*}\right) \cdot \boldsymbol{\tau}_{h}\right) \\
& \leq\left\|\nabla\left(\mathbf{u}-\mathbf{v}_{h}^{*}\right)\right\|_{0, \Omega}\left\|\boldsymbol{\tau}_{h}\right\|_{0, \Omega}+\sum_{\ell \in \mathcal{E}_{h}} \int_{\ell}\left[\left[\boldsymbol{\tau}_{h} \mathbf{n}_{\ell} \cdot \mathbf{t}_{\ell}\right]\right]\left(\mathbf{u}-\mathbf{v}_{h}^{*}\right) \cdot \mathbf{t}_{\ell} \\
& \leq C\left(h^{-1}\left\|\mathbf{u}-\mathbf{v}_{h}^{*}\right\|_{0, \Omega}+\left\|\nabla\left(\mathbf{u}-\mathbf{v}_{h}^{*}\right)\right\|_{0, \Omega}\right)\left\|\boldsymbol{\tau}_{h}\right\|_{0, \Omega} .
\end{aligned}
$$

Ahora, como $\mathbf{u} \in \mathcal{C}^{0}\left(\Omega, \mathbb{R}^{2}\right)$, la interpolada lineal de Lagrange de $\mathbf{u}$, denotémosla $\mathbf{u}^{I}$, estará bien definida. Más aún, $\mathbf{u}^{I} \in \mathbf{V}_{h} \cap H^{1}(\Omega)$. Entonces, eligiendo $\mathbf{v}_{h}^{*}=\mathbf{u}^{I}$ y usando resultados de interpolación standard, obtendremos

$$
\left|\int_{\Omega} \operatorname{div}_{h} \boldsymbol{\tau}_{h} \cdot\left(\mathbf{u}-\mathbf{v}_{h}^{*}\right)\right| \leq C h^{s}\|\mathbf{u}\|_{1+s, \Omega}\left\|\boldsymbol{\tau}_{h}\right\|_{0, \Omega} .
$$

Acotación del cuarto término. Nuevamente, sea $P_{T}$ la proyección $L_{T}^{2}$ de $H^{1}(T)$ sobre las constantes. Como ya hemos señalado, $P_{T} \mathbf{w}_{h}^{*} \in \mathbf{V}(T)$. A partir de la propiedad del diagrama conmutativo, tendremos

$$
\int_{\Omega} \operatorname{div}_{h}\left(\boldsymbol{\sigma}-\boldsymbol{\Pi}_{h}^{A W} \boldsymbol{\sigma}\right) \cdot \mathbf{w}_{h}^{*}=\sum_{T \in \mathcal{T}_{h}} \int_{T} \operatorname{div}\left(\boldsymbol{\sigma}-\boldsymbol{\Pi}_{h}^{A W} \boldsymbol{\sigma}\right) \cdot\left(\mathbf{w}_{h}^{*}-P_{T} \mathbf{w}_{h}^{*}\right) .
$$

Luego, usando la desigualdad de Cauchy-Schwarz y estimaciones de error standard para la proyección $P_{T}$, obtendremos, después de sumar sobre todos los $T \in \mathcal{T}_{h}$, usar el Lema C.4 y la estimación (4.48),

$$
\begin{aligned}
\left|\int_{\Omega} \operatorname{div}_{h}\left(\boldsymbol{\sigma}-\boldsymbol{\Pi}_{h}^{A W} \boldsymbol{\sigma}\right) \cdot \mathbf{w}_{h}^{*}\right| & \leq C\left\|\operatorname{div}\left(\boldsymbol{\sigma}-\boldsymbol{\Pi}_{h}^{A W} \boldsymbol{\sigma}\right)\right\|_{0, \Omega}\left(\sum_{T \in \mathcal{T}_{h}} h_{T}^{2}\left\|\nabla \mathbf{w}_{h}^{*}\right\|_{0, T}^{2}\right)^{1 / 2} \\
& \leq C\left\|\operatorname{div}\left(\boldsymbol{\sigma}-\mathbf{\Pi}_{h}^{A W} \boldsymbol{\sigma}\right)\right\|\left\|_{0, \Omega}\right\| \mid \mathbf{w}_{h}^{*}\|\|_{E, h} \\
& \leq C h^{1+s}\left\|\mid \mathbf{w}_{h}^{*}\right\| \|_{E, h} .
\end{aligned}
$$

Acotación del quinto término. Teniendo en cuenta que $\varepsilon(\mathbf{u})=\mathbb{A} \boldsymbol{\sigma}$, usando la desigualdad de CauchySchwarz, el Lema C.4, estimaciones del error standard para la interpolada de Lagrange $\mathbf{v}_{h}^{*}=\mathbf{u}^{I}$ y la estimación (C.5), obtendremos

$$
\begin{aligned}
\left|\sum_{T \in \mathcal{T}_{h}} \int_{T}\left(\varepsilon\left(\mathbf{v}_{h}^{*}\right)-\mathbb{A} \boldsymbol{\Pi}_{h}^{A W} \boldsymbol{\sigma}\right): \nabla\left(\mathbf{I}-\mathbf{P}_{h}\right) \mathbf{w}_{h}^{*}\right| & \leq \sum_{T \in \mathcal{T}_{h}}\left\|\varepsilon\left(\mathbf{u}-\mathbf{v}_{h}^{*}\right)\right\|_{0, T}\left\|\nabla \mathbf{w}_{h}^{*}\right\|_{0, T} \\
& +\sum_{T \in \mathcal{T}_{h}}\left\|\mathbb{A}\left(\boldsymbol{\sigma}-\boldsymbol{\Pi}_{h}^{A W} \boldsymbol{\sigma}\right)\right\|_{0, T}\left\|\nabla \mathbf{w}_{h}^{*}\right\|_{0, T} \\
& \leq c_{1}\left\|\nabla\left(\mathbf{u}-\mathbf{v}_{h}^{*}\right)\right\|_{0, \Omega}\left\|\mathbf{w}_{h}^{*}\right\| \|_{E, h} \\
& +c_{2}\left\|\boldsymbol{\sigma}-\boldsymbol{\Pi}_{h}^{A W} \boldsymbol{\sigma}\right\|_{0, \Omega}\left\|\mathbf{w}_{h}^{*}\right\| \|_{E, h} \\
& \leq C h^{s}\left\|\mathbf{w}_{h}^{*}\right\| \|_{E, h} .
\end{aligned}
$$


Acotación del sexto término. Observemos que este término ya ha sido acotado en la sección 4.1.2. En efecto, a partir de las estimaciones (4.52) y (C.5),

$$
\left|\sum_{\ell \in \mathcal{E}_{h}} \int_{\ell}\left(\mathbf{u} \cdot \mathbf{t}_{\ell}\right) \cdot\left[\left[\boldsymbol{\tau}_{h} \mathbf{n}_{\ell} \cdot \mathbf{t}_{\ell}\right]\right]\right| \leq C h^{s}\left\|\boldsymbol{\tau}_{h}\right\|_{0, \Omega} .
$$

Para completar la prueba, basta con usar la desigualdad triangular y que $\mathbf{v}_{h}^{*}=\mathbf{u}^{I}$, es la interpolada lineal de Lagrange de $\mathbf{u}$. 


\section{APÉNDICE D}

\section{Estimaciones básicas en dominios curvos}

Sea $\Omega$ un dominio simplemente conexo y acotado en $\mathbb{R}^{2}$, no necesariamente convexo. Asumiremos que el borde de $\Omega$, denotado por $\Gamma$, es Lipschitz continuo y suave a trozos; más específicamente, de clase $\mathcal{C}^{k}$ a trozos, con $k \geq 2$. Para un dado valor de $h$ (parámetro de la discretización), sea $\Omega_{h}$ una aproximación poligonal de $\Omega$. Denotaremos $\Gamma_{h}$ al borde de $\Omega_{h}$.

Comenzaremos esta sección enunciando una conjetura sobre el dominio $\Omega$ y los dominios aproximantes $\Omega_{h}$.

Sabemos que, para analizar las propiedades de aproximación de un método numérico, es sumamente importante conocer a priori la regularidad de las soluciones del problema continuo y que esta regularidad está íntimamente relacionada con la suavidad del dominio donde está definido el problema. Los problemas de aplicación que estudiaremos en este trabajo estarán planteados en polígonos curvilíneos. Estos dominios pueden definirse de la siguiente manera:

- el conjunto $\mathcal{C}$ de todas los puntos esquinas (o vértices) c en $\Omega$ es finito,

- todas los vértices c pertenecen a $\Gamma$,

- alrededor de un punto $\mathbf{x}_{0}$ en el borde $\Gamma$, tal que $\mathbf{x}_{0} \notin \mathcal{C}, \Omega$ es suave,

- para cada vértice $\mathbf{c} \in \mathcal{C}$, existe una vecindad $U_{\mathbf{c}}$ y un difeomorfismo $\varphi_{\mathbf{c}}: U_{\mathbf{c}} \rightarrow B_{R}$ de clase $\mathcal{C}^{1,1}$ $\left(B_{R}\right.$ es la bola unidad en $\left.\mathbb{R}^{2}\right)$ tal que:

$$
\begin{aligned}
& \varphi_{\mathbf{c}}(\mathbf{c})=(0,0), \\
& \varphi_{\mathbf{c}}\left(U_{\mathbf{c}} \cap \Omega\right)=K_{\mathbf{c}} \cap B_{R},
\end{aligned}
$$

siendo

$$
K_{\mathbf{c}}:=\left\{z \in \mathbb{C}: z=r e^{i \theta} ; r>0 \text { y } 0<\theta<\omega \text { para algún } \omega \in(0,2 \pi)\right\} .
$$
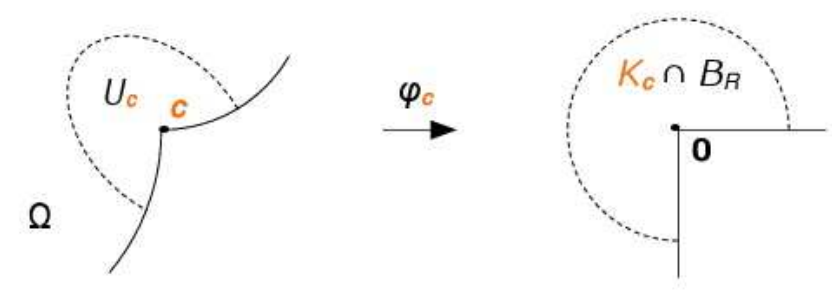

Figura D.1: Dominio poligonal curvilineo: mapeo local 
Sea ahora $\Omega_{h}$ una aproximación poligonal de $\Omega$ que satisface todas las pautas establecidas en la sección 4.2. En este contexto, tendremos

- todos los vértices de $\Omega$ son también vértices de $\Omega_{h}$,

- todos los vértices de $\Omega_{h}$ pertenecen al borde $\Gamma$.

Denotemos por $\boldsymbol{\nu}_{h}$ al vector unitario normal a $\Gamma_{h}$, positivamente orientado. En virtud de la regularidad del borde $\Gamma$,

$$
\operatorname{dist}\left(\Gamma, \Gamma_{h}\right)=\operatorname{máx}_{\mathbf{x} \in \Gamma_{h}}\left\{\left|\left(\mathbf{x}+t \boldsymbol{\nu}_{h}\right)-\mathbf{x}\right|: \mathbf{x}+t \boldsymbol{\nu}_{h} \in \Gamma\right\} \leq C h^{2} \quad \forall h<h_{0} .
$$

En esta condiciones, cada $\Omega_{h}$ representa una perturbación de $\Omega$; es decir, $\Omega_{h} \rightarrow \Omega$ cuando $h \rightarrow 0$. Esto significará, en particular, que los ángulos internos de $\Omega_{h}$ tenderán a los ángulos internos de $\Omega$ cuando $h$ vaya a cero.

En lo que sigue, $\mathcal{D}_{h}$ indicará el conjunto $\widehat{\Omega}=\Omega \cup \Omega_{h}$ o el conjunto $\Omega_{h}$, indistintamente. Sobre $\mathcal{D}_{h}$, consideraremos definidos dos problemas clásicos:

- Dirichlet: dada $f \in L^{2}\left(\mathcal{D}_{h}\right)$, encontrar $u \in H^{1}\left(\mathcal{D}_{h}\right)$ tal que

$$
\left\{\begin{aligned}
\Delta u=f & \text { en } \mathcal{D}_{h} \\
u=0 & \text { sobre } \partial \mathcal{D}_{h} .
\end{aligned}\right.
$$

- Neumann: asumiendo que $f \in L^{2}\left(\mathcal{D}_{h}\right)$ y $g \in H^{-1 / 2}\left(\partial \mathcal{D}_{h}\right)$ satisfacen la relación $\int_{\mathcal{D}_{h}} f+\int_{\partial \mathcal{D}_{h}} g=0$, encontrar $u \in H^{1}\left(\mathcal{D}_{h}\right)$ tal que

$$
\left\{\begin{array}{rll}
\Delta u & =f & \text { en } \mathcal{D}_{h} \\
\partial_{\mathbf{n}} u & =g & \text { sobre } \partial \mathcal{D}_{h}
\end{array}\right.
$$

El siguiente resultado puede encontrarse en [89] (ver también [88]).

Teorema D.1 Existe $s>3 / 2$ tal que

- Dirichlet: si $f \in H^{s-2}\left(\mathcal{D}_{h}\right)$, entonces $u \in H^{s}\left(\mathcal{D}_{h}\right)$ y vale la estimación

$$
\|u\|_{s, \mathcal{D}_{h}} \leq C_{D}\left(\mathcal{D}_{h}\right)\|f\|_{s-2, \Omega},
$$

- Neumann: si $f \in H^{s-2}\left(\mathcal{D}_{h}\right), g \in \prod_{j} H^{s-3 / 2}\left(\partial_{j} \mathcal{D}_{h}\right)$, siendo $\partial_{j} \mathcal{D}_{h}$ los lados de $\mathcal{D}_{h}$, entonces $u \in$ $H^{s}\left(\mathcal{D}_{h}\right)$ y vale la estimación

$$
\|u\|_{s, \mathcal{D}_{h}} \leq C_{N}\left(\mathcal{D}_{h}\right)\left(\|f\|_{s-2, \Omega}^{2}+\sum_{j}\|g\|_{s-3 / 2, \partial_{j} \mathcal{D}_{h}}^{2}\right)^{1 / 2} .
$$

Conjetura D.2 Sean $\Omega$ un polígono curvilíneo y $\Omega_{h}$ una aproximación de $\Omega$ construida según las pautas de la sección 3.1. Si $h<h_{0}$, las constantes $C_{D}\left(\mathcal{D}_{h}\right)$ y $C_{N}\left(\mathcal{D}_{h}\right)$ pueden acotarse uniformemente con respecto $a h$.

Observación D.3 Costabel-Dauge-McIntosh suponen que esta conjetura podría probarse, al menos en el caso bi-dimensional, formulando el problema vía integrales de borde [117].

Terminaremos esta sección exponiendo algunos resultados válidos en polígonos curvilíneos.

Dado que, en general, $\Omega_{h} \not \subset \Omega$, será necesario considerar extensiones de las funciones originalmente definidas en $\Omega$. Para ello, es conveniente introducir un dominio $\widetilde{\Omega} \subset \mathbb{R}^{d}$, con bordes suaves, tal que

$$
\widehat{\Omega} \subset \widetilde{\Omega} \quad \forall h<h_{0} .
$$


Teorema D.4 Supongamos que $\Omega$ es un dominio con borde Lipschitz. Si $0<s<1$, existe un operador (lineal) de extensión $\epsilon: H^{k+s}(\Omega) \rightarrow H^{k+s}(\widetilde{\Omega})$, definido para todos los enteros no negativos $k$, que satisface

$$
\begin{aligned}
\left.\boldsymbol{\epsilon}\right|_{\Omega} & =\varphi \quad \forall \varphi \in H^{k+s}(\Omega), \\
\|\boldsymbol{\epsilon} \varphi\|_{k-i, \widetilde{\Omega}} & \leq C\|\varphi\|_{k-i, \Omega}, \quad 0 \leq i \leq k \\
\|\boldsymbol{\epsilon} \varphi\|_{k+s, \widetilde{\Omega}} & \leq C\|\varphi\|_{k+s, \Omega},
\end{aligned}
$$

donde $C$ es una constante independiente de $\varphi$.

Prueba. En [160] se construye un operador de extensión $\mathcal{E}: H^{k}(\Omega) \rightarrow H^{k}\left(\mathbb{R}^{n}\right)$, lineal y continuo, definido para todo $k$ entero positivo. Luego, para $0<s<1$, resultados standard de interpolación en espacios de Sobolev (ver, por ejemplo, Teorema 12.2.3 en [52]) nos permiten concluir la prueba.

Por convención, si $\varphi \in H^{k+s}(\Omega), 0<s<1, \widetilde{\varphi}$ denotará siempre la aplicación $\epsilon \varphi$ definida en el Teorema D.4. Para un campo vectorial $\mathbf{v} \in H^{k+s}(\Omega)^{2}, \widetilde{\mathbf{v}}$ denotará la extensión (por componentes) definida de la siguiente manera

$$
\widetilde{\mathbf{v}}:=\left(\boldsymbol{\epsilon} v_{1}, \boldsymbol{\epsilon} v_{2}\right)
$$

Sea $\mathcal{T}_{h}$ una partición de $\bar{\Omega}_{h}$, la cual consiste en un número finito de triángulos cerrados $\bar{T}$. Asumiremos que la partición $\mathcal{T}_{h}$ fue construida de manera tal que se satisfacen todas las propiedades establecidas en la sección 4.2. A continuación, daremos algunos resultados que son consecuencia directa de esas propiedades. Preservaremos la notación introducida en la sección 4.2 .

Sea $T \in \mathcal{B}_{h}$ un elemento de borde en $\Omega_{h}$. Sea $\omega_{T}$ el dominio definido por la diferencia $T^{i d} \backslash T$ o $T \backslash T^{i d}$, según corresponda. Como consecuencia de la regularidad de $\Gamma$, valen las siguientes estimaciones.

Lema D.5 Existe una constante $C>0$, independiente de $T$, tal que

$$
\begin{gathered}
\left|\omega_{T}\right| \leq C h_{T}^{3}, \\
\left|\Omega \backslash \Omega_{h}\right|+\left|\Omega_{h} \backslash \Omega\right| \leq C h^{2} .
\end{gathered}
$$

Prueba. La primera estimación es una consecuencia directa de las propiedades de la interpolación lineal (ver, e.g., [104]). La segunda estimación, puede probarse fácilmente teniendo en cuenta que $\left(\Omega \backslash \Omega_{h}\right) \cup$ $\left(\Omega_{h} \backslash \Omega\right)=\bigcup\left\{\omega_{T}: T \in \mathcal{B}_{h}\right\}$.

Lema D.6 Existe una constante positiva $C$ tal que:

$$
\begin{array}{cl}
\|v\|_{0, \Omega \backslash \bar{\Omega}_{h}} \leq C h^{s}\|v\|_{s, \Omega} & \forall v \in H^{s}(\Omega), \quad 0 \leq s \leq 1, \\
\|v\|_{0, \Omega_{h} \backslash \bar{\Omega}} \leq C h^{s}\|v\|_{s, \Omega_{h}} & \forall v \in H^{s}\left(\Omega_{h}\right), \quad 0 \leq s \leq 1 .
\end{array}
$$

Prueba. Las desigualdades pueden probarse para $s=1$ siguiendo los argumentos usados en la prueba del Lemma 3.3.11 en [104]. Como las dos desigualdades son trivialmente ciertas para $s=0$, las estimaciones para $0<s<1$ pueden obtenerse a partir de resultados clásicos de interpolación en espacios de Sobolev (Teorema 1.4 en [109], por ejemplo).

Lema D.7 Existe una constante positiva $C$, tal que, si $h$ es suficientemente pequeño, entonces

$$
\begin{aligned}
& \|v\|_{0, \omega_{T}} \leq C\left(\|v\|_{0, T}+h_{T}^{2 s}\|v\|_{s, T^{i d}}\right) \quad \forall v \in H^{s}\left(T^{i d}\right), \quad 0 \leq s \leq 1, \quad \text { if } T \subset T^{i d} \\
& \|v\|_{0, \omega_{T}} \leq C\left(\|v\|_{0, T^{i d}}+h_{T}^{2 s}\|v\|_{s, T}\right) \quad \forall v \in H^{s}(T), \quad 0 \leq s \leq 1, \quad \text { if } T^{i d} \subset T \text {. }
\end{aligned}
$$

Prueba. Es idéntica a la prueba del Lemma 2.5 en [116].

Definición D.8 Sea $\left.v\right|_{T} \in \mathcal{P}_{k}(T), k \geq 1$, y supongamos que $T \subset T^{i d}$. Llamaremos extensión natural de $v$ de $T$ a $T^{i d}$ a la función

$$
\bar{v}: T^{i d} \rightarrow \mathbb{R} \quad \text { tal que }\left.\bar{v}\right|_{T^{i d}}=\left.p\right|_{T^{i d}}
$$

siendo $p$ el elemento de $\mathcal{P}^{k}\left(\mathbb{R}^{2}\right)$ que verifica $\left.p\right|_{T}=\left.v\right|_{T}$. 
Lema D.9 Existen constantes positivas, c y $C$, que no dependen de $T$, tales que, para $v_{h} \in \mathcal{P}_{1}\left(\mathbb{R}^{2}\right)$ y $h$ suficientemente pequeño,

$$
\begin{gathered}
c\left\|v_{h}\right\|_{0, T^{i d}} \leq\left\|v_{h}\right\|_{0, T} \leq C\left\|v_{h}\right\|_{0, T^{i d}}, \\
c\left|v_{h}\right|_{1, T^{i d}} \leq\left|v_{h}\right|_{1, T} \leq C\left|v_{h}\right|_{1, T^{i d}} .
\end{gathered}
$$

Prueba. Ver la prueba del Lema 3.3.11 en [104].

Definición D.10 Sea $w_{h} \in\left\{p \in H_{0}^{1}\left(\Omega_{h}\right):\left.p\right|_{T} \in \mathcal{P}_{1}(T) T \in \mathcal{T}_{h}\right\}$. Una función $w^{a} \in H_{0}^{1}(\Omega)$ se dice asociada a $w_{h}$ si satisface las siguientes propiedades:

- $w^{a} \in C^{0}(\bar{\Omega})$,

- $w^{a}\left(P_{i}\right)=w_{h}\left(P_{i}\right) \quad \forall P_{i} \in \mathcal{V}_{h}$,

- $w^{a}$ es lineal en cada tetraedro (triángulo) $T \in \mathcal{T}_{h} \cap \mathcal{T}_{h}^{i d}$,

- si $T \subset T^{i d}, w^{a}=0$ en $T^{i d} \backslash T$ y $w^{a}=w_{h}$ en $T$,

- si $T^{i d} \subset T,\left.w^{a}\right|_{\partial T^{i d} \subset \Gamma}=0$.

La definición anterior se debe a Feistauer-Ženíšek [104]. La construcción de tal función sigue básicamente la teoría de interpolación desarrollada por Zlámal [179] para elementos finitos curvos bi-dimensionales.

Lema D.11 Sea $w_{h}$ como en la definición anterior. Sea $w^{a} \in H_{0}^{1}(\Omega)$ la función asociada a $w_{h}$. Sea $T$ un elemento en el borde de $\Omega_{h}$. Si $T^{\text {id }} \subset T$, entonces

$$
\left\|w^{a}-w_{h}\right\|_{1, T^{i d}} \leq C h_{T}\left\|w_{h}\right\|_{1, T},
$$

donde $C$ es una constante independiente de $h_{T}$.

Prueba. Es una consecuencia directa de la Definición D.10 y de una extensión adecuada del Teorema 2 en [179].

Observación D.12 A partir de la definición D.10, aplicando el Lema D.11 y teniendo en cuenta la desigualdad de Friedrichs-Poincaré ${ }^{1}$, es inmediato que

$$
\left\|w^{a}-w_{h}^{e}\right\|_{1, \Omega}=\left(\sum_{\substack{T \in \mathcal{B}_{h} \\ T^{i d} \subset T}}\left\|w^{a}-w_{h}^{e}\right\|_{1, T^{i d}}^{2}\right)^{1 / 2} \leq C h\left|w_{h}\right|_{1, \Omega_{h}} .
$$

\footnotetext{
${ }^{1}$ Sea $\Omega$ un dominio acotado. Existe una constante positiva $C(\Omega)$ tal que, para toda $w \in H_{0}^{1}(\Omega),\|w\|_{0, \Omega} \leq C(\Omega)|w|_{1, \Omega}$. La constante $C(\Omega)$ es proporcional al diámetro del dominio $\Omega$.
} 


\section{APÉNDICE E}

\section{Propiedades de aproximación y compacidad discreta para problemas con interacción fluido-estructura}

Preservaremos la notación introducida en la sección 4.2.1.

\section{E.1 Descomposición de Helmhotz}

Comenzaremos recordando que es válida la siguiente descomposición de Helmholtz

$$
\mathcal{K}(\Omega)=\mathcal{H}(\Omega) \oplus \mathcal{G}(\Omega)
$$

y que los subespacios $\mathcal{H}(\Omega)$ y $\mathcal{G}(\Omega)$ son cerrados y ortogonales en $\mathcal{Q}(\Omega)$ (ver Lema 2.3 en [33]). Más aún, la inclusión $\mathcal{G}(\Omega) \hookrightarrow \mathcal{Q}(\Omega)$ es compacta.

Una descomposición similar también vale a nivel discreto. Para probar esto, recordemos primero que el espacio de Raviart-Thomas de orden cero verifica

$$
\mathcal{R}_{h}\left(\Omega_{\mathrm{F} h}\right)=\mathcal{Y}_{h}\left(\Omega_{\mathrm{F} h}\right) \oplus \operatorname{curl} \mathcal{Z}_{h}\left(\Omega_{\mathrm{F} h}\right),
$$

donde los espacios $\mathcal{Z}_{h}\left(\Omega_{\mathrm{F} h}\right)$ y $\mathcal{Y}_{h}\left(\Omega_{\mathrm{F} h}\right)$ están definidos por

$$
\begin{aligned}
& \mathcal{Z}_{h}\left(\Omega_{\mathrm{F} h}\right)=\left\{p_{h} \in H_{\Gamma_{\mathrm{I} h}}^{1}\left(\Omega_{\mathrm{F} h}\right):\left.p_{h}\right|_{T} \in \mathcal{P}_{1}(T), T \in \mathcal{T}_{h}^{\mathrm{F}}\right\}, \\
& \mathcal{Y}_{h}\left(\Omega_{\mathrm{F} h}\right)=\left\{\mathbf{u}_{h} \in \mathcal{R}_{h}\left(\Omega_{\mathrm{F} h}\right): \int_{\Omega_{\mathrm{F} h}} \mathbf{u}_{h} \cdot \operatorname{curl} p_{h}=0, \forall p_{h} \in \mathcal{Z}_{h}\left(\Omega_{\mathrm{F} h}\right)\right\},
\end{aligned}
$$

El espacio $\mathcal{Y}_{h}\left(\Omega_{\mathrm{F} h}\right)$ es referido usualmente como el espacio de los campos vectoriales con rotor discreto nulo. La descomposición anterior es ortogonal en $L^{2}\left(\Omega_{\mathrm{F} h}\right)^{2}$ y en $H\left(\operatorname{div}, \Omega_{\mathrm{F} h}\right)$ (ver [22]).

Luego, el espacio $\mathcal{K}_{h}\left(\Omega_{h}\right)$ podrá descomponerse de la siguiente manera

$$
\mathcal{K}_{h}\left(\Omega_{h}\right)=\mathcal{H}_{h}\left(\Omega_{h}\right) \oplus \mathcal{G}_{h}\left(\Omega_{h}\right),
$$

donde los espacios $\mathcal{H}_{h}\left(\Omega_{h}\right)$ y $\mathcal{G}_{h}\left(\Omega_{h}\right)$ estarán dados por

$$
\begin{aligned}
& \mathcal{H}_{h}\left(\Omega_{h}\right)=\left\{\left(\operatorname{curl} p_{h}, \mathbf{0}\right): p_{h} \in \mathcal{Z}_{h}\left(\Omega_{\mathrm{F} h}\right)\right\}, \\
& \mathcal{G}_{h}\left(\Omega_{h}\right)=\left\{\left(\mathbf{u}_{h}, \mathbf{w}_{h}\right) \in \mathcal{K}_{h}\left(\Omega_{h}\right): \mathbf{u}_{h} \in \mathcal{Y}_{h}\left(\Omega_{\mathrm{F} h}\right)\right\} .
\end{aligned}
$$

Por construcción, $\mathcal{G}_{h}\left(\Omega_{h}\right)$ es el complemento ortogonal de $\mathcal{H}_{h}\left(\Omega_{h}\right)$ en $\mathcal{K}_{h}\left(\Omega_{h}\right)$. Observemos que $\mathcal{H}_{h}\left(\Omega_{h}\right)$ y $\mathcal{G}_{h}\left(\Omega_{h}\right)$ son también ortogonales con respecto al producto interno en $\mathcal{Q}\left(\Omega_{h}\right)$ (ver la prueba del Teorema 5.4 en [33]). Observemos además que $\mathcal{H}_{h}\left(\Omega_{h}\right) \subset \mathcal{H}\left(\Omega_{h}\right)$, pero, en general, $\mathcal{G}_{h}\left(\Omega_{h}\right) \not \subset \mathcal{G}\left(\Omega_{h}\right)$. 


\section{E.2 Teorema de trazas para espacios de Sobolev definidos en $\Omega_{h}$}

El Teorema de trazas para espacios de Sobolev establece lo siguiente:

Teorema E.1 Sea $\Omega$ un dominio Lipschitz. El operador de traza $\left.\gamma\right|_{\partial \Omega}$ es un operador lineal y acotado de

- $H^{s}(\Omega)$ a $H^{s-1 / 2}(\partial \Omega)$ si $1 / 2<s<3 / 2$,

- $H^{s}(\Omega)$ a $H^{1}(\partial \Omega)$ si $s>3 / 2$.

Este teorema es usado frecuentemente para acotar la norma $\|\cdot\|_{s-1 / 2, \partial \Omega}$ a expensas de una constante que depende únicamente de $\partial \Omega$ [180]. Es claro, entonces, que para funciones definidas sobre $\Omega_{h}$, la aplicación de este teorema no nos proporcionará estimaciones uniformes en $h$. Sin embargo, cuando $\Omega_{h}$ es una perturbación de $\Omega$, es posible obtener una versión del Teorema de trazas con constantes independientes de $h$. Este será el objetivo de esta sección. Para ello, necesitaremos introducir algunos conceptos y establecer nuevos resultados. Seguiremos los argumentos de Bramble-King [47].

En lo que sigue, asumiremos que la aproximación poligonal $\Omega_{h}$ de $\Omega$ fue construida de acuerdo con las pautas establecidas en la sección 4.2. Recordemos que $\widehat{\Omega}=\Omega \cup \Omega_{h}$ y que $\widetilde{\Omega}$ verifica $\widehat{\Omega} \subset \widetilde{\Omega}$ para todo $h<h_{0}$. Aquí, $\Gamma_{\mathrm{I}}=\partial \Omega, \Gamma_{\mathrm{I} h}=\partial \Omega_{h}$ y $C_{\Gamma_{\mathrm{I}}}$ indicará una constante que dependerá de $\Gamma_{\mathrm{I}}$, no necesariamente la misma cada vez que aparezca, pero siempre independiente de $h$.

Indicaremos por $\left\{\mathbf{x}_{j}\right\}_{j=1}^{N(h)}$ al conjunto de puntos que definen a la poligonal $\Gamma_{\mathrm{I} h}$. De acuerdo con las pautas seguidas en la construcción de $\Gamma_{\mathrm{I} h}$ (ver sección 4.2.1), $\left\{\mathbf{x}_{j}\right\}_{j=1}^{N(h)} \subset \Gamma_{\mathrm{I}} \mathrm{y}$ contiene todos los puntos donde $\Gamma_{\mathrm{I}}$ deja de ser suave. Supongamos que los puntos $\mathbf{x}_{j}$ están ordenados de manera tal que inducen un recorrido de la curva $\Gamma_{\mathrm{I} h}$ en sentido antihorario. Haciendo $\mathbf{x}_{N(h)+1} \equiv \mathbf{x}_{1}$, definimos

$$
h_{j}:=\left|\mathbf{x}_{j+1}-\mathbf{x}_{j}\right|, \quad h:=\operatorname{máx}_{1 \leq j \leq N(h)} h_{j}, \quad \boldsymbol{\tau}_{j}:=\frac{1}{h_{j}}\left(\mathbf{x}_{j+1}-\mathbf{x}_{j}\right) .
$$

Para cada $1 \leq j \leq N(h)$, denotaremos por

- $\tilde{\ell}_{j}$ al arco de $\Gamma_{\mathrm{I}}$ delimitado por los puntos $\mathbf{x}_{j} \mathrm{y} \mathbf{x}_{j+1}$,

- $\ell_{j}$ al segmento de recta en $\Gamma_{\mathrm{I} h}$ delimitado por los puntos $\mathbf{x}_{j} \mathrm{y} \mathbf{x}_{j+1}$,

- $\boldsymbol{\nu}_{j}$ al vector unitario en la dirección normal exterior al segmento $\ell_{j}$.

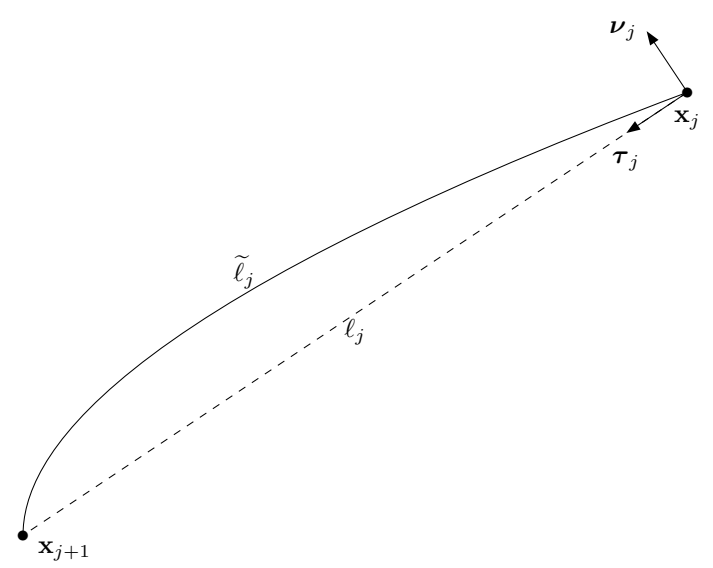

Figura E.1: Orientación de los vectores unitarios normal $\boldsymbol{\nu}_{j}$ y tangencial $\boldsymbol{\tau}_{j}$ en el punto $\mathbf{x}_{j}$.

A continuación consideraremos una parametrización de la curva $\Gamma_{\mathrm{I}}$ inducida por una parametrización standard de la curva $\Gamma_{\mathrm{I} h}$. Sea

$$
\boldsymbol{\psi}_{j}:\left[0, h_{j}\right] \rightarrow \tilde{\ell}_{j} \quad \text { definida por } \quad \boldsymbol{\psi}_{j}(t)=\mathbf{x}_{j}+t \boldsymbol{\tau}_{j}+\phi_{j}(t) \boldsymbol{\nu}_{j},
$$

donde $\phi_{j}:\left[0, h_{j}\right] \rightarrow \mathbb{R}$ es tal que $\boldsymbol{\psi}_{j}(t) \in \Gamma_{\mathrm{I}}$. Como se asumió que $\Gamma_{\mathrm{I}}$ es $\mathcal{C}^{2}$ a trozos, tendremos que 
- $\phi_{j}$ es de clase $\mathcal{C}^{2}$

- $\phi_{j}(0)=\phi_{j}\left(h_{j}\right)=0$,

- existe una constante $C_{\Gamma_{\mathrm{I}}}$ tal que $\left|\phi_{j}(t)\right|+h_{j}\left|\phi_{j}^{\prime}(t)\right| \leq C_{\Gamma_{\mathrm{I}}} h_{j}^{2}$, para todo $t \in\left[0, h_{j}\right]$.

Además, de las propiedades de $\phi_{j}$, se deduce fácilmente que la siguiente estimación es válida

$$
\sqrt{1+\left(\phi_{j}^{\prime}(t)\right)^{2}}-1 \leq C_{\Gamma_{\mathrm{I}}} h_{j}^{2} \quad \forall t \in\left[0, h_{j}\right]
$$

Definimos

$$
\boldsymbol{\Upsilon}_{h}: \Gamma_{\mathrm{Ih}} \rightarrow \Gamma_{\mathrm{I}},\left.\quad \boldsymbol{\Upsilon}_{h}\right|_{\ell_{j}}(\mathbf{x})=\left.\boldsymbol{\Upsilon}_{h}\right|_{\ell_{j}}\left(\mathbf{x}_{j}+t \boldsymbol{\tau}_{j}\right)=\mathbf{x}_{j}+t \boldsymbol{\tau}_{j}+\phi_{j}(t) \boldsymbol{\nu}_{j}=\boldsymbol{\psi}_{j}(t) .
$$

En lo que sigue, asumiremos que $h$ es suficientemente chico de manera tal que $\boldsymbol{\Upsilon}_{h}$ resulte bien definida.

Observación E.2 Si $\Omega$ fuera convexo, el mapeo $\boldsymbol{\psi}_{j}$ sería biyectivo. Lo mismo es cierto para dominios no convexos siempre que $h_{j} \leq c_{\Gamma_{\mathrm{I}}}$, siendo $c_{\Gamma_{\mathrm{I}}}$ una constante que depende únicamente de la curvatura de $\Gamma_{\mathrm{I}}$.

Sea $g$ una función definida sobre $\Gamma_{\mathrm{I}}$. Introduciremos, a continuación, una extensión de $g$ a $\Gamma_{\mathrm{I} h}$. Denotando a esta extensión por $\bar{g}$, definimos

$$
\bar{g}\left(\mathbf{x}_{j}+t \boldsymbol{\tau}_{j}\right)=g\left(\mathbf{x}_{j}+t \boldsymbol{\tau}_{j}+\phi_{j}(t) \boldsymbol{\nu}_{j}\right)=g\left(\boldsymbol{\psi}_{j}(t)\right), \quad 0 \leq t \leq h_{j}, \quad 1 \leq j \leq N(h) .
$$

Observemos que $\bar{g}\left(\mathbf{x}_{j}\right)-g\left(\mathbf{x}_{j}\right)=\bar{g}\left(\mathbf{x}_{j+1}\right)-g\left(\mathbf{x}_{j+1}\right)=0$ y que la asignación inversa también está bien definida. Entonces, dada una función $g \in L^{2}\left(\Gamma_{\mathrm{I}}\right)$, tendremos

$$
\begin{gathered}
\int_{\Gamma_{\mathrm{I}}} g(\hat{\mathbf{x}}) d s(\hat{\mathbf{x}})=\sum_{j=1}^{N(h)} \int_{0}^{h_{j}} g\left(\boldsymbol{\psi}_{j}(t)\right) \sqrt{1+\left(\phi_{j}^{\prime}(t)\right)^{2}} d t \\
\int_{\Gamma_{\mathrm{I} h}} \bar{g}(\mathbf{x}) d s_{h}(\mathbf{x})=\sum_{j=1}^{N(h)} \int_{0}^{h_{j}} g\left(\boldsymbol{\psi}_{j}(t)\right) d t .
\end{gathered}
$$

De estas expresiones, deducimos que

$$
\begin{aligned}
\left|\int_{\Gamma_{\mathrm{I}}} g(\hat{\mathbf{x}}) d s(\hat{\mathbf{x}})-\int_{\Gamma_{\mathrm{I} h}} \bar{g}(\mathbf{x}) d s_{h}(\mathbf{x})\right| & \leq \sum_{j=1}^{N(h)} \int_{0}^{h_{j}}\left|g\left(\boldsymbol{\psi}_{j}(t)\right)\right|\left(\sqrt{1+\left(\phi_{j}^{\prime}(t)\right)^{2}}-1\right) d t \\
& \leq C_{\Gamma_{\mathrm{I}}} \sum_{j=1}^{N(h)} h_{j}^{2} \int_{0}^{h_{j}}\left|g\left(\boldsymbol{\psi}_{j}(t)\right)\right| d t \\
& \leq C_{\Gamma_{\mathrm{I}}} h^{2} \int_{\Gamma_{\mathrm{I}}}|g(\hat{\mathbf{x}})| d s(\hat{\mathbf{x}}) \\
& \leq C_{\Gamma_{\mathrm{I}}} h^{2}\|g\|_{0, \Gamma_{\mathrm{I}}} .
\end{aligned}
$$

Este resultado y los que siguen a continuación (los dos primeros fueron probados en [47]) serán claves cuando tengamos que estimar los errores de la aproximación.

Lema E.3 Existen constantes c y $C$, estrictamente positivas e independientes de $h$, tales que

$$
c\|g\|_{0, \Gamma_{\mathrm{I}}} \leq\|\bar{g}\|_{0, \Gamma_{\mathrm{I} h}} \leq C\|g\|_{0, \Gamma_{\mathrm{I}}} .
$$

Lema E.4 Supongamos que g representa la traza sobre $\Gamma_{\mathrm{I}}$ de una función $w \in H^{r}(\Omega), 1 \leq r \leq 2$. Sea $\widetilde{w}$ la extensión a $\widetilde{\Omega}$ de $w$, según el Teorema D.4. Entonces, existe una constante $C$, independiente de $h$ y $w$, tal que

$$
\|\widetilde{w}-\bar{g}\|_{0, \Gamma_{\mathrm{I} h}} \leq C h^{r}\|w\|_{r, \Omega}
$$


Consideremos ahora las siguientes proyecciones ortogonales

$$
\begin{array}{ll}
P_{\ell_{j}} & : L^{2}\left(\ell_{j}\right) \rightarrow \mathcal{P}_{0}\left(\ell_{j}\right) \quad 1 \leq j \leq N(h), \\
P_{\Gamma_{\mathrm{I} h}} & : L^{2}\left(\Gamma_{\mathrm{I} h}\right) \rightarrow \mathcal{C}_{h}\left(\Gamma_{\mathrm{I} h}\right):=\left\{\chi_{h} \in L^{2}\left(\Gamma_{\mathrm{I} h}\right):\left.\chi_{h}\right|_{\ell_{j}} \in \mathcal{P}_{0}\left(\ell_{j}\right) \quad 1 \leq j \leq N(h)\right\} \subset L^{2}\left(\Gamma_{\mathrm{I} h}\right), \\
P_{\tilde{\ell}_{j}} & : L^{2}\left(\tilde{\ell}_{j}\right) \rightarrow \mathcal{P}_{0}\left(\tilde{\ell}_{j}\right) \quad 1 \leq j \leq N(h), \\
P_{\Gamma_{\mathrm{I}}} & : L^{2}\left(\Gamma_{\mathrm{I}}\right) \rightarrow \mathcal{C}_{h}\left(\Gamma_{\mathrm{I}}\right):=\left\{\chi_{h} \in L^{2}\left(\Gamma_{\mathrm{I}}\right):\left.\chi_{h}\right|_{\tilde{\ell}_{j}} \in \mathcal{P}_{0}\left(\tilde{\ell}_{j}\right) \quad 1 \leq j \leq N(h)\right\} \subset L^{2}\left(\Gamma_{\mathrm{I}}\right),
\end{array}
$$

Observemos que, en virtud de su definición, los espacios $\mathcal{C}_{h}\left(\Gamma_{\mathrm{I}}\right)$ heredan las propiedades de aproximación del espacio $\mathcal{C}_{h}\left(\Gamma_{\mathrm{I} h}\right)$. Más específicamente, para toda función $g \in H^{1}\left(\Gamma_{\mathrm{I}}\right)$, se verifica

$$
\left\|\left(I-P_{\Gamma_{\mathrm{I}}}\right) g\right\|_{0, \Gamma_{\mathrm{I}}}=\inf _{\chi_{h} \in \mathcal{C}_{h}\left(\Gamma_{\mathrm{I}}\right)}\left\|g-\chi_{h}\right\|_{0, \Gamma_{\mathrm{I}}} \leq C h\|g\|_{1, \Gamma_{\mathrm{I}}} .
$$

Además, por propiedades de la proyección ortogonal, también vale

$$
\left\|\left(I-P_{\Gamma_{\mathrm{I}}}\right) g\right\|_{0, \Gamma_{\mathrm{I}}} \leq C\|g\|_{0, \Gamma_{\mathrm{I}}} .
$$

Luego, por interpolación entre (E.3) y (E.4), tendremos

$$
\left\|\left(I-P_{\Gamma_{\mathrm{I}}}\right) g\right\|_{0, \Gamma_{\mathrm{I}}} \leq C h^{r}\|g\|_{r, \Gamma_{\mathrm{I}}} \quad 0 \leq r \leq 1
$$

Lema E.5 Sea $g \in L^{2}\left(\Gamma_{\mathrm{I}}\right)$. Sean $\bar{g}$ y $\overline{P_{\Gamma_{\mathrm{I}}} g}$ las extensiones a $\Gamma_{\mathrm{I} h}$ de g y $P_{\Gamma_{\mathrm{I}}} g$ (según la definición (E.1)), respectivamente. Existe una constante $C$, independiente de $h$, tal que

$$
\left\|P_{\Gamma_{\mathrm{I} h}} \bar{g}-\overline{P_{\Gamma_{\mathrm{I}}} g}\right\|_{0, \Gamma_{\mathrm{I} h}} \leq C h^{2}\|g\|_{0, \Gamma_{\mathrm{I}}} .
$$

Prueba. A partir de las definiciones de los operadores $P_{\Gamma_{\mathrm{I}}}$ y $P_{\Gamma_{\mathrm{I} h}}$ y de la extensión a $\Gamma_{\mathrm{I} h}$, es inmediato que

$$
\left\|P_{\Gamma_{\mathrm{I} h}} \bar{g}-\overline{P_{\Gamma_{\mathrm{I}}} g}\right\|_{0, \Gamma_{\mathrm{I} h}}^{2}=\sum_{j=1}^{N(h)}\left\|P_{\ell_{j}} \bar{g}-\overline{P_{\tilde{\ell}_{j}} g}\right\|_{0, \ell_{j}}^{2}=\sum_{j=1}^{N(h)}\left\|P_{\ell_{j}} \bar{g}-P_{\tilde{\ell}_{j}} g\right\|_{0, \ell_{j}}^{2}
$$

Luego, usando las parametrizaciones definidas anteriormente, tendremos

$$
\begin{aligned}
P_{\ell_{j}} \bar{g}-P_{\tilde{\ell}_{j}} g & =\frac{1}{\left|\ell_{j}\right|} \int_{\ell_{j}} \bar{g}\left(s_{h}\right) d s_{h}-\frac{1}{\left|\tilde{\ell}_{j}\right|} \int_{\tilde{\ell}_{j}} g(s) d s \\
& =\frac{1}{\left|\ell_{j}\right|} \int_{0}^{h_{j}} g\left(\psi_{j}(t)\right) d t-\frac{1}{\left|\tilde{\ell}_{j}\right|} \int_{0}^{h_{j}} g\left(\psi_{j}(t)\right) \sqrt{1+\left(\phi_{j}^{\prime}(t)\right)^{2}} d t \\
& =\frac{1}{\left|\ell_{j}\right|} \int_{0}^{h_{j}} g\left(\psi_{j}(t)\right)\left(1-\frac{\left|\ell_{j}\right|}{\left|\tilde{\ell}_{j}\right|} \sqrt{1+\left(\phi_{j}^{\prime}(t)\right)^{2}}\right) d t .
\end{aligned}
$$

Teniendo en cuenta que $\left|\ell_{j}\right| \leq\left|\tilde{\ell}_{j}\right|$, para todo $1 \leq j \leq N(h)$,

$$
\begin{aligned}
\left\|P_{\ell_{j}} \bar{g}-P_{\tilde{\ell}_{j}} g\right\|_{0, \ell_{j}} & \leq\left|\ell_{j}\right|^{-1 / 2} \operatorname{máx}_{t \in\left[0, h_{j}\right]}\left|1-\frac{\left|\ell_{j}\right|}{\left|\tilde{\ell}_{j}\right|} \sqrt{1+\left(\phi_{j}^{\prime}(t)\right)^{2}}\right| \int_{0}^{h_{j}}\left|g\left(\psi_{j}(t)\right)\right| d t \\
& \leq\left|\ell_{j}\right|^{-1 / 2} \operatorname{máx}_{t \in\left[0, h_{j}\right]}\left|1-\frac{\left|\ell_{j}\right|}{\left|\tilde{\ell}_{j}\right|} \sqrt{1+\left(\phi_{j}^{\prime}(t)\right)^{2}}\right| \int_{\ell_{j}}\left|\bar{g}\left(s_{h}\right)\right| d s_{h} \\
& \leq \operatorname{máx}_{t \in\left[0, h_{j}\right]} \frac{\left|\ell_{j}\right|}{\left|\tilde{\ell}_{j}\right|}\left(\sqrt{1+\left(\phi_{j}^{\prime}(t)\right)^{2}}-1\right)\|\bar{g}\|_{0, \ell_{j}} .
\end{aligned}
$$

Luego, elevando al cuadrado, sumando sobre todo $j$ y usando el Lema E.3, obtendremos

$$
\left\|P_{\ell_{j}} \bar{g}-\overline{P_{\tilde{\ell}_{j}} g}\right\|_{0, \Gamma_{\mathrm{I} h}} \leq C h^{2}\|g\|_{0, \Gamma_{\mathrm{I}}} .
$$

De esta manera, terminamos la prueba. 
Lema E.6 Sea $g \in H^{1+r}(\Omega), 0 \leq r \leq 1$. Sea $\widetilde{g}$ la extensión de $g$ a $\widetilde{\Omega}$ según el Teorema D.4. Existe una constante $C$, independiente de $h$, tal que

$$
\left\|\widetilde{g}-P_{\Gamma_{\mathrm{I} h}} \widetilde{g}\right\|_{0, \Gamma_{\mathrm{Ih}}} \leq C h^{\operatorname{mín}\{1,1 / 2+r\}}\|g\|_{1+r, \Omega} .
$$

Prueba. Sea $\bar{g}$ la extensión a $\Gamma_{\mathrm{I} h}$ de $g$. Tendremos

$$
\left\|\widetilde{g}-P_{\Gamma_{\mathrm{I} h}} \widetilde{g}\right\|_{0, \Gamma_{\mathrm{I} h}} \leq\|\widetilde{g}-\bar{g}\|_{0, \Gamma_{\mathrm{I} h}}+\left\|\overline{g-P_{\Gamma_{\mathrm{I}}} g}\right\|_{0, \Gamma_{\mathrm{I} h}}+\left\|\overline{P_{\Gamma_{\mathrm{I}}} g}-P_{\Gamma_{\mathrm{I} h}} \bar{g}\right\|_{0, \Gamma_{\mathrm{I} h}}+\left\|P_{\Gamma_{\mathrm{I} h}}(\bar{g}-\widetilde{g})\right\|_{0, \Gamma_{\mathrm{I} h}} .
$$

Usando los Lemas E.3-E.5, las propiedades aproximación de $P_{\Gamma_{\mathrm{I}}}$ y el Teorema E.1, tendremos

$$
\left\|\widetilde{g}-P_{\Gamma_{\mathrm{Ih}}} \widetilde{g}\right\|_{0, \Gamma_{\mathrm{I} h}} \leq C\left(h^{1+r}\|g\|_{1+r, \Omega}+h^{\min \{1,1 / 2+r\}}\|g\|_{1+r, \Omega}+h^{2}\|g\|_{1, \Omega}\right),
$$

expresión que nos permite concluir la prueba.

Lema E.7 Sea $g \in H^{1}(\Omega)$. Sea $\widetilde{g}$ la extensión de $g$ a $\widetilde{\Omega}$ según el Teorema D.4. Existe una constante $C$, independiente de $h$, tal que

$$
\|\widetilde{g}\|_{0, \Gamma_{\mathrm{I} h}} \leq C\|g\|_{1, \Omega} .
$$

Prueba. Sea $T$ un triángulo en el borde de $\Omega_{h}$. Sea $\omega_{T}$ el dominio definido por $T \backslash T^{i d}$ o $T^{i d} \backslash T$, según corresponda. Obviamente, para algún $1 \leq j \leq N(h), \partial \omega_{T}=\ell_{j} \cup \tilde{\ell}_{j}$. Sin pérdida de generalidad, asumiremos que $\mathbf{x}_{j}$ coincide con el origen de coordenadas y que $\mathbf{x}_{j+1}=\left(h_{j}, 0\right)$. La orientación del eje $y$ se elegirá de manera tal que que $\phi_{j} \geq 0$. En estas condiciones,

$$
\tilde{\ell}_{j}=\left\{(x, y): y=\phi_{j}(x), 0 \leq x \leq h_{j}\right\} .
$$

Aplicando el Teorema de la divergencia al campo $\left(0, \widetilde{g}^{2}\right)$, tendremos

$$
\int_{\tilde{\ell}_{j}} \frac{g^{2}}{\sqrt{1+\left(\phi^{\prime}(x)\right)^{2}}} d s-\int_{\ell_{j}} \widetilde{g}^{2} d x=2 \int_{\omega_{T}} \widetilde{g} \frac{\partial \widetilde{g}}{\partial y} d x d y .
$$

Luego, usando la desigualdad de Cauchy-Schwarz,

$$
\|\widetilde{g}\|_{0, \ell_{j}}^{2} \leq C\left(\|g\|_{0, \tilde{\ell}_{j}}^{2}+\|\widetilde{g}\|_{0, \omega_{T}}|\widetilde{g}|_{1, \omega_{T}}\right) \leq C\left(\|g\|_{0, \tilde{\ell}_{j}}^{2}+\|\widetilde{g}\|_{1, \omega_{T}}^{2}\right) .
$$

Finalmente, sumando sobre todos $\operatorname{los} T \in \mathcal{B}_{\mathrm{S} h}$, usando el Teorema D.4 y el Teorema de trazas E.1, concluimos

$$
\|\widetilde{g}\|_{0, \Gamma_{\mathrm{I} h}}^{2} \leq C\left(\|g\|_{0, \Gamma_{\mathrm{I}}}^{2}+\|\widetilde{g}\|_{1, \widehat{\Omega}}^{2}\right) \leq C\|g\|_{1, \Omega}^{2} .
$$

Así, terminamos la prueba.

Lema E.8 Sea $g \in \mathcal{L}_{h}\left(\Omega_{h}\right):=\left\{g \in H^{1}\left(\Omega_{h}\right):\left.g\right|_{T} \in \mathcal{P}_{1}(T) \forall T \in \mathcal{T}_{h}\right\}$. Sea $g^{e}$ la extensión natural de $g$ a $\widetilde{\Omega}$ (ver definición D.8). Existe una constante $C$, independiente de $h$, tal que

$$
|g|_{1 / 2, \Gamma_{\mathrm{I} h}} \leq C\left(\left|g^{e}\right|_{1 / 2, \Gamma_{\mathrm{I}}}+h|g|_{1, \Omega_{h}}\right) .
$$

Prueba. Escribamos

$$
|g|_{1 / 2, \Gamma_{\mathrm{I} h}}^{2}=\sum_{i=1}^{N(h)}|g|_{1 / 2, \ell_{i}}^{2}+\sum_{i \neq j} \int_{\ell_{i}} \int_{\ell_{j}} \frac{|g(\mathbf{x})-g(\hat{\mathbf{x}})|^{2}}{|\mathbf{x}-\hat{\mathbf{x}}|^{2}} d s_{h}(\mathbf{x}) d s_{h}(\hat{\mathbf{x}}) .
$$

En lo que sigue, acotaremos cada término que aparece en el lado derecho de la expresión anterior por separado. Para ello usaremos las parametrizaciones antes introducidas. Sea $T_{i}$ aquel triángulo en el borde del dominio $\Omega_{h}$ que verifica $\partial T_{i} \cap \Gamma_{\mathrm{I} h}=\ell_{i}$. Sea $T_{i}^{i d}$ el triángulo ideal asociado con $T_{i}$. Entonces, $\tilde{\ell}_{i}=\partial T_{i}^{i d} \cap \Gamma_{\mathrm{I}}$. Sin pérdida de generalidad, asumiremos que $\mathbf{x}_{i}$ coincide con el origen de coordenadas y que $\mathbf{x}_{i+1}=\left(h_{i}, 0\right)$. La orientación del eje $y$ se elegirá de manera tal que que $\phi_{i} \geq 0$. En estas condiciones,

$$
\tilde{\ell}_{i}=\left\{(x, y): y=\phi_{i}(x), 0 \leq x \leq h_{i}\right\} .
$$


- Caso $\ell_{i}=\ell_{j}$.

Como $g$ es lineal en cada triángulo, tendremos

$$
\frac{g(x, 0)-g(\hat{x}, 0)}{x-\hat{x}}=\frac{g^{e}\left(x, \phi_{i}(x)\right)-g^{e}\left(\hat{x}, \phi_{i}(\hat{x})\right)}{x-\hat{x}}-\frac{\partial g(x, 0)}{\partial y} \frac{\left(\phi_{i}(x)-\phi_{i}(\hat{x})\right)}{x-\hat{x}} .
$$

Como $\phi_{i}$ es $\mathcal{C}^{2}$ en $\left(0, h_{i}\right)$, existirá un punto $\varsigma_{i} \in(x, \hat{x})$ tal que

$$
\int_{0}^{h_{i}} \int_{0}^{h_{i}}\left(\frac{\partial g(x, 0)}{\partial y} \frac{\left(\phi_{i}(x)-\phi_{i}(\hat{x})\right)}{x-\hat{x}}\right)^{2} d x d \hat{x}=\left|\frac{\partial g(x, 0)}{\partial y}\right|^{2}\left|\phi_{i}^{\prime}\left(\varsigma_{i}\right)\right|^{2} h_{i}^{2} \leq C h_{i}^{2}|g|_{1, T_{i}}^{2} .
$$

Razonando de la misma manera, obtendremos

$$
\frac{(x-\hat{x})^{2}+\left(\phi_{i}(x)-\phi_{i}(\hat{x})\right)^{2}}{(x-\hat{x})^{2}} \leq 1+\left|\phi_{i}^{\prime}\left(\varsigma_{i}\right)\right|^{2} \leq 1+C h_{i}^{2} .
$$

Luego, combinando las expresiones anteriores y teniendo en cuenta que $\sqrt{1+\left(\phi_{i}^{\prime}(x)\right)^{2}} \geq 1$ para todo $x \in\left(0, h_{i}\right)$, llegaremos a

$$
|g|_{1 / 2, \ell_{i}}^{2} \leq C\left(\left|g^{e}\right|_{1 / 2, \tilde{\ell}_{i}}^{2}+h_{i}^{2}|g|_{1, T_{i}}^{2}\right)
$$

- Caso $\ell_{i} \neq \ell_{j}$ y $\ell_{i} \cap \ell_{j}=\emptyset$.

Sea $T_{j}$ un triángulo en el borde del dominio $\Omega_{h}$ tal que $\partial T_{j} \cap \Gamma_{\mathrm{I} h}=\ell_{j}$. Sea $T_{j}^{i d}$ el triángulo ideal asociado con $T_{j}$. Al igual que en el caso anterior, $\tilde{\ell}_{j}=\partial T_{j}^{i d} \cap \Gamma_{\mathrm{I}}$. Para simplificar la notación, escribiremos

$$
\begin{aligned}
& \left(x\left(t_{j}\right), y\left(t_{j}\right)\right)=\left(x_{j}+t_{j} \tau_{j 1}, y_{j}+t_{j} \tau_{j 2}\right), t_{j} \in\left[0, h_{j}\right] \\
& \left(\hat{x}\left(t_{j}\right), \hat{y}\left(t_{j}\right)\right)=\left(x_{j}+t_{j} \tau_{j 1}+\phi_{j}\left(t_{j}\right) \nu_{j 1}, y_{j}+t_{j} \tau_{j 2}+\phi_{j}\left(t_{j}\right) \nu_{j 2}\right), t_{j} \in\left[0, h_{j}\right] .
\end{aligned}
$$

Entonces, como $g$ es lineal en cada triángulo, será

$$
g(x, 0)-g\left(x\left(t_{j}\right), y\left(t_{j}\right)\right)=g^{e}\left(x, \phi_{i}(x)\right)-g^{e}\left(\hat{x}\left(t_{j}\right), \hat{y}\left(t_{j}\right)\right)-\left(\left.\phi_{i}(x) \frac{\partial g}{\partial y}\right|_{(x, 0)}-\left.\phi_{j}\left(t_{j}\right) \frac{\partial g}{\partial \boldsymbol{\nu}_{j}}\right|_{\left(x\left(t_{j}\right), y\left(t_{j}\right)\right)}\right) .
$$

De las propiedades de las funciones $\phi_{i} \mathrm{y} \phi_{j}$, obtendremos

$$
\left|\phi_{i}(x) \frac{\partial g}{\partial y}\right|_{(x, 0)}-\left.\left.\phi_{j}\left(t_{j}\right) \frac{\partial g}{\partial \boldsymbol{\nu}_{j}}\right|_{\left(x\left(t_{j}\right), y\left(t_{j}\right)\right)}\left|\leq C\left(\left.h_{i}^{2}\left|\frac{\partial g}{\partial y}\right|_{(x, 0)}\left|+h_{j}^{2}\right| \frac{\partial g}{\partial \boldsymbol{\nu}_{j}}\right|_{\left(x\left(t_{j}\right), y\left(t_{j}\right)\right)} \mid\right) \leq C h_{i} h_{j}\right| g\right|_{1, T_{i} \cup T_{j}} .
$$

Además, tengamos en cuenta que

$$
\frac{\left(x-\hat{x}\left(t_{j}\right)\right)^{2}+\left(\phi_{i}(x)-\hat{y}\left(t_{j}\right)\right)^{2}}{\left(x-x\left(t_{j}\right)\right)^{2}+\left(0-y\left(t_{j}\right)\right)^{2}} \leq C\left(1+\frac{\left(\phi_{i}(x)\right)^{2}+\left(\phi_{j}\left(t_{j}\right)\right)^{2}}{\left(x-x\left(t_{j}\right)\right)^{2}+\left(y\left(t_{j}\right)\right)^{2}}\right)
$$

y, como $\phi_{i}(0)=\phi_{j}(0)=0$ y $\operatorname{dist}\left((x, 0),\left(x\left(t_{j}\right), y\left(t_{j}\right)\right)\right.$ es mayor que $h_{i}$ y mayor que $h_{j}$, existirán puntos $\varsigma_{i} \in\left[0, h_{i}\right]$ y $\varsigma_{j} \in\left[0, h_{j}\right]$ tales que

$$
\begin{aligned}
\frac{\left(\phi_{i}(x)\right)^{2}+\left(\phi_{j}\left(t_{j}\right)\right)^{2}}{\left(x-x\left(t_{j}\right)\right)^{2}+\left(y\left(t_{j}\right)\right)^{2}} & \leq \frac{\left(\phi_{i}(x)-\phi_{i}(0)\right)^{2}}{x^{2}}+\frac{\left(\phi_{j}\left(t_{j}\right)-\phi_{j}(0)\right)^{2}}{\left(x_{j}-x\left(t_{j}\right)\right)^{2}+\left(y_{j}-y\left(t_{j}\right)\right)^{2}} \\
& \leq\left(\phi_{i}^{\prime}\left(\varsigma_{i}\right)\right)^{2}+\left(\phi_{j}^{\prime}\left(\varsigma_{j}\right)\right)^{2} \leq C\left(h_{i}^{2}+h_{j}^{2}\right) .
\end{aligned}
$$

Luego, combinando las expresiones anteriores, podremos asegurar que existe una constante $C$, que depende únicamente de $\operatorname{dist}\left(\tilde{\ell}_{i}, \tilde{\ell}_{j}\right)$, tal que

$$
\int_{\ell_{i}} \int_{\ell_{j}} \frac{|g(\mathbf{x})-g(\hat{\mathbf{x}})|^{2}}{|\mathbf{x}-\hat{\mathbf{x}}|^{2}} d s_{h}(\mathbf{x}) d s_{h}(\hat{\mathbf{x}}) \leq C\left(\int_{\tilde{\ell}_{i}} \int_{\tilde{\ell}_{j}} \frac{\left|g^{e}(\mathbf{x})-g^{e}(\hat{\mathbf{x}})\right|^{2}}{|\mathbf{x}-\hat{\mathbf{x}}|^{2}} d s(\mathbf{x}) d s(\hat{\mathbf{x}})+h_{i}^{3} h_{j}^{3}|g|_{1, T_{i} \cup T_{j}}^{2}\right) .
$$

- Caso $\ell_{i} \neq \ell_{j}$ y $\ell_{i} \cap \ell_{j} \neq \emptyset$.

En este caso, los lados $\ell_{i}$ y $\ell_{j}$ tienen un vértice en común. Supongamos que este vértice se encuentra ubicado en el punto $\left(h_{i}, 0\right)$. Tendremos 


$$
\begin{aligned}
& -\phi_{i}\left(h_{i}\right)=\phi_{j}(0)=0, \\
& \text { - } \operatorname{dist}\left((x, 0),\left(x\left(t_{j}\right), y\left(t_{j}\right)\right)>\operatorname{dist}\left((x, 0),\left(h_{i}, 0\right)\right), t_{j} \in\left[0, h_{j}\right],\right. \\
& \text { - } \operatorname{dist}\left((x, 0),\left(x\left(t_{j}\right), y\left(t_{j}\right)\right)>\operatorname{dist}\left(\left(h_{i}, 0\right),\left(x\left(t_{j}\right), y\left(t_{j}\right)\right), t_{j} \in\left[0, h_{j}\right] .\right.\right.
\end{aligned}
$$

Luego, razonando como en el caso anterior, existirán puntos $\varsigma_{i} \in\left(x, h_{i}\right)$ y $\varsigma_{j} \in\left(0, h_{j}\right)$ tales que

$$
\begin{aligned}
\frac{\left(\left.\phi_{i}(x) \frac{\partial g}{\partial y}\right|_{(x, 0)}-\left.\phi_{j}\left(t_{j}\right) \frac{\partial g}{\partial \boldsymbol{\nu}_{j}}\right|_{\left(x\left(t_{j}\right), y\left(t_{j}\right)\right)}\right)^{2}}{\left(x-x\left(t_{j}\right)\right)^{2}+\left(y\left(t_{j}\right)\right)^{2}} & \leq C\left(\left.\left|\frac{\partial g}{\partial y}\right|_{(x, 0)}\right|^{2}\left(\phi_{i}^{\prime}\left(\varsigma_{i}\right)\right)^{2}+\left.\left|\frac{\partial g}{\partial \boldsymbol{\nu}_{j}}\right|_{\left(x\left(t_{j}\right), y\left(t_{j}\right)\right)}\right|^{2}\left(\phi_{j}^{\prime}\left(\varsigma_{j}\right)\right)^{2}\right), \\
\frac{\left(x-\hat{x}\left(t_{j}\right)\right)^{2}+\left(\phi_{i}(x)-\hat{y}\left(t_{j}\right)\right)^{2}}{\left(x-x\left(t_{j}\right)\right)^{2}+\left(0-y\left(t_{j}\right)\right)^{2}} & \leq C\left(1+\left(\phi_{i}^{\prime}\left(\varsigma_{i}\right)\right)^{2}+\left(\phi_{j}^{\prime}\left(\varsigma_{j}\right)\right)^{2}\right) .
\end{aligned}
$$

Usando estos resultados, obtendremos

$$
\int_{\ell_{i}} \int_{\ell_{j}} \frac{|g(\mathbf{x})-g(\hat{\mathbf{x}})|^{2}}{|\mathbf{x}-\hat{\mathbf{x}}|^{2}} d s_{h}(\mathbf{x}) d s_{h}(\hat{\mathbf{x}}) \leq C\left(\int_{\tilde{\ell}_{i}} \int_{\tilde{\ell}_{j}} \frac{\left|g^{e}(\mathbf{x})-g^{e}(\hat{\mathbf{x}})\right|^{2}}{|\mathbf{x}-\hat{\mathbf{x}}|^{2}} d s(\mathbf{x}) d s(\hat{\mathbf{x}})+h_{i} h_{j}|g|_{1, T_{i} \cup T_{j}}^{2}\right) .
$$

La prueba se completa sumando sobre todo $j$ y sobre todo $i$.

Observación E.9 Usando el Teorema E.1 y el Lema D.9, también podremos asegurar que existe una constante $C$, independiente de $h$, tal que

$$
|g|_{1 / 2, \Gamma_{\text {Ih }}} \leq C\|g\|_{1, \Omega_{h}} .
$$

Observación E.10 Supongamos que g representa la traza sobre $\Gamma_{\mathrm{I}}$ de alguna función $w \in H^{r}(\Omega)$, con $1 \leq r \leq 2$. Sea $\bar{g}$ la extensión a $\Gamma_{\mathrm{I} h}$ de g según la definición (E.1). Razonando como lo hicimos para probar el Lema E.8, es fácil obtener el siguiente resultado: existe una constante $C$, independiente de $h$, tal que

$$
|\bar{g}|_{1 / 2, \Gamma_{\mathrm{Ih}}} \leq C|g|_{1 / 2, \Gamma_{\mathrm{I}}}
$$

En lo que sigue, necesitaremos también definir una extensión de $g$ al borde del dominio $\widehat{\Omega}$. Haremos esto utilizando la extensión (E.1). Denotando por $\check{\ell}$ a los lados que componen a $\partial \widehat{\Omega}$ (observemos que $\partial \widehat{\Omega}$ es la unión de lados rectos $\ell \subset \Gamma_{\mathrm{I} h}$ con lados curvos $\tilde{\ell} \subset \Gamma_{\mathrm{I}}$ ), definimos

$$
\left.\check{g}\right|_{\check{\ell}}:=\left\{\begin{array}{ll}
\left.g\right|_{\check{\ell}} & \text { si } \check{\ell}=\tilde{\ell} \subset \Gamma_{\mathrm{I}}, \\
\left.\bar{g}\right|_{\check{\ell}} & \text { si } \check{\ell}=\ell \subset \Gamma_{\mathrm{I} h}
\end{array} .\right.
$$

Obviamente, $\check{g} \in L^{2}(\partial \widehat{\Omega})$. Además, los mismos argumentos que los empleados en la prueba del Lema E.3, permiten probar que existe una constante $C$, independiente de $h$, tal que $\|\check{g}\|_{0, \partial \widehat{\Omega}} \leq C\|g\|_{0, \Gamma_{\mathrm{I}}}$.

Lema E.11 Supongamos que g representa la traza sobre $\Gamma_{\mathrm{I}}$ de alguna función $w \in H^{r}(\Omega), 1 \leq r \leq 2$. Sea $\check{g}$ la extensión a $\partial \widehat{\Omega}$ de $g$ definida por E.6. Entonces, $\check{g} \in H^{1 / 2}(\partial \widehat{\Omega})$; más aún, existe una constante $C$, independiente de $h$, tal que

$$
|\check{g}|_{1 / 2, \partial \widehat{\Omega}} \leq C|g|_{1 / 2, \Gamma_{\mathrm{I}}}
$$

Prueba. De acuerdo con [59], para asegurar que $\check{g} \in H^{1 / 2}(\partial \widehat{\Omega})$, solo será necesario probar que

$$
\int_{\ell_{i}} \int_{\tilde{\ell}_{j}} \frac{|\bar{g}(\mathbf{x})-g(\hat{\mathbf{x}})|^{2}}{|\mathbf{x}-\hat{\mathbf{x}}|^{2}} d s(\hat{\mathbf{x}}) d s_{h}(\mathbf{x}) \leq \infty \quad \forall i \neq j, \quad \ell_{i} \cap \tilde{\ell}_{j} \neq \emptyset .
$$

En este caso, los lados $\ell_{i}$ y $\tilde{\ell}_{j}$ tienen un vértice en común. Usaremos la misma notación y los mismos argumentos que los empleados en la prueba del Lema E.8; es decir, asumiremos que $\mathbf{x}_{i}$ coincide con el 
origen de coordenadas, que $\mathbf{x}_{i+1}=\left(h_{i}, 0\right)$ y que el vértice compartido por los lados $\ell_{i} \mathrm{y} \tilde{\ell}_{j}$ se ubica en $\left(h_{i}, 0\right)$. Tendremos

$$
\frac{\left(\hat{x}\left(t_{i}\right)-\hat{x}\left(t_{j}\right)\right)^{2}+\left(\hat{y}\left(t_{i}\right)-\hat{y}\left(t_{j}\right)\right)^{2}}{\left(x-\hat{x}\left(t_{j}\right)\right)^{2}+\left(0-\hat{y}\left(t_{j}\right)\right)^{2}} \leq C\left(1+\frac{\left(\phi_{i}(x)\right)^{2}}{\left(x-\hat{x}\left(t_{j}\right)\right)^{2}+\left(\hat{y}\left(t_{j}\right)\right)^{2}}\right) .
$$

Ahora, usando el Teorema del coseno, podremos escribir

$$
\left|(x, 0)-\left(\hat{x}\left(t_{j}\right), \hat{y}\left(t_{j}\right)\right)\right|^{2} \geq\left(\left|(x, 0)-\left(h_{i}, 0\right)\right|^{2}+\left|\left(h_{i}, 0\right)-\left(\hat{x}\left(t_{j}\right), \hat{y}\left(t_{j}\right)\right)\right|^{2}\right) \mu_{i j},
$$

donde, denotando por $\alpha_{i j}$ al ángulo con vértice en el punto $\left(h_{i}, 0\right)$, tendremos

$$
\mu_{i j}=\left\{\begin{array}{cl}
1 & \text { si } \cos \alpha_{i j} \leq 0 \\
\left(1-\cos \alpha_{i j}\right) & \text { si } \cos \alpha_{i j} \geq 0
\end{array}\right.
$$

Un cálculo directo muestra que

$$
\cos \alpha_{i j}=\frac{t_{j} \tau_{j 1}+\phi_{j}\left(t_{j}\right) \nu_{j 1}}{\sqrt{t_{j}^{2}+\left(\phi_{j}\left(t_{j}\right)\right)^{2}}}=\frac{\tau_{j 1}+\phi_{j}^{\prime}\left(\varsigma_{j}\right) \nu_{j 1}}{\sqrt{1+\left(\phi_{j}^{\prime}\left(\varsigma_{j}\right)\right)^{2}}}=\tau_{j 1}\left(\hat{\mathbf{x}}\left(\varsigma_{j}\right)\right),
$$

donde $\varsigma_{j} \in\left(0, h_{j}\right)$ y $\tau_{j 1}\left(\hat{\mathbf{x}}\left(\varsigma_{j}\right)\right)$ representa la primera componente del vector tangente unitario al lado $\tilde{\ell}_{j}$ en el punto $\hat{\mathbf{x}}\left(\varsigma_{j}\right)$. Luego,

$$
\left|(x, 0)-\left(\hat{x}\left(t_{j}\right), \hat{y}\left(t_{j}\right)\right)\right|^{2} \geq C_{\Gamma_{\mathrm{I}}}\left|x-h_{i}\right|^{2} .
$$

Entonces, como $\phi_{i}\left(h_{i}\right)=0$, existirá $\varsigma_{i} \in\left(x, h_{i}\right)$ tal que

$$
\left(1+\frac{\left(\phi_{i}(x)\right)^{2}}{\left(x-\hat{x}\left(t_{j}\right)\right)^{2}+\left(\hat{y}\left(t_{j}\right)\right)^{2}}\right) \leq\left(1+\left(\phi_{i}^{\prime}\left(\varsigma_{i}\right)\right)^{2}\right) .
$$

Combinando las expresiones anteriores con la definición (E.1), tendremos

$$
\int_{\ell_{i}} \int_{\tilde{\ell}_{j}} \frac{|\bar{g}(\mathbf{x})-g(\hat{\mathbf{x}})|^{2}}{|\mathbf{x}-\hat{\mathbf{x}}|^{2}} d s_{h}(\mathbf{x}) d s(\hat{\mathbf{x}}) \leq C \int_{\tilde{\ell}_{i}} \int_{\tilde{\ell}_{j}} \frac{|g(\mathbf{x})-g(\hat{\mathbf{x}})|^{2}}{|\mathbf{x}-\hat{\mathbf{x}}|^{2}} d s(\mathbf{x}) d s(\hat{\mathbf{x}})<\infty .
$$

Probaremos ahora que la seminorma $|\check{g}|_{1 / 2, \partial \widehat{\Omega}}$ está acotada por $|g|_{1 / 2, \Gamma_{\mathrm{I}}}$ uniformemente en $h$. Señalemos primero que, gracias a la definición (E.6), la observación E.10 y el resultado anterior, solo resta probar que existe una constante $C$, independiente de $h$, tal que

$$
\int_{\ell_{i}} \int_{\tilde{\ell}_{j}} \frac{|\bar{g}(\mathbf{x})-g(\hat{\mathbf{x}})|^{2}}{|\mathbf{x}-\hat{\mathbf{x}}|^{2}} d s_{h}(\mathbf{x}) d s(\hat{\mathbf{x}}) \leq C \int_{\tilde{\ell}_{i}} \int_{\tilde{\ell}_{j}} \frac{|g(\mathbf{x})-g(\hat{\mathbf{x}})|^{2}}{|\mathbf{x}-\hat{\mathbf{x}}|^{2}} d s(\mathbf{x}) d s(\hat{\mathbf{x}}) \forall i \neq j, \quad \ell_{i} \cap \tilde{\ell}_{j}=\emptyset .
$$

Procediendo como antes, tendremos, nuevamente,

$$
\begin{aligned}
\frac{\left(\hat{x}\left(t_{i}\right)-\hat{x}\left(t_{j}\right)\right)^{2}+\left(\hat{y}\left(t_{i}\right)-\hat{y}\left(t_{j}\right)\right)^{2}}{\left(x-\hat{x}\left(t_{j}\right)\right)^{2}+\left(0-\hat{y}\left(t_{j}\right)\right)^{2}} & \leq C\left(1+\frac{\left(\phi_{i}(x)\right)^{2}}{\left(x-\hat{x}\left(t_{j}\right)\right)^{2}+\left(\hat{y}\left(t_{j}\right)\right)^{2}}\right) \\
& \leq C\left(1+\frac{\left(\phi_{i}(x)-\left(\phi_{i}\left(h_{i}\right)\right)^{2}\right.}{\left(x-h_{i}\right)^{2}}\right) \\
& \leq C\left(1+\left(\phi_{i}^{\prime}\left(\varsigma_{i}\right)\right)^{2}\right) .
\end{aligned}
$$

De esta manera, completamos la prueba.

\section{E.3 Potencial escalar en $\widehat{\Omega}_{\mathrm{F}}$}

En esta sección vamos a construir un campo gradiente, con dominio en $\widehat{\Omega}_{\mathrm{F}}$, directamente relacionado con el campo de desplazamientos $\mathbf{u}$ en el fluido. 
Sean $(\mathbf{f}, \mathbf{g}) \in \mathcal{G}(\Omega)$ y $(\mathbf{u}, \mathbf{w})=\mathbf{T}(\mathbf{f}, \mathbf{g})$. Del Teorema 4.29 , tendremos

$$
\|\mathbf{u}\|_{s, \Omega_{\mathrm{F}}}+\|\mathbf{w}\|_{1+t, \Omega_{\mathrm{S}}}+\|\operatorname{div} \mathbf{u}\|_{1+s, \Omega_{\mathrm{F}}} \leq C\|(\mathbf{f}, \mathbf{g})\|_{0, \Omega} .
$$

Sean $\widetilde{\mathbf{w}}$ y $\widetilde{\operatorname{div} \mathbf{u}}$ las extensiones al dominio $\widehat{\Omega}_{\mathrm{F}}$ de las funciones $\mathbf{w}$ y div $\mathbf{u}$, respectivamente, de acuerdo con el Teorema D.4. Sea $\check{\mathbf{w}}$ la extensión a $\partial \widehat{\Omega}$ de la traza $\left.\mathbf{w}\right|_{\Gamma_{\mathrm{I}}}$ definida por (E.6). Consideremos la solución $\hat{q} \in H^{1}\left(\widehat{\Omega}_{\mathrm{F}}\right)$ del siguiente problema de Neumann:

$$
\left\{\begin{array}{l}
\Delta \hat{q}=\widetilde{\operatorname{div} \mathbf{u}}+\hat{c} \quad \text { en } \widehat{\Omega}_{\mathrm{F}} \\
\frac{\partial \hat{q}}{\partial \mathbf{n}}=\check{\mathbf{w}} \cdot \mathbf{n} \quad \text { sobre } \partial \widehat{\Omega}_{\mathrm{F}}
\end{array}\right.
$$

donde $\hat{c}$ es una constante elegida de manera tal que el problema resulte compatible; i.e., escribiendo $\ell=\partial T \cap \Gamma_{\mathrm{I} h}$,

$$
\hat{c}\left|\widehat{\Omega}_{\mathrm{F}}\right|:=\int_{\Omega_{\mathrm{S}} \backslash \Omega_{\mathrm{Sh}}} \operatorname{div} \mathbf{w}-\int_{\Omega_{\mathrm{F} h} \backslash \Omega_{\mathrm{F}}} \widetilde{\operatorname{div} \mathbf{u}}+\sum_{\substack{T \in \mathcal{B}_{\mathrm{Sh}} \\ T \subset T^{i d}}} \int_{\ell}(\check{\mathbf{w}}-\widetilde{\mathbf{w}}) \cdot \mathbf{n} .
$$

Nuestro próximo paso será encontrar una cota para $|\hat{c}|$ que no dependa de $h$. Usando los Lemas D.5 y D.6 y el Teorema (D.4), se obtiene fácilmente

$$
\begin{gathered}
\left|\int_{\Omega_{\mathrm{F} h} \backslash \Omega_{\mathrm{F}}} \widetilde{\operatorname{div} \mathbf{u}}\right| \leq\left|\Omega_{\mathrm{F} h} \backslash \Omega_{\mathrm{F}}\right|^{1 / 2}\|\widetilde{\operatorname{div} \mathbf{u}}\|_{0, \Omega_{\mathrm{F} h} \backslash \Omega_{\mathrm{F}}} \leq C h^{1+s}\|\operatorname{div} \mathbf{u}\|_{1+s, \Omega_{\mathrm{F}}}, \\
\left|\int_{\Omega_{\mathrm{S}} \backslash \Omega_{\mathrm{S} h}} \operatorname{div} \mathbf{w}\right| \leq\left|\Omega_{\mathrm{S}} \backslash \Omega_{\mathrm{S} h}\right|^{1 / 2}\|\operatorname{div} \mathbf{w}\|_{0, \Omega_{\mathrm{S}} \backslash \Omega_{\mathrm{Sh}}} \leq C h^{1+t}\|\mathbf{w}\|_{1+t, \Omega_{\mathrm{S}}} .
\end{gathered}
$$

A partir de la definición (E.6), tendremos $\left.\check{\mathbf{w}}\right|_{\ell}=\left.\overline{\mathbf{w}}\right|_{\ell}$. Luego, usando el Lema E.4

$$
\begin{aligned}
\left|\sum_{\substack{T \in \mathcal{B}_{\text {Sh }} \\
T \subset T^{i d}}} \int_{\ell}(\overline{\mathbf{w}}-\widetilde{\mathbf{w}}) \cdot \mathbf{n}\right| & \leq \sum_{\substack{T \in \mathcal{B}_{\text {Sh }} \\
T \subset T^{i d}}} \int_{\ell}|(\overline{\mathbf{w}}-\widetilde{\mathbf{w}}) \cdot \mathbf{n}| \leq \sum_{T \in \mathcal{B}_{\text {Sh }}} \int_{\ell}|\overline{\mathbf{w}}-\widetilde{\mathbf{w}}| \\
& \leq\left|\Gamma_{\mathrm{Ih}}\right|^{1 / 2}\|\overline{\mathbf{w}}-\widetilde{\mathbf{w}}\|_{0, \Gamma_{\mathrm{Ih}}} \leq C\left|\Gamma_{\mathrm{I}}\right|^{1 / 2} h^{1+t}\|\mathbf{w}\|_{1+t, \Omega_{\mathrm{S}}} .
\end{aligned}
$$

Sea $r:=\min \{s, t\}$. Combinando las expresiones anteriores, se sigue que

$$
|\hat{c}| \leq C h^{1+r}\|(\mathbf{f}, \mathbf{g})\|_{0, \Omega} .
$$

Gracias al Lema E.11 y a partir de las estimaciones a priori usuales para el problema de Neumann (ver, por ejemplo, [111]), sabemos que $\hat{q} \in H^{1+s_{h}}\left(\widehat{\Omega}_{\mathrm{F}}\right)$ para algún $s_{h} \in(1 / 2,1]$. Además, combinando el Lema E.11 con el Teorema de trazas E.1 y recordando D.2, tendremos

$$
\|\nabla \hat{q}\|_{s_{h}, \widehat{\Omega}_{\mathrm{F}}}^{2} \leq C\left(\|\widetilde{\operatorname{div} \mathbf{u}}+\hat{c}\|_{0, \widehat{\Omega}_{\mathrm{F}}}^{2}+\sum_{j=1}^{N(h)}\|\check{\mathbf{w}} \cdot \mathbf{n}\|_{1 / 2, \check{\ell}_{j}}^{2}\right) \leq C\left(\|\widetilde{\operatorname{div} \mathbf{u}}+\hat{c}\|_{0, \widehat{\Omega}_{\mathrm{F}}}^{2}+\|\mathbf{w}\|_{1, \Omega_{\mathrm{S}}}^{2}\right) .
$$

Finalmente, usando el Teorema (D.4) y la estimación (E.9), podremos escribir

$$
\|\nabla \hat{q}\|_{s_{h}, \widehat{\Omega}_{\mathrm{F}}} \leq C\|(\mathbf{f}, \mathbf{g})\|_{0, \Omega} .
$$

Definimos $\hat{\mathbf{u}}:=\nabla \hat{q}$. Así definido, $\hat{\mathbf{u}} \in H^{s_{h}}\left(\widehat{\Omega}_{\mathrm{F}}\right)$ y verifica

$$
\|\hat{\mathbf{u}}\|_{s_{h}, \widehat{\Omega}_{\mathrm{F}}} \leq C\|(\mathbf{f}, \mathbf{g})\|_{0, \Omega} .
$$

Además, $\operatorname{div} \hat{\mathbf{u}}=\widetilde{\operatorname{div} \mathbf{u}}+\hat{c} \in H^{1+s}\left(\widehat{\Omega}_{\mathrm{F}}\right)$. Luego, usando las mismas estimaciones que antes, tendremos

$$
\|\operatorname{div} \hat{\mathbf{u}}\|_{1+s, \widehat{\Omega}_{\mathrm{F}}} \leq C\|(\mathbf{f}, \mathbf{g})\|_{0, \Omega}
$$

En el siguiente lema mostraremos que $\hat{\mathbf{u}}$ aproxima a $\mathbf{u}$ en $\Omega_{\mathrm{F}}$. 
Lema E.12 Sea $\hat{s}_{h}:=\operatorname{mín}\left\{s, s_{h}\right\}$. Existe una constante positiva $C$ tal que

$$
\|\mathbf{u}-\hat{\mathbf{u}}\|_{H\left(\operatorname{div} ; \Omega_{\mathrm{F}}\right)} \leq C h^{\left(r+\hat{s}_{h}\right) / 2}\|(\mathbf{f}, \mathbf{g})\|_{0, \Omega}
$$

Prueba. A partir de la definición de $\hat{\mathbf{u}}$, es inmediato que $\left.\operatorname{div}(\mathbf{u}-\hat{\mathbf{u}})\right|_{\Omega_{\mathrm{F}}}=\hat{c}$. Entonces, usando la estimación (E.9), tendremos

$$
\|\operatorname{div}(\mathbf{u}-\hat{\mathbf{u}})\|_{0, \Omega_{\mathrm{F}}}=|\hat{c}|\left|\Omega_{\mathrm{F}}\right|^{1 / 2} \leq C h^{1+r}\|(\mathbf{f}, \mathbf{g})\|_{0, \Omega}
$$

Para $(\mathbf{u}, \mathbf{w}) \in \mathcal{G}(\Omega)$, consideremos el siguiente problema (compatible) de Neumann:

$$
\left\{\begin{array}{lll}
\triangle q & =\operatorname{div} \mathbf{u} & \text { en } \Omega_{\mathrm{F}} \\
\frac{\partial q}{\partial \mathbf{n}}=\mathbf{w} \cdot \mathbf{n} & \text { sobre } \Gamma_{\mathrm{I}}
\end{array}\right.
$$

De las estimaciones a priori clásicas para este problema, sabemos que $q \in H^{1+s}\left(\Omega_{\mathrm{F}}\right)$ y

$$
\|\nabla q\|_{s, \Omega_{\mathrm{F}}}^{2} \leq C\left(\|\operatorname{div} \mathbf{u}\|_{0, \Omega_{\mathrm{F}}}^{2}+\sum_{j=1}^{N}\|\mathbf{w} \cdot \mathbf{n}\|_{1 / 2, \tilde{\ell}_{j}}^{2}\right) \leq C\|(\mathbf{f}, \mathbf{g})\|_{0, \Omega}^{2}
$$

Denotaremos por $\widetilde{q}$ a la extensión al dominio $\widehat{\Omega}_{\mathrm{F}}$ de la función $q$, según el Teorema (D.4). Tendremos

$$
\begin{aligned}
& \|\mathbf{u}-\hat{\mathbf{u}}\|_{0, \Omega_{\mathrm{F}}}^{2}=\int_{\Omega_{\mathrm{F}}} \nabla(q-\hat{q}) \cdot \nabla(q-\hat{q}) \leq \int_{\widehat{\Omega}_{\mathrm{F}}} \nabla(\widetilde{q}-\hat{q}) \cdot \nabla(\widetilde{q}-\hat{q}) \\
& =\int_{\Omega_{\mathrm{F}}}^{\mathrm{F}} \nabla q \cdot \nabla(q-\hat{q})+\int_{\Omega_{\mathrm{F} h} \backslash \Omega_{\mathrm{F}}} \nabla \widetilde{q} \cdot \nabla(\widetilde{q}-\hat{q})-\int_{\widehat{\Omega}_{\mathrm{F}}} \nabla \hat{q} \cdot \nabla(\widetilde{q}-\hat{q}) \\
& =-\int_{\Omega_{\mathrm{F}}} \operatorname{div} \mathbf{u}(q-\hat{q})+\int_{\Gamma_{\mathrm{I}}} \mathbf{w} \cdot \mathbf{n}(q-\hat{q})+\int_{\Omega_{\mathrm{F} h} \backslash \Omega_{\mathrm{F}}} \nabla \widetilde{q} \cdot \nabla(\widetilde{q}-\hat{q}) \\
& +\int_{\widehat{\Omega}_{\mathrm{F}}}(\widetilde{\operatorname{div} \mathbf{u}}+\hat{c})(\widetilde{q}-\hat{q})-\int_{\partial \widehat{\Omega}_{\mathrm{F}}} \check{\mathbf{w}} \cdot \mathbf{n}(\widetilde{q}-\hat{q}) \\
& =\int_{\Omega_{\mathrm{F} h} \backslash \Omega_{\mathrm{F}}} \widetilde{\operatorname{div} \mathbf{u}}(\widetilde{q}-\hat{q})+\int_{\widehat{\Omega}_{\mathrm{F}}} \hat{c}(\widetilde{q}-\hat{q})+\int_{\Omega_{\mathrm{F} h} \backslash \Omega_{\mathrm{F}}} \nabla \widetilde{q} \cdot \nabla(\widetilde{q}-\hat{q}) \\
& +\int_{\Gamma_{\mathrm{I}}}^{\mathrm{w}} \cdot \mathbf{n}(q-\hat{q})-\int_{\partial \widehat{\Omega}_{\mathrm{F}}} \check{\mathbf{w}} \cdot \mathbf{n}(\widetilde{q}-\hat{q}) .
\end{aligned}
$$

En lo que sigue, acotaremos los términos en el lado derecho de la desigualdad anterior. Para los primeros tres términos, usaremos la desigualdad de Cauchy-Schwarz, el Lema D.6 y las estimaciones (D.4), (E.9), (E.10) y (E.14). We obtain:

$$
\begin{aligned}
& \left|\int_{\Omega_{\mathrm{F} h} \backslash \Omega_{\mathrm{F}}} \widetilde{\operatorname{div} \mathbf{u}}(\widetilde{q}-\hat{q})\right| \leq\|\widetilde{\operatorname{div} \mathbf{u}}\|_{0, \Omega_{\mathrm{F} h} \backslash \Omega_{\mathrm{F}}}\left(\|\widetilde{q}\|_{0, \Omega_{\mathrm{F} h} \backslash \Omega_{\mathrm{F}}}+\|\hat{q}\|_{0, \Omega_{\mathrm{F} h} \backslash \Omega_{\mathrm{F}}}\right) \\
& \leq C h^{2}\|\operatorname{div} \mathbf{u}\|_{1+s, \Omega_{\mathrm{F}}}\left(\|q\|_{1+s, \Omega_{\mathrm{F}}}+\|\hat{q}\|_{1+s_{h}, \widehat{\Omega}_{\mathrm{F}}}\right) \\
& \leq C h^{2}\|(\mathbf{f}, \mathbf{g})\|_{0, \Omega}^{2} \text {, }
\end{aligned}
$$

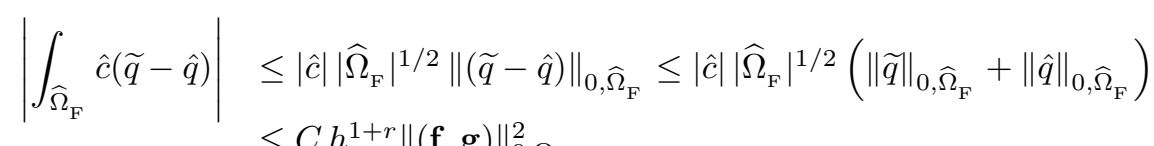

$$
\begin{aligned}
& \leq C h^{1+r}\|(\mathbf{f}, \mathbf{g})\|_{0, \Omega}^{2}, \\
& \left|\int_{\Omega_{\mathrm{F} h} \backslash \Omega_{\mathrm{F}}} \nabla \widetilde{q} \cdot \nabla(\widetilde{q}-\hat{q})\right| \leq\|\nabla \widetilde{q}\|_{0, \Omega_{\mathrm{F} h} \backslash \Omega_{\mathrm{F}}}\left(\|\nabla \widetilde{q}\|_{0, \Omega_{\mathrm{F} h} \backslash \Omega_{\mathrm{F}}}+\|\nabla \hat{q}\|_{0, \Omega_{\mathrm{F} h} \backslash \Omega_{\mathrm{F}}}\right) \\
& \leq C h^{s}\|\nabla \widetilde{q}\|_{s, \Omega_{\mathrm{F} h}}\left(h^{s}\|\widetilde{q}\|_{1+s, \Omega_{\mathrm{F} h}}+h^{s_{h}}\|\hat{q}\|_{1+s_{h}, \Omega_{\mathrm{F} h}}\right) \\
& \leq C h^{s+\hat{s}_{h}}\|(\mathbf{f}, \mathbf{g})\|_{0, \Omega}^{2} \text {. }
\end{aligned}
$$


Acotaremos ahora los dos términos restantes. Observemos primero que (recordemos que seguimos utilizando la notación $\ell=\partial T \cap \Gamma_{\mathrm{Ih}} \mathrm{y} \tilde{\ell}=\partial T^{i d} \cap \Gamma_{\mathrm{I}}$ )

$$
\int_{\Gamma_{\mathrm{I}}} \mathbf{w} \cdot \mathbf{n}(q-\hat{q})-\int_{\partial \widehat{\Omega}_{\mathrm{F}}} \check{\mathbf{w}} \cdot \mathbf{n}(\widetilde{q}-\hat{q})=\sum_{\substack{T \in \mathcal{B}_{\mathrm{Sh}} \\ T \subset T^{i d}}}\left(\int_{\tilde{\ell}}(\widetilde{q}-\hat{q}) \mathbf{w} \cdot \mathbf{n}-\int_{\ell}(\widetilde{q}-\hat{q}) \overline{\mathbf{w}} \cdot \mathbf{n}\right) .
$$

Para $T \in \mathcal{B}_{\mathrm{Sh}}$, sea $\omega_{T}$ el conjunto definido por $T^{i d} \backslash T$. Tendremos

$$
\begin{aligned}
\int_{\tilde{\ell}}(\widetilde{q}-\hat{q}) \mathbf{w} \cdot \mathbf{n}-\int_{\ell}(\widetilde{q}-\hat{q}) \mathbf{w} \cdot \mathbf{n} & \left.=-\int_{\partial \omega_{T}}(\widetilde{q}-\hat{q}) \mathbf{w} \cdot \mathbf{n}_{\mathrm{S}}=-\int_{\omega_{T}} \operatorname{div}((\widetilde{q}-\hat{q}) \mathbf{w})\right) \\
& =-\int_{\omega_{T}} \mathbf{w} \cdot \nabla(\widetilde{q}-\hat{q})-\int_{\omega_{T}}(\widetilde{q}-\hat{q}) \operatorname{div} \mathbf{w} .
\end{aligned}
$$

Sumando sobre los triángulos $T$ en el borde de $\Omega_{\mathrm{S}}$ tal que $T \subset T^{i d}$, usando la desigualdad de CauchySchwarz, el Lema D.6, el Teorema (D.4) y las estimaciones (E.10) y (E.14)

$$
\begin{aligned}
\sum_{\substack{T \in \mathcal{B}_{\mathrm{Sh}} \\
T \subset T^{i d}}}\left|\int_{\partial \omega_{T}}(\widetilde{q}-\hat{q}) \mathbf{w} \cdot \mathbf{n}\right| & \leq\|\mathbf{w}\|_{0, \Omega_{\mathrm{S}} \backslash \Omega_{\mathrm{S} h}}|\widetilde{q}-\hat{q}|_{1, \Omega_{\mathrm{F} h} \backslash \Omega_{\mathrm{F}}}+\|\widetilde{q}-\hat{q}\|_{0, \Omega_{\mathrm{F} h} \backslash \Omega_{\mathrm{F}}}|\mathbf{w}|_{1, \Omega_{\mathrm{S}} \backslash \Omega_{\mathrm{S} h}} \\
& \leq C h^{1+\min \left\{t, \hat{s}_{h}\right\}}\left(\|q\|_{1+s, \Omega_{\mathrm{F}}}+\|\hat{q}\|_{1+s_{h}, \widehat{\Omega}_{\mathrm{F}}}\right)\|\mathbf{w}\|_{1+t, \Omega_{\mathrm{S}}} \\
& \leq C h^{1+\min \left\{t, \hat{s}_{h}\right\}} \|\left(\|(\mathbf{f}, \mathbf{g})\|_{0, \Omega}^{2} .\right.
\end{aligned}
$$

Por otro lado, es inmediato que

$$
\left|\sum_{\substack{T \in \mathcal{B}_{\mathrm{Sh}} \\ T \subset T^{i d}}} \int_{\ell}(\widetilde{q}-\hat{q})(\mathbf{w}-\overline{\mathbf{w}}) \cdot \mathbf{n}\right| \leq C\|\widetilde{q}-\hat{q}\|_{0, \Gamma_{\mathrm{I} h}}\|\widetilde{\mathbf{w}}-\overline{\mathbf{w}}\|_{0, \Gamma_{\mathrm{I} h}} .
$$

Luego, usando los Lemas E.3 y E.4, el Teorema D.4, el Teorema de trazas E.1 y las estimaciones (E.10) y (E.14), tendremos

$$
\|\widetilde{q}-\hat{q}\|_{0, \Gamma_{\mathrm{I} h}}\|\widetilde{\mathbf{w}}-\overline{\mathbf{w}}\|_{0, \Gamma_{\mathrm{I} h}} \leq C h^{1+t}\|q-\hat{q}\|_{0, \Gamma_{\mathrm{I}}}\|\mathbf{w}\|_{1+t, \Omega_{\mathrm{S}}} \leq C h^{1+t}\|(\mathbf{f}, \mathbf{g})\|_{0, \Omega}^{2}
$$

Así, combinando las estimaciones encontradas, concluimos la prueba.

\section{E.4 Operadores de interpolación}

En esta sección, introduciremos un operador de interpolación para funciones vectoriales en el espacio $H^{s, 1}\left(\operatorname{div}, \widehat{\Omega}_{\mathrm{F}}\right) \times H^{1+t}\left(\widehat{\Omega}_{\mathrm{S}}\right)^{2}, s>1 / 2$ y $t>0$, y estableceremos sus propiedades de aproximación.

Comenzaremos recordando la definición del operador de interpolación de Raviart-Thomas y algunas de sus propiedades. Si u es suficientemente suave, la interpolación de Raviart-Thomas, $\boldsymbol{\Pi}_{R} \mathbf{u}$, estará bien definida por

$$
\boldsymbol{\Pi}_{R} \mathbf{u} \in \mathcal{R}_{h}\left(\Omega_{\mathrm{Fh}}\right): \quad \int_{\ell} \boldsymbol{\Pi}_{R} \mathbf{u} \cdot \mathbf{n}_{\ell}=\int_{\ell} \mathbf{u} \cdot \mathbf{n}_{\ell} \quad \forall \ell \in \mathcal{E}_{\mathrm{Fh}}
$$

donde $\mathbf{n}_{\ell}$ denota al vector unitario normal al lado $\ell$. El siguiente resultado es standard; su prueba puede encontrarse en [147], por ejemplo.

Lema E.13 El operador de interpolación de Raviart-Thomas

$$
\begin{aligned}
\boldsymbol{\Pi}_{R}: H^{1}\left(\Omega_{\mathrm{F} h}\right)^{2} & \rightarrow \mathcal{R}_{h}\left(\Omega_{\mathrm{F} h}\right) \\
\mathbf{v} & \mapsto \boldsymbol{\Pi}_{R} \mathbf{v},
\end{aligned}
$$

se extiende univocamente a $H^{s}\left(\operatorname{div}, \Omega_{\mathrm{F} h}\right), s>1 / 2$, y valen las siguientes estimaciones:

$$
\begin{aligned}
\left\|\mathbf{v}-\boldsymbol{\Pi}_{R} \mathbf{v}\right\|_{0, \Omega_{\mathrm{F} h}} & \leq C h^{s}\|\mathbf{v}\|_{s, \Omega_{\mathrm{F} h}}, \\
\left\|\operatorname{div}\left(\mathbf{v}-\boldsymbol{\Pi}_{R} \mathbf{v}\right)\right\|_{0, \Omega_{\mathrm{F} h}} & \leq C h^{s}\|\operatorname{div} \mathbf{v}\|_{s, \Omega_{\mathrm{F} h}} .
\end{aligned}
$$


Sea $\mathbf{I}_{h}: H^{s, 1}\left(\operatorname{div}, \widehat{\Omega}_{\mathrm{F}}\right) \times H^{1+t}\left(\widehat{\Omega}_{\mathrm{S}}\right)^{2} \rightarrow \mathcal{V}_{h}\left(\Omega_{h}\right)$ el operador de interpolación definido de la siguiente manera:

$$
\left.\mathbf{I}_{h}(\hat{\mathbf{u}}, \hat{\mathbf{w}})\right|_{T}:= \begin{cases}\left.\left(\boldsymbol{\Pi}_{L} \hat{\mathbf{w}}\right)\right|_{T}, & \text { if } T \subset \Omega_{\mathrm{S} h} \\ \left.\left(\boldsymbol{\Pi}_{R} \hat{\mathbf{u}}\right)\right|_{T}, & \text { if } T \subset \Omega_{\mathrm{F} h} \text { and } \partial T \cap \Gamma_{\mathrm{I} h}=\emptyset \\ \left.\left(\widehat{\boldsymbol{\Pi}}_{R} \hat{\mathbf{u}}\right)\right|_{T}, & \text { if } T \subset \Omega_{\mathrm{F} h} \text { and } \partial T \cap \Gamma_{\mathrm{I} h} \neq \emptyset\end{cases}
$$

donde $\boldsymbol{\Pi}_{L} \hat{\mathbf{w}}$ es la interpolada de Langrage de $\hat{\mathbf{w}}$ en $\mathcal{L}_{h}\left(\Omega_{\mathrm{Sh}}\right)^{2}$ y $\left.\left(\widehat{\boldsymbol{\Pi}}_{R} \hat{\mathbf{u}}\right)\right|_{T}$ es la función en $\mathcal{R}_{0}(T)$ con componentes normales definidas por

$$
\left.\left(\widehat{\boldsymbol{\Pi}}_{R} \hat{\mathbf{u}}\right)\right|_{\ell} \cdot \mathbf{n}:=\left\{\begin{aligned}
\left.\left(\boldsymbol{\Pi}_{R} \hat{\mathbf{u}}\right)\right|_{\ell} \cdot \mathbf{n} & \text { if } \ell \not \subset \Gamma_{\mathrm{I} h}, \\
\left.\frac{1}{|\ell|} \int_{\ell}\left(\boldsymbol{\Pi}_{L} \hat{\mathbf{w}}\right)\right|_{T_{S}} \cdot \mathbf{n} & \text { if } \ell \subset \Gamma_{\mathrm{I} h},
\end{aligned}\right.
$$

donde $T_{S}$ es el triángulo contenido en $\Omega_{\mathrm{Sh}}$ tal que $\partial T \cap \partial T_{S}=\ell$. Observemos que $\mathbf{I}_{h}(\hat{\mathbf{u}}, \hat{\mathbf{w}}) \in \mathcal{K}_{h}\left(\Omega_{h}\right)$ ya que la condición de compatibilidad en la interfaz $\Gamma_{\mathrm{I} h}$ está impuesta explícitamente.

Observación E.14 El operador $\mathbf{I}_{h}$ fue introducido en [33] para dominios poligonales.

El siguiente lema dará una estimación para $\left\|\boldsymbol{\Pi}_{R} \hat{\mathbf{u}}-\widehat{\boldsymbol{\Pi}}_{R} \hat{\mathbf{u}}\right\|_{H(\operatorname{div}, T)}$.

Lema E.15 Asumamos que $(\hat{\mathbf{u}}, \hat{\mathbf{w}}) \in H^{s, 1}\left(\operatorname{div}, \widehat{\Omega}_{\mathrm{F}}\right) \times H^{1+t}\left(\widehat{\Omega}_{\mathrm{S}}\right)^{2}$. Sean $T$ un triángulo en el borde de $\Omega_{\mathrm{F} h}$, $T^{i d}$ el triángulo ideal asociado con $T, \omega_{T}$ el dominio definido por $T^{i d} \backslash T, \ell:=\partial T \cap \Gamma_{\mathrm{I} h}$ y $\tilde{\ell}:=\partial T^{i d} \cap \Gamma_{\mathrm{I}}$. Entonces, existe una constante positiva $C$ tal que

$$
\left\|\boldsymbol{\Pi}_{R} \hat{\mathbf{u}}-\widehat{\boldsymbol{\Pi}}_{R} \hat{\mathbf{u}}\right\|_{H(\operatorname{div}, T)} \leq C \begin{cases}h_{T}^{1 / 2}\left(\|\operatorname{div} \hat{\mathbf{u}}\|_{0, \omega_{T}}+\|\hat{\mathbf{w}}\|_{1, \omega_{T}}\right)+\left|\frac{1}{|\ell|} \int_{\tilde{\ell}}\left(\mathbf{\Pi}_{L} \hat{\mathbf{w}}-\hat{\mathbf{u}}\right) \cdot \mathbf{n}\right|, \\ \left|\frac{1}{|\ell|} \int_{\ell}\left(\mathbf{\Pi}_{L} \hat{\mathbf{w}}-\hat{\mathbf{u}}\right) \cdot \mathbf{n}\right|, & \text { si } T \subset T^{i d}\end{cases}
$$

Prueba. Sea $\varphi_{\ell}$ la función base standard en $\mathcal{R}_{0}(T)$ asociada a $\ell$. A partir de las definiciones de las interpolaciones, tendremos

$$
\left.\left(\boldsymbol{\Pi}_{R} \hat{\mathbf{u}}-\widehat{\boldsymbol{\Pi}}_{R} \hat{\mathbf{u}}\right)\right|_{T}=\left[\frac{1}{|\ell|} \int_{\ell}\left(\hat{\mathbf{u}}-\boldsymbol{\Pi}_{L} \hat{\mathbf{w}}\right) \cdot \mathbf{n}\right] \boldsymbol{\varphi}_{\ell}
$$

Supongamos que $T \subset T^{i d}$. Usando el Teorema de Gauss, obtendremos

$$
\begin{aligned}
\int_{\ell}\left(\hat{\mathbf{u}}-\Pi_{L} \hat{\mathbf{w}}\right) \cdot \mathbf{n} & =\int_{\tilde{\ell}} \hat{\mathbf{u}} \cdot \breve{\mathbf{n}}-\int_{\omega_{T}} \operatorname{div} \hat{\mathbf{u}}-\int_{\ell} \boldsymbol{\Pi}_{L} \hat{\mathbf{w}} \cdot \mathbf{n} \\
& =\int_{\tilde{\ell}}\left(\hat{\mathbf{u}}-\boldsymbol{\Pi}_{L} \hat{\mathbf{w}}\right) \cdot \breve{\mathbf{n}}-\int_{\omega_{T}} \operatorname{div} \hat{\mathbf{u}}+\int_{\omega_{T}} \operatorname{div} \boldsymbol{\Pi}_{L} \hat{\mathbf{w}},
\end{aligned}
$$

donde $\breve{\mathbf{n}}$ denota el vector unitario normal en la dirección exterior a $\omega_{T}$. Es claro que solo será necesario acotar los dos últimos términos en el lado derecho de la desigualdad anterior. Para ello, usaremos la desigualdad de Cauchy-Schwarz y el Lema D.5. Así, obtendremos

$$
\begin{gathered}
\frac{1}{|\ell|}\left|\int_{\omega_{T}} \operatorname{div} \hat{\mathbf{u}}\right| \leq \frac{1}{|\ell|}\|\operatorname{div} \hat{\mathbf{u}}\|_{0, \omega_{T}}\left|\omega_{T}\right|^{1 / 2} \leq C h_{T}^{1 / 2}\|\operatorname{div} \hat{\mathbf{u}}\|_{0, \omega_{T}}, \\
\frac{1}{|\ell|}\left|\int_{\omega_{T}} \operatorname{div} \boldsymbol{\Pi}_{L} \hat{\mathbf{w}}\right| \leq \frac{1}{|\ell|}\left\|\operatorname{div} \boldsymbol{\Pi}_{L} \hat{\mathbf{w}}\right\|_{0, \omega_{T}}\left|\omega_{T}\right|^{1 / 2} \leq C h_{T}^{1 / 2}\left\|\boldsymbol{\Pi}_{L} \hat{\mathbf{w}}\right\|_{1, \omega_{T}} \leq C h_{T}^{1 / 2}\|\hat{\mathbf{w}}\|_{1, \omega_{T}} .
\end{gathered}
$$

La prueba termina combinando las estimaciones anteriores y recordando que $\left\|\boldsymbol{\varphi}_{\ell}\right\|_{H(\text { div } ; T)}$ está acotado por una constante positiva que solo depende de la regularidad de $T$.

Sea $\check{\mathbf{w}}$ la extensión a $\partial \widehat{\Omega}_{\mathrm{F}}$ de la traza $\left.\hat{\mathbf{w}}\right|_{\Gamma_{\mathrm{I}}}$. A las hipótesis del lema anterior, agreguemos ahora la condición $\hat{\mathbf{u}} \cdot \mathbf{n}=\check{\mathbf{w}} \cdot \mathbf{n}$ sobre $\partial \widehat{\Omega}_{\mathrm{F}}$. 
- Caso $T \subset T^{i d}$. De acuerdo con la definición $\left(\right.$ E.6), $\left.\check{\mathbf{w}} \cdot \mathbf{n}\right|_{\tilde{\ell}}=\left.\hat{\mathbf{w}} \cdot \mathbf{n}\right|_{\tilde{\ell}}$, Entonces, usando un teorema de trazas local y resultados de interpolación standard,

$$
\begin{aligned}
\frac{1}{|\ell|}\left|\int_{\tilde{\ell}}\left(\boldsymbol{\Pi}_{L} \hat{\mathbf{w}}-\hat{\mathbf{u}}\right) \cdot \mathbf{n}\right| & =\frac{1}{|\ell|}\left|\int_{\tilde{\ell}}\left(\boldsymbol{\Pi}_{L} \hat{\mathbf{w}}-\hat{\mathbf{w}}\right) \cdot \mathbf{n}\right|=\frac{1}{|\ell|}\left\|\boldsymbol{\Pi}_{L} \hat{\mathbf{w}}-\hat{\mathbf{w}}\right\|_{0, \tilde{\ell}}|\tilde{\ell}|^{1 / 2} \\
& \leq C \frac{1}{|\ell|}\left(h_{T}^{-1 / 2}\left\|\boldsymbol{\Pi}_{L} \hat{\mathbf{w}}-\hat{\mathbf{w}}\right\|_{0, T_{S}}+h_{T}^{1 / 2}\left\|\boldsymbol{\Pi}_{L} \hat{\mathbf{w}}-\hat{\mathbf{w}}\right\|_{1, T_{S}}\right)|\tilde{\ell}|^{1 / 2} \\
& \leq C h_{T}^{t}\|\hat{\mathbf{w}}\|_{1+t, T_{S}} .
\end{aligned}
$$

En el cálculo anterior hemos asumido que existe una constante $C$, independiente de $h$, tal que that $|\tilde{\ell}| \leq C|\ell|$. Dado que, por hipótesis $\Gamma_{\mathrm{I}}$ es $\mathcal{C}^{2}$ a trozos, tal resultado puede probarse fácilmente usando los argumentos introducidos en la sección E.2.

- Caso $T^{i d} \subset T$. De la definición (E.6), tendremos $\left.\breve{\mathbf{w}} \cdot \mathbf{n}\right|_{\tilde{\ell}}=\left.\overline{\hat{\mathbf{w}}} \cdot \mathbf{n}\right|_{\tilde{\ell}}$. Luego,

$$
\frac{1}{|\ell|} \int_{\ell}\left(\boldsymbol{\Pi}_{L} \hat{\mathbf{w}}-\hat{\mathbf{u}}\right) \cdot \mathbf{n}=\frac{1}{|\ell|} \int_{\ell}\left(\boldsymbol{\Pi}_{L} \hat{\mathbf{w}}-\hat{\mathbf{w}}\right) \cdot \mathbf{n}+\frac{1}{|\ell|} \int_{\ell}(\hat{\mathbf{w}}-\overline{\hat{\mathbf{w}}}) \cdot \mathbf{n} .
$$

El primer término en el lado derecho de esta ecuación puede acotarse exactamente igual a como lo hicimos en el caso anterior.

Luego, combinando los resultados del Lema E.15 con las estimaciones recién halladas, sumando sobre todos los triángulos en el borde del dominio $\Omega_{\mathrm{Fh}}$ y usando los Lemas D.6 y E.4, obtendremos

$$
\left\|\boldsymbol{\Pi}_{R} \hat{\mathbf{u}}-\widehat{\boldsymbol{\Pi}}_{R} \hat{\mathbf{u}}\right\|_{H\left(\operatorname{div}, \Omega_{\mathrm{F} h}\right)} \leq C h^{t}\left(\|\operatorname{div} \hat{\mathbf{u}}\|_{1, \widehat{\Omega}_{\mathrm{F}}}+\|\hat{\mathbf{w}}\|_{1+t, \widehat{\Omega}_{\mathrm{S}}}\right) .
$$

Este resultado nos induce a introducir el siguiente espacio:

$$
\widehat{\mathcal{K}}(\widehat{\Omega}):=\left\{(\hat{\mathbf{u}}, \hat{\mathbf{w}}) \in H\left(\operatorname{div}, \widehat{\Omega}_{\mathrm{F}}\right) \times H^{1}\left(\widehat{\Omega}_{\mathrm{S}}\right)^{2}: \hat{\mathbf{u}} \cdot \mathbf{n}=\check{\mathbf{w}} \cdot \mathbf{n} \text { sobre } \partial \widehat{\Omega}_{\mathrm{F}}\right\} .
$$

Lema E.16 Para todo $(\hat{\mathbf{u}}, \hat{\mathbf{w}}) \in \widehat{\mathcal{K}}(\widehat{\Omega}) \cap\left(H^{s, 1}\left(\operatorname{div}, \widehat{\Omega}_{\mathrm{F}}\right) \times H^{1+t}\left(\widehat{\Omega}_{\mathrm{S}}\right)^{2}\right)$, existe una constante positiva $C$, independiente de $h$, tal que

$$
\left\|(\hat{\mathbf{u}}, \hat{\mathbf{w}})-\mathbf{I}_{h}(\hat{\mathbf{u}}, \hat{\mathbf{w}})\right\|_{h, \Omega_{h}} \leq C h^{r}\left(\|\hat{\mathbf{u}}\|_{H^{s, 1}\left(\operatorname{div}, \widehat{\Omega}_{\mathrm{F}}\right)}+\|\hat{\mathbf{w}}\|_{1+t, \widehat{\Omega}_{\mathrm{S}}}\right) .
$$

Prueba. Es idéntica a la prueba del Teorema 5.2 en [33].

Observación E.17 Sea $(\hat{\mathbf{u}}, \hat{\mathbf{w}})=\left(\nabla \hat{q}, \mathbf{w}^{e}\right)$, donde $\hat{q}$ es el potencial escalar introducido en la sección previa $y \mathbf{w}$ es un campo vectorial tal que la extensión de su traza $\left.\mathbf{w}\right|_{\Gamma_{\mathrm{I}}}$ al borde $\partial \widehat{\Omega}_{\mathrm{F}}$ (denotada $\check{\mathbf{w}}$ ) es el dato de borde en el problema que define a $\hat{q}$ (ver problema (E.7)). De acuerdo con los resultados obtenidos en la sección E.3, podremos escribir

$$
\left\|\left(\nabla \hat{q}, \mathbf{w}^{e}\right)-\mathbf{I}_{h}\left(\nabla \hat{q}, \mathbf{w}^{e}\right)\right\|_{h, \Omega_{h}} \leq C h^{r_{h}}\|(\mathbf{f}, \mathbf{g})\|_{0, \Omega}
$$

donde $r_{h}=\min \left\{\hat{s}_{h}, t\right\}$.

\section{E.5 Propiedad de compacidad discreta}

La propiedad de compacidad discreta aparece frecuentemente en la literatura vinculada con la solución aproximada por elementos finitos del problema espectral de Maxwell [126, 41, 139]; pero su concepto puede ser extendido a situaciones más generales [58]. En esta sección analizaremos la propiedad de compacidad discreta en el contexto de los problemas de autovalores que surgen al estudiar ciertos sistemas con interacción fluido-estructura. Probaremos que esta propiedad es válidad para el método que acopla el espacio de Raviart-Thomas de menor orden (para discretizar los desplazamientos en el fluido) con el espacio lineal de Courant (para discretizar los desplazamientos en la estructura). Como fuera definido en la sección 4.2.1, en este esquema las condiciones de compatibilidad en la interfaz son impuestas débilmente; dando lugar así a un método no conforme. 
Recordemos que la dificultad que se presenta al tratar con el operador grad div es la falta de elipticidad que se pone de manifiesto por la presencia de un núcleo $\mathcal{H}(\Omega)$ de dimensión infinita. Es claro que, si se incorporara la condición curl $\mathbf{u}=\mathbf{0}$ sobre los campos de desplazamientos en el fluido, el espacio $\mathcal{H}(\Omega)$ quedaría eliminado; es decir, el problema recobraría sus propiedades de elipticidad gracias a la inclusión compacta $\mathcal{G}(\Omega) \hookrightarrow \mathcal{Q}(\Omega)$. Dado que la condición curl u $=\mathbf{0}$ no es fácil de imponer sobre los espacios discretos, cierto control del rotacional de sus elementos se vuelve una condición necesaria para la convergencia correcta de los modos propios aproximados. La propiedad de compacidad discreta es la expresión matemática de ese control: imita a nivel discreto la inclusión compacta $\mathcal{G}(\Omega) \hookrightarrow \mathcal{Q}(\Omega)$.

Comenzaremos dando la definición de la propiedad de compacidad discreta para problemas con interacción fluido-estructura.

Propiedad E.18 Sea $\left\{\left(\mathbf{u}_{h}, \mathbf{w}_{h}\right)\right\}_{h>0}$ una sucesión arbitraria de funciones en $\mathcal{V}_{h}\left(\Omega_{h}\right)$ tal que

$$
\int_{\Gamma_{\mathrm{I} h}}\left(\mathbf{u}_{h}-\mathbf{w}_{h}\right) \cdot \mathbf{n}=0 .
$$

Asumiremos que las funciones $\mathbf{u}_{h}$ tienen rotor discreto nulo, i.e.,

$$
\int_{\Omega_{\mathrm{F} h}} \mathbf{u}_{h} \cdot \operatorname{curl} p_{h}=0 \quad p_{h} \in \mathcal{Z}_{h}\left(\Omega_{\mathrm{F} h}\right) .
$$

Asumiremos también que el par $\left(\mathbf{u}_{h}, \mathbf{w}_{h}\right)$ está uniformemente acotado en $\mathcal{V}_{h}\left(\Omega_{h}\right)$. Entonces, existe una subsucesión, que también denotaremos $\left\{\left(\mathbf{u}_{h}, \mathbf{w}_{h}\right)\right\}_{h>0}$, tal que $\left(\mathbf{u}_{h}^{e}, \mathbf{w}_{h}^{e}\right)$ converge fuertemente a $(\mathbf{u}, \mathbf{w}) \in$ $\mathcal{V}(\Omega)$ en $\mathcal{Q}(\Omega)$ y verifica

$$
(\mathbf{u}-\mathbf{w}) \cdot \mathbf{n}=0 \quad \text { sobre } \Gamma_{\mathrm{I}} .
$$

Siguiendo la linea argumental de [58] (ver Teorema 3.5 en esa referencia), puede probarse que la Propiedad E.18, con límite fuerte en $\mathcal{G}(\Omega)$, es una consecuencia de la siguiente propiedad:

Propiedad E.19 Para todo $\left(\mathbf{u}_{h}, \mathbf{w}_{h}\right) \in \mathcal{G}_{h}\left(\Omega_{h}\right)$ existe $(\mathbf{u}, \mathbf{w}) \in \mathcal{G}(\Omega)$ tal que

$$
\left\|(\widetilde{\mathbf{u}}, \widetilde{\mathbf{w}})-\left(\mathbf{u}_{h}^{e}, \mathbf{w}_{h}^{e}\right)\right\|_{h} \leq C h^{1 / 2}\left\|\left(\mathbf{u}_{h}, \mathbf{w}_{h}\right)\right\|_{h, \Omega_{h}},
$$

donde $(\widetilde{\mathbf{u}}, \widetilde{\mathbf{w}}) \in \mathcal{X}(\widehat{\Omega})$ denota una extensión de $(\mathbf{u}, \mathbf{w})$ desde $\Omega$, su dominio original, al dominio más grande $\widehat{\Omega}$.

Prueba. Para un elemento arbitrario $\left(\mathbf{u}_{h}, \mathbf{w}_{h}\right) \in \mathcal{G}_{h}\left(\Omega_{h}\right)$, sea $\left(\mathbf{u}_{h}^{e}, \mathbf{w}_{h}^{e}\right)=\mathbf{P}_{h}\left(\mathbf{u}_{h}, \mathbf{w}_{h}\right)$ la extensión a $\widehat{\Omega}$ definida en la sección 4.2.1. Sea $o \in \mathbb{R}$ la constante que hace que el siguiente problema de Neumann sea compatible:

$$
\left\{\begin{array}{l}
\triangle q=\operatorname{div} \mathbf{u}_{h}^{e}+o \text { en } \Omega_{\mathrm{F}} \\
\frac{\partial q}{\partial n}=\mathbf{w}_{h}^{e} \cdot \mathbf{n} \quad \text { sobre } \partial \Omega_{\mathrm{F}}
\end{array}\right.
$$

Sea $\omega_{T}$ el dominio definido por $T \backslash T^{i d}$ o $T^{i d} \backslash T$, según corresponda. Entonces,

$$
o=\frac{1}{\left|\Omega_{\mathrm{F}}\right|} \sum_{T \in \mathcal{B}_{\mathrm{F} h}}\left(\int_{\omega_{T}} \operatorname{div} \mathbf{w}_{h}^{e}-\int_{\omega_{T}} \operatorname{div} \mathbf{u}_{h}^{e}\right) .
$$

Sumando sobre todos los triángulos que yacen sobre la interfaz; usando el Lema D.5 y la estimación (4.68), tendremos

$$
\begin{aligned}
|o|\left|\Omega_{\mathrm{F}}\right| & \leq\left|\int_{\Omega_{\mathrm{F}} \backslash \Omega_{\mathrm{F} h}}\left(\operatorname{div} \mathbf{w}_{h}^{e}-\operatorname{div} \mathbf{u}_{h}^{e}\right)\right|+\left|\int_{\Omega_{\mathrm{F} h} \backslash \Omega_{\mathrm{F}}}\left(\operatorname{div} \mathbf{w}_{h}^{e}-\operatorname{div} \mathbf{u}_{h}^{e}\right)\right| \\
& \leq C h\left\|\operatorname{div} \mathbf{w}_{h}^{e}-\operatorname{div} \mathbf{u}_{h}^{e}\right\|_{0, \widehat{\Omega}} \leq C h\left\|\left(\mathbf{u}_{h}, \mathbf{w}_{h}\right)\right\|_{h, \Omega_{h}} .
\end{aligned}
$$

Sea $q$ la solución (única) del problema (E.15). A partir de las estimaciones a priori clásicas para este problema, sabemos que $q \in H^{1+s}\left(\Omega_{\mathrm{F}}\right), s>1 / 2$. Más aún, usando (4.68) y (E.16),

$$
\|q\|_{1+s, \Omega_{\mathrm{F}}}^{2} \leq C\left(\left\|\operatorname{div} \mathbf{u}_{h}^{e}+o\right\|_{0, \Omega_{\mathrm{F}}}^{2}+\sum_{j=1}^{N}\left\|\mathbf{w}_{h}^{e} \cdot \mathbf{n}\right\|_{1 / 2, \tilde{\ell}_{j}}^{2}\right) \leq C\left\|\left(\mathbf{u}_{h}, \mathbf{w}_{h}\right)\right\|_{h, \Omega_{h}}^{2} .
$$


Tomemos $(\mathbf{u}, \mathbf{w})=\left(\nabla q,\left.\mathbf{w}_{h}^{e}\right|_{\Omega_{\mathrm{S}}}\right) \in \mathcal{G}(\Omega)$. Sea $\mathbf{u}^{e}$ la extensión de $\mathbf{u}$ a $\widehat{\Omega}_{\mathrm{F}}$ definida como en la sección 4.2.1. Definiremos la extensión de $(\mathbf{u}, \mathbf{w})$ a $\widehat{\Omega}$ por medio del par $\left(\mathbf{u}^{e}, \mathbf{w}_{h}^{e}\right)$. De esta manera, para probar la propiedad E.19, solo necesitaremos acotar la norma $H\left(\operatorname{div}, \widehat{\Omega}_{\mathrm{F}}\right)$ de la diferencia $\mathbf{u}^{e}-\mathbf{u}_{h}^{e}$. Obtendremos la cota buscada en los siguientes tres pasos.

Paso 1: acotación de $\left\|\operatorname{div}\left(\mathbf{u}^{e}-\mathbf{u}_{h}^{e}\right)\right\|_{0, \widehat{\Omega}_{\mathrm{F}}}$. Según el problema (E.15) y la definición de la extensión $\mathbf{u}^{e}$, tendremos

$$
\begin{aligned}
\left.\operatorname{div}\left(\mathbf{u}^{e}-\mathbf{u}_{h}^{e}\right)\right|_{\widehat{\Omega}_{\mathrm{F}}} & =\left.\operatorname{div}\left(\mathbf{u}-\mathbf{u}_{h}^{e}\right)\right|_{\Omega_{\mathrm{F}}}+\left.\operatorname{div}\left(\mathbf{u}^{e}-\mathbf{u}_{h}\right)\right|_{\Omega_{\mathrm{F} h} \backslash \Omega_{\mathrm{F}}} \\
& =\left.o\right|_{\Omega_{\mathrm{F}}}+\left.\left(\frac{1}{\left|\Omega_{\mathrm{S}}\right|} \int_{\Omega_{\mathrm{F}}} \operatorname{div} \mathbf{u}\right)\right|_{\Omega_{\mathrm{F} h} \backslash \Omega_{\mathrm{F}}}-\left.\operatorname{div} \mathbf{u}_{h}\right|_{\Omega_{\mathrm{F} h} \backslash \Omega_{\mathrm{F}}}
\end{aligned}
$$

Denotemos por $\omega_{T}$ al conjunto $T \backslash T^{i d}$. Teniendo en cuenta que $\left.\operatorname{div} \mathbf{u}_{h}\right|_{T}$ es constante para todo $T$ en $\mathcal{T}_{h}^{\mathrm{F}}$, podemos usar el Lema D.5 para obtener

$$
\begin{gathered}
\left\|\operatorname{div} \mathbf{u}_{h}\right\|_{0, \Omega_{\mathrm{F} h} \backslash \Omega_{\mathrm{F}}}^{2}=\sum_{\substack{T \in \mathcal{B}_{\mathrm{F} h} \\
T^{i d} \subset T}}\left\|\operatorname{div} \mathbf{u}_{h}\right\|_{0, \omega_{T}}^{2} \leq C h \sum_{\substack{T \in \mathcal{B}_{\mathrm{F} h} \\
T^{i d} \subset T}}\left\|\operatorname{div} \mathbf{u}_{h}\right\|_{0, T}^{2}, \\
\left\|\frac{1}{\left|\Omega_{\mathrm{S}}\right|} \int_{\Omega_{\mathrm{F}}} \operatorname{div} \mathbf{u}\right\|_{0, \Omega_{\mathrm{F} h} \backslash \Omega_{\mathrm{F}}} \leq\left|\Omega_{\mathrm{F} h} \backslash \Omega_{\mathrm{F}}\right|^{1 / 2}{\frac{\left|\Omega_{\mathrm{F}}\right|}{\left|\Omega_{\mathrm{S}}\right|}}^{1 / 2}\|\operatorname{div} \mathbf{u}\|_{0, \Omega_{\mathrm{F}}} \leq C h\left\|\operatorname{div} \mathbf{u}_{h}^{e}+o\right\|_{0, \Omega_{\mathrm{F}}} .
\end{gathered}
$$

Combinando los resultados anteriores con las estimaciones (E.16) y (E.17), tendremos finalmente

$$
\left\|\operatorname{div}\left(\mathbf{u}^{e}-\mathbf{u}_{h}^{e}\right)\right\|_{0, \widehat{\Omega}_{\mathrm{F}}} \leq C h^{1 / 2}\left\|\left(\mathbf{u}_{h}, \mathbf{w}_{h}\right)\right\|_{h, \Omega_{h}} .
$$

Paso 2: acotación de $\left\|\mathbf{u}^{e}-\mathbf{u}_{h}^{e}\right\|_{0, \widehat{\Omega}_{\mathrm{F}}}$. Consideremos nuevamente la solución $q$ del problema (E.15). Sea $\widetilde{q}$ la extensión a $\mathbb{R}^{2}$ de la función $q \in H^{1+s}\left(\Omega_{\mathrm{F}}\right)$ con las propiedades enunciadas en el Teorema D.4. Entonces, tomando $\mathbf{u}_{0}^{e}=\nabla \widetilde{q}$, tendremos

$$
\left\|\mathbf{u}^{e}-\mathbf{u}_{h}^{e}\right\|_{0, \widehat{\Omega}_{\mathrm{F}}} \leq\left\|\mathbf{u}^{e}-\mathbf{u}_{0}^{e}\right\|_{0, \widehat{\Omega}_{\mathrm{F}}}+\left\|\mathbf{u}_{0}^{e}-\mathbf{u}_{h}^{e}\right\|_{0, \widehat{\Omega}_{\mathrm{F}}}
$$

Acotaremos el primer término en el lado derecho de la expresión anterior. Observemos que, debido a la definición de $\mathbf{u}^{e}$, se cumplirá

$$
\left\|\mathbf{u}^{e}-\mathbf{u}_{0}^{e}\right\|_{0, \widehat{\Omega}_{\mathrm{F}}}=\left\|\mathbf{u}^{e}-\mathbf{u}_{0}^{e}\right\|_{0, \Omega_{\mathrm{F} h} \backslash \Omega_{\mathrm{F}}} \leq\|\nabla \xi\|_{0, \Omega_{\mathrm{S}} \backslash \Omega_{\mathrm{S} h}}+\|\nabla \widetilde{q}\|_{0, \Omega_{\mathrm{F} h} \backslash \Omega_{\mathrm{F}}}
$$

A partir del Lema D.6, la estimación (4.67), la definición de u y la estimación (E.17), tendremos

$$
\begin{aligned}
\|\nabla \xi\|_{0, \Omega_{\mathrm{S}} \backslash \Omega_{\mathrm{S} h}} & \leq C h^{\alpha}\|\nabla \xi\|_{\alpha, \Omega_{\mathrm{S}}} \leq C h^{\alpha}\left(\|\operatorname{div} \mathbf{u}\|_{0, \Omega_{\mathrm{F}}}^{2}+\sum_{j=1}^{N}\|\mathbf{u} \cdot \mathbf{n}\|_{1 / 2, \tilde{\ell}_{j}}^{2}\right)^{1 / 2} \\
& \leq C h^{\alpha}\left(\left\|\operatorname{div} \mathbf{u}_{h}^{e}+o\right\|_{0, \Omega_{\mathrm{F}}}^{2}+\sum_{j=1}^{N}\left\|\mathbf{w}_{h}^{e} \cdot \mathbf{n}\right\|_{1 / 2, \tilde{\ell}_{j}}^{2}\right)^{1 / 2} \leq C h^{\alpha}\left\|\left(\mathbf{u}_{h}, \mathbf{w}_{h}\right)\right\|_{h, \Omega_{h}} .
\end{aligned}
$$

De manera análoga, usando ahora el Lema D.6 en combinación con el Teorema D.4 y la estimación (E.17), tendremos

$$
\|\nabla \widetilde{q}\|_{0, \Omega_{\mathrm{F} h} \backslash \Omega_{\mathrm{F}}} \leq C h^{s}\|\nabla \widetilde{q}\|_{s, \Omega_{\mathrm{F} h}} \leq C h^{s}\|\nabla q\|_{s, \Omega_{\mathrm{F}}} \leq C h^{s}\left\|\left(\mathbf{u}_{h}, \mathbf{w}_{h}\right)\right\|_{h, \Omega_{h}} .
$$

Resta acotar el término $\left\|\mathbf{u}_{0}^{e}-\mathbf{u}_{h}^{e}\right\|_{0, \widehat{\Omega}_{\mathrm{F}}}$. Para ello, necesitaremos el siguiente lema.

Lema E.20 Sea $\left(\mathbf{u}_{h}, \mathbf{w}_{h}\right) \in \mathcal{G}_{h}\left(\Omega_{h}\right)$. Existen un par $\left(\mathbf{u}_{1}, \mathbf{w}_{1}\right) \in \mathcal{G}\left(\Omega_{h}\right)$ y una constante positiva $C$, independiente de $h$, tal que

$$
\left\|\left(\mathbf{u}_{1}, \mathbf{w}_{1}\right)-\left(\mathbf{u}_{h}, \mathbf{w}_{h}\right)\right\|_{h, \Omega_{h}} \leq C h^{s_{h}}\left\|\left(\mathbf{u}_{h}, \mathbf{w}_{h}\right)\right\|_{h, \Omega_{h}} .
$$


Prueba. Muchas de las ideas que usaremos en esta prueba fueron también utilizadas en las pruebas del Teorema 5.4 y del Lema 5.5 in [33]. Sea $\left(\mathbf{u}_{h}, \mathbf{w}_{h}\right) \in \mathcal{G}_{h}\left(\Omega_{h}\right)$. Consideremos la descomposición

$$
\left(\mathbf{u}_{h}, \mathbf{w}_{h}\right)=\left(\operatorname{curl} \xi_{1}, \mathbf{0}\right)+\left(\nabla q_{1}, \mathbf{w}_{h}\right)
$$

donde $\xi_{1} \in H_{0}^{1}\left(\Omega_{\mathrm{F} h}\right)$ y $q_{1}$ es la solución del siguiente problema de Neumann:

$$
\left\{\begin{array}{lll}
\triangle q_{1} & =\operatorname{div} \mathbf{u}_{h} & \text { en } \Omega_{\mathrm{F} h} \\
\frac{\partial q_{1}}{\partial \mathbf{n}}=\mathbf{w}_{h} \cdot \mathbf{n} & \text { sobre } \partial \Omega_{\mathrm{F} h}
\end{array}\right.
$$

Observemos que, como $\left(\mathbf{u}_{h}, \mathbf{w}_{h}\right) \in \mathcal{G}_{h}\left(\Omega_{h}\right) \subset \mathcal{K}_{h}\left(\Omega_{h}\right)$,

$$
\int_{\Gamma_{\mathrm{I} h}} \mathbf{u}_{h} \cdot \mathbf{n}=\int_{\Gamma_{\mathrm{I} h}} \frac{\partial q_{1}}{\partial \mathbf{n}}=\int_{\Gamma_{\mathrm{I} h}} \mathbf{w}_{h} \cdot \mathbf{n} .
$$

Por lo tanto, el problema (E.21) es compatible; tiene una única solución $q_{1} \in H^{1}\left(\Omega_{\mathrm{Fh}}\right) / \mathbb{R}$. De las estimaciones a priori clásicas, tendremos $q_{1} \in H^{1+s_{h}}\left(\Omega_{\mathrm{F} h}\right), s_{h}>1 / 2$. Más aún, usando el Lema E.8 (ver observación E.9 y recordar D.2), podremos asegurar que existe una constante $C$, independiente de $h$, tal que

$$
\left\|q_{1}\right\|_{1+s_{h}, \Omega_{\mathrm{F} h}} \leq C\left(\left\|\operatorname{div} \mathbf{u}_{h}\right\|_{0, \Omega_{\mathrm{F} h}}^{2}+\sum_{j=1}^{N(h)}\left\|\mathbf{w}_{h} \cdot \mathbf{n}\right\|_{1 / 2, \ell_{j}}^{2}\right)^{1 / 2} \leq C\left\|\left(\mathbf{u}_{h}, \mathbf{w}_{h}\right)\right\|_{h, \Omega_{h}} .
$$

Luego, la interpolada de Raviart-Thomas de $\nabla q_{1}$ estará bien definida. Además, tendremos

$$
\left\|\nabla q_{1}-\boldsymbol{\Pi}_{R} \nabla q_{1}\right\|_{0, \Omega_{\mathrm{F} h}} \leq C h^{s_{h}}\left\|q_{1}\right\|_{1+s_{h}, \Omega_{\mathrm{F} h}} \leq C h^{s_{h}}\left\|\left(\mathbf{u}_{h}, \mathbf{w}_{h}\right)\right\|_{h, \Omega_{h}} .
$$

Por otro lado,

$$
\left\|\operatorname{curl} \xi_{1}\right\|_{0, \Omega_{\mathrm{F} h}}^{2}=\int_{\Omega_{\mathrm{F} h}}\left(\nabla q_{1}-\mathbf{u}_{h}\right) \cdot\left(\nabla q_{1}-\boldsymbol{\Pi}_{R} \nabla q_{1}\right)+\int_{\Omega_{\mathrm{F} h}}\left(\nabla q_{1}-\mathbf{u}_{h}\right) \cdot\left(\boldsymbol{\Pi}_{R} \nabla q_{1}-\mathbf{u}_{h}\right) .
$$

Entonces, si pudiéramos demostrar que

$$
\int_{\Omega_{\mathrm{F} h}}\left(\nabla q_{1}-\mathbf{u}_{h}\right) \cdot\left(\boldsymbol{\Pi}_{R} \nabla q_{1}-\mathbf{u}_{h}\right)=0,
$$

el lema sería una consecuencia directa de (E.23), (E.24) y la siguiente definición

$$
\left(\mathbf{u}_{1}, \mathbf{w}_{1}\right):=\left(\nabla q_{1}, \mathbf{w}_{h}\right) .
$$

A continuación probaremos (E.25). Observemos que $\left(\boldsymbol{\Pi}_{R} \nabla q_{1}-\mathbf{u}_{h}\right) \in \mathcal{R}_{h}\left(\Omega_{\mathrm{F} h}\right)$. Por lo tanto, para todo $T \in \Omega_{\mathrm{Fh}}$, valdrá

$$
\begin{aligned}
\left.\operatorname{div}\left(\boldsymbol{\Pi}_{R} \nabla q_{1}-\mathbf{u}_{h}\right)\right|_{T} & =\frac{1}{|T|} \int_{T} \operatorname{div}\left(\boldsymbol{\Pi}_{R} \nabla q_{1}-\mathbf{u}_{h}\right)=\frac{1}{|T|} \int_{\partial T}\left(\boldsymbol{\Pi}_{R} \nabla q_{1}-\mathbf{u}_{h}\right) \cdot \mathbf{n} \\
& =\frac{1}{|T|} \int_{\partial T}\left(\nabla q_{1}-\mathbf{u}_{h}\right) \cdot \mathbf{n}=\frac{1}{|T|} \int_{T} \operatorname{div}\left(\nabla q_{1}-\mathbf{u}_{h}\right)=0 .
\end{aligned}
$$

Para todo $\ell \subset \Gamma_{I h}$, tendremos

$$
\left.\left(\boldsymbol{\Pi}_{R} \nabla q_{1}-\mathbf{u}_{h}\right)\right|_{\ell} \cdot \mathbf{n}=\frac{1}{|\ell|} \int_{\ell}\left(\boldsymbol{\Pi}_{R} \nabla q_{1}-\mathbf{u}_{h}\right) \cdot \mathbf{n}=\frac{1}{|\ell|} \int_{\ell}\left(\nabla q_{1}-\mathbf{u}_{h}\right) \cdot \mathbf{n}=0 .
$$

Consecuentemente, $\left(\boldsymbol{\Pi}_{R} \nabla q_{1}-\mathbf{u}_{h}, \mathbf{0}\right) \in \mathcal{H}_{h}\left(\Omega_{h}\right)$. Luego, a partir de la descomposición introducida en la sección E.1, sabemos que existe $p_{h} \in \mathcal{Z}_{h}\left(\Omega_{\mathrm{F} h}\right)$ tal que $\left(\boldsymbol{\Pi}_{R} \nabla q_{1}-\mathbf{u}_{h}, \mathbf{0}\right)=\left(\operatorname{curl} p_{h}, \mathbf{0}\right)$. Entonces,

$$
\int_{\Omega_{\mathrm{F} h}}\left(\nabla q_{1}-\mathbf{u}_{h}\right) \cdot\left(\boldsymbol{\Pi}_{R} \nabla q_{1}-\mathbf{u}_{h}\right)=\int_{\Omega_{\mathrm{F} h}}\left(\nabla q_{1}-\mathbf{u}_{h}\right) \cdot \operatorname{curl} p_{h}=\int_{\Omega_{\mathrm{F} h}} \nabla q_{1} \cdot \operatorname{curl} p_{h}=0 .
$$


Así, concluimos la prueba.

Paso 3: acotación de $\left\|\mathbf{u}_{0}^{e}-\mathbf{u}_{h}^{e}\right\|_{0, \widehat{\Omega}_{\mathrm{F}}}$. Sea $\left(\mathbf{u}_{1}, \mathbf{w}_{1}\right)=\left(\nabla q_{1}, \mathbf{w}_{h}\right) \in \mathcal{G}\left(\Omega_{h}\right)$, como en el lema anterior. Sea $\widetilde{q}_{1}$ la extensión a $\mathbb{R}^{2}$ de $q_{1} \in H^{1+s_{h}}\left(\Omega_{\mathrm{Fh}}\right)$ según el Teorema D.4. Entonces, eligiendo $\mathbf{u}_{1}^{e}=\nabla \widetilde{q}_{1}$, tendremos

$$
\left\|\mathbf{u}_{0}^{e}-\mathbf{u}_{h}^{e}\right\|_{0, \widehat{\Omega}_{\mathrm{F}}} \leq\left\|\mathbf{u}_{0}^{e}-\mathbf{u}_{1}^{e}\right\|_{0, \widehat{\Omega}_{\mathrm{F}}}+\left\|\mathbf{u}_{1}^{e}-\mathbf{u}_{h}^{e}\right\|_{0, \widehat{\Omega}_{\mathrm{F}}} .
$$

Los argumentos en la prueba del Lema E.12 pueden ser modificados fáclmente para probar

$$
\left\|\mathbf{u}_{0}^{e}-\mathbf{u}_{1}^{e}\right\|_{0, \widehat{\Omega}_{\mathrm{F}}} \leq C h^{\left(s+s_{h}\right) / 2}\left\|\left(\mathbf{u}_{h}, \mathbf{w}_{h}\right)\right\|_{h, \Omega_{h}}+\left\|\mathbf{u}_{0}^{e}-\mathbf{u}_{1}\right\|_{0, \Omega_{\mathrm{F} h} \backslash \Omega_{\mathrm{F}}} .
$$

Usando el Lema D.9, el Teorema D.4 y las estimaciones (E.17) y (E.21), tendremos

$$
\begin{aligned}
& \left\|\mathbf{u}_{0}^{e}\right\|_{0, \Omega_{\mathrm{F} h} \backslash \Omega_{\mathrm{F}}}=\|\nabla \widetilde{q}\|_{0, \Omega_{\mathrm{F} h} \backslash \Omega_{\mathrm{F}}} \leq C h^{s}\|\nabla q\|_{s, \Omega_{\mathrm{F} h}} \leq C h^{s}\left\|\left(\mathbf{u}_{h}, \mathbf{w}_{h}\right)\right\|_{h, \Omega_{h}}, \\
& \left\|\mathbf{u}_{1}\right\|_{0, \Omega_{\mathrm{F} h} \backslash \Omega_{\mathrm{F}}}=\left\|\nabla q_{1}\right\|_{0, \Omega_{\mathrm{F} h} \backslash \Omega_{\mathrm{F}}} \leq C h^{s_{h}}\left\|\nabla q_{1}\right\|_{s_{h}, \Omega_{\mathrm{F} h}} \leq C h^{s_{h}}\left\|\left(\mathbf{u}_{h}, \mathbf{w}_{h}\right)\right\|_{h, \Omega_{h}} .
\end{aligned}
$$

Combinando las últimas tres desigualdades,

$$
\left\|\mathbf{u}_{0}^{e}-\mathbf{u}_{1}^{e}\right\|_{0, \widehat{\Omega}_{\mathrm{F}}} \leq C h^{\min \left\{s, s_{h}\right\}}\left\|\left(\mathbf{u}_{h}, \mathbf{w}_{h}\right)\right\|_{h, \Omega_{h}} .
$$

Por otro lado, como consecuencia del Lema E.20,

$$
\left\|\mathbf{u}_{1}^{e}-\mathbf{u}_{h}^{e}\right\|_{0, \widehat{\Omega}_{\mathrm{F}}} \leq C h^{s_{h}}\left\|\left(\mathbf{u}_{h}, \mathbf{w}_{h}\right)\right\|_{h, \Omega_{h}}+\left\|\mathbf{u}_{1}^{e}-\mathbf{u}_{h}^{e}\right\|_{0, \Omega_{\mathrm{F}} \backslash \Omega_{\mathrm{F} h}} .
$$

Usando nuevamente el Lema D.9, el Teorema D.4 y la estimación (E.21), tendremos

$$
\left\|\mathbf{u}_{1}^{e}\right\|_{0, \Omega_{\mathrm{F}} \backslash \Omega_{\mathrm{F} h}}=\left\|\nabla \widetilde{q}_{1}\right\|_{0, \Omega_{\mathrm{F}} \backslash \Omega_{\mathrm{F} h}} \leq C h^{s_{h}}\left\|\nabla q_{1}\right\|_{s_{h}, \Omega_{\mathrm{F}}} \leq C h^{s_{h}}\left\|\left(\mathbf{u}_{h}, \mathbf{w}_{h}\right)\right\|_{h, \Omega_{h}} .
$$

Procediendo como en la sección 4.2.1, we obtain

$$
\left\|\mathbf{u}_{h}^{e}\right\|_{0, \Omega_{\mathrm{F}} \backslash \Omega_{\mathrm{F} h}}=\left(\sum_{\substack{T \in \mathcal{B}_{\mathrm{Fh}} \\ T \subset T^{i d}}}\left\|\mathbf{u}_{h}^{e}\right\|_{0, \omega_{T}}^{2}\right)^{1 / 2} \leq C h^{1 / 2}\left\|\left(\mathbf{u}_{h}, \mathbf{w}_{h}\right)\right\|_{h, \Omega_{h}},
$$

resultado que, combinado con las estimaciones anteriores, nos permite concluir

$$
\left\|\mathbf{u}_{1}^{e}-\mathbf{u}_{h}^{e}\right\|_{0, \widehat{\Omega}_{\mathrm{F}}} \leq C h^{1 / 2}\left\|\left(\mathbf{u}_{h}, \mathbf{w}_{h}\right)\right\|_{h, \Omega_{h}}
$$

Paso 4: conclusion. Las estimaciones (E.18), (E.19), (E.20) y (E.26) conducen a

$$
\left\|\mathbf{u}_{1}^{e}-\mathbf{u}_{h}^{e}\right\|_{H\left(\operatorname{div} ; \widehat{\Omega}_{\mathrm{F}}\right)} \leq C h^{1 / 2}\left\|\left(\mathbf{u}_{h}, \mathbf{w}_{h}\right)\right\|_{h, \Omega_{h}} .
$$

De esta forma concluimos la prueba de la Propiedad E.19.

La siguiente propiedad es también importante para el análisis de este problema.

Propiedad E.21 Para todo par $\left(\mathbf{u}_{h}, \mathbf{0}\right) \in \mathcal{H}_{h}\left(\Omega_{h}\right)$ existe un par $(\mathbf{u}, \mathbf{0}) \in \mathcal{H}(\Omega)$ tal que

$$
\left\|\left(\mathbf{u}^{e}, \mathbf{0}\right)-\left(\mathbf{u}_{h}^{e}, \mathbf{0}\right)\right\|_{h} \leq C h^{1 / 2}\left\|\left(\mathbf{u}_{h}, \mathbf{0}\right)\right\|_{h, \Omega_{h}} .
$$

Prueba. Como $\left(\mathbf{u}_{h}, \mathbf{0}\right) \in \mathcal{H}_{h}\left(\Omega_{h}\right)$, podremos escribir $\mathbf{u}_{h}=\operatorname{curl} p_{h}$, con $p_{h} \in \mathcal{Z}_{h}\left(\Omega_{\mathrm{F} h}\right)$. Sea $\hat{p}$ la función asociada a $p_{h}$ según la definición D.10. Como $\hat{p} \in H_{\Gamma_{\mathrm{I}}}^{1}\left(\Omega_{\mathrm{F}}\right),(\operatorname{curl} \hat{p}, \mathbf{0}) \in \mathcal{H}(\Omega)$. Entonces, tomando $\mathbf{u}=\operatorname{curl} \hat{p}$, tendremos

$$
\begin{aligned}
\left\|\left(\mathbf{u}^{e}, \mathbf{0}\right)-\left(\mathbf{u}_{h}^{e}, \mathbf{0}\right)\right\|_{h}^{2}= & \left\|\mathbf{u}^{e}-\mathbf{u}_{h}^{e}\right\|_{H\left(\operatorname{div} ; \widehat{\Omega}_{\mathrm{F}}\right)}^{2}=\left\|\operatorname{curl}\left(\hat{p}-p_{h}\right)\right\|_{0, \Omega_{\mathrm{F}} \cap \Omega_{\mathrm{Fh}}}^{2}+\left\|\operatorname{curl} p_{h}\right\|_{0, \Omega_{\mathrm{F} h} \backslash \Omega_{\mathrm{F}}}^{2} \\
= & \sum_{\substack{T \in \mathcal{B}_{\mathrm{Fh}} \\
T \supset T^{i d}}}\left(\left\|\operatorname{curl}\left(\hat{p}-p_{h}\right)\right\|_{0, T^{i d}}^{2}+\left\|\operatorname{curl} p_{h}\right\|_{0, \omega_{T}}^{2}\right),
\end{aligned}
$$

donde $\omega_{T}$ es el dominio definido por $T \backslash T^{i d}$. Dado que $\left.\operatorname{curl} p_{h}\right|_{T}$ es constante $\forall T \in \mathcal{T}_{h}^{\mathrm{F}}$, podremos usar el Lema D.5 para obtener

$$
\left\|\operatorname{curl} p_{h}\right\|_{0, \omega_{T}}=\left|\operatorname{curl} p_{h}\left\|\left.\omega_{T}\right|^{1 / 2} \leq C h^{1 / 2}\right\| \operatorname{curl} p_{h} \|_{0, T} .\right.
$$

Así, combinando esta estimación con el resultado de la observación D.12, concluimos la prueba. 
Observación E.22 Como surge de los cálculos anteriores, dado un par discreto $\left(\mathbf{u}_{h}, \mathbf{w}_{h}\right)$ en $\mathcal{G}_{h}\left(\Omega_{h}\right)$, es posible construir un par continuo $(\mathbf{u}, \mathbf{w})$ en $\mathcal{G}(\Omega)$ eligiendo simplemente $\mathbf{w}=\mathbf{w}_{h}^{e}$ en $\Omega_{\mathrm{S}}$. Observemos que para proveer una extensión al dominio $\widehat{\Omega}$ de este par, podríamos proceder directamente tomando

$$
\left(\mathbf{u}^{e}, \mathbf{w}^{e}\right)=\mathbf{P}(\mathbf{u}, \mathbf{w}),
$$

siendo $\mathbf{P}$ el operador de extensión definido en la sección 4.2.1. También podríamos proceder como en la prueba de la Propiedad E.19 y definir

$$
(\widetilde{\mathbf{u}}, \widetilde{\mathbf{w}})=\left(\mathbf{u}^{e}, \mathbf{w}_{h}^{e}\right)
$$

Obviamente, los pares extendidos de esta última manera no pertenecen a $\mathcal{G}^{e}(\widehat{\Omega})$. De hecho, es fácil probar que

$$
\left\|(\widetilde{\mathbf{u}}, \widetilde{\mathbf{w}})-\left(\mathbf{u}^{e}, \mathbf{w}^{e}\right)\right\|_{h}=\left\|\mathbf{w}_{h}-\mathbf{P}_{S}\left(\left.\mathbf{w}_{h}^{e}\right|_{\Omega_{\mathrm{S}}}\right)\right\|_{1, \Omega_{\mathrm{S} h} \backslash \Omega_{\mathrm{S}}}
$$

No obstante ello, los pares $(\widetilde{\mathbf{u}}, \widetilde{\mathbf{w}})$ nos permitirán definir otro espacio de funciones que será muy útil en las pruebas para establecer la convergencia del método numérico. Este nuevo espacio será denotado por $\widehat{\mathcal{G}}(\widehat{\Omega})$. Observemos que para cualquier par $(\widetilde{\mathbf{u}}, \widetilde{\mathbf{w}})$ en $\widehat{\mathcal{G}}(\widehat{\Omega})$ se verifica

$$
\|(\widetilde{\mathbf{u}}, \widetilde{\mathbf{w}})\|_{h} \leq C\left\|\left(\mathbf{u}_{h}, \mathbf{w}_{h}\right)\right\|_{h, \Omega_{h}}
$$

Observación E.23 Las Propiedades E.19 y E.21, consideradas juntas, constituyen lo que se conoce como Propiedadad de Gap para $\mathcal{K}_{h}\left(\Omega_{h}\right)$ (ver el paper [58] para su definición y una discusión más profunda sobre sus consecuencias). En ausencia de perturbaciones del dominio; i.e., cuando $\Omega_{\mathrm{F}}=\Omega_{\mathrm{F} h}$, lo cual, en particular, implica una interfaz poligonal, la Propiedad E.21 es trivialmente cierta ya que $\mathcal{H}_{h}\left(\Omega_{h}\right) \subset$ $\mathcal{H}(\Omega)$. En este último caso, el Lema E.20 y la Propiedad de Gap son equivalentes.

\section{E.6 Otras estimaciones}

Esta sección contiene algunos resultados técnicos que se usarán en las demostraciones de los lemas y teoremas enunciados en la sección 4.2.1. Preservaremos la notación de esa sección y de las secciones previas.

Lema E.24 La siguiente estimación es válida

$$
\left|\rho_{\mathrm{F}} \int_{\Omega_{\mathrm{F}} \backslash \Omega_{\mathrm{F} h}} \mathbf{f} \cdot \boldsymbol{\eta}_{h}^{e}\right| \leq C h^{s}\|(\mathbf{f}, \mathbf{g})\|_{h, \Omega}\left\|\left(\boldsymbol{\eta}_{h}, \mathbf{z}_{h}\right)\right\|_{h, \Omega_{h}} .
$$

Prueba. Como $(\mathbf{f}, \mathbf{g}) \in \mathcal{G}(\Omega)$, existe $q \in H^{1}\left(\Omega_{\mathrm{F}}\right)$ tal que $\mathbf{f}=\nabla q$. Gracias a la condición de acoplamiento en la interfaz, $q$ es una solución del siguiente problema (compatible) de Neumann:

$$
\left\{\begin{array}{l}
\Delta q=\operatorname{div} \mathbf{f} \quad \text { en } \Omega_{\mathrm{F}} \\
\frac{\partial q}{\partial n}=\mathbf{g} \cdot \mathbf{n} \quad \text { sobre } \Gamma_{\mathrm{I}}
\end{array}\right.
$$

De acuerdo con las estimaciones a priori standard para este problema, $q \in H^{1+s}\left(\Omega_{\mathrm{F}}\right)$; más aún

$$
\|q\|_{1+s, \Omega_{\mathrm{F}}} \leq C\left(\|\operatorname{div} \mathbf{f}\|_{0, \Omega_{\mathrm{F}}}^{2}+\sum_{j=1}^{N}\|\mathbf{g} \cdot \mathbf{n}\|_{1 / 2, \tilde{\ell}_{j}}^{2}\right)^{1 / 2} \leq C\|(\mathbf{f}, \mathbf{g})\|_{h, \Omega} .
$$

Usando entonces la desigualdad de Cauchy-Schwarz, los Lemas D.6 y D.9 y la estimación anterior, tendremos

$$
\begin{aligned}
\left|\rho_{\mathrm{F}} \int_{\Omega_{\mathrm{F}} \backslash \Omega_{\mathrm{F} h}} \mathbf{f} \cdot \boldsymbol{\eta}_{h}^{e}\right| & \leq \rho_{\mathrm{F}}\|\mathbf{f}\|_{0, \Omega_{\mathrm{F}} \backslash \Omega_{\mathrm{F} h}}\left\|\boldsymbol{\eta}_{h}^{e}\right\|_{0, \Omega_{\mathrm{F}} \backslash \Omega_{\mathrm{F} h}} \leq C h^{s}\|\mathbf{f}\|_{s, \Omega_{\mathrm{F}}}\left\|\boldsymbol{\eta}_{h}\right\|_{0, \Omega_{\mathrm{F} h}} \\
& \leq C h^{s}\|(\mathbf{f}, \mathbf{g})\|_{h, \Omega}\left\|\boldsymbol{\eta}_{h}\right\|_{H\left(\operatorname{div}: \Omega_{\mathrm{F} h}\right)} .
\end{aligned}
$$

Así, concluimos la prueba. 
Lema E.25 La siguiente estimación es válida

$$
\left|\rho_{\mathrm{F}} \int_{\Omega_{\mathrm{F} h} \backslash \Omega_{\mathrm{F}}} \mathbf{f} \cdot \boldsymbol{\eta}_{h}\right| \leq C h^{s_{h}}\|\mathbf{f}\|_{0, \widehat{\Omega}_{\mathrm{F}}}\left\|\left(\boldsymbol{\eta}_{h}, \mathbf{z}_{h}\right)\right\|_{h, \Omega_{h}} .
$$

Prueba. Como $\left(\boldsymbol{\eta}_{h}, \mathbf{z}_{h}\right) \in \mathcal{G}_{h}\left(\Omega_{h}\right)$, podemos proceder como en la prueba del Lema E.20 y considerar la descomposición ortogonal

$$
\boldsymbol{\eta}_{h}=\nabla q_{1}+\operatorname{curl} \xi_{1}
$$

donde $q_{1} \in H^{1+s_{h}}\left(\Omega_{\mathrm{F} h}\right) \mathrm{y}$

$$
\left\|\operatorname{curl} \xi_{1}\right\|_{0, \Omega_{\mathrm{F} h}} \leq C h^{s_{h}}\left\|\left(\boldsymbol{\eta}_{h}, \mathbf{z}_{h}\right)\right\|_{h, \Omega_{h}} .
$$

Luego, usando la desigualdad de Cauchy-Schwarz, tendremos

$$
\begin{aligned}
\left|\rho_{\mathrm{F}} \int_{\Omega_{\mathrm{F} h} \backslash \Omega_{\mathrm{F}}} \mathbf{f} \cdot \boldsymbol{\eta}_{h}\right| & \leq \rho_{\mathrm{F}}\|\mathbf{f}\|_{0, \Omega_{\mathrm{F} h} \backslash \Omega_{\mathrm{F}}}\left\|\boldsymbol{\eta}_{h}\right\|_{0, \Omega_{\mathrm{F} h} \backslash \Omega_{\mathrm{F}}} \\
& \leq C\|\mathbf{f}\|_{0, \Omega_{\mathrm{F} h} \backslash \Omega_{\mathrm{F}}}\left\|\nabla q_{1}+\operatorname{curl} \xi_{1}\right\|_{0, \Omega_{\mathrm{F} h} \backslash \Omega_{\mathrm{F}}} \\
& \leq C\|\mathbf{f}\|_{0, \widehat{\Omega}}\left(\left\|\nabla q_{1}\right\|_{0, \Omega_{\mathrm{F} h} \backslash \Omega_{\mathrm{F}}}+\left\|\operatorname{curl} \xi_{1}\right\|_{0, \Omega_{\mathrm{F} h} \backslash \Omega_{\mathrm{F}}}\right) \\
& \leq C h^{s_{h}}\|\mathbf{f}\|_{0, \widehat{\Omega}}\left(\left\|\nabla q_{1}\right\|_{s_{h}, \Omega_{\mathrm{F} h}}+\left\|\left(\boldsymbol{\eta}_{h}, \mathbf{z}_{h}\right)\right\|_{h, \Omega_{h}}\right),
\end{aligned}
$$

donde la última desigualdad se obtuvo a partir del Lema D.6 y la estimación (E.27). La prueba concluye entonces teniendo en cuenta la estimación (E.22).

Lema E.26 La siguiente estimación es válida

$$
\left|\rho_{\mathrm{S}} \int_{\Omega_{\mathrm{S}} \backslash \Omega_{\mathrm{S} h}} \mathbf{g} \cdot \mathbf{z}_{h}^{e}\right|+\left|\rho_{\mathrm{S}} \int_{\Omega_{\mathrm{S} h} \backslash \Omega_{\mathrm{S}}} \mathbf{g} \cdot \mathbf{z}_{h}\right| \leq C h\|\mathbf{g}\|_{0, \widehat{\Omega}_{\mathrm{S}}}\left\|\mathbf{z}_{h}\right\|_{1, \Omega_{\mathrm{S} h}} .
$$

Prueba. Inmediata a partir de la desigualdad de Cauchy-Schwarz y los Lemas D.6 and D.9. $\square$

Lema E.27 La siguiente estimación es válida

$$
\left|\rho_{\mathrm{F}} c^{2} \hat{c} \int_{\Omega_{\mathrm{F}}} \operatorname{div} \boldsymbol{\eta}_{h}^{e}\right| \leq C h^{1+r}\|(\mathbf{f}, \mathbf{g})\|_{0, \Omega}\left\|\boldsymbol{\eta}_{h}\right\|_{H\left(\operatorname{div} ; \Omega_{h}\right)} .
$$

Prueba. Inmediata a partir de la desigualdad de Cauchy-Schwartz y las estimaciones (E.9) y (4.68).

Lema E.28 Las siguientes estimaciones son válidas

$$
\begin{gathered}
\left|\rho_{\mathrm{F}} \int_{\Omega_{\mathrm{F}} \backslash \Omega_{\mathrm{F} h}} \hat{\mathbf{u}} \cdot \boldsymbol{\eta}_{h}^{e}\right|+\left|\rho_{\mathrm{F}} \int_{\Omega_{\mathrm{F} h} \backslash \Omega_{\mathrm{F}}} \hat{\mathbf{u}} \cdot \boldsymbol{\eta}_{h}\right| \leq C h^{s_{h}}\|(\mathbf{f}, \mathbf{g})\|_{0, \Omega}\left\|\boldsymbol{\eta}_{h}\right\|_{H\left(\operatorname{div} ; \Omega_{h}\right)}, \\
\left|\rho_{\mathrm{F}} \int_{\Omega_{\mathrm{F}}}(\hat{\mathbf{u}}-\mathbf{u}) \cdot \boldsymbol{\eta}_{h}^{e}\right| \leq C h^{\left(r+\hat{s}_{h}\right) / 2}\|(\mathbf{f}, \mathbf{g})\|_{0, \Omega}\left\|\boldsymbol{\eta}_{h}\right\|_{H\left(\operatorname{div} ; \Omega_{h}\right)} .
\end{gathered}
$$

Prueba. La primera estimación es una consecuencia directa de la desigualdad de Cauchy-Schwartz en combinación con los resultados de regularidad para las funciones $\mathbf{u}$ y $\hat{\mathbf{u}}$, el Lema D.6 y las estimaciones (4.68) y (E.11) De manera similar, la segunda estimación se obtiene a partir de la desigualdad de CauchySchwartz, el Lema E.12 y la estimación (4.68).

Lema E.29 La siguiente desigualdad es válida

$$
\left|\rho_{\mathrm{F}} c^{2} \int_{\Omega_{\mathrm{F}} \backslash \Omega_{\mathrm{F} h}} \operatorname{div} \hat{\mathbf{u}} \operatorname{div} \boldsymbol{\eta}_{h}^{e}\right|+\left|\rho_{\mathrm{F}} c^{2} \int_{\Omega_{\mathrm{F} h} \backslash \Omega_{\mathrm{F}}} \operatorname{div} \hat{\mathbf{u}} \operatorname{div} \boldsymbol{\eta}_{h}\right| \leq C h\|(\mathbf{f}, \mathbf{g})\|_{0, \Omega}\left\|\boldsymbol{\eta}_{h}\right\|_{H\left(\operatorname{div} ; \Omega_{h}\right)} .
$$


Prueba. Como $\hat{\mathbf{u}}=\nabla q$, siendo $q$ la solución del problema de Neumann (E.7), entonces $\operatorname{div} \hat{\mathbf{u}}=\widetilde{\operatorname{div} \mathbf{u}}+\hat{c}$. Usando la desigualdad de Cauchy-Schwarz, el Lema D.6 y las estimaciones (E.9) y (E.12), tendremos

$$
\begin{aligned}
\left|\rho_{\mathrm{F}} c^{2} \int_{\Omega_{\mathrm{F} h} \backslash \Omega_{\mathrm{F}}} \operatorname{div} \hat{\mathbf{u}} \operatorname{div} \boldsymbol{\eta}_{h}\right| & \leq C\left(\|\widetilde{\operatorname{div} \mathbf{u}}\|_{0, \Omega_{\mathrm{F} h} \backslash \Omega_{\mathrm{F}}}+\left|\hat{c} \| \Omega_{\mathrm{F} h} \backslash \Omega_{\mathrm{F}}\right|^{1 / 2}\right)\left\|\boldsymbol{\eta}_{h}\right\|_{H\left(\operatorname{div} ; \Omega_{h}\right)} \\
& \leq C\left(h\|\widetilde{\operatorname{div} \mathbf{u}}\|_{1, \Omega_{\mathrm{F} h}}+h^{1+r}\|(\mathbf{f}, \mathbf{g})\|_{0, \Omega}\right)\left\|\boldsymbol{\eta}_{h}\right\|_{H\left(\operatorname{div} ; \Omega_{h}\right)} \\
& \leq C h\|(\mathbf{f}, \mathbf{g})\|_{0, \Omega}\left\|\boldsymbol{\eta}_{h}\right\|_{H\left(\operatorname{div} ; \Omega_{h}\right)} .
\end{aligned}
$$

De manera idéntica, se prueba la desigualdad restante; sustituyendo, obviamente, $\Omega_{\mathrm{F} h} \backslash \Omega_{\mathrm{F}}$ por $\Omega_{\mathrm{F}} \backslash \Omega_{\mathrm{F} h}$.

Lema E.30 Las siguientes estimaciones son válidas

$$
\begin{gathered}
\left|\int_{\Omega_{\mathrm{S} h} \backslash \Omega_{\mathrm{S}}} \boldsymbol{\sigma}(\hat{\mathbf{w}}): \varepsilon\left(\mathbf{z}_{h}\right)\right|+\left|\rho_{\mathrm{S}} \int_{\Omega_{\mathrm{S} h} \backslash \Omega_{\mathrm{S}}} \hat{\mathbf{w}} \cdot \mathbf{z}_{h}\right| \leq C h^{t}\|(\mathbf{f}, \mathbf{g})\|_{0, \Omega}\left\|\mathbf{z}_{h}\right\|_{1, \Omega_{\mathrm{S} h}}, \\
\left|\int_{\Omega_{\mathrm{S}} \backslash \Omega_{\mathrm{S} h}} \boldsymbol{\sigma}(\mathbf{w}): \varepsilon\left(\mathbf{z}_{h}^{e}\right)\right|+\left|\rho_{\mathrm{S}} \int_{\Omega_{\mathrm{S}} \backslash \Omega_{\mathrm{S} h}} \mathbf{w} \cdot \mathbf{z}_{h}^{e}\right| \leq C h^{t}\|(\mathbf{f}, \mathbf{g})\|_{0, \Omega}\left\|\mathbf{z}_{h}\right\|_{1, \Omega_{\mathrm{S} h}} .
\end{gathered}
$$

Prueba. Comenzaremos recordando que $\hat{\mathbf{w}}=\widetilde{\mathbf{w}}$, siendo $\widetilde{\mathbf{w}}$ la extensión de $\mathbf{w}$ a $\widehat{\Omega}_{\mathrm{S}}$ según la definición (D.4). Usando la desigualdad de Cauchy-Schwarz, el Lema D.6 y los Teoremas D.4 y 4.29, tendremos

$$
\begin{aligned}
& \left|\int_{\Omega_{\mathrm{S} h} \backslash \Omega_{\mathrm{S}}} \boldsymbol{\sigma}(\hat{\mathbf{w}}): \boldsymbol{\varepsilon}\left(\mathbf{z}_{h}\right)\right| \leq\|\boldsymbol{\sigma}(\hat{\mathbf{w}})\|_{0, \Omega_{\mathrm{S} h} \backslash \Omega_{\mathrm{S}}}\left\|\boldsymbol{\varepsilon}\left(\mathbf{z}_{h}\right)\right\|_{0, \Omega_{\mathrm{S} h} \backslash \Omega_{\mathrm{S}}} \leq C\|\hat{\mathbf{w}}\|_{1, \Omega_{\mathrm{S} h} \backslash \Omega_{\mathrm{S}}}\left\|\mathbf{z}_{h}\right\|_{1, \Omega_{\mathrm{S} h} \backslash \Omega_{\mathrm{S}}} \\
& \leq C h^{t}\|\hat{\mathbf{w}}\|_{1+t, \Omega_{\mathrm{S} h}}\left\|\mathbf{z}_{h}\right\|_{1, \Omega_{\mathrm{S} h}} \leq C h^{t}\|(\mathbf{f}, \mathbf{g})\|_{0, \Omega}\left\|\mathbf{z}_{h}\right\|_{1, \Omega_{\mathrm{S} h}}, \\
& \left|\int_{\Omega_{\mathrm{S} h} \backslash \Omega_{\mathrm{S}}} \hat{\mathbf{w}} \cdot \mathbf{z}_{h}\right| \leq\|\hat{\mathbf{w}}\|_{0, \Omega_{\mathrm{S} h} \backslash \Omega_{\mathrm{S}}}\left\|\mathbf{z}_{h}\right\|_{0, \Omega_{\mathrm{S} h} \backslash \Omega_{\mathrm{S}}} \leq C h\|\hat{\mathbf{w}}\|_{1, \Omega_{\mathrm{S} h}}\left\|\mathbf{z}_{h}\right\|_{1, \Omega_{\mathrm{S} h}} \leq C h\|(\mathbf{f}, \mathbf{g})\|_{0, \Omega}\left\|\mathbf{z}_{h}\right\|_{1, \Omega_{\mathrm{S} h}} .
\end{aligned}
$$

Por lo tanto, la primera desigualdad se obtiene inmediatamente combinando las dos expresiones anteriores. De manera idéntica, reemplazando $\Omega_{\mathrm{S} h} \backslash \Omega_{\mathrm{S}}$ por $\Omega_{\mathrm{S}} \backslash \Omega_{\mathrm{S} h}$ y usando la estimación (4.68), se obtiene la segunda desigualdad.

Lema E.31 La siguiente estimación es válida

$$
\left|\int_{\Gamma_{\mathrm{I}}} \gamma\left(\boldsymbol{\eta}_{h}^{e}-\mathbf{z}_{h}^{e}\right) \cdot \mathbf{n}\right| \leq C h^{s}\|(\mathbf{f}, \mathbf{g})\|_{0, \Omega}\left\|\left(\boldsymbol{\eta}_{h}, \mathbf{z}_{h}\right)\right\|_{h, \Omega_{h}}
$$

Prueba. Denotemos por $\widetilde{\gamma}$ a la restricción sobre $\Gamma_{\mathrm{Ih}}$ de la función $-\rho_{\mathrm{F}} c^{2} \widetilde{\operatorname{div} \mathbf{u}} \in H^{1+s}\left(\widehat{\Omega}_{\mathrm{F}}\right)$. Sea $T$ un triángulo en el borde del dominio $\Omega_{\mathrm{F}}$. Sea $\omega_{T}$ el dominio definido por $T \backslash T^{i d} \mathrm{o} T^{i d} \backslash T$, según corresponda. El borde de $\omega_{T}$ será la unión del lado curvo $\tilde{\ell}=\partial T^{i d} \cap \Gamma_{\mathrm{I}}$ con el lado recto $\ell=\partial T \cap \Gamma_{\mathrm{I} h}$. Sea $P_{\ell}$ la proyección $L^{2}(\ell)$ de $H^{1 / 2}(\ell)$ sobre las funciones constantes definidas en $\ell$. Como $\left(\boldsymbol{\eta}_{h}, \mathbf{z}_{h}\right) \in \mathcal{G}_{h}\left(\Omega_{h}\right)$, la condición de acoplamiento (discreta) sobre la interfaz implica que $\left.\boldsymbol{\eta}_{h} \cdot \mathbf{n}\right|_{\ell}=P_{\ell}\left(\left.\mathbf{z}_{h} \cdot \mathbf{n}\right|_{\ell}\right)$. Observemos también que

$$
\int_{\ell} P_{\ell}(\widetilde{\gamma})\left(\boldsymbol{\eta}_{h}-\mathbf{z}_{h}\right) \cdot \mathbf{n}=\int_{\ell} P_{\ell}(\widetilde{\gamma})\left(P_{\ell}\left(\mathbf{z}_{h} \cdot \mathbf{n}\right)-\mathbf{z}_{h} \cdot \mathbf{n}\right)=0
$$

y, por lo tanto, podremos escribir

$$
\int_{\Gamma_{\mathrm{I}}} \gamma\left(\boldsymbol{\eta}_{h}^{e}-\mathbf{z}_{h}^{e}\right) \cdot \mathbf{n}=\sum_{\ell \subset \Gamma_{\mathrm{I} h}} \int_{\ell}\left(\widetilde{\gamma}-P_{\ell}(\widetilde{\gamma})\right)\left(\boldsymbol{\eta}_{h}-\mathbf{z}_{h}\right) \cdot \mathbf{n}-\int_{\Gamma_{\mathrm{I} h}} \widetilde{\gamma}\left(\boldsymbol{\eta}_{h}-\mathbf{z}_{h}\right) \cdot \mathbf{n}+\int_{\Gamma_{\mathrm{I}}} \gamma\left(\boldsymbol{\eta}_{h}^{e}-\mathbf{z}_{h}^{e}\right) \cdot \mathbf{n} .
$$

Sea $P_{T}$ la proyección $L^{2}(T)$ de $H^{1}(T)$ sobre las constantes. Usando un teorema de trazas local y estimaciones de error standard para $P_{T}$, tendremos

$$
\left\|\widetilde{\gamma}-P_{\ell}(\widetilde{\gamma})\right\|_{0, \ell} \leq\left\|\widetilde{\gamma}-P_{T}(\widetilde{\gamma})\right\|_{0, \ell} \leq C\left(h_{T}^{-1 / 2}\left\|\widetilde{\gamma}-P_{T}(\widetilde{\gamma})\right\|_{0, T}+h_{T}^{1 / 2}\left|\widetilde{\gamma}-P_{T}(\widetilde{\gamma})\right|_{1, T}\right) \leq C h_{T}^{1 / 2}\|\widetilde{\gamma}\|_{1, T} .
$$


Sea ahora $T_{S}$ el triángulo en el borde de $\Omega_{\mathrm{S}}$ tal que $\partial T \cap \partial T_{S}=\ell$. Procediendo de la misma manera,

$$
\left\|\mathbf{z}_{h} \cdot \mathbf{n}-P_{\ell}\left(\mathbf{z}_{h} \cdot \mathbf{n}\right)\right\|_{0, \ell} \leq C h_{T_{S}}^{1 / 2}\left\|\mathbf{z}_{h}\right\|_{1, T_{S}} .
$$

Así, sumando sobre todos los lados $\ell$ incluidos en $\Gamma_{\mathrm{I} h}$ y usando el Teorema D.4, llegaremos a

$$
\begin{aligned}
\left|\int_{\Gamma_{\mathrm{I} h}}\left(\widetilde{\gamma}-P_{\ell}(\widetilde{\gamma})\right)\left(\mathbf{z}_{h}-\boldsymbol{\eta}_{h}\right) \cdot \mathbf{n}\right| & \leq \sum_{\ell \subset \Gamma_{\mathrm{I} h}}\left\|\widetilde{\gamma}-P_{\ell}(\widetilde{\gamma})\right\|_{0, \ell}\left\|\mathbf{z}_{h} \cdot \mathbf{n}-P_{\ell}\left(\mathbf{z}_{h} \cdot \mathbf{n}\right)\right\|_{0, \ell} \\
& \leq C h\|\widetilde{\gamma}\|_{1, \Omega_{\mathrm{F} h}}\left\|\mathbf{z}_{h}\right\|_{1, \Omega_{\mathrm{S} h}} \\
& \leq C h\|\operatorname{div} \mathbf{u}\|_{1, \Omega_{\mathrm{F}}}\left\|\left(\boldsymbol{\eta}_{h}, \mathbf{z}_{h}\right)\right\|_{h, \Omega_{h}} .
\end{aligned}
$$

Usando ahora el Lema D.6, el Teorema D.4 y la estimación (4.68), tendremos

$$
\begin{aligned}
& \left|\int_{\Gamma_{\mathrm{I} h}} \widetilde{\gamma}\left(\boldsymbol{\eta}_{h}-\mathbf{z}_{h}\right) \cdot \mathbf{n}-\int_{\Gamma_{\mathrm{I}}} \gamma\left(\boldsymbol{\eta}_{h}^{e}-\mathbf{z}_{h}^{e}\right) \cdot \mathbf{n}\right|=\left|\sum_{T \in \mathcal{B}_{\mathrm{F} h}}-\rho_{\mathrm{F}} c^{2} \int_{\partial \omega_{T}} \widetilde{\operatorname{div} \mathbf{u}}\left(\boldsymbol{\eta}_{h}^{e}-\mathbf{z}_{h}^{e}\right) \cdot \mathbf{n}\right| \\
& =\rho_{\mathrm{F}} c^{2}\left|\sum_{T \in \mathcal{B}_{\mathrm{F} h}}\left(\int_{\omega_{T}} \widetilde{\operatorname{div} \mathbf{u}} \operatorname{div}\left(\boldsymbol{\eta}_{h}^{e}-\mathbf{z}_{h}^{e}\right)+\int_{\omega_{T}} \widetilde{\nabla \operatorname{div} \mathbf{u}} \cdot\left(\boldsymbol{\eta}_{h}^{e}-\mathbf{z}_{h}^{e}\right)\right)\right| \\
& \leq \rho_{\mathrm{F}} c^{2} \sum_{T \in \mathcal{B}_{\mathrm{F} h}}^{\mathrm{F} h}\|\widetilde{\operatorname{div} \mathbf{u}}\|_{0, \omega_{T}}\left(\left\|\operatorname{div} \boldsymbol{\eta}_{h}^{e}\right\|_{0, \omega_{T}}+\left\|\operatorname{div} \mathbf{z}_{h}^{e}\right\|_{0, \omega_{T}}\right) \\
& +\rho_{\mathrm{F}} c^{2} \sum_{T \in \mathcal{B}_{\mathrm{F} h}}^{\mathrm{F} h}\|\widetilde{\operatorname{div} \mathbf{u}}\|_{0, \omega_{T}}\left(\left\|\boldsymbol{\eta}_{h}^{e}\right\|_{0, \omega_{T}}+\left\|\mathbf{z}_{h}^{e}\right\|_{0, \omega_{T}}\right) \\
& \leq C \sum_{T \in \mathcal{B}_{\mathrm{F} h}}\left(\|\widetilde{\operatorname{div} \mathbf{u}}\|_{0, \omega_{T}}+\|\nabla \widetilde{\operatorname{div} \mathbf{u}}\|_{0, \omega_{T}}\right)\left\|\left(\boldsymbol{\eta}_{h}^{e}, \mathbf{z}_{h}^{e}\right)\right\|_{h} \\
& \leq C\left(\|\widetilde{\operatorname{div} \mathbf{u}}\|_{0, \Omega_{\mathrm{F}} \backslash \Omega_{\mathrm{F} h} \cup \Omega_{\mathrm{F} h} \backslash \Omega_{\mathrm{F}}}+\|\widetilde{\operatorname{div} \mathbf{u}}\|_{0, \Omega_{\mathrm{F}} \backslash \Omega_{\mathrm{F} h} \cup \Omega_{\mathrm{F} h} \backslash \Omega_{\mathrm{F}}}\right)\left\|\left(\boldsymbol{\eta}_{h}, \mathbf{z}_{h}\right)\right\|_{h, \Omega_{h}} \\
& \leq C\left(h\|\operatorname{div} \mathbf{u}\|_{1, \Omega_{\mathrm{F}}}+h^{s}\|\nabla \operatorname{div} \mathbf{u}\|_{s, \Omega_{\mathrm{F}}}\right)\left\|\left(\boldsymbol{\eta}_{h}, \mathbf{z}_{h}\right)\right\|_{h, \Omega_{h}} .
\end{aligned}
$$

Combinando las expresiones anteriores con los resultados del Teorema 4.29, concluimos la prueba.

Lema E.32 La siguiente estimación es válida

$$
\left|\rho_{\mathrm{F}} \int_{\Omega_{\mathrm{F}}}(\hat{\mathbf{u}}-\mathbf{u}) \cdot \boldsymbol{\eta}_{h}^{e}\right| \leq C h^{\left(r+\hat{s}_{h}\right) / 2}\|(\mathbf{f}, \mathbf{g})\|_{0, \Omega}\left\|\boldsymbol{\eta}_{h}\right\|_{0, \Omega_{\mathrm{F} h}} .
$$

Prueba. Sigue directamente de la desigualdad de Cauchy-Schwarz, el Lema E.12 y la estimación (4.69).

Lema E.33 La siguiente estimación es válida

$$
\left|\rho_{\mathrm{F}} \int_{\Omega_{\mathrm{F} h} \backslash \Omega_{\mathrm{F}}} \hat{\mathbf{u}} \cdot \boldsymbol{\eta}_{h}\right| \leq C h^{s_{h}}\|(\mathbf{f}, \mathbf{g})\|_{0, \Omega}\left\|\boldsymbol{\eta}_{h}\right\|_{0, \Omega_{\mathrm{F} h}} .
$$

Prueba. Sigue directamente de la desigualdad de Cauchy-Schwarz, el Lema D.6 y la estimación (E.11).

Lema E.34 La siguiente estimación es válida

$$
\left|\rho_{\mathrm{F}} \int_{\Omega_{\mathrm{F}}} \mathbf{u} \cdot\left(\boldsymbol{\eta}_{h}^{e}-\hat{\boldsymbol{\eta}}\right)\right|+\left|\rho_{\mathrm{F}} \int_{\Omega_{\mathrm{F}}} \mathbf{f} \cdot\left(\boldsymbol{\eta}_{h}^{e}-\hat{\boldsymbol{\eta}}\right)\right| \leq C h\|(\mathbf{f}, \mathbf{g})\|_{0, \Omega}\left\|\boldsymbol{\eta}_{h}\right\|_{0, \Omega_{\mathrm{F} h}} .
$$

Prueba. Comenzaremos recordando que $\boldsymbol{\eta}_{h}=\operatorname{curl} p_{h}, p_{h} \in \mathcal{Z}_{h}\left(\Omega_{\mathrm{F} h}\right)$, y que $\hat{\boldsymbol{\eta}}=\operatorname{curl} \hat{p}$, siendo $\hat{p}$ la función asociada a $p_{h}$ (ver sección D). Teniendo en cuenta las definiciones de $\boldsymbol{\eta}_{h}^{e}$ y $\hat{\boldsymbol{\eta}}$, tendremos

$$
\int_{\Omega_{\mathrm{F}}} \mathbf{u} \cdot\left(\boldsymbol{\eta}_{h}^{e}-\hat{\boldsymbol{\eta}}\right)=\int_{\Omega_{\mathrm{F}} \cap \Omega_{\mathrm{F} h}} \mathbf{u} \cdot\left(\boldsymbol{\eta}_{h}-\hat{\boldsymbol{\eta}}\right)=\sum_{\substack{T \in \mathcal{B}_{\mathrm{Fh}} \\ T \supset T^{i d}}} \int_{T^{i d}} \mathbf{u} \cdot \operatorname{curl}\left(p_{h}-\hat{p}\right) .
$$


Entonces, usando el Lema D.11 y el Teorema 4.29, podremos concluir

$$
\left|\rho_{\mathrm{F}} \int_{\Omega_{\mathrm{F}}} \mathbf{u} \cdot\left(\boldsymbol{\eta}_{h}^{e}-\hat{\boldsymbol{\eta}}\right)\right| \leq C h\|\mathbf{u}\|_{0, \Omega_{\mathrm{F}}}\left\|p_{h}\right\|_{1, \Omega_{\mathrm{F} h}} \leq C h\|\mathbf{u}\|_{0, \Omega_{\mathrm{F}}}\left\|\operatorname{curl} p_{h}\right\|_{0, \Omega_{\mathrm{F} h}} \leq C h\|(\mathbf{f}, \mathbf{g})\|_{0, \Omega}\left\|\boldsymbol{\eta}_{h}\right\|_{0, \Omega_{\mathrm{F} h}} .
$$

El término restante puede acotarse empleando argumentos idénticos.

Lema E.35 La siguiente estimación es válida

$$
\left|\rho_{\mathrm{F}} \int_{\Omega_{\mathrm{F} h} \backslash \Omega_{\mathrm{F}}} \mathbf{f} \cdot \boldsymbol{\eta}_{h}\right| \leq C h\|(\mathbf{f}, \mathbf{g})\|_{h, \Omega}\left\|\boldsymbol{\eta}_{h}\right\|_{0, \Omega_{\mathrm{F} h}} .
$$

Prueba. Sea $T \in \mathcal{T}_{h}^{\mathrm{F}}$ un triángulo en el borde de $\Omega_{\mathrm{F}}$ tal que $T \supset T^{i d}$. Sea $\omega_{T}$ el dominio definido por $T \backslash T^{i d}$. Entonces, usando la definición de la extensión dada en la sección 4.2.1,

$$
\|\mathbf{f}\|_{0, \Omega_{\mathrm{F} h} \backslash \Omega_{\mathrm{F}}}=\left(\sum_{\substack{T \in \mathcal{B}_{\mathrm{F} h} \\ T \supset T^{i d}}}\|\nabla \xi\|_{0, \omega_{T}}^{2}\right)^{1 / 2}=\|\nabla \xi\|_{0, \Omega_{\mathrm{S}} \backslash \Omega_{\mathrm{S} h}}
$$

A partir del Lema D.6 y la estimación (4.67), tendremos

$$
\|\nabla \xi\|_{0, \Omega_{\mathrm{S}} \backslash \Omega_{\mathrm{Sh}}} \leq C h^{\alpha}\|\nabla \xi\|_{\alpha, \Omega_{\mathrm{S}}} \leq C h^{\alpha}\left(\|\operatorname{div} \mathbf{f}\|_{0, \Omega_{\mathrm{F}}}^{2}+\sum_{j=1}^{N}\|\mathbf{g} \cdot \mathbf{n}\|_{1 / 2, \tilde{e}_{j}}^{2}\right)^{1 / 2} \leq C h^{\alpha}\|(\mathbf{f}, \mathbf{g})\|_{h, \Omega} .
$$

Por otro lado, teniendo en cuenta que $\boldsymbol{\eta}_{h}=\operatorname{curl} p_{h}$, con $p_{h} \in \mathcal{Z}_{h}$, podemos usar el Lema D.5 para obtener

$$
\left\|\boldsymbol{\eta}_{h}\right\|_{0, \Omega_{\mathrm{F} h} \backslash \Omega_{\mathrm{F}}}^{2}=\sum_{\substack{T \in \mathcal{B}_{\mathrm{F} h} \\ T \supset T^{i d}}}\left\|\operatorname{curl} p_{h}\right\|_{0, \omega_{T}}^{2}=\sum_{\substack{T \in \mathcal{B}_{\mathrm{F} h} \\ T \supset T^{i d}}}\left|\operatorname{curl} p_{h}\right|^{2}\left|\omega_{T}\right| \leq C h \sum_{\substack{T \in \mathcal{B}_{\mathrm{F} h} \\ T \supset T^{i d}}}\left\|\operatorname{curl} p_{h}\right\|_{0, T}^{2} \leq C h\left\|\boldsymbol{\eta}_{h}\right\|_{0, \Omega_{\mathrm{F}}}^{2} .
$$

De esta manera, el resultado que buscamos será consecuencia directa de la desigualdad de Cauchy-Schwarz y las estimaciones previas. 


\section{Bibliografía}

[1] G. Acosta and G. Armentano, Eigenvalue problems in a non-Lipschitz domain, IMA J. Numer. Anal., 34 (2014) 83-95.

[2] M. Ainsworth, Robust a posteriori error estimation for nonconforming finite element approximation, SIAM J. Numer. Anal., 42 (2005) 2320-2341.

[3] M. Ainsworth and R. Rankin, Computable error bounds for finite element approximation on non-polygonal domains, to appear.

[4] A. Alonso and A. Dello Russo, Spectral approximation of variationally posed eigenvalue problems by nonconforming methods, J. Comput. Appl. Math., 223 (2009) 177-197.

[5] A. Alonso, A. Dello Russo, C. Padra and R. Rodríguez, A posteriori error estimates and a local refinement strategy for a finite element method to solve structural-acoustic vibration problems, Adv. Comput. Math., 15 (2002) 25-59.

[6] A. Alonso, A. Dello Russo, C. Padra and R. Rodríguez, Accurate pressure post-process of a finite element method for elastoacoustics, Numer. Math., 98 (2004) 389-425.

[7] A. Alonso, A. Dello Russo and V. Vampa, A posteriori error estimates in finite element acoustic analysis, J. Comput. Appl. Math., 117 (2000) 105-119.

[8] M. Amara and J-M. Thomas, Equilibrium finite elements for the linear elastic problem, Numer. Math., 33 (1979) 367-383 .

[9] A. B. Andreev and A. H. Hristov, On the Variational Aspects for Elliptic Problems with Parameter on the Boundary, Recent Advances in Numerical Methods and Applications II, Singapore, World Scientifi, 1998.

[10] A. B. Andreev and T. D. Todorov, Isoparametric finite element approximation of a Steklov eigenvalue problem, IMA. J. Numer. Anal., 24 (2004) 309-322.

[11] P.M. Anselone and T.W. Palmer, Spectral analysis of collectively compact, strongly convergent operator sequences, Pac. J. Math., 25 (1968) 423-431.

[12] P. Antonietti, A. Buffa and I. Perugia, Discontinuous Galerkin approximations of the Laplace eigenproblem, Comput. Methods Appl. Mech. Engrg., 195 (2006) 3483-3503.

[13] G. Armentano and C. Padra, A posteriori error estimates for the Steklov eigenvalue problem, Appl. Numer. Math., 58 (2008) 593-601.

[14] D.N. Arnold, An interior penalty finite element method with discontinuous elements, SIAM J. Numer. Anal., 19 (1982) 742-760.

[15] D.N. Arnold and G. Awanou, Rectangular mixed finite elements for elasticity, Math. Models Methods Appl. Sci., 9 (2005) 1417-1429.

[16] D.N. Arnold, G. Awanou and R. Winther, Finite elements for symmetry tensors in three dimensions, Math. Comp., 77 (2008) 1229-1251.

[17] D.N. Arnold and F. Brezzi, Mixed and nonconforming finite element methods implementation, postprocessing and error estimates, R.A.I.R.O., Model. Math. Anal. Numer., 19 (1985) 7-32. 
[18] D.N. Arnold, F. Brezzi and J. Douglas Jr., PEERS: a new mixed finite element for plane elasticity, Japan J. Appl. Math., 1 (1984) 347-367 (1984).

[19] D.N. Arnold, F. Brezzi, B. Cockburn and L.D. Marini, Unified analysis of discontinuous Galerkin methods for elliptic problems, SIAM J. Numer. Anal., 39 (2001) 1749-1779.

[20] D.N. Arnold, J. Douglas Jr., and C.P. Gupta, A family of higher order mixed finite element methods for plane elasticity, Numer. Math., 45 (1984) 1-22.

[21] D.N. Arnold and R. Falk, A new mixed formulation for elasticity, Numer. Math., 53 (1988) 13-30 (1988).

[22] D. Arnold, R. Falk and R. Winther, Multigrid in H(div) and H(curl), Numer. Math., 85 (2000) 197-217.

[23] D.N. Arnold and R. Winther, Mixed finite elements for elasticity, Numer. Math., 92 (2002) 401-419.

[24] D.N. Arnold and R. Winther, Nonconforming mixed finite elements for elasticity, Math. Models Methods Appl. Sci., 13 (2003) 295-307.

[25] G. Awanou, A rotated noncomforming rectangular mixed element for elasticity, Calcolo, 46 (2009) 49-60.

[26] I. Babǔska, Error bounds for finite element methods, Numer. Math., 16 (1971) 322-333.

[27] I. Babǔska and A. Miller, A feedback finite element methods with a posteriori error estimation. Part 1: The finite element method and some basic properties of the a posteriori error estimator, Comp. Methods Appl. Mech. Engng., 61 (1987) 1-40.

[28] I. Babǔska and J. Osborn, Finte Element-Galerkin Approximation of the Eigenvalues and Eigenfunctions of Selfadjoint Problems, Math. Comp., 52 (1989) 275-297.

[29] I. Babǔska and J. Osborn, Eigenvalue Problems, in Handbook of Numerical Analysis, Vol. II, P.G. Ciarlet and J.L. Lions, eds., North Holland, 1991.

[30] G.A. Baker, Finite element methods for elliptic equations using nonconforming elements, Math. Comp., 31 (1977) 45-59.

[31] C. Beattie, Galerkin eigenvector approximations, Math. Comp., 69 (2000) 1409-1434.

[32] S. Bergman and M. Schiffer, Kernel Functions and Elliptic Differential Equations in Mathematical Physics, Academic Press, New York, NY, USA, 1953.

[33] A. Bermúdez, R. Durán, M.A. Muschietti, R. Rodríguez, and J. Solomin, Finite element vibration analysis of fluid-solid systems without spurious modes, SIAM J. Numer. Anal., 32 (1995) 1280-1295.

[34] A. Bermúdez A, R. Durán R. Rodríguez, Finite element analysis of compressible and incompressible fluidsolid systems, Math. Comp., 67 (1998) 111-136.

[35] A. Bermúdez, R. Durán, R. Rodríguez and J. Solomin, Finite element analysis of a quadratic eigenvalue problem arising in dissipative acoustics, SIAM J. Numer. Anal., 38 (2000) 267-291.

[36] A. Bermúdez, P. Gamallo, M.R. Nogueiras and R. Rodríguez Approximation of a structural vibration problem by hexahedral finite elements, IMA J. Numer. Anal., 26 (2006) 391-421.

[37] A. Bermúdez, L. Hervella-Nieto and R. Rodríguez, Finite element computation of three dimensional elastoacoustic vibrations, J. Sound Vibr., 219 (1999) 277-304.

[38] A. Bermúdez and R. Rodríguez, Finite element computation of the vibration modes of a fluid-solid system, Comp. Methods Appl. Mech. Eng., 119 (1994) 355-370.

[39] A. Bermúdez, R. Rodríguez and D. Santamarina, A finite element solution of an added mass formulation for coupled fuid-solid vibrations, Numer. Math., 87 (2000) 201-227.

[40] C. Bernardi, Optimal finite-element interpolation on curved domains, SIAM J. Numer. Anal., 26 (1989) 1212-1240.

[41] D. Boffi, Fortin operator and a discrete compactness for edge elements, Numer. Math., 87 (2000) 229-246. 
[42] D. Boffi, Finite element approximation of eigenvalue problems, Acta Numerica, 19 (2010) 1-120.

[43] D. Boffi, F. Brezzi and L. Gastaldi, On the convergence of eigenvalues for mixed formulations, Ann. Scuola Norm. Sup. Pisa, 25 (1997) 131-154.

[44] A. Bossavit, Solving Maxwell equations in a closed cavity and the question of spurious modes, IEEE Trans. Magnetics, 26 (1990) 702-705.

[45] D. Braess, O. Klaas, P. Niekamp, E. Stein and F. Wobschal, Error indicators for mixed finite elements in 2-dimensional linear elasticity, Comput. Methods Appl. Mech. Engrg., 127 (1995) 345-356.

[46] D. Braess and R. Verfürth, A posteriori error estimators for the Raviart-Thomas element, SIAM J. Numer. Anal., 33 (1996) 2431-2444.

[47] J.H. Bramble and T. King, A robust finite element method for nonhomogeneous Dirichlet problems in domains with curved boundaries, Math. Comp., 63 (1994) 1-17.

[48] J.H. Bramble and J. E. Osborn, Approximation of Steklov eigenvalues of non-selfadjoint second order elliptic operators, in Mathematical Foundations of the Finite Element Method with Applications to PDE, A. K. Aziz, ed., Academic Press, New York, 1972. 5

[49] J. Bramble and J. Osborn, Rate of convergence estimates for non-selfadjoint eigenvalue approximations, Math. Comp., 27 (1973) 525-549.

[50] J.H. Bramble and J. Xu, A local post-processing technique for improving the accuracy in mixed finiteelement approximations, SIAM J. Numer. Anal., 26 (1989), 1267-1275.

[51] S.C. Brenner and C. Carstensen, Finite Elements Methods, in Encyclopedia of Computational Mechanics, E. Stein, R. de Borst, T.J.R. Hughes, eds., John Wiley \& Sons, 2004.

[52] S.C. Brenner and R. Scott, The Mathematical Theory of Finite Elements Methods, Springer-Verlag, 1994.

[53] F. Brezzi, On the existence, uniqueness and approximation of saddlepoint problems arising from Lagrangian multipliers, R.A.I.R.O., 8 (1974) 129-151.

[54] F. Brezzi, J. Douglas, R. Durán and M. Fortin, Mixed Finite Elements for Second Order Elliptic Problems in Three Variables, Numer. Math., 51 (1987) 237-250.

[55] F. Brezzi, J, Douglas and L.D. Marini, Two Families of Mixed Finite Elements for Second Order Elliptic Equations, Numer. Math., 47 (1985) 19-34.

[56] F. Brezzi and M. Fortin, Mixed and Hybrid Finite Element Methods, Springer-Verlag, 1991.

[57] F. Brezzi, G. Manzini, D. Marini, P. Pietra and A. Russo, Discontinuous Galerkin approximations for elliptic problems, Numer. Methods for Partial Differential Equations, 16 (2000) 365-378.

[58] A. Buffa, Remarks on the discretization of some noncoercive operator with applications to Maxwell equations with jumping coefficients, SIAM J. Numer. Anal., 43 (2005) 1-18.

[59] A. Buffa and P. Ciarlet Jr.,On traces for functional spaces related to Maxwell's equations. Part I: An integration by parts formula in Lipschitz-polyhedra, Math. Meth. Appl. Sci., 24 (2001) 9-30.

[60] A. Buffa and I. Perugia, Discontinuous Galerkin approximations of the Maxwell eigenproblem, SIAM J. Numer. Anal., 44 (2006) 2198-2226.

[61] Z. Cai and X. Ye, A mixed Nonconforming Finite Element for Linear Elasticity, Numer. Methods Partial Differential Equations, 21 (2005) 1043-1051.

[62] J. Canavati and A. Minsoni, A discontinuous Steklov problem with an application to water waves, J. Math. Anal. Appl., 69 (1979) 540-558.

[63] C. Canuto, Eigenvalue approximations by mixed methods, RAIRO Anal. Numer., 12 (1978) 27-50.

[64] C. Carstensen, A posteriori error estimate for the mixed finite element method, Math. Comp., 66 (1997) 465-476. 
[65] C. Carstensen, S. Bartels and S. Jansche, A posteriori error estimates for nonconforming finite element methods, Numer. Math., 92 (2002) 233-256.

[66] C. Carstensen and G. Dotzmann, A posteriori error estimates for mixed FEM in elasticity, Numer. Math., 81 (1998) 187-209.

[67] C. Carstensen, G. Dotzmann, S.A. Funken and D.S. Helm, Looking-free adaptive mixed finite element methods in linear elasticity, Comput. Methods Appl. Mech. Engrg., 190 (2000) 1701-1718.

[68] C. Carstensen, M. Eigel and J. Gedicke, Computational competition of symmetric mixed FEM in linear elasticity, Comput. Methods Appl. Mech. Engrg., 200 (2011) 2903-2915.

[69] C. Carstensen and J. Gedicke, An oscillation-free adaptive FEM for symmetric eigenvalue problems, Numer. Math., 118 (2011) 401-427.

[70] C. Carstensen, D. Günther, J. Reininghaus and J. Thiele, The Arnold-Winther mixed FEM in linear elasticity. PartI: Implementation and numerical verification, Comput. Methods Appl. Mech. Engrg., 197 (2008) 3014-3023.

[71] C. Carstensen and R.H.W. Hoppe, Convergence analysis of an adaptive nonconforming finite element method, Numer. Math., 103 (2006) 251-266.

[72] C. Carstensen and J. Hu, A unifyng theory of a posteriori error control for nonconforming finite element methods, Numer. Math., 107 (2007) 473-502.

[73] C. Carstensen, J. Hu and A. Orlando, Framework for the a posteriori error analysis of nonconforming finite elements, SIAM J. Numer. Anal., 45 (2007) 68-82.

[74] C. Carstensen and R. Verfürth, Edge residuals dominate a posteriori error estimates for low order finite element methods, SIAM J. Numer. Anal., 36 (1999) 1571-1587.

[75] P.G. Ciarlet, Basic error estimates for elliptic problems, in Handbook of Numerical Analysis, Vol. II, P.G. Ciarlet and J.L. Lions, eds., North Holland, 1991.

[76] P.G. Ciarlet and P.A. Raviart, Interpolation theory over curved elements with applications to finite element methods, Comput. Methods Appl. Mech. Engrg., 1 (1972) 217-249.

[77] P. Clement, Approximation by finite element functions using local regularization, R.A.I.R.O., Anal. Numer., 9 (1975) 77-84.

[78] COMSOL Group, Multiphysics Cyclopedia, www.comsol.com/multiphysics/mesh-refinement.

[79] C. Conca, J. Planchard and M. Vanninathan, Fluids and periodic structures, RAM: Research in Applied Mathematics, 38, John Wiley \& Sons, Chichester, 1995.

[80] J.B. Conway, A Course in Functional Analysis, Springer, New York, 1990.

[81] M. Crouzeix and P. A. Raviart, Conforming and nonconforming finite element methods for solving the stationary stokes equations, RAIRO. Anal. Numer., 3 (1973) 33-75.

[82] F. Chatelin, Convergence of approximation methods to compute eigenelements of linear operations, SIAM J. Numer. Anal., 10 (1973) 939-948.

[83] F. Chatelin, The spectral approximation of linear operators with applications to the computation of eigenelements of differential and integral operators, SIAM Review, 23 (1981) 495-522.

[84] F. Chatelin, Spectral Approximation of Linear Operators, Computer Science and Applied Mathematics, Academic Press, New York, 1983.

[85] F. Chatelin and J. Lemordant, Error bounds in the approximation of eigenvalues of differential and integral operators, J. Math. Anal. Appl., 62 (1978) 257-271.

[86] E. Dari, R. Durán, C. Padra and V. Vampa, A posteriori error estimators for noconforming finite element methods, R.A.I.R.O., Model. Math. Anal. Numer., 30 (1996) 385-400. 
[87] E. Dari, R.G. Durán, and C. Padra, A posteriori error estimates for non conforming approximation of eigenvalue problems, Appl. Numer. Math., 62 (2012) 580-591.

[88] M. Dauge, Elliptic boundary value problems on corner domains: smoothness and asymptotics of solutions, Lecture Notes in Mathematics, 1341, Springer-Verlag, Berlin, 1988.

[89] M. Dauge, Regularity and singularities in polyedral domains. The case of Laplace and Maxwell equations, Slides of a mini-course given at the Workshop Mathematical Topics in Electromagnetic Fields and Wave Propagation in the framework of the Research Training Group Analysis, Simulation and Design of Nanotechnological Processes (University of Karlsruhe, Alemania, 2008).

[90] A. Dello Russo and A. Alonso, Hybrid finite element analysis of fluid-structure systems with coupling on curved interfaces, IMA Journal of Numerical Analysis, 31 (2011) 1636-1682.

[91] A. Dello Russo and A. Alonso, A posteriori error estimates for nonconforming approximations of Steklov eigenvalue problems, Comput. Math. Appl., 62 (2011) 4100-4117.

[92] A. Dello Russo, Eigenvalue approximation by mixed non-conforming finite element methods. The determination of the vibrational modes of a linear elastic solid, Calcolo, 51 (2014) 563-597.

[93] A. Dello Russo, A posteriori error estimates of the non-conforming Arnold-Winther method for linear elasticity eigenvalue problems, submmitted.

[94] J. Descloux, N. Nassif and J. Rappaz, On spectral approximation. Part 1: The problem of convergence, R.A.I.R.O. Anal. Numer., 12 (1978) 97-112.

[95] J. Descloux, N. Nassif and J. Rappaz, On spectral approximation. Part 2: Error estimates for the Galerkin methods, R.A.I.R.O. Anal. Numer., 12 (1978) 113-119.

[96] J. Douglas (Jr) and T. Dupont, Interior penalty procedures for elliptic and parabolic Galerkin methods, Lectures Notes in Physics, 58 Spronger-Verlag, Berlin, 1976.

[97] R. Durán, Mixed Finite Element Methods in Mixed finite elements, compatibility conditions, and applications. D. Boffi and L. Gastaldi, eds., Springer-Verlag, Berlin, 2008.

[98] R. Durán, L. Gastaldi and C. Padra, A posteriori error estimators for mixed approximations of eigenvalue problems, Math. Models Methods Appl. Sci., 9 (1999) 1165-1178.

[99] R. Durán, L. Hervella-Nieto, E. Liberman, R. Rodríguez and J. Solomin, Finite element analysis of the vibration problem of a plate coupled with a fluid, Numer. Math., 86 (2000) 591-616.

[100] R. Durán and C. Padra, An error estimator for nonconforming approximations of a non linear problem, in Finite Element Methods, Fifty years of the Courant Element, M. Krizek, P. Neittaanmaki y R. Stenberg, eds., Marcel Dekker, 1994.

[101] R. Durán, C. Padra and R. Rodríguez, A posteriori error estimates for the finite element approximations of eigenvalue problems, Math. Models Methods Appl. Sci., 13 (2003) 1219-1229.

[102] G.C. Everstine A symmetric potential formulation fot fluid-structure interaction, J. Sound Vibr., 79 (1981) 157-160.

[103] R.S. Falk and J.E. Osborn, Error estimates for mixed methods, RAIRO Anal. Numer., 14 (1980) 249-277.

[104] M. Feistauer and A. Ženíšek, Finite element solution of nonlinear elliptic problems, Numer. Math., 50 (1987) 451-475.

[105] G. Fix, Eigenvalue approximation by the finite element method, Adv. in Math., 10 (1973) 300-316.

[106] E. M. Garau, P. Morin, and C. Zuppa, Convergence of adaptive finite element methods for eigenvalue problems,Math. Models Methods Appl. Sci., 19 (2009) 721-747.

[107] F. Gardini, A posteriori error estimates for an eigenvalue problem arising from fuid-structure interaction, Istit. Lombardo Accad. Sci. Lett. Rend. A, 138 (2004) 17-34.

[108] S. Giani and I. G. Graham, A convergent adaptive method for elliptic eigenvalue problems, SIAM J. Numer. Anal., 47 (2009) 1067-1091. 
[109] V. Girault and P-A. Raviart, Finite Element Methods for the Navier-Stokes Equations, Theory and Algorithms, Springer-Verlag, Berlin, 1986.

[110] L. Grubišic and J.S. Ovall, On estimators for eigenvalue/eigenvector approximations, Math. Comp., 78 (2009) 739-770.

[111] P. Grisvard, Elliptic Problems for Non-smooth Domains, Pitman, 1985.

[112] P. Grisvard, Problémes aux limites dans les polygons. Mode d'emploi, EDF Bulletin de la Direction des Etudies et Recherches, Serie C, 1 (1986) 97-112.

[113] P. Grisvard, Singularités en elasticité, Arch. Rat. Mech. Anal., 107 (1989) 157-180.

[114] J. Gopalakrishnan and J. Guzmán, Symmetric nonconformning mixed finite elements for linear elasticity, SIAM J. Numer. Anal., 49 (2011) 1504-1520.

[115] M. Hamdi, Y. Ousset and G. Verchery, A displacement method for the analysis of vibrations of coupled ?uid-structure systems, Internat. J. Numer. Methods Eng., 13 (1978) 139-150.

[116] E. Hernández and R. Rodríguez, Finite element approximation of spectral problems with Neumann boundary conditions on curved domains, Math. Comp., 72 (2003) 1099-1115.

[117] E. Hernández and R. Rodríguez, Finite element approximation of spectral acoustic problems on curved domains, Numer. Math., 97 (2004) 131-158.

[118] R.H.W. Hoppe and B. Wohlmuth, Element-oriented and edge-oriented local error estimators for nonconforming finite element methods, RAIRO Model. Math. Anal. Numer., 30 (1996) 237-263.

[119] C. Johnson and B. Mercier, Some equilibrium finite element methods for two-dimensional elasticity problems, Numer. Math., 30 (1978) 103-116.

[120] P. Joly, C. Poirier, J.E. Roberts and P. Trouve. A new nonconforming finite element method for the computation of electromagnetic guided waves. I. Mathematical analysis, SIAM J. Numer. Anal., 33 (1996) 1494-1525.

[121] O.A. Karakashian and F. Pascal, A posteriori error estimates for a discontinuous Galerkin approximation of second-order elliptic problems, SIAM J. Numer. Anal., 41 (2003) 2374-2399.

[122] O.A. Karakashian and F. Pascal, Convergence of adaptive discontinuous Galerkin approximations of second-order elliptic problems, SIAM J. Numer. Anal., 45 (2007) 641-665.

[123] T. Kato, Perturbation theory for nullity, deficiency and other quantities of linear operators, J. Analyse Math., 6 (1958) 261-322.

[124] T. Kato, Perturbation Theory for Linear Operators, Lectures Notes in Mathematics 132, Springer-Verlag, Berlin, 1966.

[125] L. Kiefling and G.C. Feng Fluid-structure finite element vibration analysis, AIAA J., 14 (1976) 199-203.

[126] F. Kikuchi, On a discrete compctness property for the Nédélec finite elements, J. Fac. Sci., Univer. Tokio Section IA Math., 36 (1989) 479-490.

[127] K.Y. Kim, Analysis of some Low-order Nonconforming Mixed Finite Elements for Linear Elasticity Problem, Numer. Methods Partial Differential Equations, 22 (2006) 638-660.

[128] K.Y. Kim, Guaranteed a posteriori error estimator for mixed finite element methods of linear elasticity with weak stress symmetry, SIAM J. Numer. Anal., 49 (2011) 2364-2385.

[129] A. Knyazev and J. Osborn, New a priori FEM error estimates for eigenvalues, SIAM J. Numer. Anal., 43 (2006) 2647-2667.

[130] W.G. Kolata, Approximation in variationally posed eigenvalue problems, Numer. Math., 29 (1978) 159-171.

[131] C.S. Kubrusly, Spectral Theory of Operators on Hilbert Spaces, Springer Science \& Business Media, 2012. 
[132] M.G. Larson, A posteriori and a priori error analysis for finite element approximations of self-adjoint elliptic eigenvalue problems, SIAM J. Numer. Anal., 38 (2000) 608-625.

[133] M.P. Lebaud, Error estimate in an isoparametric finite element eigenvalue problem, Math. Comp., 63 (1994) 17-40.

[134] Q. Li and Y. D. Yang, A two-grid discretization scheme for the Steklov eigenvalue problem, J. Appl. Math. Comput., 36 (2011) 129-139.

[135] M. Lonsing and R. Verfürth, A posteriori error estimator s for mixed finite element methods in linear elasticity, Numer. Math., 97 (2004) 757-778.

[136] C. Lovadina, M. Lyly and R. Stenberg, A posteriori estimates for the Stokes eigenvalue problem, Numer. Methods Partial Differential Equations, 25 (2009) 244-257.

[137] C. Lovadina and R. Stenberg, Energy norm a posteriori error estimates for mixed finite element methods, Math. Comp., 75 (2006) 1659-1674.

[138] B. Mercier, J. Osborn, J. Rappaz and P.A. Raviart, Eigenvalue approximation by mixed and hybrid methods, Math. Comp., 36 (1981) 427-453.

[139] P. Monk and L. Demkowicz, Discrete compactness and the approximation of Maxwell's equations in $\mathbb{R}^{3}$, Math. Comp., 70 (2000) 507-523.

[140] H.J-P. Morand and R. Ohayon Fluid Structure Interaction, J. Wiley \& Sons, Chichester, 1995.

[141] J. Nečas, Sur une méthode pour résoudre les équations aux dérivées partielles du type elliptique, voisine de la varationelle, Annali della Scuola Normale Superiore di Pisa, Classe di Scienze $3^{\circ}$ serie, 16 (1962) 305-326.

[142] J.T. Oden and J.N. Reddy, Variational methods in theoretical mechanics, Springer-Verlag, 1976.

[143] R. Ohayon and C. Soize, Structural Acoustics and Vibration, Academic Press, 1998.

[144] J.E. Osborn, Spectral approximation for compact operators, Math. Comp., 29 (1975) 712-725.

[145] J.E. Osborn, Eigenvalue approximations by mixed methods, in Advances in Computer Methods for Partial Diferential Equations III, R. Vichnevetsky and R. Stepleman, eds., New Brunswick, 1979.

[146] J. Rappaz, Approximation of the spectrum of a non-compact operator given by the magnetohydrodynamic stability of a plasma, Numer. Math., 28 (1977) 15-24.

[147] P.A. Raviart and J.M. Thomas, A mixed finite element method for second order elliptic problems, in Mathematical Aspects of the Finite Element Method, I. Galligani and E. Magenes, eds., Lect. Notes in Math., 606, Springer-Verlag, 1977.

[148] W.H. Redd and T.H. Hill, Triangular mesh methods for the neutron transport equations, Tech. Report LA-UR-73-479, Los Alamos Scientific Laboratory, 1973.

[149] W. C . Rheinboldt, Feedback systems and adaptivity for numerical computations, in Adaptive Computational Methods for Partial Differential Equations, I. Babǔska, Chandra and Flaherty, eds., SIAM, Philadelphia, 1983.(1977)

[150] R. Rodríguez and J. Solomin, The order of convergence of eigenfrequencies in finite element approximations of fluid-structure interaction problems, Math. Comp., 65 (1996) 1463-1475.

[151] R. Scott, Interpolated boundary conditions in the finite element method, SIAM J. Numer. Anal., 12 (1975) 440-427.

[152] L.R. Scott and S. Zhang, Finite element interpolation of non-smooth functions satisfying boundary conditions, Math. Comp., 54 (1990), 483-493.

[153] P. Schieweck, A general transfer operator for arbitrary finite element spaces, Preprint 25/00 Otto-vonGuericke Universität Magdeburg, Fakultät fur Mathematik (2000). 
[154] P. Schieweck, A posteriori error estimates with post-processing for nonconforming finite elements, M2AN Math. Model. Numer. Anal., 32 (2002) 489-503.

[155] R. Stenberg, On the construction of optimal mixed finite element methods for the linear elasticity problem, Numer. Math., 48 (1986) 447-462.

[156] R. Stenberg, A family of mixed finite elements for the elasticity problem, Numer. Math., 53 (1988) 513-538.

[157] R. Stenberg, Two low-order mixed methods for the elasticity problem in The mathematics of finite elements and applications VI, Academic Press, London, 1988.

[158] R. Stenberg, Some new families of finite elements for the Stokes equations, Numer.Math., 56 (1990) 827-838.

[159] R. Stenberg, Postprocessing schemes for some mixed finite elements, RAIRO Modél. Math. Anal. Numér., 25 (1991) 151-167.

[160] E.M. Stein, Singular Integrals and Differentiability Properties of Functions, Princenton University Press, Princenton 1970.

[161] G. Stoyan, Towards discrete Velte decompositions and narrow bounds for inf-sup constants, Comput. Math. Appl., 38 (1999) 243-261.

[162] E. Süli, Lecture Notes on Finite Element Methods for Partial Differential Equations, Mathematical Institute University of Oxford, 2012.

[163] W. Tang, Z. Guan and H. Han, Boundary element approximation of Steklov eigenvalue problem for Helmhooltz equation, J. Comput. Math., 16 (1998) 165-178.

[164] G.M. Vainikko, The compact approximation principle in the theory of approximation methods, U.R.S.S. Computational Math. and Math. Phys., 9 (1969) 1-32.

[165] M. Vanmale and A. Ženíšek, External finite element approximations of eigenvalue problems, Math. Model. Numer. Anal. (M²AN) 27 (1993) 565-589.

[166] M. Vanmale and A. Ženíšek, External finite element approximations of eigenfunctions in the case of multiple eigenvalues, J. Comput. Appl. Math., 50 (1994) 51-66.

[167] M. Vanmale and A. Ženíšek, The combined effect of numerical integration and approximation of the boundary in the finite element methods for the eigenvalue problems, Numer. Math., 71 (1995) 253-273.

[168] M. Vohralík, On the discrete Poincaré-Friedrichs inequalities for nonconforming approximations of the Sobolev space $H^{1}$, Numer. Func. Anal. Opt., 26 (2005), 925-952.

[169] R. Verfürth, A posteriori error estimates for nonlinear problems, Math. Comp., 62 (1989) 445-475.

[170] R. Verfürth, A review of a posteriori error estimation and adaptive mesh-refinement techniques, Wiley \& Teubner, 1996.

[171] M. Vohralík, Unified primal formulation based a priori and a posteriori error analysis of mixed finite element methods, Math. Comput., 79 (2010) 2001-2032.

[172] X. Wang and K.J. Bathe Displacement/pressure based mixed finite element formulations for acoustic fluidstructure interaction problems, Internat. J. Numer. Methods Eng., 40 (1997) 2001-2017.

[173] V.B. Watwood Jr. and B.J. Hartz, An equilibrium stress field model for finite element solution of twodimensional elastostatic problems, Internat. J. Solids and Structures, 4 (1988) 857-873.

[174] M.F. Wheeler, An elliptic collocation finite element method with interior penalties, SIAM J. Numer. Anal., 15 (1978) 152-161.

[175] J.H. Wilkinson, The algebraic eigenvalue problem, Oxford University Press, London, 1965.

[176] Y. Yang, Q. Li and S. Li, Nonconforming finite element approximation of the Steklov eigenvalue problem, Appl. Numer. Math., 59 (2009) 2388-2401. 
[177] S.Y. Yi, Nonconforming mixed finite element methods for linear elasticity using rectangulars elements in two and three dimensions, Calcolo, 42 (2005) 115-133.

[178] K. Yosida, Functional Analysis, Springer-Verlag, New York, 1974.

[179] M. Zlámal, Curved elements in the finite element method. I, SIAM J. Numer. Anal., 10 (1973) 229-240.

[180] Z. Ding, A proof of the Trace Theorem of Sobolev spaces on Lipschitz domains, Proc. Amer. Math. Soc., 124 (1996) 591-600. 